\title{
THE PHYSIOLOGY AND PHARMACOLOGY OF THE PITUITARY BODY
}

\author{
BY \\ H. B. VAN DYKE
}




THE UNIVERSITY OF CHICAGO MONOGRAPHS IN MEDICINE

Editorial Committee

FRANKLIN C. MCLEAN, Chairman

ANTON J. CARLSON

H. GIDEON WELI.S 

THE PHYSIOLOGY AND PHARMACOLOGY OF THE PITUITARY BODY 
THE UNIVERSITY OF CHICAGO PRESS, CHICAGO THE BAKER \& TAYLOR COMPANY, NEW YORK; THE CAMBRIDGE UNIVERSITY PRESS, LONDON; THE MARUZEN-KABUSHIKI-KAISHA, TOKYO, OSAKA, KYOTO, FUKUOKA, SENDAI; THE COMMERCIAL PRESS, LIMITED, SHANGHAI 


\title{
THE PHYSIOLOGY AND PHARMACOLOGY OF THE PITUITARY BODY
}

\author{
Volume II \\ By H. B. VAN DYKE \\ Head of the Division of Pharmacology, Squibb Institute for \\ Medical Research, New Brunsicick, New Zersey \\ Honorary Professor of Physiology, Rugers Unitersity \\ Formerly Professor of Pharmacology in the University of Chicago \\ and in Peiping Union Medical College
}

THE UNIVERSITY OF CHICAGO PRESS CHICAGO - ILLINOIS 
COPYRIGHT 1939 BY THE UNIVERSITY OF CHICAGO ALL RIGHTS RESERVED. PUBLISHED MAY 1939 


\section{FOREWORD}

$\mathrm{D}$

R. VAN DYKE has again rendered a valuable service to biology and medicine in presenting in this second volume his critical digest of the experimental and clinical literature on the pituitary body that has appeared since 1935. During these four years, while public attention has been focused mainly on violence and war, on economic maladjustments and social strife in many lands, and when myopic men have clamored for a moratorium on science, biological and medical research in some countries has still gone on at a rate and of a quality which now calls for a second review volume on the pituitary body alone. I think this is clearly on the credit side of our simian ledger, even though the pace of publication of excellent research and the making of good scientific books bid fair to exceed our capacity for memory and mental digestion.

The pituitary body is still the "master" among the endocrine glands. The four additional years of research have detracted very little from its prestige, while it has extended its significance in several directions. A few pituitary hormones have become more hypothetical than they were four years ago, a few appear to have become more firmly established, but, with the possible exception of the lactogenic hormone, their chemical identity still eludes us. In fact, all the fundamental questions anent the pituitary body, in health and in disease, are still beyond the horizon. The role of this gland in the normal physiology of aging has scarcely even been formulated as a problem. 'The author's present winnowing will help to direct the next advance on many fronts.

February 1939

A. J. Carlson 



\section{PREFACE}

7 HE stream of reports on the functions and interrelationships of the pituitary body now flows at the rate of approximately 750 articles yearly. Since 19.34 research has been especially active; while new and useful contributions are much less numerous than the volume of data offered would lead one to expect, nevertheless, notable advances have been made in clarifying certain aspects of the complex functions of this organ. In this respect, several fields deserve particular mention-i.e., the physiological significance of the pars neuralis, the importance of the anterior pituitary in carbohydrate metabolism, the physiology of the breasts in relation to the anterior pituitary, and the correlation of anatomical changes in the gland with alterations of function and morphology elsewhere in the body. The great interest shown in the gonadotropic hormones appears not to have led to a commensurate return. Investigation of the thyrotropic hormone probably has been least fruitful recently.

In the present volume I have undertaken to meet the need of sifting and classifying the new data and concepts and of modifying the old by endeavoring critically to review recent work on the physiology and pharmacology of the pituitary body. A brief summary of my opinion of the present state of our knowledge has been added to each chapter. The first volume included part of the reports of 1935; the present volume is believed to include references to all important experimental work published during the remainder of 1935 and during the years 1936 and 1937 . In addition, some reports of I 938 are reviewed. The Bibliography (I,4 8 titles) represents $7^{8}$ per cent of the articles which were considered.

I wish here to record my thanks to a number of colleagues who have given me the benefit of expert and critical advice. 


\section{PREFACE}

Dr. R. O. Greep kindly assembled for me the information which appears in the Appendix. I am grateful to the authors and publishers-American, British, French, and Germanwho gave me permission to reproduce illustrations and tables. These all receive acknowledgment where the material appears.

New Brunswick, New Jersey

H. B. VAN DYKE

February 1939 


\section{TABLE OF CONTENTS}

List of Illustrations . . . . . . . . . . . . . . xiii

CHAPTER

I. The Anatomy of the Pituitary Body . . . . . . I

II. The Regulation of Growth by the Pituitary Body . 32

III. The Gonadotropic Hormones of the Pituitary Body · 47

IV. The Gonadotropic Hormones Associated with Pregnancy or Certain Neoplasms . . . . . . . . . . . 124

V. The Pars Glandularis of the Pituitary Body in Relation to the Development of the Breasts and the SecreTION OF MilK . . . . . . . . . . . . . . I5I

Vi. The Thyrotropic Hormone. . . . . . . . . . . 174

VII. The Interrelationship between the Pars Glandularis and the Adrenal Glands; the Influence of the Pars Glandularis on the Metabolism of Carbohydrates, Lipoids, Proteins, and Minerals (with Remarks on the Pituitary-Parathyroid Interrelationship) . . . . . 198

Vili. The Pituitary Body in Relation to the Regulation of the Distribution of Pigment in Chromatophores . . . 244

IX. New Observations on the Chemistry and Pharmacology of Extracts of the Pars Neuralis . . . . . . . 258

X. The Pars Neuralis as a Gland of Internal Secretion . 274

Appendix. The Structural Formulas and Principal actions of Hormones of Natural Origin . . . . . . . . 293

Bibliography . . . . . . . . . . . . . 298

INDEX . . . . . . . . . . . . 385

\section{5}

[ xi ]

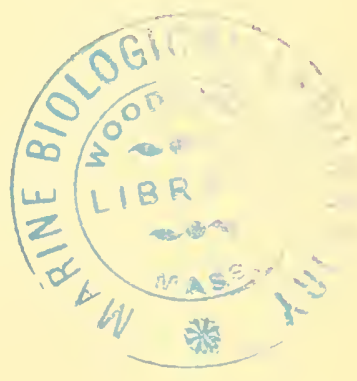





\section{LIST OF ILLUSTRATIONS}

FIGURE

I. The Blood Vessels of the Human Pituitary Body

2. The Relation of the Meninges to the Pituitary Body of THE DOg

3. The Innervation of the Pituitary Body according to Roussy and Mosinger

4. Diagram of the Hypothalamico-hypophysial Fiber System OF THE MONKEY .

5. Colloid-Formation in Neurons of the Supraoptic and Paraventricular Nuclei

6. Diagrams Illustrating Views of Different Authors on the Relationships of Cells of the Pars Glandularis.

7. Diagram of the Relationship of the Cells of the Human Pars Glandularis . . . . . . . . . facing

8. 'The Fffect of Prolonged Treatment by Oestrone on the Gross and Microscopic Appearance of the Pituitary of THE Mouse

9. The Action of Pituitary Gonadotropic Hormonfo on the Urogenital Tract of the Female Chick Embrio

io. The Action of Pituitary Gonadotropic Hormone on the Size of the Testis and the Color of the Bill of the EngLISH SPARROW

II. The Action of Pituitary Gonadotropic Hormone on the Ovary and Oviduct of the English Sparrow.

12. Changes in Ovarian Weight and in the Number, Size, and Appearance of the Ovarian Follicles Following HypophySectomy in the. lmmature. Rat

I3. Differences in the Response of the Ovary of the Imalature Rat to the Pituitary of Horses Differing in Sex and $A G E$

I4. The Relationship of the Dose of Follicle-stimulating and Hormone and the Response of the Ovaries and Uterus of

I5. the Immature Mouse. . . . . . . . . . . . . . . 108,109

16. Diagram Illustrating the Failure of the Hypophysectomized Rat To Form Antihormone in Response to the. Excessive Secretion of Gonadotropic Hormone by a Spayed Partner in Parabiotic Union 


\section{LIST OF ILLUSTRATIONS}

FIGURE

17. Potentiation of the Action of Gonadotropic Extract as a Result of the Addition of "Mierthiolate" to the Solution BEFORE INJECTION

i8. The Excretion of Gonadotropic Hormone in the Urine DURing Pregnancy.

19. The Rate of Disappearance of Gonadotropic Hormone of Pregnant-Mare Serum Following Intravenous Injection INTO THE RABBIT

20. The Effects of Oestrogen, Corpus Luteum Hormone, and Lactogenic Hormone on the Breasts of the Castrated RABBIT

21. The Relationship between the Dose of Lactogenic Hormone and the Growth of the Crop-Gland of the Pigeon .

22. The Effect of Hypophysectomy and of the Injection of Anterior Pituitary Extract on the 'Thy roid of the Newt .

23. The Effect of Hypophysectomy on the Concentration of Sugar in the Blood of the Fasting Monkey . . . . 206

24. Diagrams Illustrating the Production of Diabetes Meland litus by the Injection of Anterior Pituitary Extract 25. Into the Dog. The Effect of Insulin Also Is Shown . 222-23 26. The Effect of Hypophysectomy on the Distribution of Pigment in the Melanophores of the Angel Shark . . . 246

27. A Position of Bilateral Lesions Which Produce Diabetes INSIPIDUS IN THE CAT . . . . . . . . . . . 28 I

28. Hypertension Caused by Renal Ischemia in Relation to the Pituitary Bod 


\section{CHAP'TER I}

\section{'THE ANATOMY OF THE PITUITARY BODY}

I

$\mathrm{N}$ RECENT years the majority of the reports concerned with the anatomy of the pituitary body have dealt with its physiological aspects. The pituitary's largest and most important division - the pars glandularis - has received the greatest attention. Unfortunately the quality of the work published varies greatly, so that many of the findings scarcely deserve mention and serve only further to confuse any presentation which attempts completeness. To a varying extent, of course, the same remark is true of other pituitary investigations which are non-morphological.

The recent additions to our knowledge of the embryology of the pituitary body are few. The morphological and functional development of the pars buccalis in larvae of anuran amphibia (Rana pipiens, $R$. sylvatica) can take place without contact with nervous tissue contrary to the views of some embryologists (Atwell, 1937). This statement may not be true so far as the pars tuberalis is concerned. On the other hand, Atwell's experiments with larvae of a salamander (Amblystoma punctatum) suggested that similar development of the pars buccalis in this animal requires the presence of nervous tissue near by. Schliefer (I 935) studied the development of the pituitary of the toad (Bufo vulgaris), especially as it is related to metanorphosis. He found that development is not complete until toward the end of metamorphosis. Also he stated that the administration of pars neuralis extract ("hypophysin-feeding") is associated with some retardation of metamorphosis, an increased rate of growth, and alterations in the cells and pigment of the pars intermedia; it appears fruitless to attempt to interpret these observations. Tilney ( 1936 ) has recently made a detailed study of the de- 
velopment of the human pituitary which, he emphasizes, follows a typical mammalian pattern such as that of the cat. According to Tilney, the pars glandularis (which he terms the pars distalis) develops as a medullary core almost entirely surrounded by a cortical envelope; he believed that the functional anatomy of these developmental divisions should be carefully investigated.

There have appeared recently several contributions to the comparative anatomy of the pituitary body, particularly in fishes and amphibia. Among the fishes studied were several species of skates (Raia maculata, $R$. clavata, and $R$. brachyura [Howes, 1936]), various selachians (Ranzi, 1937), and a number of species of bony fishes (particularly Silurus glanis [Lange, 1936]), including the eel, Anguilla vulgaris (Hagen, I 936). Some of the studies embraced physiological correlations. For example, Lange described the yearly cyclic changes in the pituitary of $S$. glanis. Ranzi found that by the histological appearance of the pars glandularis and pars intermedia it was possible to decide whether the pituitary had been removed from an immature, a normal adult, or a pregnant selachian. Hagen pointed out that the pars glandularis of the eel is largely made up of oxyphils. The volume and vascularity of the pituitary markedly increases during metamorphosis. Sato (1935) studied several anuran amphibia. Among studies in birds that of Schildmacher (I937) is best mentioned here. He investigated the histology of the pituitary in the blackbird (Turdus merula) in males and females at times of annual flights (spring and autumn).

\section{THE BLOOD VESSELS OF THE PITUITARY BODY}

Wislocki and King (1936) have made an important contribution to our knowledge of the vascular connections of the pituitary body and adjacent tuber cinereum. Their results indicate that the description of Popa and Fielding, especially of the hypophysio-portal system, requires extensive modification. The pars glandularis is furnished with blood by two 
routes: afferent arteries and afferent portal veins which originate in the region of the stalk from a plexus both surrounding and penetrating the infundibular stem (see Fig. 1). The terminal parts of these arteries and veins unite to form the

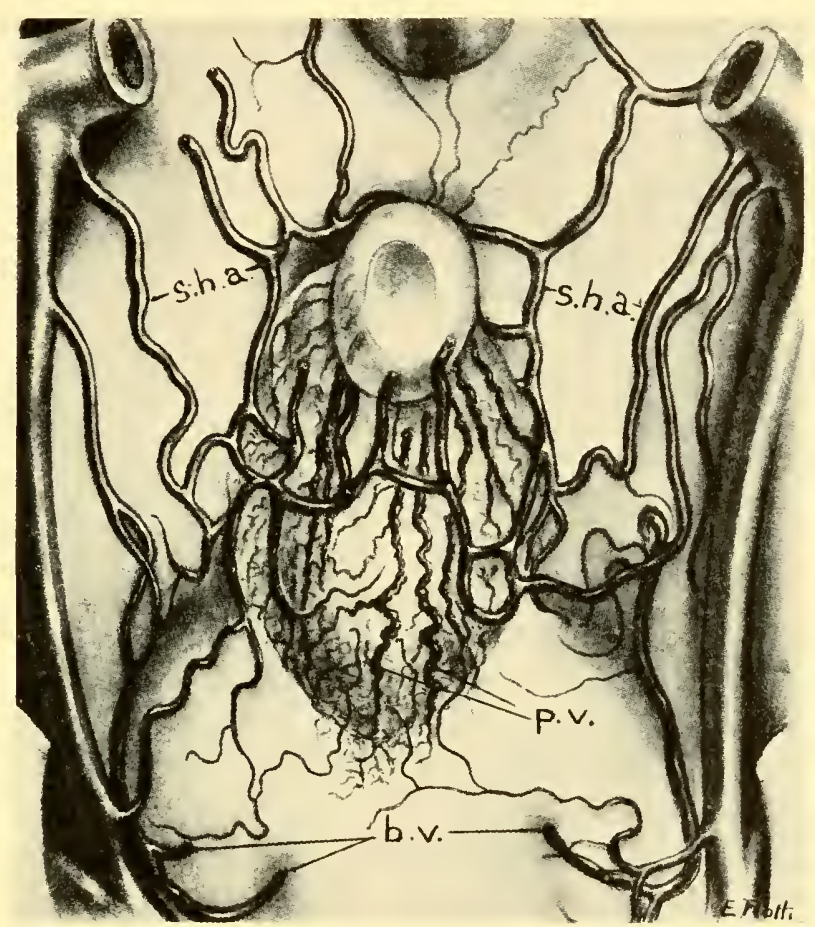

FIG. 1.-The blood vessels of the human pituitary body as revealed by a dissection of the base of the brain. s.h.a.: superior hypophysial arteries, anastomosing arteries supplying the pars neuralis, pars intermedia, and pars glandularis. p.v.: portal veins on the stalk. b.v.: basilar veins, into which blood only from the floor of the hypothalamus flows. (From Wislocki and King, Amer. J. Anat., 58, 421-72 [1936].)

sinusoids characteristic of the pars glandularis. Blood leaves the pars glandularis in veins passing from the lateral poles of the anterior lobe to the cavernous sinuses. The eminentia saccularis, infundibulum (pars neuralis), and pars intermedia are supplied with arteries and drained by veins which are 
almost independent of those of the anterior lobe. These vessels enter and leave by the posterior pole of the pars neuralis. Contrary to Popa and Fielding, as well as many other investigators, the authors concluded that the hypophysio-portal veins receive no blood from the anterior or posterior lobes and that the hypothalamus in a vascular sense is independent of the pituitary body. On the other hand, the terminal basal portion of the tuber cinereum-the eminentia saccularis - appears from its vascular supply as well as from the readiness with which it takes up vital dyes to be one part of a unit composed of the eminentia saccularis, the stalk, and the pars neuralis. In respect of their avidity for vital dyes and their unusually rich and complex blood supply, the supraoptic region of the diencephalon and the area postrema of the "hindbrain" also appear to be tissues similar to those of the unit just described.

It is emphasized by Wislocki and King that the foregoing description is particularly true of the monkey (and probably human $)^{\mathrm{I}}$ pituitary and hypothalamus. It should again be emphasized that their results indicate that blood flows from the hypophysio-portal vessels to the pars glandularis and not in the reverse direction, as was the contention of Popa and Fielding as well as others. The description of Wislocki and King, moreover, denies the assumption of many authors that secretions of the pituitary body have ready access to and specifically affect hypothalamic "vegetative centers."

The blood vessels of the diencephalic-hypophysial region in the cat are, in most respects, like those in the monkey; however, the eminentia saccularis is less well vascularized in the cat (Wislocki, I937). In the cat, also, Stevens (1937) measured the total lengths and average diameters of the capillaries per unit volume of tissue of the various parts of the pituitary body. She concluded that the pars tuberalis is supplied with sinusoids even more richly than the pars glandularis. The latter was found to have a vascular bed about

${ }^{1}$ See also the report of Wislocki (1937). 


\section{THE ANATOMY OF THE PITUITARY BODY}

six times as large as that of the pars neuralis which, in this respect, resembled the motor nucleus of the seventh nerve. The pars intermedia contained the smallest number of capillaries.

THE MENINGES OF THE PITUITARY BODY

New studies of the meningeal relations of the pituitary body in the dog (Schwartz, I936) and in a number of mam-

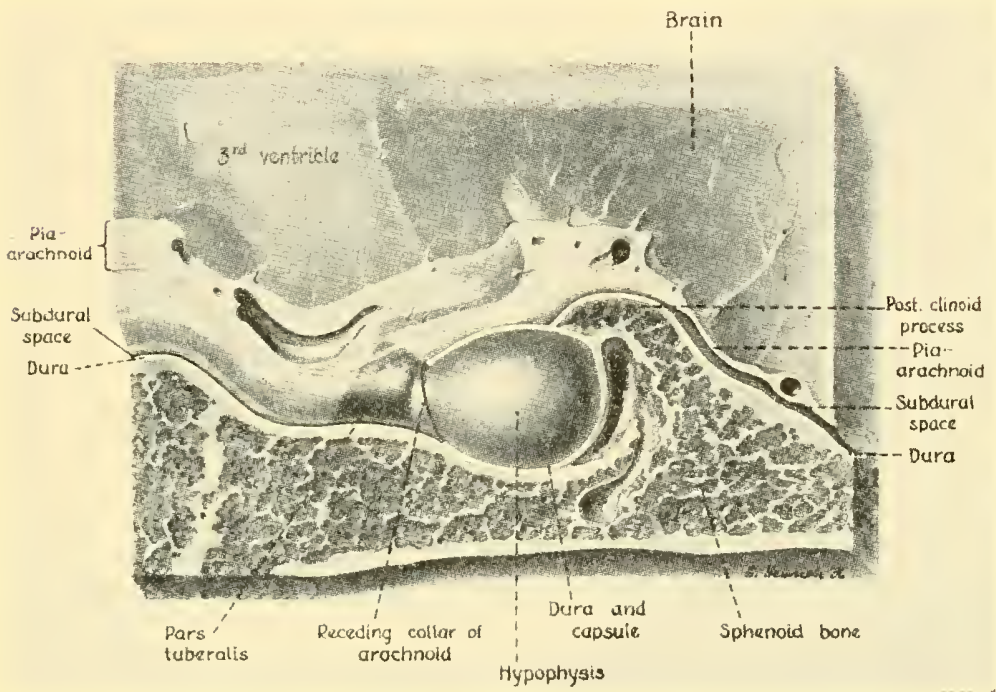

FIG. 2. - The relation of the meninges to the pituitary body of the dog. Note that the pia-arachnoid surrounds the stalk and pars tuberalis but not the remainder of the pituitary body. (From Schwartz, Anat. Rec., 67, 35-44 [1936].)

mals, including man and the human embryo (Wislocki, I937), have been reported. The subarachnoid space encircles the infundibular stalk but does not surround the pituitary body proper, although it may penetrate into the sella turcica a short distance in the cat and dog (see Fig. 2). The dura mater extends throughout the sella turcica and constitutes one layer of a fused structure consisting of periosteum, intrasellar dura, and pituitary capsule. Therefore there is no subdural space. 
THE INNERVATION OF THE PITUITARY BODY

It has been known for many years that the pars glandularis receives sympathetic nerve fibers from the carotid plexus. In the cat these non-myelinated fibers accompany the arteries and terminate chiefly in the pars glandularis and the pars neuralis. Not all the nerve fibers degenerate after cervical sympathectomy, and some may be parasympathetic in origin (Hair, 1938). The functional significance of this innervation, however, has not yet been adequately explored. Evidence of a nervous regulation of the pars glandularis is slowly accumulating. ${ }^{2}$ Concerning the pathways involved, however, little is known. The physiological importance of the nerve supply of the pars intermedia has been clearly suggested by experiments in amphibia and certain other cold-blooded animals. Here again, however, not much is known about the location of the innervating (efferent) neurones, although Hair found that some fibers of the supraoptico-hypophysial tract can be traced to the pars intermedia in the cat. Our best information deals with the nerve supply of the pars neuralis. Especially in recent studies employing physiological and morphological technics it has been possible to demonstrate the great dependence of the pars neuralis on certain hypothalamic nuclei, particularly the paired supraoptic nuclei. Necessarily, then, the greater part of the discussion will be concerned with the pars neuralis. Much of the review of the physiological experiments, together with an additional diagram of the nervous connections between the hypothalamus and the pars neuralis, will be found in chapter $\mathrm{x}$.

The diagram reproduced as Figure 3 represents the opinion of Roussy and Mosinger on the innervation of the pituitary

${ }^{2}$ Such as the effect of light on the secretion of gonadotropic hormone in birds and in one mammal, the ferret. Strong, diffuse electrical stimulation causes the liberation of gonadotropic hormone (ovulation) in the rabbit. See also the report of Collin and Hennequin (1936) on changes in the pars glandularis of the rabbit following bilateral extirpation of the superior cervical ganglia (see also chap. iii). 


\section{THE ANATOMY OF THE PITUITARY BODY}

body. Some of the interconnections depicted represent possibilities rather than probabilities inferred from correlated

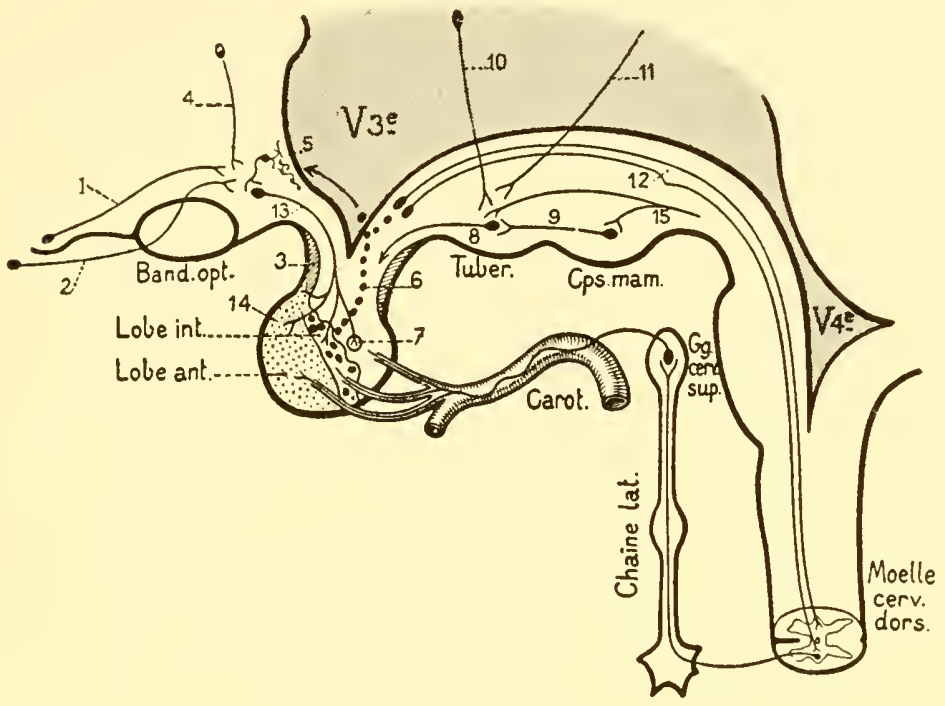

Fig. 3. - The innervation of the pituitary body according to Roussy and Mosinger. (From Presse méd., 44, $1521-23$ [1936].)

Band. opt.: optic tract; Carot.: carotid artery; Chaine lat.: cervical sympathetic; Cps. mam.: mammillary body; Gg. cerv. sup.: superior cervical ganglion; Lobe ant.: pars glandularis; Lobe int.: pars intermedia; Moelle cerv.-dors.: cervicothoracic portion of spinal cord; $V_{3}^{e}:$ third ventricle; $V_{4}^{e}$ : fourth ventricle; Tuber: tuber cinereum.

I. Amygdalo-tangential* tract (olfactory-pituitary reflexes). 2, Retino-tangential* tract (optico-pituitary reflexes). 3, Pars tuberalis. 4, Pathway by which pituitary can be influenced by corpus striatum and globus pallidus. 5, Subependymal network of sensory nerves. 6, "Neurocrinie hypophyso-hypothalamique." 7 , Islet of glandular cells in pars neuralis. 8 , Tubero-hypophysial tract. 9 , Mammillohypothalamic tract. 10 , Thalamo-hypothalamic pathways. 11 , Cortico-hypothalamic pathways. 12, Decussation of descending hypothalamic pathways. 13, Hypothalamico-hypophysial tract. 17 , Zone of transition. 15, Central sensory pathways.

* Tangential or supraoptic nucleus.

morphological-physiological experiments. An example of a diagram based upon such experiments is that of Figure 4. The function and, indeed, the morphological maintenance of the pars neuralis depends upon the normal flow of im- 
pulses from certain nuclei in the hypothalamus to the neural lobe. (The most complete experiments have been performed in cats by Fisher, Ingram, and Ranson.) The important nuclei are the paired supraoptic nuclei which supply most of the nerve fibers of the pars neuralis as well as a few fibers to the different divisions of the pars buccalis. Both of the nuclei must be destroyed or cut off from the pars neuralis before the latter undergoes atrophy and ceases to elaborate its hormones. The neurones of the supraoptic nuclei degenerate and

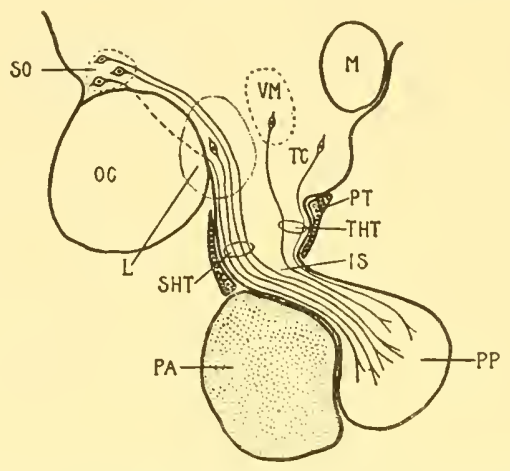

FIG. 4.-Diagram of hypothalamico-hypophysial fiber system of the monkey. (From Ingram, Fisher, and Ranson, Arch. intern. Med., 57, 1067-80 [1936].) Diabetes insipidus can be produced by lesions in the circumscribed region $L$. IS: stalk; M: mammillary body; $O C$ : optic chiasm; $P A$ : pars glandularis; $P P$ : pars neuralis; $P T$ : pars tuberalis; $S H T$ : supraoptico-hypophysial tract; $S O$ : supraoptic nucleus; VM: ventromedial component of supraoptic nucleus; TC: tuber cinereum; THT: tubero-hypophysial tract. As indicated by a broken line, it is possible that fibers of the supraoptico-hypophysial tract pass through the optic chiasm.

disappear after hypophysectomy-i.e., in the dog or rat (Hare, I937; Rasmussen, I937) or after interruption of the supraoptico-hypophysial tract. The filiform or paraventricular nuclei perhaps also directly or indirectly-i.e., by way of interposed synapses with neurones of other nuclei-innervate the pars neuralis. The morphological evidence for this interpretation is, however, better than the neurological. ${ }^{3}$

3 The following are recently published references to authors who have made observations on other parts of the pituitary and its possible central connections: Collin (1935), Collin and Fontaine (1936), Collin and Hennequin (1936), and Roussy and Mosinger (1935-36). 
Hair (1938) described knoblike enlargements projecting from nerves distributed in the pars glandularis, pars intermedia, and pars tuberalis of the cat's pituitary body. These enlargements lay in contact with the epithelial cells. In the pars neuralis the nerves terminated in bulblike structures similar to those described by others in the neural lobe of the ox and of man.

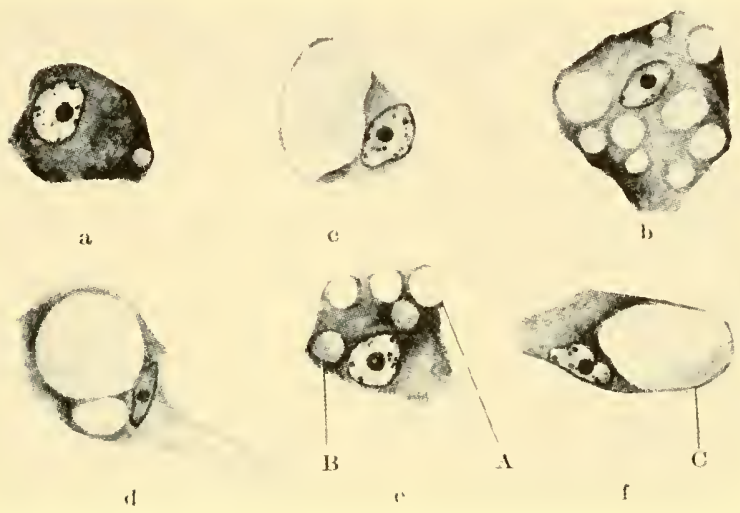

Fig. 5.-Colloid-formation in neurones of the supraoptic and paraventricular nuclei. (From Peters, Z. Neur., 154, 331-4+ [1936].) Colloid $a$ as fine droplets, $b$ diffusely distributed, and $c$ and $d$ as large accumulations believed to follow coalescence of smaller droplets. Vacuoles may appear to be empty, $A$ of $e$, or partly filled, $C$ of $f$, or completely filled, $B$ of $e$, with colloid.

A number of French and German authors ${ }^{4}$ have called attention to the peculiar morphology of the neurones of certain hypothalamic centers. Some of the morphological variations which may occur in the cells of the supraoptic and paraventricular nuclei are illustrated in Figure 5. The formation of colloid by these or homologous neurones is said to be recognizable not only in man and other mammals but also in other vertebrates such as reptiles, amphibia, and fishes, as well as in

${ }_{4}^{4}$ Collin and collaborators (see the earlier volume), Scharrer (1933-35), Gaupp (1935), Gaupp and Scharrer (1935), Peters (1935), and Roussy and Mosinger (193536). Florentin (1937) discusses changes related to secretory activity of the pars intermedia (toad). Roussy and Mosinger (1937) also review anatomical evidence for the secretion of pituitary hormones in to the cerebrospinal fluid. 
one invertebrate, the snail. Other morphological characteristics which have been described in these nuclei are multinuclear cells, endocellular capillaries, and degeneration and disintegration of the cells. All these observations seem to place the neurones of the supraoptic and paraventricular (and perhaps the tubero-mammillary) nuclei in a category different from that of other cells of the central nervous system, although Peters found colloid inclusions in cells of certain nuclei of the medulla oblongata.

The evidence that such cells secrete colloid and are to be considered a "diencephalic gland" is morphological and does not deserve acceptance at this time. 'The "secretory activity" of these cells appears to be slight in childhood; it is not otherwise correlated with age and is not altered as a result of nervous or endocrine diseases.

THE PARS INTERMEDIA, THE PARS NEURALIS

AND THE PARS TUBERALIS

It has again been emphasized that the pars intermedia of man and the anthropoid apes is a structure so rudimentary in appearance that it appears to have no important function (Berblinger and Burgdorf, 1935; Plaut, 1936; and Scriba, 1936). Frequently, also, the human pituitary contains no residual cleft of Rathke's pouch. In the pituitary of the porpoise $^{5}$ and the whale, ${ }^{6}$ the pars glandularis is completely separated from the pars neuralis by a leptomeningeal-dural fold. The embryologic development of the organ in the porpoise and whale is therefore apparently different from that in other mammals. No pars intermedia can be recognized in the cetacean pituitary. ${ }^{7}$ According to Benjamin (1935), the deposits of pigment in the pars intermedia and in the pars tuberalis of wild and hooded rats-but not present in pure

5 Tursiops truncatus.

${ }^{6}$ Balaenoptera physalus, B. sibbaldi, and Physeter megalocephalus (see Geiling, 1935; and Wislocki and Geiling, 1936).

7 Whether or not a pars intermedia can be identified in the bird's pituitary is still undecided (see the discussion of Wislocki and Geiling). 
albinos - originates in the leptomeninges and, after development, is found in the connective-tissue septa.

The cells of the pars intermedia secrete the chromatosomedispersing hormone. If, however, the pars intermedia is rudimentary and perhaps non-functional (man) or absent (fowl? cetacean), the hormone is still formed by cells of the pars glandularis.

The basophilic cells of the pars neuralis have been carefully studied by numerous authors because of their possible relationship to clinical disorders such as hypertension-a view particularly emphasized by Cushing. These cells are probably derived from the pars intermedia (Andreis, 1935; and Rasmussen, I936) and, it is generally agreed, increase in numbers particularly after middle age, when, of course, essential hypertension is more frequently present. However, it appears clear that there is no convincing correlation between the development or presence of hypertension and the ingrowth (and hyperplasia) of basophilic cells in the pars neuralis. The association of hypertension and this morphological change is fortuitous (Marcano, 1935; Hawking, I936; Rasmussen, I 936; and Scriba, 1936). Likewise these basophilic cells probably have nothing to do with the elaboration of the oxytocic and pressor-diuresis-inhibiting hormones of the pars neuralis. The cetacean pars neuralis is entirely separated from the pars buccalis by meninges and yet contains the characteristic pars neuralis hormones (Geiling, I 935). In the cat, after sufficient injury of the supraoptico-hypophysial nervous connections, the amount of oxytocic and pressor hormones in the pars neuralis is markedly reduced in association with atrophy of the pars neuralis; yet there is little change in the pars intermedia (Fisher and Ingram).

The hyaline bodies of Herring, which can be found in the pars neuralis of the whale (Wislocki and Geiling, I936), are therefore not derived from juxtaposed cells of the pars buccalis (pars intermedia) as has been suggested from studies of the pituitary of other mammals. In fact, Gersh and Tarr 
(1935) offer good evidence that such hyaline bodies are fixation artefacts and are unrelated to the amount of pressor hormone in the pars neuralis. Gersh and Tarr studied the pars neuralis of six mammals and of the fowl. True secretory cells have long been sought in the pars neuralis. Recently Gersh ( 1937) described parenchymatous "glandular" cells more or less uniformly distributed in the pars neuralis and containing granules or lipoid droplets. They could be identified in the neural lobe of nearly all mammals and of two birds (fowl and pigeon). They appear to be supplied with nerve fibers passing down the stalk and probably secrete the diuresis-inhibiting (vasopressor) hormone. They were found to undergo degeneration after section of the stalk and to increase in number and size if water was withheld. The brown and black pigment of the human pars neuralis has been studied recently by Roussy and Mosinger (1935). Trossarelli (1935) investigated the connective tissue and nerve fibers of the pars neuralis. He confirmed Tello's earlier description of the club-shape of some of the nerve endings of the pars neuralis. Although true nerve cells heretofore have not been identified in the pars neuralis, Kasahara (I 935) stated that nerve cells with growing fibers could be found in his cultures of pars neuralis tissue obtained from young rabbits. The distribution of mast cells in the infundibulum and pars neuralis of the ox, the cat, and man has been studied by Gray (1935). The mast cells often appeared to be closely associated with the primary capillary network of the hypophysio-portal system.

There have been no important additions to our knowledge of the morphology of the pars tuberalis. Its functional importance is still unknown, although Biggart believed that it secretes the diuresis-inhibiting hormone.

THE PARS GLANDULARIS

As has already been mentioned, the majority of the recent reports dealing with the anatomy of the pituitary body are chiefly concerned with the physiological anatomy of the pars 
glandularis. In addition, there are a few miscellaneous reports which will be considered at the end of this section. Some of the most interesting interpretations are the result of studies of human pituitaries. Furthermore, confidence in the validity of the conclusions reached is possible because, in the best of these reports, actual cell-counts rather than impressions were used in gathering data. Likewise, in a number of reports of an experimental nature this technic has been used. Finally, it is unfortunately also true that morphological investigations of the pars glandularis-whether of man or of animals - in which conclusions of a quantitative character are reached without any recognition of the necessity of founding these on a technic quantitatively sound are often the principal means of confusing efforts to make a modest start in satisfactorily picturing the function(s) of the cells of the anterior pituitary.

There still is no agreement in reference to the relationship between the reserve cells and the chromophil cells. ${ }^{8}$ Franck, in several reports published in $1935-37$, concluded, as many authors have before him, that the chromophil cells are derived from the reserve cells. He believed that there exist several varieties of oxyphils and basophils, and that the latter in turn are derived from oxyphils. Kirkman (1937), like Franck, also used the guinea pig. Kirkman's elaborate study included a careful analysis of previous reports and should be read by those particularly interested in the physiological anatomy of the pars glandularis. He studied the anterior pituitary of guinea pigs during the oestrous cycle, in pregnancy, postpartum, after gonadectomy, in fetal life, etc. Figure 6 is reproduced from a report by Severinghaus and indicates, in diagrammatic form, some views which have been held as to the relationship between the reserve cells (chromophobes) and the chromophils (oxyphils or acidophils and basophils). This author (1937) has recently reviewed the relationship of

${ }^{8}$ The report of Collin and Stutinsky (1937) contains a description of cellular peculiarities of the anterior pituitary of the frog.

[13] 


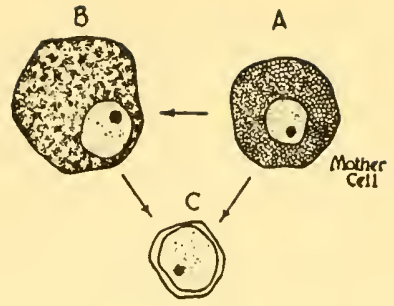

SAINT REMY
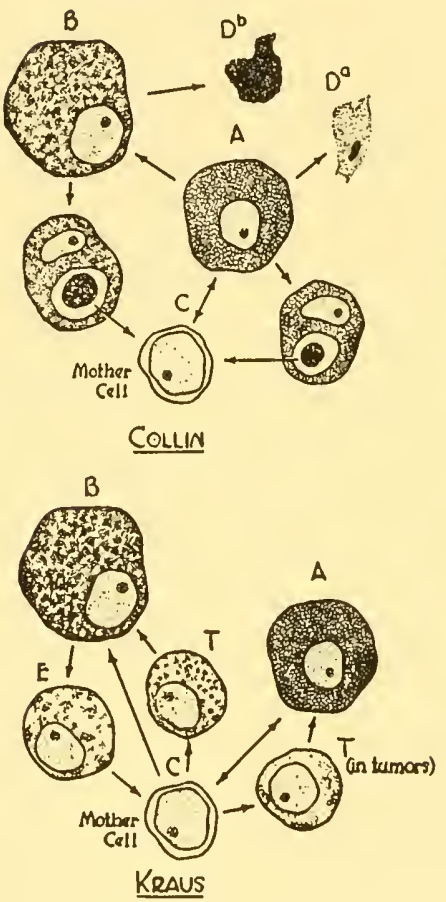

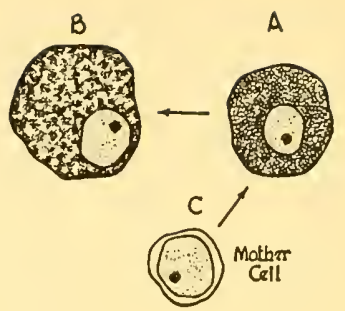

BENDA

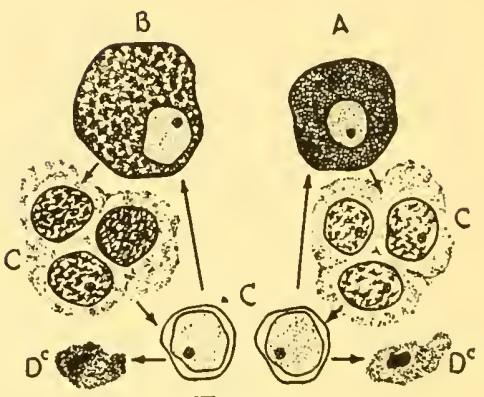

TRAUTMANW

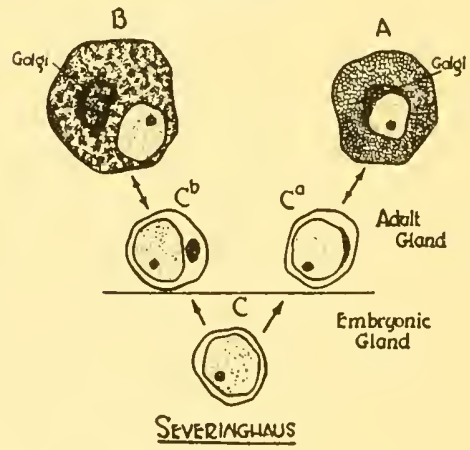
A. ACIOOPHILE
D. D $D^{\mathrm{b}} \cdot D^{\mathrm{c}} \cdot$ Degenerating Cells
B. BASOPHILE
E- Cell showimg granular elimination
C. CHROMOPHOBE
T. Transiticnal (Übergangzell)

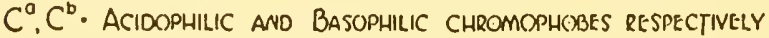

Fig. 6.-Diagrams illustrating views of different authors on the relationships of cells of the pars glandularis. (From Severinghaus, Physiol. Rev., 17, $556-88$ [1937].) 



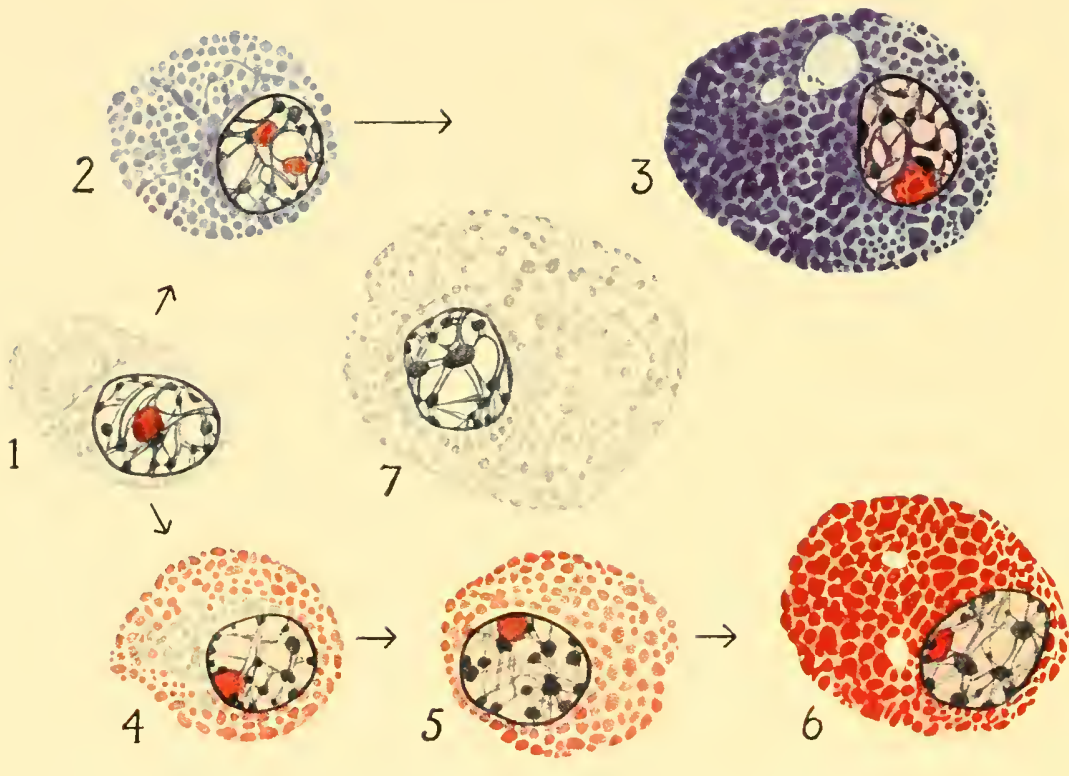

Fig. 7.-Diagram of the relationship of the cells of the pars glandularis. (From Crooke and Russell, J. Path. Bact., 40, 255-83 [1935].) Staining by authors' differential method. I, Reserve cell. 2, Transitional basophil. 3, "Adult" basophil. $f$ and 5, Oxyphils in early and later transition. 6, "Adult" oxyphil. 7, Exceptionally large reserve cell. 
the cellular morphology of the pars glandularis to the gland's physiological activity. The view of Crooke and Russell (I935) as to the cellular relationships in the human anterior pituitary is illustrated in Figure 7.

Dawson (1937) has named a specialized portion of the pars glandularis the "zona tuberalis" because of its location. In the cat and rabbit this part of the anterior pituitary consists largely of reserve cells and basophils, the proportions of which vary greatly in response to changes in the reproductive cycle, gonadectomy, adrenalectomy, etc.

According to Sanchez-Calvo (1937), if guinea pigs or rabbits are kept in a dark room, there occurs, among other changes, a marked increase in the proportion of oxyphils in the anterior pituitary. The maximum change was observed after the animals had been kept for 72 hours in darkness.

The relationship between the reproductive organs or their internal secretions and the anatomy of the pars glandularis.-The relationship between normal sexual activity and the morphology of the pars glandularis recently has been studied in the frog (Zahl, 1935) and pigeon (Mârza and Blinov, I 936). Zahl concluded that the important changes which can be correlated with the annual sexual cycle of several species of Rana involve the oxyphil and reserve cells. (The small basophils appeared to be unimportant.) During the winter months the number of oxyphils containing "fuchsinophil" droplets9 increases at the expense of the reserve cells and reaches its peak in the spring. Following the breeding season, the number of oxyphils undergoes a gradual reduction and is lowest in the late summer and autumn. According to Mârza and Blinov, the pituitary of the female pigeon enlarges at about the time of ovulation without, however, any apparent change in the number of reserve or basophil cells. Their technic did not permit them to draw any conclusions concerning the oxyphils.

Opinions on the histological appearance of the pars glandu-

${ }^{9}$ Zahl also speaks of fuchsinophil droplets in cells "otherwise chromophobic." 
laris of mammals like the rat at different stages of the oestrous cycle vary greatly. The recent careful study of Wolfe (1935), who used both qualitative and quantitative technics, led him to conclude that in the female rat the proportion of none of the cells-disregarding finer qualitative details such as the content of granules-varies at different stages of the oestrous cycle. ${ }^{10}$ He believed that the only clearcut cyclic change is in the granules of the basophils. These granules are most numerous in pro-oestrus and rapidly diminish in number during oestrus and metoestrus. Pfeiffer (1 937) supplemented his other experiments designed to alter the sexual characteristics of pituitary function in rats (see chap. iii) by anatomical studies. The distribution of cells typical of the male or female pituitary could be produced by transplanting testes into spayed females or ovaries into castrated males. The anterior pituitary of animals with both ovarian and testicular grafts tended to be of the male type. After puberty and, therefore, after sexual differentiation of the pars glandularis, the distribution of cells could not be altered.

The alterations in the pituitary accompanying parturition and the onset of lactation have interested several investigators. Collin and Florentin (1935) who used guinea pigs, as well as Weis (1935) who used rats, stated that an unusual and marked predominance of reserve cells is observed at parturition and for about 24 hours later. They believed that this change is related to the initiation of lactation. Collin and Florentin also concluded that the oxyphil is the more numerous chromophil during the first half of pregnancy, whereas the basophil predominates toward the end of pregnancy. Desclin (1936) was interested in the significance of the corpus luteum in maintaining the pituitary's lactation morphology in the rat. He found that the latter persists after ovariectomy, provided that the young continue to suckle. If, how-

${ }_{10}$ The proportions of the different cells confirmed Wolfe's previous report. The means and standard deviations were: basophils, $4.1 \pm \mathrm{I} .3$ per cent; oxyphils, $34.2 \pm 4.3$ per cent; reserve cells, $61.8 \pm 4.4$ per cent. 


\section{THE ANATOMY OF THE PITUITARY BODY}

ever, the young are removed, the pars glandularis assumes the appearance characteristic of gonadectomized animals (increased numbers of basophils, "castration-cells"). Kraus (1935) has advanced the opinion that the "pregnancy-cells" of the pars glandularis, growing initially because of placental stimulation, secrete the lactogenic hormone which is released into the circulation after the expulsion of the placenta. He believed that suckling is the stimulus responsible for the continued secretion of the lactogenic hormone by these cells.

Characteristic changes in the anterior pituitary following gonadectomy can be consistently observed in the rat and have already been described in the former volume. These changes chiefly involve the basophils which increase in number and later hypertrophy and become vacuolated. Such basophils containing a single large vacuole have been named castration-cells. In other animals, such as the guinea pig, contradictory descriptions of the changes in the pars glandularis following gonadectomy continue to appear. Unlike Severinghaus, Nelson (1935) declared that gonadectomy (or cryptorchidism) is followed by a slight but definite increase in the percentage of basophils in the guinea pig's anterior pituitary; however, Nelson agrees that castration-cells of the murine type are rare. An increased amount of colloid has been described in other studies of the pituitary of gonadectomized animals. Nelson mentions that thyroid-like follicles containing colloid are prominent in the anterior pituitary of gonadectomized or cryptorchid guinea pigs. Tuchmann (1937), who also studied the pituitary of gonadectomized guinea pigs, observed an entirely different change-i.e., a progressive, marked increase in the proportion of oxyphils. Both Nelson and Tuchmann were able to reverse the changes caused by gonadectomy by injecting oestrin. The reader is referred to the earlier volume for a description of anterior pituitary changes attributed to gonadectomy or injury of the gonads in man and other mammals.

The other recent reports deal with the correction or re- 


\section{THE PITUITARY BODY}

versal of castration changes in the pituitary of the rat. ${ }^{\text {II }}$ It is well known that several oestrogens and androgens cause reversal of the changes, so that the pituitary may assume a normal appearance both grossly and microscopically. Hohlweg (1935) insisted that progesterone alone $(0.54 \mathrm{mg}$. daily for 15 days to adult or immature spayed rats) does not alter the changes in the pituitary due to gonadectomy. The results of Migliavacca (1936) agreed with those of Hohlweg. Therefore the findings of Clauberg and Breipohl to the contrary lack confirmation. Nelson and Gallagher (1935-36), in agreement with others, found that male hormone whether from urine or testis prevents castration changes in the rat's pituitary. Similar effects with two pure androgens, androstane diol and androstene dione, were also described. ${ }^{12}$ Carcinogenic substances (benzpyrene and a dibenzanthracene diol) ${ }^{13}$ can prevent the pituitary changes due to gonadectomy (Wolfe, 1936; and Tuchmann, I937).

The effects of oestrogenic and androgenic substances on the pars glandularis are, of course, not limited to the correction of gonadectomy changes. In the past few years several authors have reported on the changes appearing in the pituitary of normal animals, both immature and adult (mouse, rat, guinea pig, and rabbit), particularly after the injection of oestradiol benzoate or oestrone. ${ }^{14}$ The alterations about to be described vary, depending upon dosage, preparation used, duration of injections, sex, age, etc. The variations for the purpose of this review, however, are not sufficiently impor-

I The relative importance of the interstitial cells and the germinal epithelium in preventing "castration" changes in the pituitary remains undecided. De Fremery (1936) minimizes the importance of the "sex hormones."

${ }^{2}$ Other experiments with androgens have been reported by Allanson, Hohlweg, Wolfe and Hamilton, and Yanagita.

${ }^{13}$ See also the report of 'Tuchmann and Demay (19.36).

I4 1935: Nelson, Wolfe, Wolfe and Phelps; 19.36: Halpern and D'Amour, Wolfe, Wolfe and Chadwick; 19.37: Wolfe. It appears that luteinization of the ovaries is definitely associated with the pituitary changes in female rats. Martins (19.36) could detect an increased vascularity of the pituitary, transplanted to the anterior chamber of the eye, $24^{-} 4^{8}$ hours after the injection of a large dose of oestradiol benzoate. 
tant to justify a detailed description. Often the dose of the oestrogen has been large (e.g., 200 rat-units of oestradiol benzoate daily for Io days), so that the effects may be such a distortion of the physiological as to be chiefly of pharmacological importance. The first and most important change is a loss of granules from the basophils; in addition, the percentage of basophils is reduced. Especially after large doses, a similar loss of granules occurs in the oxyphils. Owing to hypertrophy of the anterior pituitary, the proportion, but probably not the number, of these cells diminishes. Several of the authors concluded that "degranulated" basophils are transformed into reserve cells. Such a transformation only in a small part accounts for a simultaneous marked increase in the percentage and total number of the reserve cells in which numerous mitoses can often be observed. The hypertrophy of the pars glandularis is due to the hyperplasia of reserve cells. ${ }^{15}$

Usually the injection of a large dose of an oestrogen produces a considerable hypertrophy of the anterior pituitary (2-3 times normal size). Enormous doses of an oestrogen may correspondingly accentuate the change, so that the hyperplasia of the reserve cells is indistinguishable from a neoplasm (reserve-cell adenoma). A change of this sort was first produced by Cramer and Horning (1936) in mice (see Fig. 8). The authors were primarily interested in the production of mammary carcinoma by the application of a solution of oestrone (o.o I per cent dissolved in chloroform) to the skin, through which the hormone was readily absorbed. In several mice with large hemorrhagic adenomata composed almost entirely of reserve cells, there were cachexia and degenerative changes in the adrenal cortex, in addition to the expected regressive alterations in the gonads. The authors suggested that the mice with pituitary adenomata were suffering from

${ }_{15}$ The response of the transplanted pituitary is similar; so it is not likely that the effects depend to an important extent on the nervous system, as some authors have asserted (Desclin and Grégoire, 1936). 


\section{THE PITUITARY BODY}

a marked hypophysial deficiency, probably because of the disappearance of the chromophilic cells. Confirmatory experiments in rats have been reported by McEuen, Selye, and
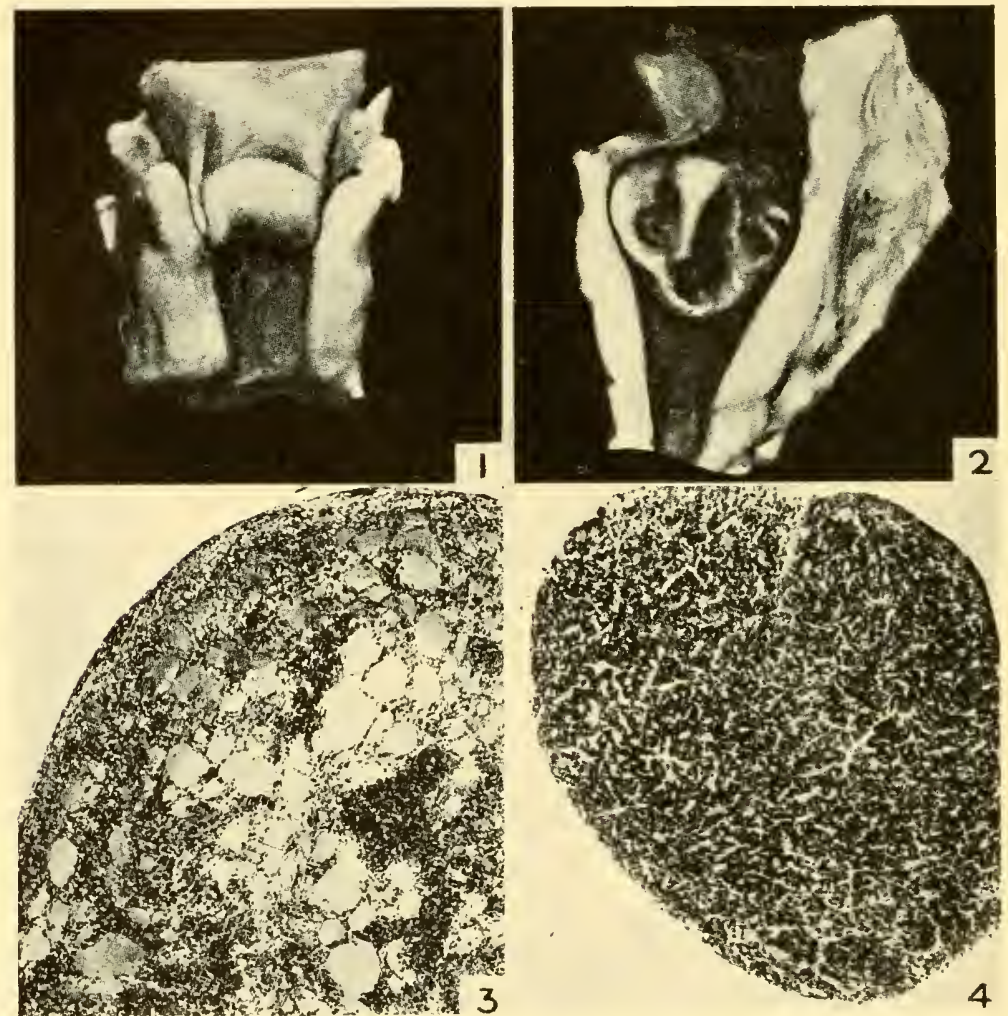

Fig. 8. - The action of large doses of oestrone, administered over a period of 6 months, on the pituitary of the mouse. (From Cramer and Horning, Lancet, 230, 247-49 [1936].) I, Gross appearance of normal pituitary and optic nerves. 2. Pituitary of treated male mouse illustrating marked congestion of the pars glandularis and compression of optic nerve. 3, Photomicrograph of pars glandularis of treated mouse (2) showing large hemorrhagic areas. A, Photomicrograph of glandularis of normal mouse at a slightly higher magnification than 3 .

Collip (1936) and by Zondek (1936). It has not been convincingly shown - as Zondek believed - that such pituitary adenomata are produced much more readily in male than in female rats. Lacassagne and Nyka (1937) concluded that the 


\section{THE ANATOMY OF THE PITUITARY BODY}

effect of an oestrogen on the pituitary varies in different strains of mice.

Selye, Browne, and Collip (I936) injected $4 \mathrm{mg}$. of progesterone into young, mature female rats for $\mathrm{I} 2$ days. This treatment was followed by some reduction in the size of the ovaries (23 per cent) and some increase in the pituitary's weight (29 per cent). In the pars glandularis of the injected animals there was found "a great number of small cells with relatively dense chromatin."

Testosterone (or its propionate) causes no pituitary hypertrophy (McEuen, Selye, and Collip, I937) and in this respect differs from oestradiol or oestrone. However, like the oestrogens it brings about a degranulation of the basophils (Wolfe and Hamilton, r937).

Wolfe (1935) and Wolfe and Phelps (1935) reported that the effects of prolan on the pars glandularis of the adult rat resemble those of an oestrogen-i.e., loss of granules from basophils (and oxyphils) and hyperplasia of reserve cells. Apparently these changes are due to the liberation of oestrogen or androgen from the gonad stimulated by prolan. In 1937 Wolfe stated that prolan prevented some of the similar effects of oestradiol in immature female rats. In comparison with the pituitary of animals given only oestradiol, that of animals receiving both prolan and oestrogen was less hypertrophied and contained more basophils which were normal in respect of granules.

The relationship between other glands of internal secretion and the anatomy of the pars glandularis. I. The thyroid.-The pars glandularis of the rabbit increases about 50 per cent in weight after thyroidectomy performed I59-333 days previously (van Dyke and Chen, 1935). Franck (1936) stated that the administration of thyroid extract or thyroxin (and, to a less extent, adrenal cortical hormone) to guinea pigs causes a reduction of the number of granules in the oxyphils. The number of basophils containing granules was thought also to be reduced. Franck also stated that an extract containing thyro- 


\section{THE PITUITARY BODY}

tropic and adrenotropic hormone caused similar changes. Iodine, KI, or diiodotyrosine were found to lessen the effects (Franck, I 937).

There has been some discussion as to the nature of the changes in basophils in thyroidectomized as compared with gonadectomized animals. Nelson and Hickman (1937), although affirming that they could distinguish between the alterations in basophils due to thyroidectomy or gonadectomy, contended that the same basophils are affected by either operation. They found that changes due to thyroidectomy, except degranulation, could be corrected by the administration of oestrone. Guyer and Claus (1937) believed that the formation of vacuoles in the basophils is due to accumulation of secretion after gonadectomy, whereas after thyroidectomy it is the result of cellular degeneration. Zeckwer (I937) particularly supports the view that the thyroidectomy and gonadectomy cells of the anterior pituitary are different and can be identified morphologically.

2. The adrenals.-Lippross (1936), using rats instead of guinea pigs, found no significant changes in the morphology of the pituitary-and adrenals and gonads-after the prolonged administration of adrenal cortical hormone or epinephrine. Therefore, his findings did not confirm those of Franck.

The compensatory hypertrophy of an adrenal gland after the extirpation of its mate depends chiefly upon the secretion of an adrenal cortical stimulating hormone by the pars glandularis. Zeckwer (I937-38), taking into account the changes occurring in both adrenals following thyroidectomy or gonadectomy, studied the effects of these operations on compensatory adrenal hypertrophy in relation to the histology of the pituitary. She concluded that the oxyphils, which tend to disappear after thyroidectomy, do not secrete the adrenotropic hormone. Also, this hormone was thought not to be secreted by the thyroidectomy-cells or castration-cells, inasmuch as the degree of compensatory hyper- 
trophy could not be correlated with the number of either cell type. Therefore, she surmised that the adrenal cortical stimulating hormone is secreted by the basophils (see also pp. I 5, 2I-22).

The change in the morphology of the pituitary characteristic of adrenal insufficiency is a reduction, sometimes very marked, in the percentage of basophils. Grollman and Firor (1935) found that this change, accompanied by an increased vascularity of the pituitary, is very marked in the dog. In the rat the reduction of the number of basophils is less prominent; however, the staining of these cells is "very abnormal." Recently, the cells of the hypophyses from patients with severe adrenal insufficiency (Addison's disease or atrophy of adrenals) have been counted by several investigators (Crooke and Russell, I935; Hawking, I936). The percentage of basophils was found to be greatly reduced (e.g., 0.06-0.5 per cent compared with $7-\mathrm{II}$ per cent in a normal series). ${ }^{16} \mathrm{~A}$ considerable number of abnormal transitional basophils was encountered in the series of Crooke and Russell. The proportion of oxyphils also was abnormally low, whereas the proportion of reserve cells was abnormally high.

3. The pancreas.-After repeated injections of insulin into guinea pigs, the pars glandularis is said to be composed of an increased number of oxyphils with a corresponding diminution in the number of reserve cells (Kahn, 1935). On the other hand, Gentile and Amato (1936) stated that pancreatectomy in the dog is also followed by an increase in the proportion of oxyphils which, however, appear relatively nongranular. The volume of all the cells was found to be increased. Kahn and Waledinskaja (1936) concluded that partial pancreatectomy in the rabbit is followed by degenerative changes in the oxyphils and that the magnitude of these changes depends upon the degree of pancreatic insufficiency. An investigation of the pituitary of normal cats and of cats

${ }^{16}$ Confirmatory reports without cell-counts have been published by Andreis (1935) and Meesen (1935). 
sacrificed at various intervals after complete pancreatectomy was made by Fichera and Ferroni (1937). 'The authors believed that the pituitary undergoes hypertrophy and that a marked increase in the proportion of reserve cells at the expense of the oxyphils takes place as a result of the operation.

4. The parathyroids.-The pituitary of male rats after parathyroidectomy or repeated injections of parathyroid hormone or a solution of $\mathrm{CaCl}_{2}$ was studied by Takahisa (I936). It was his belief that the principal alteration is in the oxyphils - the proportion increasing with parathyroid deficiency and diminishing if there is an excess of parathyroid hormone in the body's circulating fluids. The administration of a solution of $\mathrm{CaCl}_{2}$ appeared to cause changes resembling those of parathyroid deficiency.

5. The thymus.-Clark, Steinberg, and Rowntree (1936) investigated the effects of a thymus extract on the distribution of cells in the pars glandularis of the rat. Especially in very young male rats growing at a precocious rate (age: O-I 3 days) the percentage of oxyphils was almost twice that in the anterior pituitary of control animals. The authors correlate this change with the rapid growth observed. In female rats of a corresponding age, however, the increase in the proportion of oxyphils was only about 25 per cent. In older rats (age: 13-45 days) no differences were found.

6. Attempts to correlate changes in the human pituitary with alterations in other endocrine glands or with disease syndromes. - The syndrome of Cushing, which he named pituitary basophilism, now appears not to be related to a basophil adenoma of the pars glandularis as Cushing first believed. Susman (1934) found adenomata in 8 per cent of 260 human pituitaries which he examined. Nearly half the adenomata (eight of seventeen) were basophilic; yet Cushing's syndrome was not present. On the other hand, the syndrome may be present in the absence of a basophil adenoma. Crooke (1935) made the important observation that a hyaline change in the 
basophils of the anterior pituitary invariably is associated with typical pituitary basophilism ${ }^{17}$ in the absence or presence of basophil adenoma, a neoplasm of the thymus, or a hyperplasia of the adrenal cortex. A similar hyaline change in a few cells was found in 9 of 350 hypophyses of individuals without Cushing's syndrome. Another careful investigator of the pituitary, Rasmussen (1936), has fully confirmed Crooke's observations in three additional cases. Rasmussen also agreed that such hyaline changes in the basophils are rare in other diseases such as essential hypertension, eclampsia, etc.

Hawking (1936) as well as Rasmussen (1936) have made actual counts of the various cells occurring in the pars glandularis of patients afflicted with various chronic diseases. As a result, it was concluded that no convincing change in the morphology of the pars glandularis occurs as a result of essential hypertension, diabetes mellitus, and Graves's disease. Using less exact methods, Kraus (I935) ${ }^{18}$ and Spark (I935) came to a similar conclusion regarding hypertension and eclampsia. Müller (I936) stated that he discovered twelve adenomata of the anterior pituitary (mostly reserve-cell adenomata) in twenty cases of adiposity but only two in twenty control cases (normal or of cachexia). Apparently the excretion of gonadotropic hormone in the urine, revealed by the production of ovulation in rabbits, may or may not be associated with various neoplasms of the pars glandularis (McCullagh and Cuyler, I 937). According to Susman (1 935), a diminution in the number of oxyphils is characteristic of the pituitary of patients dying largely as a result of shock. Also without making cell-counts, Meesen (1935) believed that there is some correlation between basophilic invasion of the

\footnotetext{
${ }^{17}$ All twelve patients from whom Crooke's material was obtained had the following symptoms: an adiposity of the face and trunk; a plethoric, florid, or dusky complexion; a persistently high blood pressure; amenorrhea or impotence (if past puberty). In nearly all there were cutanecus striae of the abdomen or thighs and glycosuria or lowered sugar tolerance. In some there was a severe osteoporosis.

${ }^{18}$ Kraus suggested that an apparent increase in the proportion of basophils in renal disease and other conditions, including advancing age, may be of some illdefined compensatory nature.
} 
pars neuralis and essential hypertension, as well as between a proliferation of the oxyphils (secreting growth-promoting hormone?) and the growth of sarcomata.

Erdheim (I936) suggested that pregnancy-cells, which clearly are not the source of prolan, secrete a hormone promoting the growth of the fetus and some of the maternal parts. He regarded the similar cells appearing in the pituitary of patients with congenital aplasia of the thyroid as the pituitary's attempt to compensate for the harmful effect of thyroid deficiency on body-growth.

The reduction in the percentage of basophils, as well as a similar but less prominent change in the oxyphils, in the pituitary of patients with Addison's disease has already been described.

Miscellaneous observations.-Andersen, Prest, and Victor (I937) investigated the metabolism of the isolated pars glandularis of the rat at different stages of sexual activity, including pregnancy and lactation. They concluded that the metabolism of the gland could not be correlated with the percentage of basophils as determined by Wolfe and his colleagues. The pars glandularis was found to be heaviest during oestrus.

Several authors have reported their impressions of the changes in the pars glandularis due to administration or deficiency of various vitamins. Giędosz (I935) stated that repeated subcutaneous injections of a solution containing vitamin $A$ into rabbits bring about an increase in the number of oxyphils and, to a less extent, in the number of basophils. Intravenous injections of a solution of vitamin $\mathrm{C}$ were followed by similar changes. The pituitary of vitamin $B_{1}$ deficiency (six human cases of beriberi) was investigated by Marburg and Wenckebach (1936). The pars glandularis frequently contained necrotic areas; the oxyphils and basophils often were separated into adenoma-like groups. They suggested that apparent holocrine degeneration of the basophils of the pars neuralis might be related to the circulatory 
disorders of the condition. According to Nitzescu and Bratiano (1936), changes in the oxyphils follow the administration of enormous doses of vitamin D to the dog. Stein (I 935) reviewed the literature dealing with alterations in the pituitary as a result of a deficiency of vitamin E. He himself could find no difference in the size or weight of the various lobes or in the percentage of different cells by comparing the hypophyses of female rats cured of a vitamin-E deficiency with those of rats on a vitamin-E deficient diet. Confirmatory results were obtained by Müller and Müller (1937). However, in three male rats (on a diet free from vitamin $\mathrm{E}$ for 280 days) the authors found moderate castration changes in the anterior pituitary.

The pituitary has been successfully transplanted into the anterior chamber of the eyes of rabbits and guinea pigs by Haterius and his colleagues. ${ }^{19}$ Such grafts become attached to the iris and, like similar homoplastic grafts attached to the subconjunctival tissue, are composed mainly of basophils about two months after transplantation. The ocular grafts in hypophysectomized guinea pigs caused an increase in the number of ovarian follicles with uterine hypertrophy and persistent oestrus. No atrophy of the thyroid or adrenals occurred. The authors point out that inadequate vascularization and the absence of a nerve supply may account for the lack of a secretion of luteinizing hormone (no complete follicular maturation or ovulation or corpus luteum formation). Martins (1936) found that pituitary transplants (in kidney or in anterior chamber of eye) had little effect on the symptoms of hypophysial deficiency in five rats receiving the transplants I6-Ioo days after hypophysectomy. Also he transplanted the pituitary into gonadectomized nonhypophysectomized rats. Castration-cells could be found in the pituitary in situ but not in the grafts $\mathrm{I}-3$ months later. The

${ }^{19}$ Haterius, Schweizer, and Charipper (1935) and Schweizer, Charipper, and Haterius (1937). 
pars intermedia, which is the least vascular part of the pituitary, survived transplantation best.

Kasahara (1935) has studied the changes in the cells of the rabbit's pars glandularis when cultures are made of the latter. He found that the proliferating cells were neither chromophilic nor chromophobic. He described the cultured cells as "deflected epithelium" in distinction from Champy's term "dedifferentiated epithelium." Gaillard (1937) cultured juxtaposed slices of the pars glandularis and the pars neuralis of rats. He observed at the line of contact structural alterations including cysts, which led him to suggest that a similar relationship in vivo accounts for the morphological peculiarities of the pars intermedia. The same author described in another report (1937) cultures of the pars glandularis and other tissues removed from rabbits. Under proper conditions he could recognize oxyphils. He believed that suitably cultured anterior pituitary increases the rate of growth of osteoblasts. Anderson and Haymaker (I 935) found that only the chromatosome-dispersing hormone from the pars intermedia is formed in cultures of the pituitary of rats eight days old. There was no evidence of new secretion of the diuresis-inhibiting hormone (pars neuralis) or of gonadotropic, thyrotropic, or adrenal cortical stimulating hormones (pars glandularis). Their results agree with those of Engel and Werber (1937) but not with those of Nagayama (1937), who was interested only in the formation of gonadotropic hormones. The observations of Geiling and Lewis (1935) are discussed in chapter viii.

\section{SUMMARY}

The blood supply of the pituitary body is complex. The hypophysio-portal veins, concerning which so much has been written, now appear to be afferent rather than efferent as thought formerly. They correspond, as far as the pars glandularis is concerned, to the hepatic portal veins. Also, like the liver, the anterior lobe is furnished with arterial vessels. The blood supply of the pars glandularis is quite independent 
of that caring for the pars neuralis. At least in mammals like the monkey and man, it is now believed that there is no close vascular connection between the pituitary body and the hypothalamus. There is, therefore, little basis for postulating important effects of pituitary hormones on diencephalic centers.

However, certain hypothalamic centers, particularly the supraoptic nuclei, supply efferent fibers to the pars neuralis. If this innervation is completely severed, the pars neuralis atrophies and ceases to secrete its hormone in some if not all mammals. The cells of nuclei like the supraoptic nuclei morphologically are very different from most neurones but cannot yet be described as glandular cells of the diencephalon. The innervation and especially the central connections of the pars glandularis and pars intermedia are matters requiring intensive investigation.

The function and therefore the morphological significance of the pars tuberalis are unknown.

The pars intermedia, the source of the chromatosome-dispersing hormone in many animals, is, of course, a part of the pars buccalis. In certain animals lacking a pars intermedia, either because of its failure to differentiate or because of its regression in later life, the lobe's characteristic hormone is secreted by the pars glandularis.

The oxytocic and vasopressor hormones of the pars neuralis probably are not secreted or derived from cells of the pars buccalis such as invading basophils. Such invasion of the pars neuralis is not etiologically related to diseases like essential hypertension or eclampsia. Certainly, the hyaline bodies of Herring do not represent the hormones either actually or in a preformed state. A newly described glandular cell in the pars neuralis appears to be the source of the diuresis-inhibiting (vasopressor) hormone.

A great variety of changes in the pars glandularis has been related to the endocrine equilibrium of the animal, whether occurring normally or owing to a deficiency or to the injec- 
tion of hormones. Generally accepted changes in the pituitary due to a hormone-deficiency are the appearance of thyroidectomy-cells following thyroid extirpation and the appearance of castration-cells following gonadectomy. In addition, there is good evidence that a deficiency of adrenal cortical hormone is accompanied by a marked reduction in the percentage of basophils. The morphological changes in the pituitary caused by gonadectomy can be corrected by oestrogenic and androgenic substances, but not by progesterone. Injected into normal animals, large doses of male or female hormone cause a depletion of the granules of the basophils. Very large doses of an oestrogen like oestrone bring about hypertrophy of the pars glandularis because of a hyperplasia of the reserve cells. This change may culminate in the formation of a reserve-cell adenoma.

Considerable progress has been made in relating the morphology of the pituitary to disease in man. Adrenal insufficiency is clearly accompanied by a marked reduction of the percentage of basophils. The syndrome of Cushing (pituitary basophilism) is invariably associated with hyaline changes in the basophils. A basophil adenoma is not necessarily present. The best-known syndrome etiologically related to the pituitary is acromegaly (and giantism); the change here observed is the development of an adenoma composed of oxyphilic cells. Hence oxyphils are thought to secrete growth-promoting hormone. The opinion of the most careful investigators is against the view that pituitary changes (especially in the basophils sometimes in relation to their growth into the pars neuralis) are characteristic of essential hypertension, eclampsia, Graves's disease, or diabetes mellitus.

ADDENDUM

There appeared to be no great need in this volume for a chapter dealing only with the effects of hypophysectomy, inasmuch as almost all the topics would require reconsideration in the succeeding chapters. All the various effects of hy- 
pophysectomy are referred to in the Index. However, there are a few reports which should be mentioned here. Abramovitz (1937), Thomas (1937), Miyagawa (1 936), and Harris and Popa (1938) have discussed the technic of hypophysectomy in teleost fishes, in the mouse, and in the rabbit. A brief description of the effects of hypophysectomy in the mouse (in male and in normal, pregnant, and lactating female) will be found in the report of Leblond and Nelson (I 937). Observations on the effects of radon or X-rays on the pituitary of various animals have been published by Bertolotto (I 935), Cucchini (I 934), Fehr (I936), Lacassagne and Nyka (1934-35), and Franck (1937). Karlik and Robinson (1935) gave a detailed description of the changes in the central nervous system as well as the other better-known alterations in the organs and their functions appearing in a dog during and after a five-year period following hypophysectomy. According to Robinson (I 937), hypophysectomy in the pig is followed by characteristic atrophic changes in all the glands of internal secretion except the adrenal cortex. Certainly this observation requires confirmation.

The relationship of the hypothalamus to the pituitary body likewise is discussed in the appropriate chapters, especially in chapter $x$. Readers who are particularly interested in this relationship are referred to the review of Raab (1936). Raab's enthusiasm, however, has led him to many conclusions lacking a sound foundation. 


\section{CHAP'TER II}

\section{THE REGULATION OF GROW'TH BY THE PITUITARY BODY}

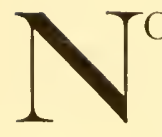

INVESTIGA'TOR has succeeded in preparing a satisfactorily "pure" growth-promoting extract of the pituitary body. There is general agreement that the pituitary, among endocrine glands, is the most important regulator of growth; but whether this regulation is effected by a specific "growth-promoting hormone" or by direct or indirect combined effects of other pituitary hormones, such as the lactogenic and the thyrotropic hormones, remains an undecided issue.

Particularly Riddle and his colleagues oppose the view that a specific growth-promoting hormone is secreted by the anterior pituitary. They suggest that the combined action probably of the thyrotropic and lactogenic hormones accounts for the principal somatic effects of growth-promoting extracts and have put forward suggestive but not conclusive evidence in favor of this view. All the potent extracts which they have examined contained both of these hormones to which they attribute any calorigenic effects such extracts may have (Riddle and others, I936). Bates and his coworkers (1937) found that the injection of "relatively highly purified preparations" of the lactogenic hormone into hypophysectomized pigeons caused body-growth as well as a marked increase in the weight of the liver. ${ }^{.}$Similar effects together with a growth of the intestine were observed in normal pigeons. The only studies which have been consid-

${ }^{2}$ Effkemann and Herold (1935) concluded that extracts of organs other than the pituitary may cause moderate or marked hypertrophy of the liver in the pigeon and rat without striking associated changes in body-weight. However, they believed that pituitary extract brought about specific morphological changes. 
ered successful in mammals were made in mice with hereditary dwarfism. According to Riddle (1935), and Bates, Laanes, and Riddle (I935), growth in such mice can be produced by thyrotropic extracts or, much less effectively, by lactogenic extracts. They observed a marked synergism of the effect if both extracts were administered. "Follicle-stimulating hormone," from the blood of the pregnant mare, was without action. Kemp and Marx (1936) agreed that lactogenic extracts produce a definite growth of mice with hereditary dwarfism. They found thyroxine more effective than thyrotropic hormone, especially in combination with a pituitary growth-promoting extract. It is of interest that the degenerative changes in the gonads of such mice were corrected and that normal gonads were maintained by a variety of treatments - growth-promoting extract or lactogenic extract or thyrotropic extract or thyroxine.

However, there are numerous objections to the view that the thyrotropic hormone or the lactogenic hormone or both account for the principal effects of growth-promoting extracts. Conclusions drawn from experiments in pigeons probably have only a limited significance in mammals. Thyrotropic hormone antagonizes the growth-promoting effect of the lactogenic hormone in pigeons (Bates, Riddle, and Lahr, I 937), whereas the two hormones were found to have a synergistic effect on growth in the dwarfed mouse. Hypophysectomized rats are very sensitive toward pituitary growthpromoting extracts; yet no investigator has succeeded in causing such animals to grow by administering either lactogenic hormone or thyrotropic hormone or a combination of the two. Although it must be admitted that partially refined grow th-promoting extracts are usually more certain and more persistent in their action than highly purified preparations, the same remark applies to comparisons of the action of such partially refined extracts with that of the combined administration of lactogenic and thyrotropic hormone to mice with hereditary dwarfism. Bates, Laanes, and Riddle (I935) ob- 
served that the maximum effect on the growth of dwarfed mice was obtained by administering a growth-promoting extract ("Phyone") which had been only partially purified and contained lactogenic, thyrotropic, and gonadotropic hormones. Moreover, some authors have reported success in extracting from the anterior pituitary a growth-promoting principle free from gonadotropic hormone (Evans and others) or gonadotropic and thyrotropic hormones (Collip, Selye, and Thomson) or lactogenic and thyrotropic hormones (Dingemanse and Freud, I935).

Some years ago Smith pointed out that the growth which occurred in hypophysectomized rats following the administration of a crude anterior pituitary extract was accompanied by no betterment of the atrophic changes in the thyroid, adrenal glands, and gonads. Also, it is agreed that the growth response to pituitary extract is not reduced as a result of thyroidectomy; in fact, acromegalic-like changes in the bones and joints of guinea pigs receiving anterior pituitary extract may be more pronounced in thyroidectomized than in normal animals (Silberberg, I936; Silberberg and Silberberg, 1937). Mortimer's observations (I 937) suggested that thyroid extract or thyrotropic hormone tends to cause a loss of minerals from certain bones, whereas growth-promoting extract may bring about a sclerosis. According to Moon (I937), suitably large doses of adrenotropic extract inhibit the somatic growth of gonadectomized animals without clearly affecting the growth of the spleen, kidneys, liver, and gastrointestinal tract; therefore, his results are in agreement with the view that effects on the adrenal cortex play no positive role in the general growth-promoting effect of a pituitary extract.

The only sensible verdict to render in answer to the plea that the anterior pituitary elaborates (or does not elaborate) a specific growth-promoting hormone is the Scotch verdict of "not proved." Although the thyroid, gonads, and adrenals undoubtedly are important in the regulation of growth, it appears unlikely that changes in the secretory performance of 
these glands account for the growth-promoting effects of anterior pituitary extract. Future work must decide the significance of the lactogenic hormone in growth, as far as growth is affected by the pituitary body. Also, without confirmation, it is hazardous to affirm or deny either that "growth-hormone" free from lactogenic (and other) hormone can be prepared or that a suitable combination of anterior pituitary "hormones," with little effect separately, can imitate all the growth-promoting effects of a suitable anterior pituitary extract. Therefore, the reader should recognize that the use of the term "growth-promoting hormone" in other parts of this chapter and book is dictated by convenience rather than by a belief that it deserves more than a qualified acceptance.

Recent reports on visceral or somatic abnormalities caused by hypophysial deficiency.-Schofield and Blount (I937) observed that the removal of the anterior pituitary from larvae of Amblystoma punctatum is followed by a general reduction in growth, including the growth in length of the digestive tract. They believed that the latter change is causally related to the former. The reports of other investigators, previously reviewed, indicated that hypophysectomy in larval or immature salamanders of a closely related species ( $A$. tigrinum) does not significantly affect growth. Aubrun (I935) removed the pars glandularis or the neurointermediate lobe from Bufo arenarum and observed cutaneous changes-hyperkeratosis and lessened secretion, or paling and capillary dilatation resembling those previously described by Giusti and Houssay.

Only a few reports dealing with mammals need be considered here. Houssay and Lascano Gonzales (1935) studied the effects of hypophysectomy on the dog's spleen. In the young dog, splenic growth was reduced; in the adult dog, splenic atrophy appeared to be associated with an increase in the size of the splenic follicle and an increase in the number of malpighian corpuscles. The authors considered that the changes resembled those characteristic of senility. The re- 
port of Freud (1935) on the rapidity of atrophy of certain endocrine organs after hypophysectomy is of interest to investigators. According to this author, complete hypophysectomy in the rat is followed by a maximum atrophy of the gonads in 8-Io days, of the secondary sexual organs in 8-25 days, of the thyroid in 10 days, and of the adrenals in $\mathrm{I}_{4}{ }^{-1} 8$ days. Lüth's report (I937) indicated that spontaneous hypophysial deficiency in man (Simmonds' disease) may occur independently of genetic constitution. He discovered cases of apparent hypophysial deficiency in individuals with normal identical twins.

The effects of growth-promoting hormone (anterior pituitary extract or tissue). - There is a number of recent observations, some clear cut, some difficult to classify otherwise, which bear on the physiology and pharmacology of the growth-promoting hormone. Murayama, Gurchot, and Guttentag (I937) concluded that a high concentration (2-4 per cent) of a commercial growth-promoting extract inhibits the root-growth of seedlings of Lupinus albus; nothing in the report indicates that this is a specific effect either of growthpromoting or, indeed, of anterior pituitary extracts. Fresh fowl pituitary was inserted into incubating hen's eggs by Pighini (I937) on the third to the fifth day of incubation. The tissue was absorbed and appeared to favor embryonic growth (seventeenth day of incubation). However, Wolff and Stoll (1937) believe that embryonic growth and differentiation, including the endocrine organs, takes place in the fowl in the absence of a functional pituitary body. All the other experiments were performed in mammals. Several investigators have found that the administration of anterior pituitary extract to pregnant animals, such as the rat, may prolong gestation and promote fetal growth; however, it usually appeared that such changes should be attributed to gonadotropic - abnormally prolonged secretion by corpora lutea-rather than to growth-promoting hormone. Recently, Watts (1935) reported that she was able to cause a significant increase in the 
weight of both fetuses and mother by administering certain growth-promoting extracts to pregnant rats. Greep (1936) studied the replacement value of pituitary grafts inserted into the sella of rats of both sexes immediately after hypophysectomy, which was performed when the animals were four weeks old. He obtained partial replacement in about threefourths of the animals ( 5 males and 32 females), in that growth occurred but did not proceed beyond one-half to twothirds the normal adult level. The performance of the sexual glands was often essentially normal and will be discussed in chapter iii.

Especially among clinicians there has always been considerable interest in the changes in bones and joints attributed to alterations in the secretory activity of the anterior pituitary. Recently Coryn (1936) has reviewed from a clinician's viewpoint the etiological relationship between diseases of bones and joints and changes in the endocrine glands. He concluded that pituitary hyperfunction-e.g., oxyphil adenoma of acromegaly-accelerates cellular proliferation and only in this way affects endochondral osteogenesis. He denied that any secretion of the normal or abnormal anterior pituitary alters hyaline cartilage or causes hypertrophy of cartilage cells or calcification of osseous tissue. Also, he believed that ankylosing or deforming arthritides do not result from a disturbance of pituitary function, contrary to experimental and clinical observations of others. Silberberg (I936), as well as Silberberg and Silberberg (1 936-37), recently have studied the changes in the bones and joints of guinea pigs receiving daily injections of an acid extract of the anterior pituitary of the ox for $\mathrm{I}-20$ weeks. The authors concluded that it is thus possible to produce changes in the joints and in the chondro-osseous junctions of the ribs resembling acromegalic arthropathia and acromegalic rosary. Also, they found that callus-formation was delayed by the administration of the extract. All these changes were equally or more pronounced in thyroidectomized guinea pigs. The reader is 


\section{THE PITUITARY BODY}

referred to the authors' publications for a detailed account of their findings, which include observations of the effects of such an extract on other phases of bone-growth in normal and ovariectomized animals. Mortimer (I937) carefully studied the changes in bones, especially those of the cranium of the albino rat, resulting from hypophysectomy or from the administration of growth-promoting extract. His observations cannot be adequately summarized in a few words, but should be read in the original communication by those who are interested in the endocrine phase of bone-growth and maintenance.

It will be recalled that the principal gross biochemical changes in the bodies of normal rats, receiving growth-promoting hormone compared with littermates not so treated, consist of a diminution in the percentage of "fat" and an increase in the percentage of water, protein, fat-free dry tissue, and ash. Lee and Ayres (1936) have recently studied some of the similar biochemical aspects of hypophysectomy in sixteen pairs of littermate rats, one of each pair being hypophysectomized at a weight of about $210 \mathrm{gm}$. All the animals received the same food in the same quantity. The weight loss of the hypophysectomized rats was about 20 per cent greater than that of the normal rats; however, the normal animals lost more fat (6o per cent loss compared with 28 per cent loss in operated rats). ${ }^{2}$ There was a 20 per cent loss of body nitrogen in the hypophysectomized rats, whereas there was no loss in the normal group. The percentage of creatine and creatinine in the carcass was the same in both groups. In general, the changes observed in hypophysectomized rats were the reverse of those following injections of growth-promoting extract. Lee and Ayres also studied a number of nitrogenous constituents, both protein and non-protein, of the liver. The only conspicuous changes were in the total non-protein nitrogen, amino acids, and urea, all of which were present in higher concentration in the liver of the hypophy-

2 Substances extracted by ether. 
sectomized animal. According to Reiss, Schwarz, and Fleischmann (1936), the administration of growth-promoting extract (containing some adrenotropic but no thyrotropic or gonadotropic hormone) to the starved dog or rabbit causes, even after a few hours, a considerable fall in the "rest" $N$ $(10-49$ per cent) and free arginine ( $19-43$ per cent) associated with a rise of 2 $\mathrm{I}-66$ per cent in the urea $\mathrm{N}$ of the blood. Their results are not fully in accord with those previously reported by others. In a study of the phosphatase activity of bone and kidney, Wilkins and others (1935) were unable to detect any significant difference in adult female rats, some of which received injections of a potent growth-promoting extract. Body, bone, and kidney weights were all increased as a result of the treatment.

The relationship between the growth-promoting hormone and other glands of internal secretion. I. The gonads. ${ }^{3}$-Perhaps the simplest interpretation of the effect of gonadectomy on growth in certain animals is that the effect is principally due to a change in the secretory activity of the anterior pituitary. Recently, studies of growth-alterations following gonadectomy in the rat have been reported by Holt, Keeton, and Vennesland (1936), Billeter (1937), Freudenberger and Hashimoto (1937), and Freudenberger and Howard (1937). Castrated and normal male rats gow at about the same rate; however, the normal male tends to grow larger. The spayed female rat clearly grows more rapidly and maintains its weight better than the normal female. The difference is not due to the deposition of fat and may amount to 20 per cent at an age of I 3 weeks ( 9 weeks after spaying). The glands of internal secretion as well as other viscera are heavier; the most striking change is in the thymus which may be 75 per cent heavier than that of the normal female.

At least in the case of the female rat, the internal secre-

3 Growth-promoting extract, like others derived from the anterior pituitary, may contain a substance inhibiting certain gonadotropic effects. This substance has been named "pituitary antagonist" by Evans and is discussed in chap. iii. 
tions of the ovary appear to inhibit the secretion of growthpromoting hormone by the pituitary. This belief is further strengthened by the observation of Spencer, D'Amour, and Gustavson that the repeated injection of oestrone in to growing rats significantly inhibits growth. Their experiments have been extended by Zondek (1936-37), Billeter (1937), and Freudenberger and Clausen (1 937). ${ }^{4}$ Small doses of oestrone, such as 3 rat-units daily, inhibit the growth of spayed rats so that they may weigh less than non-injected spayed rats and, often, less even than normal non-injected rats. However, such small doses must be given from an early age and have little effect if first injected when growth is nearing completion. The inhibition of growth due to larger doses of oestrone (e.g., $0.02 \mathrm{mg}$. on alternate days) affects the glands of internal secretion and, with the exception of the liver, almost all the other viscera including the central nervous system. Zondek used large doses of oestradiol benzoate in his experiments (e.g., I80,000 mouse-units of "Dimenformon" in I 8 weeks). He concluded that the dwarfing of rats by this treatment was caused by a marked hypophysial deficiency, which in some cases was complete, inasmuch as no further growth occurred after treatment was stopped, unless anterior pituitary growth-promoting extract was injected. Zondek, also, performed experiments with fowls in which he observed osteosclerosis (femur, tibia, and fibula). ${ }^{5}$

2. The thyroid.-The possible importance of the thyrotropic hormone as a participant in the growth effects of anterior pituitary extract has already been discussed. There can be no question concerning the inhibition of growth which may appear if complete thyroid deficiency is produced early in life- a statement again emphasized by the recent study of

${ }_{4}^{4}$ Shumacker and Lamont (1935) were unable to detect any change in the growth of 6 rats receiving 9 rat-units of oestrone daily between the ages of 23 and 90 days.

5 Numerous other observations on the effects of oestrogens on the pituitary and its secretions are discussed elsewhere (particularly chaps. $i$ and iii). Lauson, Heller, and Sevringhaus (1937) studied the effects of an oestrogen in the mature spayed rat. They particularly investigated the pituitary, adrenals, and thymus. 
Binswanger (I936), who performed his experiments in dogs. There is real doubt, however, that the thyrotropic hormone which may be found in growth-promoting extracts is of great importance so far as growth effects are concerned. ${ }^{6}$

3. The adrenals.-Moon (I 937) found that the injection of the anterior pituitary hormone stimulating the adrenal cortex inhibits somatic growth in young rats of both sexes (the author administered I $3-42$ "units" over a period of $2-4$ weeks). There was little or no effect on the grow th of the spleen, liver, kidneys, and alimentary canal. Swingle and others (1936) increased the period of survival of adrenalectomized cats by administering a growth-promoting pituitary extract; however, they attributed the beneficial effect of the extract to gonadotropic hormone rather than to a growth-promoting hormone.

4. The thymus.--The more recent observations suggest that an internal secretion of the thymus promotes growth and development. However, there are no observations indicating to what extent such an action may be related to the secretion of a growth-promoting hormone by the pituitary. Rowntree, Clark, and Hanson (1935) reported that the administration of an extract of the thymus to rats through several generations finally led to precocious growth and development which might be very marked early in life. The reverse experiment, thymic deficiency by thymectomy, produced after 2-3 generations a retardation of early growth, especially marked at an age of about I month (Einhorn and Rowntree, I936). The rate of development appeared not to be affected. Parhon and Coban (1936) also found that thymectomy retards the growth of fowls (Leghorn and Rhode Island varieties). They removed both lobes of the thymus when the birds were about 3 weeks old; at an age of 6 months the thymectomized fowls weighed about 30 per cent less than the nonoperated controls.

${ }^{6}$ The growth of the liver in the duck in relation to the thyroid, gonads, and hypophysis is discussed by Benoit (1937). 
5. The epiphysis.-Engel (1936), as well as Kup (I 936), continues to champion the view that a pineal secretion inhibits the growth-promoting effects of anterior pituitary secretion. However, the evidence which they are able to assemble does not enable one to attach even hypothetical value to this belief. Some observations suggest the opposite effect. For example, Takács (I 935) fed dried calf epiphysis to young fowls (10-35 mg. per day). The birds receiving epiphysis for 4 months weighed more than 200 per cent more than the controls. The excess weight was striking ( 102 per cent) but less pronounced after 5 months' feeding, when the experiment was terminated.

Deficiency of vitamins or minerals and the growth-promoting hormone.- It appears that failure of growth due to a deficiency of vitamin A or of the "growth factor" of casein cannot be attributed to a failure in secretion of growth-promoting hormone. This is indicated by the experiments of MargitayBecht and Wallner (I937). The authors produced growth stasis in young rats by diets deficient either in vitamin A or in the alcohol-ether extract of casein. In neither case did the administration of anterior pituitary growth-promoting extract cause a resumption of growth. There remains, of course, the possibility that the dietary deficiency had rendered the tissues refractory toward the hormone.

Orent-Keiles, Robinson, and McCollum (1937) concluded that a sodium-deficient diet, more than diets deficient in $\mathrm{Cl}$ or $\mathrm{NaCl}$, interferes with growth in the rat. Whether or not this change as well as others, particularly in the female organs of reproduction, depends to an important extent on changes in the anterior pituitary is not known. According to Hove, Elvehjem, and Hart (I 937), zinc appears to be an important factor in the secretion or peripheral action of a pituitary hormone controlling (favoring) the movement and tone of the digestive tract of the rat. With a deficiency of zinc in the diet and therefore with a deficiency of this hypothetical hormone, intestinal absorption is delayed and reduced so 
that growth is retarded. The protein phase of metabolism appeared particularly to be reduced. 'The authors' dismissal of the growth-promoting hormone as the important factor is largely based on the greater effect of whole pituitary implants in comparison with that of a growth-promoting extract ("Antuitrin G"). However, the comparison was made under different conditions as to the age of the rats and the duration of zinc-deficiency. 'Therefore, until growth-promoting hormone or other better-defined anterior pituitary hormones have been clearly excluded, it is undesirable to identify a new pituitary hormone on so slender a basis.

The effect of growth-promoting hormone on the growth of neoplasms.-Engel (1935) has made further observations on the relationship of the pituitary and of gonadotropic hormones (prolan, gonadotropic hormone of pregnant-mare serum) to the growth of transplants of Ehrlich's adenocarcinoma in mice. He concluded that gonadotropic hormones may have some inhibiting effect on the growth of the tumor and that pineal extract may have a marked inhibitory effect. He believed that the depressing effect of pineal extract is indirect and due to a secretory inhibition of, or antagonism toward, the growth-promoting hormone. Several recent authors agree that hypophysectomy retards the growth of malignant neoplasms, especially if transplantation is delayed several weeks after operation (Walker mammary carcinoma: Samuels and Ball, I935; neoplasm due to $1: 2: 5: 6$ dibenzanthracene: Ball and Samuels, I 936; transplanted neoplasm: Reiss, I936). Reiss found that the oxygen-consumption of such tumors is low, but that aerobic glycolysis remains unchanged or increases. Emge and Murphy (1936), investigating the grow th of autogenous sarcoma in the rat, observed no striking effect of hypophysectomy on tumor-growth except when transplantation was undertaken some time after operation. 'They could detect no increased rate of tumor-growth as a result of the administration of growth-promoting extract to tumor-bearing normal or hypophysectomized rats. Likewise 
Zondek (1937) concluded that the secretion of growth-promoting hormone does not influence the growth of a sarcoma due to benzpyrene; for the tumor grew no better in normal rats than in rats dwarfed because of the injection of large doses of oestradiol benzoate.

Druckrey ( 1936 ) believed that pituitary gonadotropic hormone inhibits tumor-growth in the rat. As malignant neoplasms he used the Flexner-Jobling carcinoma and the Jensen sarcoma. Shortly after castration, tumor-growth appeared to be unusually rapid. Later, however (4-6 months after castration), when the gonadectomized animal's pituitary contains and secretes the maximum amount of gonadotropic hormone, inhibition and even failure of tumor-growth appeared. Largely from this finding Druckrey reached the conclusion stated above.

Both recent and older observations permit the drawing of only limited conclusions as to the control of the growth of neoplasms by the anterior pituitary. If there is a complete pituitary deficiency, the initiation of neoplastic growth is more difficult; if the deficiency is produced after neoplastic growth is under way, further growth may take place at a slower rate. Certainly the pituitary appears not to be an important regulator of cellular proliferation in tumors. The conclusion of Engel--that pineal extract inhibits the growth of a carcinoma by acting on the pituitary-as well as the inference of Druckrey - that the growth of a sarcoma or carcinoma is inhibited by the secretion of gonadotropic hormone-hardly deserve acceptance without data from more complete experiments.

The assay of growth-promoting extracts.-Several matters of technic must be mentioned in connection with the assay of growth-promoting extract. Evans, Pencharz, and Simpson (1935) pointed out that crude rather than purified extracts seem to have more certain effects and may be active if given to animals no longer responding to a purified extract. Also, Mortimer (I937) found "resistance" appeared later toward

$$
\text { [ 44] }
$$


crude extracts than toward more purified extracts. It is difficult to correlate these observations with the conception of "antihormones."

Evans and his colleagues found that if hypophysectomized rats failed to respond to an extract, there might occur a striking response if glucose solution was also administered parenterally. ${ }^{7}$

Factors influencing the assay of growth-promoting hormone have been studied in normal and hypophysectomized rats by Chow, Chang, Chen, and van Dyke (1938). In normal rats, in confirmation of the work of others, they found the young adult female rat most suitable. Sex does not affect the response of hypophysectomized rats which, in terms of the percentage change in weight, is at least twice as great as that of normal rats. Rats with a hypophysial deficiency of 8-10 months are less sensitive than those hypophysectomized I-6 months before assay. Differences in response in relation to dosage are more easily recognized in hypophysectomized rats, whereas in normal rats a doubling of the dose may not be followed by a change in response which is convincingly greater. In either normal or hypophysectomized rats the optimum period of injection probably is not longer than 10 days. Even 6 weeks after the use of rats for assay, their response to a second course of injections is reduced.

The preparation and purity of growth-promoting extracts of the anterior pituitary. - The only recent attempts to purify growth-promoting extracts are those of Dingemanse and Freud (1935). They stated that their preparation contained neither lactogenic nor thyrotropic hormone and that 0.025 mg. administered intraperitoneally once daily to hypophysectomized rats (120-80 gm.) caused an average gain of weight amounting to $7 \mathrm{gm}$. following administration for one week. The method they described required the following

7 According to Shelton, Cavanaugh, and Long (1935), the effect of growth-promoting extract in adult female rats is potentiated by the injection of $0.5 \mathrm{cc}$. of human serum $3^{-6}$ weeks previously. 
steps: adsorption on norit from a weakly alkaline solution, elution of the hormone by phenol, and subsequent precipitation by a solution of one part of alcohol in two parts of ether. The authors also concluded that the hormone could dialyze through collodion membranes and that the nitrogen content of the material in the sac was higher than that of the dialyzed material. The dialysis was performed at a very high $\mathrm{pH}$ (o.o I N NaOH).

Summary.-The clear-cut identification of a growth-promoting hormone in the anterior pituitary is perhaps the problem of greatest immediate importance to those interested in this aspect of the physiology of the pituitary body. On the other hand, if such a hormone is not secreted by the gland, new interpretations of much satisfactory data will have to be made. It is still possible, however, provisionally to use the term "growth-promoting hormone."

Few of the recent observations require reference in this summary. Experimentally, changes in bones and joints, some resembling those of acromegaly, have been more extensively studied. Additional data on general biochemical changes due to hypophysectomy have been secured. As far as other glands of internal secretion are concerned, there is additional evidence, at least in the rat, that the oestrogenic secretion of the ovary may act as a brake on general growth. Endocrine glands such as the thyroid, adrenals, thymus, and epiphysis may affect growth-sometimes positively, sometimes negatively. How such effects are related to excessive or reduced secretion of the growth-promoting hormone in no case has been convincingly demonstrated. The growth of malignant neoplasms may be slightly favored by anterior pituitary secretion; this effect, however, appears not to be important. Recently little attention has been paid to the purification of the growth-promoting hormone. This may prove to be an impossible task made even less attractive by the difficulties of accurate assay. 


\section{CHAPTER III}

\section{THE GONADO'TROPIC HORMONES OF THE PITUITARY BODY}

$\mathrm{I}^{1}$

F THE number of articles published were acceptable as a safe basis of judgment, it could easily be concluded that the gonadotropic hormones are the most important secreted by the anterior pituitary. This field of inquiry is attractive not only because of its inherent importance but also because the probability of securing fruitful results is great. There can be no doubt that the anterior pituitary secretes gonadotropic hormone(s) essential for the normal functioning of the gonads of mammals and other vertebrates. As a result, nearly every investigator interested in the physiology of the gonads has quickly entered or wandered into the rich and diversified field of pituitary-gonad interrelationships-sometimes without realizing he is there. Furthermore, work may be greatly facilitated by the fact that the assay of the hormones with a fair degree of accuracy is often, but not always, easily accomplished.

Despite all the labor represented by a vast number of reports during the past few years, it is not yet possible to enumerate satisfactorily the gonadotropic hormones of the pituitary. Separate follicle-stimulating and luteinizing hormones are generally believed to exist; it is by no means certain that the former specifically maintains the mammalian seminiferous tubules, whereas the latter insures the normal secretion of testicular hormone by the interstitial cells. It has been reported that principles synergizing with or antagonizing gonadotropic hormone have been extracted from the pituitary; however, their physiological importance is largely postulated from deduction. Several authors have recognized the great importance of excluding the animal's own 
pituitary in determining accurately qualitative and quantitative effects of extracts. The increased accuracy of interpretation more than justifies the great increase of labor required. Final judgment on the effect(s) of an extract should be based upon experiments with hypophysectomized animals.

The follicle-stimulating hormone often excreted in human urine in considerable amounts after spaying or castration or after the menopause probably originates in the anterior pituitary. On the other hand, the gonadotropic substances discussed in chapter iv appear not to be secreted by the hypophysis. These substances are prolan (from the chorionic cells of the human pregnant uterus), the gonadotropic hormone characteristic of pregnant-mare serum (likewise probably secreted by chorionic or endometrial cells), and the gonadotropic hormones of neoplasms such as hydatidiform mole, chorionepithelioma, and testicular tumor (from the cells of the neoplasms).

In this chapter a systematic account of pituitary-gonad interrelationships, especially as these are understood from later work, will be attempted. It is hoped that the reader will recognize the diversity of the literature both in subject matter and in quality. Diversity in the former often makes arrangement difficult. Diversity in the latter may lead to definite conclusions, either sound or wrong. Sometimes, however, the only reasonable position to take is one of suspended judgment, unwelcome as this may be to many readers.

THE BIOLOGY OF THE GONADOTROPIC

HORMONES IN VERTEBRATES

Fishes.-Using an extract of ox pituitary, Young and Bellerby (I935) were unable to produce either significant changes in the gonads or metamorphosis in the lamprey (Lampetra planeri). However, changes in body-shape and marked cloacal swelling, both secondary sexual characteristics of the period preceding spawning, appeared. In earlier reports several authors had succeeded in producing spawning 
or hypertrophy of the gonads in other fishes. Usually changes in the gonads or secondary sexual organs of fishes, including spawning, can be produced by fish pituitary but not by mammalian pituitary or by extract of the mammalian pituitary. However, dosage may not have been adequate. Descriptions of experiments and references to other work can be found in the reports of Gerbilsky and Kashchenko (1937) and of von Ihering and de Azevedo (1937). Artemoff (1936) successfully used the pituitary of the frog.

Amphibia.-The majority of the reports previously reviewed indicated that the amphibian pituitary has an importance in the maintenance of the gonads corresponding to that in mammals. The most that will be attempted here is to bring the references up to date by citing the work of recent authors.

I. Amuran amphibia.-Several aspects of the biology of gonadotropic hormones have been studied in six species of frogs (Bombinator igneus, ${ }^{\mathrm{I}}$ Hyla arborea, ${ }^{\mathrm{I}}$ Pelobates fuscus, ${ }^{\mathrm{I}}$ Rana esculenta, ${ }^{1} R$. pipiens, ${ }^{2}$ and $R$. temporariain ${ }^{3,4}$. Several authors have studied ovulation or oviposition or both. Rostand administered the extract of 6-20 pituitary glands of the same species to $R$. esculenta, R. temporaria, or Bufo vulgaris. He found that ovulation could be produced 5 months before the normal time. It was more difficult to induce ovulation earlier; moreover, the ova then were often not mature and could not be fertilized. Similar results were reported by Gallien who used $R$. temporaria. The number of eggs extruded appeared to be proportional to the dose of frog pituitary (6-9 glands as a saline extract) or beef anterior lobe (1.6-3 gm. as an alkaline extract). The very low potency of the pituitary of the ox was previously reported by others (e.g., Rostand using $H$. arborea). Gallien observed no effect from the injection of urine of spayed women $(3-8 \mathrm{cc}$.) or urine of pregnancy $(7-9$ cc.). According to Rostand, extract of the pituitary of $R$. esculenta or Bufo vulgaris can readily induce

\footnotetext{
${ }^{2}$ Rostand (1934-35). ${ }^{2}$ Rugh (1937). 3 Gallien (1937). ${ }^{4}$ Shapiro (I937).
} 


\section{THE PITUITARY BODY}

ovulation in $H$. arborea but not in Bombinator igneus, unless the ovary contains ripe ova. He found P.fuscus, even when the ovaries contained ripe ova, very insensitive toward either type of pituitary extract. ${ }^{5}$

Rugh concluded that the anterior pituitary of the male frog (R. pipiens), although somewhat heavier than that of the female, contains little more than half the amount of ovulation-inducing hormone. He stated that usually within 24 hours oviposition in mature frogs followed the intraperitoneal administration of the pars glandularis of two adult females or four adult males. In the hibernating male frog of the same species, Rugh found that about 6 hours after the administration of anterior pituitary gonadotropic hormone, amplexus (with an ovulating female of the same species only) and the release of all mature spermatozoa could take place. Spermatozoa were found in the lumen of seminiferous tubules, in Bowman's capsule, the renal tubules, and the ureter. In another article, Rugh reported on seasonal changes in ovarian and pituitary weight and the relationship of such changes to alterations in pituitary gonadotropic effects. Shapiro (193637) has particularly studied the clasping reflex in R. temporaria and Xenopus laevis (a toad). He decided that secretions of both the pars glandularis and the testis or ovary are necessary for the initiation of the reflex. The reflex, followed by ovulation, oviposition, and fertilization, could be produced in Xenopus by the injection of pregnancy-urine extract or an acid extract of the pars glandularis of the sheep or goat. In $B$. arenarum the removal of the pars glandularis a few days after subtotal extirpation of testis tissue prevents the marked compensatory growth of the latter (Houssay and LascanoGonzalez, I935).

The implantation of pituitary tissue of the frog or toad can cause ovarian stimulation in the immature mouse (Benazzi, 1937; and Zwarenstein, I 937). However, the potency of amphibian pituitary is much less than that of mouse pituitary.

${ }^{5}$ See also Osima (1937). 
Shapiro (I 937) attributed the inhibiting or stimulating effects of an extract of sheep pituitary, used chiefly for its lactogenic effects, to contaminating gonadotropic hormone. The author studied the extract's effects on the amphibian testis.

2. Urodele amphibia.-Adams and Mayo (1936) investigated the effects of homo-implants on oviposition in a salamander, Triturus viridescens. They were able to produce oviposition in the nonbreeding months stretching from October to early March. The effect appeared earlier at $20^{\circ}$ than at $14^{\circ} \mathrm{C}$. (resembling the observation of Bellerby in $X$. laevis; Bellerby used beef anterior-lobe extract). The male pituitary was perhaps the more potent; the pituitaries of animals gonadectomized $4^{-} 9^{8}$ days previously were possibly, but not definitely, more potent than normal glands. Kleinschmidt (I937) studied the changes in the male gonads of hypophysectomized larvae of $T$. vulgaris for many months postoperatively. Although regressive changes in the germinal cells of the testis were clearly marked, especially $3-$ IO months after hypophysectomy, yet 20 months postoperatively islands of healthy cells with spermatogonia, resembling normal testicular tissue of animals four months old, could be found. The Wolffian and Müllerian ducts were both well developed; however, the latter was not joined to the cloaca. Some degenerative changes in the follicle-cells of the testis were found. The fat body was not altered until an age of I 4 months, after which it underwent hypertrophy because of hyperplasia.

Reptiles.-According to Evans (1935), the gonads of immature young lizards (Anolis carolinensis) of either sex could be stimulated by prolan or by extract of whole sheep pituitary. In the male the same effects were produced by either extract: testicular hypertrophy, hypertrophy of the hemipenis, epididymis, and vas deferens, spermatogenesis, elevation of the dorsal crest, frequent molting, behavior changes, etc. In female animals prolan brought about hypertrophy of the ovary and oviduct as well as more frequent molting and 


\section{THE PITUITARY BODY}

behavior changes. Only sheep pituitary extract caused, in addition to these changes, maturation of ova or ovulation and oviposition. Mellish ( I 936) investigated pituitary-gonad interrelationships in the horned lizard, Phrynosoma cornutum. Prolonged illumination of animals furnished abundant food and kept at a high temperature $\left(35^{\circ} \mathrm{C}\right.$.) for six weeks during winter had little effect on the gonads (control lizards hibernating at $5^{\circ} \mathrm{C}$.). On the other hand, if the experimental animals were also given the equivalent of $3 \mathrm{gm}$. of whole pituitary of the pig, the gonads of both sexes were stimulated so as to resemble those of animals early in the breeding season. Other experiments in similar lizards have been reported by Mellish and Meyer (I937). An increased ovarian weight, largely due to an increased deposition of yolk, was produced by the administration of various anterior pituitary extracts or pregnant-mare serum. The animals were well fed and kept at $32^{\circ} \mathrm{C}$. under continuous artificial illumination. None of the animals was hypophysectomized. An unfractionated anterior pituitary extract did not affect the ovaries of animals hibernating at $5^{\circ} \mathrm{C}$. At that temperature there appeared to be only partial absorption of the extract.

Forbes ( I 937) investigated the action of an alkaline extract of whole pituitary of sheep in immature alligators $4^{-1} 8$ months after hatching). Some hypertophy of the ovaries together with marked growth of the oviducts occurred in female animals. There was no effect on the Wolffian ducts. In males a marked hypertrophy of the testes, without spermatogenesis, was produced. The size of the mesonephron in males receiving the extract was reduced.

Birds. - In the past few years there has been reported a considerable number of new or more detailed observations on the physiology of gonadotropic hormones in birds. Almost all deal with hormones of pituitary origin. ${ }^{6}$ An interesting as-

${ }^{6}$ Later reports also support the conclusion that prolan has no significant effect on the gonads of birds (Hill and Parkes, 1935; Witschi and Keck, 1935; Uhl and others, 1937). Breneman's results (1936) apparently were inconclusive. Koch (1935) could not confirm his earlier report that prolan brings about an increase in the egg-laying performances of hens. 
pect of work in this field is the manner in which the duration, intensity, and quality of light may affect the reproductive organs of both birds and other vertebrates. There is every likelihood that such effects of light are mediated through the pars glandularis; moreover, such effects are additional evidence in favor of the physiological importance of efferent secretory fibers, innervating the anterior pituitary. In the account which follows, recent general work in birds will be considered first. At the end of this section the effects of light on the sexual organs of other vertebrates, including mammals, will be discussed.

Domm and Dennis (1937) caused definite changes in the gonads of chick embryos by administering sheep pituitary extract (see Fig. 9). Five rat-units were administered daily during the 5-9 days of incubation, the embryos being removed later (e.g., the eighteenth day). In female embryos there occurred hypertrophy of the ovary (chiefly of the medulla) and rudimentary right gonad. (Injections during post-embryonic life did not produce the latter effect.) 'Testicular hypertrophy in male embryos was less frequent and less pronounced; when present it was due principally to a change in interstitial tissue. Alterations of the Müllerian or Wolffian ducts or of the head furnishings did not occur. Hybrid embryos responded better than pure-bred Leghorns. Breneman (1936) studied the effects of follicle-stimulating and luteinizing hormones on the gonads of chicks $5^{-1} 5$ days after hatching. ${ }^{7}$ The author's experiments were often complex, and some of his conclusions appear not to be firmly established. Maximum gonad-stimulation was produced by pituitary follicle-stimulating hormone or pregnant-mare serum. He believed that, as in the mammal, the follicle-stimulating hormone affected the tubules of the testis, whereas the luteinizing hormone acted on the testicular interstitial cells. Also,

${ }^{7}$ The lactogenic hormone, which according to Riddle and others brings about a reduction in the weight of the male or female gonads of fowls or pigeons, was without effect in the very young chicks. 

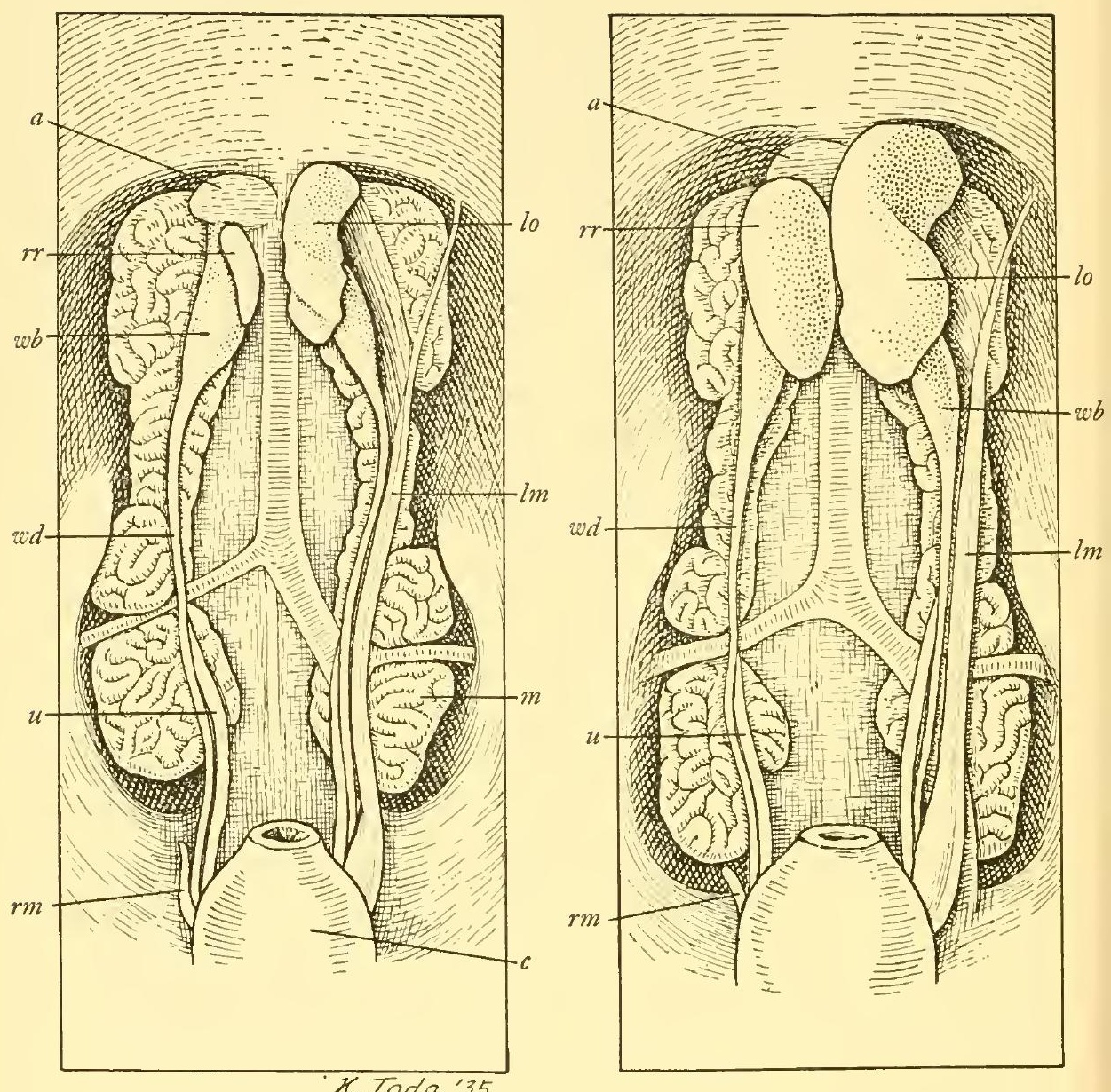

Fig. 9.-The action of pituitary gonadotropic hormone on the urogenital tract of the female chick embryo. (From Domm and Dennis, Proc. Soc. Exp. Biol. Med., 36, 766-69 [1937].)

Left: control; right: treated embryo which received 5 rat-units of sheep pituitary extract for 5 days (fifth to ninth day of incubation); incubation of both embryos continued until the eighteenth day. $r r$ : right rudimentary gonad; lo: left ovary; wb: Wolffian body; wd: Wolffian duct; $l m$ : left Müllerian duct; $r m$ : remnant of right Müllerian duct; $a$ : adrenal; $c$ : cloaca; $m$ : kidney; $u$ : ureter. 
he concluded that the action of follicle-stimulating hormone (of the pig's pituitary) is inhibited if, prior to injection, it has been mixed with luteinizing hormone, lactogenic hormone, or an extract of pregnancy-urine.

Compensatory hypertrophy of the testis as well as several other phases of the pituitary-gonad interrelationship was studied in Leghorn cockerels by Belsky (I936). The author concluded that the compensatory hypertrophy in young birds is associated with pituitary hypertrophy. Under other conditions hypertrophy of the pituitary is associated with an absence or diminution of gonad function (castration or times of seasonal rest of the gonads).

Brown Leghorn hens were hypophysectomized by Hill and Parkes (1935). The expected reversion of the feathers to the male (asexual) type was observed only in the neck hackles. Plumage changes characteristic of thyroidectomy appeared in the hypophysectomized cock. These changes as well as atrophy of the testis and comb could be lessened only for a few days by the injection of beef anterior pituitary extract. The administration of testicular hormone, but not of oestrone, caused growth of the atrophied comb.

Witschi and Keck (I935) investigated the action of extract of the pituitary of the horse and ox on the gonads of the English sparrow. Preliminary observations indicated that great seasonal variations occur in both sexes-marked growth of the gonads occurring in spring and early summer and rapid involution taking place in July and August. ${ }^{8}$ Secondary sexual characters such as the color, shape, and texture of the bill underwent corresponding alterations. The daily administration of pituitary gonadotropic hormone (e.g., 2 rat-units for $16-33$ days) could cause a sixty-fold increase in the size of the testis or ovary of birds with resting gonads. The anticipated alterations in secondary sexual characters, such as

${ }^{8}$ During involution the testis might regress to one-thousandth its previous size; the ovary might atrophy to the size of that of an immature bird $2-3$ months old. 
the color of the bill and the size of the oviduct, were observed (see Figs. IO and II).

The pituitary of birds contains gonadotropic hormone capable of stimulating the mammalian ovary (Leonard, I937, fowl pituitary, ovulation in the rabbit, or ovarian hypertrophy in the normal or hypophysectomized rat; Witschi, Stanley, and Riley, I937, turkey pituitary, ovarian hypertrophy in the normal or hypophysectomized rat). Leonard concluded that the fowl's pituitary contains luteinizing hormone as well as the hormone stimulating the adrenal cortex.

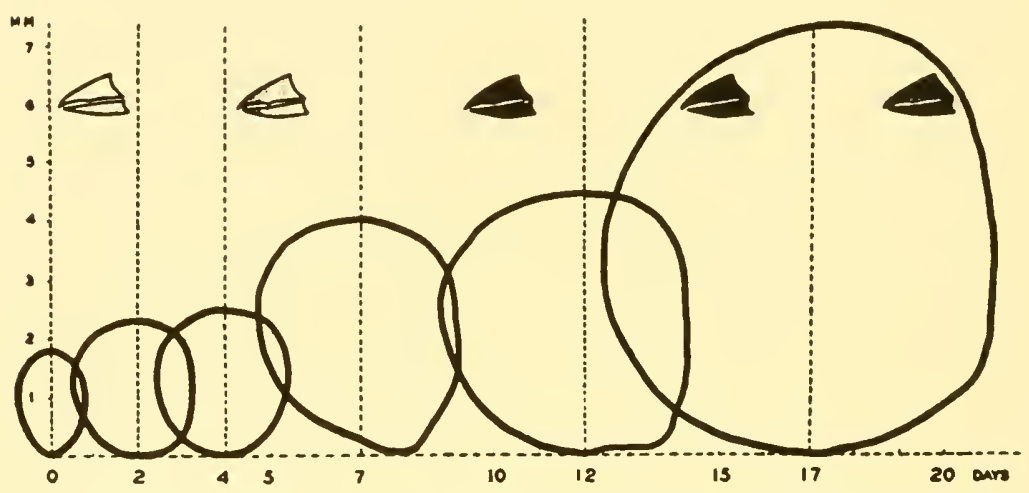

F1G. 10.-The effect of daily injections of 2 rat-units of pituitary gonadotropic hormone on the size of the testis and the color of the bill of the English sparrow. (From Witschi and Keck, Proc. Soc. Exp. Biol. Med., 32, 598-603 [1935].)

A few other observations were made, chiefly in male birds. Evans and others (I936) concluded that the apparently great sensitivity of the immature pigeon's testis toward pituitary gonadotropic hormone depends upon the lack of effect of pituitary "antagonist." If the latter is first removed from a pituitary extract, the ovary of the immature rat is equally sensitive. Riddle and Schooley (1935), basing their assays on the testicular response of the immature ring dove, studied the gonadotropic potency of the pituitary of the pigeon and the rat. The pituitary of pigeons or ring doves, I.8-2.5 months after hatching, contained no gonadotropic 
(follicle-stimulating) hormone. The pituitary of the adult pigeon was more potent than that of the immature rat. Clark, Leonard, and Bump (I937) reported that the testis of the immature pheasant responds well to the administration of sheep pituitary extract. A study of the changes in the testis and comb of chicks receiving injections of male hormone (extract of male urine, dihydroandrosterone benzoate, testosterone, or its propionate) was made by Breneman (I937). In some instances testicular growth was prevented during

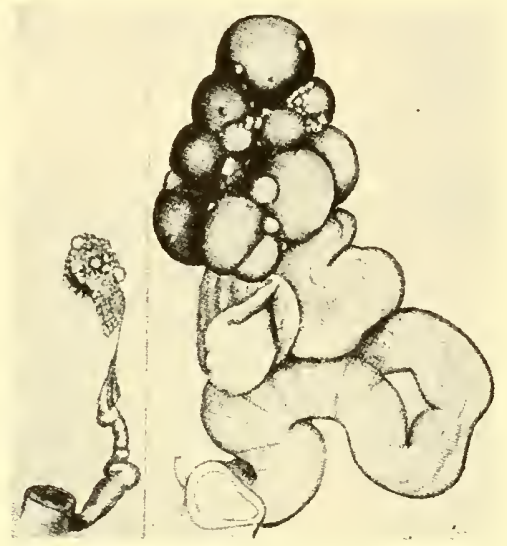

FIG. 11.-The effect of 16 daily injections of 2 rat-units of pituitary gonadotropic hormone on the ovary and oviduct of the English sparrow. (From Witschi and Keck, Proc. Soc. Exp. Biol. Med., 32, 598-603 [1935].) Left: largest of controls; right: injected bird.

the period of injection (5-10 day after hatching) but increased markedly during the three following weeks. Bagg (1936) was interested in the production of teratomata in the fowl's testis by injecting into the gland $0.3 \mathrm{cc}$. of a 5 per cent aqueous solution of $\mathrm{ZnCl}_{2}$. His injection schedules were most varied and his results permit only the following conclusions: (1) previous treatment with sheep pituitary extract made possible the production of teratoma testis in 2 of 26 birds (one in June, the other in August), resembling the effect of zinc chloride solution alone ( 2 of 20 birds in the spring); (2) 
at seasons other than spring, this effect is not produced by zinc chloride solution (200 birds); (3) although prolan was used, there is no evidence that it had any effect.

The effects of light on the activity of the gonads has received increased attention since $1934 .{ }^{\circ}$ In certain vertebrates in which sexual activity is cyclic, i.e., varies with the season, light-variation appears to be the principal environmental change responsible for the growth or decay of activity of the reproductive organs. It is reasonable to conclude that the stimulus of light reflexly initiates the secretion of gonadotropic hormones by the anterior pituitary. ${ }^{10}$ What Bissonnette termed "sexual photo-periodicity" probably depends upon the effect of radiations of different wave-lengths on an opticohypothalamo-hypophysial, nervous glandular mechanism. The most convincing data have been secured in birds and mammals.

According to Hoover (1937), precocious ovulation and spermatogenesis occur in rainbow trout (Salmo irideus), if the fish are subjected to prolonged illumination during the period of sexual inactivity. Such trout normally spawn in March (New Hampshire). In brook trout (Salvelinus fontinalis) spawning takes place when the hours of daylight are diminishing (October to December). Hoover produced in this species ovulation and spermatogenesis three months earlier than normal by increasing and then diminishing the duration of illumination. In the horned lizard, Phrynosoma cornutum, increased light and a higher temperature did not modify the size of the sexual organs during hibernation (Mellish, 1936).

Direct evidence that light may control cyclic sexual activity in birds and mammals is furnished by studies of the morphology of the gonads or of the effects of their internal

9 For recently published, general articles, see Bissonnette (1936) and Marshall (I937).

${ }^{10}$ Marshall (1937) cited evidence that in a few animals increased activity of the gonads is associated with reduced solar illumination. This also appears to be true of the macaque monkey in captivity. 
secretions on behavior or on secondary sexual characters. The inference that these changes represent the effects of a reflex release of anterior pituitary gonadotropic hormones appears to be justified, because (I) the pars glandularis is as necessary for the maintenance of the avian gonads as for the mammalian, (2) the administration of pituitary gonadotropic hormone may markedly stimulate the immature or resting gonads of birds or mammals, (3) in some experiments (in the drake, Benoit, I936; in the ferret, Bissonnette, 1935) it has been shown that the removal of the pars glandularis prevents gonad-stimulation, otherwise occurring as a result of increased illumination, and (4) the anterior pituitary contains nerve fibers, probably secretory in character, which have a likely origin in hypothalamic nuclei. To Rowan (1925) belongs the credit for making the first experimental observations in birds. He concluded that prolonged artificial illumination of the junco (Funco hyemalis) leads to precocious testicular development during the period of sexual inactivity. Recent studies bearing on the effect of light or the mechanism of this effect have been made in game birds ${ }^{\text {II }}$ and in the sparrow and duck.

In the English sparrow (Passer domesticus) the "ration of light" may largely determine testicular growth so that in midwinter increased illumination may cause precocious testicular hypertrophy with spermatogenesis and blackening of the beak. Temperature, as Rowan concluded from his study in the junco, is of little importance. In the spring, however, reduced illumination may partially but not completely suppress normal testicular development (Kirschbaum and Ringoen, 1936). From later studies, the authors (Ringoen and Kirschbaum, 1937) concluded that the testicular response to light depends almost entirely upon a "stimulus through the

"Observations of Clark, Leonard, and Bump (1937) in the grouse, pheasant, and quail. The authors suggested that regression of the gonads in the presence of an apparently adequate photostimulus is due, for unknown reasons, to a lessened anterior pituitary secretion, inasmuch as gonad-stimulation by injected extract can still be produced. 
ocular region." In contrast to this view, Ivanowa (1935) believed that an important part of the effect is due to the action of light on the whole body surface. According to Benoit (I935), neither section of the optic nerves nor enucleation of the eyes prevents precocious testicular growth caused by artificial light in the immature drake. Apparently the light had to reach the empty orbits; for if hoods were placed over the latter, testicular development was prevented. Experiments like those just cited indicate the urgent need for more observations on the afferent pathways of reflex photostimulation of the pituitary in birds. Ivanowa's experiments are open to the objection that the birds' eyes may not have been adequately hooded. It is difficult to believe that there is an inportant peripheral afferent arc other than the optic nerve.

Benoit (1935-37) has made a number of other observations in drakes either immature or during the period of normal regression of the testis. (The effects on the gonad of the female are slight and appear much more slowly.) The testicular response depends not only upon the duration of illumination but also upon the wave-lengths (quality) ${ }^{12}$ used and the distribution of "light-dosage." Testicular growth ceases if, during artificial illumination, the drake's anterior pituitary is removed. The gonadotropic potency of the drake's pituitary in immature mice may be increased if the hours of illumination are experimentally lengthened. Such experimental development of the testis and penis, for some weeks at least, was found to be lessened greatly by thyroidectomy. How thyroid deficiency acts, whether by interfering with secretion by the stimulating organ (anterior pituitary) or by diminishing the response of the end organ (testis), is not clear. The feeding of thyroid extract or the injection of thyroxine accelerates the rate of testicular growth.

Light has been shown to influence greatly the development of the gonads or secondary sexual characters including be-

${ }_{12}$ In one report, Benoit states that red and infrared radiations are especially effective. 
havior in mammals with yearly sexual cycles. Oestrus and the conditions necessary for its production by artificial illumination have been repeatedly studied in the ferret. ${ }^{13}$ Both the "quantity" and the wave-length of light are important. Variations in duration, the quantity of light remaining unaltered, do not influence the rate of acceleration of oestrus (Marshall and Bowden). These authors also concluded that, whereas infrared rays had little effect, ultraviolet rays caused a premature appearance and abnormal persistence of oestrus. Bissonnette found that the light and hair cycles of the ferret depended upon an intact pituitary. Hypophysectomy prevented both oestrus and its associated hair cycle even in animals artificially illuminated. Apparently the effect on hairgrowth may be due to the direct action of a pituitary hormone, inasmuch as cyclic changes in hair-growth are not influenced by gonadectomy. Hill and Parkes were not able to prevent or delay the onset of oestrus and gonad development at the usual time of breeding by keeping animals in darkness. Although the authors agreed that the effect of light on the anterior pituitary probably explains how oestrus is initiated in the anoestrous season by increased illumination, they denied that the increasing length of daylight is responsible for normal oestrus. Bissonnette stated that the latter may be delayed or made to persist by severing both optic nerves. Ferrets in precocious oestrus have been successfully bred by Bissonnette and Bailey.

In a preliminary report, Whitaker (1936) reported that white-footed mice (Peromyscus leucopus noveboracensis) went into oestrus 6- 8 weeks early if they were treated with a commercial ultraviolet lamp. By the increase of the daily ration of artificial illumination a similarly precocious oestrus with pregnancy was produced in the raccoon (Procyon lotor) by Bissonnette and Csech (I937). In the female rat, reversed illumination (light by night, darkness by day), darkness, or

${ }^{1_{3}}$ Late reports are those of Hill and Parkes (1934), Bissonnette (1935), Bissonnette and Bailey (1936), and Marshall and Bowden (1936). 


\section{THE PITUITARY BODY}

a constant auditory stimulus does not affect the periodicity of the oestrous cycle (Browman, 1937). However, the author found that constant illumination might cause a persistence of oestrous smears of the vaginal epithelium from a few days to several weeks. Normal cycles of oestrus, as would be expected, were present in animals blinded by cutting the optic nerves or enucleating the eyeballs. Either operation caused a resumption of normal cycles in animals in constant oestrus because of continuous illumination or prevented the development of persistent oestrus in similarly treated animals. ${ }^{14}$

Mammals.-In this section, recently acquired general information concerning the gonadotropic hormones of the pituitary body will be discussed. There will be no further consideration of the effects of light on the secretion of gonadotropic hormones; these are referred to in the preceding section.

I. Female mammals.-There is a number of new reports chiefly concerned with the effects of hypophysectomy on the female gonads. ${ }^{15}$ Swezy (1936) has again affirmed that the rate of ovogenesis is accelerated in the rat's ovary as a result of hypophysectomy. However, degeneration of the follicle appears after growth has proceeded to a depth of 6-8 rows of cells. She pointed out that this is the sole example of a

${ }^{14}$ See also Hemmingsen and Krarup (1937).

${ }_{15}$ Lacassagne (1935) concluded that the cells of the pars glandularis and other parts are quite insensitive toward radon or X-rays. His experiments were performed in rabbits. Although he was able, by the insertion of a tube containing $1 \mathrm{mg}$. of radium, to cause destruction of the gland for a distance of $1.5^{-1} .8 \mathrm{~mm}$. accompanied by the characteristic secondary changes in the ovary in about a week, he doubted that radiation of the pituitary has therapeutic possibilities so far as ovarian function is concerned. Even after complete destruction of the pars intermedia and pars neuralis together with destruction of two-thirds of the pars glandularis, ovarian function is not affected. His estimate that adequate anterior pituitary secretion is maintained by one-third of the pars glandularis agrees well with the estimates of Aschner (dog) and Smith (rat).

Newell and Pettit (1935) found that irradiation (X-rays directed toward each temple in small doses for 5 weeks) led to improvement in the subjective symptoms of two-thirds of a group of women with dysmenorrhea (26 patients). Sham irradiation had the same effect in half the proportion of a group with similar symptoms (i.e., in one-third of a group of 15 patients). 
biological process undergoing acceleration as a result of anterior pituitary deficiency. Some dilute acid extracts of the pars glandularis produced the same effect-suggesting that

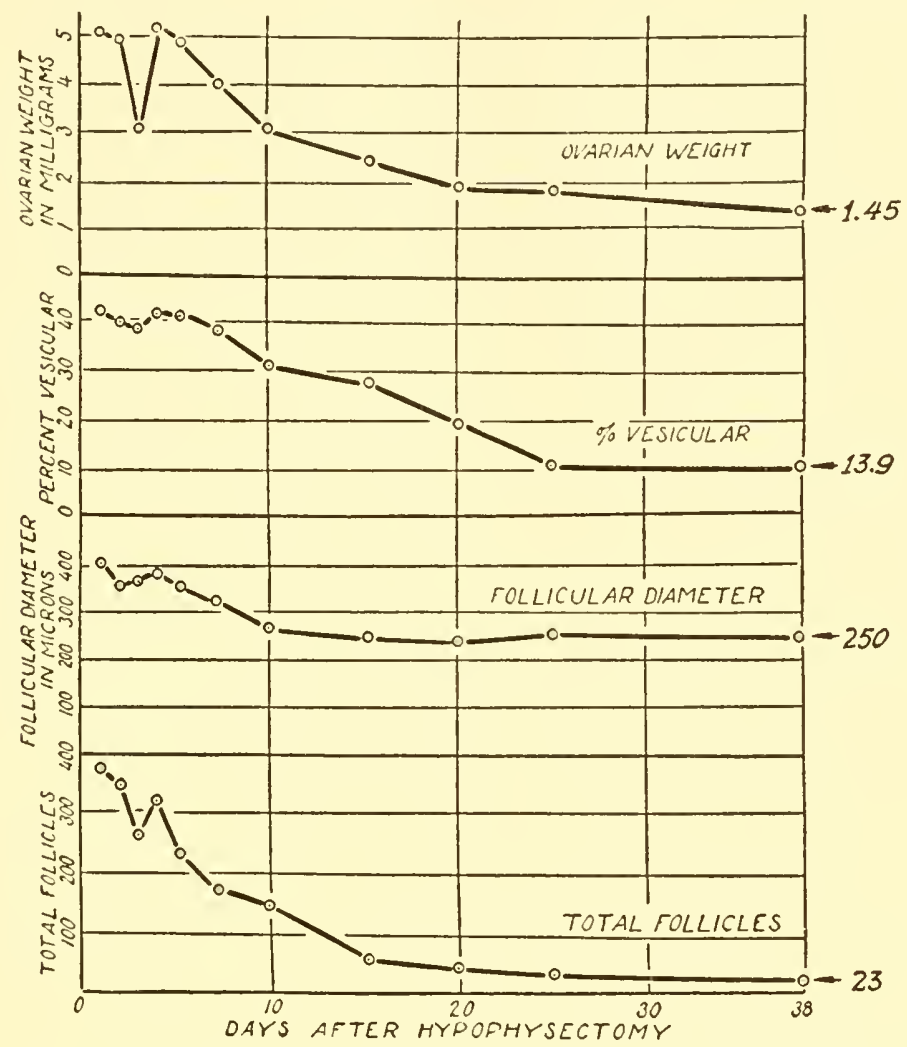

F1G. 12.- Variations in the weight, total follicle-count, mean diameter of the largest follicles, and per cent of vesicular follicles of the ovary of the rat following hypophysectomy at an age of 28 days. Each point represents the mean of determinations from 3 rats. (From Lane and Greep, Anat. Rec., 63, 139-46 [1935].)

this action was due to inhibition of anterior pituitary secretion. Lane and Greep (19.35) studied in detail the follicles and the weight of the ovary of the rat hypophysectomized at an age of 28 days. A graph summarizing their results is reproduced in Figure $\mathrm{I} 2$. It will be noted that groups of 
animals were studied for more than 6 weeks after hypophysectomy. These results are of interest to everyone employing hypophysectomized immature female rats for the assay of gonadotropic hormone. The belief that a rise in ovarian weight takes place about 4 days postoperatively requires confirmation. The authors found that the injection of follicle-stimulating hormone into hypophysectomized rats caused an increase in the total number of follicles with a decrease in the proportion of those which were vesicular. 'The proportion of vesicular follicles-without any effect on the total number - was increased by the injection of luteinizing hormone.

Because of the monkey's closer biological relationship to man, the experiments of Smith, Tyndale, and Engle (1936) are of unusual interest. These authors found that marked atresia of follicles, especially of those medium sized or large, followed hypophysectomy in the macaque (Macaca mulatta). Involutionary changes in the endometrium and vagina were as great as or greater than those following spaying. Uterine bleeding for $3-6$ days occurred $2-4$ days after hypophysectomy in the middle of the cycle (stage of follicular growth or proliferation) but was prevented by the daily administration of oestrin. No prolongation of bleeding followed hypophysectomy during menstruation. The usual changes in the uterine mucosa were produced by oestrin or progesterone. Uterine bleeding was delayed but usually followed ( 8 of Io monkeys) "oestrin-deprivation"; therefore, the authors' results speak strongly against Hartman's view that such bleeding requires a secretion of the pars neuralis.

In the hypophysectomized and ovariectomized rabbit, the injection of progestin ( $\mathrm{I}$ rabbit-unit) neither prevents the motility-increasing effect of oestrone on the uterus nor renders the latter oxytocin-insensitive as in spayed rabbits. To produce these effects in the doubly operated animals, the dose of progestin must be increased to + rabbit-units (Reynolds, Firor, and Allen, 1936). 
According to Bachman (1936) interstitial stroma may make up a large part of the ovary of the mature rabbit but largely disappears after hypophysectomy. ${ }^{16}$ Robson's experiments in the rabbit (I937) illustrate how rapid regressive changes in the ovaries may render the latter almost unresponsive to gonadotropic hormones-i.e., extract of rabbit or horse pituitary or of human pregnancy-urine. A week or more after hypophysectomy, the injection of these hormones had little or no effect on the ovaries, whereas if injections were begun at once, small doses of gonadotropic hormone maintained ovarian function as shown by functional persistence of the corpora lutea of pseudopregnancy (8 days) and by ovulation in response to gonadotropic hormone (I 2 days). Other experiments led Robson to conclude that both the pituitary and the ovary are essential for maintaining pregnancy in the rabbit. However, he was able to maintain pregnancy in the hypophysectomized rabbit, for some time at least, by injections of progesterone. The rate of atrophy of the corpora lutea apparently was not affected. It appears that among mammals, the mouse and rat are exceptional in that the corpora lutea in the latter part of pregnancy require no pituitary luteinizing hormone for their maintenance. ${ }^{17}$

Emery (1 936) reported that homo-implants of the pituitary in the peritoneal cavity or skeletal muscle of rats apparently lived from several days to a week. Often such grafts became vascularized, retained some gonadotropic potency, and appeared nearly normal histologically. Other observations on the secretory capacity of implants or transplants have been made in hypophysectomized mice, rats, and guinea pigs.

${ }^{16}$ The observations of Westman and Jacobsohn (1936) on the effects of hypophysectomy in the rabbit are largely confirmatory of earlier work.

${ }^{17}$ Houssay (1935) has confirmed the finding that hypophysectomy in the pregnant dog is followed by abortion or fetal resorption. He stated that even in late pregnancy abortion might appear in 2 days.

Bellerby (1935) found that the intravenous injection of anterior-lobe extract into pregnant rabbits almost always was followed by abortion or fetal resorption. Ovulation was often produced; hemorrhagic follicles were more frequently produced early or late in pregnancy. 
According to Hill and Gardner (1936), successful homotransplantation of the pituitary into the testis can be made in mice which are homogeneous genetically. They showed that mice carrying such grafts could be hypophysectomized, yet spermatogenesis, normal testicular germinal epithelium, and normal adrenal cortex (at least the zona fasciculata) were all maintained. If both the pituitary and ovary were transplanted, ovarian secretion was elaborated and even caused development of the branched ducts of the mammary gland. Like Hill and Gardner, Greep (1936) found that the sex of the donor of a pituitary transplant had little or no significance. For example, the male reproductive organs were as well maintained by a female pituitary as by a male gland. Greep hypophysectomized male and female rats 4 weeks old. He inserted the graft into the empty sella immediately after operation and was able to secure quite complete replacement effects in three-fourths of the animals. Oestrous cycles in the females were usually a little prolonged ( $5-7$ days) because of a longer dioestrus. However, 13 of the rats became pregnant, delivered normally, and nursed their young. Still another form of replacement-therapy in the hypophysectomized rat was studied by May (1937), who transplanted the pituitary of the new-born rat into the anterior chamber of the eye. Oestrus was first observed 77 days later; there was some increase in weight over a long period (56-119.gm. in 248 days). Oestrus might disappear after removal of the eye containing the transplant, with associated atrophy of the ovary and uterus. Schweizer, Charipper, and Haterius (1937) made ocular transplants (anterior chamber or lateral subconjunctival tissue) of the pituitary in hypophysectomized guinea pigs. After about two months the graft clearly seemed to secrete only one gonadotropic hormone-that stimulating the follicle. This was shown not only by the abnormal development of ovarian follicles and continuous oestrus in the host but also by the effect of implantation of the graft which seemed to produce only follicle-stimulation. 
Several observations of general interest should also be mentioned. Lipschütz (1936) reported additional studies in guinea pigs from which about three-fourths of the total ovarian tissue had been removed. If the operation was performed in new-born animals, it produced little disturbance of the development and function of the genital tract owing, the author concluded, to the great reserve of primordial follicles. Sometimes months after the same operation was performed in immature animals ( $13-28$ days old), the vagina remained open for abnormal periods and the uterine changes (cystic hyperplasia of the endometrium, etc.) resembled those of metropathic hemorrhage in man. It is not clear to what extent the responsibility for these changes must be attributed to the ovary or to the anterior pituitary or to both. Hamlett (1935) investigated the effects of whole pituitary extract and prolan ("Antuitrin S") on the genital tract of the armadillo (Dasypus novemcinctus). In this animal the blastocyst-i.e., fertilized ovum which has undergone cleavage-lies free in the uterine cavity for four months ("free vesicle period"). Neither hormone hastened implantation; prolan (4 cases) but not pituitary extract ( 2 cases) caused abortion or resorption of the unimplanted blastocyst. Either hormone was found to cause follicle growth, luteinization, and, occasionally, ovulation. According to Lipschütz and Oviedo (I 935), the gonadotropic potency (immature rat) of the pituitary of the ratlike South American mammal Myocastor (Myopotamus) coypu is even lower than that of the female guinea pig despite the animal's size (body-weight as great as $4.5 \mathrm{~kg}$.). Its young, like those of the guinea pig, are well developed when born. Hellbaum's demonstration of differences in the qualitative and quantitative effects of the equine pituitary in the immature rat is of great interest (see Fig. I3). If judgment be based upon ovarian hypertrophy (weight), the concentration of gonadotropic hormone is highest in the pituitary of the aged mare and lowest in that of the stallion, fetus, and colt. The potency of the pituitary of the gelding and 
mare (both pregnant and non-pregnant) is high. The qualitative effects, which are illustrated by the reproduced figure, suggest that principally follicle-stimulating hormone is produced by the pituitary of the stallion and of the aged mare and gelding, whereas luteinizing hormone also is secreted by the pituitary of the fetus, colt, young gelding, and adult preg-
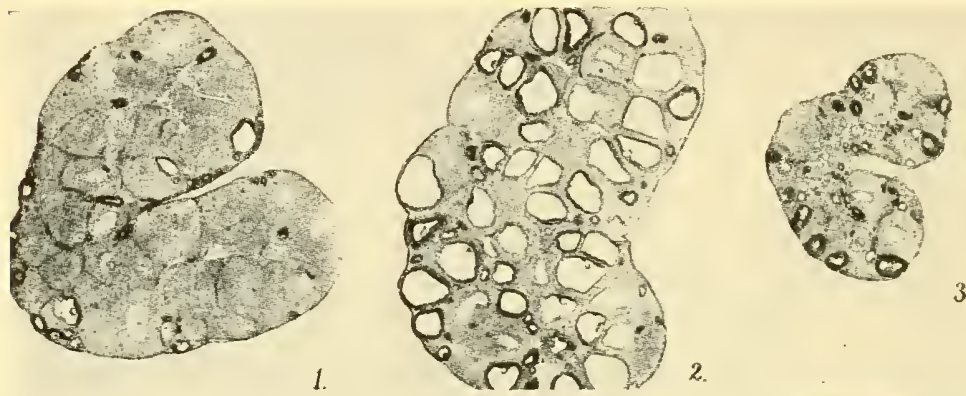

1.
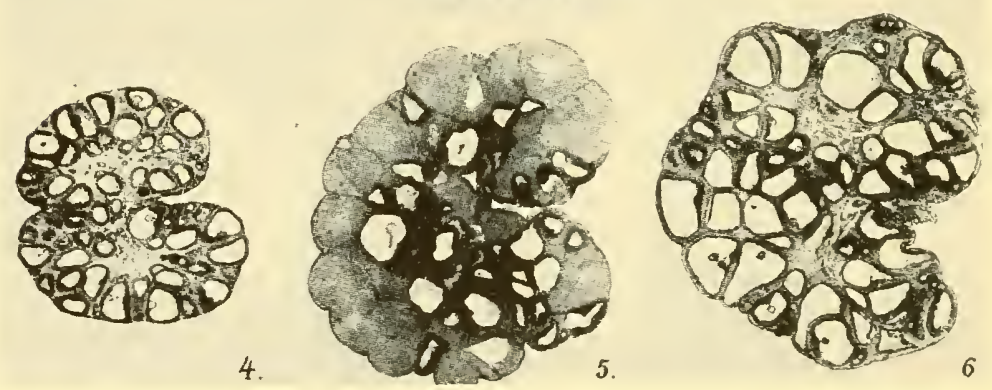

FIG. 13.- Photomicrographs illustrating the response of the ovary of the rat to equal quantities of pituitary powder from glands of horses. The magnifications are the same in all cases. (From Hellbaum, Anat. Rec., 63, I47-57 [1935].)

1 , Normal mare of reproductive age. 2, Old mare. 3, Fetus. 4, Stallion. 5, Young gelding. 6 , Old gelding.

nant or non-pregnant mare. Those interested in a recent study of the gonadotropic effects of the anterior pituitary of whales (finback, Balaenoptera physalus; sperm, Physeter megalocephalus) are referred to the report of Geiling (1935).

Cyclic changes in the pituitary in relation to those of the gonads have again been studied by several investigators. Cole and Miller ( 1935 ) could detect no change in the gonado- 
tropic potency (rabbit-ovulation test) of the ewe's pituitary during this animal's oestrous cycle. Schmidt (1937) found that, as tested in the immature female guinea pig, the gonadotropic potency of the adult female's pituitary is least during oestrus and greatest during pro-oestrus. Also she made a similar study of the pituitary of female adults receiving a minimal sterilizing dose of X-rays. The gonadotropic potency of the pituitary did not always correspond to the normal sexual cycle as judged by the vagina. For example, the pituitary, although removed from an animal in prolonged oestrus, might contain much gonadotropic hormone. The metabolism of the pars glandularis of the rat in different phases of reproductive activity has been investigated by Victor and Andersen (1936) and Andersen, Prest, and Victor (19.37). Their results can be summarized as follows:

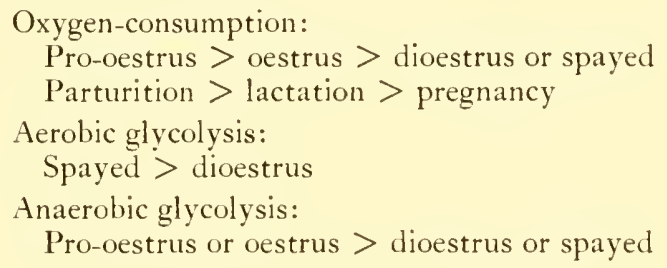

The highest oxygen-consumption was found in parturient animals, the lowest in spayed animals. It was about the same in lactating rats and rats in pro-oestrus. 'There were no significant differences in the aerobic and anaerobic glycolysis of pregnant, parturient, and lactating rats.

The effect of pregnancy on the assay of gonadotropic hormones has been investigated by Rowlands (1935), who used rabbits and expressed dosages in terms of the amount of extract causing ovulation in 50 per cent of each group. 'Table I is a summary of Rowlands' results.

These observations suggest the following conclusions: (I) The "ovulating dose" of a gonadotropic hormone is higher in pregnancy than in oestrus. (2) Pituitary extracts, but not prolan, may vary enormously, depending upon their source, 


\section{THE PITUITARY BODY}

in their relative gonadotropic potency in rabbits and rats. These observations are not new but are here more accurately confirmed. It is not possible exactly to state how differences are related to the distribution of various pituitary gonadotropic hormones in the extracts. Ox pituitary extract is probably much less potent in follicle-stimulating hormone and richer in luteinizing hormone; perhaps it contains more gonadotropic hormone "antagonist," the effects of which would be more marked in the rat (repeated subcutaneous injections) than in the rabbit (single intravenous injection). The reader is also referred to later discussion (pp. I I 5-17).

TABLE 1

\begin{tabular}{|c|c|c|c|c|}
\hline \multirow{4}{*}{ ExTract of } & \multicolumn{4}{|c|}{ Relative Dose Causing } \\
\hline & \multicolumn{3}{|c|}{ Ovulation in 50 Per Cent of Rabbits in } & \multirow{3}{*}{$\begin{array}{c}\text { Ovarian Hyper } \\
\text { trophy to } 40 \mathrm{Mg} \text {. } \\
\text { in Immature } \\
\text { Rats }\end{array}$} \\
\hline & \multirow{2}{*}{ Oestrus } & \multicolumn{2}{|c|}{ Pregnancy of Duration of } & \\
\hline & & 15 Days & 25 Days & \\
\hline Anterior lobe $(o x) \ldots \ldots$. & I & 2 & 5 & 100 \\
\hline Whole pituitary (horse). & 20 & 70 & 60 & 100 \\
\hline Pregnancy-urine $1 . . .$. & 25 & $\cdots \cdots$ & 50 & 100 \\
\hline Pregnancy-urine 2. & 20 & 45 & 35 & 100 \\
\hline
\end{tabular}

One other report, unrelated however to pregnancy, may be mentioned here. Bachman (1936) observed that in very young or juvenile rabbits ( $15^{-90}$ days old) the outstanding ovarian change in response to sheep pituitary extract or prolan might be limited to the interstitial stroma in which there appeared large polyhedral cells taking lipoid stains. If, on the other hand, prompt and extensive luteinization of the membrana granulosa occurred, there might be no change in the interstitial tissue. Also, in very young rabbits gonadotropic hormone could cause the formation of corpora lutea without associated progestational changes in the uterus.

According to Morgan (1935), extract of beef anterior lobe or prolan alters the motility of the uterus of the nonanesthe- 
tized rabbit according to the predominant change in the ovaries. Increased motility is associated with follicle growth; the change is in the opposite direction, if ovulation and corpus luteum formation occur. ${ }^{18}$ Several other possible interrelationships between the uterus and gonadotropic hormones have been studied. ${ }^{19}$ Gillard (I937) concluded that hysterectomy in the rabbit delays degeneration of the corpora lutea, thus prolonging pseudopregnancy with hyperemia of the mammary gland. In his experiments, pseudopregnancy lasted about 25 days instead of 18 days in normal animals. Krane's (I937) observations in hysterectomized women, some of whom had been studied as long as 6 years postoperatively, indicated that in the absence of the uterus there may be cyclic excretion of oestrogen, resembling that in normal women, without any increased excretion of gonadotropic hormone. Therefore, hysterectomy is not necessarily associated with a disturbance of the pituitary-gonad interrelationship.

In conclusion, data bearing on the number of gonadotropic hormones or their specific effects will be briefly considered. Loeb and his collaborators, ${ }^{20}$ who performed most of their experiments with immature female guinea pigs, describe the following principal gonadotropic effects: (I) the production of follicular atresia or destruction by "atresin," (2) luteinizing effects on the cells of the theca interna or premature luteinization of these cells or maturation of the granulosa of immature follicles, and (3) maturation of the granulosa of large follicles. From a study of the effects of pituitary glands of various animals, of serial implantation, etc., they suggested that these effects might be due to at least three different gonadotropic hormones. More often it is assumed that the

${ }^{18}$ Morgan found that no effects were produced in spayed rabbits. The earlier work of Reynolds indicated that a reduction of motility is to be expected in normal or spayed rabbits after the injection of beef pituitary extract. However, Reynolds referred to tests made 5-7 hours after injection.

19 See also Hauptstein and Bühler (1936).

${ }^{20}$ Kunkel and Loeb (1935); Loeb, Saxton, and Hayward (1936); Saxton and Loeb (1937). 
important gonadotropic hormones are the follicle-stimulating and luteinizing hormones. Fevold and Hisaw and their coworkers have emphasized that the production of ovulation requires both hormones. Other experiments indicate that the secretion of luteinizing hormone in appropriate amount and at the proper time following follicle growth also is essential for normal oestrus, so that without this hormone, ovulation, mating, corpus luteum formation and maintenance-and hence pregnancy-cannot occur (Casida, I934; Witschi and Pfeiffer, I935; Dempsey, Hertz, and Young, 1936). Casida suggested that atretic corpora lutea are due to the administration of an excessive amount of luteinizing hormone. Riddle and others ( 1936 ), unable to demonstrate augmentation of the ovulation-producing effect of follicle-stimulating hormone by luteinizing hormone, concluded that the latter is not necessary for ovulation. Inasmuch as their experiments were not performed in hypophysectomized rabbits, this conclusion does not appear to be warranted. The difficulty of the experimental production of ovulation in the oestrous cat was emphasized by Foster and Hisaw (I 935), who concluded that at least some luteinizing hormone-even in minute amount relative to the dose of follicle-stimulating hormone-must be administered to cause ovulation. The authors also studied the duration of pseudopregnancy which persisted $40-44$ days. In the cats of van Dyke and Li (I938) pseudopregnancy persisted only about 20 days; however, they produced a much smaller number of corpora lutea than did Foster and Hisaw. Van Dyke and Li used prolan also. Experiments in the immature monkey have been described by Hisaw (1935).

Pfeiffer (1937) believed that the male rat pituitary secretes or is able to release much less luteinizing hormone than the female. According to Bunde and Greep (1936), luteinizing hormone or some substance associated with it can cause the rapid regression of corpora lutea in hypophysectomized young adult rats. The earlier experiments of Smith demonstrated how remarkably long the corpora lutea persist after hypophy- 
sectomy in the rat. According to Lane and Greep (I935), the effects of separated pituitary hormones in immature hypophysectomized rats are as follows: follicle-stimulating hormone causes an increase in the total number of follicles (numerous small primary follicles) and a decrease in the percentage of vesicular follicles; luteinizing hormone has no action on the total number of follicles but brings about an increase (35-II2 per cent) in the proportion of vesicular follicles.

2. Male mammals. ${ }^{21}$-A study of the rate of regression of the testes and accessory organs following the removal of the pituitary from 9 guinea pigs has been made by Allanson, Hill, and McPhail (I935). They concluded that maximum atrophy of the testes, epididymides, prostate, and seminal vesicles occurred after about 45 days, although there was marked atrophy of the seminal vesicles after 20-25 days. Atrophy of the secondary sexual organs occurred at about the same rate after castration. According to Leonard and Hamilton (I 937), the testis which has been made cryptorchid experimentally degenerates more rapidly (peak at 6 days after operation instead of Io days), if hypophysectomy is also performed. The authors used rats.

Wells and Moore (1936) found that in the adult or young male ground squirrel (Citellus tridecemlineatus), ${ }^{22} \mathrm{kept}$ in the laboratory, spermatozoa were produced in December and January. Precocious spermatogenesis and full development of the accessory organs were produced weeks or months in advance of the normal time by gonadotropic substances (pitui-

${ }^{21}$ Koch, Schreiber, and Schreiber transplanted pituitary and testis of immature animals into the anterior chamber of the same eye. They observed, in comparison with control experiments, a definite effect on the germinal epithelium. Guinea pigs were used.

Bastenie and Zylberszac (I937) injected an anterior pituitary extract into male or female guinea pigs, which also were given colchicine to arrest the mitoses. There were no striking changes in the gonads. Mitotic division was markedly increased in the epithelium of the seminal vesicles and the uterine mucosa.

22 Wells and Gomez (1937) describe a technic of hypophysectomy in this animal as well as the effects of the operation in males. 


\section{THE PITUITARY BODY}

tary implants, prolan, or pregnant-mare serum) or by androgens (androsterone or extract of male urine or bull testis). Why androgens should have this effect on spermatogenesis is not known; in view of other work in the rat it is reasonable to believe that an action on the testicular germinal epithelium is important. Recent studies of the action of gonadotropic substances $^{23}$ in immature rats have been made by Moore (1936) and Price (1936). Price compared the effects of pituitary implants and prolan in very young rats. The indirect effect on the seminal vesicles was accompanied by less change in the apparent amount of interstitial tissue after pituitary implants than after prolan. Prolonged injection of the latter caused damage to the germinal epithelium. Moore found that sheep pituitary and pregnant-mare serum, like prolan, brought about no precocious spermatogenesis in normal immature rats. The most marked effects were on the interstitial cells and secondary organs; although the seminal vesicles might weigh fifty times as much as those of non-injected rats, the testicular weight was never more than doubled. Little change of either type was produced in adult animals.

Follicle-stimulating extract (from the urine after gonadectomy or after the menopause) was injected by Huberman, Israeloff, and Hymovitz (I 937) into men with sterility caused by an endocrine disturbance (300-2,000 rat-units as 50 ratunits twice weekly). The treatment appeared to cause an increase in the number and motility of the spermatozoa but did not correct the sterility.

Using normal rats $2 \mathrm{I}$ days old and hypophysectomized rats 35 days old (7 days after hypophysectomy), Greep, Fevold, and Hisaw (1936) compared the effects on the male reproductive organs of follicle-stimulating extract and luteinizing extract made by them from sheep pituitary. They concluded that the follicle-stimulating extract had no effect on the interstitial tissue but caused increased mitotic division of the cells of the germinal epithelium so that spermatogenesis could be

${ }_{23}$ Pituitary homo-implants, sheep pituitary, prolan, and pregnant-mare serum. 
carried as far as the secondary spernatocyte stage. The luteinizing extract brought about growth of the interstitial tissue but left the germinal epithelium as "degenerate" as in control animals. Increased diameter of the tubules in rats receiving luteinizing extract was believed to be the result of generalized swelling of the tubules partly owing to the accumulation of fluid in the lumen. The administration of a mixture of the two extracts apparently produced an augmented effect on the secondary organs. The general results are in agreement with the views of many authors: a pituitary follicle-stimulating hormone maintains gametogenesis in the male; a pituitary luteinizing hormone is essential for the normal functioning of the interstitial tissue. However, proof to silence all arguments to the contrary awaits the isolation of the hormones in pure form.

Pfeiffer (I936-37) offered experimental evidence that the pituitary of the male rat secretes only follicle-stimulating hormone, whereas the female pituitary secretes both folliclestimulating and luteinizing hormone. His conclusions are supported by a different type of data-i.e., effects of extracts or implants of the pituitaries of male or female rats-gathered by others. Thus it would appear that follicle-stimulating hormone alone maintains both the germinal epithelium and the interstitial cells of the testis of the normal rat. Greep has suggested that minute continuous secretion of luteinizing hormone (the male rat's pituitary does contain a very small amount of this hormone) by the male pituitary may be sufficient for the needs of the interstitial cells, which probably secrete continuously rather than in a cyclic fashion. The cyclic course of ovarian activity as well as the action of oestrogen on the pituitary might account for the much larger amount of luteinizing hormone secreted by the female pituitary. A later report of Greep and Fevold (1 937) further complicates discussion. Follicle-stimulating hormone or luteinizing hormone was administered to adult hypophysectomized male rats. Spermatogenesis could be maintained by either 
extract, whereas regression of the interstitial cells was prevented only by luteinizing hormone.

SPECIAL CONSIDERATIONS

The secretion of gonadotropic hormones in relation to sex and the internal secretions of the gonads. I. Differences related to sex and age.-McQueen-Williams (I935) studied the gonadotropic potency of the pituitary of rats of different ages and sexes. Her data supplement those of Clark previously referred to. McQueen-Williams performed her assays by means of intramuscular implants in immature female rats, whereas Clark used immature mice for assay. Both authors found that the female pituitary is much the more potent in rats about 3 weeks old, whereas in adult rats the male pituitary is richer in gonadotropic hormone. One important change encountered by McQueen-Williams was a remarkable increase in the potency of the pituitary of male rats 27-30 days old, the pituitary then being more potent than at any other age studied. The gonadotropic potency of the pituitary of male rats only a week older was again low and did not rise until the animals were more than four months old. ${ }^{24}$ Bates, Riddle, and Lahr (1935) compared the concentrations of gonadotropic hormone in the pituitary of the ox (embryo, calf, adult steer [castrated male], adult bull, normal cow, and cow in early and late pregnancy.) Gonadotropic hormone was determined by its effect on the testis of the immature dove. The authors concluded that the only significant differences were (I) the low potency of the steer's pituitary (concentration about 23 per cent less than the average of others) and (2) the high potency of the pituitary of the cow in early pregnancy (concentration about $3^{6}$ per cent more than the average of others). The low potency of the steer's pituitary is surprising; also, it might be expected that the pituitary of pregnancy would be poor rather than rich in gonadotropic hormone. In

24 The pituitaries of rats between the ages of forty-four days and four months were not investigated. 


\section{THE GONADO'TROPIC HORMONES}

TABLE 2

The Gonadotropic Potency of the Anterlor Pituitary

in Relation to Sex*

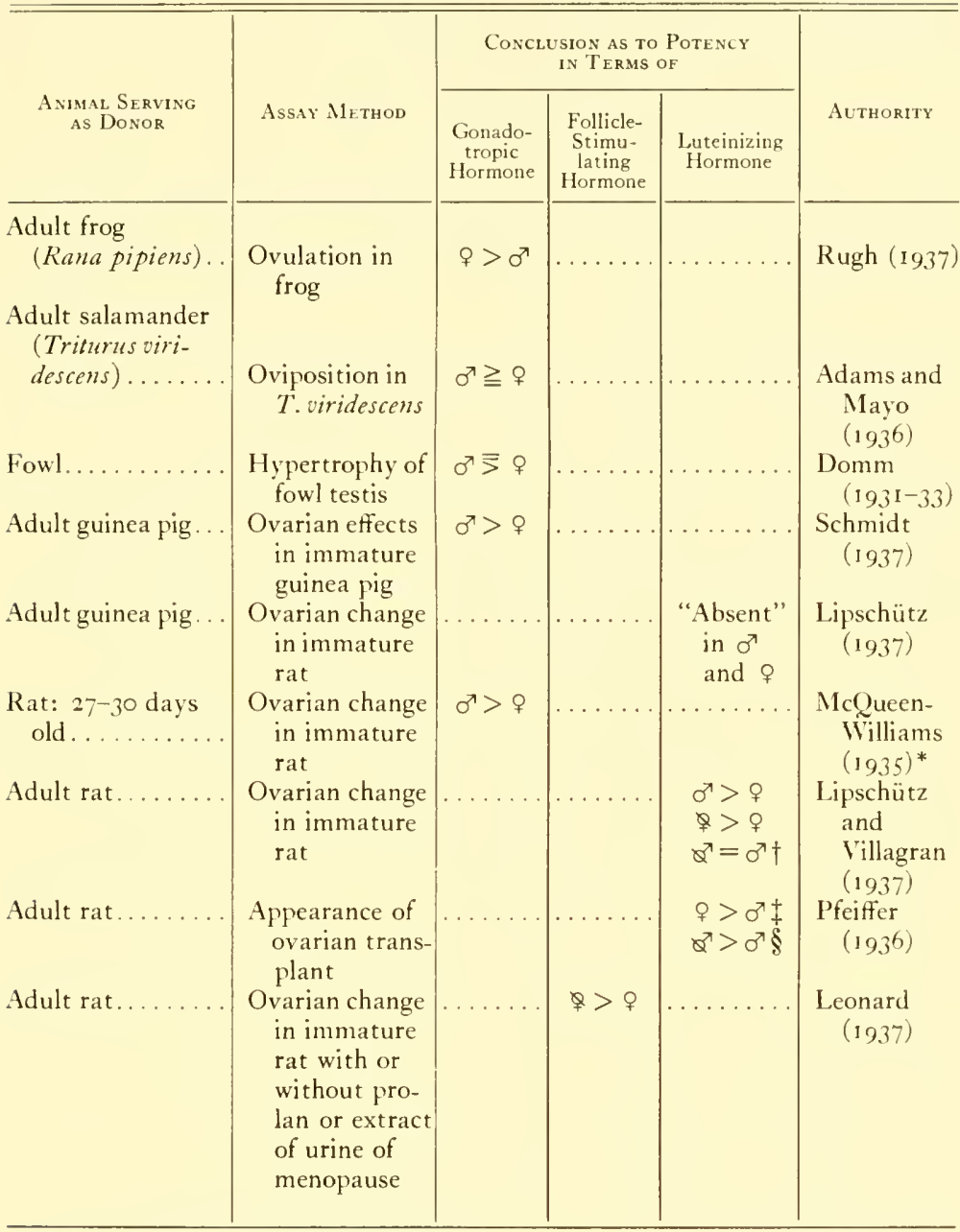

* See also Table V, p. 143, of earlier volume.

† In terms of concentration. After castration the male pituitary increases in size. Gonadectomized animals are indicated by $Q$ or $Q^{7}$.

‡ The author believed that the pituitary of the normal male secretes only follicle-stimulating hormone.

§ Castrated at birth. 


\section{THE PITUITARY BODY}

the guinea pig, the presence of a gonad, functioning definitely but at a low level, appears not to lessen the gonadotropic potency of the pituitary in comparison with that of gonadectomized animals (Lipschütz, I 936).

Other observations on the relation of sex to the pituitary's content of gonadotropic hormone have attempted to distinguish between the follicle-stimulating and luteinizing hormones. According to Lipschütz and Del-Pino (1936), man resembles the rat and guinea pig in that (tested in the immature rat) the male pituitary causes more formation of lutein tissue than the female. (The pituitary of women in the first month of pregnancy caused luteinization of the ovaries; in the later months this effect diminished or disappeared.) The opposite view was expressed by Pfeiffer (1936-37), as well as by others, whose experiments were performed in rats. According to this author, the male pituitary is capable of secreting very little luteinizing hormone. Moreover, Pfeiffer's conclusion appears to be based on a more "physiological" method (the behavior of ovarian grafts as affected by the gonadotropic hormone secreted by the host's pituitary). The conflicting views expressed in recent reports are summarized in Table 2. Some of these results will be considered later when an evaluation will be attempted. Pfeiffer (I936), relying on the effect on ovarian transplants of gonadotropic hormone released by the rat's pituitary in situ, concluded that the normal male pituitary secretes follicle-stimulating hormone with little or no luteinizing hormone, whereas the female pituitary is "bipotential"-i.e., secretes both hormones. Also he believed that this sexual difference is hormonal rather than genetic and that after puberty the pattern of pituitary secretion is practically fixed; Pfeiffer (1936-37) cites other experiments in support of these views.

2. Experiments with animals in parabiosis. ${ }^{25}$-No attempt

${ }_{25}$ Unless there is a statement to the contrary, all the animals used are rats. References to other experiments employing the method of parabiosis will be found both in this chapter and in the Index. 9 refers to a normal female; refers to a 


\section{THE GONADOTROPIC HORMONES}

will be made to recapitulate the older experiments with parabiotic animals; an outline of the results of these experiments will be found in the earlier volume. The outstanding fact previously demonstrated is that, if a gonadectomized and a normal animal are joined in parabiosis, an abnormal stimulation of the gonads occurs and persists for months, because, in all likelihood, abnormal amounts of pituitary gonadotropic hormone are present in the blood. This is particularly true of the experiment $q \Phi^{7} \cdot{ }^{26}$ In the experiment $\sigma^{7} \Phi^{7}$, the accessory organs of the castrated male remain atrophic. However, according to McCullagh and Walsh (I935), the injection of a comb-growth-stimulating hormone ("androtin") prevents both a gonadotropic effect in the normal male and a regression of the accessory organs in the castrated male. ${ }^{27}$ De Mello (I936) placed an ovarian graft in the normal male of the parabiotic pair $\sigma^{7} \sigma^{\top}$. A hypertrophy of the prostate and seminal vesicles of the normal male, abnormal even for such an experiment, apparently was caused by the combined effects of ovarian and testicular secretions. Both the graft and the testes in situ were stimulated.

Recently additional observations have been made on the results of parabiosis between hypophysectomized and normal or gonadectomized rats. In experiments of the type $\sigma^{7} \mathrm{~h} \boldsymbol{s}^{\mathrm{T}}$,

spayed female; $\sigma^{7}$ refers to a normal male; $Q^{7}$ refers to a castrated male; h refers to a hypophysectomized female, etc. $\sigma^{7} \mathrm{~s}^{\mathrm{T}}$ would refer to a parabiosis between a hypophysectomized male and a castrated male.

${ }^{26}$ In this experiment, the ovaries become cystic and remain in this condition for months. Coincidently, the changes characteristic of oestrus are observed in the uterus and vagina. Evans, Simpson, and Pencharz (1935) suggest that the castrated male pituitary contains (secretes?) but does not release luteinizing hormone. They implanted the pituitary of castrated male rats in to female rats hypophysectomized at an age of 26 days. Follicle-stimulation followed the smaller dose of pituitary tissue, whereas corpora lutea were formed after the larger dose ( 4 glands of young males 40 days after castration). Four glands of normal males caused only folliclestimulation in accordance with the belief of some authors that the normal male pituitary "releases" only follicle-stimulating hormone.

27 The authors stated that a specific substance in testicular extract ("inhibin") prevents oestrous cycles in the female rat and causes atrophy of the male accessory organs. 
both the germinal epithelium and the interstitial tissue were stimulated by the gonadotropic hormones of the castrated rat's pituitary. The effects were much less evident if the hypophysectomized male rat was joined to a normal rat or a rat with experimental cryptorchidism (Cutuly and Cutuly, I 937; Cutuly, McCullagh, and Cutuly, I 937). From a study of the changes in the accessory organs and testes which tended to be parallel, the authors suggest that the testicle requires only one gonadotropic hormone, whereas the ovary may require two. Mфller-Christensen (I935) has published a long report of his experiments with rats in parabiosis. He has advanced the view that in the experiment $q$, the pituitary of the normal rat inhibits the activity of its own ovaries. He cites the experiment $\$ \mathrm{~h} \phi$ : cystic ovaries without corpora lutea are found in the hypophysectomized rat; atrophy of secondary organs in the spayed female is moderate and not advanced. ${ }^{28}$ In the experiment $\$$ 우, of course, the ovaries of the normal female may become cystic; this change can be observed more certainly in the experiment $q q^{\top}$. The pituitary of the normal female of the partners $q$ or $q q^{\gamma}$ undergoes hypertrophy and contains no gonadotropic hormone (M $\phi$ ller-Christensen). Another type of parabiosis investigated by the author was that symbolized by 우 . Normal oestrous cycles and normal sexual organs were observed in the normal rat; the gonads and accessory organs of the hypophysectomized rat underwent atrophy. The interpretation of all these results, especially in terms of an inhibiting effect of the pituitary on ovarian function, is a suggestion requiring more supporting data. The contrary effect-increased release (and diminished utilization) of gonadotropic hormone by the pituitary after gonadectomy - still appears to be the best interpretation of most experiments with parabiotic rats.

3. The effects of the internal secretions of the gonads on pitui.

${ }^{28}$ Oestrus in the spayed partner is observed more frequently in the experiment 애 $\$$ than in the experiment $ᄋ$ (Møller-Christensen, 1933). 
tary function. (a) Hormones of the oestrin group.29,30-The effects of hormones of the oestrin group on the secretory performance of the pituitary, aside from their pharmacological interest, are important because they furnish a basis for interpreting the interplay of ovarian and pituitary (gonadotropic) hormones. This is true although only one member of the group (oestradiol) probably is a normal secretion of the ovary. Particularly in the preceding sections, other aspects of this problem have been mentioned. The experimental data about to be taken up here extend our knowledge by other technics. The early experiments of Meyer, Leonard, Hisaw, and Martin (1930, I932) demonstrated the diminished gonadotropic potency of the pituitary of rats which had received injections of oestrin for $4^{-}$Io weeks. The publication of their work was followed by numerous reports of related phenomena, which, as far as recent articles are concerned, now require discussion.

Berkowitz (1937) repeatedly added tablets of "Progynon" (oestrone?) for a long period to the tank water in which he kept immature male guppies (Lebistes reticulatus). In size, shape, and color the fish ultimately resembled females. No spermatogenesis appeared. The author believed that the treatment accelerated grow th. He was unable to demonstrate that the tablets had any effect on adult male fish. The injection of large doses of oestradiol benzoate into the cock causes regression of the comb and spurs (Zondek, I936). Bates, Riddle, and Lahr (I937) concluded that testicular atrophy occurs if oestrone be injected into adult male ring doves $(4 \mathrm{O}$ rat-units per day for Io days). All these effects, like similar effects in mammals, probably depend upon an interference with the secretion of gonadotropic hormones by the pituitary body. Oestrone is said not to affect the egg-laying performance of the fowl (Unik and Liptschina, 1934).

${ }^{29}$ Particularly oestradiol, oestrone, and oestriol, and their esters.

$3^{\circ}$ According to Müller (I937), the dose of oestradiol necessary to produce the vaginal signs of oestrus in the thymectomized or spayed rat is much higher as a result of hypophysectomy. These results are contrary to those of Smith (I932), who used oestrin. 
According to Bokslag (I 937), a number of hormones ${ }^{31}$ reduce the gonadotropic potency of the pituitary of rats of both sexes. However, without critical use, his method of assay is objectionable (indirect effects of implants on the uterus and vagina of immature mice). He believed that gonadotropic activity might be increased following the administration of thyroid extract, but not thyroxine. Other studies in rats have been made by Bühler (1936), Fischer and Engel (1936), Halpern and D'Amour (1936), and Emery (1937). Oestrin was found to cause marked atrophy of the testes sometimes comparable to that in hypophysectomized rats. ${ }^{32}$ Halpern and D'Amour again reported on the effect of oestrin on the mammary gland. Robson and Henderson (I 936) concluded that the pituitary plays no direct part in bleeding like that occurring before oestrus in the bitch. Oestrone or oestriol produced this effect in the absence of the pituitary. ${ }^{33}$ As far as the monkey is concerned, moderate doses of oestrin ( $1,265^{-1}, 390$ rat-units during 28-39 days) cause a decrease in the number of large follicles and a slight increase in the rate of follicular atresia. Because of this indirect action of oestrin on the rate of secretion of gonadotropic hormone by the anterior pituitary, the damage to the ovary is unimportant (Allen and Diddle, I935). Of interest and practical importance to the gynecologist are the experiments which have been performed in women. Frank and Salmon (1935) reported that menopausal symptoms were associated with the excretion of gonadotropic hormones. Both the symptoms

\footnotetext{
${ }^{3}$ Such as oestrone, oestrone benzoate, progesterone, testosterone, androsterone, prolan, etc.

${ }^{32}$ Shumacker and Lamont (1935) found that the administration of 9 rat-units of oestrone daily for nearly 10 weeks was without effect on the microscopic appearance of the ovary or testis of the rat. Emery's experiments indicated that the secretion of gonadotropic hormone by the young female $(130-50 \mathrm{gm}$.) rat's pituitary is scarcely affected by doses of oestrone as high as 20 rat-units daily for 6 weeks. Halpern and D'Amour injected 5 rat-units daily for 3 weeks followed by 20 ratunits daily for 5 weeks.
}

${ }_{33}$ Oestrin seemed to reduce the reactivity of the uterus to the oxytocic principle of the pars neuralis. The opposite effect follows the administration of oestrin to other animals such as the rabbit. 


\section{THE GONADOTROPIC HORMONES}

and the excretion of gonadotropic hormones disappeared after the administration of oestradiol benzoate (4,000-22,000 rat-units; apparently as many as 4,000 R.U. were administered at one time). Enormous doses of oestradiol benzoate have been administered to women ${ }^{34}$ so that the excretion of gonadotropic hormone is prevented and uterine bleeding may appear following treatment $(500,000-1,500,000$ mouse-units during 20-60 days: Jones and MacGregor, 1936; 200,000 [or more] mouse-units with or without progesterone: Zondek, I937). According to Zondek ( 1936 ), 200,000,-300,000 mouseunits of "follicle-hormone" may delay menstruation 6-70 days. He contended that this treatment prevented the secretion of the pituitary luteinizing hormone so that the development of the corpus luteum was inhibited. Also he believed that the treatment increased the rate of secretion of folliclestimulating hormone. His results should be compared with those of others who used animals and thus could control their experiments better (vide infra). ${ }^{35}$

Other experiments in male animals will be referred to briefly. ${ }^{36}$ Clauberg (1936) believed that a single dose of 5,000 mouse-units of "follicle-hormone" to adult male mice increased their sexual activity and fertility, partly by causing an increased liberation of gonadotropic hormone by the pituitary and partly by bringing about a hyperemia of the genital tract. According to Tuchman (1936), the administration of $0.4 \mathrm{mg}$. of "folliculin benzoate" (or I mg. of I-2-benzpyrene) once weekly for 4 weeks causes a cessation of spermatogenesis and a hypertrophy of interstitial tissue in the male guinea pig. In male animals oestrogens usually bring about regressive changes in the testes, especially in the germinal epithe-

${ }^{3+}$ In the menopause or with primary or secondary amenorrhea or with amenorrhea following ovariectomy.

35 Marx, Catchpole, and McKennon (19.36) concluded that the uterus retards the onset of the menopause. For example, the clinical and hormonal disturbances characteristic of the menopause appeared earlier after complete hysterectomy than after supravaginal hysterectomy.

${ }^{36}$ See also p. 8 I. 
lium. However, oestrogens are also known to cause hypertrophy of some of the male accessory organs. It is probable that this is a direct effect, although an indirect action on the testicular interstitial cells may be a participating cause.

The effect of oestrogens on the secretion of follicle-stimulating and especially luteinizing hormones has interested a number of recent authors. Usually it has been concluded that enhanced luteinizing effects or abnormally long persistence of corpora lutea already formed may occur as a result of the injection of an oestrogen. For example, in the adult mouse, Clauberg (1936) reported that sterility for as long as 29 days occurred after the injection of one or two large doses of "follicle-hormone." The sterility was attributed to an abnormal persistence and growth of corpora lutea which might be larger than those of pregnancy. In the rat, ovarian hypertrophy following the injection of various oestrogens is due to corpora lutea (Ellison and Burch, I936; Mazer, Israel, and Alpers, 1936). Ellison and Burch found that this effect could be prevented by hypophysectomy. The same conclusion was reached by Fevold, Hisaw, and Greep (1936) as well as Hohlweg and Chamorro (I937), so that it appears that oestrogens increase the rate of secretion of luteinizing hormone by the pituitary body. ${ }^{37}$ Hohlweg and Chamorro injected $\mathrm{i} i \gamma$ of oestradiol benzoate in to immature female rats; the customary appearance of corpora lutea after this treatment could be prevented by hypophysectomy on the second day after injection but not if operation was delayed to the fourth day.

The experiments of Fevold, Hisaw, and Greep were designed to detect changes due to injected oestrin, in the amounts of follicle-stimulating or luteinizing hormone liberated by the pituitary. After the injection of oestrin (O.I-4 R.U.), a constant dose of pituitary follicle-stimulating hormone was administered. The increased ovarian weight, in comparison with immature animals receiving no oestrin, was

${ }^{37}$ Lipschütz has again reported that oestrogens lower the luteinizing potency of the adult male rat's pituitary (e.g., see Lipschütz, Palacios, and Akel, 1936). 


\section{THE GONADOTROPIC HORMONES}

considered to be due to the luteinizing hormone liberated by the pituitary in situ and to some extent was related to the size of the oestrin dose. ${ }^{38}$ No such increased effect of folliclestimulating hormone after oestrin occurred in hypophysectomized rats. In normal animals, the injection of prolan after oestrin produced no ovarian hypertrophy greater than after prolan alone. If prolan be considered a luteinizing hormone, this result indicates that oestrin does not facilitate the secretion of a synergizing, follicle-stimulating hormone. If oestrin was injected for 8 days, the ovarian response to pituitary follicle-stimulating hormone or prolan was reduced in normal but not in hypophysectomized rats, indicating that this antagonistic effect is on the pituitary, by which some gonadotropic hormone is secreted even in immature animals, and not on the ovary. All these results suggest the following conclusions: (I) oestrin in small doses increases the rate of secretion of luteinizing, but not of follicle-stimulating, hormone; (2) larger doses of oestrin (8-day injection period) diminish the rate of secretion of both gonadotropic hormones; and (3) oestrin appears to have no effect on the ovary. ${ }^{39}$

Leonard's results (1937) led to a different interpretation as far as conclusion (2) is concerned; however, he used a different method. He estimated the quantity of follicle-stimulating or luteinizing hormone in the pituitary of the rat receiving oestrin by determining the effect of a maximum dose of prolan (50 rat-units) or menopausal urine (equivalent to Ioo cc.) and comparing these effects (I) with those of pituitary tissue from control animals and animals receiving oestrin and (2) with the effects of pituitary tissue in addition to prolan or extract of menopausal urine. The degree of augmentation of the effect of prolan on the ovary was taken as a measure of the quantity of follicle-stimulating hormone present in the ad-

38 The maximum effect apparently was produced by 0.5 R.U. of oestrin.

${ }^{39}$ Earlier work had indicated that ovarian regression due to a "sex hormone" is an indirect result of diminished pituitary function. See, however, the results of Robson in the rabbit discussed on pp. 86-87. 
ministered pituitary tissue, whereas similar augmentation of the action of extract of menopausal urine indicated the amount of luteinizing hormone administered. The author administered to rat-units of oestrin daily for Io days. Pituitary tissue in a constant dose of $3.5 \mathrm{mg}$. dissolved in O. I per cent $\mathrm{NaOH}$ was always given, so that the results refer to the concentration of a particular gonadotropic hormone. The administration of oestrin to adult, spayed female rats brought about a reduction in the concentration both of follicle-stimulating hormone and of luteinizing hormone. $4^{40}$ Contrary to the results of Fevold, Hisaw, and Greep, repeated injections of oestrin into immature rats had no effect on the concentration of follicle-stimulating hormone present; however, Leonard agreed that the amount of luteinizing hormone was reduced. It must be remembered that the pituitary body was intact in the immature rats used by Leonard for assay-perhaps constituting a further complicating variable.

Allen and Heckel (I936) reported that pseudopregnancy in the rabbit can be prolonged to 25 days after a sterile mating, provided that oestrin be injected; the authors gave no data on the dosage used. Hidaka (1937) produced pseudopregnancy in rabbits by injecting pregnancy-urine; large doses of an oestrogen caused a prolongation of the condition (5,000-10,000 international units of "Gynandol benzoate" on alternate days). Klaften (1937) injected various doses of oestrone (sometimes as much as $\mathrm{I}, 200,000 \mathrm{I}$.U.) to produce corpora lutea. No corpora lueta could be observed in infantile or juvenile rabbits (doses as high as 150,000 I.U.). ${ }^{4 \mathrm{I}} \mathrm{In}$ adult rabbits there was found, in addition to corpora lutea, glandular cystic hyperplasia of the uterus with loss of sensitivity to the oxy tocic principle of the pars neuralis. Robson's results (1937) raise the question of the site of action of an

${ }^{40}$ Castration alone caused a marked increase in the concentration of folliclestimulating hormone (comparison with normal animals).

4r Mazer, Israel, and Alpers (19.36) stated that mature ovarian follicles could be produced by the injection of large doses of oestrogens into immature rabbits. 
oestrogen in causing persistence of corpora lutea. The other evidence available indicates that the secretion of luteinizing hormone by the pars glandularis is responsible for the appearance of corpora lutea, when these follow the injection of an oestrogen. According to Robson, oestrone (Io $\gamma$ daily) or oestradiol ( $5 \gamma$ daily) maintains the structure and function of pseudopregnant corpora lutea of hypophysectomized rabbits for as long as 13 days. ${ }^{42}$ As in hypophysectomized rabbits receiving no oestrogen, the ovary of these animals did not respond to gonadotropic hormone. The observations of Westman and Jacobsohn (1937) are fully in agreement with those of Robson.

Late in pregnancy in the rat, the administration of $0.5 \mathrm{mg}$. of oestrone daily increases the duration of pregnancy to about $24^{-26}$ days (Selye, Collip, and Thomson, 1935). The authors concluded that this treatment prolongs the life of the corpora lutea, giving rise to interference with parturition and fetal death. Pincus and Kirsch (1936) studied the effect of oestrogens on ovulation and implantation in the rabbit. As much as 3,000 rat-units of oestrone, given before mating, did not prevent ovulation. The administration of oestrone during the $3^{-6}$ days after coitus caused a considerable reduction in the number of implantation sites. The authors concluded that ova were killed in the early blastocyst stage but that cleavage was not affected. Some oestrogens were more harmful than others. Implanted ova, like the fetuses into which they developed, were normal. The experiments of Courrier and Gros (1935) in the cat indicated that "folliculin" prevents nidation in the cat if a total dose of 500-1,000 rat-units be administered on the $5^{-1} 8$ days following coitus. Abortion was caused by 1,000 rat-units during the $38-43$ days, although other doses at other times might not have this effect.

Experiments in parabiotic rats indicate that oestrone interferes with the liberation of gonadotropic hormone by the gonadectomized partner. Meyer and Hertz (1937) injected

${ }^{2} \mathrm{In}$ one experiment $1 \mathrm{mg}$. of testosterone daily was without effect. 
$0.05-5.00 \gamma$ of oestrone daily into the gonadectomized partners of the pairs $q Q$ and $q \alpha^{7} .{ }^{43}$ Rats $30-33$ days old were used and received injections for I I days. The spayed female appeared to be more sensitive than the castrated male (e.g., weight of both ovaries of $q$ of $q$ q without injection, 7I.0 mg.; similar weight, 우 of $q$ \& , if $q$ received oestrone, 24.0 mg.). This would be expected from the fact that ovarian changes are usually more pronounced in the female of the pair $\$ Q^{\top}$. Also the authors concluded that by the technic of parabiosis, a castration change in pituitary function is revealed earlier than by studies of pituitary histology.

According to Victor and Andersen ( I 937), oestrone or oestradiol causes a significant increase in the oxygen-consumption of the pituitary of the rat, whether the hormone be added to the surviving pituitary in vitro or administered to the spayed rat about 6 hours before the gland is removed. No such phenomenon was observed in control tissues (liver, kidney).

b) Progesterone.44-Progesterone, like oestrone and androsterone, may cause regression of the testes in adult ring doves (Bates, Riddle, and Lahr, 1937). To obtain this effect the authors injected 0.25 Clauberg-unit each day for Io days.

According to Hohlweg (I 935) the characteristic effects of gonadectomy on the pituitary are not corrected by the injection of progesterone, despite reports to the contrary. Hohlweg found that the administration of $0.54 \mathrm{mg}$. of progesterone daily for two weeks to adult spayed rats did not alter the histologic changes in the pars glandularis. He attributed the positive results of other investigators to contamination of their extracts with an oestrogen or an androgen. Large doses of progesterone inhibit oestrus in the rat (Selye, Browne, and Collip, I 936; Phillips, 1937). Associated with this effect are moderate ovarian atrophy and some pitui-

${ }_{43}$ For the meaning of the symbols, see pp. $78-79$, n. 25.

${ }_{44} \mathrm{Zwarenstein}$ (1937) concluded that progesterone causes ovulation in the toad (Xenopus laevis) by its direct action on the ovary. Shapiro previously had found that testosterone, androsterone, or certain derivatives of these, adrenal cortical extract, etc., can cause ovulation in this amphibian. 


\section{THE GONADO'TROPIC HORMONES}

tary hypertrophy. There is little basis for interpreting these observations, although Bokslag believed that progesterone, like many other substances, lessens the gonadotropic potency of the pituitary. Moreover, Laroche, Simmonet, and Bompard (I 937) found that progesterone $e^{45}$ lessens the urinary excretion of gonadotropic hormone in spayed women or women past the menopause and concluded that its effect therefore resembles that of oestrone. The corpus luteum hormone appears not to affect the testis or secondary sexual organs of immature male rats (Fels, I936). Fels, however, injected small doses of progesterone (1.2-1.7 $\mathrm{mg}$. as the total dose during I I-22 days).

A few observations in other animals require mention. Dempsey (1937) reported that preovulatory swelling and ovulation are prevented in the guinea pig by the administration of progesterone (0.05 I.U. daily for 20 days). Follicular grow th was not inhibited by the hormone. In the rabbit, also, the injection of progesterone prevents ovulation after coitus, although it appears not to diminish the ovulation-producing effect of prolan ${ }^{46}$ (Makepeace, Weinstein, and Friedman, I 936-37). These observations extend and confirm earlier reports such as that of Mahnert.

c) Androgenic substances such as hormones extracted from the testes or urine or derivatives of these. ${ }^{47}$-Breneman (1937) studied the effects of androgens on the chick receiving injections between the fifth and tenth days after hatching. Observations were continued to an age of 30 days. Substances such as testosterone or dihydroandrosterone benzoate prevented testicular growth during the injection period. However, three weeks after injections were stopped, the testes weighed almost twice as much as those of non-injected chicks. The author also concluded that dihydroandrosterone ben-

45 Total doses of $25^{-5} 8 \mathrm{mg}$. of progesterone as $5^{-23}$ injections over $\mathrm{II}^{-} \mathrm{I}_{44}$ days.

${ }^{4}$ In pregnant does, the dose of prolan or extract of the pars glandularis required to produce ovulation is larger than in oestrous rabbits.

${ }_{47}$ 'Testosterone or androstenedione can cause ovulation by acting on the excised ovary of the toad, X. laevis (Shapiro and Zwarenstein, 1937). 
zoate potentiates the response of the chick's testis to sheep pituitary extract.

Observations like those of Breneman illustrate the complexity and difficulty of interpreting the reported effects of "sex" hormones on the gonadotropic phase of pituitary function. It is necessary to take into account depressant (and perhaps excitatory) effects ${ }^{4}$ on the secretion of gonadotropic hormones as well as substitution-effects on the gonad itself after the removal of the pituitary. For some years the view that "male sex hormone" may lessen the secretion of gonadotropic hormone and thus bring about testicular or ovarian damage has been accepted and can again be illustrated by recent observations in rats. Oestrus, as judged by the vaginal smear, may be prevented in the adult female rat by large doses $^{49}$ of several androgens such as testosterone, androsterone, ${ }^{50}$ androstanedione, and androstenedione (Browman, I 937; Nelson and Merckel, 1937). Androgens may completely prevent ovarian hypertrophy in the female of the parabionts o $\varangle^{\top}$, if administered to the castrated male (Hertz and Meyer I 937). The inhibitory effect of three androgens corresponded to their potency as "male hormones" (e.g., testosterone propionate $>$ testosterone $>$ dehydroandrosterone). Hain (1937) concluded that testosterone propionate antagonizes the oestrogenic effect of oestrone in the spayed rat-a conclusion not in accord with the usual view that antagonism of the secretion of the intact ovary by a male hormone is the indirect result of depression of pituitary function. Hain also found that large doses of androgens such as testosterone or its propionate, and transandrostenediol cause abortion in the rat

${ }^{48}$ Pfeiffer's results led to the conclusion that the normal internal secretion of the rat's testis prevents or inhibits the liberation of luteinizing hormone by the pars glandularis.

${ }^{49} 0.5-5 \mathrm{mg}$. daily. Nelson and Merckel usually injected $0.5^{-1.5} \mathrm{mg}$. daily for as long as 30 days.

${ }^{50}$ According to Bühler (1936) crystalline "Proviron" (androsterone?) does not inhibit the growth of the uterus and ovaries of immature rats receiving $30-50$ caponunits in 8 days. 
if injected during midpregnancy or late pregnancy. Oestrone caused the same effect in 0.003 the dose (or less) of androgen required. Recently, Moore and Price (1937) studied the effect of androsterone on the testes and on the gonadotropic potency of the pituitary of young rats. The daily injection of $0.5^{-1.5} \mathrm{mg}$. of the androgen for 20 days inhibited testicular growth $12-50$ per cent and reduced the gonadotropic action of the injected rat's pituitary. There was no stimulation of spermatogenesis. The authors concluded that the testicular damage was the indirect result of pituitary injury. On the other hand, enormous doses of androsterone $\left(4^{-6} \mathrm{mg}\right.$. daily for 20 days) appeared not to affect the testes of adult rats.

It will be recalled that the gonadotropic potency of pituitary implants increases after gonadectomy. Therefore, the normal internal secretions of the ovary or testis appear to inhibit the secretion (storage) of gonadotropic hormone. Clark had earlier found that, judged by the gonad-stimulating effect of pituitary implants, secretion of testicular hormone occurs much earlier than secretion of ovarian hormone in the rat. In other words, castration at an early age is followed by an increased gonadotropic effect of the pituitary, whereas spaying produces no change. Stein (1935) has shown by this technic that there is considerable testicular secretion even in the first week of the male rat's life. A confusing exception to the generalization that gonadectomy is followed by increased storage or secretion of gonadotropic hormone is that reported by Bates, Riddle, and Lahr (1935). They found that the concentration of gonadotropic hormone in the pituitary of the ox, as indicated by testicular hypertrophy in immature doves, is lowest in the castrated animal (adult steer), apparently being lower than in the pituitary of the bull, calf, embryo, etc.

Another aspect of the problem which can be interpreted as indicating an inhibiting effect of androgens on pituitary gonadotropic function is the prevention or correction of castration changes in the pars glandularis. This has been ac- 
complished in castrated male rats by injecting androsterone (Hohlweg, 1937) and in spayed females by injecting androsterone as well as dehydroandrosterone, androstanedione, or testosterone (Nelson and Merckel, I937). All the authors injected large doses of the androgens used. Schoeller, Dohrn, and Hohlweg (1936) compared the doses of oestradiol benzoate, androsterone, testosterone, and testosterone propionate needed to correct gonadectomy changes in the pituitary of immature or young adult castrated or spayed rats. Oestradiol benzoate (total dose $0.15-0.3 \gamma$ ) was $200-500$ times as potent as the androgens in immature animals, whereas in young adult gonadectomized animals of both sexes its potency was 500-1 3,000 times greater. In the older animals at least, testosterone propionate (total dose $150 \gamma$ ) was found to be the most potent of the androgens, its activity being twice that of testosterone and IO-I 3 times that of androsterone. Frank and Salmon (1936) found that androgens affected symptoms due to castration only slightly and did not influence the excretion of gonadotropic hormone. Their experiments were performed in two castrated men who received total doses of androgens such as $20 \mathrm{mg}$. of androsterone, 25 $\mathrm{mg}$. of dihydroandrosterone benzoate, or I I $5 \mathrm{mg}$. of testosterone. Apparently androgens are much less potent than oestrogens in correcting pituitary castration changes, whether reference is made to altered morphology or to the storage or rate of secretion of gonadotropic hormones. Salmon's report (I 937) also supports this belief. The injection of 8 I $5 \mathrm{mg}$. of testosterone propionate over a period of about 4 weeks into a spayed woman produced effects - i.e., amelioration of menopausal symptoms, disappearance of gonadotropic hormone in urine-corresponding to those of approximately $4 \mathrm{mg}$. of oestradiol benzoate.

Androgens may cause oestrus, prolonged or only lengthened periodically, in animals with intact ovaries (Nelson and Merckel, 1937). Hypophysectomy seemed to increase this effect of dehydroandrosterone. Hohlweg (I 937) believed that 
corpus luteum formation may result from the injection of dehydroandrosterone or testosterone into rats. Obviously suppression of oestrus by an androgen might be related to such an action. There is no evidence that the anterior pituitary plays an important part in these effects of androgens. It has been suggested that the ovary may convert an androgen into an oestrogen.

There is good evidence from recently published reports that androgenic substances also directly affect the testes. ${ }^{51}$ Wells and Moore (1936) found that androsterone or extract of male urine or bull testis (like gonadotropic extracts) might cause precocious spermatogenesis in the ground squirrel (Citellus tridecemlineatus) weeks or months before the germinal epithelium normally becomes active. None of their animals was hypophysectomized. Confirming the work of Walsh, Cuyler, and McCullagh, Nelson and Gallagher (1936) as well as Nelson and Merckel (1937) concluded that "male hormone" (extract of urine, crystalline androgens) maintains spermatogenesis in the hypophysectomized rat. ${ }^{52}$ Injection must be started a day or two after hypophysectomy; if there is an interval of 3 weeks between hypophysectomy and initiation of treatment, spermatogenesis cannot be initiated perhaps because of irreparable damage to the germinal epithelium. The treatment does not correct the degenerative changes in the interstitial cells. Cutuly, McCullagh, and Cutuly ( 1937) believed that the maintenance of scrotal function accounted for the favorable action of androgens on spermatogenesis; however, Nelson and Merckel pointed out that scrotal function

${ }^{5 \pi}$ For studies comparing the effect of several androgens on the secondary sexual organs of normal and hypophysectomized male rats, see Freud (s935) and Laqueur, Dingemanse, and Freud (1935). For example, although (with a cosubstance) testosterone and dihydroandrosterone might produce typical responses in hypophysectomized animals, androsterone was almost without action.

${ }^{52}$ McEuen, Selye, and Collip (1937) concluded that normal testicular structure in the hypophysectomized rat is not maintained by the injection of testosterone. Cutuly, McCullagh, and Cutuly (1937) prevented testicular atrophy in hypophysectomized rats by androsterone (1.50 mg. daily) and testosterone $\left(0.45^{-1.50} \mathrm{mg}\right.$. daily) but not by dihydroandrosterone benzoate (1.25 mg. daily). 
but not spermatogenesis may persist in hypophysectomized rats after the injection of oestrone ( 1,000 I.U. daily). The fact that certain androgenic substances maintain spermatogenesis in hypophysectomized rats suggests that the normal pituitary gonadotropic hormone necessary for the testis is only that stimulating the interstitial cells. If pituitary secretion insures normal function on the part of the interstitial cells, perhaps the secretion of the latter is all that is needed to maintain spermatogenesis.

The interrelationship between the secretion of gonadotropic hormones and the internal secretions of other glands. I. The thyroid gland.-Reports published after those previously reviewed still indicate that only exceptionally does there appear to be an important interrelationship between the secretion of gonadotropic hormones and that of the thyroid gland. Gonadectomy in the guinea pig may be followed by moderate proliferative changes in the thyroid according to Kippen and Loeb (1936). Several authors have studied the effect of thyroidectomy on the gonads or their response to gonadotropic hormone. Leonard and Leonard (I937) found that thyroid deficiency of about one week's duration probably had no effect on the number of follicles (including vesicular follicles) of the immature rat's ovary. According to Friedgood and Cannon (1936), a marked maturation of the ova in the rabbit's ovary can be observed several weeks after thyroidectomy. Another aspect of the problem is the testing of the ovarian response to gonadotropic hormones after thyroidectomy. ${ }^{53}$ Leonard (1936) tested pituitary or urinary extracts in thyroidectomized or normal rats. The extracts themselves were free from thyrotropic hormone. He concluded that the follicle-stimulating phase of the response is greater in thyroidectomized animals and that thyroid hormone inhibits the action of follicle-stimulating hormone but not that of luteiniz-

${ }_{53}$ Benoit (19.36) observed that the response of the testes and secondary sexual organs of the drake to intensive illumination - probably by an indirect neurohumoral mechanism involving the optic nerves and the pars glandularis-could be prevented by thyroidectomy. 
ing gonadotropic hormones such as prolan or pregnant-mare serum. ${ }^{54}$ The observations of 'Tyndale and Levin (1 937) support Leonard's conclusions. These authors found that the ovarian response to follicle-stimulating hormone (extract of "menopause urine") may be much greater in hypophysectomized than in normal immature rats. Inasmuch as the injection of thyroxine reduced the ovarian response of similarly treated hypophysectomized rats, they concluded that poor function on the part of the thyroid may account for the difference found. Obviously this suggestion does not exhaust the list of possible explanations. Thyroid hormone probably produces an inhibitory effect by its action on the ovary (see also Fischer and Engel, I936). On the other hand, the results of Morrin and Loeb (1935) indicated that the response of the guinea pig's ovary to implants of the pars glandularis (guinea pig, ox, rabbit, and rat) was the same whether or not the thyroid had been removed previously.

Halpern and Hendryson (1935) reported that dinitrophenol, a general stimulant of metabolism, does not significantly affect the oestrous cycles of rats and that changes in the oestrous cycles caused by thyroid extract therefore cannot be attributed to the stimulating effect of the extract on metabolism.

2. The adrenal glands.-The hormonal variables requiring attention in a consideration of the interrelationship of the pituitary (gonadotropic function) and adrenal glands are pituitary gonadotropic hormones, adrenal cortical stimulating hormone, and the internal secretions of both the gonads and the adrenal cortex. ${ }^{55}$ Moreover, the internal secretions of the "end-organs," the gonads and the adrenal cortex, are probably so similar in structure that, under suitable conditions,

54 Loeb, Saxton, and Hayward (1936) believed that thyrotropic hormone usually is associated with luteinizing hormone and "atresin" in the pituitary. The concentration of follicle-stimulating hormone tends to be low if that of the other three substances is high.

5s Epinephrins, the important medullary secretion, appears to require no consideration. 
they may possess substitutional properties. For example, it appears that the internal secretion of the corpus luteum, progesterone, may be a much less potent but fairly satisfactory substitute for adrenal cortical hormone after adrenalectomy. Therefore, experiments dealing with the interplay of so many variables, to which are added the necessary inaccuracies of biological work, must be interpreted with reserve.

Complete adrenalectomy may affect adversely sexual and, presumably, pituitary gonadotropic function. Until there is better evidence to the contrary, these actions are best interpreted as resulting from the general, nonspecific, harmful effects of a deficiency of the adrenal cortical hormone. Martin and Fazekas (1937) concluded that salt therapy of bilaterally adrenalectomized adult female rats facilitated the normal cyclic sexual phenomena (normal oestrous cycles in 55 per cent of animals receiving salt solution in comparison with normal cycles in 2 I per cent of control animals observed for a much shorter period). What observations they made with pituitary implants are not of much value as an aid in interpretation. According to Fitzhugh (1937), adrenalectomy in the rat is followed by a disappearance of oestrous cycles in the female or atrophy and degenerative changes in the testes of the male; he reported that both of these changes could be corrected by the injection of adrenal cortical extract. Britton and Kline (1936) concluded that in the presence of adrenal insufficiency the female rat is usually sterile; if adrenal insufficiency is produced in pregnant animals, abortion commonly occurs and there is no lactation. All these harmful changes can be prevented by adrenal cortical extract. Fertility also is reduced in the adrenalectomized male rat surviving because of accessory tissue. Friedgood (1937) studied the effect of adrenalectomy on ovulation in the cat following coitus. He found that removal of the second adrenal $15-55$ minutes after mating was not followed by ovulation (9 cats), whereas if the operation was delayed until 6 hours after mating, normal ovulation occurred (3 cats). Bilateral adrenal- 
ectomy did not prevent ovulation due to injected pituitary gonadotropic hormones; however, there appeared to be a retardation of ovulation and interference with the subsequent formation of corpora lutea (Friedgood and Foster, 1937).

Pituitary implants may markedly prolong the life of adrenalectomized young female rats. It is likely that this effect is due to an indirect action on the ovaries: luteinization with abnormal production of corpus luteum hormone occurs (Emery and Schwabe, 1936; Cavanaugh and Gaunt, I 937)..$^{6}$

Several authors have declared that extract of the adrenal glands can cause a moderate gonadotropic effect in rats. ${ }^{57}$ Extract of the adrenal of the gelding or ox in a dose equivalent to II + mg. of dried gland was found by Deanesly (1935) to cause oestrous changes in the uterus and enlarged or cystic follicles in the ovaries of 3 of 10 immature rats. The experiments of Allen and Bourne (I936) are of little significance because their female rats were 56-70 days old when used. Fitzhugh (1937) believed that extract containing adrenal cortical hormone causes a slight hypertrophy of the ovaries and uterus or a moderate atrophy of the testes of rats. In the female rat the results were obtained by injecting extract on the 21-44 days of life. Hoffmann (1937) concluded that cortical extracts containing no cortical hormone cause a gonadotropic effect or a potentiation of the action of prolan in immature rats. The maximum effect appeared after 65 hours; the dose used was equivalent to $600 \mathrm{mg}$. of dried adrenal cortex; the substance responsible for the effect was insoluble in lipoid solvents. Perhaps these effects, elicited with difficulty, are due to an action on the pars glandularis. Corey (I 937) was un-

${ }^{56}$ Swingle and others (1937) showed that anterior pituitary extract may prolong the life of bilaterally adrenalectomized cats independently of sex or the gonads. Also they found that the bitch during pseudopregnancy requires no adrenal cortical hormone, although bilaterally adrenalectomized. Their negative results with progesterone in cats were perhaps due to insufficiently large doses.

57 The secondary sexual organs of castrated, hypophysectomized rats may respond to adrenal cortical stimulating hormone by a stimulation of growth (Davidson, 1937). 
able to produce oestrus by injecting potent cortical adrenal extract into adult hypophysectomized female rats.

3. The epiphysis.--Despite Engel's statement to the contrary (review, 1936), there is not yet satisfactory evidence that pineal secretion antagonizes the gonadotropic secretion of the pars glandularis. Recent observations are those of Fleischmann and Goldhammer (1936), Tarkhan (1937), and Wade (1937). Wade also used prolan and pregnant-mare serum as gonadotropic substances.

4. The lactogenic hormone of the anterior pituitary. ${ }^{58,59}$ Lactogenic extracts of the pars glandularis, injected into female rats, may cause a prolonged period of dioestrus (Dresel, 1935). Similar results were obtained by Nathanson, Fevold, and Jennison (1937) both with pituitary extract and with an extract of the urine of lactating women. They suggest that luteinizing hormone rather than lactogenic hormone is responsible for the effect. According to Engelhart (1936), the lactogenic hormone brings about extensive luteinization of the rabbit's ovary. However, not all his extracts were free of gonadotropic hormone. Lahr and Riddle (1936) injected lactogenic extract into rats and caused a temporary suppression of oestrous cycles in adult females. Large ovaries containing corpora lutea were present after $8-12$ days' treatment. The authors doubted that progesterone-secretion was responsible for the disappearance of oestrous cycles and suggested that either an alteration of ovarian function or an interference with the liberation of follicle-stimulating hormone accounted for the change produced. Desclin and Grégoire (1937) transplanted ovaries to the kidneys of female rats spayed a few days postpartum. Lactation was permitted to continue in half of the group. A comparison of the ovaries of the lactating and nonlactating groups 15 days later led the

${ }^{58}$ Leblond and Nelson (1936) concluded that the maternal instinct depends neither on lactation nor on internal secretions of the anterior pituitary, although these may reinforce or reduce manifestations of the instinct.

59 See also chap. $v$. 
authors to conclude that the secretion of gonadotropic hormone is inhibited during lactation. According to De Fremery and Denekamp (I935), doses of pituitary lactogenic hormone, large enough to initiate the secretion of milk, cause abortion or fetal death in utero if administered to pregnant goats, guinea pigs, rabbits, or rats. The lactogenic extract, prolactin, of Bates, Riddle, and Lahr (I 937) caused as marked a regression of the testes of adult pigeons as hypophysectomy. They believed that the extract did not affect the testes but interfered with the liberation (or formation?) of "follicle-stimulating" (gonadotropic) hormone.

The statements in the foregoing paragraph can soon be tested accurately, inasmuch as the isolation of crystalline, lactogenic hormone has been announced recently by White, Catchpole, and Long (I 937).

The nervous control of the secretion of gonadotropic hormones. - In the discussion of the effect of light and related radiations on the gonads it was emphasized that many of the observations indicate that the secretion of gonadotropic hormones by the pars glandularis may be reflexly stimulated, the afferent arc being the optic nerves carrying impulses to the hypothalamus, whence secretory fibers pass to the pars glandularis. Here it is proposed to review other data which also indicate that impulses from the central or peripheral nervous systems may control the secretion by the anterior pituitary of gonad-stimulating hormones into the blood stream.

Rosen and Shelesnyak ( 1937 ) produced pseudopregnancy in more than one-half of a group of rats as a result of the intranasal instillation of a solution of silver nitrate. This effect possibly is related to a reflex effect on the anterior pituitary. Other investigators have studied peripheral sympathetic nerves. Friedgood and Pincus (1935) stated that bilateral faradic stimulation of the cervical sympathetic nerves caused ovulation in 3 of 6 adult rabbits as well as clear-cut maturation of ova. In later work Friedgood and Cannon (I936) reported that bilateral cervical sympathetic stimulation by a 
condenser-discharge method failed to cause ovulation but did bring about maturation of ova. They believed that the cervical sympathetics have only a limited control over the discharge of gonadotropic hormones by the anterior pituitary. Brooks (1937) was unable to prevent the ovulation following coitus in the rabbit by removing sense organs or parts of the central and peripheral nervous systems (such as the olfactory bulbs or the cerebral cortex or the sacral cord, the abdominal sympathetics, the uterus, and part of the vagina).

Diffuse powerful electrical stimulation applied to the brain or lumbosacral cord of the rabbit can cause ovulation, although the latter occurs $7^{-I} 4$ hours later than after coitus (Marshall and Verney, 1936). Harris (1936) applied a similar stimulus to the head of the adult female rat; as a result pseudopregnancy occurred in about 70 per cent of the animals. The same author (1937) was able to produce ovulation, sometimes associated with the formation of cystic or hemorrhagic follicles, by applying an electrical stimulus to the hypothalamus or pituitary of the rabbit. According to Haterius and Derbyshire (1937), who used a small bipolar electrode, a sharply localized area $5 \mathrm{~mm}$. below the surface above and anterior to the optic chiasm causes ovulation when stimulated. There is an associated motor response (flexion of hind legs, pelvis, and trunk; elevation of tail). The authors used rabbits. Cahane and Cahane (1935-36) described histologic changes in the anterior pituitary and genital atrophy in 2 of 8 rats surviving a lesion made in the infundibulo-tuberal region of the hypothalamus.

Presumably impulses from the hypothalamus (or thalamus) pass down the stalk and provoke secretion of gonadotropic hormones when central stimulation is followed by ovulation and pseudopregnancy. However, the evidence is much less complete than that afforded by similar studies of the secretion of the diuresis-inhibiting hormone of the pars neuralis. Harris (1937) stated that lesions of the stalk were followed by genital atrophy in both male and female rabbits. According 
to Westman and Jacobsohn (1937), this occurs in female rats as well as female rabbits; the effects, after section of the stalk, resemble those of hypophysectomy except that degeneration of the cells of the membrana granulosa does not appear. However, Brooks (1937-38), who used only female rabbits, concluded that the severing of the stalk, although preventing ovulation due to coitus and causing atrophy of the pars neuralis, had no other effect including possible actions on growth, maintenance of the genital tract, ovaries, thyroid, adrenal, etc. The ovaries contained ripe follicles from which ovulation occurred after the injection of pregnancy-urine. Brooks emphasizes that operations on the stalk may also seriously impair the vascular supply of the pituitary body. According to Keller and Hamilton (I937), breeding, pregnancy, parturition, and lactation can occur normally in the dog after the hypophysial stalk has been severed.

$V$ itamins and minerals in relation to the gonadotropic hormones of the anterior pituitary. - It is natural that the vitamin considered to be necessary for normal reproductive performance, vitamin $E$, should receive the most attention of those interested in the possible effects of vitamin deficiency on the pars glandularis. Extracts, such as wheat germ oil, containing a high concentration of vitamin $\mathrm{E}$, possess no gonadotropic action (Verzár and others; Saphir, 1936). Marchesi (I935) stated that adult female rats on a diet deficient in vitamin $\mathrm{E}$ and otherwise sterile became pregnant and delivered the young normally after a suitable course of treatment either with anterior pituitary extract or prolan. The young died shortly after birth. Diakov and Křiženecký (1935), however, believed that the vitamin is necessary for the successful completion of pregnancy, although the administration of either prolan or anterior pituitary extract might bring about follicle growth and ovulation, and make possible fertilization and implantation in female rats receiving no vita$\min \mathrm{E}$. The gonadotropic hormone(s) causing ovulation in oestrus rabbits is reduced in amount in the pituitary of the 
adult female rat on a diet contraining no vitamin $\mathrm{E}$ (Rowlands and Singer, 1936). The reduction is comparable to that occurring on the sixteenth day of pregnancy in normal rats (50-60 per cent of the normal value). The pituitary of animals which recover from the vitamin deficiency contains as much as or more hormone than the normal female gland. Vitamin-E deficiency is accompanied by no conspicuous change in pituitary weight. ${ }^{60}$

According to Orent-Keiles, Robinson, and McCollum (I937) a sodium-deficient diet brings about, in the female rat, a delay in sexual maturity and a serious disturbance in the oestrous rhythm as well as other phases of reproductive physiology. The animals do not mate. Males, however, remain fertile for $2.5-3$ months. To what extent pituitary function is altered is not known.

Riddle and Dotti ( 1936 ) concluded that pituitary gonadotropic hormone causes an increase in the concentration of serum calcium in the pigeon (normal, hypophysectomized, or thyroidectomized) but not after gonadectomy. Other pituitary extracts, not containing gonadotropic hormone, were without action. They believed that oestrogens but not androgens had a similar action in the pigeon, fowl, and dog (but not the rabbit); often their results were sufficiently irregular as not to be convincing. Kozelka and Tatum (I937) found that the injection of I 50 rat-units (total dose?) of gonadotropic hormone lowered the serum calcium of rachitic dogs I.7 mg. per cent (from IO. I to $8.4 \mathrm{mg}$. per cent).

Neoplastic growths and the gonadotropic hormones of the pituitary body.-Both Katz (1936) and Druckrey (1936-37) concluded that pituitary gonadotropic hormones antagonize

${ }^{60}$ Selye and Collip (1 936) reported that rats on a "deficent diet" ( 50 per cent ground beans, 50 per cent "Purina") went into permanent dioestrus with ovarian atrophy. Inasmuch as the ovaries of such animals responded to prolan in a typical fashion, the authors concluded that the diet led to a failure of secretion of gonadotropic hormone by the pituitary.

Teresa (I937) believed that the amount of gonadotropic hormone in the pituitary of the mouse fed a diet lacking vitamin B is less than normal. This observation is contrary to that of Marrian and Parkes in rats. 
the growth of malignant neoplasms. Their reasons were the following: gonadectomy is followed by an inhibition of growth (including metastases) of such malignant tumors as tar cancer, the Jensen sarcoma, and the Flexner-Jobling carcinoma; this inhibitory effect is at its peak when the amount of gonadotropic hormone in the gonadectomized animal's pituitary is highest; the inhibitory effect of gonadectomy may be somewhat antagonized by an oestrogen; the injection of a gonadotropic extract (prolan, 5 rat-units on alternate days) also may inhibit tumor-growth in both normal and gonadectomized rodents. The report of Bischoff and Maxwell (1936) directly contradicts this interpretation as far as transplanted sarcomata ( 180 and RIO) are concerned. Although injections of prolan or pituitary gonadotropic hormone produced marked changes in the gonads, they did not inhibit tumor-growth.

The metabolism of the gonadotropic hormones of the anterior pituitary. ${ }^{6}$ - Freed (1935), supplementing previous reports, concluded that gonadotropic hormone, probably originating in the pars glandularis, is found in the urine of children more than 4-5 years old. The urine of children 10 years old contains as much hormone as the urine of adults. According to Frank and Salmon (1935), cyclic variations in the concentration of both follicle-stimulating hormone and luteinizing hormone can be detected in the blood and urine of normal women. They reported that the concentration of the hormones in the blood increased about the $9^{-12}$ day of the menstrual cycle and that a day or two later (IO-I 4 day) there occurred

6r Engel and Werber (1937), confirming Anderson and Haymaker (1935), found that tiss' 2 cultures of the pituitary of the mouse contain no detectable amount of gonadotropic hormone. The degree of growth of the typical epithelial cells is not affected by the previous treatment (e.g., gonadectomy) of the mouse furnishing the pituitary tissue (see also chap. i).

The relationship between the pituitary and the embryonic development of the gonads in the goat, ox, pig, and sheep was investigated by Daineko (1936).

Saxton and Loeb (1937), using the female guinea pig for assay, studied the gonadotropic effects of the pituitary of man in relation to sex, age, pregnancy, and lactation. Those interested should read the original report.

$$
\text { [103] }
$$


a rise in the urinary concentration of the hormones. ${ }^{62} \mathrm{It}$ must be emphasized that these reports may not be in accord with those of other investigators. The reports and discussions of Österreicher (1935) and Fluhmann (1937) should be read to correct any belief that various authors are in reasonable agreement as to the concentration of gonadotropic hormones in the blood or the excretion of the hormones in the urine of both children and adults. This is also true of the cyclic variations which may be found in the blood and urine of women.

The daily urinary excretion of gonadotropic hormone by normal men is probably greater than 6 rabbit-units (Friedman and Weinstein, 1937).

Anselmino and Hoffmann (1936) concluded that early in pregnancy small amounts of pituitary gonadotropic hormone are excreted in the urine. In the urine of patients with hyperemesis gravidarum an increased amount of pituitary gonadotropic hormone was believed to be excreted, whereas none of the hormone could be found in the urine of patients with eclampsia. These conclusions must be regarded only as suggestions, because detection of the pituitary hormones was based on the production of ovarian hypertrophy greater than $55^{-60} \mathrm{mg}$. in rats $30-35$ days old $(28-35 \mathrm{gm}$.). It was assumed that the prolan present produced a limited hypertrophy. A total number of only 54 rats was used and the possible synergistic effect of the secretion of the rats' pituitaries was disregarded. According to Tenney and Parker (1937), the urine of parturient women contains a pituitary-stimulating substance causing indirect gonadotropic effects. They believed that this substance could be distinguished from prolan which

${ }_{62}$ The authors as well as Frank, Salmon, and Friedman (1935) performed their assays in normal rats. Their results should be confirmed (e.g., the presence of luteinizing hormone) in hypophysectomized rats (see also Guyénot and others, 1936). Evans and Simpson (1935) produced superovulation and even corpus luteum formation with extract of "menopause urine." 'To what extent these phenomena would have been observed in hypophysectomized rats is not known. Also, they agreed with others in their description of the synergistic effects of such extracts with prolan. 
was completely precipitated by 5 volumes of alcohol, whereas under similar conditions only 20 per cent of the newly described substance could be recovered. Obviously, better data are needed to establish their conclusion.

Monnier (1936) found that lumbar or ventricular cerebrospinal fluid of patients with brain tumor (9 of 20) or other cerebral disease ( 3 of Io) stimulated the gonads of male or female immature mice. Such effects were never produced by normal cerebrospinal fluid. (Other reports are mentioned in the previous volume.)

Miscellaneous observations.-Emery (1937) reported that splenectomy in the rat does not alter various gonadotropic effects of "minimal strength" pituitary transplants. Gordon and others had reported differently but used prolan instead of a true anterior pituitary hormone.

THE PREPARATION, ASSAY, AND SPECIAL EFFECTS OF GONADOTROPIC PITUITARY EXTRACTS

The preparation and chemical nature of pituitary gonadotropic hormones. - The complex effects of pituitary gonadotropic extracts have led to equally complex interpretations of the number of hormones secreted. Even the generally accepted belief that separate follicle-stimulating and luteinizing hormones are secreted by the pars glandularis has not been proved with convincing thoroughness. Therefore, once a gonadotropic hormone has been isolated as a crystalline, chemically pure substance, it will be possible rapidly to decide many vexing questions of interpretation.

What data have been published recently still indicate that pituitary gonadotropic hormone(s) are proteins or substances of protein-like nature. Tryptic digestion (or heat to a far less extent) rapidly destroys pituitary hormones, causing ovulation in rabbits or testis-stimulation in immature doves (Riddle and others, 1936). On the other hand, tryptic digestion under proper conditions appears to bring about destruction of nearly all the luteinizing action of anterior pituitary extract 
in the rat but permits the retention of the extract's folliclestimulating effect (McShan and Meyer; Chen and van Dyke). Rowlands (1935) found that extracts of the beef anterior pituitary, tested by their ovulation-producing effect in rabbits, were not affected by "Merthiolate" ( 0.02 per cent) but deteriorated rapidly at room temperature $(50$ per cent loss in 9 days, 75 per cent loss in 23 days). Stored at $-2^{\circ} \mathrm{C}$, such extracts lost less than 50 per cent of their activity after a year. Maxwell and Bischoff (I935), who doubt that there are two gonadotropic hormones, found that their pituitary extract was inactivated by o. I N NaOH ( 3 hours at $37^{\circ} \mathrm{C}$.) but was only partially destroyed by treatment with $0.033 \mathrm{~N}$ $\mathrm{NaOH}$ or o. I $\mathrm{N} \mathrm{HCl}$ under the same conditions. Mild oxidizing or reducing agents did not affect the hormone(s), whereas it was partially or completely inactivated by reagents reacting with amino, imino, or hydroxyl groups. Formalin treatment $\left(4-10\right.$ per cent at $\left.\mathrm{pH} 7^{-8}\right)$ did not prevent luteinizing effects. ${ }^{63}$

The extraction procedure of Bates, Riddle, and Lahr ( 935 ) utilized as an initial solvent 60 per cent aqueous alcohol at a pH of 9-9.5. Guyénot, Ponse, and Dottrens (I935) described methods - differential filtration, hydrolysis by acid, autolysis, peptic digestion - of separating follicle-stimulating ("auxogenic") hormone from luteinizing ("crinogenic") hormone. Evans and others, in two reports published in 1936, described methods for isolating from pituitary tissue specific extracts comprising ( $\mathrm{I}$ ) a substance stimulating the interstitial cells of the ovary or testis, (2) a luteinizing substance, (3) a follicle-stimulating substance which also stimulates the testicular germinal epithelium, and (4) a substance inhibiting or antagonizing the action of the follicle-stimulating substance (or of prolan or of the gonadotropic hormone of pregnant-mare serum). Revised methods, including necessary precautions, of extracting gondaotropic hormones from urine have recent-

${ }^{63}$ Hayward and Loeb (1937) studied the effects of pituitary tissue implanted after immersion for hours to days in strong solutions of sucrose, glycerine, or urea. 
ly been published by Levin and Tyndale (1935), Thomsen and Pedersen-Bjergaard (1935-36), Palmer (1937), and Katzman (1937).

The assay of the gonadotropic hormones of the anterior pituitary.-The "unit" of a gonadotropic hormone, whether the assay be performed in the immature rat or in some other animal, is a term which remains without precision. Recently a start has been made by the National Institute for Medical Research (Great Britain) to set up provisional standards and to determine from the experience of widely separated investigators what methods of assay should be recommended. If standard preparations for assay and standard procedures of performing assays are agreed upon and adopted, units of gonadotropic activity designated by different authors can be evaluated with far more assurance than at present. Assay is affected by many factors, the importance of which varies with the source and nature of the gonadotropic agent. For example, the frequency of injection and the route of injection may greatly modify the response of the immature rat's ovary to pituitary gonadotropic hormone; however, Bates and Riddle (1936) found this not to be true of the effect of hormone on the pigeon's testis. ${ }^{64}$ Other obvious factors are the size (or litter-size $)^{65}$ of the immature rat, diet, racial strain, ease with

${ }^{64}$ Bates, Riddle, and Lahr (1935) stated that the response of the immature dove's testis to gonadotropic extract is not affected by the presence or absence of luteinizing hormone.

65 An example of work bearing on this factor is the report of Engle, Crafts, and Zeithamel (1937). The effect of the rat's rate of growth on the age and body-weight of vaginal canalization was investigated by comparing groups of individuals from litters of varied size - the rate of growth usually being inversely proportional to litter-size. The results were as follows:

\begin{tabular}{|c|c|c|}
\hline \multirow{2}{*}{ LitTER-Size } & \multicolumn{2}{|c|}{$\begin{array}{l}\text { Opening of Vaginal Orifice } \\
\text { (First Oestrus) }\end{array}$} \\
\hline & Age in Days & Weight in Grams \\
\hline 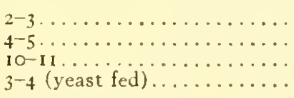 & $\begin{array}{l}46.5 \\
52.9 \\
77.9 \\
41.1\end{array}$ & $\begin{array}{l}109.9 \\
114.8 \\
115.1 \\
127.0\end{array}$ \\
\hline
\end{tabular}




\section{THE PITUITARY BODY}

which the preparation used is metabolized, possible synergistic or antagonistic effects of substances in the extract, etc. There is increasing recognition of the inability to control the effects of the anterior pituitary secretion if normal animals are used for assay. Especially is it necessary to use hypophysectomized animals in testing extracts considered to have specific qualitative effects.

Investigations of the relationship between dosage of pituitary gonadotropic hormone and the response of the gonads

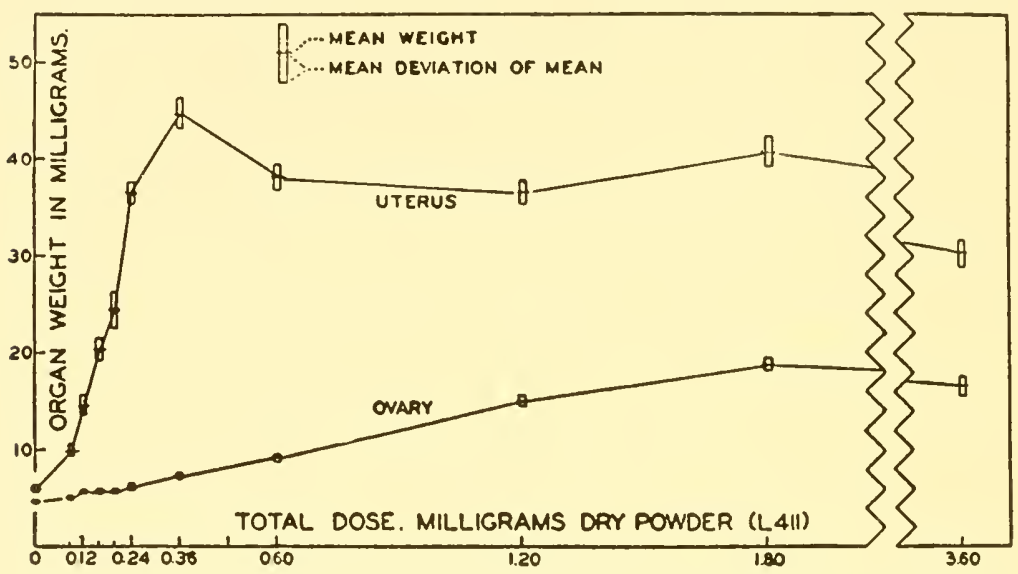

FIG. I 4.- The effect of various doses of follicle-stimulating hormone of "castrate" urine on the weight of the ovary and uterus of the mouse. (From Levin and Tyndale, Endocrinology, 21, 619-28 [1937].)

have been made recently by Deanesly (1935), Chow and Liu (1937), and Levin and Tyndale (1937). Deanesly recommended that the ovarian hypertrophy produced for quantitative assay of pituitary gonadotropic hormone should be represented by paired ovarian weights within a range of about $30-$ $80 \mathrm{mg} .{ }^{66}$ However, it has become increasingly clear that the use of ovarian weight as a basis for assay may have only a limited value. Levin and Tyndale found that the response of the uterus of the immature albino mouse to the follicle-

${ }^{66}$ After fixation in Bouin's fluid and partial dehydration (70 per cent alcohol). 
stimulating hormone of "castrate" urine to be $5^{- \text {IO }}$ times greater than that of the ovaries. They obtained data on the relationship between dose and uterine hypertrophy (see Figs. $I_{4}$ and 15 ). In a later report Heller, Lauson, and Sevringhaus (1938) particularly studied the response of the immature rat's uterus. According to Bachman (1936), corpora lutea

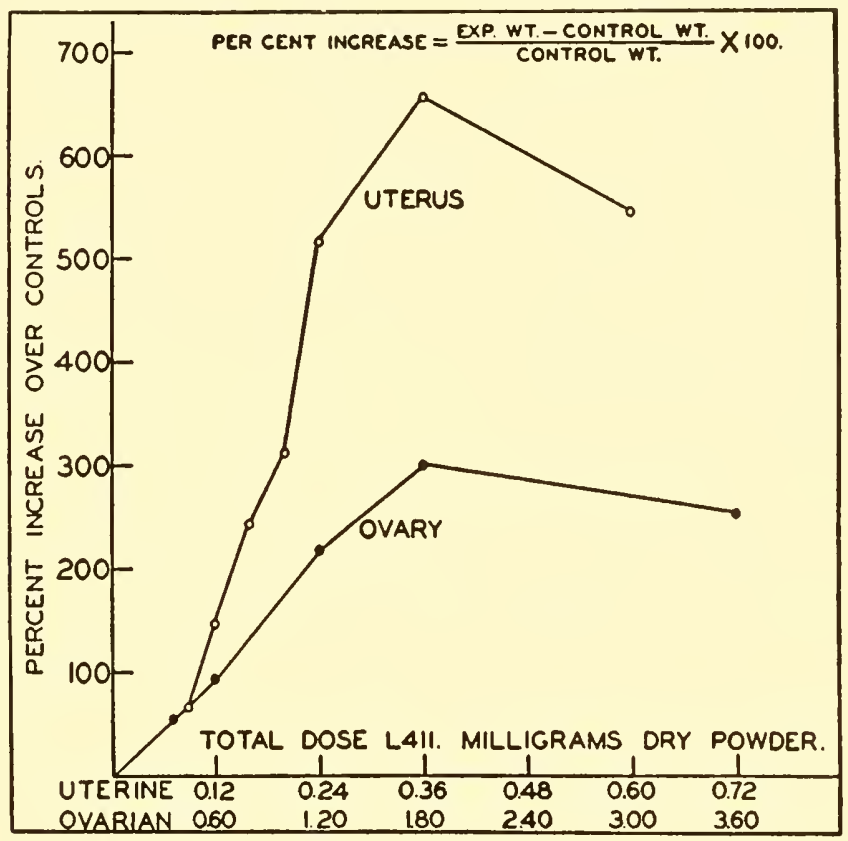

Fig. I 5.- The change in weight of the ovary and uterus of the mouse, in terms of control weights, following various doses of follicle-stimulating hormone of "castrate" urine. (From Levin and Tyndale, Endocrinology, 21, 619-28 [1937].)

without uterine changes may appear after the injection of gonadotropic extract (sheep pituitary extract, prolan) into very young rabbits. No effects are produced in rabbits less than $1.5^{-2}$ months old.

Special effects of gonadotropic pituitary extracts or related substances. 1. The doctrine of antihormones.- Since the publication of reports by Collip and his collaborators indicating 
that the prolonged injection of gonadotropic extracts is followed by the production, at some unknown site, of "antihormone" which can be detected in the serum, numerous authors have made many further investigations of the phenomenon. The production of antihormone is of practical importance; for, if an injected extract produces antihormone, successful therapy may be thwarted and the patient's condition even may be worsened. ${ }^{67}$ The other important question is the significance of possible antihormone balance to gonadotropic and other hormones secreted by the gland in situ. Chiefly from experiments in parabiotic animals there is good evidence against the view that the body may produce its own antihormone to inhibit excessive secretion by a gland like the anterior pituitary. Also the evidence from artificially introduced hormone is by no means concordantly in favor of the antihormone hypothesis.

Usually the prolonged injection of a gonadotropic extract is followed by progressively less effect, until finally the ovaries or testes of the injected animals may actually be smaller than those of animals not receiving treatment. Antihormone can then be readily detected in the serum by its inhibitory effect on the gonadotropic action of the extract when the serum and extract are injected into fresh animals. An attempt again to stimulate the gonads of the animals receiving the prolonged injections after a period of rest either fails or produces only a slight change. Most investigators are in agreement with these general statements. However, the interpretation of the phenomenon is a matter of disagreement which perhaps cannot be settled from a consideration of the published data.

One obvious basis for the development of antihormone in response to the long-continued injection of gonadotropic ex-

${ }^{67}$ Spence, Scowen, and Rowlands (1938) concluded that the injection into human beings of prolan $(\mathrm{I}, \infty 00$ rat-units weekly) or extract of the pig pituitary (30-50 R.U. weekly) for varying periods up to 7 months was followed by the appearance of no "serious amount of anti-gonadotropic activity" in the serum.

Rowlands and Parkes (19.37) suggested that antihormone obtained from serum of suitably injected animals might be used to lessen the effects of hormones secreted by the hyperactive pituitary. 
tract is the production of immune bodies by specific or nonspecific protein included in impure extract. Max, Schmeckebier, and Loeb (1935), for example, believed that extraneous protein was responsible for the refractory state of their guinea pigs to extract injected after $4^{-6}$ weeks of treatment. Others have attempted, without success, to correlate the presence or development of typical immune bodies with the development of antihormone. Usually the development of antibodies does not parallel the development of antihormone (Collip, I935; Gustus, Meyer, and Dingle, 1935; Brandt and Goldhammer, 1936). Sulman (1937) who used prolan or pregnant-mare serum as possible antigens concluded that these substances, injected into rabbits, behaved neither as antigens (reaction with antisera, including complement-fixation) nor as haptenes. ${ }^{68}$

A great deal of work is represented by investigations of the species (and source) specificity of antihormones due to the injection of gonadotropic extracts. ${ }^{69}$ If gonadotropic extracts were highly species specific, this fact would constitute evidence that they are artificially produced by some mechanism analogous to antigen-antibody reactions. The results are most varied and indicate that, in the hands of different investigators-and sometimes in the hands of the same investigator-gonadotropic extracts may or may not be species specific. ${ }^{70}$ Chen (1937) found that the ordinary proteins of sheep or human serum, injected as serum repeatedly into

${ }^{68} \mathrm{~B}$. F. Chow informs me that failure to secure a complement-fixation reaction does not necessarily indicate that a substance is not antigenic and that the antigenic effects of a highly potent antigen (e.g., prolan) may not be recognized unless relatively large doses are injected.

${ }^{69}$ Gonadotropic extracts, both for producing antihormone and for detecting the presence of antihormone, have been obtained from the following sources: pituitary tissue of man, the horse, ox, pig, or sheep; blood, placenta, or urine of human pregnancy; serum or urine of equine pregnancy; urine of women past the menopause.

${ }^{70}$ Parkes and Rowlands (1937) concluded there is at least class specificity toward antihormones. They were unable to prevent the thyrotropic or gonadotropic effects of mammalian extracts in fowls by first injecting rabbit serum rich in antihormone as judged by tests in mammals. 
rabbits, do not cause the production of substances behaving like antihormone toward gonadotropic extracts of sheep or human pituitary. Several recent authors concluded that various gonadotropic extracts from the pituitary, blood, or urine of different animals could produce antihormone which is not species specific (Gregerson, Clark, and Kurzrok; Parkes and Rowlands, I936; Collip, Rowlands, Thompson and Cushing, 1937). These experiments are the most complete and extensive. However, mixed results may be obtained, so that some extracts produce antihormone which appears to be partially or completely species characteristic (Collip, Rowlands, I937). Finally, several observers found, at least with their preparations, that antihormones toward gonadotropic extracts inhibit the effect only of extracts of tissue or urine of the animal of the same species (Fluhmann, 1935; Gustus, Meyer, and Dingle, I935; Brandt and Goldhammer, 1936). As far as pituitary gonadotropic extracts are concerned, the weight of evidence appears to be against the view that antihormone is species specific. Perhaps there is true "species specificity" when extract of human pregnancy-urine (prolan) or pregnant-mare serum is used as an "antigen" (Rowlands). "Source specificity" is believed by Rowlands (1938) to be complete in the case of pregnant-mare serum, although others (e.g., Thompson and Cushing) have obtained different results. It is probable that, if antihormone formation is analogous in some phase or phases to the formation of immune bodies, the problem is rendered more complex and more difficult to interpret than most immune reactions, because of the possibility that the hormone "antigens" are more complex than is usually suspected (see Rowlands, 1938).71 'The con-

${ }^{71}$ 'The method of detecting antihormone (cf. failure of ovarian hypertrophy in the rat and failure of ovulation in rabbit) may determine whether the result is positive or negative. Using an extract of the anterior pituitary of the ox as "antigen," Rowlands (1937-38) found that its antihormone antagonized the gonadotropic action of sheep pituitary extract (ovarian hypertrophy in rats), whereas Parkes and Rowlands (1936) found this not to be the case when they used the production 
sidered opinion of investigators like Rowlands is that an immune-like reaction has been neither proved nor ruled out.

Several attempts to inhibit the effects of gonadotropic hormones secreted by the pars glandularis in situ have been successful and constitute evidence favorable to the view that antihormone-formation is important physiologically. Parkes and Rowlands (I 936) showed that the ovulation which usually follows coitus in the rabbit can be prevented by the intravenous injection, I 5 minutes after mating, of rabbit serum containing a high titer of antihormones toward the gonadotropic principles in ox anterior pituitary extract. An extension of this study by Rowlands (1937) indicated that such serum containing antihormone, although not interfering with corpus luteum formation, pregnancy, or lactation in the mouse or rat, did have the following antigonadotropic effects: (I) it prevented ovulation in the prepubertal rat, (2) it produced atrophy of the reproductive organs of the adult male rat, and (3) it could prevent, in the rabbit, ovulation or corpus luteum formation or, in pregnant animals, implantation of the blastocyst or fetal survival. According to Collip (1937), antihormone-containing serum prevents oestrous cycles in rats. Also he believed (I935) that antihormone-like substances may appear in the blood spontaneously. Thompson and Cushing (1937) caused marked ovarian atrophy in growing rats by injecting serum containing antihormone (sheep pituitary extract as "antigen" for about two months). Hisaw, Hertz, and Fevold (I 936) concluded that, although the continued injection of sheep pituitary extract might be accompanied by a refractory condition of the ovaries in juvenile monkeys (Macaca mulatta), only temporary effects were produced in adults. They believed that the antihormone pro-

of ovulation in the rabbit as an indication of gonadotropic effect. However, Parkes and Rowlands used a saline suspension of the sheep pituitary body.

More clear cut are the experiments of Rowlands (1938) who found that the serum of an animal receiving a course of injections of pituitary extract might augment the effects of the extract in immature rats (ovarian hypertrophy) and yet inhibit the action of the extract in rabbits (ovulation response). 
duced in response to sheep pituitary extract does not inhibit the action of gonadotropic hormone secreted in situ by the monkey's pars glandularis.

The strongest evidence against antihormone-production as a physiological mechanism is afforded by experiments with animals living parabiotically, as has been emphasized re-

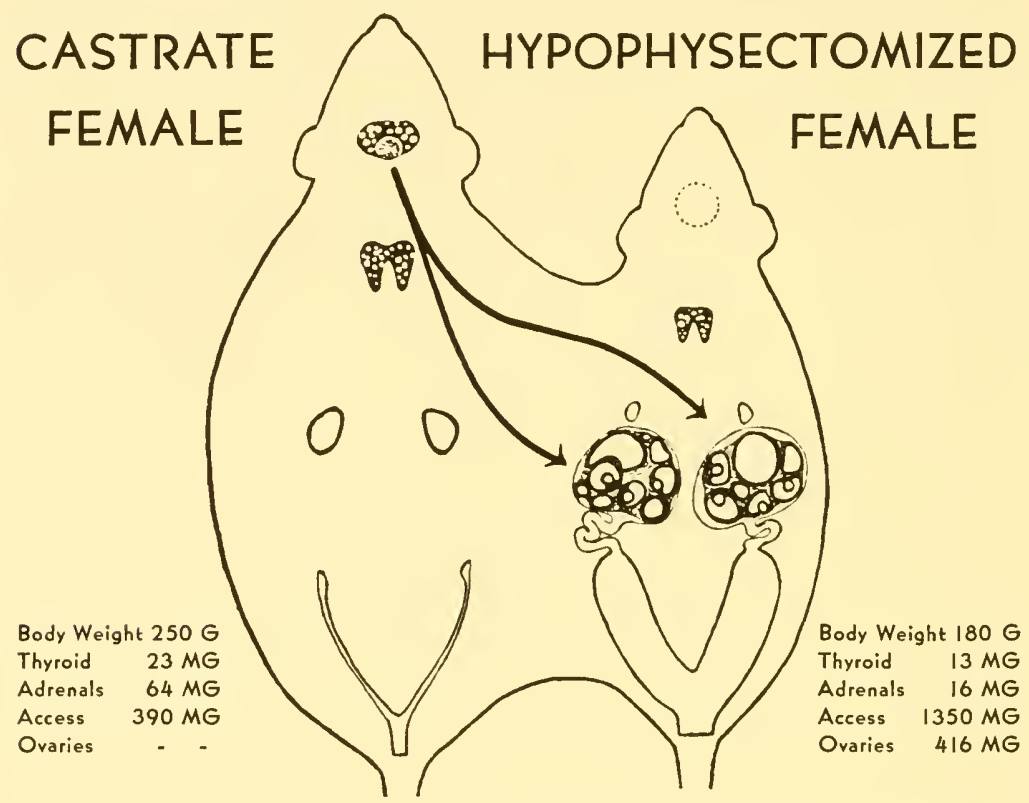

FIG. I6.-Diagram illustrating the ovarian changes which occur as a result of parabiosis between a hypophysectomized rat with intact ovaries and a spayed rat which otherwise is normal. No antihormone antagonizing gonadotropic hormone is produced. (From DuShane, Levine, Pfeiffer, and Witschi, Proc. Soc. Exp. Biol. Med., 33, 339-45 [I935].)

peatedly both by the author and by other investigators (e.g., Doisy, Martins, Witschi). Du Shane and others (I935) therefore consider antihormone formation a type of immune-body reaction. Parabiosis between a spayed or castrated rat and a normal or hypophysectomied female (e.g., $\&$ \& , $\Phi^{\top}$, 우 $\mathrm{h}$ ) is accompanied by a marked follicle-stimulating effect of the gonadotropic hormone secreted principally by the 
gonadectomized partner's anterior pituitary. Large cystic ovaries are developed - a phenomenon which apparently proceeds indefinitely (e.g., I 5 months). In response to this endogenous gonadotropic hormone formed in great excess there is no compensatory production of antihormone (see Fig. I6). Finally, it must be realized that potent gonadotropic preparations, although administered for a prolonged period, do not necessarily bring about refractoriness of the "end-organ" (e.g., the ovary) with atrophy as is so commonly the case. Katzman, Wade, and Doisy (I 9.37) inserted homo-implants of the pituitary into female rats for 210-75 days. At the end of the period of treatment the ovaries still were enlarged, and there was no gonadotropic (or thyrotropic) antihormone in the blood. ${ }^{72}$ Fluhmann (1936) observed that a gonadotropic extract of the blood of pregnant women still maintained a marked ovarian hypertrophy in rats receiving injections for as long as a year. ${ }^{73}$ In Thompson's experiments (1 937) sheep received, for six months, injections of a sheep pituitary extract which readily produced gonadotropic antihormone in other species of animals. Yet none could be clearly detected in the blood of the sheep.

2. The inhibition of gonadotropic effects by substances other than antihormones. ${ }^{74}$-Extracts of the pituitary body may, under suitable conditions, antagonize the action of gonadotropic substances such as prolan, pregnant-mare serum, and gonadotropic pituitary extract. To this hypothetical substance Evans has given the name "pituitary antagonist." Its inhibition of gonadotropic effect is most characteristically manifested when administration is intraperitoneal. Evans and others (1936) have described methods of preparing the substance free from follicle-stimulating or luteinizing effects.

${ }^{72}$ There was no significant alteration in the weight of the adrenals, thyroid, or pituitary (see also the similar experiments of Artemov, 1937).

${ }^{73}$ The ovaries contained large corpora lutea, lutein cysts, and developing follicles. There was hypertrophy of the pituitary with associated histologic changes.

${ }^{74}$ See also the effects of lactogenic extracts (pp. 98-99). 
The authors concluded that it antagonizes the action of follicle-stimulating hormone but not luteinizing hormone and that it acts independently of the pituitary, thyroid, or adrenals. Some of its described effects suggest that it is luteinizing hormone: it has no action on the ovaries of normal immature rats, but it causes or permits extensive luteinization of the ovaries of adult rats and prolongs pregnancy, if injected in the last half of pregnancy. Evans and his colleagues suggest that such a substance may be important physiologically. It is obvious that its presence in extracts may complicate all studies of the effects of gonadotropic extracts, including investigations of antihormones (see the following section). ${ }^{75}$ Leonard, Hisaw, and Fevold (1935) concluded that the inhibitory substance is associated with luteinizing hormone, although follicle-stimulating hormone, also injected intraperitoneally, might produce some inhibition. It is the belief of Jensen (personal communication) that luteinizing hormone, "pituitary antagonist," and "interstitial cell stimulating hormone" are one and the same principle.

Freud (1937) believed that he had detected an "antiluteogenic factor" in extract of the pars glandularis of the ox. He suggested that the absence of this substance accounts for the persistence of corpora lutea in hypophysectomized rats. (Bunde and Greep [1936] considered this to be owing to a lack of luteinizing hormone or some substance associated with it.) Two early pregnancies in rats were interrupted by intraperitoneal injections of the extract - a fact which the author interprets as supporting his view that the injected substance antagonized the luteinizing hormone.

3. The angmentation of the effects of gonadotropic extract.A variety of substances may potentiate or augment the action of a gonadotropic extract. For example, the hypertrophy

75 Evans concluded that the immature pigeon's testis is very sensitive toward gonadotropic extract, because it is unaffected by "pituitary antagonist" (see also Bates, Riddle, and Lahr, 1935). 
of the immature rat's ovary, caused by a follicle-stimulating extract, is greatly increased if a luteinizing substance also be given. A puzzling recent observation of great interest is the potentiating action, at certain times, of the serum of animals receiving a prolonged course of injections of gonadotropic pituitary extract to produce antihormone which, of course, has the opposite action. This phenomenon has been studied by Collip (1937), Thompson (1937), and Rowlands (1938). Thompson's experiments illustrate how this information was obtained. A horse and two dogs received repeated injections of sheep pituitary gonadotropic extract. The effects of their sera on the gonadotropic action of the extract in hypophysectomized or normal immature rats was ascertained at intervals of 7-20 days. Finally, the sera were found to contain a substance or substances which augmented the action of the extract on the immature rat's ovary about threefold. Sera which were obtained 50-80 days after the injections were started had the maximum augmenting effect. The active substance(s) was in the pseudoglobulin fraction which also contains immune bodies and antihormones, if these are present. Thompson suggested that an antihormone to "pituitary antagonist" (see the foregoing section) may have been formed. Rowlands is inclined to concur with this view and enumerates reasons in support (specificity of effect for pituitary gonadotropic extract only, especially of extracts probably rich in "antagonist," lack of phenomenon with nongonadotropic pituitary extracts with other effects, etc.). ${ }^{76}$

By direct extraction of the anterior pituitary, a substance augmenting the action of certain gonadotropic extracts (e.g., follicle-stimulating extract as extract of urine of women after spaying or after the menopause, endometrium of the preg-

${ }^{76}$ In Collip's experiments (sheep pituitary extract as "antigen" injected into lambs) antihormone was demonstrated later. Katzman, Wade, and Doisy (1937) who unusuccessfully attempted to produce antihormone effects by pituitary homoimplants in rats, remarked that the serum obtained at the end of treatment $\left(7^{-} 9\right.$ months) augmented the gonadotropic effects of implants. 


\section{THE PITUITARY BODY}

nant mare, etc.) can be secured. This was named "pituitary synergist" by Evans and others. There is not satisfactory evidence that this substance is different from what others call "luteinizing hormone." Recent work has been reported by Evans and Simpson (1935), Saunders and Cole (1935), and Lein (1937).

A number of substances, if injected with pituitary extract, is capable of augmenting the action of pituitary gonadotropic hormone. In addition to tannic acid (recent studies by Bischoff, I937; and Fevold, Hisaw, and Greep, 1937) and $\mathrm{ZnSO}_{4}$ (recent studies by Saunders and Cole, I935; Fevold, Hisaw, and Greep, 1936; and Emery, 1937), which were previously known to have this action, approximately a dozen new substances have been shown also to be effective. These include $\left(\mathrm{CH}_{3} \mathrm{COO}\right)_{2} \mathrm{Cu}$ and $\mathrm{CuSO}_{4}$ (Fevold, Hisaw, and Greep, I 936-37; Emery, I937; and Pfeiffer, I937), "Merthiolate" $\left(\mathrm{C}_{2} \mathrm{H}_{5} \mathrm{HgSC}_{6} \mathrm{H}_{4} \mathrm{COONa}\right.$ ), a germicide (Chen and van Dyke, 1938), yeast extract and yeast ash (Fevold and others, 1936), blood, hemoglobin, or heme (Casida, I936; McShan and Meyer, 1937), casein and egg-albumin (Saunders and Cole, I935), and a miscellaneous group of substances only crudely characterized (Hellbaum, I 936).

Figure 17 illustrates how strikingly the gonadotropic effect of an extract may be augmented by a foreign chemical mixed with the extract before injection. Until there is better evidence to the contrary, it is probable that this group of potentiating substances acts by interfering with the absorption of hormone, thus decelerating excretion (and possibly destruction) and prolonging the action of each dose. ${ }^{77}$

77 Fevold, Hisaw, and Greep ( 19.36 ) were inclined to believe that $\mathrm{CH}_{3} \mathrm{COOCu}$ catalyzes the synergistic effects of follicle-stimulating and luteinizing hormones. In their earlier work, they reported that the intravenous injection of yeast extract ( $10-15$ "grams-equivalent") or $\left(\mathrm{CH}_{3} \mathrm{COO}\right)_{2} \mathrm{Cu}(10 \mathrm{mg}$.) can cause ovulation in the rabbit. Pfeiffer (I 937) concluded that the injection of a solution of $\mathrm{CuSO}_{4}$ has no effect on gonadotropic hormone secreted by the rat's pituitary in situ. 


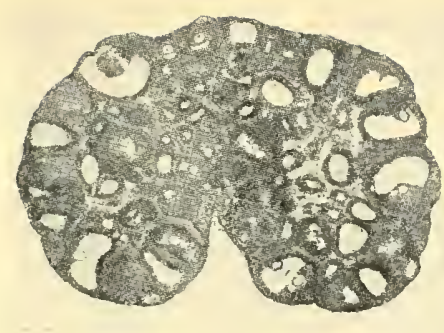

A
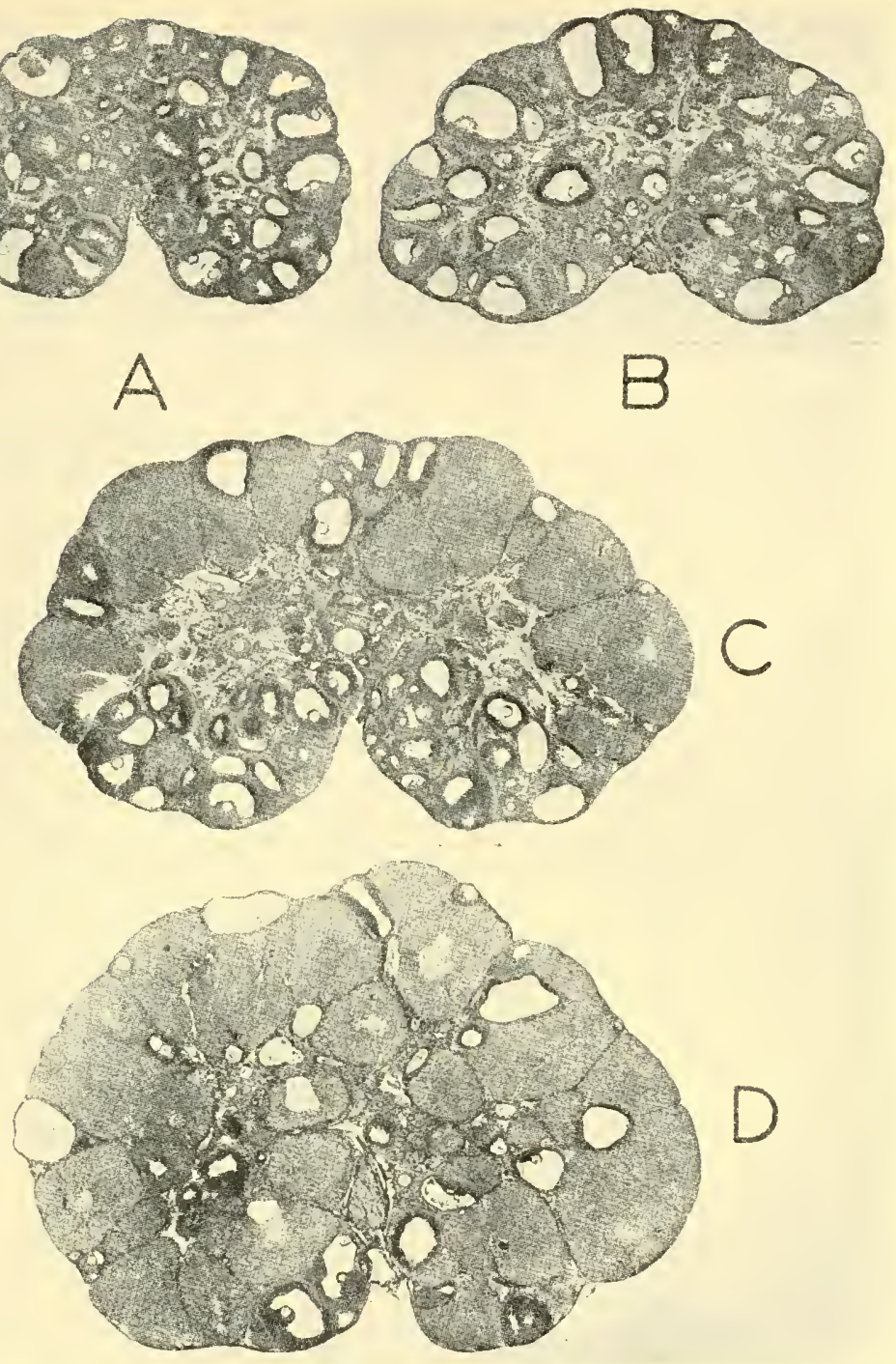

Fig. 17.-The potentiation of the response of ovaries of littermate rats as a result of the addition of "Merthiolate" to the pituitary gonadotropic extract injected. The rats were 26 days old at death and weighed $68 \mathrm{gm}$., except $D$, which weighed $54 \mathrm{gm}$. (From Chen and van Dyke, J. Pharmacol. exp. Therap., 62, $333^{-}-45[1938]$.)

\begin{tabular}{l|c|c|c}
\hline \hline Rat & $\begin{array}{c}\text { Total Dose of } \\
\text { Pituitary } \\
\text { Gonadotropic } \\
\text { Extract (Mg.) }\end{array}$ & $\begin{array}{c}\text { Percentage } \\
\text { Merthiolate in } \\
\text { Solution of } \\
\text { Extract }\end{array}$ & $\begin{array}{c}\text { Weight of } \\
\text { Both Ovaries } \\
\text { (Mg.) }\end{array}$ \\
\hline$A \ldots \ldots \ldots \ldots \ldots \ldots$ & 0 & 0 & 17.27 \\
$B \ldots \ldots \ldots \ldots \ldots \ldots$ & 2.0 & 0 & 25.71 \\
$C \ldots \ldots \ldots \ldots \ldots$ & 2.0 & 0.02 & 46.90 \\
$D \ldots \ldots \ldots \ldots$ & 2.0 & 0.075 & 83.59 \\
\hline
\end{tabular}


Anterior pituitary secretion is a necessary condition of normal function of the gonads of all classes of vertebrates. How this necessary condition is implemented in the living animal has been of prime interest to a host of biological investigators, both seasoned and newly recruited. Although experiments have been performed in all classes of vertebrates-fishes, amphibia, reptiles, birds, and mammals - there still remain lamentably great gaps in our knowledge of the physiology of gonadotropic hormones. It is an unenviable task to summarize the work, because unifying working concepts either are lacking or may be widely used without equally wide recognition of their inadequate experimental foundations.

Discussion is difficult if there is not agreement on the probable number of gonadotropic hormones. Yet no investigator has succeeded in preparing a gonadotropic hormone in pure form. Partial purification has apparently been achieved and there continues to be evidence that there are two gonadotropic hormones: one facilitating follicle-growth and maturation (follicle-stimulating hormone), the other promoting the conversion of the cells of the membrana granulosa and thecae into lutein cells (luteinizing hormone). Both hormones are said to be necessary to produce ovulation. 'There is evidence that what is called follicle-stimulating hormone maintains spermatogenesis in the testis, whereas luteinizing hormone nurtures the interstitial cells of Leydig, whence arises "male hormone." 78 Some authors would lengthen the list further

${ }^{78}$ However, other data suggest different interpretations. Observations which must be reconciled are as follows: (1) follicle-stimulating hormone maintains the germinal epithelium, whereas the interstitial cells require luteinizing hormone for normal function (immature hypophysectomized male rats); (2) either hormone will support spermatogenesis, but only luteinizing hormone maintains the interstitial cells (mature hypophysectomized male rats); (3) spermatogenesis can take place normally in the absence of the pituitary, provided that a suitable androgen ("male hormone") is injected early enough after operation (mature hypophysectomized male rats); and (4) the pituitary of the normal male rat secretes only follicle-stimulating hormone (at least the secretion of luteinizing hormone has not been detected in the living animal, although small amounts of luteinizing hormone may be recognized by implanting the male pituitary). 
with newly named (but not necessarily newly described) hormones, so that its total would be five or more instead of two. To recall only the example of the number of "corpus luteum hormones" once thought to exist before the pure substance had been isolated is to recognize the continued need for caution in discussing the number of gonadotropic hormones secreted by the pars glandularis. At present, there is probably nothing that will further rational interpretation and real advancement in this field as much as the isolation of a gonadotropic hornione of the anterior pituitary in a satisfactorily pure state.

Much of the recent data requires no reconsideration here. Descriptions of the effects of destruction or removal of the pituitary body supplement former reports as to the atrophic changes which dramatically follow in the ovaries, testes, and secondary sexual organs. Other experiments have increased our knowledge of the effects of pituitary tissue or extracts on the sexual organs of many different animals. It is known more accurately that even a short period of pituitary deficiency may markedly lessen the sensitivity of the gonads toward gonadotropic hormone. Also it is of interest that the persistence of corpora lutea in hypophysectomized rats appears to be due to the lack of an anterior pituitary hormone.

Physiological evidence of the nervous control of the secretion of gonadotropic hormones has been greatly strengthened in the past few years. In birds, and at least in some mammals, photic stimuli may elicit the secretion of gonadotropic hormones, which in turn stir the gonads into activity. Probably "light" reflexly stimulates the secretory nerves of the pars glandularis by impulses arising in the optic nerves, whence they are guided down fibers of the stalk by one or more groups of neurons in the diencephalon. In animals without precise seasonal variations in sexual, and hence pituitary, activity photic stimuli do not play such an evident role. However, diffuse or sometimes sharply localized stimulation of the brain may be followed by the release of gonado- 
tropic hormone from the anterior pituitary as shown by ovulation in an animal like the rabbit. Other peripheral nerves like those of the sympathetic system may be part of an afferent arc, but their significance is not clear.

Great interest has been shown in the hormonal control of the secretion of gonadotropic hormones, especially as far as the internal secretions of the ovaries and testes may here be important. There is much data to suggest that surges or recessions of secretion by the anterior pituitary causing the growth or decay of gonadal activity (e.g., oestrous cycles) may be related to the absence or presence of internal secretion(s) of the gonads such as oestrogen from the ovaries. The regulation of the secretion of gonadotropic hormones by the pars glandularis may well depend upon a delicate, complex mechanism, partly hormonal and partly nervous. Also it is probable that the relative importance of the nervous system and of hormones of the gonads varies in different animals. The reader can easily diagrammatize a self-regulating physiological unit consisting of the pars glandularis, the gonads, and the nervous system. However, he cannot safely picture this unit in any detail. The known hormones characteristic of the gonads vary enormously in their apparent "regulating" (usually depressing) effect on the formation of gonadotropic hormone by the anterior pituitary. Oestrogens far excel androgens in potency; progesterone (from the corpus luteum) appears to be of slight importance.

The relation of adrenal cortical secretion to the phase of pituitary physiology under discussion is obscure because so many variables affect experimentation. Thyroid secretion perhaps is not of great significance; its usual effect, if any, is to lessen the action of follicle-stimulating hormone. How much regulation of the liberation of gonadotropic hormones by the pars glandularis is effected by the gland's own lactogenic hormone can be judged better when experiments have been performed with the recently isolated lactogenic principle. 


\section{THE GONADO'TROPIC HORMONES}

Much has been written and continues to be written concerning "antihormones." 'There can be no question that gonadotropic extracts, if repeatedly administered in their present state of impurity, may cause at an unknown site the production of substances circulating in the blood and antagonizing the effects of pituitary extracts. However, other experiments furnish arguments, so far not refuted, that such substances are not formed in response to gonadotropic hormone secreted by the intact gland. 


\section{THE GONADOTROPIC HORMONES ASSOCI- ATED WITH PREGNANCY OR CERTAIN NEOPLASMS}

YONADOTROPIC hormones associated with pregT nancy or with neoplasms are not secreted by the pars glandularis of the pituitary body. In pregnant women, as well as in other pregnant mammals in which their presence has been demonstrated, the characteristic hormone probably originates in the epithelial cells of the chorion. Neoplasms, such as certain tumors of the testis, also may secrete gonadotropic hormones; neoplasms originating in the chorion almost invariably liberate large amounts of such substances into the blood stream. The effects of all these substances differ in one or more ways from those of gonadotropic hormones secreted by or extracted from the pars glandularis.

Most of the published reports deal with the gonadotropic hormones of the pregnant woman and the pregnant mare. Presumably, the important function of such hormones in pregnancy is to insure the maintenance of an ovarian function favorable to the pregnancy. Inasmuch as the secretion of the corpus luteum has been shown-especially in the earlier part of pregnancy - to be vital for the continuance of pregnancy in many mammals, the luteinizing effects of the chorionic hormones have been especially emphasized. In what mammals, other than man and the horse, is there new evidence for the secretion of chorionic hormone in pregnancy? Heretofore there has been doubt as to the presence of gonadotropic hormone in the urine of the pregnant monkey. How-

${ }^{x}$ See also chaps. $\mathrm{i}$ and iii or refer to the Index. 
ever, Hamlett (1937) has shown that the urine of the pregnant macaque about the $19^{-25}$ day of pregnancy may contain detectable amounts of gonadotropic hormone. It is probable that gonadotropic principles secreted by the chorion will be demonstrated in other pregnant mammals by future investigation. ${ }^{2}$

No gonadotropic hormone could be found in the serum of the pregnant ewe (Cole and Miller, 1935) or in the milk of the pregnant cow (total dose of $1.2 \mathrm{cc}$. in immature mice initially 17 days old; Weisman, Kleiner, and Allen, I935). These reports supplement those reviewed in 1936.

THE GONADOTROPIC HORMONE (PROLAN) ASSOCIATED WITH PREGNANCY IN WOMEN

New observations on the metabolism of prolan. ${ }^{3-A}$ - All the recent observations support the view that prolan is secreted by the chorionic cells (Bourg and Legrand, I935; Philipp and Huber, 1936; Kido, I937). Most of the experiments were performed with implants. Kido observed the action of human placenta which survived transplantation in the anterior chamber of the female rabbit's eye. The secreted hormone affected the animal's ovary and could be detected in the urine.

The most striking new fact clearly demonstrated in respect of the urinary excretion of prolan is that an enormous excretion is observed about the 50-60 day following the last menstrual period (Browne and Venning, I936; Evans, Kohls, and Wonder, 1937). Figure 18 is reproduced from the article of Browne and Venning. The highest figures reported by these authors are $100,000-300,000$ rat-units per liter of urine in comparison with a later fall to about 3,000 rat-units per

2 E.g., see Astwood and Greep (1938).

${ }^{3}$ See chap. $v$ and Table VI of the earlier volume. Guercio (1936) believed that prolan can be detected in the saliva of pregnant women; however, this was denied by Weisman and Yerbury (1936). 

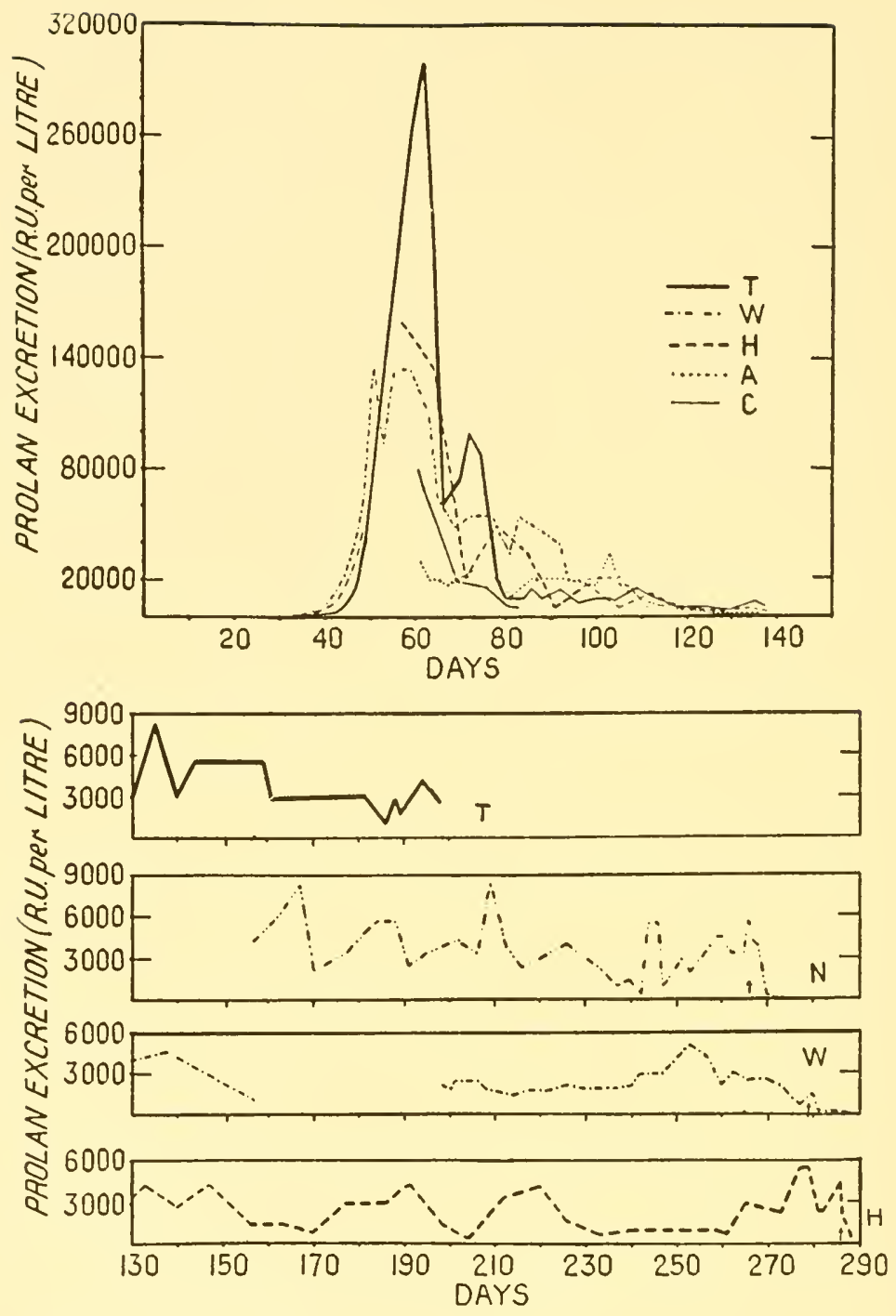

FIG. 18.-The excretion of gonadotropic substance(s) in the urine during pregnancy. Days are counted from the first day of the last menstrual period. The termination of pregnancy is indicated by arrows. (From Browne and Venning, Lancet, 231, I 507-11 [1936].) 


\section{THE GONADOTROPIC HORMONES}

liter, a concentration which persists after 120 days. ${ }^{+}$The rapid rise is prevented by fetal death or if the fetus is dying. Less accurate studies have been reported by Bourg and Legrand (1935), Ehrhardt (1936), and Spoto (1936). Bourg and Legrand also investigated the concentration of prolan in the serum and placenta. Their results justify the belief that the peak of urinary excretion coincides with high concentrations of prolan in the serum and placenta. (Ehrhardt likewise concluded that the concentration of prolan in the urine and serum is about the same.) The concentration of prolan in the amniotic fluid was found to be $7-33$ per cent of that in serum..$^{5}$

Smith and Smith (1935) have again pointed out that high concentrations of prolan in the serum are associated with toxemias of pregnancy, including eclampsia. However, Bourg and Legrand (1935) as well as Ehrhardt concluded that, although this is true of what are termed toxemias, an abnormally high prolan titer may not be found in the serum in eclampsia.

Some aspects of the fate of prolan after its administration to normal men or animals have been investigated. Friedman and Weinstein (1937) found that no more than 20 per cent of a dose of prolan injected intramuscularly into men could be recovered in the urine. After large doses by mouth $(8,000-42,500$ rat-units), none was detected in the urine. Stamler (I937) made other observations in the dog and the gelding. New experiments in rabbits were reported by Lip-

4 Evans, Kohls, and Wonder observed maximum excretions of 75,c00-1,040,000 rat-units in 24 hours compared with $2,600-15,000$ rat-units at other times.

$s$ 'The convenient and accurate methods of diagnosing pregnancy are those depending upon ovarian changes in immature rodents or ovulation in adult isolated rabbits. Intracutaneous tests in the patients themselves are of no value (Schneider and Cohen, I937). Although chromatosome-dispersing ("melanophore-expanding") hormone is found more frequently in the urine of pregnant women, its presence is not reliable as indication of pregnancy (Mandelstamm, 1935; Jores, 1936; Bruckmann, I937; Raza and Spurrell, 1937). Dychno (1936) considered such a test reliable.

For descriptions of a method based upon a color-reaction in urine see Visscher and Bowman (1934) and Friedrich (1936). 
schütz, Fuente-Alba, and Vivaldi (1935). 'Tissue fixation or destruction of the hormone was indicated by the fact that Io hours after intravenous injection, 30 per cent of the prolan had disappeared from the blood of nephrectomized rabbits; 80 per cent was lost in normal rabbits. Nizza and Berutti (I936) compared the changes in one intact ovary with the other which was transplanted into a second rabbit 30-r 20 minutes after the injection of urine of pregnancy. The differences appeared to be quantitative.

The gonadotropic effects of prolan in fishes, amphibia, and reptiles. - Some of the effects of prolan in cold-blooded animals have been described already. ${ }^{6}$ Schreiber (1935) reported that the administration of prolan to immature eels can cause changes characteristic of testicular maturation, including the differentiation of spermatogonia into normal spermatozoa. According to Morosowa ( 1936 ), perch (November to March; weight $250-75^{\circ} \mathrm{gm}$.) receiving prolan, although kept in water at $6-9^{\circ} \mathrm{C}$., produce ripe eggs and spermatozoa, from which fertilized eggs can be secured. Shapiro (1936) demonstrated that in the toad, Xenopus laevis, the injection of prolan evoked amplexus in animals which otherwise were in a state of complete sexual inactivity. Ovulation, oviposition, and fertilization occurred. The offspring were raised to the tadpole stage. Turner's experiments (1935) were performed with the lizard, Eumeces laticeps. During the season of involution of the gonads, the injection of prolan (20 rat-units twice daily for 20 days) caused hypertrophy of the testes and the epididymides with the production of mature spermatozoa. Such treatment caused enlargment of the oviducts but had no effect on the ovaries. When the animals were active sexually, the response was much smaller.

The effects of prolan in warm-blooded animals. ${ }^{7}$ I. Male

${ }^{6}$ See chap. iii and the Index.

7 Prolan is without demonstrated action on the gonads of birds (see pp. 52, 58). Schunterman (1935) declared that an intravenous injection of an extract of digitalis evoked emesis less frequently in pigeons which had received 2 units of prolan intramuscularly 10 minutes before. 
mammals.-In normal male mammals, the typical effects of prolan-best observed in immature specimens-are on the interstitial cells of the testis (cells of Leydig). These secrete "male hormone," which is probably testosterone, at a more rapid rate so that the secondary sexual organs, such as the epididymides, seminal vesicles, and prostate, undergo abnormal hypertrophy and, histologically, may resemble the organs of adult animals. Such changes, of course, are absent, if the prolan-sensitive cells have been removed by castration. In immature mammals prolan does not initiate spermatogenesis. In adult animals, however, prolan, if administered immediately after operation, will maintain spermatogenesis after hypophysectomy (Smith and Leonard). This probably is due to the fact that the secretion of androgenic hormone by the interstitial cells continues as a result of the injection of prolan. It is well known that androgens by themselves may have this effect (see chap. iii). This fact probably accounts for the spermatogenesis which may be initiated in the ground squirrel (Citellus tridecemlineatus) by the injection of prolan at times of seasonal involution of the gonads (Baker and Johnson, 1936; Wells and Moore, 1936).

Other reports not reviewed previously are those of van Os (1936) and Kuschinsky and Tang (1936). Van Os could not bring about spermatogenesis in the testes of rats in which testicular degeneration had followed vitamin-A deficiency or cryptorchidism. Kuschinsky and Tang stated that the typical effects of prolan (seminal-vesticle hypertrophy, no consistent effect on testicular weight) could be observed in rats receiving prolan when only $6-\mathrm{I} 3$ days old.

2. Female mammals.-In female mammals the most striking effect of prolan is on the cells of the corpus luteum or on the cells of the theca interna-parent cells of corpora lutea. It may appear that follicle growth is stimulated, and some authors believe that prolan is composed of an "A" component, stimulating the follicle, and a " $\mathrm{B}$ " component, causing luteinization. Good evidence that both components 
exist has never been offered. It is more simple and more accurate to consider that prolan is primarily a luteinizing gonadotropic hormone. Its presumed follicle-stimulating effects are seen in animals with intact hypophyses which contribute the important, and perhaps the only, share in bringing about follicle-stimulating effects. Anterior pituitary secretion is of great importance in permitting the recognition of the luteinizing effects of prolan. In hypophysectomized female rats, prolan may cause hypertrophy of the cells of the thecae and interstitium, with which may be associated a persistent oestrus; it does not bring about follicular growth.

Kuschinsky and Tang (1935) described luteinization of the ovaries of rats receiving large doses (25 rat-units daily) during the second week of life. A deciduoma reaction of the uterus could not be produced. Hohlweg (1936) commented on the large doses of prolan required to produce luteinization or corpora lutea in very young or abnormally small immature rats. Probably the important factor is the inability of the pituitary to secrete satisfactory amounts of follicle-stimulating hormone at this age. Ljachowezki and Chwatow (1937) believed that the administration of prolan in small doses (2-5 mouse-units daily) permitted successful transplantation of the ovary or testis, if injections were begun immediately after transplantation and continued for IoI 2 days. They stated that such transplants could function (apparently judged by the morphology of the transplant) for more than 2 months in animals (mice, rats, guinea pigs, and cats) with their own gonads intact.

According to Desaive (I935), although repeated injections of prolan (25 rat-units daily) increase markedly the rate of development of primordial follicles, they produce an even greater rate of follicular atresia, which was considered to be the important effect in the rabbit. Moricard (1935) injected prolan for $2-5$ months into rabbits. After about 3 weeks enormous numbers of corpora lutea might be found; later the predominant effect was a pronounced development of 
interstitial tissue which gradually receded as "antihormone" was produced. The experiments of Bachman (1936) indicated that prolan might produce hypertrophy of the interstitial stroma or non-functional corpora lutea, if injected into young rabbits. Padootcheva and her colleagues (1935) believed that prolan is of practical value in rearing rabbits. Pregnancy was successfully initiated by causing ovulation by the injection of prolan after a suspension of spermatozoa had been injected into the uterus. The vaginal instillation of spermatazoa also was successful. The authors stated that on a rabbit farm pregnancy occurred in 74 per cent of animals receiving an ovulating dose of prolan, whereas the proportion was only 30 per cent without such treatment. 'The injection of prolan by various routes brings about ovulation in sheep without signs of oestrus, according to Zawadowsky and others (1935). They recommended the administration of I00-500 mouse-units and found that corpora lutea due to this treatment did not interfere with expected oestrous cycles. $^{8}$

Comparisons of the dose of prolan necessary to cause ovulation in pregnant and non-pregnant rabbits have been made by Rowlands (1935) and are discussed on pages 69-70.9 Prolan has some replacement value in pregnant rabbits which have undergone hypophysectomy, as was shown by Robson (1937). The same author found that, although hypophysectomy of the rabbit is followed by a rapid loss of ovarian sensitivity toward gonadotropic hormone, the injection of prolan will postpone the appearance of this refractory condition for nearly two weeks. In hypophysectomized rabbits

${ }^{8}$ Markee and Hinsey (I936) injected urine of pregnancy in to rabbits, so that the course of ovulation could be carefully studied by observing the ovary through an abdominal window or in the anterior chamber of the eye after transplantation.

Holtz and Wöllpert (1937) used prolan to induce the formation of luteal tissue in the ovaries of guinea pigs and cats. They were interested in the uterine response to epinephrine under these conditions.

9 Weinstein and Makepeace (1937) estimated that the dose of prolan causing ovulation in oestrous rabbits must be doubled or trebled in pseudopregnant rabbits.

$$
[13 \mathrm{I}]
$$




\section{THE PITUITARY BODY}

also, the administration of prolan will maintain the corpora lutea of pseudopregnancy for more than a week. These effects of prolan apparently are due to its luteinizing properties; it replaces the pituitary luteinizing hormone which normally is so important for corpus luteum function and the maintenance of pregnancy. On the other hand, von Arvay (1937), who was able to prevent normal parturition and cause the prolonged retention of the dead fetuses by injecting prolan into pregnant rabbits, contended that these changes were not due to the persistent action of corpus luteum secretion. ${ }^{\mathbf{0}}$

Reynolds' observations on the inhibitory action of prolan on the motility of the uterus of the unanesthetized rabbit were confirmed by Sager and Leonard (I 936), who observed a similar effect following the injection of extract of urine of patients with testicular tumor or of others following gonadectomy or the menopause. The inhibitory effect could be prevented by the injection of oestrin. ${ }^{\text {II }}$

There are other miscellaneous observations which are difficult to classify. Vercesi and Guercio (1935) stated that the growth in vitro of ovarian fragments from rabbits is poor even in the presence of prolan; however, the administration of prolan for several days before ovarian removal greatly facilitates the growth of fibroblasts, which the authors considered is an important part of the response in vivo. According to Kiyohara and Isawa (1936), the oxygen-consumption of the isolated ovary of the guinea pig ( $300 \mathrm{gm}$.) is nearly doubled $10-15$ hours after the injection of $7-8 \mathrm{cc}$. of urine of pregnancy. Some effect could be observed 2-3 hours after injection or as long as to days later. There were no similar changes in the metabolism of liver and kidney.

Hamblen in association with Ross (1937) reviewed the

10 See also Spreng ( 1937) who investigated the action of a single intravenous dose of prolan on the duration of pseudopregnancy in the rat and the rabbit.

"Pierson (1936) produced cancer-like adenomata of the uterus in 2 of I6 rabbits by injecting a concentrated extract of pregnancy-urine on alternate days. However, it is not certain that her extract was free of oestrin. 


\section{THE GONADOTROPIC HORMONES}

effects of prolan in a large number of patients. In a smaller series of 7 patients, in whom ovarian specimens were available in 3 instances and endometrial samples were available in all, they found no evidence of ovulation and corpus luteum formation as a result of the injection of prolan. The authors injected total doses of $6,000-24,000$ rat-units $(400-8,000$ ratunits daily). Among other recent descriptions of the clinical effects of prolan in women are those of Anselmino and Hoffmann (1935), Moricard (I936), and Trettenero (1936). The effects of prolan (2,000-22,000 rat-units in $4^{-6}$ days) are difficult to evaluate in the report of Anselmino and Hoffmann, because the patients all suffered from carcinoma of the uterus. Apparently the important changes were atresia of the follicles and luteinization of the thecae. Moricard transplanted the ovary into the vulva. The injection of an extract of pregnancy-urine was followed by hypertrophy of the transplant, from which oestrin-containing fluid could be aspirated. Trettenero believed that his extract of pregnancyurine produced ovarian changes - chiefly persistence or new formation of luteal tissue in the ovaries - which modified the menstrual rhythm.

Modifications of the action of prolan may occur as a result of the injection of an oestrogen. The augmentation of the size of the corpora lutea, if an oestrogen also is administered, is probably due to the added effect of luteinizing hormone liberated from the injected animal's anterior pituitary (Wolfe, I 936; see chap. iii). It could be inferred from other data that progesterone might lessen the action of prolan. Recently, Jacobsen (I 936) found that the injection of progestin (0.03$0.0_{4}$ rabbit-unit daily), if made before and during the administration of prolan, markedly interfered with the formation of corpora lutea in immature mice.

In confirmation of previous work, Connon (I 937) reported that large doses of prolan (80-125 rat-units daily for $3-5$ days) inhibit lactation in rats, as shown by the failure of the young to grow and by the histological appearance of the 
breasts. The author injected the hormone intraperitoneally from the day of delivery. The treatment caused considerable ovarian hypertrophy owing, apparently, to new formation of lutein tissue. Howard (I 936) as well as Jadassohn, Uehlinger, and Zürcher (I937) observed that the injection of prolancontaining extracts caused enlargement and elongation of the nipples in rats and guinea pigs. The effects, which can also be produced by an oestrogen, were more marked in female animals. The prolonged injection of Howard's acid extract of the blood of pregnant women caused growth and differentiation of the mammary glands which often secreted milk (after injection for 90 days). The injection of the extract for 6.5-9 months was accompanied by regression of the secretory phenomena and the development of adenomata in the breasts. Howard stated that all these changes were absent in gonadectomized rats.

The effects of prolan in relation to glands of internal secretion other than the gonads. I. The thyroid.-Thyroidectomy does not alter the response of the rabbit's ovary to prolan (Pitzorno and Serra, 1936: 20 rat-units on alternate days for Io days). The same is true of the hormone's gonadotropic effect in male or female rats (Leonard, I936; Leonard and Hansen, i 936).

2. The adrenals.-Several recent authors agree in their description of the effects of prolan on the adrenal glands in such mammals as the guinea pig, rabbit, rat, and mouse (Inohara, Savona, 1935; De Boissezon, I936). 'The hypertrophy which occurs is limited to the cortex and has been compared with that of pregnancy. The chief alteration is in the zona fasciculata, the cells of which enlarge and contain more lipoid than usual. The zona glomerulosa may be narrowed. ${ }^{12}$ According to Pitzorno and Serra (see above),

12 Takewaki (1935) stated that the prolonged injection of prolan or pregnancyurine had little effect on the adrenal of the female mouse, whereas in males it caused a disappearance of the zona reticularis with the formation of a membrane in its place.

Bau (1936) declared that pregnancy-urine, extract of corpora lutea, pars neuralis 
the cortical hypertrophy due to prolan in the rabbit is prevented by thyroidectomy.

De Fremery (1934) stated that the weight of the male rat's adrenal before puberty is correlated with body-weight, whereas after puberty adrenal weight does not increase as rapidly as that of the rest of the body. (In adult female animals, adrenal weight is proportional to body-weight.) The injection of doses of prolan causing an enormous hypertrophy of the seminal vesicles did not cause any fall in the weight of the adrenals in immature male rats; as in normal immature males, there was found still to be a correlation between the weight of the body and of the adrenal glands.

3. The thymus.-Klein (1935-36) investigated the action of prolan on the thymus of guinea pigs. Atrophic changes were noted in both sexes. In females, however, the number of Hassal's corpuscles appeared to be increased and was accompanied by the appearance of cells described by Fulci as characteristic of the thymus of pregnant guinea pigs. Prolan did not produce such changes in male animals.

4. The epiphysis.-There is not acceptable evidence that pineal extract antagonizes the gonadotropic action of prolan. Vinals ( I935) even believed that a suspension of beef pineal synergizes with prolan in some of its ovarian effects (see also the reports of Engel, 1936; Engel and Buño, I936; Wade, I 937; and others).

The metabolic effects of prolan.-Prolan (20 rat-units daily for 7 days) does not alter the oxygen-consumption of albino female rats (Danforth, Greene, and Ivy, I937). Although the administration of desiccated thyroid appeared to produce less of a calorigenic effect after the injection of oestrogens, this was not shown to be true after the injection of prolan.

Osada (1935) believed that an increased deposition of

extract, etc., but not prolan, cause a development of the zona reticularis in the adult male mouse. In normal adult mice this zone is readily identified in females but not in males. 
glycogen in the liver was the result of the injection of prolan into normal or gonadectomized rabbits of both sexes.

Creatine-creatinine metabolism in relation to age, sex, and the effect of prolan was studied in a small number of rabbits and dogs by Bühler (I935). He concluded that gonadectomy of adult animals is followed after $2-3$ months by an increased urinary excretion of both creatine and creatinine. Later only the excretion of creatinine remained elevated. (Gonadectomy in immature animals did not result in such changes.) The administration of prolan was found not to affect creatinecreatinine excretion in immature dogs. In the sexually mature dog it caused a disappearance of creatine from the urine. Although prolan did not affect the urinary excretion of creatine in adult normal rabbits, it caused an increased excretion after castration. This effect could not be observed in castrated dogs. The dose of prolan used by the author in dogs or rabbits was I 20 rat-units administered twice. Nitzescu and Gontzea (1937) studied creatine-creatinine metabolism in an achondroplastic, infantile dwarf. The injection of 200-300 rat-units of prolan intramuscularly brought about a reduction in the excretion of creatine but scarcely affected that of creatinine. Also there appeared to be an increased creatine-tolerance. The same phenomena were more pronounced following the administration of male hormone (4 capon-units of "Erugon").

The effects of prolan on the metabolism of only one lipoid, cholesterol, ${ }^{\mathrm{x} 3}$ have been investigated recently. Szpidbaum (I935) found that the blood-cholesterol rose (e.g., 40-50 per cent or more) after the injection of prolan into patients recovering from typhoid fever. He believed that the hormone caused a mobilization of cholesterol reserves with consequent benefit to patients because of an increased non-specific immunity. Szpidbaum injected total doses of $1,500-3,000$ ratunits as 500 rat-units daily or on alternate days. Other observations have all been made in rabbits (Cioglia, I935-36;

${ }^{\text {r3 }}$ Presumably all the authors determined the total cholesterol.

$$
\text { [ } 136]
$$


Teilum, I936; and Tramontana, 1936). There was observed consistently a hypercholesterolemia following the injection of prolan. Cioglia concluded that hypercholesterolemia of early pregnancy is due to prolan, whereas the reduced or abnormally low concentration of the lipoid in later pregnancy depends upon the effect of an oestrogen.

Two reports deal with the action of prolan on the concentration of inorganic substances in the blood. According to Klodt (I 937), the subcutaneous administration of prolan to rabbits (500 rat-units daily for 6 days) brings about a transient rise in the concentration of $\mathrm{Na}$ associated with a fall of $\mathrm{K}$ and inorganic P. Less water and $\mathrm{Na}$ are excreted by the kidneys. In the other report Dell'Acqua (I935) confirmed Cannavó's statement that prolan causes a rise in the concentration of $\mathrm{Mg}$ in the blood. The average increase was 38 per cent (20-78 per cent; 6 experiments in 2 cats).

Marchesi (1935) reported that female rats on a diet free from vitamin E, and otherwise sterile, could become pregnant (by normal males) following the administration of prolan. The young died shortly after birth, because of the absence of lactation. A glycerol extract of the placenta was even more effective than prolan. Van Os (1936) studied the effect of prolan on the degenerated testis of the rat on a vitamin-A-free diet. The interstitial cells were stimulated, but there was not resumption of spermatogenesis.

The growth of neoplasms in relation to prolan.-Prolan appears not to have important inhibitory effects on the growth of neoplasms. Magath and Smoilowskaia (I935) concluded that the injection of 50-100 rat-units daily for as long as a month might cause a maximum inhibition of 50 per cent in the growth of a neoplasm. These investigators used mice and rats (transplanted mammary adenocarcinoma and Jensen sarcoma). Other reports of Bischoff and Maxwell, Druckrey, Engel, and Katz are discussed in chapters ii and iii.

Miscellaneous observations.-Prolan does not affect the coagulation-time of the blood in man ( $125^{-250}$ rat-units 


\section{THE PITUITARY BODY}

daily for 9-20 days; Chew and others, I935). Nicolle (1936) concluded that the hormone causes a reticulocytosis in the rabbit, whereas oestrone has the opposite effect. In the rabbit, also, Wilson (1937) observed a marked leukocytosis in normal female rabbits $5^{-8}$ hours after injection. Repeated injections did not produce this effect. Wilson was not convinced that this was a specific effect.

Prolan appears not to be of value in the treatment of acne vulgaris (total dose about 5,700 rat-units over 8-I I weeks; Williams and Nomland, I937). Moffat (I937) believed that small doses of prolan may cure "menstrual migraine"; however, he gave no evidence that such treatment is of specific importance. According to Steinbach and Klein (1937), experimental tuberculosis caused by a standardized dose of bovine bacilli in guinea pigs takes a much less severe course, if extract containing prolan or pregnant-mare serum be injected repeatedly. A similar effect could not be observed following the administration of anterior pituitary extract.

The contractile activity of the isolated ureter of the pig is not affected by the addition of 500 rat-units of prolan to I 50 cc. of bath fluid (Schmitz, 1937).

The experimental augmentation or inhibition of the gonadotropic effects of prolan. I. Augmentation.- It is well known that gonadotropic extracts of the pituitary (or probably derived from the pituitary as in the urine of spayed women) may enormously enhance the gonadotropic action of prolan, as indicated by hypertrophy of the ovary of the immature rodent. Probably the follicle-stimulating component of such extracts is reponsible for this synergism. The luteinizing effects of prolan can be manifested to a maximum extent, if the ovaries are properly prepared by widespread growth of follicles. Non-specific augmentation, as with $\mathrm{ZnSO}_{4}$, usually cannot be demonstrated if prolan instead of anterior pituitary extract is used as the gonadotropic agent. ${ }^{14}$

${ }^{14}$ For an apparent exception see Emery (1937). 
2. Inhibition.-The administration of certain anterior pituitary extracts may inhibit the gonad-stimulating effect of prolan. This phenomenon is best observed after the intraperitoneal administration of the anterior pituitary extract. The mechanism of this inhibitory action is not known; the obvious possibility that a local action on the ovary is important has not been investigated.

The doctrine of antihormones as related to prolan. ${ }^{15}$ - The degree of purity of the best preparations of prolan is not known. Like similarly impure anterior pituitary extracts, preparations of prolan, if repeatedly administered for weeks or longer, produce progressively reduced gonadotropic effects until the organ affected - whether ovary or testis - is refractory to further treatment. "Antihormone" or a substance inhibiting the action of the extract in fresh, non-injected animals can be detected in the serum at this time. Its site of formation may be the reticulo-endothelial system. ${ }^{16}$ Are these phenomena important physiologically? Probably not. The chorionic epithelium of the placenta of women secretes enormous quantities of prolan into the blood throughout pregnancy, although the peak of formation suddenly appears at about the end of the second lunar month. There is no evidence of any formation of antihormone by the tissues of pregnant women. It appears that the numerous investigations of the formation and characteristics of prolan-antihormone represent, at most, contributions to a new field of immunology.

Eichbaum and Kindermann (1935) and Kindermann and Eichbaum (1936) have published several communications on

${ }^{15}$ Other experiments in which prolan was used are discussed in chap. iii (pp. $110 \mathrm{ff}$.).

${ }^{16}$ Experiments of Gordon, Kleinberg, and Charipper (1937) in splenectomized rats to some of which trypan blue was also administered to "block" the reticuloendothelial system. The refractory (non-responsive) condition of the ovary appeared much later in splenectomized rats also receiving trypan blue. Ten rat-units of prolan were injected daily. When refractoriness of the ovary finally appeared, it was attributed to the production of antithormone by redeveloped reticulo-endothelial tissue. 
the immune aspects of antihormone due to prolan prepared from urine of pregnancy. They found that the apparent concentration of antihormone present in the serum of rabbits could not be correlated with precipitin or complement-deviation reactions. Such immune reactions appeared to depend upon (I) an antibody specific for human protein and (2) an antibody specific for an antigen associated with prolan but occurring in human urine irrespective of the presence or absence of prolan. The results of Bachman (19.35), although less complete, are in agreement with those of Kindermann and Eichbaum. Twombly (1936) favored the view that antihormone toward prolan is similar to an antibody formed in response to a foreign protein. The precipitin-reaction (rabbits were given an extract of pregnancy-urine) was parallel to the antihormone content of sera. Prolan inactivated because of heat or age was about as efficient in evoking antihormone formation as were potent preparations. The serum of patients receiving prolan (Ioo rat-units daily) for 2-6 weeks or for more than a year contained no antihormoneindicating that the protein, being homologous, evoked no antibody reaction. In confirmation of Twombly, De Fremery and Scheygrond (I937) reported that an extract of male urine, if injected into rabbits, caused the production of substances preventing the gonadotropic effects of prolan. It seemed unlikely that the small amount of gonadotropic activity in the "antigen" could be responsible for the effect. The urine contained non-specific substances with the antigenic properties of prolan. ${ }^{x 7}$

Harington and Rowlands (1937) investigated the chemical nature of gonadotropic antihormone in the serum of the goat or the rabbit which had received repeated injections of prolan or extract of pregnant-mare serum. The antihormone of prolan was recovered quantitatively in the globulin fraction of serum and was distributed between the pseudoglobulin

${ }_{17}$ See also Laroche and Simmonet (1936) who injected "Antélobine" into patients and subsequently tested their serum for antihormone.

$$
\text { [ I } 40 \text { ] }
$$




\section{THE GONADOTROPIC HORMONES}

and euglobulin fractions. The antihormone inhibiting the action of the extract of pregnant-mare serum could not be completely recovered; most of it appeared also to be associated with the globulin fraction of serum. Zondek and Sulman (1937) have published several reports on biochemical reactions between prolan and its antihormone. They concluded that the antihormone is similar to an antibody. The reaction between prolan and its antibody is reversible. ${ }^{8}$ The authors were unable to detect antihormone in tissues (liver, spleen, muscle) or in the urine of animals whose serum contained the substance. Biochemical differences between prolan and its antihormone were also described.' ${ }^{9}$

The assay of prolan. ${ }^{20}$ - The remarks which were made in chapter iii in reference to the assay of pituitary gonadotropic hormone apply with equal force to prolan. As soon as suitable standards have been assayed by different technics and the results evaluated, it will be possible to recommend preferred assay technics. Among reports published recently are those of Davy (1935), Korenchevsky, Dennison, and Simpson (1935), Owen (1936), Morosowa (1936), and Kelly and Woods (1937). Nelson and Overholser (1935), basing their results on various criteria-i.e., opening of vaginal orifice, oestrus, ovulation, follicular stimulation, luteinizationestimated the relationship between the mouse-unit and the rat-unit for several gonadotropic hormones to be as given in Table 3. Their belief in the remarkable lack of potency of prolan in the mouse as compared with the rat is in agreement with most of the older reports.

Levin and Tyndale (I937) found that the increase of uter-

${ }^{18}$ In one report the authors state that the antihormone destroys prolan.

${ }_{19}$ Zondek and Sulman also studied biological effects of the antihormone of prolan. Antihormone produced no effect after administration by way of the gastrointestinal tract. After subcutaneous injection, antihormone could be retained in the body for several days. Also it could inhibit the effect of prolan, if injected 24 hours after the hormone was administered. They regarded it as being both species and organ specific (it did not antagonize the gonadotropic action of extract of blood of pregnant women or of extract of the human anterior pituitary).

${ }^{20}$ See also chap. iii. 
ine weight in the immature rat, although suitable for the biological estimation of pituitary follicle-stimulating hormone, cannot be used for the accurate assay of prolan or the active principle of pregnant-mare serum.

The chemistry of prolan.-Prolan has not been prepared as a pure substance. There is not good additional evidence that it is composed of more than one gonadotropic principle, although Brindeau, Hinglais, and Hinglais (1936) contend that it is a mixture of 3 principles. Reports of the hormone's preparation by modifications of the tannic acid, tungstic acid, or benzoic acid methods as applied to urine have been made by Hellbaum, Fevold, and Hisaw (I 935), Freud and Hechter (1936), and Ito, Hajazu, and Ueno (1936). Also, Fevold and

TABLE 3

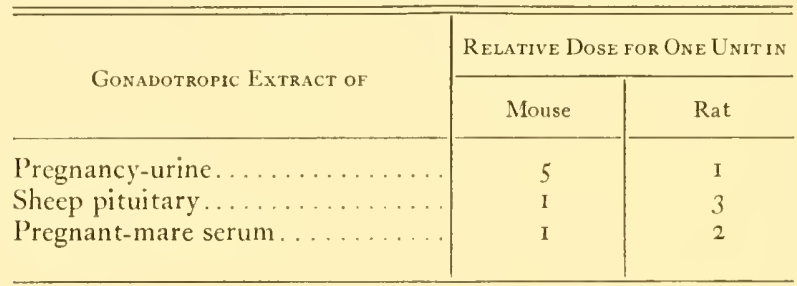

Hisaw (1936) described a method based on the extraction of the hormone from urine by means of io per cent cresol. The effect of hydrogen-ion concentration, ${ }^{2 \mathrm{~T}}$ various organic reagents, etc., on the hormone has been studied by Bischoff and Long ( 1936 ).

\section{GONADOTROPIC HORMONES ASSOCIATED WITH NEOPLASMS IN MAN}

The neoplasms with which the excretion of large quantities of what is probably prolan are most definitely associated are hydatidiform mole and chorionepithelioma. Malignant testicular neoplasms likewise may produce large quantities of prolan-like hormone which, however, may differ from pro-

${ }^{2 t}$ See also Elden and Fellows (1935). 
lan in important aspects of its action (see the earlier volume). Blümel (1935) emphasized the diagnostic significance of the presence of considerable quantities of gonadotropic hormone in the urine. However, cerebral disease with increased intracranial tension as well as the disappearance of internal secretory activity by the gonads, as after the menopause, must be ruled out, because these conditions may give rise to the excretion of gonadotropic hormone in quantities greater than normal. Search should then be made for hydatidiform mole, chorionepithelioma, or teratoma. The last-named tumors may, of course, also occur in males. Provided the neoplasm secretes gonadotropic hormone, determinations of the latter during treatment have obvious prognostic significance.

Testicular tumors in relation to hormone-excretion have been recently discussed by Freed and Coppack (1935), Zondek (1937), and Hinman and Powell (1938). Fifty-eight patients with testicular neoplasms were investigated by Hinman and Powell. The greatest amount of hormone was excreted by patients with teratoid chorionepithelioma of the testis (20,000 to 3,000,000 mouse-units per liter of urine). Large amounts might be secreted by teratoid adenocarcinomata, by "differentiated" (partly carcinomatous) teratoid neoplasms or by primitive monocellular carcinomata. Only small amounts of hormone were excreted by the large number of patients with differentiated monocellular carcinoma of the testis. No hormone could be found in the urine of patients with dysgerminoma (Zondek) or "adult" tumors classified as adult teratoma or adult seminoma (Hinman and Powell). Benign hypertrophy and carcinoma of the prostate or hypernephroma do not secrete gonadotropic hormone, as indicated by its absence in the urine of patients (Owen and Cutler, I 936$){ }^{22}$

So-called prolan A or B may be found in women with genital carcinoma other than the tumors already discussed. It has not been shown that such hormone is not secreted by

${ }^{22}$ See also Aron (1935) and Baudler (I936). 
the pars glandularis. Baudler (I935-36) in recent reports associates such findings with destructive malignant tumors such as carcinoma of the cervix, vulva, or ovary. Sometimes the same is true of extragenital malignant tumors of men or women. It must be remembered that the tumor itself or radiation therapy of such tumors might interfere with the secretion of male or female hormone by the gonads, so that the increased excretion of gonadotropic hormone could resemble that following gonadectomy.

Lewis and Geschickter ( 1936 ) investigated the concentration of gonadotropic hormone in various tumors. Some of the results, expressed as rat-units per kilogram tumor, were as follows: breast tumors-carcinoma (5 negative), 6,0007,000 rat-units, fibromyxoma, 60,000 rat-units, fibroadenoma, 2,500 rat-units; uterine myomata (2 negative), 4,500-I I,000 rat-units; irradiated sclerosing osteogenic sarcoma, 4,500 ratunits.

THE GONADOTROPIC HORMONE OF PREGNANT-MARE SERUM ${ }^{23}$

In several respects, the gonadotropic hormone of the serum of the pregnant mare is strikingly different from prolan. Considerable quantities are present in the serum only during a limited part of the gestation period. It is not excreted in significant amounts in the urine even at times when its concentration is highest in the serum. It can produce marked stimulation of the gonads of birds, whereas prolan is without action in this class of animals. As might be inferred from its failure to appear in the urine, it is slowly metabolized, so that the administration of a single dose, during a period of several days, may be as effective as repeated doses. It apparently can replace the gonadotropic hormones no longer secreted because of hypophysectomy, at times when prolan is patently inadequate. It produces unquestionable folliclestimulating effects, whereas prolan does not.

${ }^{23}$ See the Index for references to other reports.

$$
\text { [ I } 4 \text { ] }
$$


Methods of securing purified hormone have been described lately by Evans and others (1936), Gustus, Meyer, and Woods (1936), and Cartland and Nelson (1937). Gustus, Meyer, and Woods adsorbed the hormone on a suspension of $\mathrm{Al}(\mathrm{OH})_{3}$ from which elution was accomplished by means of dilute $\mathrm{NH}_{4} \mathrm{OH}$. Cartland and Nelson not only described in detail a method of making very potent extracts but also studied some of the hormone's properties. It was found to be soluble in serum containing $5 \circ$ per cent acetone or 60 per cent ethanol. When the percentage of acetone or alcohol was raised to 70 per cent $(\mathrm{pH} 6)$ the greater part of the gonadotropic substance was precipitated. Further manipulation permitted the isolation of preparations of a potency of 0.05 $\mathrm{mg}$. for each rat-unit. (In terms of ovarian effect, their ratunit was 2-Io times as potent as that used by others.) The hormone could be inactivated by $\mathrm{HCHO}$ (4 per cent, $\mathrm{pH}$ 8.०, 3 hours) or heat at $70^{\circ} \mathrm{C}$. or above. Inactivation by heat was studied at three hydrogen-ion concentrations: $\mathrm{pH} 6.0,7.0$, and 8.o. Destruction, especially at $70^{\circ} \mathrm{C}$., was greatest at $\mathrm{pH}$ 6.0 and least at $\mathrm{pH}$ 8.0. Enzymic destruction took place in the presence of trypsin (also reported by others), but not in the presence of emulsin or invertin. The high concentration of hydrogen ions necessary for investigating the effect of pepsin produced inactivation in the absence of the enzyme.

The investigation of Evans and his colleagues is of interest because of their finding that suitable concentrations of $\left(\mathrm{NH}_{4}\right)_{2} \mathrm{SO}_{4}$ can be used to fractionate pregnant-mare serum into two components, one chiefly stimulating follicle-growth and the other causing a "hypertrophy of the theca interna and interstitial tissue" without follicle growth or the formation of lutein tissue. The second fraction restored the function of the interstitial cells of hypophysectomized rats. The follicle-stimulating fraction apparently was not free from the second principle; its action in male rats after hypophysectomy was not described. Hellbaum (1937) declared that follicle-stimulating, luteinizing, and augmenting substances can

$$
\text { [ } 145 \text { ] }
$$


be separated by suitable methods; however, his views have been given only in the form of an abstract. Although Cole earlier believed that more than one gonadotropic principle is present in pregnant-mare serum (e.g., Saunders and Cole, I 935), he later reported ( 1936 ) that he was unable to confirm this belief. Also, Cartland and Nelson at no time secured evidence of the presence of more than a single principle. For purposes of discussion it will be assumed that there is but one gonadotropic principle, although new work may support the belief that a mixture of principles really is secreted.

The slow metabolism of the hormone and the failure of the kidneys to secrete appreciable amounts have already been mentioned. In this connection the experiments of Catchpole, Cole, and Pearson (1935) are of interest. The authors found that about 50 per cent of the hormone disappeared from the rabbit's blood 26 hours after intravenous injection (see Fig. I9). In the gelding the same proportion disappeared in 6 days. The hormone probably was destroyed, inasmuch as none could be found in the urine or feces or in tissues such as the uterus, lungs, kidneys, spleen, or liver. The rate of destruction was not affected by gonadectomy.

Extract of the serum of pregnant mares stimulates the gonads of male or female fowls. ${ }^{24}$ In the immature cockerel the homone causes testicular hypertrophy (without precocious spermatogenesis) and a marked growth of the comb. ${ }^{25}$ In immature females there is only moderate hypertrophy of the ovary without ovulation but with marked growth of the oviduct; the head furnishings are female in type.

Cole (1936) as well as Hamburger (1936) investigated the action of the hormone in both sexes of several species of mam-

${ }^{24}$ Recent reports are those of Asmundson and Wolfe (1935), Hamburger (1936), Uhl, Engelbreth-Holm, and Rothe-Meyer (1937), and Zavadowsky and others (1937).

${ }_{25}$ The cockerels used by Zavadowsky and his colleagues were 50 days old. Injections of 50-200 mouse-units of the hormone for 20-40 days produced tremendous comb growth, testicular hypertrophy, and spermatogenesis. Regression and even degenerative changes appeared after long-continued administration of the hormone. 


\section{THE GONADOTROPIC HORMONES}

mals. ${ }^{26}$ Saunders and Cole (I 936) showed that only the ovarian interstitial tissue is stimulated in rats $10-15$ days old, whereas follicular stimulation was marked in animals i 8 days

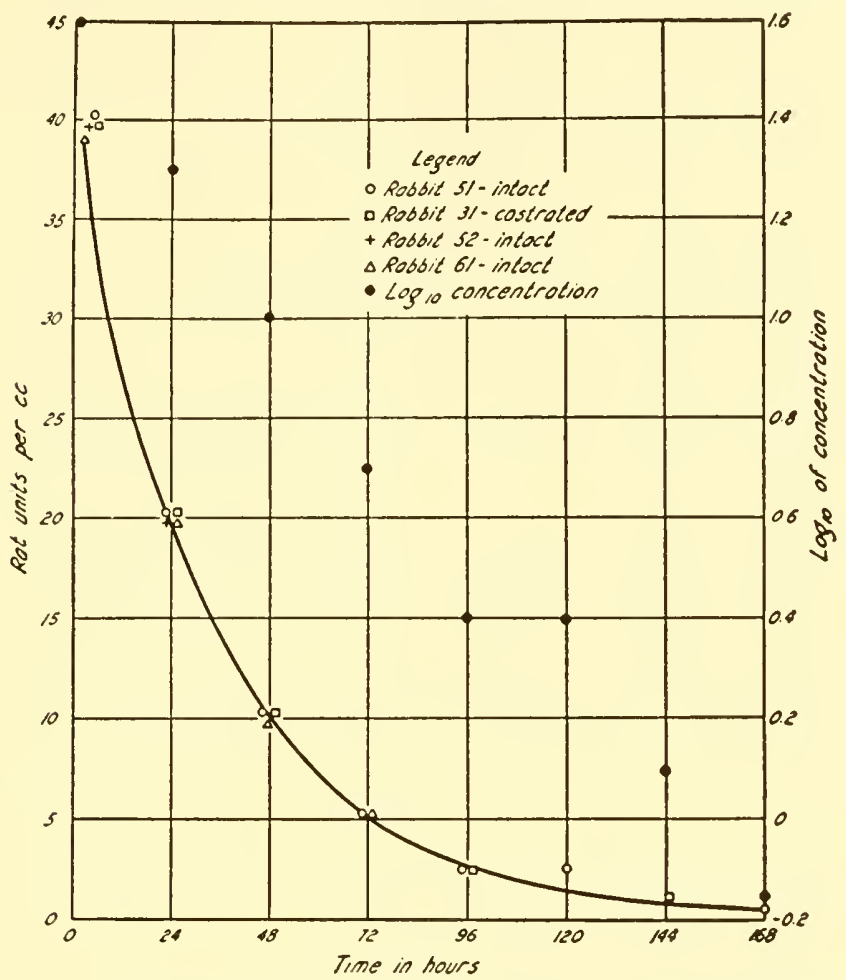

Fig. I9.-The rate of disappearance of the gonadotropic hormone of pregnantmare serum following the intravenous injection of 3,000 rat-units into rabbits. (From Catchpole, Cole, and Pearson, Amer. J. Physiol., I I2, 2I-26 [1935].)

old, and ovulation and corpus luteum formation appeared with increasing frequency in still older rats ( $19^{-25}$ days old. $)^{27}$

${ }^{26}$ Wells (1937) describes the action of the hormone in a hermaphrodite ground squirrel.

Engel (1936) and Engel and Buño (1936) believed that certain pineal extracts antagonize the gonadotropic action of the hormone.

${ }^{27}$ None of the rats was hypophysectomized. However, others, including Hamburger, have observed the formation of lutein tissue in hypophysectomized rats receiving an extract of pregnant-mare serum.

$$
[+7]
$$




\section{THE PITUITARY BODY}

Cole was able to produce ovulation in the rat, ewe, cow, and sow. Both Cole and Hamburger agree that the seminiferous tubules and interstitial cells are affected in male rats; however, the greater effect is on the interstitial cells. The hypertrophy of the testis in relation to that of accessory sexual organs is greater after pregnant-mare serum than after prolan; however, anterior pituitary extract excels both in this respect (Leonard and Hansen, I936). The same authors found that the action of pregnant-mare serum is not altered after thyroidectomy.

Cole (I 937) reported that the injection of the hormone into adult female rats at a suitable time (I 2 rat-units during metoestrus) was followed by pregnancies resulting in an increased percentage of large litters. Matings occurred in about half of a series of immature rats receiving daily injections when 26-3I days old. At necropsy about the Io-I2 day of pregnancy, more than 20 implanted fetuses could be counted in $3^{8}$ per cent of the immature rats. There were 17 young in the largest litter born alive. The percentage of fertile matings in immature or adult rats was reduced by large doses of the hormone.

\section{SUMMARY}

Large quantities of gonadotropic hormone may be liberated into the blood stream during pregnancy in man and other primates and in the mare. Presumably, such hormone is important in insuring the maintenance of pregnancy which, especially at first, requires adequate amounts of the internal secretion of the corpus luteum of the ovary. The corpus luteum in turn cannot flourish unless it is furnished with "luteinizing" gonadotropic hormone either by the anterior pituitary or by some other organ. In some animals, for at least part of the period of gestation, the anterior pituitary appears to supply most of the gonadotropic hormone required. In man, however, the chorionic epithelium of the placenta furnishes enormous quantities of luteinizing hormone (pro- 
lan), especially toward the end of the second lunar month, and thus possibly replaces the anterior pituitary. When gestation is completed or fails because of fetal death, this hormone is no longer secreted, either because the placenta, together with the other products of gestation, leaves the body or because the placenta degenerates and is resorbed or removed.

Conditions in the pregnant mare appear to be different, although the function of the gonadotropic hormone probably is similar. The period of secretion is more sharply limited (especially between the 40-80 days of gestation). The endometrium as well as the chorionic epithelium secretes a hormone which is far more complete in its gonadotropic effects than is prolan. Whereas prolan is chiefly a hormone facilitating the growth and function of lutein tissue in the ovary or of the interstitial cells in the testis, the gonadotropic principle(s) of pregnant-mare serum resembles anterior pituitary gonadotropic principles in respect of the adequacy and completeness of its effects. The chorionic-endometrial hormone of the pregnant mare can maintain the gonads of hypophysectomized male or female animals and therefore affects the follicles, corpora lutea, and interstitial tissue of the ovary or the interstitial cells and germinal epithelium of the testis. Likewise this hormone stimulates the gonads of birds, whereas prolan does not. Some believe that the hormone is a mixture of gonadotropic principles. However, more evidence is needed before this belief can be accepted. On the other hand, prolan appears to be a single gonadotropic substance.

In this chapter and in others which have preceded it, the recent investigations of the effects of these hormones characteristic of pregnancy are discussed. Neither hormone has been isolated as a pure substance. If either is injected repeatedly, the gonadotropic effects disappear, and an associated production of "antihormone," which can be found in the globulin fraction of serum, takes place. The hormone of pregnant-mare serum appears to have much the greater 


\section{THE PITUITARY BODY}

promise as a therapeutic agent, both because its gonadotropic effects are nearly complete and because it is remarkably slowly metabolized, apparently not being excreted but only undergoing a slow destruction.

Gonadotropic hormone can be found in certain neoplasms in man such as hydatidiform mole, chorionepithelioma, certain malignant tumors of the testis (especially teratoid neoplasms), ${ }^{28}$ and in some extragenital tumors (e.g., sarcoma of breast or bone). Urinary excretion of hormone may be marked in patients with hydatidiform mole, chorionepithelioma, or testicular tumor; its course in relation to treatment may furnish information of great value with respect to prognosis. The excreted gonadotropic hormones may be different-that of chorionepithelioma or hydatidiform mole resembles the prolan of normal pregnancy, whereas those associated with testicular neoplasms may have different or additional biological effects.

It has not been shown that the moderate quantities of gonadotropic hormone excreted by women with other types of carcinoma of the genital tract do not arise in the anterior pituitary.

${ }^{28}$ The absence of gonadotropic hormone in the urine does not indicate that a testicular tumor is not malignant. 
THE PARS GLANDULARIS OF THE PITUITARY BODY IN RELATION TO THE DEVELOPMENT OF THE BREASTS AND THE SECRETION OF MILK ${ }^{\mathrm{I}}$

7 HE relationship between lactation and the secretory activity of the pars glandularis is complex and only 1 incompletely understood. ${ }^{2}$ It is agreed that the lactogenic hormone initiates and maintains lactation. However, its presence in adequate concentration in the blood is a necessary but not a sufficient condition of lactation. Moreover, preceding lactation there must be proper development of the mammary glands. The extent to which this prelactation grow th depends either directly on an internal secretion of the pars glandularis or indirectly on gonadotropic hormones which increase gonadal function is still a problem undergoing active investigation.

Lactation cannot continue in the absence of the pituitary body. The correctness of this statement which has been generally held for some years was recently again confirmed in the guinea pig (Gomez and Turner, 1936; Macchiarulo, 1936) and in the dog (Houssay, 1935). The other aspect of this discovery is represented by the following question: After hypophysectomy in a lactating animal, can lactation be maintained by the lactogenic hormone alone, or are several anterior pituitary hormones required? In the pigeon, according to Schooley, Riddle, and Bates (1937), proliferation of the crop-glands and the formation of crop-milk, which are changes homologous to breast development and lactation in

I The pars neuralis, pars intermedia, and pars tuberalis have not been shown to be of significance.

${ }^{2}$ See also reviews of other work in chaps. iii and iv. 
mammals, can be produced by the injection of lactogenic hormone into hypophysectomized birds. The authors mention that the response to this hormone in hypophysectomized rabbits is a small fraction (about one-eighth) of that in normal rabbits. Other authors agree that the lactogenic hormone, by itself, can neither initiate nor maintain lactation in hypophysectomized mammals, although the breasts may appear to be fully prepared for stimulation or continuance of stimulation. It appears that the initiation or continuance of lactation requires at least two hormones of the pars glandularisthe lactogenic hormone and the hormone stimulating the adrenal cortex ("corticotropic" hormone). Some authors would further lengthen the list, especially with respect to a separate action of the anterior pituitary on carbohydrate metabolism. The importance of all these factors will be considered after the conditions necessary for breast development prior to lactation have been discussed.

THE INFLUENCE OF THE ANTERIOR PITUITARY ON THE DEVELOPMENT OF THE BREASTS PRIOR TO LACTATION

Before lactation can occur, there must be suitable development of the breasts. Such development cannot occur in hypophysectomized animals, probably because of two factors: (I) any favorable influence of the gonad will be prevented by the lack of gonadotropic hormone, and (2) the anterior pituitary appears to secrete a hormone essential for mammary development, even when the latter follows the administration of an oestrogen.

The development of the mammary gland in relation to oestrogens. ${ }^{3}$ - In a mammal like the rabbit the repeated injection of an oestrogen such as oestrone may cause a marked development of the mammary tree, especially of the ducts. The

3 Debré, Marie, and Bernard (1935) described marked development of the breasts in a girl eight years of age without any other signs of puberty. The development of the vulva and bones corresponded to the child's age. There had been no uterine bleeding. 
growth of alveoli appears to depend to an important extent on the action of progesterone, although this may not be true of other mammals, e.g., the guinea pig. Gardner, Gomez, and Turner (1935) produced mammary development in normal male rabbits or in normal or spayed (multiparous) female rabbits by injecting 20-25 rat-units of oestrone daily for about 3 weeks. If lactogenic hormone was injected within not more than 3 days after oestrone treatment was stopped, lactation promptly appeared. The observations of others who used rabbits do not require a detailed description. ${ }^{4}$ Similar experiments have been performed in castrated male rabbits. In the normal monkey (Allen, Gardner, and Hill, I935) and in the goat (De Fremery, 1936) the administration of an oestrogen will likewise suitably prepare the mammary gland so that lactogenic hormone is then effective. Gardner (1935) produced general development of the duct system, as well as some localized growth of alveoli in male mice with ovarian grafts in the testis. In the depancreatized bitch treated with insulin, lactogenic hormone produces lactation, provided that mammary development corresponds to that of pregnancy or pseudopregnancy (Nelson, Himwich, and Fazekas, I936). Houssay (I 935) used only an oestrogen to bring about mammary development in normal or gonadectomized dogs of both sexes ("folliculin" as $\mathrm{I}, 000-10,000$ international units daily for 49-90 days). The intraperitoneal administration of what was probably a crude extract of beef anterior lobe then caused lactation. ${ }^{5}$

Certain experiments in the mouse, rat, and guinea pig are of particular interest because they indicate that mammary development following the administration of an oestrogen cannot take place in the absence of the pituitary body. ${ }^{6}$

4 Anselmino, Herold, and Hoffmann (1935), Macdonald (1936), Margulis (1936), Pallot (1936), and Gillard (1937).

5 The effects were present after removal of the lumbar sympathetic chains.

${ }^{6}$ Houssay stated that the pituitary is not necessary in the dog. Nelson (1935) reported that oestrone produced about the same degree of mammary development in male guinea pigs, whether or not the pituitary had been removed. Also, Asdell and 
Gomez, Turner, Gardner, and Hill (I937) observed no development of the rudimentary mammary glands in completely hypophysectomized male mice receiving injections of various oestrogens (50-500 international units weekly). If, however, a very minute fragment of the pars glandularis remained, the injection of oestrogen brought about mammary growth and development. Likewise in male or female rats oestradiol benzoate will cause no growth of the breasts after hypophysectomy, although this action is not prevented by gonadectomy (Reece, Turner, and Hill, 1936). Atrophy of the mammary glands due to the removal of the pituitary from the rat cannot be halted by oestrone; however, inanition seems to be an important factor (Astwood, Geschickter, and Rausch, I937). Other experiments by Gomez and Turner ( 1936 ), who used guinea pigs, also lead to the conclusion that growth and development of the mammary ducts and parenchyma following the injection of moderate to enormous doses of oestrone or oestradiol benzoate (e.g., 25-I,000 international units daily) cannot take place after hypophysectomy has been performed. ${ }^{7}$ If, however, hypophysectomized male guinea pigs receive implants of the pituitary of male rats in which breast development has been induced by injections of oestrone, growth of the nipples and breasts including the development of alveoli occurs, whereas implants from rats to which no oestrone has been given do not affect the recipient guinea pig's rudimentary nipples and breasts (Gomez, 'Turner, and Reece, I937). According to Robson (1936), little or no development of the mammary glands occurs in rabbits hypophysectomized about the 22-24 day of pregnancy de-

Seidenstein (1935) found that oestrone in combination with progestin caused about equally good development of the mammary glands in spayed rabbits with an intact pituitary or after partial or complete hypophysectomy. Do these findings indicate that the animals used were not completely hypophysectomized? According to Gomez and others (1937), remnants of the anterior pituitary, too small to maintain the gonads or the adrenals, permit the response of the mammary gland of the mouse to an oestrogen.

? The growth of the nipple due to administered oestrogen is not affected by hypophysectomy (Gomez and Turner, 1936). 
spite the injection of progestin or progesterone to term. Gomez and Turner (1938) suggest that a pituitary "mammogenic" hormone is secreted in response to an oestrogen or to an oestrogen and progesterone and that this hormone, perhaps made up of two components, brings about the growth of the mammary ducts and the development of the lobules and their alveoli. The authors injected simple preparations of the anterior pituitary of pregnant or non-pregnant heifers into a small number of rats and rabbits which had been spayed when immature. The administration of anterior pituitary from non-pregnant heifers - although lactogenic hormone was present in the injected anterior pituitary-did not affect the mammary gland. On the other hand, if anterior pituitary of pregnant heifers was injected for several weeks, mammary development corresponding to advanced pseudopregnancy or pregnancy could be produced both in rabbits and rats.

These results indicate that the growth and development of the breasts are as dependent upon the pars glandularis as lactation itself. A complex interplay of the hormones of the anterior pituitary and the ovary may take place normally. Gonadotropic hormone secreted by the anterior pituitary maintains ovarian function, whence arises the secretion of oestrogen and progesterone. In non-pregnant animals oestrogen chiefly causes the formation and liberation of a substance from the anterior pituitary, so that partial growth and development of the breasts occur. In pregnant animals the production of "mammogenic" hormone(s) is increased further not only by the increased quantities of oestrogen produced in the ovaries and placenta but also by the added stimulation afforded by the prolonged secretion of corpus luteum hormone. As a result, full development of the breasts preparatory to lactation occurs. The lactogenic hormone of the anterior pituitary then initiates the secretion of milk.

Progesterone as a factor in the development of the mammary glands.-Progesterone, the secretion of the corpus luteum, is 
believed to vary in significance as a cause of breast development. Certainly, it is much less important than oestrogen and is thought to act only after some growth, especially of the ducts, has already occurred, following the secretion or administration of oestrogen. Most authors have concluded that the secretion of the corpus luteum is of definite importance in bringing about alveolar development in the rabbit's breast, provided that growth of the ducts has been stimulated by oestrogenic hormone. ${ }^{8}$ Lactogenic hormone then readily produces or "releases" the secretion of milk. On the other hand, in the guinea pig and rat, progesterone appears to be of little significance in supplementing the effects of oestrogens on mammary growth and development (Gardner and others, 1935; Repetti, 1936; Astwood and others, 1937; and Nelson, 1937). ${ }^{9}$

The effect of androgens on the development of the mammary glands.-As in female rats, the post-pubertal development of the breasts in male animals is prevented by gonadectomy. Testosterone propionate, although without oestrogenic effects, will cause mammary development in spayed rats (McEuen, Selye, and Collip, i936). The authors injected $3 \mathrm{mg}$. of testosterone propionate as the total dose over II days. Astwood, Geschickter, and Rausch (1 937) concluded that testosterone can bring about, in young male rats, mammary development corresponding to that in adult males. Large doses of the hormone, although causing cyst formation and an increase in the fibrous tissue, produced no development of the mammary tree such as followed the administration of oestrone. Apparently the effects of testosterone on the breast, like those of an oestrogen, are secondary to the secretion of an anterior pituitary hormone; hypophysectomy prevents such effects (McEuen, Selye, and Collip, I937).

\footnotetext{
${ }^{8}$ Gardner, Gomez, and Turner (1935); Anselmino, Herold, and Hoffmann (1935); Macdonald, Pallot (1936); Gillard (1937).

${ }^{9}$ Such observations should be made in spayed animals, inasmuch as the oestrogen may bring about the new formation of lutein tissue by increasing the rate of liberation of luteinizing hormone from the anterior pituitary.
}

$$
\text { [ } 156 \text { ] }
$$



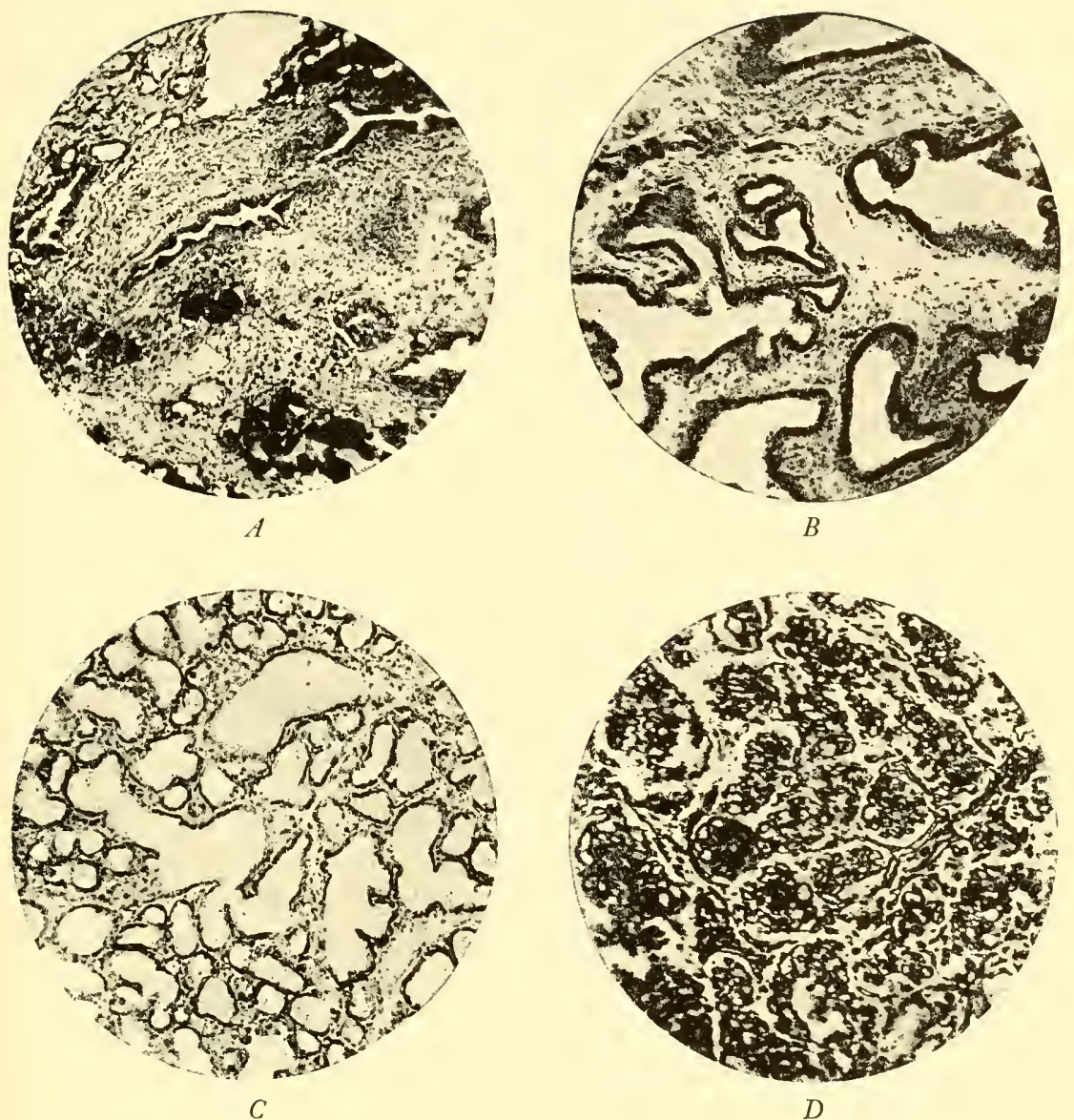

F1G. 20. - Photomicrographs of biopsy specimens of breast from a single castrated male rabbit. (From Anselmino, Herold, and Hoffmann, Zbl. Gynäkol., 59, 963-69 [1935] ) $A$, After castration. $B$, After treatment for 25 days with oestrogen. Development of the ducts has taken place. $C$, After further treatment for 6 days with corpus luteum hormone. There is marked development of the alveoli. $D$, After the administration of anterior pituitary extract (lactogenic hormone) for 5 days. Lactation is under way.

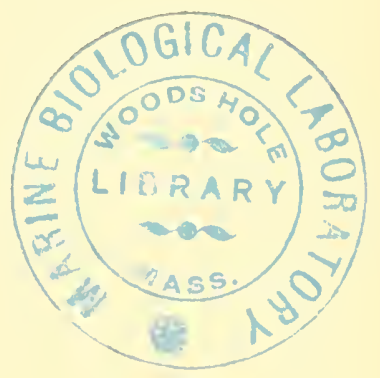


The effects of various hormones on the morphology of the breast are well illustrated by figures reproduced from a report by Anselmino, Herold, and Hoffmann (1935). All the samples of breast tissue were removed from the same castrated male rabbit after castration (Fig. 20, $A$ ) and after treatment for nearly 4 weeks with oestrin (Fig. 20, $B$ ), followed by the daily injection of progesterone for 6 days (Fig. $20, C)$. Finally, extract containing anterior pituitary lactogenic hormone was administered for 5 days; lactation then appeared (Fig. 20,D).

THE LACTOGENIC HORMONE ${ }^{\text {IO }}$

Provided that there is adequate development of the breasts, the secretion of milk in normal mammals probably takes place because of the release and continued secretion of the lactogenic hormone of the anterior pituitary. However, this statement is not meant to exclude the participation of other hormones, including, particularly, the adrenal cortical hormone. After hypophysectomy, with which is associated a marked reduction of the activity of the rest of the endocrine system, lactogenic hormone alone will not initiate or support lactation, although the breasts may be suitably developed or secreting milk at the time of operation.

It is now proposed to discuss factors which influence, both positively and negatively, the action or the conditions necessary for the action of the lactogenic hormone.

The effect of suckling on the secretion of lactogenic hormone.Selye, Collip, and Thomson had earlier demonstrated that the stimulus of suckling, without the escape of milk, maintains the secretion of lactogenic hormone, as shown by lactation in breasts from which the nipples had been excised. ${ }^{\mathrm{II}}$ Ingelbrecht's experiments (I935) indicate that the reflex

ro Synonymous terms are galactin, mammotropic hormone, and prolactin.

"In the guinea pig, unlike the rat, ligation of the galactophores is rapidly followed by failure of lactation in the corresponding breast, although suckling and lactation continue in the other breast with a normal nipple (Hesselberg and Loeb, 1937). 
stimulation of the release of lactogenic hormone from the pars glandularis is prevented by section of the spinal cord. The author severed the spinal cord between the last thoracic and first lumbar segments in lactating rats and covered the 6 upper nipples, which were still sensitive, with tape. Despite vigorous suckling of the insensitive nipples, the young died within $4^{8}$ hours. However, if only two sensitive nipples were left exposed or if a hemisection of the spinal cord was performed, lactation continued in the breasts of the anesthetized and paralyzed abdomen. Reece and Turner (1936) investigated the effect of suckling and removal of the secreted milk on the amount of lactogenic hormone in the pituitary of lactating rats. In terms of pigeon-units, the results obtained in one set of observations are given in Table 4 . Thus, it ap-

TABLE 4

Units per Gland

I. Normal suckling . . . . . . . . . . . $7 \cdot 7$

2. Accumulation of milk without suckling for 15 hours 9.2

3. No suckling for $\mathrm{I} 2$ hours followed by suckling for 3 hours. . . . . . . . . . . . . . .

4. Suckling (as in 3) but without removal of milk due to ligation of main galactophores......... 5.2

pears that suckling causes a marked release of stored lactogenic hormone and that the removal of accumulated milk may be an additional factor.

Lactogenic hormone as a factor in promoting the secretion of milk in hypophysectomized animals.-It is well known that lactation promptly ceases after the pituitary body has been removed from lactating animals. For the restoration of lactation in such animals the lactogenic hormone is necessary but not sufficient. Several authors have pointed out that crude anterior pituitary extracts will again initiate lactation in hypophysectomized mammals, whereas refined lactogenic extracts will not (Gomez and Turner, I936; Nelson and Gaunt, I936). 
It is probable that the successful initiation of lactation by means of the crude anterior pituitary extracts depends upon the presence of adrenal cortical stimulating hormone (as well as, perhaps, that affecting carbohydrate metabolism) in such extracts. ${ }^{\mathrm{I2}}$ According to Gaunt and Tobin (1936), the adrenal glands contain no substance with lactogenic effects. However, after bilateral adrenalectomy has been performed in lactating rats, the secretion of milk ceases but can be restored by the administration of about twice the dose of adrenal cortical extract necessary to prevent death. Smaller doses can be used in conjunction with salt therapy, and sometimes salt therapy alone is sufficient. After hypophysectomy in lactating guinea pigs, lactation ceases but can be initiated again by the administration of refined lactogenic extract and adrenal cortical hormone (Gomez and Turner, 1936; Nelson and Gaunt, I 936. ${ }^{x_{3}}$ However, this treatment permits lactation for only a few days and does not prevent the involution of the breasts. Either extract by itself is of no value. Gomez and Turner (1937) later reported on the use of adrenal cortical stimulating hormone, obtained from the anterior pituitary, in place of adrenal cortical extract. By injecting an extract containing this hormone as well as lactogenic extract and glucose, they could maintain lactation in hypophysectomized guinea pigs for as long as 8-I 5 days.

The effects of "sex hormones" on lactation. I. Oestrogens.Numerous earlier reports indicated that the secretion of lactogenic hormone as indicated by lactation is inhibited by the internal secretions of the gonads. For example, ovariectomy may be followed promptly by lactation. On the other

12 Thyrotropic hormone may be of importance but certainly is not essential, inasmuch as lactation takes place in thyroidectomized mammals (see pp. 165-66).

Schooley, Riddle, and Bates (1937) found that the response of the crop-glands of one pigeon following the injection of lactogenic hormone was not prevented by adrenalectomy four days before.

${ }^{13}$ Gomez and Turner $\left(193^{6-37)}\right.$ believed that it is important also to administer glucose, which they injected in amounts as high as $200 \mathrm{mg}$. per $100 \mathrm{gm}$. of bodyweight daily. 
hand, lactation may occur in spite of the continued injection of an oestrogen into mammals like the rat and rabbit.

A distinction should be made between the formation and storage of lactogenic hormone and its release in adequate amounts in to the circulating blood. Recent experiments furnish us with some information on the effects of oestrogens on the storage of lactogenic hormone. Reece and Turner (I 937) reported that the total amount (and often the concentration) of lactogenic hormone in the rat's anterior pituitary is increased following the injection of oestrone or oestradiol benzoate. The pituitary of the ovariectomized rat contained less lactogenic hormone in a lower concentration. Margulis (1936) concluded that the injection of "folliculin" into castrated rabbits is accompanied by an increase in the content of lactogenic hormone in the pituitary. However, Margulis used only a few animals. ${ }^{14}$

The inhibitory effect of oestrogens on lactation has been studied by other authors. Folley and White (1937) injected into male or female pigeons $5 \mathrm{mg}$. of oestradiol benzoate, followed a few days later by a dose of lactogenic hormone sufficient to cause a marked hypertrophy of the crop-glands. In birds also receiving oestradiol benzoate the hypertrophy of the crop-glands was only about one-half (males) to three-fourths (females) that in control birds. The injection of large doses of oestrogens may inhibit normal lactation in rats, guinea pigs, and rabbits (Folley and Kon, I938; Mugnai, I937; Smith and Smith, I933; Custo, 1937). Folley and Kon ob-

${ }^{14}$ In Wiegand's experiments (1937) ovarian secretion was stimulated in rats, initially immature, by the injection of 100 rat-units of prolan daily for $15^{-20}$ days. The amount of lactogenic hormone in the pituitary was much greater than in control animals receiving no prolan. If, however, the injections were stopped and the ovaries were removed, lactation appeared after about 36 hours. At that time there had occurred a marked fall in the lactogenic action of the pituitary in agreement with the view that stored hormone had been discharged. Such an effect of gonadectomy was not observed in normal rats.

In other reports (1937) Wiegand attributed the marked increase in the amount of lactogenic hormone in the pituitary immediately following delivery to an increased formation of the hormone caused by the loss of the inhibitory effect of oestrone believed to be present during pregnancy in the rat. 
served a definite but much less marked inhibition, if the ovaries were removed immediately following parturition in rats. Nelson (1937) observed lactation in guinea pigs (normal or gonadectomized of both sexes), when the injection of oestrone, which had caused a complete development of the breasts, was stopped. Also he reported that lactation after oestrone continued in hypophysectomized guinea pigs, if crude pituitary extract was injected; if injections of oestrone were again administered, the lactogenic action of the crude pituitary extract was inhibited. According to Richter (1936), spaying is not followed by any improvement in the quantity or fat-content of the milk in lactating cows; also the duration of lactation is not affected. However, the injection of an oestrogen like oestradiol benzoate is followed by a reduction in the quantity of milk produced by cows (Folley, I9.36; Waterman, Freud, and Vos-De Jongh, I 936). Folley studied the effects of oestrogen on some of the constituents of milk and serum. The percentage of fat and non-fatty solids of milk was increased, whereas the nitrogen-partition was not affected, indicating that the milk was not colostrum-like. In the serum the treatment appeared to cause some increase in the concentration of inorganic $\mathrm{P}$ and, transiently, of phosphatase; also there was observed a temporary fall in the concentration of Ca.

Other investigators have studied the practical importance of oestrogens as means of suppressing lactation after abortion or stillbirth (Snoeck, 1935; Hoffmann, Mayor, van Tongeren, I 936; Mugnai, I 937). Such treatment is of value only when enormous doses are injected. Snoeck injected 100,000 international units of oestrone (?) within three days. Mayor as well as Mugnai concluded that the injection of 250,000 units (mouse-units [Mayor], international units [Mugnai]) of oestradiol benzoate can completely suppress lactation following delivery. Breast tension may be lessened by smaller doses. Mugnai recommended the use of oestrogen to inhibit 
the discharge from fistulas of the breast following incision for abscess.

2. Progesterone.-Folley and Kon (1937-38) particularly doubt that the inhibition of lactation during pregnancy is caused by the secretion of progesterone. However, they admit that the inhibiting effect of oestrogen on lactation may be reinforced by progesterone, which by itself is without such action. In their experiments with lactating rats, they injected I mg. of progesterone daily to the mothers from the fifth day after parturition. There was no detectable effect on the weight-curve of the nursing young. Anselmino, Herold, Hoffmann, and Pencharz (I936) also used rats but concluded that progesterone can cause marked inhibition of lactation. The authors injected 200 rat-units of prolan daily for $\mathrm{I}_{4}-\mathrm{I} 8$ days to bring about breast development in immature rats. The animals were then spayed, following which (36-48 hours) lactation appeared. Almost complete inhibition of lactation then followed the injection of two doses of 0.5 unit each of progesterone, whereas the administration of 4,000 units of oestrogen was without effect. ${ }^{15}$

3. Androgens.-Suitable doses of testosterone propionate apparently can completely inhibit lactation in intact lactating mice or rats, whereas androsterone has practically no effect (Robson, I937; Folley and Kon, I938). Robson injected O.I mg. of testosterone propionate in oil daily to lactating mice; nearly all the young died in less than three weeks. Similar injections of 0.2 to $0.4 \mathrm{mg}$. of androsterone were without action. Lactation (but not suckling or maternal care) rapidly disappeared, if $0.05 \mathrm{mg}$. of testosterone propionate suspended in $0.05 \mathrm{cc}$. of $\mathrm{I} 0$ per cent alcohol were injected every 2 hours. Control experiments indicated that the solvent did not contribute to the effect. The observations

${ }^{35}$ Folley and Kon (1938) found that the inhibitory action of oestrogen on lactation is less readily observed in spayed animals. The possible luteinization of the ovaries owing to the liberation of luteinizing hormone from the pituitary following the injection of a large dose of an oestrogen seems to have been left out of consideration. 


\section{THE PITUITARY BODY}

of Folley and Kon were made in lactating rats. Lactation was markedly inhibited by the injection of $0.4 \mathrm{mg}$. of testosterone propionate per $100 \mathrm{gm}$. body-weight; on the other hand, three-fourths of this dose of androsterone had no effect. The authors suggest the generalization that substances which cause growth and development of the breasts also inhibit lactation.

OTHER BIOLOGICAL INTERRELATIONSHIPS

OF THE LACTOGENIC HORMONE

New observations on the distribution of the hormone.-The concentration of lactogenic hormone in the pars glandularis of the sperm whale is less than 1.5 per cent of that in the anterior pituitary of the ox (Geiling, 1935). Leblond and Noble (I937) attempted to determine the amount of lactogenic hormone in the pituitary of animals of several classes. Their assays were performed in pigeons, into which they made injections intradermally over a crop-gland. They doubted the specificity of some responses because, although undulated thickenings were produced, the cells of the cropgland contained few or no fat-granules (Scharlach R). The clearest responses were obtained by the injection of the pituitary of mammals (mice, rats, rabbits) or of birds (fowls, pigeons). Glands from various fishes were implanted, sometimes as many as 100 in a single assay. Attempts also were made to detect and determine the amount of hormone in the pituitary of an amphibian (Rana pipiens) and a reptile (Kinosternon odoratum).

The secretion of milk in response to the injection of extract containing the lactogenic hormone.-Grant (1936-37) has investigated the action of lactogenic extract on the regressing mammary glands of the guinea pig. Large doses of the hormone caused the transient reappearance of a small amount of mammary secretion which, however, contained little or no lactose (0.04-0.24 per cent). The volume of secretion was increased tremendously if preliminary treatment with oestra- 
diol ( $0.5 \mathrm{mg}$. daily for 7 days) followed by progesterone ( 0.4 mg. daily for 4 days) was given. ${ }^{16}$ De Fremery (1936) concluded that lactogenic extract can initiate the secretion of milk in the goat, irrespective of the season or the phase of the oestrous cycle. Other studies in lactating cows have been made by Waterman, Freud, and Vos-De Jongh (1936) and by Asimov and Krouze (1937). Only two cows were used by Waterman and others whose experiments indicated that lactation is favorably influenced by the hormone. Asimov and Krouze concluded that milk production is increased approximately ${ }^{20-} 40$ per cent by the injection of a crude anterior pituitary extract every to days. Five hundred and ten lactating cows which received injections were compared with $9 \circ$ control animals. The effect of the extract was much greater in the first half of the normal period of lactation. Except for a temporary rise of $0 . \mathrm{I}-0.8$ per cent in the concentration of fat, the milk was found to resemble normal milk. (The $\mathrm{pH}$ and the concentration of lactose and chloride were also studied.)

Among observations in primates are those of Geschickter and Lewis (1936), who studied the action of lactogenic extract in women who had received injections of oestrin for a month previously. The administration of a total dose of $600-1,120$ bird-units of lactogenic hormone during a week was followed by the elaboration of a secretion which persisted only a few days, despite further injections or mechanical stimulation. Histological examination of breast tissue indicated that true lactation had not appeared. The authors believed that such combined treatment might cause changes resembling cystic disease of the breast.

The thyroid gland in relation to lactation. ${ }^{17}$-.-Lactation and,

${ }^{16}$ The injection of 80 units of pregnant-mare serum daily for 3 days prior to the injection of lactogenic extract appeared not to be of value.

${ }_{17}$ Riddle and others $(1936-37)$ pointed out that lactogenic extracts may have a marked calorigenic action in pigeons. This effect, like similar effects of thyroid extract or thyrotropic hormone, can be observed at $30^{\circ} \mathrm{C}$. At $20^{\circ} \mathrm{C}$. the change may be slight, and at $15^{\circ} \mathrm{C}$. it may be in the opposite direction. The calorigenic 
therefore, the action of the lactogenic hormone do not depend on the normal secretion of thyroid hormone to any important extent. Schooley, Riddle, and Bates (I937) were able to produce a typical crop-gland response to lactogenic hormone in a thyroidectomized pigeon. In mammals like the dog, guinea pig, and rat, lactation following parturition or the withdrawal of oestrin or the injection of anterior pituitary extract is not prevented by thyroidectomy (Houssay, 1935; Nelson and Tobin, 1937). Likewise the development of the breasts during pregnancy is not significantly inhibited by thyroidectomy in the rat (Nelson and Tobin, I937). Unlike adrenal cortical extract, thyroid extract or thyroxine administered coincidently with lactogenic extract will not initiate lactation in suitable hypophysectomized animals (Gomez and Turner, I937; Nelson and Tobin, 1937).

De Fremery (1936) reported that doses of thyroxine sufficient to cause a severe hyperthyroidism (I $5 \mathrm{mg}$. daily) in goats brought about a reduction in the volume of milk secreted daily. No change in the composition of the milk was noted. After the administration of thyroxine was stopped, the output was not restored to its original level. Van Tongeren (1936) could demonstrate no action of thyroxine in lactating women. Probably the doses he used were too small.

Miscellaneous observations.-Additional evidence in favor of the view that broody behavior of fowls depends upon the lactogenic hormone has been gathered by Burrows and Byerly (1936). The authors compared the proliferative changes in the crop-glands of pigeons by implanting one pituitary (e.g., from a broody hen) over one crop-gland and the other (e.g., from a laying hen) over the other crop-gland.

action of lactogenic extract is not prevented by thyroidectomy. Riddle, Dotti, and Smith suggest that a moderate increase (about 20 per cent) in the blood-sugar concentration as well as a calorigenic effect are caused by an action of lactogenic hormone on adrenal cortical tissue.

The concentration of sugar in the blood of the normal rabbit or of the normal or depancreatized monkey is not affected by the injection of lactogenic extract (Nelson, Turner, and Overholser, I935). 
The pituitary gland of broody hens produced much more hypertrophy of the crop-gland than that of laying hens or of cocks. Other comparisons between the pituitaries of hens of broody or non-broody genetic constitution indicated again that broodiness is associated with the larger amount of lactogenic hormone in the pituitary.

Leblond and Nelson (1937) concluded that the maternal instinct in lactating mice and rats does not depend upon the secretion of lactogenic hormone because the instinct persists after hypophysectomy.

De Fremery (1936) was able to terminate pregnancy, either as fetal death or abortion, by the injection of lactogenic extract into pregnant goats, guinea pigs, rabbits, or rats. He stated that the effect was not due to gonadotropic or thyrotropic hormone.

According to Salle and Schechmeister (1936), embryonic crop-gland cultured in vitro is not affected by lactogenic extract. The growth of the Simpson mammary carcinoma in mice is not altered after the injection of 60 bird-units of lactogenic hormone (Bischoff and Maxwell, I936).

The metabolism of the lactogenic hormone.-Wiegand (1937) found that little change in the amount of lactogenic hormone is found in the pituitary body of the rat during pregnancy. Immediately after parturition the amount present is doubled or trebled; however, the quantity of the stored hormone falls as it is liberated into the blood stream and lactation appears. Perhaps a similar change occurs in women. At any rate, several authors have demonstrated lactogenic hormone in the urine of lactating women. Like Wiegand, these authors relied on the hypertrophy of the pigeon's crop-gland to detect the hormone. ${ }^{8}$ Lactation in new-born children-the secretion of “witches' milk"--is attributed by Lyons (I 937) to the action

${ }^{18}$ Lyons and Page (1935), Hoffmann (1936), and Tesauro (1936). Lyons and Page estimated that the daily excretion of hormone in the urine of women $4^{-1} 3$ days after parturition was at least equivalent to what can be extracted from one pars glandularis of the ox. Liard's experiments (1937) appear not to be adequately controlled. 
first of oestrin and then of lactogenic hormone. He was able to detect lactogenic hormone $\left(0.03-0.5\right.$ unit $^{19}$ per cc.) in the urine of new-born babies. Two units ${ }^{\mathrm{Ig}}$ per cc. of urine were excreted by a lactating baby boy.

Reece and his associates investigated the concentration and total amount of lactogenic hormone, as assayed in pigeons, in the pituitary of rats with various vitamin deficiencies. There appeared to be a reduction in the total amount of hormone, if the diet was deficient in vitamins $A$, $\mathrm{B}$ (as B complex), and D; however, the concentration of the hormone was increased in vitamin-B deficiency, whereas if vitamin $A$, or especially vitamin $D$, was lacking, the concentration was diminished. No change in the total amount or concentration of the hormone accompanies vitamin-E deficiency. ${ }^{20}$

According to Lewis and Geschickter (1936), cyst fluid of cystic disease of the breast, although sometimes without action, contains on the average about 500 bird-units of lactogenic hormone per liter. Colostrum as well as the tissue of the udders of lactating cows contain lactogenic hormone (Geschickter and Lewis, 1936).

The lactogenic extract used by Giuffrida (I937) was administered repeatedly to pigeons. The maximum hypertrophy of the crop-glands was observed after seven daily injections. The effects disappeared despite injections for a longer period. Refractoriness toward the extract appeared earlier if larger doses were used. Young (I937) prepared an extract with lactogenic and diabetogenic effects but without significant action on either the thyroid or the gonads. Although this extract was injected daily-in some instances for as long as 9 weeks - into rabbits, dogs, or monkeys, the sera of these animals not only contained no lactogenic "an-

19 Intradermal "micro-units" in the pigeon.

${ }_{20}$ Marchesi (1935) administered gonadotropic extract, so that pregnancy and parturition took place in animals presumably receiving no vitamin E. Lactation either did not appear or was hopelessly inadequate. 
tihormone" but even seemed to augment the action of the hormone.

The assay of lactogenic hormone.--The only satisfactory methods of assaying the lactogenic hormone are based upon the response of the crop-glands of pigeons - a test introduced by Riddle and his colleagues. After the injection of extract containing lactogenic hormone, the crop-glands undergo hypertrophy which may be limited to only one of the two glands, provided that not too large a dose is injected into the skin overlying the gland. Pigeons of the same stock vary greatly, of course, in sensitivity. Some varieties of pigeons are more suitable than others; Bates and Riddle (1935) found that a tenfold variation in sensitivity may be encountered in different races of pigeons (see also Evans, 1937). Wolff ( I 937) concluded that the response of young birds is more regular than that of adults. Birds of either sex can be used.

The effect of a dose of lactogenic hormone depends upon the route of absorption. The greatest response follows the intracutaneous administration of extract over a crop-gland. The dose causing a given response is about I per cent of that necessary, if injection is subcutaneous, intramuscular, or intraperitoneal (Lyons and Page, 1935; Bates and Riddle, 1936; and Chasin, 1936). According to Bates and Riddle (1936), the relative efficacy of injected hormone is approximately the following: intracutaneous, I,000; subcutaneous, IO; intramuscular, 2; intraperitoneal, I.3; and intravenous, 0.9 .

Varous authors ${ }^{21}$ have devised methods of assay and have often determined the relationship between dose and response, which usually is given in terms of crop-gland weight, sometimes in relation to body-weight. Potency also may be estimated from the percentage of birds in which a minimum response is produced. Probably few investigators will oppose the view that a standard preparation, perhaps crystalline

${ }_{21}$ Bates, Riddle, and Lahr (1936), Dyer (1936), McShan and Turner (1936), Lyons (1937), Rowlands (1937). 
hormone, must be introduced. An example of the relationship between dose and response is the graph of Rowlands reproduced in Figure 21. Rowlands' extract, which contained neither gonadotropic nor thyrotropic hormones, was injected subcutaneously once daily for 6 days when the maximum effect was observed. The crop-glands were removed on the seventh day, fixed in Bouin's fluid, and weighed after they had been carried to 70 per cent alcohol. Weights of

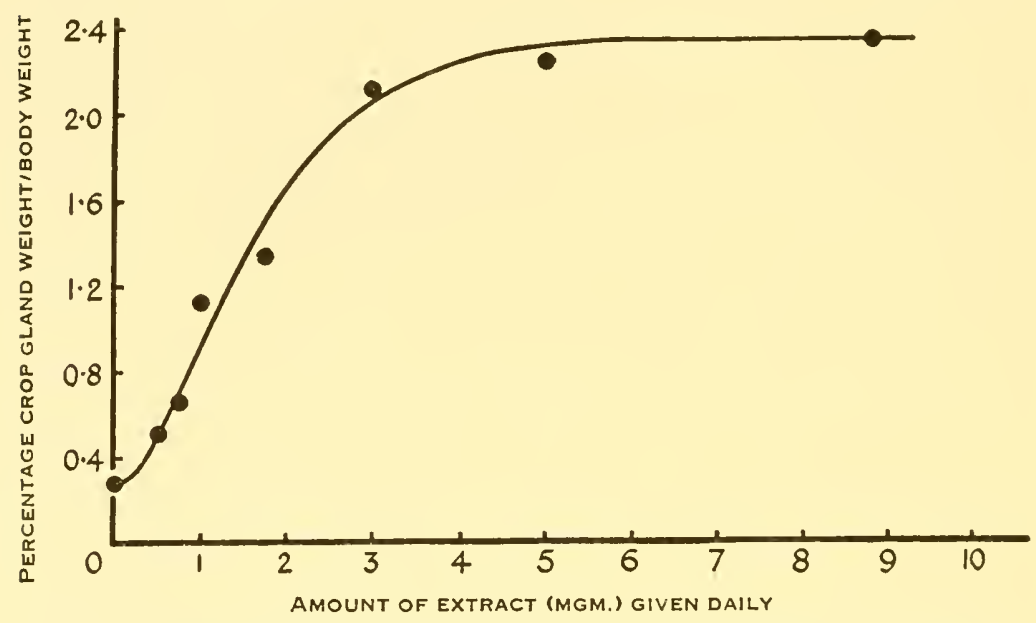

FIG. 21.-The relationship between the dose of anterior pituitary extract (containing lactogenic hormone) and the grow th of the crop-gland of the pigeon. (From Rowlands, Quart. J. Pharm. Pharmacol., 10, 216-21 [1937].)

crop-glands are expressed as percentages of body-weight. In birds receiving no injections the crop-glands constitute about 0.3 per cent of the body-weight. Rowlands recommended that the glands of injected birds should fall within a weightrange represented by $0.8-2.0$ per cent of the body-weight.

Leblond and Allen (I937) stated that the action of lactogenic hormone could be detected as soon as Io hours after injection, if $0.5 \mathrm{mg}$. of colchicine also was administered. After the injection of either the hormone or colchicine, about 2 per cent of the cells of the crop-glands were in mitosis; however, if both were injected, "arrested" mitoses were present

$$
\text { [ } 170]
$$


in about 20 per cent of the cells. According to Valle (I 937), smears of the cells of crop-gland secretion can be obtained by means of a fistula. Especially $4^{8-72}$ hours after the injection of lactogenic extract, the cells are found to contain orange-colored droplets of various sizes after fixation in formalin and staining by sudan III and methylene blue. ${ }^{22}$

The chemistry of the lactogenic hormone.-White, Catchpole, and Long (1937) recently described a method of isolating a crystalline lactogenic principle which presumably is the pure hormone. By the two-day intracutaneous test of Lyons and Page a "unit" was found to be 0.05-0.1 $\gamma$; by a systemic test, also in pigeons, O.I mg. was designated a unit. The method used was briefly as follows: $100 \mathrm{mg}$. of purified extract was dissolved in $2 \mathrm{cc}$. of $\mathrm{I} 3$ per cent acetic acid to which was added $2 \mathrm{cc}$. of 13 per cent pyridine. The cloudy mixture was set aside and later centrifugated. The precipitate was subjected to the same treatment - the whole process being repeated ten times. Crystalline hormone was finally obtained either by allowing the acetic acid-pyridine mixture to stand in the refrigerator or by the careful addition of 1 per cent $\mathrm{NH}_{4} \mathrm{OH}$ to the mixture followed by centrifugation and refrigeration of the turbid mother-liquor. The crystalline material yielded on analysis 5I.I I per cent of C, 6.76 per cent of $\mathrm{H}, \mathrm{I} 4.3^{8}$ per cent of $\mathrm{N}$, and $\mathrm{I} .77$ per cent of $\mathrm{S}$. It appeared to be protein or protein-like. The following reactions were positive: xanthoproteic, biuret, labile S, Millon, and Hopkins-Cole.

Other reports are of interest so far as the preliminary purification of the hormone is concerned. ${ }^{23}$ The most varied media have been used for initial extraction: 60-70 per cent alcohol at "pH" 9-IO (this was used by Bates and Riddle

${ }^{22}$ Leblond and Nelson (1937) and Leblond and Noble (1937), who injected material intracutaneously, concluded that non-specific proliferation of the crop-glands can be produced (e.g., by liver). In proliferation not due to lactogenic hormone, fatgranules are not found in the growing epithelium.

${ }^{23}$ Bates and Riddle (1935), Bergman and Turner (1937), Evans (1937), Lyons (1937), and McShan and French (1937). 


\section{THE PITUITARY BODY}

and is recommended by Bergman and 'Turner), dilute acetic acid or acidified acetone (Lyons, and McShan and French), $0.05 \mathrm{~N} \mathrm{NaOH}$ (Evans). The further details of preparation can be found in the reports of the various authors.

McShan and French confirmed the statement of Bates and Riddle that purified extracts withstand boiling in a solution of $\mathrm{pH}$ 8. If the substance is a protein, this is a remarkable property. So far, this experiment has not been performed with crystalline hormone. In a solution at a lower or a higher $\mathrm{pH}$, the hormone is inactivated at a temperature of $100^{\circ} \mathrm{C}$.

\section{SUMMARY}

The manner in which the anterior pituitary controls the development of the breasts and the secretion of milk is more complex than was suspected a few years ago. The important and probably essential glands of internal secretion are the pars glandularis of the pituitary body, the ovaries, ${ }^{24}$ and the cortex of the adrenal glands.

For the growth and development of the breasts, an oestrogen analogous to what is obtained from ovarian tissue must be secreted or injected. In some mammals like the guinea pig this appears to be the only ovarian secretion required for prelactation development. In other mammals like the rabbit it is believed that corpus luteum hormone (progesterone) is also required later. However, development of the breasts due to an oestrogen is prevented by hypophysectomy. Also, there is other evidence that an anterior pituitary secretion, which brings about development of the mammary glands, is formed and liberated into the blood stream in response to ovarian (or placental) secretion or to the injection of an oestrogen. A working hypothesis regarding the growth and development of the breasts is as follows: gonadotropic hormones from the anterior pituitary are essential for the

${ }^{24}$ For the purposes of this summary, breast development and lactation in male animals will not be specifically considered. It may be mentioned that the development of the rudimentary breasts of male animals appears to depend upon the testes and the anterior pituitary. 
normal secretory activity of the ovaries; ovarian secretions (or placental secretions, or both in pregnant animals) bring about the elaboration of a new anterior pituitary secretion which causes growth and development of the mammary glands.

Provided that prelactation development has occurred in the breasts, another anterior pituitary hormone-the lactogenic hormone- brings about lactation. However, this hormone alone cannot initiate lactation in hypophysectomized animals. Perhaps because of its effects on salt metabolism, adrenal cortical hormone must also be available to the organism. In hypophysectomized animals lactation from adequately developed breasts can be initiated by the injection of lactogenic hormone in combination with adrenal cortical hormone from the adrenals or adrenal cortical stimulating hormone from the pituitary. It is probable that the maintenance of lactation depends upon additional hormones, including those responsible for the continued development of the breasts.

The greater part of the attention of investigators has been focused on the lactogenic hormone. This substance apparently has been isolated as a crystalline substance, which is either a protein or is closely related to proteins. It is conveniently assayed by its stimulating effect on the growth of the crop-glands in the pigeon. In the rat, at least, its liberation from the pars glandularis largely depends upon centripetal nervous impulses due to suckling. The amount of hormone secreted during lactation may exceed the needs of the mammary glands, so that the unused portion is excreted in the urine. Other data concerning the physiology of the lactogenic hormone are discussed not only in this chapter but also in the two which precede it. 


\section{CHAP'TER VI}

\section{THE THYRO'TROPIC HORMONE}

$\mathrm{O}$ $\mathrm{NE}$ of the impressive effects of hypophysectomy in mammals is a marked fall in the rate at which heat is produced. This change is due principally to inadequate function of the thyroid gland and can be correlated with morphological changes in the thyroid-i.e., undischarged colloid accumulates in vesicles lined by flat, "inactive," epithelial cells. A specific substance called the thyrotropic hormone is secreted only by the anterior pituitary; ${ }^{\mathrm{I}}$ it is responsible for the maintenance of normal thyroid function and may be important in disorders attributed to deficient or excessive thyroid secretion. In classes of animals other than mammals different changes, likewise dependent on the virtual absence of thyroid secretion, follow the removal of the pars glandularis. A well-known example is the absence of metamorphosis after the removal of the gland from anuran larvae.

The thyrotropic hormone has not been isolated as a pure substance. Nearly all the recent progress is concerned with its biological action.

The biology of thyrotropic hormone in fishes, amphibia, and reptiles.-Young and Bellerby (1935) were not successful in attempts to produce metamorphosis in lampreys (Lampetra planeri) by the injection of an extract of the anterior pituitary of the ox.

The pioneer work of Adler, Smith, and Allen clearly demonstrated that metamorphosis in anuran amphibia is pre-

I Sturm and Schöning (1935) believed that extracts of the ovary or the medulla of the adrenal gland may act like true thyrotropic hormone. Repetition of their work with ovarian tissue yielded no confirmation (Ballif and Gherscovici, 1936; McGinty and McCullough, 1936; and Emerson, 1937). 
vented by the removal of the anterior lobe or the buccal anlage of the pituitary, because what is now termed thyrotropic hormone is no longer available. Without thyrotropic hormone from the anterior pituitary, thyroid function is so deficient that metamorphosis is prevented. The experiments of Voitkevič (1937), who used larvae of Rana esculenta and $R$. temporaria, confirm observations-i.e., such as the greater thyrotropic effect of implants of basophilic chromophils in comparison with oxyphilic chromophils - which have already been discussed in my monograph of 1936. The author's observation that implants of beef anterior pituitary containing oxyphils as the principal chromophil antagonize spontaneous metamorphosis or metamorphosis caused by anterior pituitary basophils or thyroid extract has not been independently confirmed. Etkin's study (I935), in which larvae of $R$. sylvatica were used, led to the following conclusions: (I) The pars buccalis is self-differentiating (i.e., differentiation occurs after its transplantation to a new site), (2) thyrotropic hormone is secreted in an amount proportional to the amount of actively secreting pituitary tissue, and (3) the liberation of the hormone appears not to depend on nerves. Metamorphosis occurred at about the normal age and stage of growth in embryos which had been hypophysectomized and given replacement therapy in the form of a transplant of the removed pituitary which was placed in the eyecup or under the adhesive disk. If similar embryos received three transplants of the pars buccalis under the adhesive disks after hypophysectomy, precocious metamorphosis (at 8 instead of the normal i 8 days) followed; also, the length of the larvae was less than that of normal larvae at the time of the corresponding change (29 instead of $44 \mathrm{~mm}$.). According to Atwell (1935), the differentiation and subsequent secretory activity of the anterior pituitary in larvae of $R$. sylvatica or $R$. pipiens can take place without contact with nervous tissue or foregut. (Atwell could not be sure that this statement holds for the pars intermedia; also, he stated that Etkin's 
transplants were not free from nervous tissue.) Similar experiments in Amblystoma punctatum were unsuccessful. ${ }^{2}$

Atwell (1937) found that compensatory hypertrophy of thyroid fragments in larvae of $R$. pipiens is prevented by hypophysectomy. This finding is in accord with other work in amphibia and mammals.

Another group of authors has published observations largely confirming and extending previously reported experiments in urodele amphibia. Uhlenhuth and his collaborators $^{3}$ again described the stimulating effect of anterior pituitary extract on the thyroid as indicated by morphological changes in the latter, by increased oxygen-consumption or by precocious metamorphosis. Most of their observations were made in Amblystoma tigrinum. The photomicrographs of Figure 22 are reproduced from those published by Adams and Martindale (1936). The injection of an alkaline extract of the pars glandularis of the ox ("Phyone") produced a marked stimulation of thyroid function in hypophysectomized newts (Triturus viridescens). Maximum changes were produced after daily injections had been given for about three weeks. The thyroid underwent regression to its former condition, characteristic of hypophysectomy, only weeks after injections were stopped.

Hypophysectomy prevents molting in adult urodele amphibia and, in this respect, resembles thyroidectomy. The cornified cells of the epidermis are retained as successive layers. These effects of extirpation of the pituitary do not occur in larval urodeles or in neotenous forms, except perhaps in Necturus maculosus (Osborn, 1936). Adams and Gray ( 1936 ) were able to cause molting of the layers of cornified epidermis of hypophysectomized newts ( $T$. viridescens) by the administration of anterior pituitary extract, thyroxine, or

2 Blount's results in Amblystoma indicated that the pars neuralis is required for the differentiation of the pars glandularis. In his animals there also were a pronounced intensification of pigmentation and distortions of growth.

3 Uhlenhuth and Schwartzbach (1935); Uhlenhuth, Schwartzbach, and Thompson (1935); and Schwartzbach and Uhlenhuth (1936).

$$
[176]
$$



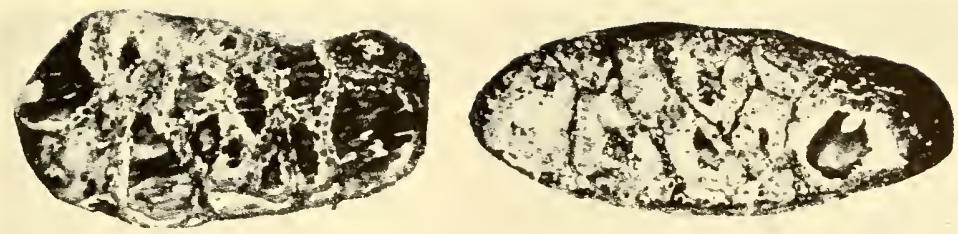

1

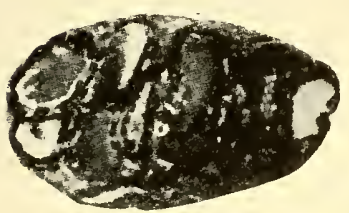

2
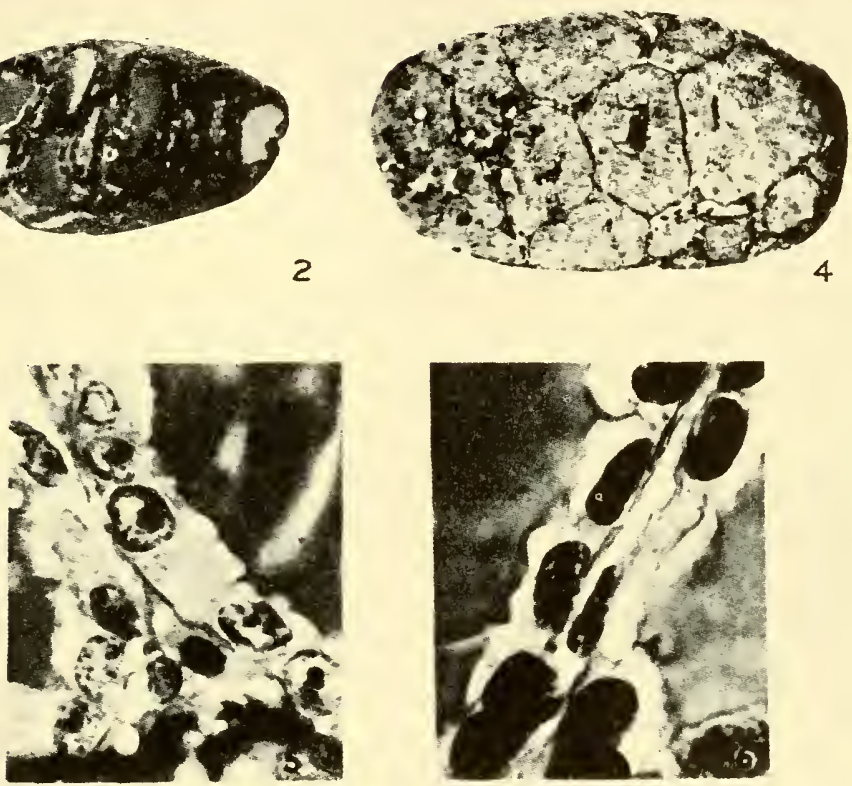

FIG. 22.- The effects of hypophysectomy and of the injection of anterior pituitary extract after hypophysectomy on the thyroid gland of the newt, Trimurus viridescens. (From Adams and Martindale, Anat. Rec., 65, 319-31 [1 936].)

$I, 5$, Photomicrographs of thy roid of normal newt at low and high magnifications. 2, 6, Photomicrographs of thy roid of newt 5 weeks after hypophysectomy. 3 , Photomicrograph of thyroid of newt receiving anterior pituitary extract for 10 days. Treatment was begun 5 weeks after hypophysectomy. f, Treatment begun as in 3 , but continued for 20 days. Photomicrographs $I-f$ were made at the same low magnification, photomicrographs $5^{-6}$ at the same high magnification. 
iodine, or by grafts of the thyroid. Anterior pituitary extract was effective because it contained thyrotropic hormone. The mechanism by which iodine facilitates molting in such animals is not clear; the element produced no morphological change in the thyroid gland.

After hypophysectomy, repeated molting occurs in snakes (Thamnophis sirtalis, T. radix) (Schaefer) - the reverse of the effect induced in urodele amphibia. Hellbaum (1936) recently described the effects of hypophysectomy as well as those following the injection of anterior pituitary extract into such snakes. The cytological changes in the anterior pituitary of thyroidectomized snakes ( $T$. radix) were investigated by Siler (1936).

Recent observations in birds.-After the complete removal of the pituitary from Brown Leghorn cocks, the feathers finally resemble those of thyroidectomized cocks-loss of black pigment of feathers of part of the neck, of the breast, and of the legs. Hill and Parkes (1935), who made this observation, were able to restore the pigment to normal by administering thyroxine to a hypophysectomized cock.

The biology of the thyrotropic hormone in mammals.Numerous experimental observations with a great variety of objectives have been made in mammals. Much of the work only adds adornment to knowledge previously available; in addition, however, new facts have been gathered. The classification of extensions of our knowledge as well as additions to it will now be attempted.

The effects of thyroidectomy on the anterior pituitary. $-\mathrm{Hy}$ pertrophy of the pars glandularis, which is often more marked in male than in female animals, usually follows thyroidectomy. It is generally agreed that an important histological change in the anterior pituitary is a marked reduction in the proportion of oxyphils. Also there is an increase in the percentage of cells with an affinity for basic dyes; such cells, often first undergoing hypertrophy, may become vacuolated or appear to be filled with hyaline material and usually are 
identified as "thyroidectomy-cells." Their origin is still a matter of disagreement. Some authors believe that they arise at least partly from basophils and either are identical with "castration-cells" or are completely different. ${ }^{4}$ Their relationship to the hypertrophied reserve cells which other authors have so frequently noted in the anterior pituitary of animals with thyroid deficiency probably also is close. ${ }^{5}$

Lebedewa (1936) believed that the pituitary of young thyroidectomized rats contains less thyrotropic hormone than normal. However, her conclusion is not based on enough experiments to warrant its acceptance. According to Zeckwer (I936), although the concentration of thyrotropic hormone in the pituitary of thyroidectomized rats is greater than normal in terms of body-weight, the amount present is less than normal in terms of age. ${ }^{6}$ Chen and van Dyke (1936) investigated the anterior pituitary of normal and thyroidectomized rabbits. In littermate normal animals there was no sexual difference in thyrotropic potency. Three months or more after thyroidectomy an increase in the total amount of hormone in the hypertrophied anterior pituitary of female rabbits was noted. Although hypertrophy of the gland was greater in male rabbits, a similar change in potency could not be found. In both sexes the hypertrophied anterior pituitary associated with thyroid deficiency contained more water than the normal gland (total solids I9. I-I9.4 per cent compared with the normal of 22.2-23.7 per cent).

The effects of hypophysectomy on thyroid function.-There is little to add to the previous account. Normal thyroid function and all that this implies is markedly but not completely deficient after hypophysectomy. Low-grade thyroid

${ }_{4}^{4}$ See chap. i, p. 22.

5 Altschule and Cooper (1937) recently reported on the changes in the human pituitary associated with operative or spontaneous hypothyroidism. They concluded that the number of basophils, of ten markedly vacuolated, may be $2-4$ times as great as normal. Like other authors they believed that hypothyroidism may be due to a primary disturbance of either the pars glandularis or the thyroid.

${ }^{6}$ See also Zeckwer and others (1935). 
activity is indicated morphologically by the atrophy of the epithelium of the thyroid follicles and the distention of the latter with dense, non-vacuolated colloid. Such changes were again reported by Rowlands (I 935), who hypophysectomized fowls and mammals of several species (ferret, guinea pig, and hedgehog). It is of interest that weight-atrophy does not follow hypophysectomy in the guinea pig. The morphological signs of inactivity of the thyroid could be observed in the various animals $\mathrm{I}-2$ weeks after hypophysectomy. ${ }^{7}$

Other aspects of the biology of thyrotropic hormone.-The most significant action of thyrotropic hormone is to facilitate or promote the discharge of thyroid hormone from the thyroid gland. The colloid becomes vacuolated; the thyroid vesicles diminish in size; the epithelial cells become hypertrophied. Its further effect is to promote hyperplasia of the epithelium, so that doses much larger than those causing the discharge of stored hormone may bring about marked hypertrophy of the thyroid. There still is disagreement as to the unity or duality of the anterior pituitary hormone(s) causing these two principal effects.

Several new reports concerning the amount of hormone in the pituitary body of various mammals have been published. Müller, Eitel, and Loeser (1935) declared that the human gland contains 5-30 "guinea pig units." Undoubtedly there was great variation in the degree of postmortem autolysis in their material. The variations in potency were great and appeared not be be related to age or sex; high values were obtained if death was due to tuberculosis or other infections. According to Saxton and Loeb (1937), the amount of thyrotropic hormone in the human pars glandularis is quite constant irrespective of age or other factors such as pregnancy and lactation. Rowlands (1936) used a reliable uni-

${ }^{7}$ Reiss and Fischer-Popper (1936) injected $0.1-0.5 \mathrm{mg}$. of thyroxine daily into rats. They concluded that after hypophysectomy the sensitivity of such animals was greatly increased (loss of body-weight, increased rate of urinary excretion of $\mathrm{N}$, increased basal metabolic rate), because compensating "antithyroid" effects of the pituitary, mediated through the adrenal glands, were absent. 


\section{THE THYROTROPIC HORMONE}

form method of assay (hypertrophy of the guinea pig thyroid according to Rowlands and Parkes). Two methods of initial extraction of the same acetone-desiccated powders were employed with the results shown in Table 5. From this data it appears that the best practical sources of thyrotropic hormone are the pituitaries of cattle and pigs. ${ }^{8}$

TABLE 5

\begin{tabular}{|c|c|c|}
\hline \multirow{2}{*}{ Powdered Pituitary of } & \multicolumn{2}{|c|}{$\begin{array}{c}\text { Units PER Gram of Desiccated } \\
\text { PoWDer after InItial } \\
\text { EXtraction WITH }\end{array}$} \\
\hline & $\mathrm{N} / 10 \mathrm{NaOH}$ & $\begin{array}{l}\text { 50 Per Cent } \\
\text { Pyridine }\end{array}$ \\
\hline 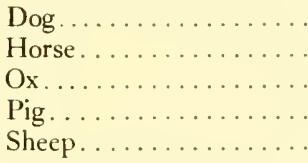 & $\begin{array}{r}43 \\
5^{*} \\
20 \\
40 \\
8\end{array}$ & $\begin{array}{l}\cdots \cdots \\
\cdots \cdots \\
28 \\
\\
54 \\
16\end{array}$ \\
\hline
\end{tabular}

* Anterior lobe only. Apparently the other figures refer to whole pituitary.

The effects of the injection of pituitary extracts containing thyrotropic hormone. I. Morphological changes in the thyroid gland.-The response of the thyroid of different animals to thyrotropic hormone probably varies widely. A good example is the great susceptibility of the guinea pig's gland in comparison with the insensitivity of the rat's thyroid. With doses near the threshold, all parts of the gland are not equally sensitive; in the thyroid of the guinea pig the first and more pronounced changes due to thyrotropic hormone are observed in the central part of the thyroid lobes. Compensatory hypertrophy of the thyroid following partial extirpation depends upon the secretion of thyrotropic hormone by the anterior pituitary. Albani's recent report (1936) indicated that in young dogs this may be a very slow process, different from

8 The anterior pituitary of the ox contains a higher concentration of thyrotropic hormone than that of the guinea pig (Emerson, I 937); however, the rat's gland contains 7-9 times as much as that of the ox (McQueen-Williams, 1935). 
the rapid, dramatic changes which can be induced by anterior pituitary extract; however, this is hardly proof, as the author suggests, that the mechanisms involved are different. Extracts containing thyrotropic hormone probably facilitate the survival and growth of transplants as has again been reported by Eitel (1936). According to Koch, Schreiber, and Schreiber (I937), the peripheral growth of a transplant of the thyroid in the guinea pig is facilitated, if thyroid and pituitary tissue are transplanted together.

For some years it has been known that the rate of mitotic division of thyroid epithelium may be greatly increased in a mammal like the guinea pig, if anterior pituitary extract (thyrotropic hormone) be injected. An enormous change was reported by Kippen and Loeb (I935), who injected anterior pituitary extract into immature guinea pigs and removed the thyroid gland 48 hours later. In comparison with the normal thyroid in which was found an average of about 150 mitotic figures, the thyroid of injected animals contained as many as I 90,000 mitotic figures. The effects observed by Bastenie and Zylberszac (I937) were clear cut but much less pronounced. These authors also injected colchicine (about 0.8 mg. per kg. body-weight) to bring out clearly the action of thyrotropic hormone on mitotic division. 9 Guinea pigs weighing $220-250 \mathrm{gm}$. were used. In thyroid tissue from control animals 6.3 mitotic figures per 100 follicles were found. The number increased to 16.8 after the injection of thyrotropic hormone. However, if both thyrotropic hormone and colchicine were injected, I I 9 mitotic figures - in the prophase for the most part-were found in roo follicles.

Halpern (1935) investigated the action of anterior pituitary extract or KI on the appearance of the mitochondria and Golgi apparatus of epithelium of the thyroid of rats 2.5-3 months old.

9 Bastenie and Zylberszac mention two interpretations of the manner in which colchicine acts: it may bring about an increased rate of karyokinesis (Dustin) or it may arrest karyokinesis before the process is completed (Allen and others). 
2. Physiological, pharmacological, and biochemical aspects of the action of thyrotropic hormone. - The administration of sufficiently large doses of anterior pituitary extract rich in thyrotropic hormone into a susceptible animal like the guinea pig may produce a "syndrome" strikingly resembling Graves's disease in man. 'These changes, of course, are prevented by thyroidectomy. Friedgood's article (1934) contains a discussion of the manner in which symptoms in guinea pigs may develop in relation to the administration of extract toward which refractoriness finally appears. The basal metabolic rate rises at first but later returns to normal, whence recrudescences of an elevated rate may appear. The rate is not necessarily high, although a well-pronounced thyroid hyperplasia may persist. Exophthalmos likewise is not necessarily associated with an increased rate of basal metabolism and may still be present after a long series of injections. Pathological changes in organs like the heart, kidneys, and liver are not like those of Graves's disease in man (Heinemann, 1937); however, this is not surprising. A number of authors have offered evidence indicating that an increased concentration of thyroid hormone is present in the blood after the gland has been stimulated by thyrotropic hormone. Zunz. and La Barre (1935) found that the concentration of thyroid hormone in the serum of dogs is increased $2-4$ times only 30 minutes after the injection of $60-80$ guinea pig units of thyrotropic hormone. (Thyroid hormone was estimated by the method of von Euler and Holmquist.)

Several authors have paid particular attention to the indirect effect of thyrotropic hormone on the oxygen-consumption of man and animals under basal conditions after the administration of thyrotropic hormone. ${ }^{10}$ Observations in man

ro According to O'Donovan and Collip (1937), pituitary extract may contain a substance causing an elevation of the basal metabolic rate persisting only a few hours after injection. The authors concluded that this substance is not thyrotropic hormone but probably is related to or identical with the melanosome-dispersing hormone of the pars intermedia and that it accelerates the rate of oxidation of fat.

Kuschinsky (1935) reported that if rats were kept at $4^{\circ} \mathrm{C}$., their pituitaries con- 
have been reported by Lederer (I935), Sylla (1935), Thompson and others (1936), and Scowen (1937). Thompson and his colleagues found that extracts containing thyrotropic hormone were without action, if thyroid tissue capable of function was lacking, as in certain patients with marked myxedema. The basal metabolic rate could be raised to normal (mild or moderate hypothyroidism) or elevated (non-toxic goiter), sometimes strikingly (exophthalmic goiter). Apparently because of the development of "antihormone," the change induced was only temporary and could not be elicited by a second course of injections. Sylla listed three therapeutic uses to which he put an extract containing thyrotropic hormone: (I) to cause complete recovery from pituitary cachexia, (2) to cause a loss of weight in obesity of certain types, and (3) to inhibit diuresis in a single patient with diabetes insipidus, as effectively as after the injection of extract of the posterior lobe. Lederer as well as Scowen has described the beneficial effects of the treatment of hypothyroidism due to pituitary insufficiency by the injection of extracts containing thyrotropic hormone. Measurements of the basal metabolic rate furnished objective proof of the favorable action of the hormone. Scowen found that much larger doses of thyrotropic hormone did not affect the basal metabolism of patients with classical myxedema, whereas the administration of thyroxine Na caused a prompt response. Presumably

tained about the same amount of thyrotropic hormone in association with histologic signs of diminished (at first) or increased (later) thyroid function. However, thyrotropic hormone might almost disappear from the pituitary of animals with inactive thyroids because of an environmental temperature of $38^{\circ}-40^{\circ} \mathrm{C}$.

See also the reference to the observation of Riddle and others (pp. 165-66). Riddle, Smith, and Moran (1935) found that the basal metabolic rate of pigeons is reduced as a result of hypophysectomy $\left(-33\right.$ per cent at $30^{\circ} \mathrm{C}$. or -17 per cent at $20^{\circ} \mathrm{C}$.). If $10-23$ per cent of the gland remained, there was a definite, but less pronounced, fall.

The hypophysectomized rat adapts itself slightly but very poorly to a cold environment (Wolf and Greep, 1937). Its body temperature is abnormally low (e.g., $34^{\circ}-35^{\circ} \cdot 5$ C. after 29 days in an environment at $2^{\circ}-4^{\circ} \cdot 5$ C.). In the thyroids of such animals is found some colloid absorption at the periphery; the central part of the gland is atrophic. 
such patients suffer from a purely "thyrogenic" hypothyroidism.

Mahaux (1937) found that the elevation of the basal metabolic rate of guinea pigs, an effect ordinarily appearing about 48 hours after the injection of a large dose of thyrotropic hormone, was absent if I mg. of thyroxine had been administered. (The calorigenic action of thyroxine was allowed to decay before thyrotropic hormone was administered.) Likewise using guinea pigs, Gessler ( 1936 ) reported that oestradiol or its benzoate $(5.0 \mathrm{mg}$.) caused a fall in the basal metabolic rate of $3^{-2} 3$ per cent. 'The oestrogen also appeared to lessen the calorigenic action of thyroid extract. Sherwood and Bowers ( 1936 ) stated that the injection of oestrone or oestrin may lower the basal metabolic rate of rats as much as $28-54$ per cent. Also, the return of the rate to normal, after a marked elevation had been caused by thyroid extract, was accelerated by the injection of oestrin. Oestrogens appear to antagonize the action of thyroid hormone, not that of thyrotropic hormone as Gessler (1937) suggested. In the dog, according to Zajic (I935), the increased metabolic rate following the injection of thyrotropic hormone is not accompanied by any change in the respiratory quotient. ${ }^{\text {II }}$

Extracts containing thyrotropic hormone readily cause exophthalmos in thyroidectomized guinea pigs (Smelser, 1936; Paulson, 1937); therefore, this change cannot be caused by an increased secretion of thyroid hormone. Smelser even found that exophthalmos is more readily produced in thyroidectomized guinea pigs. He studied anatomically the contents of the orbit in normal animals and those with experimental exophthalmos. The increase in the bulk of the retrobulbar tissues amounted to as much as 40 per cent, because of hypertrophy of the fatty connective tissue, the dorsal lacrimal gland, the extraocular muscles, etc. As in certain instances of exophthalmos associated with a low basal metabolic rate in man, a fluid containing lipoid droplets and gran-

"I See also O'Donovan (1937) and Sinha (1937). 
ules as well as lymphocytes was found to infiltrate the orbital tissues. Paulson particularly described degenerative changes in the lacrimal glands sometimes associated with similar alterations in the extraocular muscles and, occasionally, in the orbicularis oculi. Altered activity of the sympathetic nervous system appears not to be a factor (Smelser).

Eitel's observations (1936) are in accord with other views that the action of thyrotropic hormone does not depend on peripheral nerves innervating the thyroid. On the other hand, Uhlenhuth (1937) believed that the effectiveness of thyrotropic hormone in salamanders or guinea pigs is increased if epinephrine or pilocarpine is also injected; however, he was not certain that this phenomenon was due to action on peripheral fibers of the autonomic nervous system. The experiments of Scharrer and Gaupp (1935) led them to conclude that thyrotropic hormone is not secreted by diencephalic "glands" (neurons of the supraoptic and paraventricular nuclei). As a result of hypophysectomy, toads do not molt. This defect is due to a deficiency of thyrotropic hormone and is not corrected by the administration of as many as three diencephalic "glands" rich in colloid.

One aspect of the possible interrelationship of the gonads and thyrotropic hormone has already been discussed in the section dealing with the action of thyrotropic hormone on the gaseous metabolism (p. 185). Certain other aspects of this interrelationship remain for consideration. ${ }^{\text {22 }}$ Voss (1935) was able to produce oviposition in the axolotl as many as three times a year by the injection each time of 120 guinea pig units of thyrotropic extract. ${ }^{\mathrm{x}}$ In confirmation of Riddle and Krizenecky, Mârza and Blinov (1936) found that the thyroid of the pigeon appears histologically to be more active at times of sexual activity and ovulation. According to Chouke, Friedman, and Loeb (1935), mitotic activity in the guinea

${ }^{12}$ See chap. iii, pp. 94-95.

${ }^{13}$ Adams and Hilsman had shown that pituitary transplants cause oviposition. 
pig's thyroid is highest during the ovarian luteal stage and lowest during the follicular stage. Female thyroids appeared to be more active than those of male animals. Likewise, Franck (1937) declared that the injection of oestrone (2502,500 international units) into guinea pigs is followed by histological signs of thyroid inactivity. The young male albino rat was the experimental animal chosen by Amilibia, Mendizábal, and Botella-Liusiá (1936). They believed that the daily injection of 200 mouse-units of oestrin ("Progynon") for 5 days causes the histologic changes commonly described as characteristic of increased thyroid activity and that this is due to an effect on the anterior pituitary. Also, they stated that a stage of thyroid inactivity followed that of activity. ${ }^{14}$ According to Cramer and Horning (1938), the injection of extracts containing thyrotropic hormone prevents the following effects of the prolonged administration of oestrin to mice: (I) the spontaneous development of mammary carcinoma in a special strain of mice with a high, spontaneous incidence, (2) the development of the mammary gland in male mice, and (3) alterations of the anterior pituitary, likewise caused by oestrin, such as marked congestion and disappearance of the oxyphils.

Oehme (1936) concluded that one adrenal cortical extract ("Cortidyn"), but not a second called "Pancortex," prevents the elevation of basal metabolism due to thyrotropic hormone. However, the histological signs of thyroid stimulation appeared as usual. The effect observed appeared not to be due either to ascorbic acid or to tyrosine.

An increased rate at which isolated strips of the auricle beat can be observed, if guinea pigs receive injections either of thyroxine or of thyrotropic hormone (Ferrannini, I936). From observations of the heart rate in rabbits, Rihl, Oestreicher, and Reiss (1936) concluded that the effects of thyroxine are strikingly different from those of thyroid hormone

${ }^{14}$ The similar injection of progesterone as I Clauberg-unit daily was found to have no effect. 
secreted in response to the injection of thyrotropic hormone. After the injection of the latter, the heart rate begins to rise within an hour and is $30-50$ per cent more rapid after $7-9$ hours. On the other hand, even after $4 \mathrm{mg}$. of thyroxine the heart rate is not significantly changed within a day. The authors' other comparisons of the effects of repeated doses appear to be less significant. Page and Sweet (1937) produced hypertension in dogs by means of Goldblatt's clamp. After hypophysectomy the blood pressure fell but could be raised moderately by the daily administration of $0.8 \mathrm{gm}$. of desiccated thyroid. The authors suggest that the lack of thyrotropic hormone following the removal of the hypophysis may account partly for the fall in blood pressure in dogs with experimental hypertension.

Anderson and Alt (1937) found that the addition of thyrotropic hormone to slices of isolated canine thyroid increased the oxygen-consumption of the tissue 17-120 per cent per hour during the first 3 hours; a similar change could not be produced if isolated slices of liver or kidney were used. These observations confirm the earlier report of Eitel, Krebs, and Loeser.

Either thyrotropic hormone or thyroid extract brings about a reduction in the concentration of hepatic glycogen. This effect appears not to be modified by the administration of KI (Holden and Thurston, 1935). Another action on carbohydrate metabolism attributed to the thyrotropic hormone is an increased rate of liberation of insulin in dogs, whether or not the vagi have been cut (Zunz and La Barre, I935). Confirmatory experiments were performed in non-anesthetized dogs by Kotchneff and London (1937), who concluded that the concentration of insulin in the blood may be quadrupled 30 minutes after the injection of extract containing 20-60 units of thyrotropic hormone. There appeared to be no effect on the rate of liberation of epinephrine. After the repeated injection of thyrotropic hormone into guinea pigs, the heart undergoes considerable hypertrophy (about 60 per cent in 
excess of its initial weight); coincidently, the concentration of glycogen is reduced to about one-fourth its former value (Lederer, 1935). Both of these effects can be observed after the basal metabolic rate has returned to normal but are less pronounced as the rate falls below normal with continued injection of the extract and the probable formation of "antihormone."

The metabolism of proteins or of substances related to proteins is referred to in a few reports. ${ }^{\mathrm{I} 5}$ Schönholzer (1 937) fed casein to rats and relied upon the Unna-Pappenheim histological technic for recognizing protein in the liver. The injection of $100-300$ units of thyrotropic extract for $\mathrm{I}-7$ days was followed by markedly diminished numbers or almost a complete absence of the Eiweissschollen (but see also the report of Liang and $\mathrm{Wu}$ [1937], who considered that a special hormone is responsible for such effects). According to Bühler (1935), thyrotropic hormone, by its action on the thyroid, increases the rate of excretion of creatine and creatinine in the dog but not in the rabbit. Maloberti (1936) believed that an increase in the concentration of glutathione in the blood and certain tissues of the guinea pig parallels the symptoms of hyperthyroidism provoked by the injection of thyrotropic hormone. Maloberti's results often were variable and would be more convincing if he had secured more supporting data.

Pugsley (I935) reported that thyrotropic hormone causes a fall of approximately $35^{-5}$ o per cent in the concentration of serum-cholesterol in both rats and dogs. Injections were made intraperitioneally, sometimes twice daily for as long as 8-1o days. Only total cholesterol was determined. The effect could not be produced in one dog after thy roparathyroidectomy. Rothschild and Staub (I935) were not able to detect any effect of thyrotropic hormone on the lipoids of the blood of rabbits.

The relationship of the anterior pituitary, the thyrotropic

${ }^{15}$ For reports dealing with the specific dynamic response in patients, see Sylla (1935) and Mahaux (1936). 
hormone, and the thyroid gland to the metabolism of water is discussed in chapter $\mathrm{x}$.

In the immature guinea pig the administration of a large dose of vitamin A ( 1,800 units daily for Io days) prevents the action of 6 guinea pig units of thyrotropic hormone (Fellinger and Hochstädt, I 936). ${ }^{16}$ Elmer, Giedosz, and Scheps (I 935) concluded that both vitamin $\mathrm{A}$ and ascorbic acid inhibit the action of thyrotropic hormone in the guinea pig but are much less effective than iodide. Vitamin D appeared to be without action. ${ }^{17}$

According to Eitel and Lexer (I936), the healing of bone fractures in rabbits is facilitated by the administration of thyrotropic hormone or thyroid extract. ${ }^{8}$ Likewise the healing of wounds in guinea pigs takes place at a faster rate, if thyrotropic hormone be administered (Eitel and Riecker, I936). Arsenic (as $0.003-0.3 \mathrm{mg}$. $\mathrm{As}_{2} \mathrm{O}_{3}$ per $\mathrm{kg}$. rat for 20 days) or $\mathrm{CO}$ (as repeated exposure of guinea pigs to an atmosphere containing 0.5 per cent $\mathrm{CO}$ ) was believed to bring about a reduction in the amount of thyrotropic hormone (Kampelmann, and Kampelmann and Schulze, 1937).

The metabolism of thyrotropic hormone.-Fellinger (I936) and Bodart and Fellinger (I936) have undertaken the assay of thyrotropic hormone in the blood of patients. Before the material was injected, an attempt was made to remove thyroid hormone which would be a source of interference with the assays, as they were performed in guinea pigs. The authors concluded that a positive histological response of the guinea pig's thyroid can be secured from the thyrotropic hor-

${ }_{10}$ The authors do not mention whether or not the presence of iodine in the vitamin-A preparation was excluded.

17 Demole and Ippen (1935) stated that ascorbic acid can prevent death after a fatal dose of thyroxine.

The experiments of Loeser and Trikojus (1937) indicated that thyrotropic hormone does not alter to any important extent the concentration of ascorbic acid in the liver and the adrenals. The authors found that adrenal hypertrophy followed the long continued administration of thyrotropic hormone. This effect was not prevented by the administration of ascorbic acid.

${ }^{18}$ See also chap. ii. 
mone in 5-8 cc. of blood. Generally the blood of patients with hyperthyroidism contained less hormone than normal; the findings were similar in patients thought to be suffering from hypothyroidism due to hypofunction of the anterior pituitary. On the other hand, the concentration of thyrotropic hormone in the blood of patients with hypothyroidism of purely thyroid origin or in that of patients with a pituitary tumor associated with an elevated basal metabolic rate was higher than normal. In investigating the thyrotropic hormone of serum, Hertz and Oastler (1936) relied upon an exceedingly specific test-i.e., the induction of histological signs of thyroid activity in hypophysectomized rats. Thyrotropic hormone was detected in the serum of 9 patients with myxedema, whereas none could be found in the serum of 5 normal individuals or of 7 patients with hyperthyroidism. Serum in doses of $\mathrm{I}-2 \mathrm{cc}$. twice daily was administered intramuscularly for 5 days.

There is still controversy concerning the excretion of thyrotropic hormone in the urine. Antognetti and Geriola (1936) could secure no convincing evidence that thyrotropic hormone can be extracted from the urine of normal persons or of patients with Graves's disease or myxedema, whether spontaneous or resulting from thyroidectomy. In the work of Hertz and Oastler referred to in the preceding paragraph the detection of thyrotropic hormone in urine was also undertaken. The authors usually injected $5 \mathrm{cc}$. of urine twice daily for 5 days by an intramuscular route into hypophysectomized male rats. Thyrotropic hormone could be detected in the urine of patients with myxedema, whereas none could be found in the urine of patients with hyperthyroidism or in that of normal persons. Niţescu and Timuş (1938) agree that the hormone cannot be found in normal urine or in urine of patients with Graves's disease; however, they did detect thyrotropic hormone in the urine of an acromegalic with symptoms of hyperthyroidism. Grumbrecht (1935) believed that the urine of women past the menopause contains thyro- 
tropic hormone; he performed assays in the rabbit by injecting the extract of urine intravenously.

Goiter was produced by Remington (1937) in rats by feeding a diet containing too little iodine. The effects of thyrotropic hormone were no less difficult to elicit in such rats than in normal rats. On the other hand, thyroids of rats, goitrous for unknown reasons, were found by Anderson and Collip to be abnormally sensitive to the thyrotropic action of anterior pituitary extract.

The effects of iodides will be considered in this section. Friedgood (1936) reported that the administration of sodium iodide caused a remission of the symptoms of hyperthyroidism in guinea pigs receiving anterior pituitary extract. He concluded that the remission resembled that produced in human beings with exophthalmic goiter or with the hyperthyroidism of early acromegaly. Weil and Bernheim (1936) made autotransplants of the thyroid after total thyroidectomy in the guinea pig. The effect of $70-400$ units of thyrotropic hormone, indicated by histological signs. of stimulation of the thyroid, could be antagonized by the administration of KI; however, a much greater antagonistic action was produced by thyroxine and, to a less extent, by diiodotyrosine. 'The results of Anderson and Evans (1937) were different from those of the authors just cited. Anderson and Evans concluded that the coincident administration of KI does not interfere with the production of thyroid hyperplasia by thyrotropic hormone but that the iodide prevents the liberation of thyroid hormone into the blood, so that a calorigenic effect does not appear. This conclusion was based on experiments in normal guinea pigs and hypophysectomized rats. ${ }^{19}$

Extracts containing thyrotropic hormone in relation to antihormone effects. - Some authors believe that a small amount of antithyrotropic substance is present in normal serum

${ }^{19}$ See also Franck's interpretation (1937) of histological changes in the pituitary after the injection of anterior pituitary extract with or without Lugol's solution or diiodotryosine. 
(Herold, I934; Scowen and Spence, I936); however, this appears doubtful and was not confirmed by Rowlands and Parkes (1936). Various investigators ${ }^{20}$ have experienced no difficulty in confirming earlier experiments on the production of thyrotropic antihormone; even with the best extracts available, repeated injections soon appear to lose their thyroid-stimulating properties, as is shown by the absence of anatomical signs of thyroid stimulation and of physiological changes, such as an increased basal metabolic rate due to the increased liberation of thyroid hormone. The serum of blood removed at this time prevents the thyrotropic action of anterior pituitary extract. Sometimes the antihormone effect appears to be specific for the animal species furnishing the pituitary tissue from which the extract was made (Eichbaum and Kindermann, I936; Oudet, 1937); in other experiments, as in those of Thompson (I 937), there seems to be no species specificity, and the serum alone seems to cause atrophic changes in the thyroid which resemble the condition following hypophysectomy. An intermediate phenomenon was described by Oudet, who concluded that species specificity is present after a short course of injections but not after a long course. A later report by Eichbaum and others (1937) suggested that two types of antibody are formed - one characteristic of the proteins of the species, the other characteristic of thyrotropic hormone.

Loeser (1936) has confirmed earlier experiments indicating that hypophysectomy does not interfere with the formation of thyrotropic antihormone. He continues to affirm (Eitel and Loeser, 1935; and Loeser, 1936) that the presence of the thyroid gland greatly facilitates the formation of antihormone; but this is improbable and could not be confirmed either by Oudet (1936) or by Chou (1937).

Okkels' experiments (I937) likewise indicate that tissues

${ }^{20}$ Extracts with thyrotropic effects were used in a number of the experiments discussed in chap. iii. 
other than the thyroid are sources of antihormone and that the neutralization of thyrotropic hormone does not occur in the thyroid. After anterior pituitary extract had been injected into rabbits for 2 months and the thyroid was then refractory toward further treatment, the gland was removed and, presumably still living, was perfused by the CarrelLindbergh technic with normal serum containing anterior pituitary extract. Thyroid-stimulating effects could be observed within 24 hours. In other experiments the author was surprised by the fact that a large volume of rabbit serum containing antihormone only partially neutralized the thyrotropic action of anterior pituitary extract with which it was mixed. The mixture was perfused through the isolated thyroid of the normal rabbit.

Werner's experiments (1936) strongly support the view that thyrotropic antihormone is an artifact characteristic of relatively crude anterior pituitary extracts. Equivalent doses (in terms of weight of crude gland) of extract of the beef anterior pituitary were injected in to guinea pigs and led to the following conclusions: ( $\mathrm{I}$ ) whether or not antihormone is produced depends on the type of extract not on its potency, (2) it may be possible to produce antihormone by doses of extract without thyroid-stimulating effects, (3) the thyroids of animals completely refractory to one extract may be readily stimulated by the other-yet both were obtained from ox pituitary - and (4) an extract may cause the formation of no antihormone in the majority of animals even after injection for nearly 3 months. As a basis for these conclusions the author relied upon the basal metabolic rate and the histology and total iodine-content of the thyroid.

The assay of thyrotropic hormone.-Among mammals no animal is as convenient for the assay of thyrotropic hormone as the immature guinea pig. The histological signs of thyroid stimulation-vacuolization of the colloid, hypertrophy, and later, hyperplasia (increased mitotic division) of the epithe- 
lial cells - were first investigated by Aron and Loeb ${ }^{2 x}$ and have been extensively used as a means of assay. According to Wilcke (1935), fixation is of great importance, if an attempt is made to grade the histologic effects by the plan of Heyl and Laqueur; Wilcke recommended ro per cent formalin as a fixative. The production of thyroid hypertrophy as a means of assay (Rowlands and Parkes) is more objective but requires much larger doses of hormone. There is no confirmatory evidence to support the contention of Heyl and Laqueur that a different principle is responsible for hypertrophy.

Smelser ( 1 937) recommended the use of day-old White Leghorn chicks. His practice was to divide the total dose into five daily injections and to perform the necropsy 24 hours after the last injection, when the thyroid lobes were dissected out under a binocular microscope. The method was found to be more sensitive than a similar technic applied in guinea pigs.

Other methods which may be sensitive are either inconvenient or are difficult to evaluate. For example, the decrease in the total amount of iodine in the thyroid of chicks or guinea pigs as a result of thyroid stimulation is a method suggested by Cuyler, Stimmel, and McCullagh (1936) and Stimmel, McCullagh, and Picha (1936). Atwell (1935) recommended the hypophysectomized tadpole as a very sensitive biological indicator; the prominent change which occurs if thyrotropic (or thyroid) extract is administered is metamorphosis. Collip and his collaborators have frequently detected the presence of thyrotropic hormone by its calorigenic action in hypophysectomized rats.

The chemistry of thyrotropic hormone.-Thyrotropic hormone is available only as an impure extract. It is a heatsensitive substance which, like other anterior pituitary hormones, appears to belong to the group of proteins. Readers interested in a new report on the preparation of extract are referred to the article of Lambie and Trikojus (1 937). 'There

21 The following are late reports by these authors: Kippen and Loeb (1935) and Aron (1936). 
are no new experimental data enabling us to decide whether or not thyroid stimulation in amphibian larvae is due to a principle different from that stimulating the mammalian thyroid.

\section{SUMMARY}

The recent investigations of the various aspects of the biology of the thyrotropic hormone have yielded a disappointingly small crop of new facts. Much of the effort has been expended in consolidating or extending slightly knowledge which was already available.

Probably there is general agreement on the principal functions of the thyrotropic hormone. Its importance is great in nearly all classes of vertebrates-i.e., to insure normal thyroid function, especially the discharge of thyroid secretion. Unless thyroid hormone is actually liberated from the gland, various striking effects occur in cold-blooded animals-i.e., the metamorphosis of tadpoles cannot take place; in both urodele and anuran amphibia molting or the desquamation of cornified epidermis is absent; on the other hand, in reptiles molting may either be accelerated or take place less frequently. In both classes of animals, as in mammals, the rate of heat-production is abnormally slow and cannot be adapted readily to the demands of the environment. Atrophy of the thyroid gland may or may not rapidly appear after hypophysectomy. The invariable effect of the operation is to produce all the histological signs of inactivity of the thyroid, such as the accumulation of densely staining colloid in vesicles lined by flat epithelium, together with physiological evidences of thyroid deficiency, such as a low rate of metabolism.

Changes in the opposite direction are readily produced by the injection of extracts of the anterior pituitary. The colloid becomes vacuolated and may almost disappear. The epithelium, now active, is cuboidal or columnar and begins to proliferate. Animals of various classes exhibit changes dependent upon thyroid secretion. Metamorphosis can be pro- 
duced in hypophysectomized anuran larvae. Adult amphibia, subjected to the same operation, shed the accumulated layers of cornified epidermis. Heat-production is raised to normal or can be elevated above normal.

These facts have led to efforts to attribute deficiency or hyperfunction of the thyroid in man to a deficient or abnormally rapid rate of secretion of thyrotropic hormone. Such an explanation, it appears, may be of value in unraveling the genesis of certain types of hyperthyroidism in man. The hyperthyroidism associated with acromegaly probably is the result of the secretion of excessive amounts of thyrotropic hormone. On the other hand, thyroid deficiency in man less often seems to depend upon a disturbance of the anterior pituitary.

The effects of anterior pituitary extracts containing thyrotropic hormone have been studied extensively in mammals. Occasionally all the histological signs of thyroid stimulation are present without the expected general physiological changes, such as an elevated basal metabolic rate. Usually, however, the phenomena are associated. Under suitable conditions thyroid hormone is found to be liberated within a few hours or less following the injection of thyrotropic extract and acts more rapidly than its essential fraction, thyroxine. Antagonistic substances such as oestrogens, thyroxine, iodides, etc., appear to act in a variety of ways. Sometimes the action is peripheral to the thyroid; at other times the thyroid itself or the interaction between thyrotropic hormone and the thyroid appears to be affected.

Thyrotropic extracts contain both the hormone and other substances. If such extracts are repeatedly injected, "antihormone" may be produced. This phenomenon has not been shown to be of physiological importance.

Exophthalmos can be produced by the injection of anterior pituitary extract into normal or thyroidectomized animals. It is not known whether or not thyrotropic hormone is responsible for its appearance. 
THE INTERRELATIONSHIP BETWEEN THE PARS GLANDULARIS AND THE ADRENAL GLANDS; THE INFLUENCE OF THE PARS GLANDULARIS ON THE METABOLISM OF CARBOHYDRATES, LIPOIDS, PROTEINS, AND MINERALS (WITH REMARKS ON THE PITUITARY-PARATHYROID INTERRELATIONSHIP)

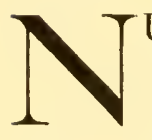

TUMEROUS interrelationships between the anterior pituitary and the adrenal glands have been suggested or investigated in almost every field of interest to the endocrinologist. Much of this work is discussed elsewhere and, for complete references, the reader is referred to the Index. The review in this section will be principally confined to a discussion of the part taken by the anterior pituitary in preserving the function of the adrenal cortex as demonstrated by the morphology of that structure. The important interrelationships affecting the metabolism of carbohydrates and fats are taken up in the sections dealing with the metabolism of these foodstuffs.

It is well known that the cortex of the adrenal glands undergoes a pronounced atrophy after hypophysectomy, whereas the medulla is affected scarcely at all. Likewise extracts of the anterior pituitary affect the cortex chiefly, if not entirely. Therefore, almost all the discussion will refer to the adrenal cortex.

New observations on the effects of hypophysectomy or of anterior pituitary extract ${ }^{\mathrm{x}}$ on the morphology of the adrenal glands. -Adrenal cortical stimulating hormone is probably as wide-

INo short, unobjectionable term to describe the hypothetical adrenal cortical stimulating hormone has been devised. Such terms as "adrenotropic" and "corticotropic" are undesirable from an etymological standpoint. 
ly distributed as other hormones of the anterior pituitary. Leonard (1937) concluded that it is present in the pituitary of the fowl. According to the assays of McQueen-Williams (1935) in rats, the anterior pituitary of the ox contains a higher concentration of adrenal cortical stimulating hormone than that of the rat. The author's preliminary experiments indicated that the removal of both adrenal glands is followed by an increase in the amount of the hormone in the pituitary.

Extracts of the anterior pituitary readily cause enlargement of the adrenal glands of normal or hypophysectomized animals chiefly by bringing about hypertrophy of the cortex. The effects of extracts in normal animals are, of course, difficult to evaluate accurately because the pituitary is intact. Among such observations are those of Bierring (1935), Friedgood (1936), and Latyszewski (1937) who used rats, guinea pigs, and rabbits. Bierring, who injected crude anterior pituitary extract into rats-sometimes for monthsconsidered that the important effect is on the zona glomerulosa but that the three cortical zones were more clearly demarcated in the treated animals. Some of Moon's observations (1937) also were made in normal rats. He reported that cells of the glomerular and fasciculate zones underwent both hypertrophy and hyperplasia after the injection of extract. In the guinea pig, according to Friedgood, the left adrenal undergoes more hypertrophy than the right. Latyszewski did not feel convinced that the changes he observed in the guinea pig and rabbit after the injection of extract were specific or easily reproducible. The principal effects he described were in the zona fasciculata and consisted of the loss of lipoids and cellular hypertrophy affecting both the protoplasm and the nuclei.

The effects of extracts in hypophysectomized animals are more easily analyzed, because normally secreted corticalstimulating hormone is not an unknown and therefore con- 


\section{THE PITUITARY BODY}

fusing factor. ${ }^{2}$ Davidson (I937) produced a marked hypertrophy of the adrenal glands in hypophysectomized rats by injecting anterior pituitary extract. He concluded that the cortical enlargement was due to cellular hypertrophy, not hyperplasia. 'The histological appearance of the cortical lipoids has received the special attention of Reiss and others (1936) and of Moon (1937). In normal rats there exists a narrow band of tissue between the zone fasciculata and the zona glomerulosa, which histologically appears to contain little or no lipoid. Deposition of lipoids in the cells of this band is one of the earliest signs of a cortical-stimulating effect (Moon). After hypophysectomy the outer part of the zona fasciculata ${ }^{3}$ rapidly loses its lipoids, which are specifically restored by the injection of pituitary extract containing adrenal cortical stimulating hormone (Reiss and others; Moon).

The development of the "X-zone" of the mouse adrenal, located as a central fringe of the cortex, appears to depend on sex, inasmuch as it is found in immature or young female mice, whereas it fails to develop in male mice unless castration is performed. Deanesly (I938) suggested that the development of the $\mathrm{X}$-zone probably depends upon a secretion of the anterior pituitary. She found that the castration of mice with hereditary dwarfism and with partial pituitary deficiency is not followed by the development of an $\mathrm{X}$-zone.

${ }^{2}$ Cutuly (1936) studied quantitatively the morphology of the adrenal glands of rats 30 days after hypophysectomy. At death, the male rats weighed an average of $166 \mathrm{gm}$., the female rats, $139 \mathrm{gm}$. Atrophy appeared to be due solely to cortical shrinkage and was relatively greater in female rats. His results were as follows (the figures refer to the mean of the calculated weights in milligrams):

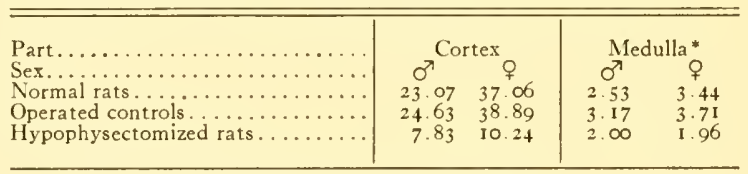

* Differences in weight not statistically significant.

3 Moon's Fig. 3 indicates that this does not represent a widening of the lipoidfree band of normal animals. 
Lacassagne and Raynaud (1937) stated that, although oestrone or oestradiol do not disturb the development of the $\mathrm{X}$-zone, these oestrogens do cause regressive changes in the adrenals, e.g., loss of lipoids. The regressive changes were attributed to an interference with pituitary secretion.

Several reports on the morphology of the adrenal cortex in relation to the pars glandularis also mention the medulla. In Cutuly's quantitative morphological study (1936) of the adrenals of normal and hypophysectomized rats of both sexes the mean weight of the medulla of operated rats was less than that of control animals. However, the difference was not statistically significant. Davidson (1937) stated that after the injection of adrenal cortical stimulating extract into hypophysectomized rats the medulla appeared more normal. Specifically, he mentioned the absence of vacuoles, which sometimes appeared in the adrenal medulla of operated, non-treated rats. Normal rats received daily injections of a crude anterior pituitary extract for as long as 9 months in the experiments of Bierring (1935). He reported that the medulla appeared hyperplastic and that enlarged chromaffin cells were crowded with granules. The changes observed by this author are the reverse of those attributed by Anselmino and Hoffmann to medulla-stimulating ("adrenotropic") hormone. The observations do not offer convincing evidence that the pars glandularis has an important influence on the morphology of the adrenal medulla.

Physiological and pharmacological correlations.-Grollman and Firor (1935) used several methods to produce chronic adrenal insufficiency in rats, cats, and dogs. They concluded that if no attempt was made to treat the cortical deficiency for a considerable time, adrenal cortical extract could not cause a resumption of growth, a reappearance of normal sexual performance, or the maintenance of a normal body-temperature. The lack of benefit of adrenal cortical hormone under such conditions was attributed to irreparable damage of the pars glandularis; for anterior pituitary extract repaired 


\section{THE PITUITARY BODY}

the defects. Early treatment by cortical hormone prevented pituitary damage, i.e., the defects of growth, gonadal function, and metabolism did not appear. In the experiments of Gaunt, Remington, and Schweizer the metabolism of water after the intraperitoneal injection of isotonic glucose solution was investigated. At least in respect of the ease with which "water intoxication" was produced or ameliorated or prevented by cortical extract, it appeared that hypophysectomized rats suffered from a deficiency of adrenal cortical hormone.

All recent work indicates that thyroidectomy interferes neither with the release of adrenal cortical stimulating hormone from the pituitary nor with the peripheral action of the hormone. Cortical-stimulating hormone produces about the same adrenal hypertrophy and increased accumulation of osmiophilic material in the cortical cells of tadpoles, whether or not the thyroid is present (Atwell, 1937). Thyroidectomy appears not to affect the adrenal-stimulating effects of anterior pituitary extract in guinea pigs (Jores and Boecker, 1937). ${ }^{4}$ Compensatory hypertrophy of an adrenal gland after the removal of its mate depends upon the liberation of adrenal cortical stimulating hormone from the pituitary; however, thyroidectomy does not interfere with this phenomenon in the male rat (Winter and Emery, 1936). ${ }^{5}$

4 These authors found that adrenal hypertrophy following the administration of thy roxine was associated with a loss of lipoids from the cortex. This effect, of course, is the reverse of that considered to be typical of adrenal cortical stimulating hormone.

5 These authors found that gonadectomy does not interfere with compensatory adrenal hypertrophy in rats of either sex. If both adrenals are intact, castration is followed by adrenal hypertrophy, spaying by adrenal atrophy.

Elmer, Giedosz, and Scheps (I937) concluded that thyrotropic hormone played the important part in the adrenal hypertrophy which follows the administration of acid extract of the anterior pituitary, because the effect was prevented by the administration of jodide. Friedgood (I936) also administered iodides and alkaline anterior pituitary extract to guinea pigs and found that adrenal hypertrophy and splenomegaly were less pronounced or even absent. However, Friedgood did not feel that the $h$ pothes is that thyrotropic hormone is responsible for the corticalstimulating effects of pituitary extract is warranted. 
Perla (1935-36) and Perla and Rosen (1935) have reported new experiments indicating that the increased toxicity of histamine in hypophysectomized rats is due to a deficiency of adrenal cortical hormone resulting from the absence of cortical-stimulating hormone. According to Selye and Collip (1936), large doses of oestrone or the administration of formaldehyde bring about hypertrophy of the adrenal cortex of the rat. These changes appear to be due to the increased liberation of adrenal cortical stimulating hormone, because they cannot be produced in hypophysectomized rats.

Some aspects of the metabolism of adrenal cortical stimulating hormone.- Several authors have shown that the pars glandularis must be intact if compensatory hypertrophy of an adrenal is to take place after the other has been removed. Reiss, Bálint, and Aronson ( I 936) found that compensatory hypertrophy amounts to about 95 per cent if partial hypophysectomy has been performed, whereas in normal animals the compensatory hypertrophy is only 20 per cent. Apparently operative trauma is followed by changes facilitating the formation or liberation of adrenal cortical stimulating hormone. The adrenal cortices of the female rat are considerably larger than those of the male. This suggests that the female pituitary liberates more cortical-stimulating hormone than the male. This hypothesis was tested by Wyman and tum Suden (1937) by determining the survival of homotransplants. Regeneration of such transplants occurred in 7 I per cent of female recipients but in only 20 per cent of male recipients. The sex of the donor appeared not to affect the results. These authors also showed (1937) that the successful transplantation of the adrenal requires pituitary secretion. The adrenal of the hypophysectomized rat can be successfully transplanted into a normal rat. Regeneration of a transplant is particularly favored by adrenal insufficiency (Wyman and tum Suden, Ingle and Higgins), probably because the rate of formation and liberation of cortical-stimu- 


\section{THE PITUITARY BODY}

lating hormone is accelerated. ${ }^{6}$ On the other hand, the rate of secretion of cortical-stimulating hormone is so diminished as a result of the administration of adrenal cortical extract that cortical atrophy appears but can be prevented, if cortical-stimulating extract be simultaneously administered (Ingle and Kendall, i 937).

In human beings with increased intracranial tension, usually the result of primary cerebral tumors, Kraus (1937) observed a hyperplasia of the pars glandularis associated with a similar change in the cortex of the adrenal glands, including an increased accumulation of lipoids. ${ }^{7}$ Kraus believed that the cortical hyperplasia was due to an increased secretion of adrenal cortical stimulating hormone by the anterior pituitary and described the phenomena as "corticotropic hyperpituitarism." He pointed out that cortical atrophy occurs in the pituitary cachexia of Simmonds. The reports of Giordano and Zeglio (1936) were available only in the form of abstracts. These authors reported that adrenal cortical stimulating hormone, recognized by its effect on the adrenal of the guinea pig, can be extracted from the urine of patients with hypertension. They believed that effects of urinary extracts on the adrenal medulla could be detected but were less important.

The chemistry and assay of adrenal cortical stimulating hormone.-Little is known concerning the chemistry of corticalstimulating hormone. Although incidental observations on the extraction and some properties of potent extracts are mentioned in several reports, it appears that little is to be gained from a review at this time. One method of extraction which should be cited is that of Moon (1937), who reported that by Lyons' method of preparing lactogenic extract the material which is insoluble at $\mathrm{pH} 6.5$ is rich in cortical-stimulating hormone. Extract rich in lactogenic hormone is, by this method of extraction, least soluble at $\mathrm{pH} 5 \cdot 5$.

${ }^{6}$ See also McQueen-Williams (1935).

2 The weight of the adrenal glands was $30-40$ per cent greater than normal. 
The methods which have been used for the assay of adrenal cortical stimulating extract have received no careful quantitative study. Unquestionably, hypophysectomized animals should be used, if there is to be the least possible doubt as to the specificity of the effects. Reiss and others (1936), Collip (1937), and Moon (1937) have all used hypophysectomized rats for assay and usually have relied upon both the gross change-hypertrophy-and microscopic evidences of repair-especially the reappearance of lipoids distributed generally. Methods based upon the use of normal mice or rats have been described by Jores and Beck (1936) and by Moon (1 937).

THE METABOLISM OF CARBOHYDRATES IN RELATION

TO THE PARS GLANDULARIS

Great interest in the importance of the pituitary body in carbohydrate metabolism was aroused by Houssay and Biasotti, who discovered that the course of diabetes following pancreatectomy in the dog is greatly ameliorated by removal of the hypophysis. Without doubt the extirpation of the pars glandularis is responsible for this change. The problem, in general terms, is: How and to what extent is the metabolism of carbohydrates regulated by the pars glandularis? Numerous aspects of this problem have been studied, especially since I934. However, it will be seen that our knowledge of the mechanisms in operation is still regrettably limited.

The metabolism of carbohydrates after hypophysectomy.Previous work had demonstrated several important changes consequent to hypophysectomy-i.e., the blood sugar of fasting animals falls to abnormally low levels; the sensitivity toward insulin is greatly increased; the regulation both of the absorption of glucose and of the formation and degradation of glycogen is disturbed.

If animals are fed adequate amounts of carbohydrate, the concentration of sugar in the blood is not strikingly different 
in hypophysectomized animals from that in normal animals. Shortly after food is withheld, however, a marked hypoglycemia appears in successfully operated animals. For example, Russell (1936) found that the concentration of sugar in the blood of young male rats, 3-4 weeks after hypophysectomy, was reduced approximately 50 per cent after a fast of 8 hours, whereas the reduction occurring in normal rats was only 20 per cent. If the fast was continued for 10 hours longer (total i 8 hours) a greater change then occurred in normal

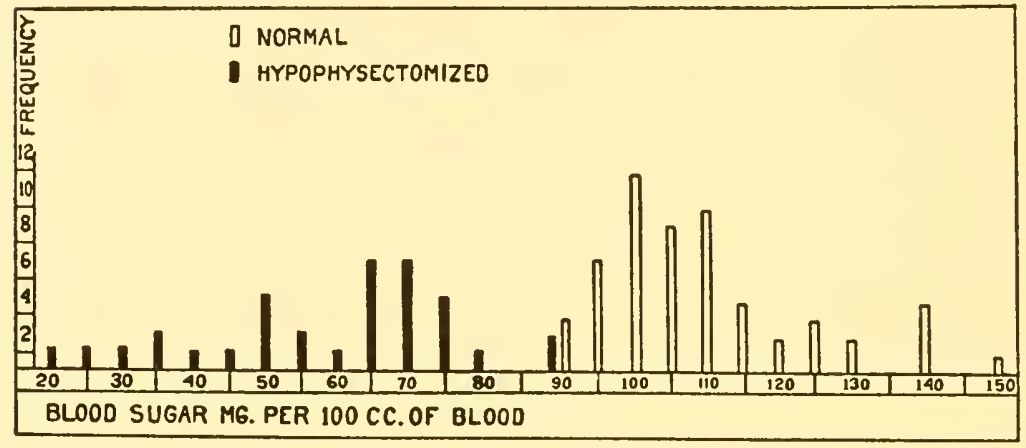

F1G. 23.-The concentrations of sugar in the blood of normal and hypophysectomized monkeys in relation to their frequency. The monkeys were first starved 1618 hours. (From Smith, Dotti, Tyndale, and Engle, Proc. Soc. Exp. Biol. Med., 34, $247-49$ [1936].)

rats (total reduction, 32 per cent) than in hypophysectomized animals (total reduction, 54 per cent). The results of the withdrawal of food are similar in the rabbit (Cope, 1937) and monkey (Smith and others, 1936). Figure 23 illustrates the distribution of concentrations of sugar in the blood of normal and hypophysectomized monkeys, as determined by Smith, Dotti, Tyndale, and Engle after the animals had been fasted for 16-18 hours. The concentration of sugar in the blood of partially hypophysectomized monkeys was found to lie $\left(87 \pm 2.7^{8} \mathrm{mg}\right.$. per cent) between that of normal monkeys ( $110 \pm 2.0^{8} \mathrm{mg}$. per cent) and that of hypophysec-

8 Standard error of the mean. 
tomized monkeys $\left(59 \pm 3.1^{8} \mathrm{mg}\right.$. per cent $) .{ }^{9}$ The reasons for the precipitate diminution of the sugar of the blood of hypophysectomized animals as a result of fasting will be discussed later.

The rate at which glucose is absorbed from the digestive tract by hypophysectomized animals is abnormally slow, as was first demonstrated by Phillips and Robb in rats. Bennett (1936), Fisher and Pencharz (1936), Russell (1937), and Russell and Bennett (I937) all used rats and agree with the conclusion of Phillips and Robb. According to Bennett, the amount of glucose absorbed in an arbitrary period is about 35 per cent less in hypophysectomized than in normal rats. Russell and Cori (I937) reported that the tolerance for intravenously injected glucose is reduced in hypophysectomized rats to the same extent that oxygen-consumption suffers a reduction. ${ }^{\text {Io }}$ The renal threshold of glucose was found to be abnormally high in operated rats. Russell and Bennett found that as early as 24 hours after hypophysectomy, ${ }^{11}$ fasting is followed by an abnormally rapid decrease in the concentration of glucose in the blood and of glycogen in the liver and striated muscle. If such animals are then fed, the return of these carbohydrates to normal levels is abnormally slow, principally because the reserves are abnormally low and because the absorption of glucose is abnormally slow.

${ }^{9}$ Scott (1937) investigated the concentration of lactic acid in the blood of normal and hypophysectomized monkeys and obtained high values, which she attributed to muscular activity which was beyond her control. Blood obtained from the heart of normal monkeys contained $10_{4} \pm 6.5 \mathrm{mg}$. per cent of lactic acid, whereas similar blood of hypophysectomized monkeys contained $37 \pm 4.5 \mathrm{mg}$. per cent.

See the article of Marenzi (1936) for an account of the changes in, and the effects of pituitary (extracts) on, the following constituents of striated muscle of the toad after hypophysectomy: glycogen, inorganic P, phosphocreatine, glutathione, and lactic acid.

ro There are few new data on changes in glucose-tolerance as a result of hypophysectomy. For example, Slome (1936) found that glucose-tolerance is increased after hypophysectomy in the toad, Xenopus laevis. There are other references in the text.

II Inanition or brain injury or the absence of the pars neuralis was not responsible for the change. 
After hypophysectomy, rats continue to oxidize glucose readily. In fact, when such animals depend upon their own reserves of foodstuffs, as in fasting, they depend to an unusual extent on carbohydrate-oxidation for their energy requirements (Fisher and Pencharz, 1936). Fisher and Pencharz also found that if hypophysectomized rats are fed glucose, they oxidize more carbohydrate to supply energy than do normal rats, despite the fact that the rate of oxygenconsumption and of absorption of glucose is lower after hypophysectomy. ${ }^{12}$ A high-fat diet prior to glucose feeding appeared to lessen the oxidation of glucose only slightly. Greeley (1935) attempted to determine the rate of utilization of glucose by hypophysectomized rabbits at the time of maximum need during fasting. He considered that such a need occurred 5-8 hours after a drop in blood sugar appeared ( $\mathrm{I}-32$ hours of fast) in fasting hypophysectomized rabbits. He concluded that at least $0.5^{0-0.67} \mathrm{gm}$. of glucose per $\mathrm{kg}$. body-weight per hour is required by an intravenous route, if the blood sugar is to be maintained near (usually below) the normal level. Russell ( I 937) investigated the action of thyroxine on the metabolism of glucose by hypophysectomized rats. From comparisons with operated animals receiving glucose alone, she concluded that thyroxine causes an increase in the rate of absorption of glucose and in the rate of oxidation of glucose, roughly proportional to the increase in oxygen consumption caused by the hormone.

According to Weichselbaum, Heinbecker, and Somogyi (I937), tolerance toward glucose is improved in hypophysectomized dogs by a high-carbohydrate diet compared with a high-fat diet. The diets did not uniformly cause this sort of a difference in normal dogs. These authors also found (Somogyi, Weichselbaum, and Heinbecker, 1937) that hyperglycemia following hypoglycemia due to the administration

${ }_{12}$ During the first 4 hours after the feeding of glucose, normal rats might oxidize $\mathrm{I}+4 \mathrm{mg}$. of the sugar per $100 \mathrm{gm}$. body-weight in comparison with $\mathrm{Ig} \mathrm{mg}$. per $100 \mathrm{gm}$. body-weight in hypophysectomized rats. 
of either glucose or insulin appeared both in normal and in hypophysectomized dogs.

Abnormalities in the deposition and degradation of glycogen in hypophysectomized animals have already been referred to briefly. Bennett (1936) as well as Russell (1936) determined the concentration of sugar in the blood and of glycogen in the liver and striated muscle of normal and $h y-$ pophysectomized rats under various conditions. After a fast of 8 hours, the operated rats metabolized much more of the glycogen of the liver (95 per cent compared with 27 per cent in normal rats) and of striated muscle $(24$ per cent compared with 8 per cent). If the fast was prolonged to 18 hours, both normal and operated animals had consumed most of the hepatic glycogen, whereas a further loss of muscle glycogen occurred only in hypophysectomized rats. These observations were made by Russell, who also reported that the chief abnormality found in hypophysectomized rats fed 2 gm. of starch within 8 hours after a fast of 18 hours was a low concentration of glycogen in the liver. Likewise, after the feeding of glucose, partly because of slow absorption, hypophysectomized rats restore the glycogen of the liver and striated muscle only slightly (Bennett). Bennett's results are summarized in Table 6 . His calculations of the proportion of absorbed glucose converted into glycogen indicated that the percentage so converted is much higher in normal animals-especially in respect of hepatic glycogen. Cope ( 1937 ) has reported further experiments in rabbits from which he concluded that hypophysectomy interferes with the endogenous formation of carbohydrate and that when hepatic glycogen is exhausted, the concentration of sugar in the blood rapidly falls to levels associated with the onset of convulsions so that the animal, in this respect, behaves like a hepatectomized animal. In the hypophysectomized animal the hepatic glycogen appears to be only of exogenous origin. According to Soskin and others (1935), the hypophysectomized dog, 
unlike the normal, cannot form carbohydrate from fat. ${ }^{{ }^{3}}$ Ketonuria does not appear readily in hypophysectomizeddepancreatized dogs even in the presence of hyperglycemia and glycosuria. The authors concluded that glucose can be endogenously derived only from carbohydrate and protein after hypophysectomy. Crandall and Cherry (1 937) likewise

TABLE 6

\section{Carbohydrate Metabolism in Normal and in HyPophysectomized RATS*}

\begin{tabular}{|c|c|c|c|c|}
\hline \multirow{3}{*}{ All. Rats Fasted for 24 Hours } & \multirow{3}{*}{\begin{tabular}{|c|} 
Gl,UCOSE \\
ABSORBED \\
Mg.per 100 \\
Gm. Body- \\
Weight in \\
2 Hours
\end{tabular}} & \multirow{3}{*}{$\begin{array}{c}\begin{array}{c}\text { Glucose } \\
\text { IN BLOOD }\end{array} \\
\begin{array}{c}\text { Mg. Per } \\
\text { Cent }\end{array}\end{array}$} & \multicolumn{2}{|c|}{ Glycogen in } \\
\hline & & & Liver & $\begin{array}{l}\text { Striated } \\
\text { Muscle }\end{array}$ \\
\hline & & & $\underset{\text { Cent }}{\mathrm{Mg} . \mathrm{Per}}$ & $\underset{\text { Cent }}{\text { Mg. Per }}$ \\
\hline 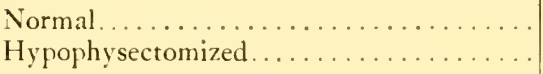 & & $\begin{array}{l}80 \\
51\end{array}$ & $\begin{array}{l}23 \\
15\end{array}$ & $\begin{array}{l}502 \\
322\end{array}$ \\
\hline 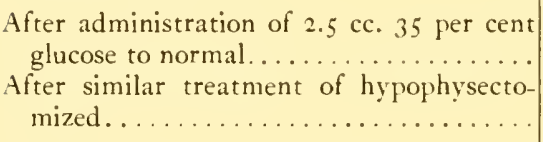 & 412 & I 40 & $\begin{array}{r}, 348 \\
194\end{array}$ & $\begin{array}{l}673 \\
378\end{array}$ \\
\hline
\end{tabular}

*From Bennett, Proc. Soc. Fxp. Biol. Med., 34, 277-79 (1936).

performed experiments with dogs and believed that their results indicated that the formation of glucose from one aminoacid, glycine, is not prevented by hypophysectomy. The technic of their experiments is described in the section dealing with the effects of insulin. ${ }^{14}$

The recent experimental work so far reviewed, as well as

${ }_{13}$ However, even in normal dogs, it is not generally believed that proof of the formation of carbohydrate from fat, other than glycerol, is available.

${ }^{14}$ According to Schott, Samuels, and Ball (1937), the Walker tumor No. 256 in male rats contains significantly more glyocgen (but grows more slowly) in hypophysectomized than in normal rats. The amount of glycogen in the tumor of rats was: (I) + hours after feeding: normal, 0.026 per cent; hypophysectomized, 0.046 per cent, and (2) on high caloric diet: normal, 0.058 per cent; hypophysectomized, 0.186 per cent. 
previous work, indicates that hypophysectomy leads to the following changes in the metabolism of carbohydrates. ${ }^{15}$

I. The rate of absorption of glucose is abnormally slow.

2. Fasting leads to an abnormally rapid utilization of glycogen both of the liver and of striated muscle. The more labile hepatic glycogen is more strikingly depleted. In the opinion of Fisher, Russell, and Cori (1937), a secretion of the pars glandularis regulates the (formation and) utilization of glycogen in fasting animals and spares carbohydrates so that fat and protein are utilized to a greater extent as sources of energy. This regulatory influence is lost after removal of the gland, so that carbohydrate oxidation continues at an abnormally rapid rate until even the concentration of sugar in the blood falls to low levels.

3. A further metabolic abnormality probably is an interference with gluconeogenesis from non-carbohydrate sources. It appears that the glycerol of fats cannot be used to form carbohydrate in hypophysectomized animals. The degree of disturbance of gluconeogenesis from proteins is not known with any accuracy. Apparently glycine can be utilized. Also, Fisher, Russell, and Cori found that the excretion of nitrogen in the urine is not affected by hypophysectomy. However, the $\mathrm{G} / \mathrm{N}$ ratio of hypophysectomized dogs receiving phlorhizin is lower than normal and the quantity of $\mathrm{N}$ excreted is less (Houssay and others), which suggest a lowered rate of formation of glucose from protein.

4. All the foregoing changes help to explain the slow rate at which carbohydrate reserves are replenished when food is again furnished to fasting hypophysectomized animals. Absorption is slow; the reserves are low or, in the case of the liver, may be virtually exhausted; the animal continues to depend to an abnormal extent on carbohydrate-oxidation as a source of energy; and, finally, carbohydrate from non-carbohydrate sources can be secured only to a limited extent.

\section{The effects of epinephrine in hypophysectomized animals.-} So far as carbohydrate metabolism in normal animals is concerned, epinephrine appears to produce the following changes:

I. A hyperglycemia appears and persists. The increased concentration of blood sugar is partly due to increased hepatic glycogenolysis and partly due to diminished utilization of sugar by the tissues.

2. Simultaneously epinephrine promotes the formation of glycogen in the liver from lactic acid, the formation of which depends upon the degradation of muscle glycogen. In the formation of lactic acid from muscle glycogen, hexosemonophosphate must be formed; phosphate is therefore mobilized, and the concentration of inorganic $\mathrm{P}$ in the blood falls.

${ }_{5}$ The most numerous experiments have been performed with rats in which, as Fisher, Russell, and Cori emphasize, experimental procedures such as fasting may produce changes different from those observed in larger animals. 
Therefore, the injection of epinephrine produces a hyperglycemia, a fall in the hepatic glycogen (which is restored later), a fall in the glycogen but an increase in the hexosephosphate of striated muscle, a fall in the concentration of inorganic $\mathrm{P}$ in the blood, and a change in the reverse direction of lactic acid. It is proposed to examine, in the light of new work, how these effects are modified by hypophysectomy.

Years ago Aschner learned that the subcutaneous injection of epinephrine produces glycosuria in hypophysectomized

TABLE 7

\begin{tabular}{|c|c|c|}
\hline & \multicolumn{2}{|c|}{ Change as Mg. Per Cent in } \\
\hline & $\begin{array}{c}\text { Normal } \\
\text { Rats }\end{array}$ & $\begin{array}{l}\text { Hypophysecto- } \\
\text { mized Rats }\end{array}$ \\
\hline 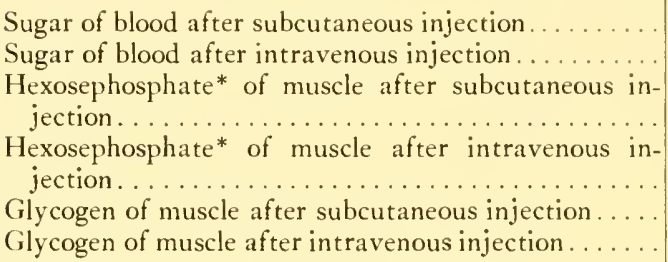 & $\begin{array}{l}+103 \\
+\quad 68 \\
+\quad 43 \\
+\quad 53 \\
-182 \\
-130\end{array}$ & $\begin{array}{l}+38 \\
+\quad 78 \\
+\quad 13 \\
+\quad 70 \\
-\quad 56 \\
-158\end{array}$ \\
\hline
\end{tabular}

* The figures refer to the concentration of hexose.

dogs much less readily than in normal animals. This observation applies with equal force to the behavior of the sugar in the blood and has been extended to other mammals. Deficient absorption as an important factor in explaining the small response was not considered experimentally until Russell and Cori (1937) undertook their work in rats. These authors observed changes after the injection of epinephrine into anesthetized rats as shown in Table 7. This brief summary of the results indicates that the delayed absorption of epinephrine from the subcutaneous tissues is of great importance in accounting for the apparent insensitivity of hy- 
pophysectomized animals toward changes in carbohydrate metabolism caused by epinephrine. ${ }^{16}$

In all other work epinephrine was administered subcutaneously. Obviously, then, if observations were made in wellfed animals, differences in the reponse of normal and hypophysectomized animals cannot be attributed to a fundamental change in the action of epinephrine, unless the factor of deficient absorption has been ruled out. Cope (1937) and Cope and Thompson (I 9.37) used rabbits. They believed that storage of hepatic glycogen does not follow the subcutaneous injection of epinephrine, but they did find in a different series of animals that the hormone causes an increase in the concentration of lactic acid in the blood comparable to that produced before hypophysectomy. (It appeared that less glycogen was formed by the liver from intravenously injected lactate as a result of hypophysectomy.) The lactic acid of the blood of hypophysectomized rats, which were fasted but received no epinephrine, began to rise when the concentration of sugar in the blood fell below $40 \mathrm{mg}$. per cent. Cope and Thompson believed that hypophysectomy does not affect the mobilization of the glycogen of muscle. Chaikoff and others (I 935) used dogs for their experiments. They injected epinephrine subcutaneously and concluded that the response of hypophysectomized animals - in respect of increase in the concentration of sugar and lactic acid in the blood and of decrease in inorganic $\mathrm{P}$-is indefinitely smaller than that of

${ }^{16}$ Heinbecker and Weichselbaum (1937) found that the intraperitoneal injection of epinephrine provokes hyperglycemia with equal effectiveness in normal and hypophysectomized dogs, provided that the nutritional condition is good.

Collip, Thomson, and Toby (1936) injected epinephrine subcutaneously and concluded that, as a result of hypophysectomy, hyperglycemia and reduction of muscle glycogen are prevented but that restoration of the effects appears if anterior pituitary extract be injected. The authors used rats. Bachman and Toby (1936) reported that hypophysectomy interferes with the mobilization of glycogen in muscle caused by the injection of epinephrine into rabbits. These authors injected the hormone subcutaneously but sometimes observed a satisfactory hyperglycemic response, if the liver contained an adequate amount of glycogen. 


\section{THE PITUITARY BODY}

normal animals or of animals subjected to all the operative procedures except hypophysectomy. ${ }^{17}$

The effects of insulin in hypophysectomized animals.-It has long been known that, as a result of the extirpation of the pituitary body, sensitivity toward insulin is greatly increased. The change is due to the removal of the pars glandularis and not to removal of the pars neuralis (see the recent articles of Pencharz, Cori, and Russell, I 936; and of Karlik, I936). However, Chaikoff, Reichert, Larson, and Mathes (1935) showed that retraction of the right temporal lobe of the dog (after craniotomy and incision of the dura mater) alone was sufficient frequently to increase insulin-sensitivity even 3 months after operation. There occurred in some animals as much fall in the concentration of sugar and inorganic $\mathrm{P}$ in the blood as in hypophysectomized animals. In the insulin-sensitive animals, in which operation had been carried only to the stage of temporal-lobe retraction, the pituitary appeared normal; however, the authors suggest that an unrecognized injury of the region about the pituitary had occurred. The quantitative studies of Heinbecker, Somogyi, and Weichselbaum (I937) indicated that in the dog insulinsensitivity is approximately doubled 4 weeks after hypophysectomy and, after a year, is quadrupled.

From the discussion of the metabolism of carbohydrates in hypophysectomized animals it is clear that such animals tend rapidly to use up all available carbohydrate reserves when fasting is imposed, whereas in normal animals some carbohydrate is spared and more fat and protein are oxidized. These facts alone would justify the expectation that the blood sugar would fall to lower levels and that shock would be more easily induced in hypophysectomized animals receiving insulin. Kater (1936) reported new experiments on the insulin-sensitivity of hypophysectomized rats. Whereas

${ }_{17}$ According to Di Benedetto and Di Benedetto (1935), hyperglycemia caused by ether is less in hypophysectomized dogs than in control aimals. However, this would be expected if carbohydrate reserves were low as a result of hypophysectomy. Hyperglycemia caused by morphine ( $30 \mathrm{mg}$. per $\mathrm{kg}$.) appeared not to be affected. 
after the injection of $\mathrm{I}-5$ (?) units of insulin into normal fasting rats, there appeared to be no other change except a fall in the concentration of sugar in the blood from $90 \mathrm{mg}$. per cent to $60-70 \mathrm{mg}$. per cent, the injection of 0.05 unit into fasting hypophysectomized rats depressed the level of the blood sugar to $30-40 \mathrm{mg}$. per cent, with which were associated myasthenia, rapid, shallow respiration, and coma often with convulsions. The injection of I cc. of 20 per cent glucose I 5 minutes after the administration of insulin maintained the level of the blood sugar above normal; the rats appeared normal and did not lapse into coma-yet they usually died within about 5 hours. Smith and others (1936) tested the insulin-sensitivity of normal, partially hypophysectomized, and completely hypophysectomized monkeys which first underwent a fast of I 6 hours. Thirty minutes after the injection of 0.06 unit of insulin per kilogram body-weight, the following changes were observed in the concentration of sugar in the blood of the heart (the figures refer to milligrams per cent; the pre-injection concentration is given first): normal, I I 5 then 99; partially hypophysectomized, 78 then 66; completely hypophysectomized, 57 then 37 . According to Crandall and Cherry (1937), who performed their experiments in dogs, either hypophysectomy or denervation of the adrenals - i.e., excision of one adrenal and splanchnotomy on the other side-prevents an increased liberation of glucose from the liver after the injection of insulin. The authors secured blood from the hepatic and portal veins and arterial blood without recourse to anesthesia.

The effect of diet on the response of normal and hypophysectomized dogs to insulin was studied by Heinbecker, Somogyi, and Weichselbaum (I937). In operated, but not in normal, dogs the response to insulin was uniformly better if the animals were fed a high-carbohydrate, low-fat diet in comparison with a diet low in carbohydrate and high in fat. On the other hand, Himsworth and Scott found that normal rabbits, on a diet low in carbohydrate, exhibited a disturbed 
sugar-tolerance and a diminished sensitivity toward insulin. Diet was without effect in hypophysectomized rabbits which, irrespective of food high or low in carbohydrate, were found to possess a high sugar-tolerance and an increased insulinsensitivity. However, anterior pituitary extract altered the response of hypophysectomized rabbits to a high-carbohydrate diet, so that it resembled that of normal animals on a low-carbohydrate diet. Cope (1937) was interested in the effect of hypophysectomy on the rabbit's ability to store glycogen in the liver. There seemed to be no interference with this process after the intravenous injection of glucose into fasting animals. However, unlike normal animals receiving no food for $4^{8}$ hours, hypophysectomized rabbits stored no glycogen in the liver after the injection either of insulin or of epinephrine. After the intravenous injection of lactate, less glycogen was stored by hypophysectomized rabbits than by normal rabbits.

The interrelationship of the pars glandularis and pancreas as revealed by extirpation experiments. ${ }^{18}$ - It is well known that Houssay and Biasotti first demonstrated that the course of diabetes mellitus due to pancreatectomy is greatly ameliorated by the removal of the pars glandularis. This observation has been confirmed by a number of investigators who have added new data on the manner in which hypophy-

${ }^{18}$ According to Krichesky (1936), the volume of islet tissue in the pancreas of the rat increases after hypophysectomy. The increase in the volume of islet tissue perhaps was less (in terms of body-weight), if hypophysectomized rats were given injections of anterior pituitary extract. Hypophysectomy was performed 30-60 days before observations were made.

Fichera and Aldoni (1937) studied the pituitary body of depancreatized cats. The pituitary was hypertrophied (20.80 mg. per $\mathrm{kg}$. body-weight compared with $9.69 \mathrm{mg}$. per $\mathrm{kg}$. body-weight in normal cats). Histological changes were believed to be a marked reduction or even a disappearance of oxyphils and a pronounced increase of the percentage of reserve cells.

The observations of Jacobs and Colwell (1936) are believed to have a bearing on the subject matter of this chapter. These authors infused glucose solution intravenously into non-anesthetized dogs until death occurred as a result of a non-ketogenic acidosis. There was congestion of all the tissues; however, specific, marked hemorrhage and evidences of destruction occurred in the pars glandularis and pancreas. 
sectomy modifies pancreatic diabetes. So far as the toad is concerned, Slome (1936) confirmed in another species, Xenopus laevis, observations of Houssay and Biasotti who used Bufo arenarum. If hypophysectomy be performed first and the pancreas be removed later, the concentration of sugar in the blood of Xenopus does not rise above about $40 \mathrm{mg}$. per cent about $\mathrm{I} 2$ hours after the second operation, whereas after pancreatectomy alone it reaches a level of about $230 \mathrm{mg}$. per cent under similar conditions. ${ }^{19}$

All the other observations were made for the purpose of studying different aspects of metabolism in hypophysectomized-depancreatized dogs ("Houssay dogs"). ${ }^{20}$ The metabolism of isolated tissues of hypophysectomized-depancreatized dogs was studied by Shorr, Richardson, and Sweet ( 1936) and by Fazekas, Campbell, and Himwich (1937). According to Shorr and his colleagues, the metabolism of excised skeletal muscle of doubly operated dogs is like that of normal dogs rather than that of depancreatized dogs, e.g., in capacity to utilize added lactate. On the other hand, Fazekas, Campbell, and Himwich concluded that the metabolism of isolated renal tissue of hypophysectomized-depancreatized dogs resembles that of depancreatized dogs, because oxidation of lactate but not of glucose takes place.

The experiments of Chambers, Sweet, and Chandler (1937) led them to conclude that relatively little carbohydrate can be oxidized by hypophysectomized-depancreatized dogs. If such dogs were on a diet of meat and carbohydrate, the postabsorptive respiratory quotient was only 0.76 . The injection of $50 \mathrm{gm}$. of glucose daily might or might not elevate the respiratory quotient $(+0.05)$; however $20-80$ per cent of the glucose administered appeared in the urine. Carbohydrate-

I9 Slome found that the level of the blood sugar of fasting normal toads was 35 mg. per cent in animals on a black background, and $26 \mathrm{mg}$. per cent in animals on a white background.

${ }^{20}$ Képinov (1936) reported that the blood of depancreatized dogs contains a substance producing hyperglycemia but that this substance cannot be detected in the blood if hypophysectomy has also been performed. 
balance studies indicated that doubly operated dogs might oxidize as much as $16 \mathrm{gm}$. of glucose per day. Despite the absence of insulin, hypophysectomized-depancreatized dogs can deposit glycogen in the liver-even in excess of 2 per cent (Chaikoff, Gibbs, Holtom, and Reichert, 1936). These authors also concluded that insulin is essential for the survival of the doubly operated dog but that survival is long if the animal is in an excellent nutritional condition at the time of operation. Chaikoff and his colleagues observed in hypophysectomized-depancreatized dogs all the changes characteristic of pancreatic diabetes, including ketonuria. However, Soskin and others (1935) denied that ketonuria can be detected in such animals.

Interrelationships of the pars glandularis and other glands of internal secretion in affecting carbohydrate metabolism. ${ }^{21}$ I. The adrenals.-Problems which have interested investigators in this complex field are illustrated by experiments which Houssay and Biasotti undertook in the toad, Bufo arenarum. They relied on quantitative determinations of the blood sugar to measure changes in carbohydrate metabolism. Some fall in the concentration of sugar in the blood appeared after the destruction of all adrenal tissue. Diabetes caused by pancreatectomy was less pronounced, in terms of the level of blood sugar, if either hypophysectomy or adrenalectomy also was performed. In either case, the administration of anterior pituitary but not of adrenal cortical extract caused a further elevation of the level of the blood sugar. The findings were similar if all three structures were removed. The authors suggest the possibility that adrenalectomy may favorably influence the course of diabetes because it lessens the

${ }^{21}$ It appears that for purposes of discussion there is still too little experimental basis for understanding the manner in which the central nervous system-especially the hypothalamus-affects carbohydrate metabolism, as this is related to the functional activity of the anterior pituitary, the adrenal medulla, and the pancreas. References to recent work in this field are the following: Davis, Cleveland, and Ingram (1935); Dawson and Milne (1935); Houssay (1935); Cleveland and Davis (1936); Ingram and Barris (1936); Lucke (1937). 
secretion of the anterior pituitary hormone affecting carbohydrate metabolism.

It is still the belief of Lucke (Lucke, I936; Lucke and Kröger, 1936) that the diabetogenic effects of anterior pituitary extract are due ultimately to an action on the adrenal glands - presumably because unusual amounts of epinephrine are liberated. This view appears to be untenable, because extract of the pars glandularis causes typical diabetogenic effects, including, of course, an elevation of the blood sugar after removal of all adrenal medullary tissue or bilateral splanchnotomy (Houssay and Leloir, 1935). However, Houssay and Leloir do agree that an immediate temporary rise in the concentration of sugar in the blood due to the injection of anterior pituitary extract requires the presence of adrenal tissue. ${ }^{22}$

Confusion is the chief result of considering reports on the effects of adrenal cortical extract or of extracts of the anterior pituitary with cortical-stimulating effects. Anselmino, Hoffmann, and Rhoden (1936) concluded that adrenal cortical extract prevented the loss of hepatic glycogen which otherwise occurred after the injection of an anterior pituitary extract into rats. Corey's experiments (1937), likewise in rats, were performed under different conditions. Adrenal cortical extract caused hyperglycemia in non-fasted hypophysectomized rats and in normal and hypophysectomized rats fasted for $4^{8}$ hours. ${ }^{23}$ In the latter group there was no apparent effect on the glycogen of the liver or muscle; however, the concentrations of glycogen before injection are not given

${ }^{22}$ Képinov (1936) concluded that the liberation of epinephrine from the adrenal in response to the injection of insulin is not disturbed by hypophysectomy in the dog. The liberated epinephrine, however, affected the level of blood sugar much less. The same author (1937) also believed that anterior pituitary extract or extract of liver or muscle restores epinephrine gly cogenolysis in the liver of the frog after a long perfusion. An obvious objection to this conclusion is the author's failure to estimate the lactic acid, glucose, or glycogen in his extracts, although extracts of liver or muscle of hypophysectomized animals were said not to contain the necessary substance.

${ }_{23}$ The extracts did not alter the blood pressure of cats anesthetized by "Amytal." 
but presumably were low. The observations of Bennett (1937) are difficult to evaluate. An extract of the anterior pituitary causing stimulation of the adrenal cortex was found to prevent a fall in the concentration of glycogen of muscle of hypophysectomized rats, whereas it did not affect hepatic glycogen or blood sugar. However, the effect was not attributed to adrenal cortical stimulating hormone. Treatment for longer periods (10-24 days) did cause an elevation both of the level of glucose in the blood and of the concentration of hepatic glycogen. The effects of repeated injections of the extract on the glucose of the blood and on the glycogen of the liver did not appear in "demedullated," hypophysectomized rats; but the author attributed this finding to cortical deficiency rather than to loss of medullary tissue.

Both the pituitary body and the adrenal glands have been removed from rats by Corey and Britton (1937) and by Samuels, Schott, and Ball (1937). Corey and Britton did not fast their rats and found that doubly operated animals, in comparison with hypophysectomized animals, were unable to maintain the levels of blood sugar $\left(6_{4} \mathrm{mg}\right.$. per cent compared with I2 I mg. per cent), muscle glycogen (0.24 per cent compared with 0.43 per cent), or hepatic glycogen ( 0.23 per cent compared with 0.94 per cent). According to Samuels and his colleagues, the double operation did not affect glucose tolerance (in comparison with the effect of hypophysectomy alone) but, not remarkably, did hasten death when fasting was instituted. Neither group of authors was in a position to state the relative importance of the cortex and medulla.

Long and Lukens ${ }^{24}$ have published further work on the amelioration of pancreatic diabetes by adrenalectomy both in cats and in dogs. The effects resemble those of hypophysectomy in depancreatized animals to such an extent that it is possible to raise the question of whether or not marked lower-

${ }_{24}^{4}$ Long (1935), Long and Lukens (1936), Long (1937), and Long, Lukens, and Dohan (1937). 
ing of adrenal function as a result of hypophysectomy accounts for the amelioration due to the latter operation. (Another possibility suggested by Long and Lukens is that adrenalectomy suppresses the secretion of diabetogenic hormone by the pars glandularis.) Insufficiency of epinephrine appeared not to be responsible for the prolonged survival of depancreatized-adrenalectomized cats. On the other hand, adequate doses of adrenal cortical extract or of Kendall's crystalline compound B prevent or restore, at least in rats, the marked loss of the glycogen of liver or muscle and the fall in the level of the blood glucose of fasting hypophysectomized animals (Long and Katzin, I938).

It is, of course, important that there be agreement as to the action or lack of action of anterior pituitary extract after complete adrenalectomy. Houssay and Leloir (I935) concluded that extract can cause a definite increase in the concentration of sugar in the blood of bilaterally adrenalectomized dogs. On the other hand, the more extensive experiments of Long and his associates in the cat and rat indicate that, in the absence of adrenal cortical tissue, glycosuria or an increase of glycosuria does not follow the injection of a potent extract. ${ }^{25}$

2. The effects of oestrogens.-Evidence that the injection of large doses of oestrogen prolongs the survival period and ameliorates the symptoms of pancreatic diabetes in the dog and monkey was offered several years ago. Recently, Nelson and Overholser (1936) published further details of their experiments in monkeys and concluded that secretion of the diabetogenic hormone by the pituitary is depressed, with consequent improvement of the symptoms appearing after pancreatectomy, if oestrogen in doses such as 300 rat-units of oestrone be injected daily. Unfortunately, their observations could not be confirmed by Collip, Selye, and Neufeld (I 937), who concluded that as much as I mg. of oestrin (oestrone?)

${ }^{25}$ See also Anselmino and Hoffmann (1936) and Russell (1936). 
daily affected neither the hyperglycemia nor the glycosuria due to pancreatectomy. Depancreatized monkeys survived even several months without insulin and irrespective of oestrin treatment. The depancreatized monkey resembled the dog or cat after removal of the pancreas and adrenals or of the pancreas and hypophysis.

The effects of extracts of the pars glandularis on the metabolism of carbohydrates.-Young's experiments in dogs (I937)

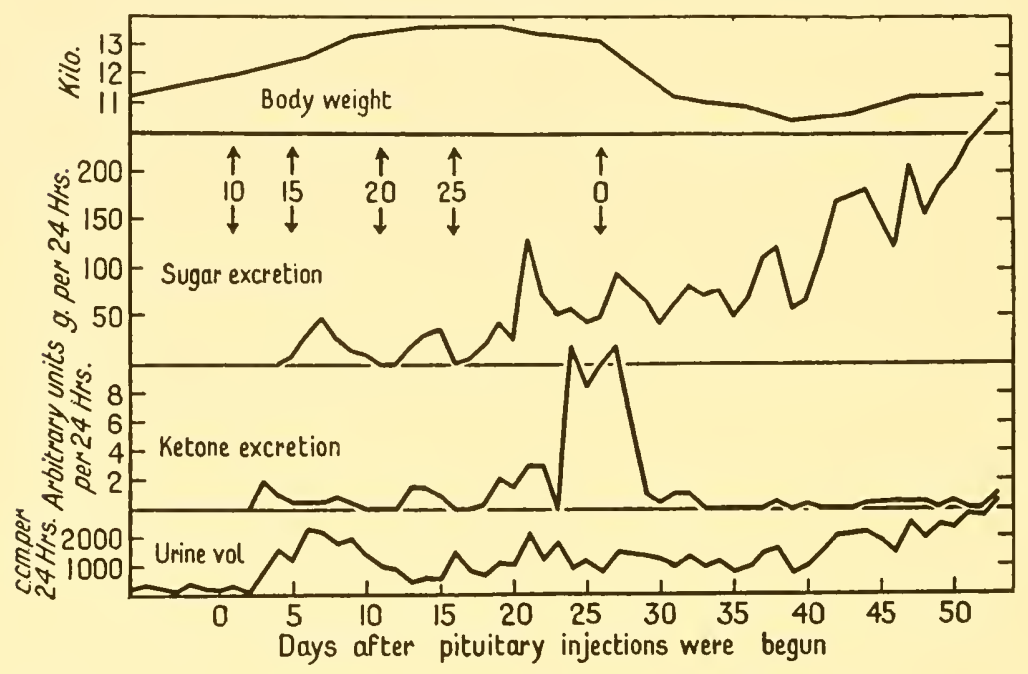

Fig. 24.-Diagram illustrating the production of a condition resembling diabetes mellitus by repeated injections of anterior pituitary into a dog. The numerals between arrows refer to the grams of fresh anterior pituitary of the ox used to prepare the extract injected daily until the next larger amount was used. Injections were stopped on the twenty-sixth day. (From Young, Lancet, 233, 372-74 [1937].)

indicate that anterior pituitary extract can be truly diabetogenic. Figures 24 and 25 are reproduced from his report. After repeated injections of anterior pituitary extract were stopped, a permanent diabetes mellitus appeared in 2 dogs. However, the animals remained vigorous without the administration of insulin, and in one there was no loss of weight. If insulin was injected to prevent almost entirely the excretion of glucose, it appeared that more was required (4.4 units 
per $\mathrm{kg}$. body-weight per day) than in the depancreatized dog. However, Young emphasizes that this is only his impression. Among other general observations are those of Etcheverry (I937) and Foglia and others (1937). Etcheverry concluded that bilateral supra-diaphragmatic vagotomy with or without removal of the abdominal sympathetics does not modify the course of diabetes caused either by pancreatec-

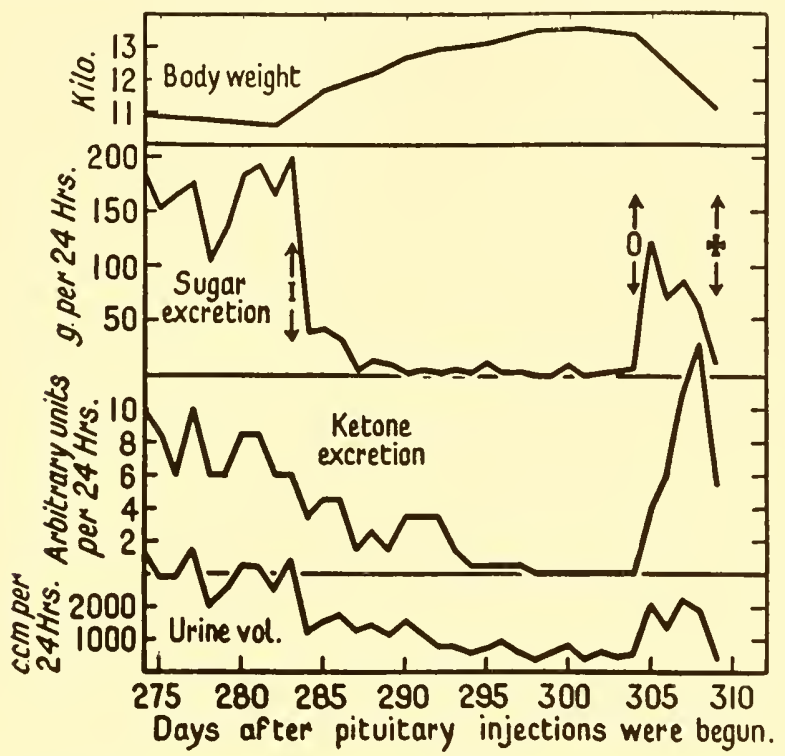

FIG. 25.-Continuation of Figure 24 after a long interval not shown in the diagrams. The daily injection of 60 units of insulin was begun at the first arrow and stopped at the second arrow. Death occurred on day 309. (From Young, Lancet, 233, 372-74 [1937].)

tomy or by the injection of anterior pituitary extract. Foglia and others injected crude anterior pituitary extract into dogs with diabetes following pancreatectomy. The extract shortened survival time, increased the formation of acetone bodies and the deposition of lipoids in the liver, and antagonized the action of insulin.

The effects of anterior pituitary extract in experiments of short duration were observed in normal and hypophysecto- 
mized rats by Fisher, Russell, and Cori (1936) and Russell (1936). To some groups of animals glucose was fed after fasting; the percentage which underwent oxidation was reduced. The respiratory quotient of fasted, hypophysectomized rats receiving injections tended to be lower, i.e., some carbohydrate was spared as is the case when a normal animal fasts. Also, less muscle glycogen disappeared. Russell concluded that the normal rat can deposit much more carbohydrate than the hypophysectomized animal, if anterior pituitary extract be injected after a fast but before the administration of glucose. These observations were extended by Meyer, Wade, and Cori (1937) who used normal rats. The animals were fasted 24 hours, after which they received anterior pituitary extract intraperitoneally. A short time later glucose was fed. The extract produced two principal effects: (I) the amount of carbohydrate oxidized was markedly reduced (following the injection of active extract, the respiratory quotient was 0.77 , whereas after inactivated extract it was 0.88 ), and (2) the carbohydrate spared appeared to be distributed among the liver, striated muscle, and blood and tissue fluids. More than two-thirds was stored in the liver and striated muscle. Bennett (I937) found that, for a short time only, a crude anterior pituitary extract will restore the muscle glycogen to a normal level in hypophysectomized rats. He could not demonstrate "antihormone" in the blood of animals which received much less benefit from repeated injections. An increase in the deposition of glycogen in the liver and probably in striated muscle was demonstrated by Young (1937), who injected anterior pituitary extract into fasting mice and rabbits. He suggests that gluconeogenesis from fat (glycerol?) may be an important part of the effect (rather than a depression of carbohydrate oxidation) or that another principle is responsible for glycogen deposition.

"Pancreatropic" effects of extracts of the pars glandularis.Several years ago Anselmino and Hoffmann published their first reports on the "pancreatropic" hormone of the pars 
glandularis. Extracts of the anterior pituitary were found to cause hypertrophy and hyperemia of the islet tissue of the pancreas of the rat. New formation of islet tissue was also reported. Changes in the level of the blood sugar or hepatic glycogen were found to conform to the interpretation that the extract also promoted the secretion of insulin.

So far as the histological change is concerned, confirmatory reports have been published by the authors themselves, by Picinelli (1936), Chrzanowski and Grzycki (1937), and Roussy and Mosinger (1937). Roussy and Mosinger believed that the effect is associated with suitable contact with neuroganglionic tissue (neurocrinie pancréatique). On the other hand, despite efforts to duplicate exactly the experiments of Anselmino and Hoffmann, neither Elmer, Giedosz, and Scheps (1937) nor Santo (I 938) could offer confirmatory observations. The obvious importance of an effort to evaluate "pancreatropic" effects quantitatively has been neglected by all authors except Richardson and Young (1937). These authors demonstrated that a saline extract of fresh anterior pituitary of the ox can cause, after daily injection for $2-3$ weeks, an increase in the absolute amount of islet tissue as well as a doubling of the ratio of islet tissue to acinar tissue. However, in another strain of rats, receiving no treatment, the ratio was found to be just as high.

There appeared to be little or no effect of the extract of Richardson and Young on the blood-sugar level of their rats. Elmer and his colleagues (I937) likewise could demonstrate no effect with extracts made according to the directions of Anselmino and Hoffmann. Positive results were obtained by Zunz and La Barre (I935). Pancreatropic extract furnished by Anselmino and Hoffmann was injected intravenously into dogs with the pancreatic vein anastomosed with a jugular vein of a second animal which, preferably, was prevented from liberating epinephrine. The injection of the extract caused a lowering of the level of the blood sugar in the second 
animal. No noteworthy effect on the thyroid or adrenals (epinephrine-liberation) could be detected.

Anselmino and Hoffmann ( 1936 ) injected pancreatropic extract in to anesthetized dogs and concluded that the drop in blood sugar produced was increased if the adrenals were removed. These authors also detected the principle in serum and in urine of normal and diabetic individuals and of pregnant women (see also Picinelli, I936).

The effects of hypophysectomy and of the injection of alkaline anterior pituitary extract (ox) were investigated in adult male newts (Triturus viridescens) by Adams and Ward (1936) and appear to be different from those in mammals. For example, the liver was found to weigh more in hypophysectomized newts than in normal animals. After hypophysectomy the liver by histological examination appeared to contain more fat. The authors also investigated the weight, number of islets, and cytology of the islets of the pancreas as well as the concentration of glycogen in the liver by a method of doubtful quantitative value. Although the pancreas was smaller after hypophysectomy, yet it was found to contain an increased number of islets. The reader is referred to their report for a detailed description of their results.

Observations on the effects of serum, cerebrospinal fuid, or urine on the metabolism of carbohydrates.-The observations to which reference is made in the heading of this paragraph are commonly interpreted by the authors as evidence that anterior pituitary hormones affecting carbohydrate metabolism have been identified. This view, of course, may be entirely incorrect. Harrow and others (1936) extracted from the urine of normal young men a substance which causes a marked hyperglycemia in rabbits. Elmer, Giedosz, and Scheps (I937) reported that the serum of a patient with acromegaly produced a similar effect after injection into the rabbit. According to De Wesselow and Griffiths (1936), if the serum of aged individuals with glycosuria be injected 
subcutaneously into rabbits, there is definitely less hypoglycemia produced by a subsequent dose of insulin. This finding suggested that diabetes in the aged may be the result of a relative excess of diabetogenic hormone in the body fluids, because the serum of normal individuals or of young people with diabetes produced almost no antagonism of insulin. ${ }^{26}$

Anselmino and Hoffmann (1936), Anselmino and Rhoden (1936), and Anselmino (I937) detected "carbohydratemetabolism hormone" of the anterior pituitary by the decrease of hepatic glycogen following administration to rats. In terms of such an effect, the substance has been detected in the serum and urine of young persons with severe diabetes and in the serum of normal individuals or dogs after the administration of I $25^{-} \mathrm{I} 50 \mathrm{gm}$. of glucose by mouth. The latter change is said not to occur if fat also is fed or if large doses of insulin are given. ${ }^{27}$ Anselmino and Hoffmann have not defined in terms of accurate experiments how "carbohydratemetabolism hormone" is related to the diabetogenic hormone of the majority of authors (see also the report of Anselmino and Hoffmann as published in 1935). Future work must decide whether or not "carbohydrate-metabolism hormone" really exists. This is doubted by Singer and Taubenhaus (1937). Diabetes mellitus in relation to "carbohydratemetabolism" and "fat-metabolism" hormones is discussed by Anselmino and Hoffmann (1935-36) and Effkemann (1936).

In general, diabetogenic extracts of the anterior pituitary do not readily produce changes which can be attributed to antihormone-formation. Although the effects of an extract

${ }^{26}$ Hahndel (1935) stated that the cerebrospinal fluid of normal men, if injected intravenously into rabbits, does not affect the level of the blood sugar. However, if insulin be injected first, the cerebrospinal thuid then causes hyperglycemia which the author attributes to contra-insulin hormone liberated into the cerebrospinal fluid.

${ }_{27}$ The serum of cattle suffering from "paresis puerperalis"-vegetative endocarditis with an associated disturbance of mineral metabolism-is reported to produce a decrease of the concentration of liver glycogen in rats without affecting the blood-sugar level (Seekles, 1937). 
may appear to diminish progressively, the serum of such animals is reported not to antagonize the hormone's effects in fresh animals (particularly see Young, 1937).

THE METABOLISM OF LIPOIDS IN RELATION TO

THE PITUITARY BODY 28

According to Lee and Ayres (1936), hypophysectomized rats lose less "fat," i.e., substances extracted by ether, than normal rats when both groups are fed identical amounts of the same diet. The results of Reiss, Epstein, and Gothe (I937) were variable. A few weeks after hypophysectomy the total fat of the body appeared to have fallen to about 40 per cent of the normal value, to which it returned several weeks later ( 8 weeks after operation). On the basis of other experiments the authors suggest that adrenal cortical stimulating hormone is of great importance in facilitating the deposition of fat and that this action ultimately depends upon the liberation of adrenal cortical hormone. The administration to man or the dog either of adrenal cortical extract or of pituitary extract causing cortical stimulation was followed by a fall of 25 per cent or less in the concentration of fat in the blood because, according to the authors' interpretation, more fat is deposited in the tissues. In the dog, Chaikoff and his colleagues (1936) found that long after hypophysectomy all the lipoids ${ }^{29}$ of the blood may be present in normal concentrations; in about one-third of the animals, however, the lipoids of the blood were present in higher concentrations than were ever encountered in normal animals.

A number of reports are concerned with the behavior of the fat of the liver in relation to the anterior pituitary. In the terminology of Benoit (1937) the "hepatotropic function" of the pituitary is increased in the duck after thyroid-

${ }^{28}$ Oestreicher (1936) reported that the oxygen-consumption of isolated fatty tissue of the rat (white subcutaneous fat or testicular fat body) is increased by the addition of "thyrotropic hormone" or "fat-metabolism hormone."

${ }_{29}$ Total fatty acids, phosphatide, and free and ester cholesterol. 
ectomy; in other words, the removal of the thyroid is followed by enlargement of the liver. The experimentally enlarged liver contains about twice as high a concentration of lipoids (I0.4 per cent) as the normal liver (5. I per cent), but if, in addition to thyroidectomy, anterior pituitary extract be injected into the duck, there is further hypertrophy and the concentration of lipoid rises to 38.6 per cent (Benoit, 1936). The observations of Best and Campbell (1936) were made in rats. Large doses of anterior pituitary extract were followed by the deposition of large amounts of fat (total fatty acids together with unsaponifiable material) in the liver. Associated changes in fasting rats were a decrease in the fat of other parts of the body and an increased excretion of acetone bodies. The accumulation of fat in the liver as a result of the injection of anterior pituitary extract is prevented by adrenalectomy but not by demedullation of the adrenals (Fry, 1937). Likewise, Mackay and Barnes (1937) showed that adrenalectomy prevents the deposition of fat in the liver, caused either by anterior pituitary extract or by fasting.

Pancreatectomy is ordinarily followed by an accumulation of lipoids in the liver of animals such as the dog. This change is not prevented if hypophysectomy also is performed (Chaikoff and others, 1936).

The relation of what Anselmino and Hoffmann term the "fat-metabolism hormone" to the accumulation of lipoids in the liver is not clear. Pituitary extract may cause, under proper experimental conditions, an accumulation of acetone bodies in the blood and an increased excretion of the bodies in the urine. An extract with such effects is what Anselmino and Hoffmann have called the "fat-metabolism hormone." Its other effects come up for discussion shortly. Although Anselmino, Hoffmann, and Rhoden (1936) state that extract with the previously described properties is identical with the active substance present in the extracts of Best and Campbell and causes an increase of about 50 per cent in the concentration of ether-soluble substances in the liver dried at 
$60^{\circ} \mathrm{C}$., their earlier report (Anselmino, Effkemann, and Hoffmann, 1935) emphasized that (I) the consistent effect of the extract is an unsaturation of fatty acids of the liver and (2) the concentration of total fatty acids in the liver falls or rises depending upon the presence of high or low initial levels. ${ }^{30}$

It must be remembered that, if the concentration of acetone bodies in the blood increases or if the urinary excretion of these bodies becomes markedly elevated after the injection of anterior pituitary extract, these facts do not justify naming the causative substance "fat-metabolism hormone." The experimental data indicate that its action depends upon the presence of intact adrenal tissue. Dingemanse (1936) is not convinced that a ketogenic hormone exists and found ( 1 ) that only an occasional extract raises the level of acetone bodies in the blood of the rat (but not the rabbit), (2) its detection is difficult because of the effect of fasting, time of day, etc., and (3) the ratio of $\beta$ hydroxybutyric acid to acetoacetic acid and acetone is the same, whether ketogenesis is caused by fasting or by extract.

Mirsky (1936) takes the position that inasmuch as ketogenesis cannot occur in the absence of the liver, a ketogenic effect depends upon the utilization of non-carbohydrate foods in the absence of ample carbohydrate available for oxidation by the cells of the liver. He prevented the ketogenic action of anterior pituitary extract in rabbits fasted for 24 hours by the injection of insulin ( $1 \cdot 3-3$ units per $\mathrm{kg}$. body-weight) or ergotamine tartrate ( $2 \mathrm{mg}$. per $\mathrm{kg}$. body-weight). Both of these substances were considered to prevent glycogenolysis, thus acting as ketolytic agents. Anselmino and Hoffmann (1936) concluded that insulin inhibits the liberation of a ketogenic hormone occurring otherwise as a result of feeding butter. The authors used dogs.

\footnotetext{
${ }^{30}$ The same authors (1935) stated that "carbohydrate-metabolism hormone" causes an effect the opposite of ( $\mathrm{I}$ ) and also brings about a fall in the concentration of total fatty acids.
} 
There appears to be little doubt that the ketogenic effect of anterior pituitary extract, as determined by ketonuria in the rat, is prevented by the removal of the adrenal glands (Fry, I937; Houssay and Rietti, 1937; Mackay and Barnes, 1937). The acetoacetic acid of blood was determined by Houssay and Rietti, whereas the other investigators estimated the acetone bodies of urine. Cortical tissue alone seemed to be necessary for a ketogenic action, inasmuch as the latter is not affected by demedullation (Fry). Houssay and Rietti stated that the administration of adrenal cortical extract restores the ketogenic action of anterior pituitary extract in adrenalectomized rats, whereas this is not accomplished by means of salt therapy. ${ }^{3 r}$

According to Fry (1937), the ketogenic effects of anterior pituitary extract can be observed in the rat even 7 weeks after thyroidectomy. However, Best and Campbell (1936), who performed their experiments in rabbits, found that as hypothyroidism became more pronounced (e.g., 4-6 weeks postoperatively) no ketogenic effect could be produced unless thyroid extract was administered.

Anselmino and Hoffmann (1936) discuss the extraction of ketogenic principle from the anterior pituitary and from blood and urine. Its detection in the blood and urine of diabetic individuals has been reported (Anselmino and Hoff-

${ }^{31}$ Mirsky (1938) suggested that adrenalectomy in the rat does not affect ketogenesis caused by anterior pituitary extract as measured by the acetone bodies of the blood, but that the operation does raise the threshold of renal excretion of acetone bodies. Thus, authors investigating only ketonuria would erroneously conclude that the operation prevented the ketogenic action of extract. However, Houssay and Rietti studied the acetoacetic acid of blood and found that adrenalectomy largely prevented (or even reversed) the ketogenic effect of extract 6-I 4 days after operation. Earlier than 6 days after adrenalectomy, when Mirsky made his observations ( 48 hours after operation), Houssay and Rietti found that the ketogenic action of extract was unimpaired. If the method of estimating acetoacetic acid used by Houssay and Rietti be considered accurate, their extract produced an adequate ketosis, inasmuch as acetoacetic acid probably represents less than 20 per cent of the total acetone bodies.

Anselmino, Hoffmann, and Rhoden (1936) concluded that adrenal cortical extract fully antagonizes the ketogenic action of anterior pituitary extract.

$$
\text { [23I] }
$$


mann, I936; Taubenhaus, I936). ${ }^{32}$ If a ketogenic pituitary extract be injected repeatedly, the animal becomes refractory to this effect (Bennett, I937).

Teilum (1937) attributes to the pituitary the hypercholesterolemia which he observed invariably in gonadectomized persons or patients with hypogonadism. He offers no satisfactory evidence for this interpretation. If we accept the data of Cioglia and Tore (I936), gonadectomy is followed by hypocholesterolemia (rabbit). These authors observed hypercholesterolemia after the administration of prolan or pituitary gonadotropic hormone.

THE METABOLISM OF PROTEIN AND SOME NON-PROTEIN NITROGENOUS COMPOUNDS IN RELATION TO

THE ANTERIOR PITUITARY

The metabolism of protein in relation to the pituitary body. In addition to references in other chapters, there remain a few general reports on the metabolism of protein. Gaebler and Price (1 937) studied the action of single large doses of anterior pituitary extract (with growth-promoting properties) on the metabolism of protein in dogs. The authors found that a fall in the rate of excretion of $\mathrm{N}, \mathrm{S}$, and phosphate was associated with a rise in weight. At the same time the ratio of $\mathrm{N}$ to $\mathrm{S}$ increased and more $\mathrm{N}$ was retained. All these changes are similar to those occurring when synthesis of protein is in progress and suggest that the extract had, in fact, brought about an increased rate of protein synthesis. ${ }^{33}$

${ }^{32}$ The obesity of a patient with Cushing's syndrome was considered by Freyberg and Newburgh (1936) not to be caused by any unusual metabolic derangement, because loss of weight due to undernutrition took place exactly as in normal persons.

33 See also the report of Paschkis and Schwoner (1937), who investigated the action of commercial anterior pituitary extracts on the level of the amino acids in the blood of patients who had been given gelatine by mouth.

Binet, Képinov, and Weller (1935) published determinations of reduced and total glutathione in the liver, thyroid, and testis of normal and hypophysectomized dogs. Hypophysial deficiency was found to be accompanied by reductions in the concentration of glutathione in all three tissues; the most marked reduction occurred in the thyroid, the least in the testis.

$$
\text { [232] }
$$


Perla and Sandberg (1936) observed that the excretion of $\mathrm{N}$ in the urine is increased by 100 per cent during the first few weeks after hypophysectomy and remains elevated for weeks later. The observations of Yokoyama (I935) indicated that, several weeks after hypophysectomy, the dog excretes considerably less total $\mathrm{N}$, uric acid, and urea. Slighter but similar changes in the behavior of allantoin, ammonia, and creatinine were also reported.

The metabolism of creatine and creatinine in relation to the anterior pituitary.-The excretion of nitrogen and creatinine are about the same in normal and hypophysectomized rats, provided that the diet contains sufficient protein. However, if the diet is free from nitrogen, less of both constituents is excreted by hypophysectomized animals. Braier and Morea ( 1935 ) interpret these findings as further evidence that endogenous protein metabolism is reduced as a result of hypophysectomy.

The concentration of creatine in the hamstring muscle of the toad (Xenopus laevis) falls about I 5 per cent I 8-22 weeks after the removal of the pars glandularis or the whole pituitary. The opposite effect is produced by the injection of anterior pituitary extract - the concentration of creatine may increase about 30 per cent 3-5 weeks after injections are started in normal toads. These observations were reported by Shapiro and Zwarenstein (1936), who believed that the effect is mediated through another gland. ${ }^{34}$ Nitzescu and Gontzea (I937) concluded that the anterior pituitary extract "Antuitrin G," although without effect in normal individuals, increases the creatinuria of patients classified as suffering from infantilism, dwarfism, and dystrophia adiposogenitalis. The extract also lessened the tolerance for creatine

3. The secretions of the gonads are of great importance in the metabolism of creatine; e.g., Kun and Peczenik (1936) recently concluded that, at least in the rat, creatinuria appears if there is testicular deficiency and disappears after the injection of male hormone, whereas spayed females excrete no creatine in the urine unless an oestrogen be injected. The male and female hormones, therefore, appeared to affect creatine-metabolism in opposite directions. 
and antagonized the effect of male hormone ("Erugon") on creatine-excretion.

According to Perla and Sandberg (1936) creatinuria transiently appears in male but not in female rats after hypophysectomy.

THE ANTERIOR PITUITARY AND THE METABOLISM OF MINERALS; THE PITUITARY-PARA-

THYROID INTERRELATIONSHIP

The pituitary body and the metabolism of minerals (except calcium).--Gerschman and Marenzi (I935) reported that the injection of large doses of alkaline anterior pituitary extract into hypophysectomized dogs was followed by a diminished concentration of $\mathrm{Na}$ and $\mathrm{Cl}$ in the blood, whereas the concentration of " $\mathrm{CO}_{2}$," glucose, $\mathrm{Ca}, \mathrm{Mg}$, and inorganic $\mathrm{P}$ was elevated following this treatment. Friedgood and McLean (I937), however, could not observe any change in the concentration of serum phosphate of guinea pigs receiving daily injections of alkaline anterior pituitary extract for more than a week. Perla and Sandberg (I 936) found that hypophysectomy accelerates the loss of $\mathrm{P}$ in the feces (but not in the urine) of rats. Later, a study of the effects of partial or complete hypophysectomy on the metabolism of water, $\mathrm{Na}, \mathrm{K}$, $\mathrm{Cl}$, and $\mathrm{S}$ was made in rats by Sandberg, Perla, and Holly (I937). The reader is referred to their article for a description of their findings. That the concentration of $\mathrm{Mg}$ in blood is greatly affected by anterior pituitary secretion is the belief of Cannavò and Beninato (1935), who extended the work of their laboratory in this aspect of mineral metabolism. The pituitary of rabbits was irradiated by X-rays so that the pars glandularis was severely injured and a cachexia followed by death in 2-3 weeks appeared. The serum-concentration of neither $\mathrm{Ca}$ nor inorganic $\mathrm{P}$ was clearly affected. About 10 days after irradiation the level of $\mathrm{Mg}$ was reduced from the normal level $(3.25-4.04 \mathrm{mg}$. per cent) to a new, lower level (1.83-2.54 mg. per cent). The authors believed that the ex- 
cretion of $\mathrm{Mg}$ both in the urine and in the feces was reduced in irradiated animals.

According to the interpretation of Brull (1937) the pituitary body is important in the maintenance of the level of inorganic $\mathrm{P}$ in the blood, because after hypophysectomy the renal threshold for $\mathrm{PO}_{4}$ rises. In the dog anesthetized by chloralose, the excretion of $\mathrm{PO}_{4}$ almost disappears but can be reinstated by the injection of parathyroid hormone, which is believed to act directly on the kidneys. If hypophysectomy is then performed, urine is excreted at an increased rate, but $\mathrm{PO}_{4}$ almost disappears from the urine, although its concentration in the blood rises from a level of $8.4 \mathrm{mg}$. per cent to a level of $10.0 \mathrm{mg}$. per cent.

The pituitary body and the metabolism of calcium. The interrelationship of the pars glandularis and the parathyroid glands. ${ }^{35}$-Some further evidence that parathyroid function is affected by the anterior pituitary has been published. Gerschman and Marenzi ( I935) concluded that large doses of alkaline anterior pituitary extract can cause a small elevation (1.9 mg. per cent) of the level of $\mathrm{Ca}$ in the blood of dogs and that such an effect still can be produced after hypophysectomy or thyroidectomy but not after thyroparathyroidectomy. However, the authors' data do not include the level of $\mathrm{Ca}$ after thyroparathyroidectomy but before injection; after injection the concentration of $\mathrm{Ca}$ was $5.02 \mathrm{mg}$. per cent. The reports of Speransky-Stepanova (1936) (Speranskaia-Stepanowa) were based upon observations in two hypophysectomized dogs. The level of $\mathrm{Ca}$ in the serum was not affected by hypophysectomy but promptly fell after subsequent thyroparathyroidectomy which, of course, caused tetany. ${ }^{36}$ Both in rats (Friedgood, 1936) and in guinea pigs (Friedgood and

35 Freyberg and Grant (1936) studied the metabolism of $\mathrm{Ca}$ and $\mathrm{P}$ in a patient described as suffering from pituitary basophilism. The changes, contrary to what might be expected, were found not to resemble those of hyperparathyroidism (see also the review of Rivoire, I935).

${ }^{36}$ Tetany was not accompanied by an inhibition of diuresis as in dogs with an intact pituitary. 
McLean, 1937) alkaline anterior pituitary extract can cause an elevation of $0.9^{-2.0} \mathrm{mg}$. per cent in the concentration of $\mathrm{Ca}$ in the serum.

An increased excretion of $\mathrm{Ca}$ in the urine following hypophysectomy has been observed in the rat by Perla and Sandberg (1936). The change in absolute terms was slight but represented an increase of several hundred per cent. Perla and Sandberg found that the principal loss of $\mathrm{Ca}$ occurred by way of the digestive tract and that fecal loss was increased after hypophysectomy. Teel and Cushing had found that the urinary loss of $\mathrm{Ca}$ by the dog is increased after the injection of anterior pituitary extract.

Anselmino, Hoffmann, and Herold, who first described histological signs of a parathyrotropic effect of anterior pituitary extract have investigated the action of an aqueous extract of acetone-desiccated anterior pituitary powder (ox) in various animals (Anselmino, Herold, and Hoffmann, 1936). 'They stated that macroscopic enlargement could be produced in the rat and rabbit but not in the cat, dog, or guinea pig. In all the species, histological changes appeared-principally hyperplasia of cells resembling the chief cells of the normal parathyroid.

\section{MISCELLANEOUS OBSERVATIONS}

Perla (1936) investigated the relationship between the spleen and the anterior pituitary and concluded that the gland is required for the normal maintenance of the spleen. ${ }^{37}$ However, it is not possible to state that the anterior pituitary secretes a separate spleen-stimulating principle. Hypophysectomy in rats is followed by splenic atrophy, which regresses considerably if an emulsion of pituitary be injected. Regeneration of a remnant of the spleen after partial splenectomy likewise is prevented by hypophysectomy but takes place as a result of administration of pituitary emulsion. The injection of an emulsion of anterior pituitary of the ox into ${ }^{37}$ See also Friedgood (1936) and earlier reports on the effect of hypophysectomy. 
normal rats - or of an alkaline extract of fresh or desiccated gland-can cause a marked hypertrophy of the spleen due chiefly to hyperplasia and hypertrophy of the reticulo-endothelial cells of the red pulp. In addition, there is some growth of follicles, but the Kupffer cells are not affected. Hyperplasia of the reticular cells of the bone marrow likewise occurs. Wetzler-Ligeti and Wiesner (1937) measured the effect of anterior pituitary extract on the reticulo-endothelial system by the rate at which Congo red was removed from the blood after the dye had been injected intravenously. They concluded that anterior pituitary extracts can either favor or interfere with the removal of Congo red from the circulating blood (positive and negative "restropic" effects).

The relation of the pituitary to erythrocyte- (and leucocyte-)formation was investigated by Kapran (1935) and Flaks, Himmel, and Zlotnik (1937). Kapran reported on the changes following hypophysectomy in the dog-i.e., diminished number of erythrocytes, elevated color-index, interference with reticulocyte formation, slight leucopenia, and eosinophilia even to 20 per cent; but that these changes were specifically due to hypophysectomy was not shown. On the other hand, McFarlane and McPhail (1937) were unable to detect any change in erythrocyte count or content of hemoglobin in guinea pigs because of the removal of the pituitary. According to Flaks and his colleagues, a thermostable substance can be extracted from the anterior pituitary which directly stimulates the red bone marrow even after administration by mouth. Reticulocytosis and later polycythemia can be observed in rats receiving the extract.

Keller and D'Amour ( 1936 ) occasionally found hemorrhage into and ulceration of the gastrointestinal tract of dogs in which hypophysectomy had been undertaken. However, they were not certain that these pathological changes were not caused by injury of the central nervous system. Belief in the efficacy of heterotransplants of the anterior pituitary was expressed by Kylin ( 1937 ), who concluded that they were 
of great value in the treatment of Simmonds' disease; he also described the successful transplantation of calf pituitary into the peritoneal cavity of the rabbit.

According to Gagyi (I 936), who performed titrations with 2,6 dichlorophenolindophenol, the pars neuralis (contrary to Giroud and Leblond) contains about three times as much ascorbic acid as the pars buccalis in terms of concentration (pars neuralis 7.2 and the pars glandularis-pars intermedia $2.3 \mathrm{mg}$. per gm). Also he stated that the gland of immature animals, especially females more than males, contains a higher concentration of the vitamin than the gland of adults. The observations were made in guinea pigs. Two other reports refer to constituents of the pituitary. Regnier (I936) found that sulfonal (diethylsulfonmethane) was deposited in the pars glandularis to a greater extent than in any other tissue after the drug had been administered by mouth to a horse ( $4 \circ \mathrm{gm}$. to a horse weighing $450 \mathrm{~kg}$.). The tissues containing the highest concentrations of this hypnotic, expressed as milligrams per 100 gm., were: pars glandularis, 750; adrenals, 426; pars posterior, 200; blood, 29.4. The brain contained $7.8 \mathrm{mg}$. per $100 \mathrm{gm}$. It is extremely unlikely that the presence of bromine in the pituitary body has any significance. Dixon's recent report (I935) indicated that the pituitary of the normal pig contains about $0.27 \mathrm{mg}$. of $\mathrm{Br}$ per $\mathrm{s} 00$ $\mathrm{gm}$. of fresh tissue. As in other organs the ratio of $\mathrm{Br}$ to $\mathrm{Cl}$ roughly corresponded to what was probably the ratio of these elements in the diet. According to Moruzzi and Guareschi (1936), the fresh pituitary of the ox and of man contains respectively about I or about $0.6 \mathrm{mg}$. of $\mathrm{Br}$ per $\mathrm{I} 00 \mathrm{gm}$.

\section{SUMMARY}

The morphological and functional maintenance of the cortex of the adrenal glands depends upon secretion(s) of the pars glandularis. (The medulla of the adrenal gland may have important indirect interrelationships with the anterior pituitary; its direct dependence on the anterior pituitary prob- 
ably is slight and unimportant.) Without pituitary secretion either from the intact gland or from administered extract, the adrenal cortex rapidly diminishes in size and performs its usual functions only imperfectly. The principal morphological evidences of atrophy are in the outer part of the thickest layer of the cortex, the zona fasciculata, although the zona glomerulosa also is affected. There is a striking loss of lipoids. The administration of a suitable anterior pituitary extract not only restores the lipoids lost but may increase their concentration in association with hypertrophy and hyperplasia of cortical cells. In this way an extract may cause cortical enlargement in hypophysectomized rats, so that the adrenal glands are larger than any ever encountered in normal rats receiving no treatment.

The $\mathrm{X}$-zone of the innermost part of the cortex of young female mice or of castrated males seems also to depend upon an anterior pituitary hormone for its development (Deanesly).

The physiological interrelationships of the adrenal cortex and the anterior pituitary are important and numerous; unfortunately, however, they have been explored only imperfectly. Compensatory adrenal hypertrophy requires adrenal cortical stimulating hormone. This hormone probably is secreted at an increased rate if there is cortical deficiency, whereas a change in the opposite direction takes place if abnormally large amounts of adrenal cortical hormone(s) are present in the body-fluids. Some of the most important correlations appear to affect the metabolism of carbohydrates and fats and are mentioned in later discussion.

The pars glandularis appears to be the principal division of the pituitary body important in the metabolism of carbohydrates. The substance or substances responsible for participation in this important phase of metabolism has been detected in anterior pituitary extracts which, for the most part, are quite crude. The isolation of these substances must 
be carried much farther before definite statements can be made concerning either their number or their other biological effects (e.g., on the mammary glands or adrenal cortices). However, a number of other methods has been employed to learn how regulation by the anterior pituitary is effected. Carbohydrate metabolism has been studied after hypophysectomy; in such studies, epinephrine or insulin also has been used. The remarkable amelioration of diabetes which follows the removal of the hypophysis from depancreatized animals has received further attention. The most important interrelationships with other glands of internal secretion are those with the pancreas and adrenal glands.

The anterior pituitary appears to be necessary for the absorption of glucose at a normal rate from the digestive tract; however, there is no evidence that a specific hormone, such as that affecting the metabolism of carbohydrate after absorption, alters the process of absorption. The "diabetogenic" hormone of the anterior pituitary probably prevents the prodigal waste of important, but sometimes small, carbohydrate reserves which, for example, are maintained in spite of fasting. An animal isolated from all sources of the hormone (as after hypophysectomy) and dependent upon its own reserves of food, dangerously uses up all available carbohydrate. It is able readily to mobilize the glycogen of both the liver and striated muscle and to oxidize glucose. Perhaps another important metabolic function of anterior pituitary secretion is to facilitate the new formation of carbohydrate from both fat (glycerol) and protein.

The diabetogenic hormone can be viewed as an antagonist of insulin, the internal secretion of the pancreas. There is evidence that this antagonism is indirect and depends upon the support of adequate adrenal function. However, there is not complete agreement that bilateral adrenalectomy prevents the disturbing effects of anterior pituitary extract on carbohydrate metabolism - e.g., hyperglycemia in the dog. On the other hand, the glycosuria which follows the injection of an-

$$
\text { [2+0] }
$$


terior pituitary extract into cats or rats is not observed after complete adrenalectomy. Insulin-sensitivity is increased by hypophysectomy, because an important physiological antagonist of insulin-diabetogenic hormone-is no longer available, and carbohydrate reserves both disappear at an abnormally rapid rate and probably are restored from other endogenous sources only with great difficulty. After pancreatectomy, secretion(s) of the anterior pituitary is not antagonized (however indirectly) by insulin and may be regarded as an important contributor to changes which threaten life-e.g., the accumulation of acetone bodies. Anterior pituitary secretion can also be pictured as interfering with the oxidation of glucose which otherwise is facilitated by insulin. If, however, both glands are removed, the important means of regulating carbohydrate metabolism are lost. A unique derangement of metabolism appears and, depending upon conditions such as nutritional state before operation, fasting, diet, etc., the animal resembles sometimes the diabetic animal-e.g., with hyperglycemia and glycosuria-and sometimes the animal with hypophysial deficiency-e.g., with hypoglycemia.

Although the effects of subcutaneously injected epinephrine are less pronounced after hypophysectomy, it is probable that delay in absorption accounts for most of this change, provided that reserves of carbohydrate are adequate. Any diminished effect of epinephrine is observed in respect of all the expected changes-elevation of the level of sugar and lactic acid in the blood, depression of the level of inorganic $\mathrm{P}$, lowering of the concentration of glycogen in muscle, etc.

New observations support the belief that anterior pituitary extract can cause hypertrophy of the islet tissue of the pancreas. The physiological significance of this effect has not been adequately explored.

Any attempt briefly to summarize our knowledge of the relation of the anterior pituitary to carbohydrate metabolism is necessarily confusing, because we know only some phases 
of processes of unknown complexity. However, the potential value of what can be learned is immense in its implications not only to physiology but also to clinical medicine.

The metabolism of fat, depending upon conditions, is probably closely interrelated with that of carbohydrates. Since little is known concerning the nature of the substance(s) affecting the metabolism of carbohydrates and fats which can be extracted from or are secreted by the pars glandularis, it is by no means clear whether or not one or more substances is responsible for the effects, or, indeed, whether the effects are due to a hormone to which another name has already been given. Perhaps an anterior pituitary hormone which depresses the rate of catabolism of carbohydrate (and increases the rate of protein anabolism) indirectly necessitates the mobilization and oxidation of an increased proportion of fat, so that ketogenesis is facilitated. The outstanding facts concerning the effects of pituitary extract on the metabolism of fat appear to be (I) large doses of anterior pituitary extract bring about a deposition of fat in the liver which, in fasted animals at least, is enriched at the expense of other tissues and (2) anterior pituitary extract produces ketosis which probably is associated with the changes described under (I). (It is generally believed that acetone bodies are formed in the liver.) It is of great interest that both of these changes are said to be prevented by adrenalectomy, apparently because of the removal of the cortex. This, in turn, suggests that an adrenal cortical stimulating substance is the pituitary secretion affecting the metabolism of fats.

There still is not a sufficiently large body of data to enable one to discuss with any assurance the manner in which the metabolism of proteins is influenced by secretion(s) of the pars glandularis. It appears that hypophysial deficiency probably is accompanied by increased catabolism of proteins of endogenous origin and imperfect utilization of proteins of 
exogenous origin, whereas anterior pituitary extract favors protein synthesis.

Additional reports on the interrelationship of the anterior pituitary and the metabolism of minerals have been made. However, there frequently is a lack of agreement. Possible effects of extracts on the metabolism of water are usually neglected. There is evidence that the activity of the parathyroid glands is regulated to some extent by the pars glandularis. 'This control appears to be slight and is much less effective than that exercised over the adrenals, gonads, or thyroid. 


\section{CHAPTER VIII}

\section{THE PITUITARY BODY IN RELATION TO THE REGULATION OF THE DISTRIBUTION OF PIGMENT IN CHROMATOPHORES}

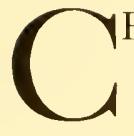

YELLS containing granules of pigment of various colors may be found in the skin and other tissues of cold-blooded animals. These cells often are of great importance in the adaptation of color to background or in effecting color changes for other purposes - e.g., that associated with the breeding season. The change of color is effected by the movement of the granules of pigment, chromatosomes, in cells which are called chromatophores. These cells commonly are named in accordance with the color of the pigment they contain. Melanophores are so named because they contain melanin-granules for which the term melanosomes will be used. Likewise erythrosomes refer to the granules of red pigment in erythrophores, etc.

The pituitary body is of importance in this aspect of biology because extracts of the pars intermedia (or of the pars glandularis) may bring about a striking dispersion of chromatosomes, especially melanosomes or erythrosomes. The removal of most of the pituitary may be followed by the opposite effect, e.g., a marked concentration of the melanosomes may follow hypophysectomy in the frog. In addition, Hogben and his collaborators have inferred that a hormone of the pars tuberalis is important in certain amphibia in bringing about adaptation to light backgrounds.

The degree to which the position of pigment-granules in chromatophores is regulated by the pituitary body varies greatly in different classes of animals, such as fishes, amphibia, and reptiles. The importance of the gland generally 
is more easily demonstrated in certain amphibia than in animals of other classes. ${ }^{\text {. }}$

The importance of the pituitary body in regulating chromatosome dispersion in fishes.-In fishes hypophysectomy often is followed by a marked concentration of the melanosomes (the "melanophore contraction" of some authors). However, the change appears more sluggishly than in amphibia. Most of the successful experiments have been performed in various elasmobranchs. Lundstrom and Bard had shown that the removal of the neuro-intermediate lobe from the dogfish, Mustelis canis, is followed by a marked pallor of the skin due to the concentration of the melanosomes in the central part of the melanophores. As a phase of his investigation of the physiology of the chromatophores of various animals, Hogben (1936) studied the effects of hypophysectomy in several elasmobranchs. Figure 26 is reproduced from Hogben's article and illustrates the cutaneous pallor of Rhina squatina following hypophysectomy. The adaptation of fishes such as dogfishes (Scyllium catulus, $S$. canicula), the angel shark (Rhina squatina), and rays (Raia maculata, $R$. brachiura) to a black background is abolished by hypophysectomy. However, in some species the operation may not be followed by as striking a macroscopic effect as is illus-

Abramovitz (1936-37) has published several reports extending the observations of Megasŭr and of Carlson on the hormone in the eye-stalks of crustacea. This hormone appears to be responsible for melanosome dispersion in crustacea such as Callinectes, Libinia, Palaemonetes, and $U c a$. Extirpation of the eye-stalks of the crab, $U c a$, is followed by paleness of the integument (Megasurr); if an extract of eye-stalks be injected into such pale crabs, dispersion of the melanosomes occurs and the animal becomes dark (Carlson). Abramovitz found that an extract of the eye-stalks of Palaemonetes vulgaris produces effects similar to those of the chromatosome-dispersing hormone of the pituitary. This was shown by the dispersion of the pigment in the melanophores following injection of the extract into light-adapted or hypophysectomized fishes (Ameiurus nebulosus, Mustelis), frogs (Rana pipiens), or reptiles (Anolis carolinensis). The erythrophores and melanophores of the dace (Chrosomus erythrogaster) were similarly affected.

The substance responsible for these effects was found to be remarkably stable. It could survive boiling in water or in aqueous solutions of I per cent $\mathrm{HCl}$ or $\mathrm{KOH}$. It appeared to be soluble in water and in methanol or ethanol-although the extent to which this is true was not accurately described. 


\section{THE PITUITARY BODY}

trated in Figure 26. As in the dogfish, the loss of the neurointermediate lobe is responsible for the failure of the melanosomes to disperse when the background is black. Hogben is of the opinion that adaptation of elasmobranchs to light backgrounds, due to a concentration of the melanosomes, de-

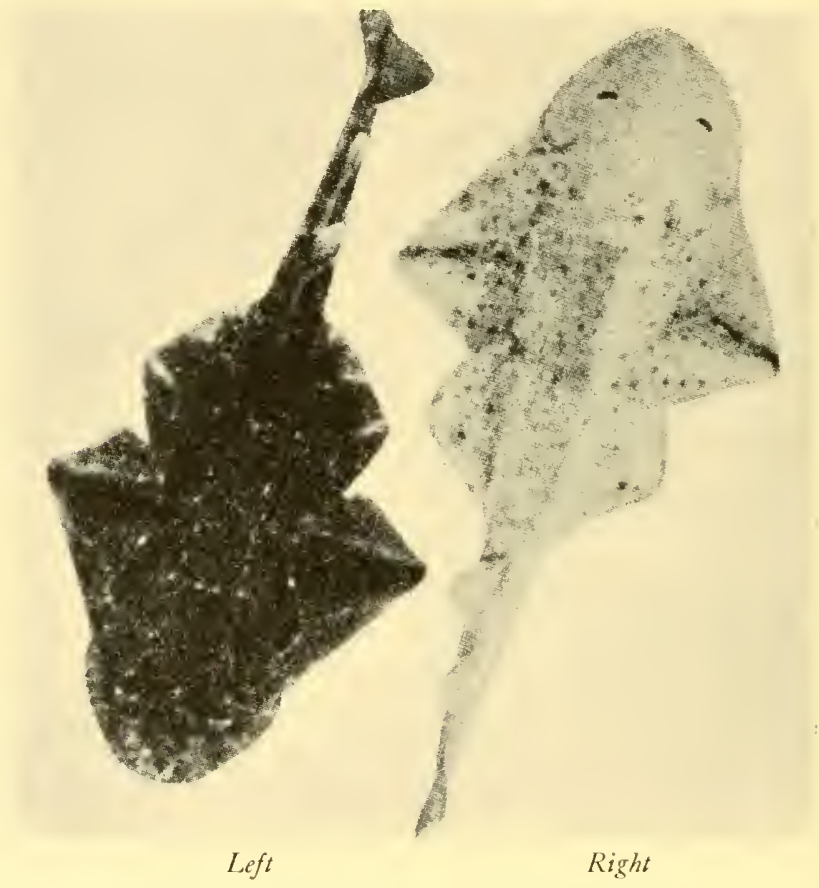

Fig. 26. - The effect of hypophysectomy on the appearance of the adult angelshark, Rhina squatina. Left: normal; right: hypophysectomized 10 days before. Both kept continuously in black tank. (From Hogben, Proc. Roy. Soc., B, I20, I $42-58$ [1936].)

pends upon a secretion from a part of the pars buccalis homologous with the mammalian pars tuberalis. However, adaptation to light backgrounds may be slight, e.g., in Raia clavata. Hogben's results support the general belief that the pars intermedia of the pituitary body of animals in which it can be identified morphologically elaborates the secretion

$$
[2+6]
$$


causing dispersion of the melanosomes. ${ }^{2}$ In fishes, as in amphibia, optic stimuli chiefly determine whether or not the secretion will be released.

Likewise in elasmobranch fishes, other authors have demonstrated that hypophysectomy is followed by a cutaneous pallor which persists irrespective of the background. Barry ( 937 ) described a dogfish (Scylliorhinus canicula), the cutaneous melanophores of which resembled those of a hypophysectomized fish in respect of the unchanging concentration of the melanosomes and their rapid dispersion after the injection of a pituitary extract. However, his description of the histological appearance of the pituitary body and its related structures is of little assistance in explaining the phenomenon. The removal of the pituitary from Torpedo marmorata causes a light coloration of the skin as a result of melanosome concentration (Veil and May, 1937). Vilter (I937) attributed the "melanophore contraction" which appears as a result of hypophysectomy in two rays, Trigon pastinaca and Raia undulata, to a pronounced tonus of the sympathetic nervous system because ergotamine, which may cause paralysis of sympathetic terminal fibers, brought about a marked dispersion of the melanosomes in either normal or hypophysectomized fishes of these species. ${ }^{3}$

In teleost fishes the role of the pars intermedia in effecting color changes by means of the melanosome-dispersing hormone is less important and often is difficult to evaluate. Veil recently stated (I 937) that the removal of the gland from the catfish (Ameiurus) is followed by blanching of the skin, which can again be made dark by the administration of fish pituitary or an extract of the mammalian posterior lobe. According to Parker (I935), chromatosome dispersion in Ameiurus

${ }^{2}$ Neither the oxytocic nor the vasopressor hormone of the pars neuralis is responsible for melanosome dispersion.

${ }^{3}$ For years, epinephrine has been regarded as a substance causing melanosome concentration. So far as fishes are concerned, it appears that the importance of a sympathetic innervation of the melanophores is greater in teleosts than in elasmobranchs. 
is effected both by nervous impulses and by the hormone; however, he regarded the latter as the less important. Parker believed that melanosome concentration is chiefly under the control of the (sympathetic) nervous system in both teleosts and elasmobranchs, whereas in elasmobranchs the dispersion of pigment-granules is due to a hormone of the pituitary. In another teleost, Fundulus heteroclitus, both the light and dark phases of pigment distribution appeared to be regulated by nerves (Parker). However, Kleinholz (I935) showed first that the pituitary of Fundulus contains melanosome-dispersing hormone as demonstrated by experiments in catfish, frogs, and lizards, and second, that denervated melanophores of Fundulus responded in a typical fashion (melanosome dispersion) to extracts of the pituitary of Fundulus, of the frog, or of the mammal. 4 Such an effect was never observed in melanophores with a normal innervation. Abramovitz (1937) concluded that the melanosome-dispersing hormone of the pituitary probably is of real significance in the physiology of the melanophores in Fundulus, despite the fact that adaptation to light and dark backgrounds ordinarily is not affected by hypophysectomy. Abramovitz found that the operation prevented the complete dispersion of the melanosomes of denervated melanophores, when the fish were placed on a black background.

In other teleost fishes such as Phoxinus laevis the distribution of pigment in erythrophores is principally affected by the chromatosome-dispersing hormone of the pars intermedia. Fleischmann and Kann (1937) injected posteriorlobe extract into several species of marine fishes (Coris julis, Crenilabrus pavo, Serranus scriba, and Tripterygium nasus) and found that the changes in coloration strongly resembled those appearing in fishes in full "wedding dress." They were unable to detect an effect on the melanophores. Erythro-

${ }_{4}$ The melanosomes of isolated scales of Fundulus generally become concentrated, if the scales are placed in a solution of posterior-lobe extract. Such an observation is of little assistance for purposes of interpretation. 
some dispersion in the erythrophores of intact fish or of isolated scales has again been used in recent experiments for purposes of assay.

The regulation of chromatosome dispersion in amphibia. ${ }^{5-}$ Especially in anuran amphibia past work has shown that adaptation of the skin's color to dark or black backgrounds chiefly depends upon an internal secretion of the pars intermedia. After the removal of the pars intermedia (or the neuro-intermediate lobe), marked aggregation of the melanosomes takes place, so that animals like the frog remain pale indefinitely, irrespective of the background. Atwell and Holley (1936) found that the pars intermedia may be removed from tadpoles, and yet sufficient tissue of the pars glandularis is spared, so that in such silvery tadpoles complete or partial metamorphosis occurs and normal development of the thyroid, gonads, and adrenals takes place. The authors suggested that the pars intermedia of tadpoles of Rana sylvatica can undergo development without contact with nervous tissue.

New studies by Hogben and Slome (1936) support their conclusion that both the white- and black- background responses of the toad, Xenopus laevis, depend upon hormones. Their study was limited to the dermal melanophores and indicated that dispersion of the melanosomes is caused by a hormone of the pars intermedia, whereas concentration of the melanosomes is dependent upon an intact pars tuberalis. Their results are summarized in Table 8. Presumably the low sensitivity of the melanophores toward the melanosomedispersing hormone in animals without a pars intermedia (and pars neuralis) is due to the pars tuberalis, the effects of the secretion of which are no longer antagonized by the normal secretion of the pars intermedia. On the other hand, the great sensitivity of the melanophores of toads after the re-

5 The chromatosome-dispersing hormone can cause dispersion of the pigmentgranules in the melanophores of certain reptiles such as lizards (e.g., Anolis carolinensis [Kleinholz, 1935]). 


\section{THE PI'TUITARY BODY}

moval of all parts of the pituitary body is the result of the removal of the antagonistic influence of the pars tuberalis. ${ }^{6}$

According to Jores and Caesar (1935), pigment of the retina of the frog's eye assumes the dark position at a more rapid rate, if the eye be treated by a solution of the "melanophore hormone" in the dark. Similar treatment of the eye in the light only rarely is followed by an effect. Hypophysectomy, however, does not alter the movement of retinal pig-

\section{TABLE 8}

The Effects of Extirpation of Various Divisions of the Pituitary Body on the Condition and Response of the Dermal MelanoPHORES IN Xenopus laeris*

\begin{tabular}{|c|c|c|c|}
\hline \multirow{2}{*}{ Condition of TOAD } & \multicolumn{2}{|c|}{ MELANOPHORE-INDEX $†$ ON } & \multirow{2}{*}{$\begin{array}{c}\text { SENSITIVITY } \\
\text { TOWARD } \\
\text { MELANOSOME- } \\
\text { DIIPERSING } \\
\text { HORMONE }\end{array}$} \\
\hline & $\begin{array}{c}\text { White } \\
\text { Background }\end{array}$ & $\begin{array}{c}\text { Black } \\
\text { Background }\end{array}$ & \\
\hline 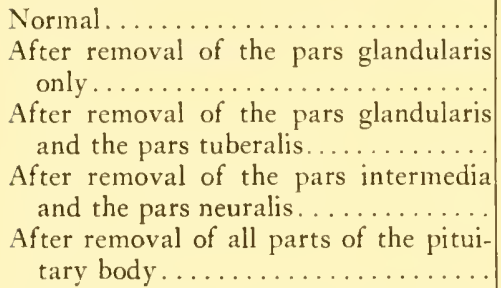 & $\begin{array}{l}\text { I. } 4 \\
5.0 \\
\text { I.O } \\
\text { I. } 8\end{array}$ & $\begin{array}{l}4.5 \\
4.5 \\
5.0 \\
1.0 \\
1.8\end{array}$ & $\begin{array}{c}+ \\
+++\end{array}$ \\
\hline
\end{tabular}

* From Hogben and Slome, Proc. Roy. Soc., London, B, r 20, 158-73 (1936).

$\dagger$ The higher the melanophore-index, the greater the degree of dispersion of the melanosomes, i.e., the greater the "expansion" of melanophores.

ment in response to light or darkness in either frogs (Jores and Caesar, I935; Matuo, I935) or toads (Okamato, I937). Dubois-Poulsen (1937) also agreed that chromatosome-dispersing hormone facilitates the wandering of retinal pigment into the position characteristic of darkness. ${ }^{7}$ The author used frogs (Rana temporaria) and concluded that epinephrine less effectively produces a change in the opposite direction.

${ }^{6}$ The authors also investigated the manner in which optic stimuli affect the background response. They concluded that the behavior of the melanophores of toads on white or black backgrounds depends upon retinal localization.

${ }^{7}$ See also Matuo (1935). 
New observations on the pharmacology of melanophores or of the melanosome-dispersing hormone.-Shen (1937) has studied the effects of a number of drugs on the behavior of the granules of pigment in the melanophores of the skin of frogs. Substances like F 933 and $\mathrm{F} 883^{8}$ bring about marked dispersion of the melanosomes, provided that the pituitary is intact. After hypophysectomy, "expansion" of the melanophores does not appear. The conditions under which chloralosane, nicotine, or yohimbine cause melanosome dispersion are similar. Therefore, all these substances appear to act either by increasing the rate of secretion of the hormone or by markedly potentiating its peripheral effects or by both mechanisms. Shen found that melanosome concentration is reversed by $\mathrm{F} 933$, if it is due to epinephrine but not if it follows stimulation of "pigmento-motor" nerves. Hence, in respect of a congregating effect on the melanosomes, he describes F 933 as a substance which is adrenolytic but not sympatholytic. The injection of either atropine sulphate or ergotamine tartrate into normal frogs adapted to a dark background is followed by paling; in hypophysectomized frogs, however, either drug causes some melanosome dispersion. The author's experiments yield no specific information on the possible importance of effects on the secretion or peripheral action of a hormone of the pars tuberalis in relation to melanosome concentration. Hypophysectomy does not affect the dispersion of the melanosomes which is the result of the administration of amyl nitrite, chloroform, ether, or strychnine.

According to Jores (1935), the injection of extract containing melanophore hormone into rabbits by either an intraventricular or an intravenous route is followed by a fall in

${ }^{8} \mathrm{~F}$ 933: piperidino-methyl-3-benzodioxane; F 883: diethylamino-methyl-3-benzodioxane.

9 The injection of ergometrine, "Ephetonin" (synthetic ephedrine), or lobeline is followed by a similar effect. 


\section{THE PITUITARY BODY}

body-temperature $(0.7-0.8 \mathrm{C}$.) and a rise in the concentration of sugar in the blood. Atropinization or treatment by "Somnifen" lessened or abolished the apparent depressing effect on temperature. Both Jores (1936) and Parhon and Cahane (1936) reported that the repeated administration of epinephrine to rats is followed by an increase in the amount of melanosome-dispersing hormone in the pituitary body. ${ }^{\text {10 }}$ Jores attributed this change to a corresponding hypertrophy of the pars intermedia. In the mouse, on the other hand, a single large dose either of epinephrine or of "Cortidyn" (an extract of the adrenal cortex) caused an immediate reduction of the amount of melanosome-dispersing hormone in the pituitary. Jores also found that similar treatment of the rabbit or of man brought about an acute fall in the concentration of melanophore hormone in the blood. ("Cortidyn" alone or in combination with epinephrine had the same action in rats as epinephrine.)

Melanosome-dispersing hormone in relation to the metabolism of water.-The belief of some observers, such as Sulzberger, that the chromatosome-dispersing hormone (or some other new principle) of the pars intermedia is an agent capable of inhibiting diuresis cannot be accepted. Böttger (I936) even called this substance "adiuretin," but his evidence that it differed from the vasopressor principle of the pars neuralis consisted of comparisons of vasopressor and antidiuretic effects of extracts. Later (I937), he agreed that chromatosomedispersing hormone is diuresis-inhibiting in proportion to its contamination by the vasopressor principle. Dischreit (I935) was unable to produce diuresis inhibition by "Intermedin" free from vasopressor hormone, although he used much larger doses, in terms of melanosome-dispersing hormone, than did Sulzberger. Fraser (1937) also was convinced that

\footnotetext{
${ }^{10}$ Parhon and Cahane believed that testicular or corpus luteum hormone potentiates the action of melanosome-dispersing hormone, whereas thyroid hormone or oestrin is without such action.
} 
the vasopressor principle is responsible for any antidiuretic effects of chromatosome-dispersing hormone. ${ }^{\text {I }}$

The metabolism of chromatosome-dispersing hormone.Chromatosome-dispersing hormone can be detected in the urine of normal men and women. It appears with increased frequency in the urine of pregnant women; this fact, however, does not make tests for its presence of much value in diagnosing pregnancy (see p. 127, n. 5).

Lewis, Lee, and Astwood (I937) have extended the observations of others on the distribution of chromatosome-dispersing hormone in the pituitary body and adjacent structures of cattle. The unit used by them was the minimum amount of material causing an erythrosome dispersion in all fish (Phoxinus laevis) receiving that dose. ${ }^{12}$ The error of assay apparently was high. Their estimate of the concentration of the hormone in various parts of the gland was as follows (all figures refer to units per gram fresh tissue): puslike material in cleft consisting chiefly of desquamated cells of the pars intermedia, 270,000; pars intermedia, 255,000; colloid in cleft, I23,000; pars neuralis, 33,000; pars glandularis, 20,000-31,000; inferior part of stalk, 3,500; superior part of stalk, 300 ; tuber cinereum, 3०; region about third ventricle, I 2 ; cerebrospinal fluid, 0 . The authors concluded that typical basophils could not be responsible for the secretion of the hormone by the pars intermedia, inasmuch as these were absent from that division of the gland. Their results are in agreement with the best data which had been gathered previously: in the pituitary of the ox, the chromatosome-dispersing hormone is secreted by the pars intermedia. Posterior-lobe extract contains the hormone because

II Jones and Steggerda (1935) could detect no change in the rate of loss of weight of frogs in water in relation to color adaptation to light, dark, or neutral backgrounds. In fact, hypophysectomized frogs lost weight at a similar rate.

${ }_{12}$ Böttger (1937) assayed chromatosome-dispersing hormone by using the isolated fin of male specimens of Phoxinus laevis. He observed microscopically the number of erythrophores affected as well as the degree of dispersion of the erythrosomes. Maximum effects occurred in $30-45$ minutes. The error of determination was believed to be \pm 20 per cent. 
it is commonly made from tissue containing the pars intermedia and because the hormone readily diffuses into it and into other parts of the pituitary body. It is interesting that Lewis and his colleagues could furnish no support for the view, which has been popular with some European authors, that chromatosome-dispersing hormone may be secreted by way of the stalk into the cerebrospinal fluid.

The chromatosome-dispersing hormone is secreted by cells of the pars buccalis. In animals in which the pars intermedia can be identified morphologically there can be little doubt that that division of the pars buccalis secretes the hormone. In animals like the whale in which no pars intermedia can be found, the hormone can be extracted from the pars glandularis (Geiling, 1935). Fisher (1937) reported that in the posterior lobe of the pituitary of cats in which marked atrophy of the pars neuralis had been produced experimentally, there appeared to be no reduction in the amount of chromatosomedispersing hormone as determined by the response of the melanophores of frogs or the erythrophores of the red-bellied dace (Chrosomus erythrogaster). ${ }^{13}$ The atrophied pars neuralis contained no oxy tocic or vasopressor (and diuresis-inhibiting) hormone. The results of Geiling and Lewis (1935) also indicated that the pars intermedia is the site of formation of the chromatosome-dispersing hormone. The authors undertook to make tissue cultures of the pars glandularis, the pars intermedia, or the pars neuralis of the mouse and rat. The pituitary of the mouse was preferred as a source of the tissues because the various divisions could be separated more completely. After culture for 50 days, the pars intermedia contained chromatosome-dispersing hormone but no vasopressor hormone, indicating that the latter is not secreted by the pars intermedia. Both hormones could be detected in cultures of the pars neuralis not entirely free from pars inter-

${ }^{13}$ Fisher was not satisfied that accurate results could be obtained by the use of this fish. 
media. Cultures of the pars glandularis contained neither chromatosome-dispersing hormone nor vasopressor hormone.

Jores (1935) was convinced that melanophore hormone accumulates in the pituitary of mice kept in darkness ( 1.45 units), whereas the amount falls rapidly when animals are exposed to light ( 0.27 unit). Responsibility for this change appeared to rest chiefly with light of shorter wave-lengths (blue), although exposure of mice to yellow and red light was followed by a slower and less pronounced reduction.

According to Rodewald (1935), if frogs are kept in darkness, their blood contains a substance "binding" chromatosome-dispersing hormone. The author believed that this substance is closely associated with the erythrocytes. It was not found in the blood of light or dark frogs exposed to light. Rodewald believed that two mechanisms operate to prevent any action of chromatosome-dispersing hormone in frogs kept in darkness: (I) no hormone is formed by the pituitary body, ${ }^{14}$ and (2) a substance inactivating the hormone is liberated into the blood. Jores and Hoeltje (1936) believed that they confirmed the observation of Rodewald. 'Their results varied greatly and the differences found appear to be small. However, the authors reported that the blood of mammals (the rabbit and man) kept in darkness does not contain the inactivating substance but, on the other hand, seems to contain a higher concentration of the hormone itself. Later, Rodewald (I936) reported that the serum of patients with cancer contains a substance binding (or inactivating) melanophore hormone. Such a result was obtained by the use of the serum of 105 of 109 patients investigated ( 96 per cent). The reaction of the serum was positive in 2 of 50 pregnant women (4 per cent) but was negative in patients with carcinoma of the skin.

New observations on the chemistry of the chromatosome-dispersing hormone.-Among principles which can be extracted from

${ }^{1}+\mathrm{A}$ change in the opposite direction was recorded by Jores, who used mice. 
the pituitary body, none withstands chemical manipulation better than the chromatosome-dispersing hormone. The hormone is soluble in dilute aqueous solutions of acid or alkali and in certain organic solvents such as methanol and ethanol. Like the hormones of the pars neuralis, it is not destroyed by boiling in acidified water. However, it is remarkable that the hormone not only survives treatment by, or boiling in, fairly strong alkaline solutions, e.g., N/ı。, but also that its effects appear to be much more pronounced after this treatment, as was first shown by Hogben and Gordon. Böttger (I937) believed that after alkali has acted upon the hormone, the latter is metabolized more slowly, as shown by changes in the intensity and duration of its effects. According to Stehle (1936), the hormone is altered qualitatively, i.e., the initial effect is less intense but the duration of action is much longer. The degree of potentiation of the action of the hormone as a result of treatment by alkali has not been exactly defined. Stehle is of the opinion that it is much greater than Jores' estimate of 200-300 per cent. According to Abramovitz (1937), the chromatosome-dispersing effect of the pituitary of Fundulus is increased about twenty-five fold by boiling in $\mathrm{N} / \mathrm{I} O \mathrm{NaOH}$.

It is probable that the same hormone causes dispersion of both melanosomes and erythrosomes. This view has recently been supported by Böttger ( I 937). New methods of purifying chromatosome-dispersing hormone have been described by Stehle (1936) and Böttger (1937). Stehle's product was about twenty-five times as active as International Standard Powder (posterior-lobe).

\section{SUMMARY}

The pituitary body or homologues of its divisions may be of great importance in the regulation of the distribution of pigment-granules in chromatophores of cold-blooded animals. Almost all the detailed studies are concerned with melanophores (fishes, amphibia, reptiles) and erythrophores (fishes). 
Chromatosome dispersion is caused by a secretion of the pars intermedia or of the pars glandularis in animals otherwise lacking the anatomical equivalent of the pars intermedia. ${ }^{15}$ The experiments of Hogben and Slome suggest that a secretion of the pars tuberalis causes the opposite effect-a concentration of the chromatosomes.

The significance of the chromatosome-dispersing hormone in vertebrates with functional chromatophores varies greatly. In some fishes, e.g., dogfishes, the hormone may be the principal means of chromatosome dispersion and, hence, of adaptation to dark backgrounds. In others, such as the catfish, nerves also play a part in melanosome dispersion. Finally, in a teleost fish like Fundulus, the hormone is present in the fish's pituitary but plays a negligible part in the regulation of chromatosome distribution, which is largely under the control of nerves. In the fish Phoximus laevis the erythrophores and xanthophores are more specifically affected by the chromatosome-dispersing hormone than the melanophores. In amphibia like the frog the hormone appears to be the principal means of effecting dispersion of melanosomes. The action of the hormone in some reptiles - e.g., the lizard, Anolis carolinensis-may be similar, but its importance in normal animals has not been precisely investigated. Dispersion of either melanosomes or erythrosomes appears to be due to the same hormone.

The chromatosome-dispersing hormone can be extracted from the mammalian pars intermedia or pars glandularis. A substance with similar properties has been recovered from the blood and urine of mammals. If the substance has a function of importance to mammals, this has not been convincingly demonstrated. There probably is no foundation for the belief that melanosome-dispersing hormone can inhibit diuresis.

I5 The eye-stalks of crustacea appear to be homologous with the pars intermedia in this respect. 


\section{CHAPTER IX}

\section{NEW OBSERVATIONS ON THE CHEMISTRY}

\section{AND PHARMACOLOGY OF EXTRACTS}

OF THE PARS NEURALIS

7 HE purpose of this chapter is to review recent investigations of the chemistry and pharmacology of extracts of the pars neuralis. There is no satisfactory additional evidence that more than two hormones-the oxytocic hormone and the vasopressor or diuresis-inhibiting hormone-are secreted by the pars neuralis. The advances which have been made in elucidating the cellular origin of the hormones are described in chapter i. The physiological importance of these hormones is discussed in chapter $\mathrm{x}$.

The chemistry of the active principles. - The isolation of either the oxytocic or the vasopressor principle as a pure, crystalline substance is a goal yet to be attained. Stehle and Fraser (1935) have described new methods for separating and concentrating both hormones. However, these methods are too complicated for detailed review here. The authors found that, after the removal of most of the impurities, both of the hormones are soluble in 96 per cent methanol. Subsequent separation and concentration of the hormones was effected by the addition of ethyl acetate to solutions of both or one hormone in methanol ( 96 per cent) or ethanol ( $c a .9^{8}$ per cent). The pressor hormone was concentrated to about 100 times the value of International Standard Powder, the oxytocic hormone to about I 25 times that value. Therefore, the potency of the preparations of Stehle and Fraser was about the same as that of the extracts of Kamm and his colleagues (1928). Tests for various amino-acids in the purified extracts were reported. The depressor substances in the posterior lobe were studied by Larson (1936), who attributed 
most of the depressor action to histamine. This organic base, of course, was isolated from the posterior lobe years age. ${ }^{\mathrm{I}}$

Gulland and others (1935) have particularly studied the oxytocic principle. They were unsuccessful in attempts to employ adsorbents to effect a high degree of purification. Electrodialysis also was found not to be of value. At $\mathrm{pH}$ 6.0-6.5 or less the hormone migrated toward the cathode; at a higher $\mathrm{pH}$ ( 8 or greater) the hormone remained in the central cell. No ultraviolet absorption spectrum characteristic of the hormone could be detected.

Gulland and Randall, Freudenberg, Weiss, and Biller, and Sealock and Du Vigneaud all published in the same year (1935) evidence that posterior-lobe hormone(s) contain an oxidation-reduction system probably dependent upon a disulphide linkage. According to Gulland and Randall, the $\mathrm{E}^{\prime}$ 。 of the oxytocic principle is -0.025 volts at $\mathrm{pH} 6$. They found the activity of the principle to be 100 per cent in the oxidized state and 50 per cent in the reduced state and offered an interpretation of this finding. Also, they suggested that there may be a second oxidation-reduction system in the molecule of the oxytocic hormone. Sealock and Du Vigneaud concluded that an $-\mathrm{SH}$ group, real or potential (-S-S-group), must be present in active extracts of either oxytocic or vasopressor hormone. Activity was preserved after reduction by cysteine or after reoxidation following such reduction but not after benzylation or methylation of the reduced compounds. The activity of the oxidized forms was not affected by treatment with methyl iodide or benzyl chloride. The activity of the oxytocic and vasopressor principles seems to be less dependent on the state of their oxidation-reduction systems than is the case with insulin.

The assay of the active principles of the pars neuralis.-In addition to the procedures reviewed previously, a few new methods have been employed for the assay of the hormones.

${ }^{I}$ It is not clear whether the che nical studies of Downes and Richards (I935) are of any' significance. 
Sealock and Du Vigneaud (I935) assayed the oxytocic principle by means of its depressor action on the blood pressure of fowls. As the authors point out, troublesome difficulties due to the action of foreign substances (such as those used for chemical manipulation of the hormone) may appear if the isolated guinea pig uterus be employed, whereas these substances may be without effect on the fowl's blood pressure. In addition, several authors have introduced refined methods of determining oxytocic effects both in vitro and in vivo.

For the assay of the vasopressor principle, Simon (I937) recommends that the effect on the blood pressure of the decapitated rat be determined. By this method as little as $0.0025^{-0.005}$ unit (0.00125-0.0025 mg. equivalent of International Standard Powder) can be detected. Small doses of histamine were found to be without action. The most sensitive test for vasopressor (diuresis-inhibiting) hormone is based upon the inhibition of water diuresis in the nonanesthetized dog. If the extract be given intravenously, as little as $0.000 \mathrm{I}-0.0003$ unit may cause a recognizable effect.

The pharmacology of the oxytocic principle. ${ }^{2}-\mathrm{Newton}$ (1934, 1937) found that the cervix of the uterus of the pregnant goat or of the rat and guinea pig in pregnancy or in other stages of sexual activity is very insensitive toward the oxytocic principle in comparison with the uterine cornua. According to the findings of $\mathrm{Li}$ (1935), the isolated fallopian tube of the monkey is much more sensitive toward posteriorlobe extract during the secretory (corpus luteum) or menstrual phases of the uterus than during the proliferative (follicular) phase. Also, spontaneous activity of the fallopian tube tended to be lower during the growth of the ovarian follicle.

${ }^{2}$ The intact, but not the isolated, uterus of the cat, dog, or rabbit relaxes and becomes quiescent in response to the administration of vasopressor hormone. This effect is most readily observed in animals which have received oestrogen previously and is said not to appear if the uterus is pregnant (cat and dog). Robson and Schild (1937) believed that the effect should not be attributed to the vascular action of the hormone (see also Azuma and Kumagai, 1935; Robson, 1936; and Morgan, 1937, as well as earlier reports previously reviewed.) 
Nearly all the other observations on the pharmacology of the oxytocic principle were made to ascertain the uterine response after the secretion or injection of oestrogen or progesterone or both. For example, under the influence of oestrogen the uterus of some species of animals may exhibit a heightened sensitivity toward the oxytocic principle. Abortion, as shown by Parkes in the pregnant mouse, may occur after the administration of oestrogen followed by oxytocic principle, although either substance by itself does not interrupt pregnancy. To some authors (e.g., Druckrey and Bachmann, 1937) it has seemed that these and related observations satisfactorily explain the initiation of parturition and perhaps its continuation. However, the experimental basis for such a belief is far from satisfactory. ${ }^{3}$ 'The later experiments of Marrian and Newton (1935) and of Robson (I935) in pregnant mice fully confirm Parkes's findings. The increased sensitivity to oxy tocic principle after the administration of oestrogen can also be observed in the isolated uterus. ${ }^{4}$ From experiments in other animals ${ }^{5}$ such as the rabbit and man there is also evidence that both spontaneous activity of the uterus and its sensitivity toward the oxytocic principle are increased when follicular hormone (oestradiol) is predominantly affecting the uterus. The specificity of this increased sensitivity has not been proved. For example, ergotamine, like posterior-lobe extract, has been found to elicit uterine contraction more readily during ovarian follicular growth.

Undoubtedly, the uterus of the rabbit is less sensitive toward the oxytocic principle during pseudopregnancy or

3 'The reader is particularly referred to the articles of Marrian and Newton (1935), D'Amour and Dumont (1937), and Robson (1937).

4 The isolated pregnant uterus of the mouse or rabbit is more sensitive toward the oxytocic principle after ovariectomy but not if the uterine contents also have been removed (Robson, I936).

s Robson and Henderson (1936) reported that the oxytocin-sensitivity of the uterus of the hypophysectomized $\operatorname{dog}$ is, if anything, reduced after the administration of oestrin. 
during much of pregnancy - an effect which is usually attributed to the internal secretion of the corpus luteum, progesterone. The effect can be produced in rabbits, spayed immediately after mating, by the injection of $4-5 \mathrm{mg}$. of natural or synthetic progesterone distributed over 5 days (Makepeace, Corner, and Allen, 1936). No corresponding decrease in the sensitivity of the urinary bladder or colon was observed by Podleschka and Dworzak (1936), who used posterior-lobe extract; their results, therefore, have no apparent significance. Robson (I935-36) found that it was difficult to demonstrate any change in the activity of oxytocic hormone on the uterus of pregnant or pseudopregnant rabbits as a result of the administration of oestrogen.

It is probable that the decreased oxytocin-sensitivity of the pregnant or pseudopregnant rabbit's uterus has no general significance. When the uterus of other animals is under the influence of the corpus luteum, there may be no evidence of diminished uterine sensitivity. This is true of the cat (van Dyke and Li, I938), guinea pig (Bell and Robson, I936), mouse and rat (Brooksby, I937). From observations previously reviewed, as well as from new experiments, it may be concluded that the human uterus likewise is not less sensitive toward the oxytocic principle during the luteal phase of ovarian secretion. Although Kraul (1935) and Podleschka ( 1935 ) believed that the oxytocin-sensitivity of the uterus is reduced in the latter part of the menstrual cycle, their findings were not confirmed by the experiments of Kurzrok and others (1937), Miller, Cockrill, and Kurzrok (1937), and Robertson (1937).

Androsterone appears not to affect the sensitivity of the rabbit's uterus toward posterior-lobe extract (van Bokkum, I936).

The pharmacology of the vasopressor hormone apart from its metabolic effects.- Following the intravenous injection of posterior-lobe extract or of an extract containing principally the vasopressor principle, a contraction of capillaries and arteri- 
oles takes place as shown most simply by a rise in the blood pressure. The degree and the duration of the contraction depend to a considerable extent on the dose administered. Moreover, the vascular spasm produced may affect the coronary vessels, especially the left coronary artery, ${ }^{6}$ so that tachycardia, disturbances of conduction, and cardiac dilatation appear. As a result, the blood pressure may fall initially but rises later as the coronary circulation is adequately reestablished and an increased or normal volume of blood is pumped against the increased peripheral resistance. This effect on the heart may or may not be observed, if small doses of vasopressor principle are used. If posterior-lobe extract is injected, it may be absent or less evident, perhaps because the oxytocic principle antagonizes the action of vasopressor principle on the coronary arteries.

According to Watrin and François (1937), repeated subcutaneous injections of posterior-lobe extract may be followed by cardiac hypertrophy in the guinea pig. The doses used (4-10 units on alternate days for $49^{-90}$ days) were large enough to cause convulsions occasionally. The maximum change observed was represented by a cardiac weight of 2.75 gm. (The weight of the heart of control animals was about I. 6 gm.) Enlargement of the heart was found to be due to hypertrophy, not hyperplasia, of the muscle fibers. The toxicity of vasopressor principle is known to be increased after the administration of thyroid extract, chiefly because of a change in cardiac response. Gruber, Moon, and Sufrin (1935) reinvestigated this problem by studying the electrocardiographic abnormalities in non-anesthetized rabbits. The histology of the hearts was also described.

Byrom (I937) injected enormous doses of vasopressor principle (5-40 units once or twice daily for 2 days or longer) subcutaneously into rats. Apparently marked arterial spasm locally gave rise to such pathological changes as infarction and necrosis in the kidneys, liver, etc. Like other authors,

${ }^{6}$ See the recent articles by Frommel and Zimmet (1937). 
Byrom was impressed by the analogy between the effects of the vasopressor principle and the symptoms and pathology of eclampsia. Later (I938), he observed that the prior injection of oestrogen sensitized the rat toward the vasopressor principle, so far as the production of eclampsia-like changes in the kidneys are concerned. The author believed that this later finding strengthens the view that the vasopressor hormone is etiologically important in eclampsia.

Several authors have published new reports on the action of posterior-lobe extract or of purified vasopressor principle on the medulla7. If $5^{-10}$ units be injected intracisternally into the dog, a pressor effect immediately appears, probably because of stimulation of bulbar centers such as the vasomotor center. In comparison with intravenous injection, (I) the pressor effect is less pronounced but persists longer in terms of the rise in blood pressure and (2) a preliminary fall (coronary constriction) is not observed. It is probable that the effects cannot be attributed to the absorption of the hormone into the blood stream.

In the experiments of Daly, Mark, and Petrovskaia (I937) posterior pituitary extracts were added to the blood used to perfuse the lungs of dogs. A suitable period later it was found that there was a reversal of the usual action of epinephrine on the bronchi-i.e., broncho-constriction instead of broncho-dilatation was produced. This effect was prevented by ergotoxine. The extracts, of which "Pitocin" appeared the least active and "Pituitrin" the most active, also potentiated the action of epinephrine on the vascular system of the lungs.

Among new observations on the effects of posterior-lobe extracts on the eye are those of Holtz and Jancke (1936). Only the vasopressor principle was found to cause definite changes. For example, the instillation or subconjunctival injection of a large dose of the hormone (Io units) caused a

7 Van Bogaert, Sacchi (1935); Deleonardi, Seligsohn (1936). 
fall in intraocular pressure and a miosis which was present after the administration of 2 drops of 1 per cent atropine sulphate. The fall in intraocular pressure apparently was due to vasoconstriction.

Reports on the action of posterior-lobe extract or of vasopressor principle on the movements of the gastrointestinal tract appear to require only brief mention. ${ }^{8}$ Posterior-lobe vasopressor extract appears to be of considerable clinical value in stimulating peristalsis of the lower part of the small intestine and of the colon.

It seems unlikely that the enormous doses of vasopressor principle - e.g., 200 units subcutaneously in rabbits - used by Dodds and his colleagues to produce gastric lesions such as hemorrhage and ulceration are of any etiological significance in the formation of such lesions under other circumstances. ${ }^{9}$ Large doses of posterior-lobe extract did not cause gastric lesions in the guinea pig (McFarlane and McPhail, 1937). Various studies of Dionessov (1936), Ejdinova (1936), Langeron, Paget, and Danès (1 936), Merkulow (1936), and Cutting and others ( I 937) on the secretion of saliva, gastric juice, succus entericus, and pancreatic juice indicate that the effect of vasopressor principle is largely or entirely due to its vasoconstrictor action on the blood vessels supplying the glandular secreting cells.

The effects of posterior-lobe extracts on the metabolism of water and minerals.-The important changes in the metabolism of water and minerals following the injection of an extract of the posterior lobe are usually attributed to the vasopressor principle. In anesthetized or, under certain conditions, in non-anesthetized animals, the principal effect is an increased rate of urinary secretion which recently has been attributed to a change in the metabolism of salt, for which water is required for renal excretion. In non-anesthetized

${ }^{8}$ De Biasio (1935), Guthrie and Bargen (1936), Melville, Necheles, and others (1936), and Frazier (1937). See also Schofield and Blount (1937).

9 See also Laporta, Pepe, and Marinelli (1936). 
mammals water diuresis is markedly and specifically inhibited by the vasopressor principle; simultaneously certain salts, particularly $\mathrm{NaCl}$, are excreted at an increased rate. Diuresis inhibition usually is attributed to an increased reabsorption of water by the tubular epithelium of the kidneys (loop of Henle?). In addition, however, Fraser (I 937) has found that the oxytocic principle appears to cause an increased secretion of urine in both "hydrated" and "nonhydrated" rats without clearly affecting the chloride in the urine. Fraser could not demonstrate any diuretic effect of oxytocic principle in the non-anesthetized dog with a fistula of the bladder. (Whether or not water was administered is not stated.)

The observations of Unna and Walterskirchen (1935-36) were made in non-anesthetized dogs with a permanent fistula of the bladder. Of particular interest is their report on Leerdiurese. The animals ${ }^{10}$ were fasted i $6-20$ hours but were allowed water until 2 hours before the experiment. The injection of as little as $0.0 \mathrm{I}$ unit of posterior-lobe extract subcutaneously or $0.000 \mathrm{I}$ unit intravenously could cause a definite increase in the excretion of $\mathrm{Cl}$. If a diuresis appeared, it was thought to be caused by the $\mathrm{Cl}$ excreted. Both the $\mathrm{Cl}$ excretion and the diuresis were higher if the diet was high in $\mathrm{Cl}$; likewise they were low in animals on a low $\mathrm{Cl}$ diet. Also Melville ( I 936) was of the opinion that a "salt-mobilizing" action is responsible for any diuretic action of vasopressor hormone. In anesthetized or non-anesthetized dogs, the diuretic effect of extract could be increased by the administration by mouth or by intravenous infusion under anesthesia of solutions of $\mathrm{NaCl}, \mathrm{KCl}$, or $\mathrm{KNO}_{3}$ but not $\mathrm{Na}_{2} \mathrm{SO}_{4} \cdot{ }^{.1}$

Further investigations of the mechanism of the diuresis-

${ }^{10}$ Dogs weighing 6-I $2 \mathrm{~kg}$.

${ }^{11}$ See also Frey (1937) for a discussion of factors influencing the diuretic action of posterior-lobe extract. Frey believed that increased glomerular filtration accounts for the change. It is not possible to state that this view is correct. 
inhibiting effect of vasopressor principle (either as purified principle or as a simple extract of the pars neuralis) have been reported in the past few years by Samaan (1935), Handovsky and Samaan (I936), and Walker, Schmidt, Elsom, and Johnston (1937). Samaan confirmed previous observations on the lack of effect of renal denervation on diuresis inhibition due to the hormone. He found that posterior-lobe extract could cause very constant effects under standardized conditions. The extract caused no effect on the secretion of urine in dogs receiving repeated large doses of a solution of urea. The other authors reported on the behavior of the renal blood flow in non-anesthetized dogs and rabbits and in anesthetized dogs as revealed by the thermostromuhr. It appears that Handovksy and Samaan injected extract intravenously and found that the renal blood flow might or might not diminish during diuresis inhibition because of the extract. Walker and his colleagues used only non-anesthetized dogs and rabbits into which they injected extract subcutaneously. No change in renal blood flow occurred in association with the inhibition of diuresis.

If a large enough dose of vasopressor principle is injected subcutaneously into the non-anesthetized animal, hydremia is observed as in the experiments of Yanagi (1936). The author also found that the concentration of $K$ in plasma is increased ${ }^{2}$ and suggested that vasopressor extract brings about a transfer of water and $K$ from the interior of cells to the extracellular fluid. ${ }^{3}$ Anemia due to the injection of large doses of posterior-lobe extract was first reported by Dodds and his colleagues who used rabbits. It can also be produced in normal or hypophysectomized guinea pigs (Mc-

12 According to Blazsó (1936), this change is observed in anesthetized animals, whereas the opposite change--i.e., decreased concentration of $\mathrm{K}$ in serum-takes place in the absence of anesthesia.

${ }_{3}$ The skin, which is important as a depot for chlorine, is believed by Toxopéus (1935) to deposit less of the element after the administration of $\mathrm{NaCl}$ if posteriorlobe extract also is injected. In addition, the author studied the chlorine of muscle without much success. 
Farlane and McPhail, I937). Gilman and Goodman (1935, I937) attributed the effect to marked water retention, so that dilution of the blood renders the environment of the erythrocytes so unfavorable that a hemolytic anemia is produced. Using rats, Arnold and Marx (1937) observed hemoglobinuria which they also believed to be due to intravascular hemolysis. Arnold and Marx administered subcutaneously I 5 units of posterior-lobe extract for each $100 \mathrm{gm}$. of bodyweight.

In patients with edema chiefly caused by renal disease considerable excess water can be removed during the diuresis which follows diuresis inhibition induced by vasopressor principle (McQuarrie, Thompson, and Ziegler, 1936). "Pitressin" was repeatedly injected, until the retention of water was represented by an increase of $3-4$ per cent in weight. Administration of the hormone was then stopped abruptly. During the subsequent diuresis, edema fluid often was removed, especially if the diet was low in $\mathrm{NaCl}$. The balance of $\mathrm{NaCl}$ was negative during both phases of renal secretion, whereas that of $\mathrm{K}, \mathrm{Ca}, \mathrm{P}$, and $\mathrm{N}$ appeared not to be effected. ${ }^{\mathrm{I}}$

It will be recalled that, if frogs are kept in water, the injection of posterior-lobe extract promotes the transfer of water through the skin (and perhaps its retention), so that the body-weight increases. In confirmation of earlier work, Oldham (1936) found that the oxytocic principle is $2.5-5.0$ times as effective as the vasopressor hormone in causing this effect. The chromatosome-dispersing hormone probably is not a factor (Jones and Steggerda, I935; Oldham, 1936). There is no agreement as to the action of posterior-lobe extract on the kidneys of the frog. Rey (1935) concluded that diuresis inhibition can be produced in either dry or wet frogs by a large dose of extract (about I unit injected into the

${ }^{{ }^{4}}$ See also the preliminary report of Smith and Mackay (1936), who studied the action of posterior-lobe extract on the intake and urinary excretion of $\mathrm{NaCl}$ in a normal man and in a patient with diabetes insipidus. Beltrametti (1935) believed that oestrogens or androgens favor the secretion of vasopressor hormone and thus are useful in the treatment of diabetes insipidus. 
lymph sac). On the other hand, Granaat and Hillesum (1937) reported that doses of "Pitressin" which cause retention of water absorbed through the skin were without appreciable effect on the secretion of urine. If posterior-lobe extract produces anuria in frogs, it probably arrests the circulation of blood in the glomeruli (Adolph, 1936).

The effects of posterior-lobe extracts on the metabolism of carbohydrates.- It is not yet known to what substance the principal effects of posterior-lobe extract on carbohydrate metabolism should be attributed. In the rabbit, at least, extracts rich in the vasopressor principle usually appear to be the most powerful both in causing an elevation of the level of the blood sugar and in antagonizing the hypoglycemic action of insulin or the hyperglycemic action of epinephrine. ${ }^{15}$ In this last case the vasopressor hormone perhaps facilitates liberation of insulin; however, there may be a simpler explanation such as an effect on the absorption of epinephrine. According to Ellsworth (1935-36), who confirmed the results of others in rabbits, it is the oxytocic fraction and not the vasopressor fraction which is responsible both for the production of hyperglycemia and for the antagonism of insulin in the dog. Moreover, Ellsworth found that the effect of the purified oxytocic extract in the dog was produced by much smaller doses (in terms of units) than are necessary to cause corresponding changes in the rabbit, which apparently is sensitive only to the vasopressor principle. Further studies are greatly needed to decide conclusively whether or not the action on carbohydrate metabolism is caused by some substance not identifiable otherwise.

Experimental work in animals indicates that hyperglycemia or insulin antagonism caused by posterior-lobe extract is the result of an action on the liver from which an increased quantity of glucose is liberated into the hepatic veins and thence into the general circulation because of glycogenolysis

${ }^{x 5}$ See the recent article of Gurd (1934). 
(see reports such as those of Clark, 1928, and of Högler and Zell, 1935). However, it is the opinion of Cohen and Libman (1937), who performed their experiments in healthy men, that posterior-lobe extract lessens the utilization of glucose by the tissues and thus antagonizes the action of insulin. This belief arises from the following observations and reasoning: in comparison with the results of injecting only glucose, the injection of both glucose and posterior-lobe extract is followed by a longer and more pronounced rise in the level of the sugar of the blood; at the height of the effect the arteriovenous difference in the concentration of sugar in the blood is reduced; the arteriovenous difference in the level of the blood sugar is an index of the activity of insulin, which should be secreted at an increased rate in response to the hyperglycemia.

According to Ferrannini (I937), poisoning by phlorhizin in the rabbit affects only slightly the diuresis-inhibiting action of posterior-lobe extract. The latter in turn appears not to influence the glycosuria. ${ }^{16}$

Other effects of posterior-lobe extracts on metabolism.-Bühler (1935) reported that oxytocic extract can cause creatinuria in dogs or rabbits to disappear. In the toad, Xenopus laevis, neither the injection of posterior-lobe extract nor the removal of the pars neuralis seems to affect the creatine of muscle (Shapiro and Zwarenstein, 1937).

Blotner (I935) concluded that posterior-lobe extracts, after administration intranasally, prevent the rise- or even cause a fall-in the concentration of cholesterol in the plasma of patients with obesity or diabetes insipidus to whom $500 \mathrm{cc}$. of "20 per cent" cream had been given.

Some recent observations on the metabolism of the principles of the pars neuralis.-As is the case with anterior pituitary gonadotropic extracts, certain salts of metals delay absorption and thus prolong the action of the hormone(s). Dodds

${ }^{16}$ The effect of posterior-lobe extract on the glucose, $\mathrm{Cl}$, and protein of the blood of normal and diabetic persons was investigated by Dell'Acqua (1935). 
and others (1937) demonstrated this phenomenon by observing the diuresis-inhibiting action of posterior-lobe extract with and without the addition of acetates of $\mathrm{Zn}, \mathrm{Ni}$, or $\mathrm{Cd}$ to the extract.

Jones and Schlapp (1936) found that both principles are metabolized alike after their intravenous injection in a large dose (about 20 units per kilogram body-weight) into decapitated cats. Within 20 minutes most of the hormones $(85$ per cent) had disappeared from the blood; there was none present after 2 hours. About 30 per cent of the extract in terms of vasopressor hormone could be recovered from the urine. The work of Heller (1937) indicated that, after the intravenous injection of posterior-lobe extract into the rabbit (and rat), the vasopressor hormone, measured by its diuresisinhibiting effect, is excreted in the urine up to about 30 minutes after injection. Apparently, the amount excreted is not proportional to the dose but is limited because of unknown factors. Consequently, the higher the injected dose, the lower is the proportion of hormone recovered in the urine. Heller has found that blood adsorbs the hormone and has concluded that the kidney liberates the adsorbed hormone which is excreted at its point of action, the renal tubules. ${ }^{17}$

Certain tissues, especially the liver, can rapidly adsorb vasopressor hormone. According to Heller and Urban (1935), the adsorbing substance is heat labile and can be removed by animal charcoal. The hormone can be released from the adsorbent by boiling in saline solution. In addition, blood or liver contains an enzyme-like substance which destroys the hormone. Destruction by such a mechanism is rapidly accomplished by human blood, but not by cerebrospinal fluid. About $0.025^{-0.05}$ unit per cc. of blood is thus inactivated after I.5-2 hours (see also Jones and Schlapp, I936; and Heller, I937).

${ }_{17}$ See also pp. $265-66,278-79$. 
Only two active principles have been identified as characteristic secretions of the pars neuralis: the oxytocic principle and the vasopressor or diuresis-inhibiting principle. It is not certain whether there is a third principle affecting carbohydrate metabolism. The oxytocic and vasopressor principles have been markedly concentrated and nearly completely separated from each other but are not yet available as pure substances. In both, an oxidation-reduction system dependent upon the disulphide linkage appears to be present; however, the state of this system seems to be much less important in determining activity than is true of insulin.

The physiological importance of these hormones is discussed in chapter $\mathrm{x}$.

The oxytocic principle is usually more effective when the uterus is under the influence of oestrogen. In the rabbit the activity of the principle is markedly reduced if the condition of the uterus corresponds to that of pregnancy (except near term) and pseudopregnancy. However, this relationship is not found in other animals, including, probably, man. The important vascular effects of oxytocic principle appear to be (I) a depressor effect in birds and (2) possibly a relaxing effect on the coronary arteries. Recently it has been reported that the principle can promote diuresis in the rat.

The vasopressor principle is most important, not for its pressor action, but because it brings about water retention by facilitating the tubular reabsorption of water in the kidneys. It is difficult to describe the significance of its action on mineral metabolism. The hormone usually increases the rate of excretion of sodium chloride especially. Some evidence suggests that actions on tissues other than the kidneys are important; however, the findings so far are inconclusive. Larger doses of the vasopressor hormone are required to elicit a pressor effect, which initially may be masked by a fall in blood pressure caused by coronary constriction, which 
in turn may markedly reduce the efficiency of the heart. Stinulation of peristaltic movements of the lower intestine and colon is produced by the vasopressor hormone. Also, the vasopressor hormone may cause an inhibition of movements and a relaxation of the uterus in situ. Very large doses of vasopressor hormone may cause anemia or lesions of the gastrointestinal tract. These effects probably are not of physiological interest. In the case of anemia, the change perhaps is due to facilitation of hemolysis by water retention. Lesions, especially of the stomach, probably are the result of a prolonged spasm of the organ's arterial supply.

Posterior-lobe extract may affect carbohydrate metabolism by (1) producing a moderate hyperglycemia, (2) antagonizing the hypoglycemic action of insulin, and (3) antagonizing the hyperglycemic action of epinephrine. The explanation of these phenomena cannot be satisfactorily given after a consideration of the data which have been published.

It is not yet possible to ascribe consistent effects on the metabolism of lipoids or proteins to extracts of the pars neuralis. 


\section{CHAPTER X}

\section{THE PARS NEURALIS AS A GLAND OF INTERNAL, SECRETION}

7 HE belief that the pars neuralis is an important gland of internal secretion has been greatly strengthened by recent experimental work on the physiological importance of the diuresis-inhibiting (or vasopressor) principle, confirming the earlier views of Starling and Verney, to mention only two of many investigators. Some of the reported experiments also indicate that secretion(s) of the pars glandularis is responsible or necessary for the marked polyuria and polydipsia accompanying the suppression of pars neuralis secretion. On the other hand, the importance of a secretion of the oxytocic principle has not yet been demonstrated and no data of great significance have been added to those previously reviewed. To a less extent this is also true of the possibly important vascular effects of the vasopressor principle.

Recent attempts to detect the active principles of the pars neuralis in cerebrospinal fuid or blood. New studies of the principles in the pituitary body.-Attempts to detect the active principles of the pars neuralis in cerebrospinal fluid have not met with convincing success. In a recent report Deleonardi (I 936) concluded that oxytocic, pressor, and diuresisinhibiting effects can be produced both by the cisternal fluid of the dog and rabbit and by the ventricular fluid (second and third ventricles) of the human cadaver but only exceptionally by human lumbar fluid. His observations on the oxy tocic and pressor effects were not adequately controlled, because he failed to take into account the oxytocic effect of calcium ions in cerebrospinal fluid (he mentions that he employed a Tyrode's solution low in calcium for the uterine bath) and 
because he did not recognize the possibility that cerebrospinal fluid may contain a pressor substance which is not identical with that of the pars neuralis (Page, I935 and later). (The pressor substance described by Page is without effect after the complete destruction of the central nervous system.) Therefore, there remains from Deleonardi's observations the suggestion that cerebrospinal fluid may produce in rats, in a dose of I cc. per hundred gm., a slight diuresisinhibiting effect similar to that following minute doses of pars neuralis extract. Levitt's observations ( 1936 ) must be added to those of others who, contrary to Anselmino and Hoffmann, have been unable to detect the diuresis-inhibiting (vasopressor) hormone in the blood of patients with hypertension or eclampsia. Simon (1937), using Marx's method, was unable to extract detectable quantities of vasopressor hormone from $100-200 \mathrm{cc}$. of human or canine blood (see also Neubach, 1937). The oxytocic effects of blood-extracts (women in second stage of labor, the pregnant cow, the rabbit before and after injection of pars neuralis extract) have been further investigated by Bell and Robson (1935). They concluded that the oxytocic effects of such extracts, if present, probably were neither caused by the oxytocic principle of the pars neuralis nor related to parturition.

Fisher and Ingram (1936) have shown that the atrophic pars neuralis of cats with diabetes insipidus due to a sufficiently extensive lesion of the supraoptico-hypophysial system contains only one-tenth or less of the normal total amount of the principal hormones of the pars neuralis. The concentration of active principles in the pars neuralis cannot be calculated from their data. The results of Fisher and Ingram are given in Table 9 .

Jores (I 935) reported that, if guinea pigs are kept in darkness for about 6 hours, the concentration of oxytocic and vasopressor principles in their pituitary bodies increases markedly. This observation was not confirmed by Simon (1936), who used guinea pigs, rabbits, and rats for his ex- 


\section{THE PITUITARY BODY}

periments. Simon also found that the amount of hormones in the gland is not disturbed by the injection of posteriorlobe extract, thyroid extract, thyrotropic hormone extract, or insulin. Likewise, the amount of the vasopressor principle in the male guinea pig's pituitary is not altered following the injection of large doses of oestradiol benzoate or thyroxine (Schockaert and Lejeune, I 935). Determinations of the amount of oxytocic principle in the pituitary of adult and fetal sheep and pigs were made by Bell and Robson (I 937). In terms of international units the pituitary of the adult sow contained $2-4.5$ times as much oxytocic principle as that of

TABLE 9

\begin{tabular}{|c|c|c|c|}
\hline & \multicolumn{3}{|c|}{ Total Units in Pituitary of } \\
\hline & Pressor Activity & $\begin{array}{l}\text { Diuresis-inhibiting } \\
\text { Activity }\end{array}$ & Oxytocic Activity \\
\hline 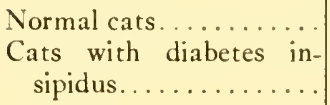 & $\begin{array}{l}5.4-7.5(3)^{*} \\
0.25-0.39(4)\end{array}$ & $\begin{array}{r}+.0(1) \\
0.23-0.29(2)\end{array}$ & $\begin{array}{l}2.2-3.5 \quad(3) \\
0.18-0.25(4)\end{array}$ \\
\hline
\end{tabular}

* The number of cats is indicated by the numerals in parentheses.

the adult ewe ( $14-32$ international units compared with 7$)$. The maximum amount of the principle in the gland of newborn animals ranged from 3-7 per cent of the figure for adults. The pituitary of young fetuses often contained much less.

Bickenbach (1936) could find no difference in the diuresisinhibiting (and chloride-secreting) effects of the pituitaries of 2 patients with eclampsia compared with the effects of 2 normal human pituitaries.

The vasopressor principle. ${ }^{\mathrm{I}}$ A. As a regulator of water metabolism in the body.--Ranson and his co-workers have called attention to the work of von Hann (I9I8) who investigated the pathology of diabetes insipidus in several human cases

${ }^{1}$ Nearly all the evidence indicates that the diuresis inhibiting effects of pars neuralis extracts are due to the vasopressor principle.

$$
\text { [ } 276 \text { ] }
$$


and compared his results with the findings of others. Von Hann concluded that diabetes insipidus in man can occur only if (1) the pars neuralis is destroyed or severely damaged, (2) the pars glandularis is functionally active, and (3) there is no serious disorder of the heart and kidneys. Richter (1934) also maintained that diabetes insipidus in rats is readily produced, if all the posterior lobe but only part of the anterior lobe is removed. The majority of the recent reports support the von Hann-Richter hypothesis that typical diabetes insipidus requires the abolition of pars neuralis secretion but the persistence of pars glandularis secretion.

According to this hypothesis the development of diabetes insipidus may proceed in the following way. Either by disease or by experiment the internal secretion(s) of the pars neuralis no longer escapes into the blood or body-fluids. This may be accomplished by interruption of the secretory fibers from hypothalamic nuclei to the pars neuralis as by injury to the nuclei or to the supraoptico-hypophysial tract in the tuber cinereum or stalk, or by removal or destruction of the pars neuralis itself. Abnormal depletion (polyuria) of the body water then occurs, because the vasopressor hormone, which by its effect on the loop of Henle normally insures an adequate reabsorption of water during the secretion of urine, no longer finds its way into the blood. Compensation is then effected by the drinking of large amounts of water (polydipsia). Why, then, is a normally secreting pars glandularis also necessary for the maintenance of diabetes insipidus? Presumably a normal rate of formation of glomerular filtrate in the kidneys depends upon normal metabolic activity, which in turn depends to an important extent on the glands of internal secretion, particularly the thyroid. If the anterior pituitary, without whose secretion the principal other endocrine glands become inactive, is also removed, metabolic activity is reduced and probably abnormal. Consequently, a marked reduction in the volume and rate of formation of glomerular filtrate takes place, so that the lack of an adequate 
reabsorption of water is masked and no clear-cut polyuria and polydipsia occur. A view favored by many is that the thyrotropic hormone, by its indirect effect on the thyroid, is chiefly responsible for the maintenance of a normal rate of glomerular filtration. ${ }^{2}$ In the pages which follow the evidence in favor of the hypothesis just outlined as well as some observations to the contrary will be reviewed. Some of the best observations have been reported only recently and are impressive because of the care with which they have usually been made.

I. The secretion of the vasopressor principle as a means of preventing dangerous loss of body water.-Gilman and Goodman (1936-37) performed a series of interesting experiments in rats. They found that, if water and food were withdrawn from normal rats, the rate of secretion of the urine, of course, fell, depending upon the period of thirst. At the same time diuresis-inhibiting effects, analogous to those produced by pars neuralis extracts, could be secured by injecting suitably prepared urine. The amount of diuresis-inhibiting principle present increased as the period of water withdrawal was prolonged, so that the equivalent of as much as 5 units of hormone was excreted by 20 rats during a period of 72 hours without water. ${ }^{3}$ No detectable amounts of the principle could be discovered in the urine of rats drinking water ad libitum. If rats were hypophysectomized and then subjected to water-withdrawal, they secreted three times as much urine as normal rats treated similarly; no diuresis-inhibiting hormone could be found in their urine. Control experiments indicated that the procedure used was suitable for identifying diuresis-inhibiting (vasopressor) hormone. Moreover, the diuresis-inhibiting substance in the urine of rats receiving

${ }^{2}$ Another possibility, in favor of which there is little evidence, is that a secretion of the pars glandularis has diuretic effects not mediated through another gland of internal secrecion. White and Heinbecker suggested that a secretion of the thyroid sensitizes the animal toward an anterior pituitary diuretic factor.

3 Diuresis caused by the administration of a solution of $\mathrm{NaCl}$ to rats from which water was witheld likewise was characterized by the rapid appearance of diuresisinhibiting principle in the urine. 
no water was destroyed in strong solutions of acid or alkali, as is that in an extract of the pars neuralis. The conclusions of Gilman and Goodman from these experiments seem logical. The amount of vasopressor or diuresis-inhibiting principle secreted by the pars neuralis fluctuates with the body's need for water conservation. Normally, the amount secreted is so small that none can be detected in the glomerular filtrate. If there is a shortage of water, its retention in the body is largely due to a reduction in the volume of urine secreted. And the emergency secretion of an increased amount of diuresis-inhibiting hormone by the pars neuralis is perhaps the important means of accomplishing this by increasing the reabsorption of water in the renal tubules. The unusual amount of hormone secreted filters in part through the glomeruli and can then be detected in the urine.

2. The importance of hypothalamic nuclei, especially the supraoptic nuclei, for maintaining the secretion of the vasopressor principle.-In several recent reports of experiments in cats and monkeys, Fisher, Ingram, and Ranson ${ }^{4}$ have shown that the supraoptic nuclei of the hypothalamus may be essential not only to liberate suitable amounts of vasopressor hormone - to prevent undue loss of water by way of the kidneysbut also to maintain the pars neuralis anatomically. Cats were used for most of their experiments. Employing the Horsely-Clarke stereotaxic instrument as a means of placing lesions in different parts of the hypothalamus, they produced a severe permanent diabetes insipidus in a number of cats. ${ }^{5}$ Later, sometimes after months of severe diabetes insipidus, the animals were killed, and the hypothalamus and pituitary

4 Fisher, Ingram, Hare, and Ranson (1935), Fisher, Ingram, and Ranson (1935), Fisher and Ingram (1936), Ingram and Fisher (1936), Ingram, Fisher, and Ranson (1936).

5 The diaberes insipidus appeared about 8-I2 days after a suitable lesion(s) had been made. Polyuria appeared to precede polydipsia (see also Fisher, Magoun, and Hetherington, 1938). In addition, a transient polydipsia and polyuria were commonly observed immediately after operation. These initial transient changes have been observed by a number of authors using different animals. Usually they are attributed to an effect on the nervous system alone. 
body were carefully studied anatomically to determine how the lesions found could be correlated with animals' symptoms. As a result of this study, the authors have reached the following conclusions: ${ }^{6}$ ( $\mathrm{I}$ ) diabetes insipidus is due to a deficiency of pars neuralis secretion, presumably the vasopressor principle; (2) the secretion of the pars neuralis is not formed and liberated unless at least one supraoptic nucleus is functionally active and connected with the pars neuralis. The fibers from the supraoptic nuclei to the pars neuralis appear to be efferent. If the tract(s) is interrupted, the nerve cells of the nuclei degenerate. The anatomical changes in the hypothalamic-hypophysial region accompanying diabetes insipidus may be due to $(a)$ a lesion causing destruction of both supraoptic nuclei, $(b)$ a lesion interrupting the tract fibers arising from both nuclei as in the tuber cinereum or the stalk, or $(c)$ destruction or removal of the pars neuralis; lesions $(a)$ or $(b)$ are followed by atrophy of the pars neuralis. (3) The pars tuberalis and probably the pars intermedia are of no significance in the genesis and maintenance of diabetes insipidus; and, finally, (4) the pars glandularis probably is important or essential, if pronounced diabetes insipidus occurs.

The relationship of hypothalamic nuclei to the pituitary as well as the most convenient position in which suprapituitary lesions can be placed to produce diabetes insipidus are illustrated in Figures 27 (cat) and 4 (monkey). Destructive lesions of the mammillary bodies or of nuclei other than the supraoptic nuclei (such as the paraventricular nuclei, the ventrolateral hypothalamic nuclei, etc.) are not followed by diabetes insipidus.

Farr, Hare, and Phillips (1937), using cats, have confirmed the observations of Ranson and his colleagues. In reports of the anatomical changes in 4 human cases of diabetes in-

${ }^{6}$ The conclusions refer to the experiments of Fisher, Ingram, and Ranson. The authors do not hold that other mechanisms for producing diabetes insipidus-especially in other animals - do not exist. 


\section{PARS NEURALIS AND INTERNAL SECRETION}

sipidus, Biggart (1935-36) also observed changes in the supraoptic nuclei and stalk consistent with the views expressed by Fisher, Ingram, and Ranson. There is little evidence to

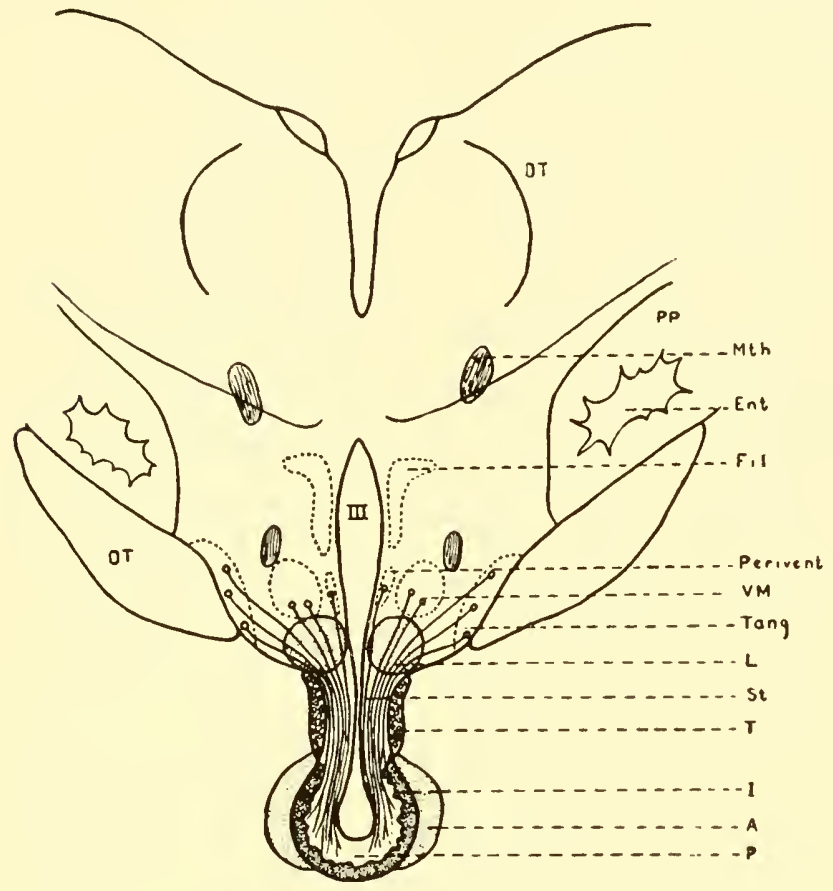

Fig. 27.- One position in which bilateral lesions of the hypothalamus can be placed to produce diabetes insipidus in the cat by interrupting fibers of hypothalimic hypophysial tracts. (From Fisher, Ingram, and Ranson, Arch. Neurol. Psychiat., 34, 1 $2.4-63$ [1935].)

.1: pars glandularis; Ent: entopeduncular nucleus; Fil: filiform (paraventricular) nucleus; $I$ : pars intermedia; $L$ : lesion as stippled area; $M$ th: mammillothalamic tract; OT: optic tract; $P$ : pars neuralis; Perivent: periventricular nucleus; $P P$ : pes pedunculi; $S t:$ stalk; $T$ : pars tuberalis; Tang: caudal part of tangential (supraoptic) nucleus; VM: ventromedial hypothalamic nucleus.

favor Biggart's opinion that the pars tuberalis is etiologically important in diabetes insipidus. There is still less justification for his belief that diuresis inhibition by the vasopressor principle depends to an important extent on an action of the hormone on tuberal nuclei (in Biggart's Case 5 the polyuria 
"was not controlled by injections of pituitrin"). The report of Adlersberg and Friedmann (1935) deals with changes in the metabolism of water, under various conditions, in patients with lesions of the mesencephalon or diencephalon or both. In some instances the response to posterior-lobe extract was clearly diminished. Keller, Noble, and Hamilton (1936) stated that a "complete" separation of the pituitary from the hypothalamus (including "the greater portion of the infundibulum") in the dog is not followed by any increased water or food consumption by the animals. More recently, Keller and Hamilton (1937) observed complete degeneration of the nerve fibers in the pars neuralis as a result of lesions of the tuber cinereum in 7 cats. In 4 of the cats there was no diabetes insipidus; the authors concluded that degeneration of the nerves of the pars neuralis - contrary to the views of Ranson and his colleagues - is not necessarily accompanied or followed by diabetes insipidus. Other data of less recent origin, some of which also can be interpreted as opposing the beliefs of Fisher, Ingram, and Ranson, have been reviewed already (van Dyke, 1936).

3. To what extent does diabetes insipidus depend upon a functioning pars glandularis? - The hypothesis of von Hann, supported by the later experimental work of Richter, has already been discussed (pp. 276-77). It is proposed here to review briefly a number of recent communications bearing on this question.

In a few reports diabetes insipidus - or the secretion of an increased volume of urine - has been observed in completely hypophysectomized animals (toad: Pasqualini, I935; rat:7 Dodds, Noble, and Williams, I937; dog: Page and Sweet, Reichert and Dandy, 1936). On the other hand, Buratschewski and Rappoport (1936) found that the metabolism of salt and water in the dog was not disturbed by either hypophysectomy or the removal of the pars neuralis. Such data contradict most of the recent findings of others.

7 See also Sandberg, Perla, and Holly (1937). 
The work of Pencharz, Hopper, and Rynearson (1936) in the rat as well as that of Keller, Noble, and Hamilton (1936) and of White and Heinbecker (1937) in the dog and that of Dodds and his colleagues in the cat (1937) indicate that diabetes insipidus follows the excision of the pars neuralis, but that it promptly ceases if the pars glandularis is later removed. Pencharz and others were unable to provoke polydipsia in completely hypophysectomized rats by repeatedly administering homo-implants $(\mathrm{I}-3$ anterior lobes on alternate days for 3 weeks). However, Keller (I937) observed a dog whose diabetes insipidus disappeared as a result of complete hypophysectomy. Inasmuch as the diabetes insipidus reappeared following the administration of anteriorlobe extract or the feeding of thyroid extract, Keller concluded that the maintenance of polyuria in dogs with an intact anterior lobe is due to the thyrotropic hormone. White (I 937), like Pencharz and his colleagues, was unable to cause, by the administration of anterior pituitary extract, ${ }^{8}$ any convincing change in the amount of urine secreted by rats from which the pars neuralis or the whole pituitary had been removed a year previously. The administration of thyroid extract with or without the injection of anterior-lobe extract likewise was without significant effect. ${ }^{9}$ Other experiments of White and Heinbecker (I 937) in dogs led to the conclusion that the pars glandularis secretes a diuretic principle which is not thyrotopic hormone but, nevertheless, at first is ineffective in the absence of the thyroid. After the removal of the thyroid from the dog, the diuretic effect of anterior pituitary extract gradually reappeared, perhaps, as the authors suggest, owing to regeneration of thyroid tissue or to some unknown readjustment. Hypophysectomized or normal dogs were about equally sensitive toward the diuretic

${ }^{8}$ White injected an acid extract of beef anterior lobe.

9 Farr, Hare, and Phillips (1937) stated that cats with diabetes insipidus caused by lesions of the supraoptico-hypophysial tract exhibited a pronounced increase in the polyuria, if a saline suspension of beef anterior lobe had been injected. 
action of anterior pituitary extract. ${ }^{\mathrm{IO}}$ Thyroid extract caused no change in the metabolism of water either in thyroidectomized or in hypophysectomized-thyroidectomized dogs. As a result of their experiments in cats and rats, Dodds, Noble, and Williams (1937) also suggested that the diuretic action of anterior pituitary secretion after the removal of the posterior lobe is not due to thyrotropic hormone.

Evidence from other types of experiments speaks more in favor of than against the belief that a secreting thyroid gland is responsible to an important extent for the polyuria of diabetes insipidus. It is necessary also to bear in mind that the importance of the thyroid gland may vary in different animals. Mahoney and Sheehan (1935) occluded the pituitary stalk in dogs by means of a silver clip. The subsequent severe polyuria and polydipsia could be abolished by thyroidectomy and later re-established by the feeding of thyroid extract. The removal of the thyroid in cats with diabetes insipidus caused by a lesion of the supraoptico-hypophysial system "may somewhat reduce but does not abolish the polyuria" (Fisher and Ingram, I936; Ingram and Fisher, 1937). On the other hand, the feeding of thyroid to such cats causes a marked diuresis; after the withdrawal of thyroid-medication, the polyuria may be as great as before thyroidectomy. Findley and Heinbecker (1937) completely removed the thyroid from a man with syphilis of the central nervous system and with diabetes insipidus. The important changes observed subsequent to the operation were some reduction of the polyuria when the amount of salt in the diet was high or unrestricted and some increase in the diuresis-inhibiting effect of "Pitressin" (see also Findley, I937).

4. Miscellaneous observations.-According to Pickford (1936) as well as others, the action of posterior-lobe extract after intravenous administration is, within certain limits, inversely proportional to the "water load" of the body. This

so The extract sometimes caused an initial decrease in the rate of formation of urine. This always occurred in the monkey and might be the only change observed. 


\section{PARS NEURALIS AND INTERNAL SECRETION}

conclusion was based upon experiments in dogs with intact pituitaries. Presumably more vasopressor hormone was secreted as the water load diminished.

By means of acute experiments in rabbits which had received neither food nor water for $24^{-4} 8$ hours, Janssen (I 935) found that afferent stimuli could cause an increased rate of secretion of urine in which the concentration of chloride rose. The fact that this effect occurred after renal denervation led to the conclusion that it was due to the reflex secretion of a hormone. Theobald and Verney (1935) measured the action of afferent stimulir on the secretion of urine by the denervated kidney of the dog in which diuresis was provoked by water. Inhibition of diuresis was readily produced and sometimes persisted for 5-20 minutes after the removal of the stimulating agent. The authors concluded that the diuresis inhibition was not caused by epinephrine and suggested that the hormone responsible for the effect was secreted by the pars neuralis. This suggestion seems logical and is in agreement with both the earlier work of Verney and his colleagues and with nearly all recent observations.

Brull (1937) concluded that an unknown pituitary principle (not those recognized in the pars neuralis and neither gonadotropic nor thyrotropic hormone) can lower the threshold of renal excretion of inorganic phosphate, provided that the parathyroid hormone is also present.

The vasopressor principle. B. As a regulator of the cardiovascular system.-Blount (1935) transplanted two pituitary anlagen (including the future pars neuralis) into individual urodele larvae, Amblystoma punctatum, and concluded that the symptoms appearing later were analogous to hypertension in man. He based this conclusion particularly on the reduction in the size and number of the peripheral capillaries associated with "labored attempt(s) at propulsion of corpuscles." and an "increased back-flux of diastole." The heart

II E.g., by the insertion of a hypodermic needle in the region of the fourth lumbar interspace. 
rate was found to be reduced; also, there were enlargement of the heart and hypertrophy of the ventricular wall. Because of contraction of the vessels, the spleen was less than half the normal size. The glomeruli of the kidneys resembled those of hypertension in man. The profound peripheral vascular disturbance apparently interfered with the growth of some of the peripheral structures. Blount also had the impression that the vascularity of the structures studied was increased after hypophysectomy.

All the other observations indicating that the cardiovascular effects of pars neuralis secretion may be physiologically important have been made in mammals. Chang and his colleagues ( I937) discovered that stimulation of the central end of the vagus in the dog whose head is connected with the body only by blood vessels, causes a rise in blood pressure resembling that following the injection of vasopressor principle. The effect could be abolished either by hypophysectomy or by cutting the pituitary stalk. Thus it appears that a reflex secretion of vasopressor principle can be demonstrated in the dog. The vagus nerve is the only part of the afferent arc known at present; probably the terminal efferent arc is the supraoptico-hypophysial tract. The experiments of Schockaert and Lambillon (I935-36), although of more deductive significance, are also of considerable interest. They showed that the serum of pregnant women seemed to bind or inactivate, i.e., prevent, the vasopressor effect of a pars neuralis extract in the decapitated cat. Later they demonstrated that the intravenous injection of 5 units of a purified solution of the vasopressor principle ("Tonephin") caused much more severe symptoms such as precordial and abdominal pain, vomiting, and involuntary defecation in normal women than in women in the last third of pregnancy. The average elevation of the systolic blood pressure in non-pregnant women was $43 \mathrm{~mm}$. Hg. ${ }^{\mathrm{I}}$ In the pregnant women, the

I2 In spite of the recent statement of Gilman and Goodman (1937) that “. . . in unanesthetized dogs and humans, pituitary extract is not pressor," this observation 
injection caused an average elevation of $15 \mathrm{~mm}$. $\mathrm{Hg}$ in the systolic blood pressure; only rarely were symptoms other than paleness of the face and cyanosis of the lips present. These observations suggest that the diminished response of pregnant women to the vasopressor principle is due to a substance liberated in to the blood. Whether or not a disturbance in its formation may be etiologically important in eclampsia, as Schockaert and Lambillon suggest, is unknown.

The experiments of Anselmino and Hoffman were the basis for the attractive hypothesis that the reduced urinary secretion and the hypertensive symptoms of certain diseases such as eclampsia and essential hypertension are due to a "hypervasopressinemia." However, satisfactory confirmatory data are almost entirely lacking. Dieckmann and Michel (I937) as well as others have pointed out that the pregnant woman in the pre-eclamptic condition is markedly hypersensitive to the pressor effect of pars neuralis extract; moreover, they concluded that this abnormal response constitutes a useful diagnostic test for pre-eclampsia, but that it is not free from danger. Byrom (1938) has suggested that abnormally large amounts of free oestrin may circulate in the blood of patients with eclampsia and that this oestrin synergizes with vasopressor hormone in causing the important pathological changes (see pp. 263-64). According to Coester (1935), purified extracts of urine from normal individuals or from patients with two varieties of hypertension produced about the same diuresis-inhibiting and pressor effects. Still other observations have been made by Jores (1936), who concluded that abnormal amounts of anterior pituitary hormones stimulating the adrenals (cortex and medulla) circulate in the blood in certain hypertensive disorders, such as essential hypertension and pituitary basophilism (Cushing), and that these

and others which have been made before indicate that man, like other animals, exhibits a clear-cut pressor response to intravenous injections of pars neuralis extracts. 


\section{THE PITUITARY BODY}

hormones are causally related to the symptoms. ${ }^{13}$ Van Bogaert (1936) was convinced that neither the pituitary nor the adrenals is etiologically important in hypertension but that the pituitary may be important in maintaining the symptoms.

The relationship between the pituitary and the experimental hypertension due to renal ischemia produced by the clampof Goldblatt and others has been investigated in the dog by Page and Sweet (1936-37). In normal animals hypophysectomy caused some reduction of the arterial blood pressure. In dogs in which hypertension $(240 / 160 \mathrm{~mm}$. Hg) had been produced by means of Goldblatt's clamp, the high level of arterial pressure was maintained for several months unless hypophysectomy was performed. Some weeks after hypophysectomy, the blood pressure fell, sometimes nearly to normal ( $50 / 100 \mathrm{~mm}$. Hg), sometimes below normal $(90 / 40$ $\mathrm{mm} . \mathrm{Hg}$ ). Subsequent further constriction of the renal arteries again produced a rise in blood pressure which tended to be transient. The administration of thyroid extract also might be followed by a moderate increase in arterial pressure (see Fig. 28). The authors concluded that the effects of hypophysectomy were indirect and depended probably upon the removal of the pars glandularis without which the thyroid and adrenal glands performed their functions imperfectly. It is regrettable that this interpretation was not strengthened by observing the effects of extirpation of either the pars glandularis or the pars neuralis alone, because their observations do not satisfactorily exclude the possibility that the pars neuralis plays some part in maintaining the hypertension. Page (1936) was not able to detect any increased amount of hypertensive principle in the blood of dogs with the hypertension of renal ischemia; however, his method of

${ }_{13}$ Positive results were obtained in 20 of 28 patients with essential hypertension and in 6 with Cushing's syndrome. The findings were negative in 8 patients with eclampsia or renal disease of pregnancy. 

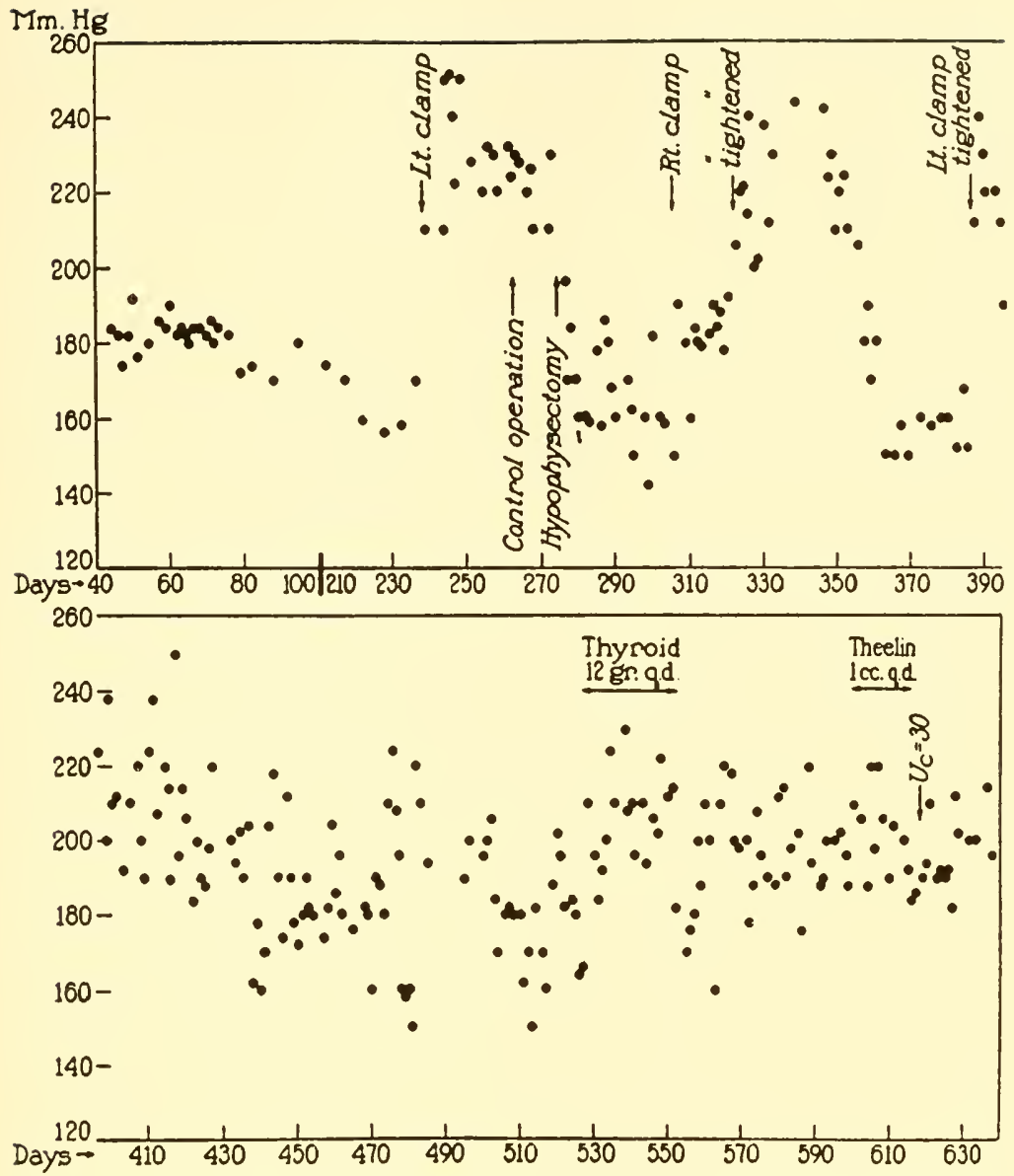

Fig. 28. - The relation of the pituitary body to hypertension caused by renal ischemia following the application of Goldblatt's clamp to the renal arteries. (From Page and Sweet, Amer. J. Physiol., x20, 238-45 [1937].)

Ordinate: arterial blood pressure; abscissa: time in days. Without hypophysectomy, the hypertension following the application of the first clamp probably would have persisted some months. Hypertension subsequently produced by tightening the right and left clamps was transient. The administration of thyroid gland in other experiments caused a recurrence of the hypertension. 
making extracts (alcoholic extracts of plasma) may not have been suitable for the pituitary vasopressor principle.

The oxytocic principle.-The additions to our knowledge of the possible physiological importance of the oxytocic principle in parturition are, for the most part, only of inferential value. Newton (1937) found that the isolated cervix uteri of the rat and guinea pig, in any stage of sexual activity including pregnancy, is very insensitive toward the oxytocic principle (concentration of 40 units per liter of bath-fluid). Newton considers that this fact strengthens the evidence favoring the importance of oxytocic-principle secretion in normal parturition. Possibly important interrelationships between the secretion of oestrogens or progesterone and the action of the oxytocic principle are discussed on pages 26 I-62. According to Ingram and Fisher (1937), if diabetes insipidus is produced in pregnant cats by a lesion of the supraopticohypophysial system, the subsequent parturition is incomplete and terminates in death. However, others (including Houssay, 1935, and Robson, I936-37) have demonstrated that normal parturition can take place in the cat, dog, mouse, rabbit, and rat after the removal of the posterior lobe or after hypophysectomy. This fact must be taken into account if the initiation or continuation of labor is to be explained as the result of oestrogen-sensitization of the uterus toward the oxytocic principle or if the interference with parturition observed by Ingram and Fisher is to be ascribed to a deficiency in the secretion of the oxy tocic principle.

The pars neuralis and menstruation.-Hartman and Firor (1935) have again suggested that menstruation possibly requires a functioning pars neuralis. However, the evidence offered does little to commend this hypothesis: of four immature monkeys in which complete hypophysectomy was attempted, the operation was successful in only one; "oestrindeprivation" bleeding after 640 rat-units of oestrin was observed in all the animals except the one completely hypophysectomized. In the experiments of Smith, Tyndale, and 
Engle (1936), 400-500 rat-units of oestrin were injected daily for Io days into young or adult hypophysectomized monkeys. After injections were stopped, delayed bleeding was observed in 8 instances. In 2 instances bleeding did not occur. The authors were loath to attribute the delay in or absence of bleeding to a specific factor.

\section{SUMMARY}

Contrary to the view previously expressed (van Dyke, I936), there is now good evidence that the pars neuralis is an important gland of internal secretion. The best data have been gathered in studies of the metabolism of water. In the normal mammal, it seems probable that the reclamation of water filtered through the glomeruli of the kidney when urine is secreted depends upon the diuresis-inhibiting (vasopressor) hormone of the pars neuralis. If a dangerous loss of water from the body is threatened, diuresis-inhibiting hormone is secreted at an increased rate and by its local action on the tubular epithelium of the kidneys increases the rate of reabsorption, and hence the conservation, of water. The nervous control of the secretion of diuresis-inhibiting hormone appears to be of the greatest importance and explains how afferent stimuli can affect (inhibit) the secretion of urine after renal denervation. In an animal like the cat the paired supraoptic nuclei of the hypothalamus apparently supply most of the secretory fibers of the pars neuralis which undergoes atrophy and secretes little or no hormone if the nuclei have been destroyed or the fibers have been cut, as after section of the stalk. Under such circumstances, the kidneys lose their ability to concentrate urine, so that water loss, polyuria, is abnormally rapid. In compensation the animal drinks an increased volume of water (polydipsia). The changes, therefore, are analogous to diabetes insipidus in man.

Furthermore, it appears that the function of another part of the pituitary, the pars glandularis, is necessary for the 


\section{THE PITUITARY BODY}

complete development of diabetes insipidus. Generally, it has been found extremely difficult to produce diabetes insipidus after complete hypophysectomy, whereas, if the pars glandularis is intact, the suppression of posterior-lobe secretion readily produces that condition. ${ }^{14}$ (Also, if diabetes insipidus has been produced, it can be abolished by removal of the pars glandularis.) Most observations indicate that the thyroid gland also must be intact. However, it is not certain that the diuretic principle of the anterior pituitary is identical with thyrotropic hormone.

Diuresis-inhibiting hormone facilitates the excretion of certain cations and anions, especially $\mathrm{Na}$ and $\mathrm{Cl}$. Future work will have to decide what is the best interpretation of this phenomenon in terms of the physiological importance of the hormone.

Whether or not the vasopressor hormone is physiologically important because of its cardiovascular effects is not known. Recently it has been shown that the hormone probably is liberated by stimulation of the central end of a vagus nerve. Its possible clinical significance in disorders like hypertension and, especially, eclampsia, however great it may be, has not been demonstrated.

In view of the highly specific action of the oxytocic principle on the uterus, it is to be expected that new observations on its importance in normal parturition will be gathered. At present it appears that the hormone, i.e., the pars neuralis, is not necessary for parturition. It is possible that parturition in normal animals may be greatly facilitated by its action, perhaps in synergism with oestrogen.

Although changes in the metabolism of carbohydrates can be produced by posterior-lobe extracts, it is not possible to state whether these effects are physiologically important.

${ }^{14} \mathrm{It}$ is to be hoped that older observations indicating that diabetes insipidus can be produced by hypothalamic lesions after complete hypophysectomy will be either disproved or confirmed, so that they can be evaluated. 


\section{APPENDIX}

\section{THE STRUCTURAL FORMULAS AND PRIN- CIPAL ACTIONS OF HORMONES OF NATURAL ORIGIN}

Notable advances have been made in isolating and determining the structure of hormones and of substances necessary for their synthesis or produced in their degradation. Moreover, a number of synthetic hormones has been made. The skill of chemists has been most fruitfully applied in studying substances with a nucleus derived from cyclopentenophenanthrene.

The table and formulas of the Appendix deal only with hormones or related substances of natural origin and of known structure. Although the list is believed to be complete at this time, it undoubtedly will be lengthened as a result of future research. The substances are listed alphabetically in the table which includes data believed to be of greatest interest to readers of this book. The formulas likewise are arranged alphabetically and follow the table. 


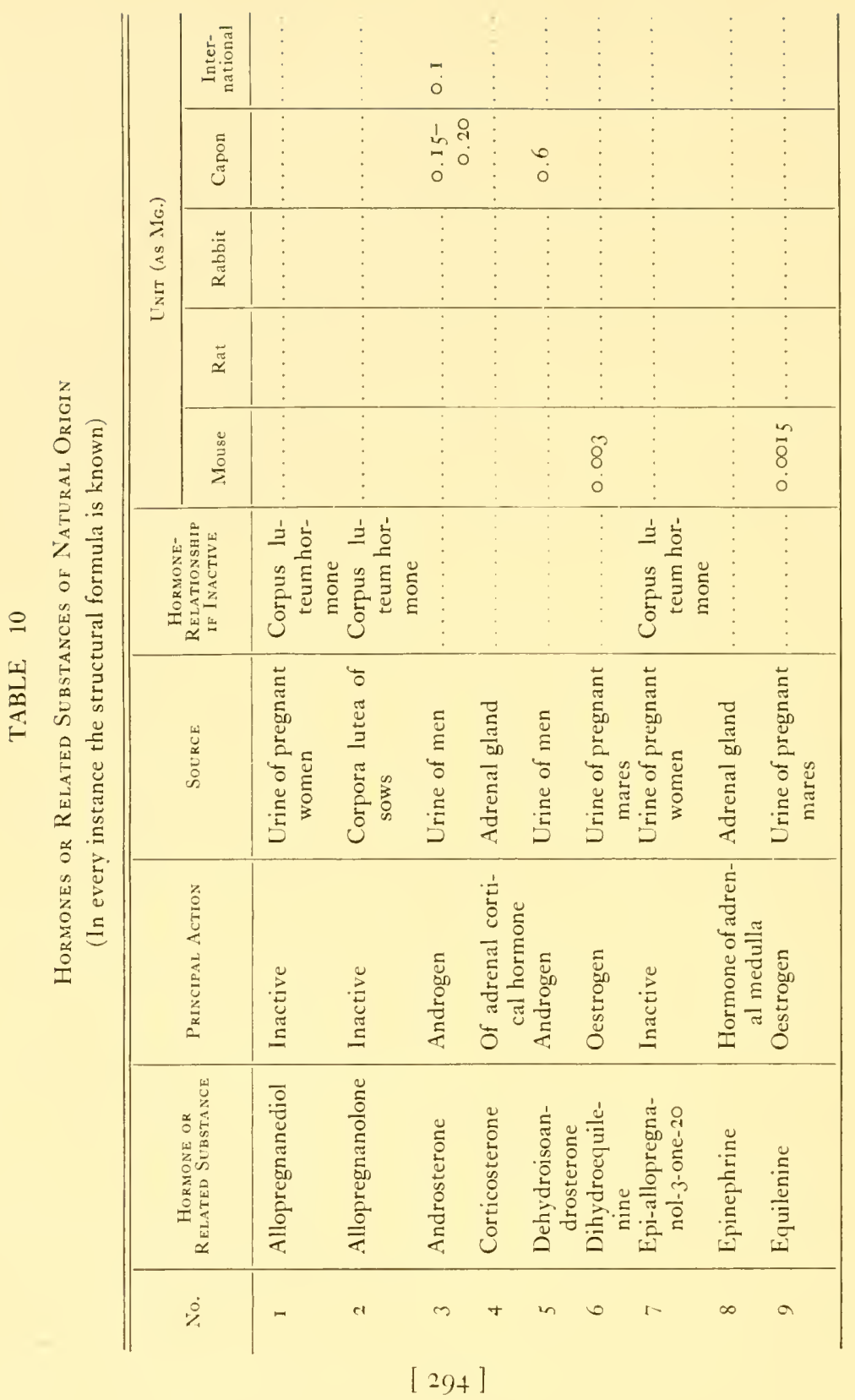




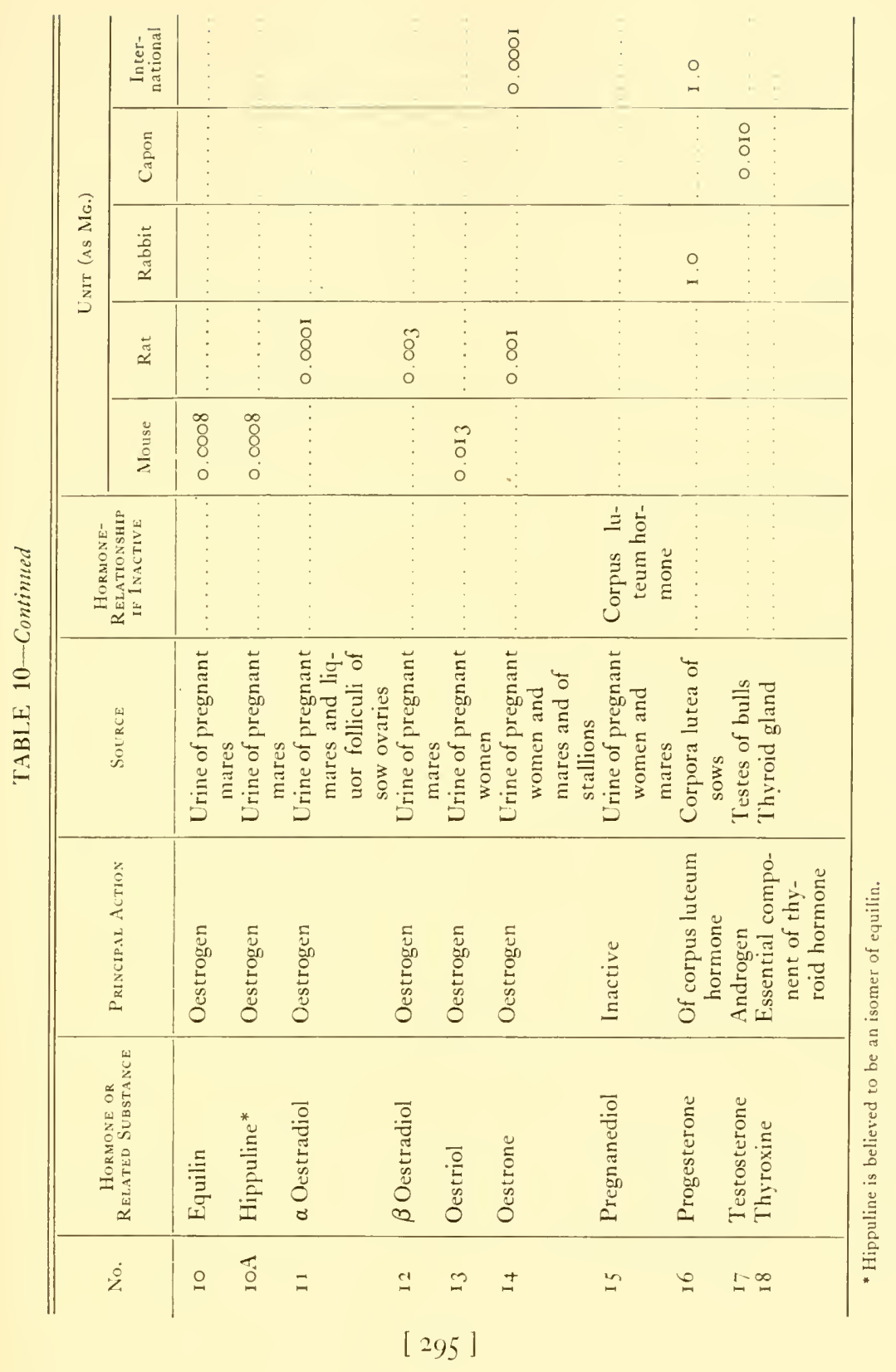


STRUCTURAL FORMULAS OF HORMONES
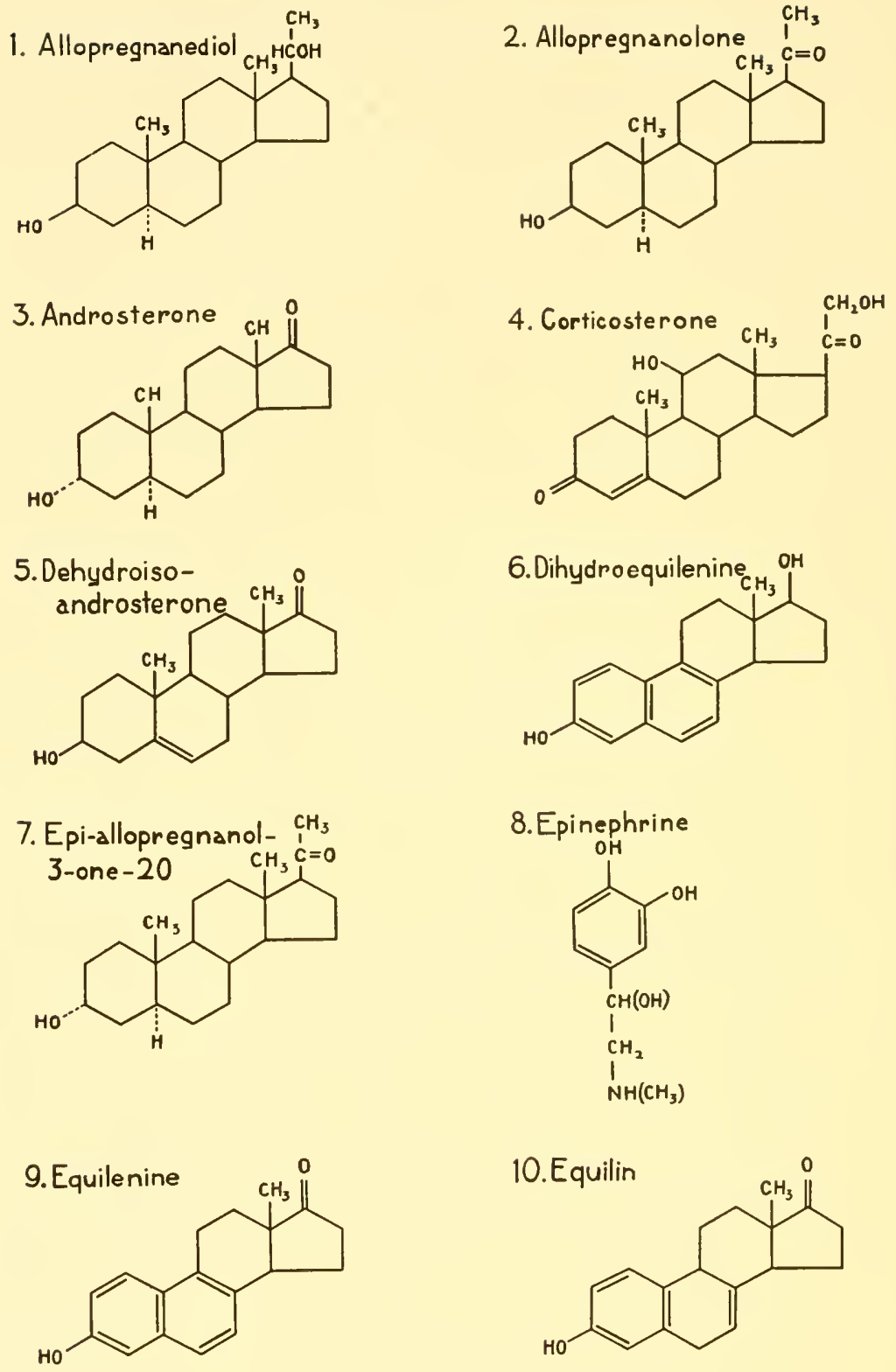
STRUCTURAL FORMULAS OF HORMONES<smiles></smiles><smiles></smiles><smiles>CC12CCC3c4ccc(O)cc4CCC3C1CC(O)C2O</smiles><smiles></smiles><smiles>CC(O)C1CCC2C3CCC4C[C@@H](O)CCC4(C)C3CCC12C</smiles>

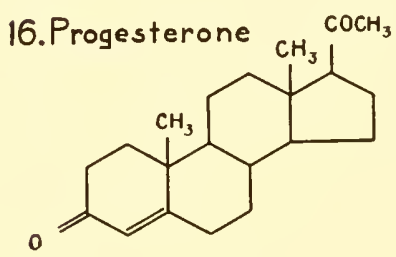

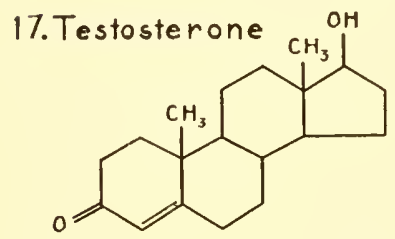

18.Thyroxine<smiles>NC(=O)C(N)Cc1cc(I)c(Oc2cc(I)c(O)c(I)c2)c(I)c1</smiles> 


\section{BIBLIOGRAPHY}

A bramowitz, A. A.: The action of intermedin on crustacean melanophores and of the crustacean hormone on elasmobranch melanophores. Proc. Nat. Acad. Sci. Wash., 22, 521-23 (1936).

Abramowitz, A. A.: Action of crustacean eye-stalk extract on melanophores of hypophysectomized fishes, amphibians, and reptiles. Proc. Soc. exp. Biol., N.Y., 34, 7 1 4-16 (1936).

Aвramowitz, A. A.: The chromatrophorotropic hormone of the Crustacea: standardization, properties and physiology of the eye-stalk glands. Biol. Bull. Wood's Hole, 72, 344-65 (I937).

Abramowitz, A. A.: The role of the hypophyseal melanophore hormone in the chromatic physiology of Fundulus. Biol. Bull. Wood's Hole, 73, 134-42 (1937).

Авramowitz, A. A.: The opercular approach to the pituitary. Science, 85,609 (1937).

Adams, A. E. and B. Gray: A comparative study of the thyroid glands of hypophysectomized newts after treatment with anterior pituitary, thyroid and iodine. Anat. Rec., 65, 69-8 I (1936).

Adams, A. E. and F. Martindale: The response of thyroid glands of hypophysectomized newts to injections of phyone and their reaction after cessation of treatment. Anat. Rec., 65, 319-3I (1936).

Adams, A. E. and V. MaYo: The gonad-stimulating potency of the pars anterior in normal and castrated newts. Proc. Soc. exp. Biol., N.Y., $35,227-3$ I (1936).

ADams, A. E. and E. N. WARD: The effect of hypophysectomy and of phyone injections on the pancreas and liver of the newt. Endocrinology, 20, 496-502 (1936).

Adlersberg, D. and R. Friedmann: Beiträge zur neuro-hormonalen Regulation des Wasserhaushaltes. II. Klinisch-experimentelle Untersuchungen über Störungen der Diurese nach Wasserbelastung. Z. klin. Med., I 29, 327-62 (I 935).

Adolph, E. F.: Control of urine formation in the frog by the renal circulation. Amer. J. Physiol., I 17, 366-79 (1936).

Agduhr, E.: Fortgesetzte Untersuchungen über die Bedeutung sexueller Funktionen für die Widerstandskraft des Organismus gegen schädliche Stoffe. I. Gesteigerte Widerstandskraft gegen Überdosierung von aktiviertem Ergosterin. Upsala Läkaref. förh. Ny följd, 42, I-8 (1937).

Agduhr, E.: Über die Steigerung der Widerstandskraft der Organismen gegen schädigende Faktoren durch das Zusammenleben der Geschlechter. Upsala Läkaref. förh. Ny följd, 43, 1-48 (1937).

Albani, L.: Ricerche sull'azione tireotropa degli ormoni ipofisari. Z. Zellforsch., 24, 42-63 (1936). 


\section{BIBLIOGRAPHY}

Allanson, M.: The effect of androgenic compounds on the histological structure of the pituitary in the castrated rat. Proc. Roy. Soc., B, 124, I 96-209, ( I 937).

Allanson, M., R. T. Hill, and M. K. McPhail: 'The effect of hypophysectomy on the reproductive organs of the male guinea-pig. J. exp. Biol., I 2, 348-54 (1935).

Allen, E. and A. W. Didde: Ovarian follicular hormone effects on the ovaries. Amer. J. Obstetr., 29, 83-87 (I935).

Allen, E., IV. U. Gardner, and A. W. Diddle: Experiments with theelin and galactin on growth and function of the mammary glands of the monkey. Endocrinology, I9, 305-13 (1935).

Allen, R. and G. Bourne: An extract from the adrenal gland causing luteinisation of the ovaries and endometrial hyperplasia. Aust. J. exp. Biol., I4, 45-50 (1936).

Allen, IV. M. and G. P. Heckel: Prolongation of the corpus luteum in the pseudopregnant rabbit. Science, 84, I6I-62 (1936).

Allen, W. M. and R. K. Meyer: Physiology of the corpus luteum. IX. The inhibition of oestrin by progestin-containing extracts of the corpus luteum. Anat. Rec., 61, 427-39 (1935).

Altschule, M. D. and P. Cooper: Changes in the pituitary gland following total thyroidectomy. Arch. Path., 24, 443-53 (1937).

Amilibia, E. de, M. M. Mendizábal, and J. Botella-Llusiá: Ovarialhormone und Schilddrüsenfunktion. Klin. Wschr., I5, I00I-4 (1936).

Andersen, D. H.: The effect of ovarian hormone on the pituitary, thyroid, and adrenal glands of spayed female rats. J. Physiol., 83, I 5-25 (I934).

Andersen, D. H., M. R. Prest, and J. Victor: Metabolic changes in liver, kidney and anterior hypophysis of pregnant, parturient and lactating rats. Amer. J. Physiol., I I9, 445-54 (1937).

Anderson, E., and W. Haymaker: Elaboration of hormones by pituitary cells growing in vitro. Proc. Soc. exp. Biol., N.Y., 33, 31 3-16 (1935).

Anderson, E. M. and H. M. Evans: The effect of thyreotropic hormone combined with small amounts of iodine upon the function of the thyroid gland. Amer. J. Physiol., I 20, 597-603 (I 937 ).

Anderson, R. K. and H. L. Alt: 'The effect of thyrotropic pituitary hormone on the oxygen consumption of thyroid tissue in vitro. Amer. J. Physiol., i 19, 67-69 (I937).

Andreis, N.: Contributo allo studio istologico delle manifestazioni di senescenza dell'ipofisi umana. Arch. ital. Anat. Istol. pat., 6, 44-72 (1935).

Anselmino, K. J.: Über die Wirkung des Kohlehydratstoffwechselhormons des Hypophysenvorderlappens auf den Blutzucker. Endokrinologie, 19, 30-33 (1937).

Anselmino, K. J., G. Effkemann, and F. Hoffmann: Über die Wirkung des Fettstoffwechselhormons des Hypophysenvorderlappens auf die gesättigten und ungesättigten Fettsäuren der Leber. Z. ges. exp. Med., 96, 209-20 ( I 935 ). 


\section{THE PITUITARY BODY}

Anselmino, K. J., G. Efrkemann, and F. Hoffmann: Über die Wirkung des Kohlehydratstoffwechselhormons des Hypophysenvorderlappens auf die gesättigten und ungesättigten Fettsäuren der Leber. Z. ges. exp. Med., 97, 44-50 (I 935 ).

Anselmino, K. J., L. Herold, and F. Hoffmann: Über die Wirkung des parathyreotropen Hormons des Hypophysenvorderlappens bei verschiedenen Tierarten. Z. ges. exp. Med., 97, 5 I-59 (1935).

Anselmino, K. J., L. Herold, and F. Hoffmann: Über die Wirkung des pankreatropen Hormons des Hypophysenvorderlappens bei verschiedenen Tierarten. Z. ges. exp. Med., 97, 329-35 (1935).

Anselmino, K. J., L. Herold, and F. Hoffmann: Studien zur Physiologie der Milchbildung. II. Mitt. Experimenteller Aufbau der Brustdrüse und Erzeugung von Lactation bej männlichen Tieren. Zbl. Gynäkol., 59, 963-69 (1935).

Anselmino, K. J., L. Herold, F. Hoffmann, and R. I. Pencharz: Studien zur Physiologie der Milchbildung. III. Mitt. Über den Hemmungsstoff der Milchbildung. Zbl. Gynäkol., 6o, 7-15 (1936).

Anselmino, K. J. and F. Hoffmann: Über die Blutzuckerwirkungen von Hypophysenvorderlappenfraktionen. Arch. exp. Path. Pharmak., I79, $273-85$ (1935).

Anselmino, K. J. and F. Hoffmann: Über das Stoffwechselhormon und die insulinogene Substanz des Hypophysenvorderlappens. Bemerkungen zu der gleichnamigen Arbeit von Prof. O. Steppuhn. Wien. Arch. f. inn. Med., 26, 87, 1934. Wien. Arch. inn. Med., 28, 117-20 (1935).

Anselmino, K. J. and F. Hoffmann: Über die Wirkung des Prolans am menschlichen Ovar und über seine Beziehung zum Hypophysenvorderlappen. Z. Geburtsh. Gynäkol., I I I, 26-36 (1935).

Anselmino, K. J. and F. Hoffmann: Über die Beteiligung der Hypophyse an der Entstehung des menschlichen Diabetes mellitus. I. Mitt. Nachweis einer Störung hypophysärer Regulationsmechanismen beim Diabetes mellitus. Z. klin. Med., 129, 24-51 (1935).

Anselmino, K. J. and F. Hoffman: Darstellung und Wirkungsprüfung des Fettstoffwechselshormons und des Kohlehydratstoffwechselshormons des Hypophysenvorderlappens. Handbuch der biologischen Arbeitsmethoden. Hrsg. von Emil Abderhalden. Abt. V, Tl. 3B, H6, Liefg. 454 (1936).

Anselmino, K. J. and F. Hoffmann: Über die Blutzuckerwirkung von Hypophysenvorderlappen-Fraktionen nach Ausschaltung der Nebennieren. Arch. exp. Path. Pharmak., r81, 674-80 (1936).

Anselmino, K. J. and F. Hoffmann: Zur Darstellung des Fettstoffwechselhormons des Hypophysenvorderlappens. Endokrinologie, I7, 1-8 (1936).

Anselmino, K. J. and F. Hoffmann: Zur Darstellung des Kohlehydratstoffwechselhormons des Hypohysenvorderlappens. Endokrinologie, 17, 289-9 I ( 1936$)$. 


\section{BIBLIOGRAPHY}

Anselmino, K. J. and F. Hoffmann: Über den Nachweis des pankreatropen Hormons des Hypophysenvorderlappens im Blut und Harn unter normalen und krankhaften Bedingungen. Klin. Wschr., I5, 999-100 I (1936).

Anselmino, K. J. and F. Hoffmann: Über die Ausscheidung von hypophysär gebildeten, gonadotropen Hormonen in der normalen Schwangerschaft und bei den Schwangerschaftstoxikosen. Z. Geburtsh. Gynäkol., I 14, 52-62 (1936).

Anselmino, K. J. and F. Hoffmann: Über die Beteiligung der Hypophyse an der Entstehung des menschlichen Diabetes mellitus. III. Mitt. Untersuchungen beim Pankreasdiabetes sowie beim Phloridzin- und Adrenalindiabetes des Hundes. Z. klin. Med., I29, 733-38 (I 936).

Anselmino, K. J. and F. Hoffmann: Über die Beteiligung der Hypophyse an der Entstehung des menschlichen Diabetes mellitus. IV. Mitt. Weitere Untersuchungen über die vermehrte Ausscheidung des Fettstoffwechselhormons des Hypophysenvorderlappens im Harn von Diabeteskranken. Z. klin. Med., I30, 424-28 (1936).

Anselmino, K. J. and F. Hoffmann: Über die Beteiligung der Hypophyse an der Entstehung des menschlichen Diabetes mellitus. V. Mitt. Über die Hemmung der Ausschüttung des Fettstoffwechselhormons und des Kohlehydratstoffwechselhormons des Hypophysenvorderlappens durch Insulin. Z. klin. Med. r30, 588-94 (1936).

Anselmino, K. J., F. Hoffmann, and E. Rhoden: Über die antagonistische Beeinflussung der Wirkung des Fettstoffwechselhormons und des Kohlehydratstoffwechselhormons des Hypophysenvorderlappens durch das Hormon der Nebennierenrinde. Arch. exp. Path. Pharmak., I8I, $325^{-27}(1936)$.

Anselmino, K. J., F. Hofrmann, and E. Rhoden: Über Leberverfettung durch Behandlung mit dem Fettstoffwechselhormon des Hypophy-

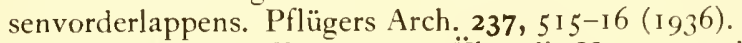

Anselmino, K. J. and E. Rhoden: Über die Hemmung der Ausschüttung des Fettstoffwechselhormons und des Kohlehydratstoffwechselhormons des Hypophysenvorderlappens bei kombinierter Zufuhr von Fett und Zucker. Z. ges. exp. Med., 98, 762-68 (1936).

Antognetti, L. and F. Geriola: Studi sui "tests" ormonici. Nota tredicesima. L'ormone tireotropo preipofisario. Endocrinologia, I I, 395-410 (1936).

ARNOLD, O. and H. MARx: Über Hämoglobinurie nach Zufuhr von Hypophysen-Hinterlappen-Hormon und Wasser. Z. ges. exp. Med., Ioo, $393-98$ (1937).

Aron, M.: Action d'extraits urinaires sur les surrénales et sur l'ovaire du lapin. Son application au diagnostic du cancer. Presse méd., 43, IO4446 (1935).

Aron, M.: Sur le titrage biologique de la thyréo-stimuline préhypophysaire: le "seuil des mitoses" dans la thyroïde des cobayes traités. C. R. Soc. Biol., Paris, 123, 250-53 (1936). 


\section{THE PITUITARY BODY}

Artemoff, N. M.: Über die Wirkung der Hypophyse auf das Genitalsystem der Fische. I. Mitt. Versuche an Weibchen von Bitterlingen. Bull. Biol. Méd. exp., URSS, 2, 25-26 (1936).

Artemov, N. M.: The influence of chronic homo-implantations of the pituitary body upon the genital system of female rats. A contribution to the problem of anti-hormones. Bull. Biol. Méd. exp. URSS., 3, $642-45$ (I937).

Arvay, A. von: Über die hormonalen Ursachen der Übertragung. Zbl. Gynäkol., 6I, 2900-29II (1937).

Asdell, S. A. and H. R. Seidenstein: Theelin and progestin injections on uterus and mammary glands of ovariectomized and hypophysectomized rabbits. Proc. Soc. exp. Biol., N.Y., 32, 931-33 (1935).

Asimov, G. I. and N. K. Krouze: The lactogenic preparations from the anterior pituitary and the increase of milk yield in cows. J. Dairy Sci., 20, 289-306 (1937).

Asmundson, V.S., and M. J. Wolfe: Effect of pregnant mare's serum on the immature fowl. Proc. Soc. exp. Biol., N.Y., 32, I 107-9 (1935).

Astwood, E. B., C. F. Geschickter, and E. O. Rausch: Development of the mammary gland of the rat. A study of normal, experimental and pathologic changes and their endocrine relationships. Amer. J. Anat., 6r, 373-405 (1937).

Astwood, E. B., and R. O. Greep: A corpus luteum-stimulating substance in the rat placenta. Proc. Soc. exp. Biol., N.Y., 38, 713-16 (1938).

Atwell, W. J.: Effects of thyreotropic and adrenotropic principles on hypophysectomized amphibia. Anat. Rec., 62, 36I-79 (1935).

ATWELL, W. J.: Differentiation and function of heterotopic autoplastic transplants of the amphibian hypophysis. Proc. Soc. exp. Biol., N.Y., $33,224-26$ (1 9.935).

ATwELL, W. J.: Effects of administering adrenotropic extract to hypophysectomized and thyroidectomized tadpoles. Amer. J. Physiol., I I8, $452-56$ (1937).

Atwell, W. J.: Functional transplants of the primordium of the epithelial hypophysis in Amphibia. Anat. Rec., 68, $431-47$ (1937).

Atwell, IV. J. and E. Holley: Extirpation of the pars intermedia of the hypophysis in the young amphibian with subsequent silvery condition and metamorphosis. J. exp. Zoöl., 73, 23-41 (I936).

Aubrun, E. A.: Symptômes cutanés du crapaud hypophysoprive. C. R. Soc. Biol., Paris, I 20, 734-35 (1935).

Azuma, R. and H. Kumagar: On the effects of posterior pituitary preparations and oestrone upon the uterine activity in the unanaesthetized dog. Jap. J. med. Sci., Trans. IV. Pharmacol., 8, $87 \dagger-89 \dagger$ (I 935).

BaARLE, F. van: Influence de l'hormone thyréotrope sur le métabolisme du cobaye. Arch. internat. Méd. exp., II, 763-89 (I936).

Bachman, C.: Immunologic studies of anti-gonadotropic sera. Proc. Soc. exp. Biol., N.Y., 32, 85 I-54 (I 935 ). 


\section{BIBLIOGRAPHY}

Bachman, C.: Reactions of immature rabbit ovary to gonadotropic extracts. Proc. Soc. exp. Biol., N.Y., 34, 33-37 (1936).

Bachman, C. and G. Tову: The responses of normal and hypophysectomized rabbits to adrenaline. J. Physiol., 87, I-10 (1936).

BAGG, H. J.: Experimental production of teratoma testis in the fowl. Amer. J. Canc., 26, 69-84 (1936).

BAKER, B. L. and G. E. Johnson: The effect of injections of antuitrin-S on the sexually inactive male ground squirrel. Endocrinology, 20, 2 I 9-23 ( I 936).

BALL, H. A. and L. T. Samuels: The relation of the hypophysis to the growth of malignant tumors. III. The effect of hypophysectomy on autogenous tumors. Amer. J. Canc., 26, 5+7-5 I (1936).

BALLif, L. and 1. Gherscovici: L'Hormone thyréotrope dans les tissus non hypophysaires. C. R. Soc. Biol., Paris, 121, 1437-38 (1936).

BARRIS, R. W. and W. R. Ingram: The effect of experimental hypothalamic lesions upon blood sugar. Amer. J. Physiol., I 14, 555-6I (I936).

BARRY, D. R.: Changes of colour by injection of pituitary extracts in a dogfish (Scylliorhinus canicula). Nature, r40, 769-70 (I 937 ).

Bastenie, P. and S. Zylberszac: Mise en évidence de stimulations hormonales par la colchicine. Détection de stimulation thyroïdienne par l'extrait antéhypophysaire. C. R. Soc. Biol., Paris, 126, $4_{4} 6^{-4} 8$ (19.37).

Bastenie, P. and S. Zylberszac: Mise en évidence de stimulations hormonales par la colchicine. Action de l'extrait anté-hypophysaire sur l'appareil génital du cobaye impubère. C. R. Soc. Biol., Paris, r26, 1 282-83 (1937).

Bates, R. W., T. LaAnes, and O. Riddle: Evidence from dwarf mice against the individuality of growth hormone. Proc. Soc. exp. Biol., N.Y., 33, 446-50 (1935).

Bates, R. W. and O. Riddle: The preparation of prolactin. J. Pharmacol. exp. Therap., 55, 365-7 I (I935).

BAtEs, R. W. and O. Riddle: Effect of route of administration on the bioassay of prolactin. Proc. Soc. exp. Biol., N.Y., 34, 847-49 (1936).

Bates, R. IV., O. Riddle, and E. L. LAHR: An assay of three hormones present in anterior pituitaries of seven types of cattle classified for age, sex, and stage of reproduction. Amer. J. Physiol., I I3, 259-64 (1935).

Bates, R. W., O. Riddle, and E. L. LAHR: Racial variation in the cropgland response of doves and pigeons to prolactin. Amer. J. Physiol., I $16,7^{-8}$ (1936).

Bates, R. IV., O. Riddle, and E. L. LaHR: The mechanism of the antigonad action of prolactin in adult pigeons. Amer. J. Physiol., I19, 610-14 (1937).

Bates, R. W., O. Riddle, E. L. Lahr, and J. P. Schooler: Aspects of splanchnomegaly associated with the action of prolactin. Amer. J. Physiol., 119, 603-9 (1937).

BAu, K. T.: Über die Wirkung des Harns von Schwangeren auf die Neben nierenrinde der männlichen Maus. Z. Zellforsch., 24, 714-26 (1936). 


\section{THE PITUITARY BODY}

Baudler, U.: Der Prolannachweis bei Tumoren. Arch. Gynäkol., I59, IOI-25 (I935).

Baudler, U.: Gonadotrope Wirkstoffe im Urin von Tumorkranken. Mschr. Geburtsh. Gynäkol., 102, I 56-60 (1936).

Bell, G. H. and J. M. Robson: Oxytocic properties of blood extracts and their physiological significance. J. Physiol., 84, 35 I-6I (I935).

Bell, G. H. and J. M. Robson: The effect of certain hormones on the activity of the uterine muscle of the guinea pig. J. Physiol., 88, 31227 (1936).

Bell, G. H. and J. M. Robson: The oxytocin content of the foetal pituitary. Quart. J. exp. Physiol, 27, 205-8 (1937).

BellerBY, C. W.: Termination of pregnancy in the rabbit by intravenous injection of anterior lobe pituitary extract. J. exp. Biol., I2, 286-95 (1935).

Berskr, N. W.: Die Analyse der Hypertrophieerscheinung der Gonaden hei einseitiger Kastration der Vögel. Z. vergl. Physiol., 23, 578-9I (1936).

Beltrametti, L.: Die Sexualfunktionen in ihren Beziehungen zum Wasserhaushalt, insbesondere zum Diabetes insipidus. Endokrinologie, r6, $241-56$ (19.35).

Benazzi, M.: La Preipofisi di Rana non provoca l'estro nella femmina impubere di topo. Atti Accad. naz. Lincei, 6th ser., 26, I I2-I 5 (1937).

Benedetto de Sabelli, Elena di, and E. J. di Benedetto: Hyperglycémie morphinique et éthérée chez les chiens hypophysoprives. C. R. Soc. Biol., Paris, I 20, 738-39 (1935).

Bennett, L. L.: Glucose absorption and glycogen formation in the hypophysectomized rat. Proc. Soc. exp. Biol., N.Y., 34, 277-79 (1936).

BennetT, L. L.: Failure of ant. pituitary extracts to maintain fasting carbohydrate levels of hypophysectomized rats after preliminary treatment. Proc. Soc. exp. Biol., N.Y., 37, 29-31 (1937).

BennetT, L. L.: Hyperglycemia and increased liver glycogen values in hypophysectomized rats on chronic administration of adrenocorticotropic hormone. Proc. Soc. exp. Biol., N.Y., 37, 50-51 (1937).

Benort, J.: Rôle de l'hypophyse dans l'action stimulante de la lumière sur le développement testiculaire chez le canard. C. R. Soc. Biol., Paris, I $8,672-74$ (1935).

Benoit, J.: Influence de la lumière naturelle sur la croissance testiculaire chez le canard au cours de la reprise sexuelle saisonnière. C. R. Soc. Biol., Paris, 120, 131-33 (1935).

Benoit, J.: Stimulation par la lumière artificielle du développement testiculaire chez des canards a veuglés par section du nerf optique. C. R. Soc. Biol., Paris, 1 20, 133-36 (1935).

Benoit, J.: Facteurs externes et internes de l'activité sexuelle. I. Stimulation par la lumière de l'activité sexuelle chez le canard et la cane domestiques. Bull. biol. France Belg., 70, 487-533 (1936). 


\section{BIBLIOGRAPHY}

Benoit, J.: Hypertrophie du foie chez le canard thyroïdectomisé. Rôle de la préhypophyse dans son enrichissement en lipides. C. R. Acad. Sci., Paris, 203, 468-70 (1 936).

BenoIt, J.: Sur le caractère quantitatif de la réponse à l'excitation par la lumière artificielle du mécanisme gonado-stimulant chez le canard domestique. C. R. Soc. Biol., Paris, 123, 241-43 (1936).

Benoit, J.: Rôle de la thyrö̉de dans la gonado-stimulation par la lumière artificielle chez le canard domestique. C. R. Soc. Biol., Paris, I23, 243-46 (1936).

BenoIT, J.: Activation du fonctionnement hypophysaire par des radiations lumineuses chez le canard domestique. Rev. Physiothérap., I2, 86-94 (1936).

Benoit, J.: Facteurs externes et internes de l'activité sexuelle. II. Etude du mécanisme de la stimulation par la lumière de l'activité testiculaire chez le canard domestique. Rôle de l'hypophyse. Bull. biol. France Belg., 71, 393-437 (1937).

Benoit, J.: Thyroide et croissance testiculaire chez le canard domestique. C. R. Soc. Biol., Paris, I25, 459-60 (I 937).

Benoit, J.: Sur les relations entre le foie et quelques glandes endocrines (thyroides, hypophyse, glandes génitales) chez le canard domestique. C. R. Soc. Biol., Paris, 1 25, 887-91 (1937).

Benolt, J.: Relation between thyroid and growth of testes and penis when stimulated by electric light. Proc. Soc. exp. Biol., N.Y., 36, $782-84$ (1937).

BenOIT, J.: Influence of hypophysis and thyroid glands on the liver of the duck. Proc. Soc. exp. Biol., N.Y., 36, 784-86 (1937).

Bentivogio, F.: Alcune azioni collaterali dell'ormone galattogeno preipofisario. Fol. demogr. gynaec. (Genova), 34, 363-96 (1937).

Berblinger, W . and Burgdorf: Neue Färbemethode zur Darstellung der Gewebsbestandteile der Hypophyse des Menschen. Endokrinologie, I 5, 38 I-88 (1935).

Bergman, A. J. and C. W. Turner: Comparison of methods of extraction of the lactogenic hormone. J. biol. Chem., I I8, 247-51 (1937).

Berkowitz, P.: Effect of estrogenic substances in Lebistes reticulatus (Guppy). Proc. Soc. exp. Biol., N.Y., 36, 416-18 (1937).

Bertolotto, U.: Ricerche sperimentali sull'irradiazione della ipofisi. Fol. Gynaecol. (Genova), 32, 173-206 ( I 935).

Best, C. H., and J. Campbell: Anterior pituitary extracts and liver fat. J. Physiol., 86, I 90-203 (1936).

Biasio, B. DE: Sull'azione della retroipofisi sulla muscolatura dello stomaco. Arch. Farmacol. sper., 6o, 305-I I (1935).

Bickenbach, W.: Über den Gehalt der Hypophysen eklamptischer Frauen an Hinterlappenhormon. Z. Geburtsh. Gynäkol., I I4, 29-36 (I 936).

BierRing, K.: Action de l'extrait préhypophysaire sur les surrénales chez le rat. Bull. Histol. appl., I 2, 269-73 (19.35). 


\section{THE PITUITARY BODY}

Biggart, J. H.: Diabetes insipidus. Brain, 58, 86-96 (1935).

Biggart, J. H.: Diabetes insipidus. The site of formation of the antidiuretic hormone. Edinb. med. J., 43, 4'7-25 (I936).

Biggart, J. H.: The anatomical basis for resistance to pituitrin in diabetes insipidus. J. Path. Bact., London, 44, 305-14 (1 937 ).

Billeter, O. A.: The effect of spaying and theelin injections on body growth and organ weights of the albino rat. Amer. J. Anat., 60, 36795 (1937).

Binet, L., L. Képinov, and G. Weller: Le Glutathion dans les tissus du chien hypophysoprive, nouvelles preuves en faveur d'une association hypophyso-thyroïdienne et hypophysotesticulaire. C. R. Soc. Biol., Paris, 120, 589-90 (1935).

Binswanger, F.: Studien zur Physiologie der Schilddrüse. III. Schilddrüse und Wachstum (Studien am Hund). Endokrinologie, I7, 22-35, 1 $50-6$, ( 1936).

Bischof , F.: The influence of divided dosage of gonadotropic extracts in the immature male rat. Amer. J. Physiol., I I4, 483-87 (I936).

Bischoff, F.: Histone combinations of the protein hormones. Amer. J. Physiol., I I7, I 82-87 (1936).

Bischoff, F. and M. L. Long: Chemical studies on prolan (from urine of pregnancy). J. biol. Chem., i $16,285-90$ ( 1936).

Bischoff, F. and L. C. MAxwell: Effect of sex hormones on transplanted neoplasms. Amer. J. Canc., 27, 87-90 (1936).

Bissonnette, T. H.: Relations of hair cycles in ferrets to changes in the anterior hypophysis and to light cycles. Anat. Rec., 63, I59-68 (1935).

Bissonnette, T. H.: Modification of mammalian sexual cycles. IV. Delay of oestrus and induction of anoestrus in female ferrets by reduction of intensity and duration of daily light periods in the normal oestrous season. J. exp. Biol., I 2, 31 5-20 (I 935).

Bissonnette, T. H.: Sexual photoperiodicity. Quart. Rev. Biol., II, 37 I-86 (1936).

Bissonnette, T. H. and E. E. Bailey: Litters from ferrets in January induced by increased exposures to light after night-fall. Amer. Naturalist, $70,454-58$ (1936).

Bissonnette, T. H. and A. G. Csech: Modifications of mammalian sexual cycles, VII-Fertile matings of raccoons in December instead of February induced by increasing daily periods of light. Proc. Roy. Soc., B., 122, 246-54 (1937).

Blazsó, S.: Wirkung des Hypophysenhinterlappenhormons auf den Kaliumspiegel des Serums. Cited by Ber. ges. Physiol., 94, 672 (1936).

BLOTNER, H.: The effect of pitocin, pitressin and antuitrin on fat tolerance tests. Endocrinology, 19, 587-91 (1935).

Blount, R. F.: Size relationships as influenced by pituitary rudiment implantation and extirpation in the urodele embryo. J. exp. Zoöl., 70, $131-85$ (1935). 


\section{BIBLIOGRAPHY}

Blount, R. F.: The influence of additional pituitary anlagen upon the circulatory system of the developing urodele. A condition paralleling hypertension in the mammal. J. exp. Zoöl., 7 I, 42I-48 (I9.35).

Blümel, P.: Die Prolanausscheidung beim Mann. Zbl. Chir., 62, 301925 (I935).

Bock, K. A.: Über die Änderung der Wirkung des Ovarialhormones und des gonadotropen Anteils des Hypophysenvorderlappens durch Störung des Säurebasengleichgewichts. Klin. Wschr., I4, I750-5.3 (1935).

Bodart, F. and K. Feli.inger: Über die thyreotrope Wirkung des Serums bei endokrinen Erkrankungen. Wien. klin. Wschr., 49, I28687 (1936).

Böttger, G.: Pigmenthormon und antidiuretisches Prinzip der Hypophyse. Klin. Wschr., I5,73-76 (1936).

Böttger, G.: Über das Pigmenthormon. I. Mitt. Der Test. Z. ges. exp. Med., ror, 42-47 (1937).

Böttger, G.: Über das Pigmenthormon. II. Mitt. Zur Darstellung und zur Frage der Diuresewirkung. Z. ges. exp. Med., ror, 48-54 (1937).

Böttger, G.: Über das Pigmenthormon. III. Mitt. Zur Frage der Einheitlichkeit und über die aktive Substanz alkalischer Extrakte. Z. ges. exp. Med., I01, 55-6I (1937).

Bogaert, A. van: Action des extraits de l'hypophyse sur le diencéphale et sur la tension artérielle. C. R. Soc. Biol., Paris, 120, 450-52 (1935).

Bogaert, A. van: Hypothalamus et réactions cardio-vasculaires d'origine centrale. Arch. int. Pharmacodyn., 53, 137-76 (1936).

Bogaert, A. van: Régulation hypothalamo-hypophysaire de l'appareil circulatoire. Arch. Mal. Cœur, 29, I 5-38, 109-37 (1936).

Bogaert, A. van: Hypothalamus und centralnervöse Blutdruckregulation. Wien. klin. Wschr., 49, I06I-67 (1936).

Bogaert, A. van and L. van Meel: Lipémie, calcémie et potassémie au cours de l'excitation expérimentale de l'hypothalamus. C. R. Soc. Biol., Paris, r21, I99-201 (1936).

Borssezon, P. DE: La Corticale surrénale du cobaye et ses modifications, après injection d'urine de femme enceinte. Bull. Histol. appl., I3, I 29-36 (1936).

Bokkum, C. van: Neue Versuche über die Empfindlichkeit des Uterus für Pituitrin unter Finfluss von Hormonen. Acta brev. neerl., 6, 100IOI (I936).

Bokslag, J. G. H.: Die gonadotrope Funktion der Rattenhypophyse unter dem Einfluss von Hormonen. Acta brev. neerl., 7, 87-9I (I937).

Bourg, R. and G. LEgRAND: Etude comparative de la teneur en gravidine du placenta et des humeurs maternelles et ovulaires au cours de l'accouchement normal et pathologique. Arch. internat. Méd. exp., Io, 55 I-68 (I 935).

Bourg, R. and G. Legrand: Etude comparative de la teneur en gravidine du placenta et des humeurs maternelles et ovulaires au cours de l'ac- 


\section{THE PITUITARY BODY}

couchement normal et pathologique. Bull. Soc. belge Gynéc., I I, I97206 (1935).

Bowman, D. E., J. P. Visscher, and J. W. Mull: Properties of hormones in the female urine. J. biol. Chem., ro9, xii (1935).

Braier, B. and R. Morea: Métabolisme azoté endogène des rats hypophysoprives. C. R. Soc. Biol., Paris, I 19, 88 I-82 (1935).

Brander, J.: Studies of the human pituitary in health and disease. Proc. Roy. Soc. Med., 29, 609-22 (1936).

Brandt, R. and H. Goldhammer: Die Spezifität der gonadotropen Hormone und ihrer Antiseren. Z. Immunitätsforsch., 88, 79-90 (1936).

Braun-Menéndez, E.: Rev, argent. Cardiol., I, 4J5-49 (I935) (available as an abstract).

Breneman, IV. R.: The effect on the chick of some gonadotropic hormones. Anat. Rec., 64, 2 I 1-20 (1936).

Breneman, IV. R.: Male hormone and the testis-comb relationship in the chick. Endocrinology, 2I, 503-IO (1937).

Brindeau, A., H. Hinglais, and M. Hinglais: Essai de fractionnement des prolans par voie chimique, action de l'aldéhyde formique. C. R. Soc. Biol., Paris, 123, 393-94 (1936).

Britton, S. IV. and R. F. KLine: Relation of the adrenal cortex to reproduction and lactation. Amer. J. Physiol., I I5, 627-31 (1936).

Brooks, C. M.: Studies on the neural basis of ovulation in the rabbit. Amer. J. Physiol., I I9, 280-8 I (I937).

Brooks, C. M.: The rôle of the cerebral cortex and of various sense organs in the excitation and execution of mating activity in the rabbit. Amer. J. Physiol., I20, 544-53 (1937).

Brooks, C. M.: A study of the mechanism whereby coitus excites the ovulation-producing activity of the rabbit's pituitary. Amer. J. Physiol., 121, 1 57-77 (1938).

BrooksBy, J. B.: 'The reactivity during oestrus and pregnancy of the rat uterus to the oxytocic principle of the posterior pituitary gland. J. Physiol., 90, 365-70 ( 1937 ).

Browman, L. G.: Light in its relation to activity and estrous rhythms in the albino rat. J. exp. Zoöl., 75, 375-88 ( 1937 ).

Browman, L. G.: Effects of androsterone and testosterone on oestrous cycle of rats. Proc. Soc. exp. Biol., N.Y., 36, 205-8 (1937).

Browne, J. S. L. and E. M. Venning: Excretion of gonadotropic substances in the urine during pregnancy. Lancet, 23I, 1507-1 I (1936).

Bruckmann, N.: La Melanoforo-reazione nella rana non è specifica per la diagnosi di gravidanza. Riv. Ostetr., I9, I09-19 (1937).

BrulL, L.: Hypophysectomie et excrétion urinaire du phosphore. C. R. Soc. Biol., Paris, 1 24, I 242-44 ( 19.37 ).

BRULL, L.: 'The excretion of inorganic phosphate in the heart-lung-kidney preparations. J. Physiol., 90, 70P-7IP (1937).

BüHlER, F.: Tierexperimentelle Untersuchungen über den Einfluss von verschiedenen Hormonen auf die Ausscheidung von Kreatin und Kreatinin im Urin. Z. ges. exp. Med., 96, 821-44 (1935). 


\section{BIBLIOGRAPHY}

BüHLER, F.: Über den Einfluss der Keimdrüsenhormone auf den Geschlechtsapparat, den Thymus und die Hypophyse infantiler Ratten. Z. ges. exp. Med., 98, 1 5 1-63 (1936).

BÜLBRING, E., and J.H. BURN: The estimation of oestrin and of male hormone in oily solution. J. Physiol., 85, 320-33 (I935).

Bulliard, H.: Hypophyse et priapisme expérimental. Bull. Ass. Anat., $83-88$ (1936).

Bunde, C. A. and R. O. Greep: Suppression of persisting corpora lutea in hypophysectomized rats. Proc. Soc. exp. Biol., N.Y., 35, 235-37 (1936).

Buratschewsi, I. I. and A. N. Rappoport: Bull. Biol. Méd. exp. URSS., 2, 359-60 (1936).

BURN, J. H.: The modification of the action of insulin by pituitary extract and other substances. J. Physiol., 57, 318-29 (1923).

Burrows, W. H. and T. C. Byerly: Studies of prolactin in the fowl pituitary. I. Broody hens compared with laying hens and males. Proc. Soc. exp. Biol., N.Y., 34, 84 I-44 (1936).

Byerly, T. C. and W. H Burrows: Studies of prolactin in the fowl pituitary. II. Effects of genetic constitution with respect to broodiness on prolactin content. Proc. Soc. exp. Biol., N.Y., 34, 844-46 (1936).

Bугом, F. B.: Morbid effects of vasopressin on the organs and vessels of rats. J. Path. Bact., London, 45, I-I6 (I937).

Byrom, F. B.: The effect of oestrogenic and other sex hormones on the response of the rat to vasopressin. Lancet, 234, I29-3I (I938).

Cahane, M. and T. Cahane: Sur certaines modifications de l'hypophyse après une lésion du centre infundibulaire régulateur de la fonction génitale. (Note prélim.) Rev. franç. Endocrin., I3, 366-7I (1935).

Cahen, R. and P. Ardoint: Détérmination de l'activité gonadotrope de l'extrait préhypophysaire. C. R. Soc. Biol., Paris, I23, 547-49 (I936).

Cannavò, L. and R. Beninato: Untersuchungen über die Röntgenzerstörungen des Hypophysenvorderlappens. I. Röntgenzerstörung des Hypophysenvorderlappens und Mg-, Ca-, und P-Stoffwechsel. Endokrinologie, 15, 389-404 (1935).

Cartland, G. F. and J. W. Nelson: The preparation and purification of extracts containing the gonad-stimulating hormone of pregnant mare serum. J. biol. Chem., I 19, 59-67 (1937).

Casida, L. E.: Production of ovulation by gonadotropic extracts. Endocrinology, I8, $714^{-20}$ (1934).

CASIDA, L. E.: Relative gonadotropic augmentive action of plasma and formed elements from blood of cattle. Proc. Soc. exp. Biol., N.Y., 33, $570-72(1936)$.

Catchpole, H. R., H. H. Cole, and P. B. Pearson: Studies on the rate of disappearance and fate of mare gonadotropic hormone following intravenous injection. Amer. J. Physiol., I12, 2 I-26 (1935).

Cavanaugh, C. J. and R. Gaunt: Effect of pituitary substances on adrenalectomized rats. Proc. Soc. exp. Biol., N.Y., 37, 226-28 (1937). 


\section{THE, PITUITARY BODY}

Chaikoff, I. L., G. E. Gibis, G. F. Ногтом, and F. L. Reichert: The lipid metabolism of the hypophysectomized dog and the lipid and carbohydrate metabolism of the hypophysectomized-depancreatized dog. Amer. J. Physiol., i 16, 543-50 (I 936).

Chaikoff, I. L., F. L. Reichert, P. S. Larson, and M. E. Mathes: The effect of hypophysectomy and cerebral manipulation in the dog upon the response of the blood sugar and inorganic phosphorus to insulin. Amer. J. Physiol., I I2, 493-503 (I935).

Chaikoff, 1. L., F. L. Reichert, L. S. Read, and M. E. Mathes: The influence of epinephrine on the blood sugar, lactic acid and inorganic phosphorus of completely hypophysectomized dogs. Amer. J. Physiol., II3, 306-I I (I 935).

Chambers, IV. H., J. E. Sweet, and J. P. Chandler: Carbohydrate metabolism in the hypophysectomized-depancreatized dog. Amer. J. Physiol., I 19, 286-87 (1937).

$\mathrm{C}_{\text {hampy }}, \mathrm{C}_{\mathrm{H}}$. and $\mathrm{C}$. Champy: Fluctuations de la valeur endocrine de l'ovaire chez les jeunes oiseaux et à la suite d'injections chaloniques intenses. C. R. Soc. Biol., Paris, r22, $108_{4}-86$ (1936).

Сhang, H. C., K. F. Chia, C. H. Hsü, and R. K. S. Lim: A vagus-postpituitary reflex. I. Pressor component. Chinese J. Physiol., I2, 30926 ( 1937 ).

Chang, H. C., K. F. Chia, C. H. Hsü, and R. K. S. Lim: Reflex secretion of the posterior pituitary elicited through the vagus. J. Physiol., 90,

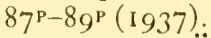

Chasin, P. S.: Über die Testierung des Lactationshormons des Hypophysenvorderlappens. Arch. Gynäkol., I62, 476-78 (1936).

Chen, G.: Attempts to produce antigonadotropic substance by the use of serum or blood extract. Chinese J. Physiol., I I, 329-33 (I 937).

Chen, G. and H. B. vaN Drke: The amount of thyroid-stimulating hormone in the anterior lobe of the pituitary of the thyroidectomized rabbit. Chinese J. Physiol., ro, 285-96 (1936).

Chen, G. and H. B. van Dyke: Action of merthiolate on gonadotropic effect of anterior pituitary extract. Proc. Soc. exp. Biol., N.Y., 35, 49I-92 (1936).

Chen, G. and H. B. van Dyke: The action of merthiolate on the gonadotropic effect of anterior pituitary extract. I. Pharmacol. exp. Therap., $62,333-45$ (1938).

Chen, G. and H. B. van DYKe: The gonadotropic action of anterior pituitary extract after tryptic digestion. Proc. Soc. exp. Biol., N.Y., 40, $172-76$ (1939).

Chew, W. B., R. P. Stetson, G. van S. Smith, and O. W. Smith: Estrogenic, luteal and gonadotropic hormones in hemophilia. Arch. int. Med., 55, 43 I-44 (1935).

Chiodi, V. and R. Pugliese: L'Istofisiologia dell'ipofisi in rapporto al metabolismo dell'acqua. Ricerche sperimentali. Endocrinologia, I2, 198-2 10 ( 1936). 


\section{BIBLIOGRAPHY}

Choay, A. and P. Desoille-Merlhes: Effets gonadotropes de la poudre de lobe antérieur d'hypophyse insérée sous la peau. C. R. Soc. Biol., Paris, 124, I 286-87 (1937).

CHou, C. H.: The antithyrotropic effect of the serum of normal and thyroidectomized rabbits. Chinese I. Physiol., I2, I 55-62 (I937).

Chou, C., C. Chang, G. Chen, and H. B. van Dyke: Observations on the quantitative assay of growth-promoting extract of the hypophysis. Endocrinology, 22, 322-34 (I938).

CHou, S. K. and S. H. LIU: Comparison of pituitary gonadotropic extract and prolan on ovarian and uterine response in immature rats. Proc. Soc. exp. Biol., N.Y., 37, 228-34 (1937).

Chouke, K. S., H. Friedman, and L. Loeb: Proliferative activity of the thyroid gland of the female guinea pig during the sexual cycle. Anat. Rec., 63, I3I-37 (1935).

Chrzanowski, B. and S. J. Grzycki: Das pankreotrope Hormon des Vorderlappens der Hypophyse und die Langerhansschen Inseln der Bauchspeicheldrüse. Klin. Wschr., 16, 488-91 (1937).

Croglia, L.: Ormoni dell'urina gravidica e colesterolemia. Boll. Soc. ital. Biol. sper., 10, 890-92 (1 935 ).

Cioglia, L.: Increti sessuali femminili e colesterolemia. V. Azione delle iniezioni di urina di donna gravida. Riv. Pat. sper., 5, 373-85 (19.36).

Cioglia, L. and D. Tore: Increti sessuali femminili e colesterolemia. II. Azione di preparati prolanici e preipofisari. Riv. Pat. sper., 5, 237-50 (1936).

Clark, J. H.: "Three dimension" graphs for correlating "age-weightgland" relationships. Proc. Soc. exp. Biol., N.Y., 35, I.39-42 (I936).

Clark, J. H., A. Steinberg, and L. G. Rowntree: Three dimension graphs for contrasting various endocrine organs of thymus rats with "age-weight-gland" controls. Proc. Soc. exp. Biol., N.Y., 35, I $43-47$ ( I 936$)$.

Clark, J. H., A. Steinberg, and L. G. Rowntree: Differential cell counts of the pituitary in the thymus treated strain of rats. Proc. Soc. exp. Biol., N.Y., 35, 239-42 (1936).

Clark, L. B., S. L. Leonard, and G. Bump: Light and the sexual cycle of game birds. Science, $85,339^{-40}$ ( 1937 ).

Clauberg, C.: Experimentelle Untersuchungen zur hormonalen temporären Sterilisierung und zur Behebung hormonalbedingter Sterilität. I. Mitt. Kurzdauernde temporäre Sterilisierung bei Weibchen durch "Follikelhormonstoss." Z. Geburtsh. Gynäkol., I I2, 4-23 (1935).

Clauberg, C.: Die Stimulierung der männlichen Geschlechtsdrüse durch weibliches Sexualhormon. (Tierexperimentelle Untersuchungen.) Zbl. Gynäkol., 60, I 457-64 ( I 936).

Clauberg, C. and W. Breipohl: Spezielles zur biologischen Wirksamkeit des Dihydrofollikelhormonbenzoats. Arch. Gynäkol., 160, 26377 (1935). 


\section{THE PITUITARY BODY}

Cleveland, D. and L. Davis: Further studies on the effect of hypothalamic lesions upon carbohydrate metabolism. Brain, 59, 459-65 (1936).

Coester, C.: Über biologisch wirksame Substanzen im Harn. III. Mitt. Vasoaktive und antidiuretische Stoffe im Harn bei Kreislaufgesunden und Hochdruckkranken. Z. klin. Med., I 28, 665-69 (I 935).

Conen, H. and J. Libman: Observations on the site of the antagonistic action of posterior pituitary extracts on insulin hypogylcaemia. Quart. J. Med., 6, I 57-63 (1937).

Cole, H. H.: On the biological properties of mare gonadotropic hormone. Amer. J. Anat., 59, 299-331 (1936).

Cole, H. H.: Superfecundity in rats treated with mare gonadotropic hormone. Amer. J. Physiol., 119, 704-12 (1937).

Cole, H. H. and R. F. Miller: Changes in the reproductive organs of the ewe with some data bearing on their control. Amer. J. Anat., 57, 3997 ( 1935 ).

Collin, R.: Sur l'existence probable d'une voie réflexe courte optohypothalamo-pituitaire. C. R. Soc. Biol., Paris, I 18, I 560-62 (I935).

Collin, R. and P. Florentin: La Structure de la glande pituitaire du cobaye dans le post-partum. C. R. Soc. Biol., Paris, I 20, I 43-46 (1935).

Collin, R. and T. Fontaine: L'Innervation de l'épendyme neuro-hypophysaire chez le chat et sa signification. C. R. Soc. Biol., Paris, r22, $1087-89$ (1936).

Collin, R. and L. Hennequin: Réactions tardives de la glande pituitaire à la gangliectomie cervicale supérieure chez le lapin. C. R. Soc. Biol., Paris, I 21, I 405-7 ( 1936 ).

Collin, R. and F. Stutinsky: Amitoses, endocytoses, endocytogenèses dans la glande pituitaire de la grenouille. C. R. Soc. Biol., Paris, r26, 334-36 (1937).

Collip, J. B.: Recent studies on anti-hormones. Ann. int. Med., 9, I 506r (1935).

Collup, J. B.: The standardization of anterior pituitary hormones. Amer. J. Obstetr., 33, 1010-16 (1937).

Collip, J. B.: Results of further experiments with the antimaturity hormone. J. Canad. Med. Ass., 36, I99-200 (1937).

Collip, J. B., H. Selye, and A. Neufeld: Experimental pancreatic diabetes in the monkey. Amer. J. Physiol., I 19, 289-90 (1937).

Collip, J. B., D. L. Thомson, and G. Tову: The effect of adrenaline on muscle glycogen in adrenalectomized, thyroidectomized, and hypophysectomized rats. J. Physiol., 88, i91-98 (1936).

Connon, F. E.: Effect of anterior pituitary-like hormone on lactation in the albino rat. Proc. Soc. exp. Biol., N.Y., 37, 52-55 (1937).

Cope, O.: The relation of the pituitary to liver glycogen production and utilization. J. Physiol., 88, 40I-16 (I 937).

Cope, O., and R. H. Thомpson: Adrenaline and the blood lactic acid level in hypophysectomized rabbits. J. Physiol., 88, 4I 7-24 (1937). 


\section{BIBLIOGRAPHY}

Corey, E. L.: Effects of cortico-adrenal extracts on the carbohydrate metabolism of hypophysectomized rats. Amer. J. Physiol., II9, 29 I (1937).

COREY, E. L.: Effects of cortico-adrenal extract on the estrus cycle of hypophysectomized rats. Proc. Soc. exp. Biol., N.Y., 36, 4 I-43 (I 937 ).

Corey, E. L. and S. W. Britton: Carbohydrate metabolism of hypophysectomized and hypophyso-adrenalectomized rats. Amer. J. Physiol., I I8, I 5-20 ( I 937).

Coryn, G.: Etude de l'influence des glandes endocrines sur le squelette. Arch. internat. Méd. exp., II, I35-290, 291-403 (1936).

Courrier, R. and G. Cohen-Solal: Sur les rapports des hormones mâle et femelle. Testostérone et folliculine. Etude quantitative de leur antagonisme. C. R. Soc. Biol., Paris, I 24, 925-28 (1937).

Courrier, R. and G. Gros: Action de la folliculine chez la chatte gestante. C. R. Soc. Biol., Paris, I 20, 8-9 (1 935).

Crainiceano, A., L. Copelman, E. Banu, and Sarbou: L'Action de l'hormone gonadotrope sur la corticale surrénale. C. R. Soc. Biol., Paris, 125, 227-28 (1937).

Cramer, W. and E. S. Horning: Experimental production by oestrin of pituitary tumours with hypopituitarism and of mammary cancer. Lancet, I, 247-49 (1936).

Cramer, W. and E. S. Horning: The effect of oestrin on the pituitary gland. Lancet, I, 1056-57 (1936).

Cramer, W. and E. S. Horning: The prevention of spontaneous mammary cancer in mice by the thyrotropic hormone of the pituitary gland. Lancet, I, 72 (I938).

Crandall, L. A., Jr. and I. S. Cherry: Effect of insulin and glycine on hepatic carbohydrate metabolism in unanesthetized normal, hypophysectomized, and adrenal denervated dogs. Amer. J. Physiol., I I9, 291-92 (1937).

CRooKe, A. C.: A change in the basophil cells of the pituitary gland common to conditions which exhibit the syndrome attributed to basophil adenoma. J. Path. Bact., London, 4I, 339-49 (1935).

Сrooke, A. C. and D. S. Russell: The pituitary gland in Addison's disease. J. Path. Bact., London, 40, 255-83 (1935).

Cucchini, F.: La Radiosensibilità del tessuto ipofisario. Scr. ital. Radiobiol. med., I, 21 5-2I (1934).

Cushing, H.: The basophil adenomas of the pituitary body and their clinical manifestations (pituitary basophilism). Johns Hopk. Hosp. Bull., 50, I 37-95 (1932).

Custo, E. L.: Comportamento della ghiandola mammaria in seguito ad iniezione di ormone follicolare. Fol. demogr. gynaec. (Genova), 34, $641-65$ (1937).

Cutting, W. C., E. C. Dodds, R. L. Noble, and P. C. Williams: Pituitary control of alimentary blood flow and secretion. The effect of posterior pituitary extract on the alimentary secretions of intact animals. Proc. Roy. Soc., B, 123, 27-38 (1937). 


\section{THE PITUITARY BODY}

Cutting, W. C., E. C. Dodds, R. L. Noble, and P. C. Williams: Pituitary control of alimentary blood flow and secretion. The effect of alterations in blood flow on gastric secretion. Proc. Roy. Soc., B, r23, 39-48 (1937).

Cutting, W. C., .E C. Dodds, R. L. Noble, and P. C. Williams: Pituitary control of alimentary blood flow and secretion. Gastric secretion and blood flow in hypophysectomized animals. Proc. Roy. Soc., B, I23, 49-59 (1937).

Cutuly, E.: Quantitative study on the adrenals of hypophysectomized rats. Anat. Rec., 66, i i 9-22 (1936).

Cutuly, E. and E. C. Cutuly: Gonadokinetic effects in parabiotic rats. Proc. Soc. exp. Biol., N.Y., 37, 477-80 (1937).

Cutuly, E., D. R. McCullagh, and E. C. Cutuly: Effects of androgenic substances in hypophysectomized rats. Amer. J. Physiol., I I9, I II-26 (1937).

Cutuly, E., D. R. McCullagh, and E. C. Cutuly: The type and degree of gonadal stimulation induced in hypophysectomized male rats parabiotically joined with castrated, cryptorchid, and normal partners. Endocrinology, 21, 241-48 (1937).

Cuyler, W. K., B. F. Stmmel, and D. R. McCullagh: Quantitative studies with the thyrotropic hormone. J. Pharmacol. exp. Therap., 58, 286-93 (1936).

Dahlberg, G.: Follicular hormone and ovulation inhibition. J. Obstetr. Gynecol., 42, 953-6i (I 935).

DaINeko, L. N.: Does the anterior lobe of the pituitary of the mammal embryos participate in the development of the genital apparatus. Bull. Biol. Méd. exp. URSS, 2, 434-35 (1936).

Daly, I. de B., E. G. L. Mark, and B. Petrovskaia: A bronchoconstrictor action of adrenaline following injections of pituitrin, pitocin or pitressin in isolated perfused lungs. J. Physiol., 89, 26 $\mathrm{P}-28^{\mathrm{P}}$ (1937).

D'Amour, F. F. and C. Dumont: Hormonal factors involved in parturition in the rat. Quart. J. exp. Physiol., 26, 21 5-24 (1937).

Danforth, D. N., R. R. Greene and A. C. Ivy: The effect of female sex hormones upon the oxygen consumption rate of normal rats, and upon the tolerance to desiccated thyroid. Endocrinology, 2I, 36I-67 (I9.37).

D Ausset, H., M. Ferrier, and H. Ucko: Action de la d'arsonvalisation endocrinienne sur le temps de réduction de l'oxyhémoglobine. C. R. Soc. Biol., Paris, r2 r, 631-34 (1936).

DAvidson, C. S.: Effect of adrenotropic extract upon the accessory reproductive organs of castrated rats. Proc. Soc. exp. Biol., N.Y., 36, 7035 (1937).

1) Avidson, C. S. and H. D. Moon: Effect of adrenocorticotropic extracts on accessory reproductive organs of castrate rats. Proc. Soc. exp. Biol., N.Y., 35, 28 I-82 (1936).

DAvis, D. D. and C. R. LAw: Gonadectomy and a new secondary sexual character in frogs. Science, $8 \mathbf{I}, 562-64$ (I935).

$$
\text { [3It] }
$$




\section{BIBLIOGRAPHY}

Davis, L., D. Cleveland, and W. R. Ingram: Carbohydrate metabolism. The effect of hypothalamic lesions and stimulation of the autonomic nervous system. Arch. Neurol., 33, 592-61 5 (1935).

DAvy, L.: Factors to be considered in immature female rat titration of pregnancy urine. Proc. Soc. exp. Biol., N.Y., 32, 927-3I (1935).

Dawson, A. B.: The relationships of the epithelial components of the pituitary gland of the rabbit and cat. Anat. Rec., 69, 47I-85 (1937).

DAwson, D. J. and A. Milne: Hyperglycaemia in fasted rabbits following injury in the pituitary region. Quart. J. exp. Physiol., 25, 69-76 (1935).

DEANESLY, R.: The response of immature rats to various gonadotropic substances. Quart. J. Pharm. Pharmacol., 8, 65 I-68 (1935).

Deanesly, R.: Adrenal cortex differences in male and female mice. Nature, I4I, 79 (1938).

Deanesly, R. and A. S. Parkes: Comparative activities of compounds of the androsterone-testosterone series. Biochem. J., 30, 291-303 (1936).

Deanesly, R. and A. S. Parkes: Note on male hormones and the question of accessory substances. Lancet, r, 837-39 (19.36).

Debré, R., J. Marie, and J. Bernard: Hypertrophie mammaire isolée chez une enfant de huit ans. Bull. Soc. Pédiatr., Paris, 33, 460-62 (1935).

DEleonardi, S.: Nachweis der oxytocischen, blutdrucksteigernden und diuresehemmenden Komponenten des Hypophysenhinterlappensekretes im Liquor cerebrospinalis. Arch. exp. Path. Pharmak., r8o, I35-4I (1936).

Deleonardi, S.: Azione sul circolo degli estratti neuroipofisari iniettati nel liquor con puntura sottoccipitale. Boll. Soc. ital. Biol. sper., Ir, 702-4 (1936).

Dell'Acqua, G.: Curve glico-cloro-proteinemiche negli individui normali e nei diabetici dopo iniezioni di estrat to ipofisario posteriore. Boll. Soc. ital. Biol. sper., ro, 423-27 (1935).

Dell'Acqua, G.: Über den Einfluss einiger Hormone auf den Mg- und Ca-Gehalt des Blutes. Z. ges. exp. Med., 96, 357-6I (I 935).

Demole, V. and F. Ippen: Die antithyreotoxische Wirkung von Ascorbinsäure. Z. physiol. Chem., 235, 226-32 (1935).

DEMPSEY, E. W.: Follicular growth rate and ovulation after various experimental procedures in the guinea pig. Amer. J. Physiol., 120, I2632 ( 1937 ).

Dempsey, E. W., R. Hertz, and W. C. Young: The experimental induction of oestrus (sexual receptivity) in the normal and ovariectomized guinea pig. Amer. J. Physiol., r 16, 201-9 (1936).

Desaive, P.: Effets des doses fractionnées de prolan sur la morphologie de l'ovaire de lapine adulte. Essai d'interprétation statistique des phénomènes observés. Arch. Biol., 46, 429-73 (1935).

Desclin, L.: A propos de l'influence de la lactation sur la structure du lobe antérieur de l'hypophyse du rat blanc. C. R. Soc. Biol., Paris, I22, 447-49 (I936). 


\section{THE PITUITARY BODY}

Desclin, L. and C. Grégoire: Etude de l'influence des gonades sur l'hypophyse transplantée chez le rat blanc. Bull. Acad. Méd. belg., 6th ser., I, 249-66 (1936).

Desclin, L. and C. Grégoire: Influence de l'hormone folliculaire sur l'hypophyse transplantée. C. R. Soc. Biol., Paris, I21, 1366-68 (I936).

Desclin, L. and C. Grégoire: Influence de la lactation sur les fonctions gonadotropes du lobe antérieur de l'hypophyse chez le rat blanc. C. R. Soc. Biol., Paris, 126, 250-52 (1937).

DIAKOv, F. A. and J. KŘIŽENECKÝ: The relation of the gonadotropic hormones to vitamin E. Biol. generalis (Wien), II, I 49-58 (I935).

Dieckmann, W. J. and H. L. Michel: Vascular-renal effects of posterior pituitary extracts in pregnant women. Amer. J. Obstetr., 33, I3I-37 (1937).

Dingemanse, E.: Über die Wirkung von Hypophysenvorderlappenextrakten auf den Acetonkörpergehalt im Blute. Endokrinologie, I7, 292-301 (1936).

Dingemanse, E. and J. Freud: Purified growth hormone from beef anterior pituitary. Acta brev. neerl., 5, 39-40 (1935).

Dingemanse, E. and J. Freud: Purified growth hormone from beef anterior putuitary. II. Dialysable growth hormone. Acta brev. neerl., 5, 109-II (1935).

Dionessov, S. M.: Zur Frage nach dem Mechanismus der Hemmungswirkung von Hypophysenpräparaten auf die Sekretion der Drüsen des Verdauungskanals. Fiziol. Ž., 20, 405-17 (1936).

Dischreit, J.: Beitrag zur Prüfung der therapeutischen Anwendbarkeit des Intermedins bei Diabetes insipidus. Klin. Wschr., I4, 629-32 ( 1935 ).

Dixon, T. F.: Bromine in the tissues. Biochem. J., 29, 86-89 (1935).

Dodds, E. C., R. L. Noble, H. Rinderknecht, and P. C. Williams: Prolongation of action of the pituitary antidiuretic substance, and of histamine, by metallic salts. Lancet, 233, 309-1 I (1937).

Dodds, E. C., R. L. Noble, R. W. Scarff, and P. C. Willíams: Pituitary control of alimentary blood flow and secretion. Changes in the stomach produced by the administration of posterior pituitary extract. Proc. Roy. Soc., B, 123, 22-26 (1937).

Dodds, E. C., R. L. Noble, and P. C. Williams: The pituitary gland and the control of urinary secretion. J. Physiol., 9I, 202-I I (I 937).

Dodero, G.: L'Azione dell'estratto di lobo posteriore di ipofisi sul comportamento dei fosfati inorganici del sangue e dell'orina. Arch. Farmacol. sper., 6o, 422-29 (1935).

DoI, A.: Über die Wirkung der Hypophysensubstanz auf die Bewegung des Uterus in den sexuellen Cyclen. II. Mitt. Über die Wirkung des Hormonpräparates, Antuitrin, auf die Bewegung des isolierten Rattenuterus in den fünf verschiedenen Sexualcyclen. Mitt. med. Ges. Chiba, 14, 37-38 (1936).

Dor, A.: Über die Wirkung der Hypophysensubstanz auf die Bewegung des Uterus in den sexuellen Cyclen. III. Mitt. Über die Wirkung des 


\section{BIBLIOGRAPHY}

Hormonpräparates, Puberogen, auf die Bewegung des isolierten Rattenuterus in den verschiedenen Sexualcyclen. Mitt. med. Ges. Chiba, 14, 38-39 (1936).

Domm, L. V. and E. A. Dennis: Effect of pituitary hebin upon reproductive system of the chick embryo. Proc. Soc. exp. Biol., N.Y., 36, 76669 (1937).

Downes, H. R. and L. Richards: A note on the concentration of the antidiuretic factor of the anterior lobe of the pituitary. J. biol. Chem., I I0, 8 I-90 (1935).

Dresel, I.: The effect of prolactin on the estrus cycle of nonparous mice. Science, 82, I73 (1935).

Druckrey, H.: Die Wirkung der Hypophyseninkretion auf Geschwülste. Arch. exp. Path. Pharmak., I80, 367-80 (1936).

Druckrey, H.: Hypophysenvorderlappen und Krebs. Bemerkungen zu der gleichnamigen Arbeit von K. Katz in Bd. 45 dieser Zeitschrift. Z. Krebsforsch., 45, 352-54 (1 937).

Druckrey, H. and H. Bachmann: Über die wehenauslösende Wirkung des Follikelhormons. Zbl. Gynäkol., 6r, 109 I-93 (1937).

Dubois-Poulsen, A.: Effets de l'extrait hypophysaire et de l'adrénaline sur les franges de l'épithélium pigmentaire de la rétine de la grenouille. C. R. Soc. Biol., Paris, 125, 248-49 (1937).

Dunlop, G.: The effect of the growth-promoting, appetite-stimulating or "physin" factor on the live-weight increase of swine. J. agricult. Sci., 25, 445-59 (1935).

Du Shane, G. P., W. T. Levine, C. A. Pfeiffer, and E. Witschi: Experimental "constant oestrus" and the notion of anti-gonadotropic hormones. Proc. Soc. exp. Biol., N.Y., 33, 339-45 (1935).

Dychno, A.: Akuš. i. Ginek., pp. 542-50 (1936) (abstract).

Dychno, M.: Akuš. i. Ginek., pp. 923-30 (1936) (abstract).

Dyer, F. J.: Standardization of prolactin. J. Physiol., 88, 6P-7P , (1936).

Effkemann, G.: Über die Beteiligung der Hypophyse an der Entstehung des menschlichen Diabetes mellitus. II. Mitt. Wirkung der gesteigerten Ausschüttung des Fettstoffwechselhormons und des Kohlehydratstoffwechselhormons auf die gesättigten und ungesättigten Fettsäuren der Leber. Z. klin. Med., r 29, 585-92 (1936).

Effremann, G. and L. Herold: Über Lebervergrösserung nach Zufuhr von Hypophysenvorderlappen- und Organextrakten. Z. ges. exp. Med., 96, $195^{-208}$ (1935).

Eggert, B.: Zur Morphologie und Physiologie der Eidechsen-Schilddrüse. II. Über die Wirkung von hohen und niedrigen Temperaturen, von Thyroxin und von thyreotropem Hormon auf die Schilddrüse. Z. Zool., 149, 537-94 (1936).

Eнrнardt, C.: Quantitative Untersuchungen über die Ausscheidung von Hypophysenvorderlappen-hormon im Harn. Klin. Wschr., 15, 514-16 (1936). 


\section{THE PITUITARY BODY}

Eichiaum, F. and V. Kindermann: Untersuchungen über die antigenen Funktionen von Hormonpräparaten. I. Gonadotropes Hypophysenvorderlappenhormon (Prähormon). Z. Immunitätsforsch., 86, 284-99 (1935).

E.ich ваUm, F. and V. Kindermann: Untersuchungen über die antigenen Funktionen von Hormonpräparaten. II. Thyreotropes Hypophysenvorderlappenhormon. Z. Immunitätsforsch., 89, 498-5 II (I936).

Eichbaum, F., E. Kindermann, F. Oestreicher, and M. Reiss: Zur Frage der Unwirksamkeit des thyreotropen Wirkstoffes bei andauernder Zufuhr. Endokrinologie, 18, 375-78 (1937).

Einarson, L. and H. OkKels: Les Glandes endocrines et le cerveau dans la vieillesse. Ann. d'Anat. path., 13, 557-80 (1936).

Einhorn, N. H. and L. G. Rowntree: 'The biologic effects of thymectomy. Accruing retardation in growth in succeeding generations. Endocrinology, 20, 342-47 (1936).

Eitel, H.: Schilddrüse und Nervensystem. Dtsch. Z. Chir., 247, 5758 I (1936).

Eitel, H.: Der Einfluss von thyreotropen Hormon auf die Einheilung verpflanzten Schilddrüsengewebes im Tierversuch. Dtsch. Z. Chir., $247,647^{-56}(1936)$.

Eitel, H. and E. W. Lexer: Schilddrüsentätigkeit und Frakturheilung. Arch. klin. Chir., 185, 587-98 (1936).

Eitel, H. and A. Loeser: Die Bedeutung der Schilddrüse für die antithyreotrope Schutzkraft des Blutes. Arch. exp. Path. Pharmak., I79, 440-47 (1935).

Eiter, H. and O. E. Riecker: Schilddrüsentätigkeit und Wundheilung. Bruns Beitr. klin. Chir., 164, 69-78 (1936).

EjDinova, M.: The action of hormones upon excitability of the digestive glands. III. Action of pituitrine $\mathrm{P}$ upon pancreatic secretion, as determined by the state of the glandular apparatus. Bull. Biol. Méd. exp. URSS, I, 354-55 (1936).

Elden, C. A. and M. D. Fellows: Relation of potency of anterior pituitary-like hormone to hydrogen ion concentration. Proc. Soc. exp. Biol., N.Y., 32, 1 597-99 (1935).

Ellison, E. T. and J. C. Burch: The effect of estrogenic substances upon the pituitary, adrenals and ovaries. Endocrinology, 20, 746-52 (1936).

Ellsworth, H. C.: The action of posterior pituitary hormone upon the blood sugar of the rabbit. J. Pharmacol. exp. Therap., 55, 435-38 (I935).

Elloworth, H. V.: The antagonism between posterior lobe pituitary hormones and insulin. J. Pharmacol. exp. Therap., 56, 417-20 (1936).

Elmer, A. W., B. Giedosz, and M. Scheps: Action des vitamines A, C, D sur la thyroïde normale et sur la thyroïde hyperactivée par la thyréostimuline préhypophysaire, effet inhibiteur des vitamines A et de l'acide ascorbique dans l'hyperthyréose expérimentale. C. R. Soc. Biol., Paris, г 20, 560-62 (1935). 


\section{BIBLIOGRAPHY}

Elmer, A. W., B. Giendosz, and M. Scheps: The anterior pituitary and its diabetogenic and pancreatotropic (blood-sugar decreasing) activity. Acta med. scand., 93, 487-98 (I 937).

Elmer, A. W., B. Gidęosz, and M. Scheps: Sur ]'hormone pancréatostimulante du lobe antérieur de l'hypophyse. C. R. Soc. Biol., Paris, I 24, 823-26 ( I 937).

Ermer, A. W., B. Giędosz, and M. Scheps: Sur l'action cortico-stimulante et médullostimulante du lobe antérieur de l'hypophyse. C. R. Soc. Biol., Paris, I25, $1082-85$ (1937).

Fimer, A. W., B. Gideosz, and M. Scheps: L'Action immédiate hyperglycémiante et anti-insulaire de la préhypophyse et du sang dans l'acromégalie. C. R. Soc. Biol., Paris, I25, I086-88 (I 937).

Emerson, K., Jr.: On the specificity of the thyreotropic action of the anterior pituitary gland. Johns Hopk. Hosp. Bull., 60, 358-67 (I 937).

Emery, F. E.: Potency of pituitary implants after several days in the muscles or peritoneal cavity. Anat. Rec., 66, 253-55 (I936).

EMERY, F. E.: A study of the augmentation of ovarian weights as affected by zinc sulphate, antuitrin $\mathrm{S}$ and thyroid implants. Amer. J. Physiol., I I 8, 3 I6-20 (I 937).

Emery, F. E.: Augmentation of gonad stimulating hormone of the hypophysis by copper. Proc. Soc. exp. Biol., N.Y., 36, 73 I-33 (I 937 ).

EmerY, F. E.: Effects of splenectomy on pituitary gonadotropic substances. Proc. Soc. exp. Biol., N.Y., 37, 455-57 (I937).

F.MERY, F. E.: Studies on hypertrophy, regeneration, and retardation of ovarian weights in growing rats after oestrone injections. Quart. J. exp. Physiol., 27, 17-26 (1937).

EMERY, F. E. and E. L. SchwaBe: The vaginal smears of rats as influenced by frequent examinations. Anat. Rec., 64, I47-54 (1936).

Emery, F. E. and E. L. Schwabe: The rôle of the corpora lutea in prolonging the life of adrenalectomized rats. Endocrinology, 20, 550-55 (1936).

Emge, L. A. and K. M. Murphy: The relation of the endocrine system to tumor growth. The effect of hypophysectomy and pituitary growth hormone on transplantable rat sarcoma. Amer. J. Obstetr., 32, 5936 I i ( 936$)$.

Engel, P.: Gegenhormone und Zirbeldrüse. Klin. Wschr., I4, 970-7I (I 935 ).

Engel, P.: Über die antigonadotrope Wirkung des Epiphysens. Wien. klin. Wschr., 48, I 160-6 I ( I 935 ).

ENGEL, P.: Weitere Untersuchungen über die bjologischen und chemischen Eigenschaften des antigonadotropen Hormons der Zirbeldrüse. Z. ges. exp. Med., 96, 328-36 (I 935 ).

ENGEL, P.: Wachstumsbeeinflussende Hormone und Tumorwachstum. 7. Krebsforsch., 4I, 488-96 (I 935 ).

F.ngel, P.: Die physiologische und pathologische Bedeutung der Zirbeldrüse. Erg. imn. Med., 50, I I6-7I (1936). 


\section{THE PITUITARY BODY}

Engel, P.: Zur Frage der hormonalen Wirkung der Zirbeldrüse. Bemerkungen zu der Arbeit von Walter Fleischmann und Helene Goldhammer in Jg. 1936, S. 1047 dieser Wochenschrift. Klin. Wschr., I5, I28 I (1936).

Engel, P. and W. Buño: Zur Wirkung des antigonadotropen Hormons der Zirbeldrüse am Kaninchen. Wien. klin. Wschr., 49, Iо 18-I9 (1936).

Engel, P. and E. Werber: Über das Wachstum der Mäusehypophyse in der Gewebekultur. Arch. exper. Zellforsch., 20, 194-97 (1937).

Engel, P. and E. Werber: Über das Wachstum der Mäusehypophyse in der Gewebekultur. Klin. Wschr., I6, I35 (I937).

Engelhart, E.: Über den antagonistischen Einfluss des Schilddrüsenhormons auf das Corpus luteum und des Follikelhormons auf den scheinschwangeren Uterus. Klin. Wschr., I4, Io68-70 (I935).

Engelhart, E.: Über eine neue Wirkung des Lactations-Hormons des Hypophysenvorderlappens auf Ovar und Uterus. Klin. Wschr., I5, $424(1936)$.

Engelhart, E. and H. Häusler: Die Bedeutung der Hypophyse für den Beginn des Klimakteriums mit experimentellen Untersuchungen über den Einfluss der H.V.I..-Implantation auf die Ovarialfunktion und den Stoffwechsel seniler Ratten. Arch. Gynäkol., 163, 643-6 i (1937).

Engle, E. T., R. C. Crafts, and C. E. Zeithaml: First estrus in rats in relation to age, weight, and length. Proc. Soc. exp. Biol., N.Y., 37, 42732 (1937).

Erdheim, J.: Biologie der Schwangerschaftszellen und ihre Beziehung zum Skelet. Frankf. Z. Path., 49, 452-78 (1936).

Etcheverry, A. O.: Diabètes pancréatique et hypophysaire chez les chiens vagotomisés. C. R. Soc. Biol., Paris, 126, 159-60 (I 937 ).

Eтkin, W.: Effect of multiple pituitary primordia in the tadpole. Proc. Soc. exp. Biol., N.Y., 32, 1653-55 (1935).

Evans, E. I.: The lactogenic hormone of the anterior pituitary. Amer. J. Physiol., I I9, 303-4 (1937).

Evans, E. I.: The assay of the lactogenic hormone. Amer. J. Physiol., II9, 304 (I937).

Evans, H. M., C. L. Kohls, and D. H. Wonder: Gonadotropic hormone in the blood and urine of early pregnancy. The normal occurrence of transient extremely high levels. J. Amer. med. Ass., 108, 287-89 (1937).

Evans, H. M., K. Korpi, R. I. Pencharz, and M. E. Simpson: On the separation and properties of the antagonist, a pituitary substance inhibiting ovarian responses to gonadotropic hormones. Univ. Calif. Publ. Anat., 1, 237-54 (1936).

Evans, H. M., K. Korpi, M. E. Simpson, and R. I. Pencharz: Fractionation of the gonadotropic hormones in pregnant mare serum by means of ammonium sulfate. Univ. Calif. Publ. Anat., I, 275-8 I (1936).

Evans, H. M., K. Korpi, M. E. Simpson, R. I. Pencharz, and D. H. Wonder: On the separation of the interstitial cell-stimulating, luteinizing, and follicle-stimulating fractions in the anterior pituitary gonadotropic complex. Univ. Calif. Publ. Anat., I, 255-74 (1936). 


\section{BIBLIOGRAPHY}

Evans, H. M., R. I. Pencharz, and M. E. Simpson: On the conditions necessary for the continuous growth of hypophysectomized animals. Endocrinology, 19, 509-14 (1935).

Evans, H. M. and M. E. Simpson: Production of superovulation in normal immature rats by injection of the principle in menopause urine. Proc. Soc. exp. Biol., N.Y., 32, 1046-47 (1935).

Evans, H. M. and M. E. Simpson: Synergism or augmentation produced by the addition of an hypophyseal synergist to menopause or castration urine. Proc. Soc. exp. Biol., N.Y., 32, 1047 (1935).

Evans, H. M. and M. E. Simpson: A sensitive biological test for meno. pause or castration prolan. Proc. Soc. exp. Biol., N.Y., 32, 1048 (1935).

Evans, H. M., M. E. Simpson, and R. I. Pencharz: Gonadotropic effects in hypophysectomized female rats of implants of pituitaries from castrated males. Proc. Soc. exp. Biol., N.Y., 32, 1048-49 (1935).

Evans, L. T.: The effects of pituitary implants and extracts on the genital system of the lizard. Science, 8r, 468-69 (1935).

Evans, L. T.: The effect of antuitrin $\mathrm{S}$ on the male lizard, Anolis carolinensis. Anat. Rec., 62, 21 3-22 (1935).

Evans, L. T.: The effects of antuitrin $\mathrm{S}$ and sheep pituitary extract on the female lizard, Anolis carolinensis. Biol. Bull. Wood's Hole, 68, 355-59 (1935).

Farr, L. E., K. Hare, and R. A. Phillips: Production of experimental diabetes insipidus in cats. Amer. J. Physiol., I I9, 305-6 (1937).

Fazekas, J. F., E. H. Campbell, Jr., and H. E. Нimwich: The respiratory quotient of renal tissue of Houssay dogs. Amer. J. Physiol., ir8, 29799 (1937).

Ferr, A.: Experimentelle Röntgenbestrahlung der Hypophyse bei Kaninchen. Schweiz. med. Wschr., pp. 289-9I (1936).

Feldinger, K.: Klinische und experimentelle Untersuchungen über das Verhalten und die Bedeutung des thyreotropen Hormons im Blute. Wien. Arch. inn. Med., 29, 375-406 (1936).

Fellinger, K. and O. Hochstädt: Über die antithyreoidale Wirkung des Vitamins A. Wien. klin. Wschr., 49, 1339-40 (I936).

Fels, E.: Röntgenkastration und Parabiose. Centralbl. allg. Path. path. Anat., 58, 69-74 (1933).

FEls, E.: Zur Testierung und Aktivierung der Corpus luteum-Hormone (Luteosteron C und D). Zbl. Gynäkol., 59, 2420-27 (I 935).

FELs, E.: Corpus luteum-Hormon (Progesteron) und männlicher Genitaltrakt. Arch. Gynäkol. 160, 460-66 (1936).

Ferrannini, A.: Über die Wirkung von Thyroxin und thyreotropem Hormon auf die Frequenz des isolierten Herzvorhofstreifens. Arch. int. Pharmacodyn., 54, 299-301 (1936).

Ferrannini, A.: Gibt es eine gegenseitige Beeinflussung der Phlorrhizinund Pituitrin-Wirkung auf den tubulären Nierenapparat? Arch. exp. Path. Pharmak., r84, 580-86 (1937). 


\section{THE PITUITARY BODY}

Fevold, H. L. and F. I. Hisaw: Concentration of gonadotropic substance from pregnancy urine. Proc. Soc. exp. Biol., N.Y., 34, 7I 2-I 4 (1936).

Fevold, H. L., F. L. Hisaw, and R. Greep: Effect of oestrin on the activity of the anterior lobe of the pituitary. Amer. J. Physiol., Ir4, 508$13(1936)$ :

Fevold, H. L., F. L. Hisaw, and R. Greep: Augmentation of the gonad stimulating action of pituitary extracts by inorganic substances, particularly copper salts. Amer. J. Physiol., II7, 68-74 (1936).

Fevold, H. L., F. L. HisAw, and R. O. Greep: Comparative action of gonad-stimulating hormones on the ovaries of rats. Endocrinology, 2 I, $343^{-}+5$ (1937).

Fichera, G. and A. Ferroni: Sui rapporti tra ipofisi e pancreas. I. Gli effetti della pancreasectomia sulla ipofisi. Pathologica (Genova), 29, $432-36$ ( 1937 ).

Findeey, T., JR.: Thyroid-pituitary relationship in diabetes insipidus. Ann. int. Med. I I, 701-13 (1937).

Findeey, T., Jr. and P. Heinbecker: Total thyroidectomy for human diabetes insipidus. Proc. Soc. exp. Biol., N.Y., 36, 448-49 (1937).

Fischer, A. and N. Engel: L'Influence des hormones sexuelles sur l'hypophyse. Rev. franç. Endocrin., I4, 203-25 (1936).

Fisher, C.: The site of formation of the posterior lobe hormones. Endocrinology, 2I, 19-29 (1937).

Fisher, C. and W. R. Ingram: Effect of feeding of thyroid or salt and of thyroidectomy on fluid exchange of cats with diabetes insipidus. Arch. intern. Med., 58, 117-29 (1936).

Fisher, C. and W. R. Ingram: The effect of interruption of the supraoptico-hypophyseal tracts on the antidiuretic, pressor and oxytocic activity of the posterior lobe of the hypophysis. Endocrinology, 20, $762-68$ (1936).

Fisher, C., W. R. Ingram, W. K. Hare, and S. W. Ranson: The degeneration of the supraoptico-hypophyseal system in diabetes insipidus. Anat. Rec., 63, 29-52 (1935).

Fisher, C., W. R. Ingram, and S. IV. Ranson: Relation of hypothalamico-hypophyseal system to diabetes insipidus. Arch. Neurol. Psychiat., Chicago, 34, I $24^{-63}$ (1935).

Fisher, C., H. W. Magoun, and A. Hetherington: The effect of water deprivation on the fluid exchange of cats with diabetes insipidus. Amer. J. Physiol., I 2 I, I I 2-22 (1938).

Fisher, R. E. and R. I. Pencharz: Carbohydrate oxidation in hypophysectomized rats. Proc. Soc. exp. Biol., N.Y., 34, 106-7 (1936).

Fisher, R. E., J. A. Russell, and C. F. Cori: Glycogen disappearance and carbohydrate oxidation in hypophysectomized rats. J. biol. Chem., I $15,627-34$ (1936).

Fitzhugh, O. G.: Effects of cortico-adrenal extract on growth and sexual activities. Amer. J. Physiol., I I8, 677-89 (1937).

Flaks, J., I. Himmel, and A. Zlotnik: Sur l'existence d'une hormone hemopoiétique dans l'hypophyse. Presse méd., 45, 1261-62 (1937). 


\section{BIBLIOGRAPHY}

Fleischmann, IV. and H. Goldhammer: Zur Frage der hormonalen Wirkung der Zirbeldrüse. Klin. Wschr., x 5, 1047-48 (1936).

Fleischmann, IV. and H. Goldhammer: Nachweis einer oestrushemmenden Substanz im Kinderharn. Klin. Wschr., I5, I730-31 (1936).

Fleischmann, W. and S. KanN: Wirkung von Hypophysenhormonen auf den Farbwechsel einiger Adriafische. Z. vergl. Physiol., 25, 25 I-55 (I937).

Florentin, P.: La Neurocrinie hypophysaire chez le crapaud (Bufo vulgaris Laur.). Etude expérimentale. C. R. Soc. Biol., Paris, I26, 33 I34 (1937).

Flummann, C. F.: Ovary-stimulating factors and antihormones. Amer. J. Obstetr., 30, 584-89 (1935).

Fluhmann, C. F.: Species-specificity in production of antigonadotropic substances. Proc. Soc. exp. Biol., N.Y., 32, I 595-96 (1935).

Fluhmane, C. F.: Comparative studies of gonadotropic hormones. IV. Ovaries and hypophyses of rats in chronic experiments. Proc. Soc. exp. Biol., N.Y., 34, 691-94 (1936).

Flummans, C. F.: The demonstration of gonadotropic substances in the blood and urine. Amer. J. Obstetr., 33, 93 I-4I (I 937).

Foerster, O., O. Gagel, and IV. Mahoney: Vegetative Regulationen. Verh. dtsch. Ges. inn. Med., pp. I65-87 (1937).

Foglia, V. G., R. Gerschman, A. D. Marenzi, J. M. Muñoz, and C. T. Rietti: L'Aggravation du diabète pancréatique par l'extrait antérohypophysaire. C. R. Soc. Biol., Paris, I 26, I 5 2-53(I937).

Fol.LEY, S. J.: The effect of oestrogenic hormones on lactation and on the phosphatase of the blood and milk of the lactating cow. Biochem. J., 30, 2262-72 (1936).

Folley, S. J. and S. K. Kon: Effect of progesterone on lactation in the rat. Nature, I40, I 107 (1937).

Foldey, S. J. and S. K. Kon: The effect of sex hormones on lactation in the rat. Proc. Roy. Soc., B, I 24, 476-92 (1938).

Folley, S. J. and P. White: Response of the pigeon crop gland to prolactin: inhibition by oestradiol monobenzoate. Nature, I40, 505 (1937).

Forbes, T. R.: Studies on the reproductive system of the alligator. I. The effects of prolonged injections of pituitary whole gland extract in the immature alligator. Anat. Rec., 70, I I 3-37 ( I 937).

Foster, M. A. and F. L. Hisaw: Experimental ovulation and the resulting pseudopregnancy in anoestrous cats. Anat. Rec., 62, 75-93 (I935).

Franck, S.: Histophysiologie de la préhypophyse. L'hypophyse du cobaye normal. C. R. Soc. Biol., Paris, I I9, 4 I I-I 5 (I935).

Franck, S.: Histophysiologie de l'hypophyse du cobaye. Cycle sécrétoire et régénération de la cellule hypophysaire chez le cobaye. C. R. Soc. Biol., Paris, I I9, 416-18 (I935).

Franck, S.: Histophysiologie de la préhypophyse. Action de l'extrait préhypophysaire alcalin sur l'hypophyse du cobaye. C. R. Soc. Biol., Paris, I19, 419-2I (I935). 


\section{THE PITUITARY BODY}

Franck, S.: Histophysiologie de la préhypophyse. Action de quelques fractions isolées de l'extrait préhypophysaire sur l'hypophyse du cobaye. C. R. Soc. Biol., Paris, I23, 33 I-34 ( I 936).

Franck, S.: Histophysiologie de la préhypophyse. Action de l'hormone thyroïdienne et de l'hormone de la cortico-surrénale sur l'hypophyse du cobaye. C. R. Soc. Biol., Paris, 123, 335-38 (1936).

Franck, S.: Histophysiologie de la préhypophyse. La cytologie dynamique de la préhypophyse. C. R. Soc. Biol., Paris, r23, 729-32 (1936).

FRANCK, S.: Studies on the thyroid gland. VII. Histophysiology and endocrine interrelationship of the anterior pituitary. Acta path. scand., 14, 339-82 (1937).

Franck, S.: Studies on the thyroid gland. VIII. Anterior pituitarythyroid. I) After combined treatment with injection of alkaline hypophyseal extract and iodine and 2) after $\mathrm{X}$-ray treatment of the anterior pituitary. Acta path. scand., 14, 538-52 (1937).

Franck, S.: The dynamic cytology of the anterior pituitary gland. Arch. exp. Zell forsch., 19, 380-83 (1937).

Franck, S.: Histophysiologie de la préhypophyse. Préhypophyse et glande thyroïde soumises à l'action de l'iode. C. R. Soc. Biol., Paris, I 25, 569-73 (1937).

Franck, S.: Histophysiologie de la préhypophyse. Préhypophyse et glande thyroïde soumises à l'action de la folliculine. C. R. Soc. Biol., Paris, I25, 573-76 (1937).

Frank, R. T. and U. J. SAlmon: Gonadotropic blood and urine cycles in normal menstruating woman. Proc. Soc. exp. Biol., N.Y., 32, 1237-39 (I935).

Frank, R. T. and U. J. SALmon: Effect of administration of estrogenic factor upon hypophyseal hyperactivity in the menopause. Proc. Soc. exp. Biol., N.Y., 33, 31 I-I 2 (1935).

Frank, R. T. and U. J. SALMON: Gonadotropic excretion in the male castrate. Effect of the male sex hormones. Proc. Soc. exp. Biol., N.Y., 34, $80_{4}-5$ ( I 936 ).

Frank, R. T. and U. J. SAlmon: Time factor relationship of follicle stimulation and luteinization in the immature rat. Proc. Soc. exp. Biol., N.Y., 35, 493-95 (1936).

Frank, R. T., U. J. Salmon, and R. Friedman: Determination of luteinizing and follicle-stimulating principles in castrate and menopause urine. Proc. Soc. exp. Biol., N.Y., 32, 1666-67 (1935).

Fraser, A. M.: The alleged antidiuretic action of the pigmentary hormones of the pituitary gland. J. Pharmacol. exp. Therap., 6o, 82-88 (I 937).

Fraser, A. M.: 'The diuretic action of the oxytocic hormone of the pituitary gland and its effect on the assay of pituitary extracts. J. Pharmacol. exp. Therap., 60, 89-95 (1937).

Frazier, W. D.: Use of Pitressin for control and relief of distention. Amer. J. Surg. , 36, 672-78 (I 937 ). 
Freed, S. C.: Gonadotropic substance in urine of normal children. Proc. Soc. exp. Biol., N.Y., 33, 35-36 (1935).

Freed, S. C. and A. Coppock: Gonadotropic substance from teratoma of the testis. Proc. Soc. exp. Biol., N.Y., 32, I 589-9I (I935).

Freed, S. C. and O. Hechter: The extraction of both the gonadotropic and (free or total) estrogenic hormones from a single urine sample. Endocrinology, 20, 396-97 (1936).

Fremery, P. DE: Experimentelle Frühreife und deren Einfluss auf die Nebennierengrösse der männlichen Ratte. Acta brev. neerl., 4, 7-9 (I934).

Fremery, P. DE: Über die Kastrationshypophyse infantiler Ratten. Acta brev. neerl., 6, I 5-I7 (I936).

Fremery, P. DE: On the influence of different hormones on lactation. J. Physiol., 87, 50P-5 IP (1936).

Fremery, P. de and P. J. Denekamp: Experimentelle Untersuchungen über Lactation und Schwangerschaft. Acta brev. neerl., 5, 44-46 (I935).

Fremery, P. De and B. Scheygrond: Inhibition of the gonadotropic activity of pregnancy urine extract by the serum of rabbits injected with an extract of male urine. Nature, I 39, IOI 5-I6 (I 937).

FREUD, J.: Continued observations with reference to the effect of various male hormones in hypophysectomized animals. Acta brev. neerl., 5, 97-98 (1935).

Freud, J.: Künstliches Wachstum und Wachstumshemmung. Ned. T. Geneesk., pp. 4977-90 (I935) (available as an abstract).

Freud, J.: An antiluteogenic factor in the anterior pituitary. Nature, I39, 880-8 I ( I 937).

Freud, J., E. Dingemanse, and J. Polak: Assay of co-substance $\mathrm{X}$ of male hormones. Acta brev. neerl., 5, 179-80 (1935).

Freudenberg, K., E. Weiss, and H. Biller: Notiz über Oxytocin. Z. physiol. Chem., 233, I72-73 (I935).

Freudenberger, C. B. and F. IV. Clausen: The effect of continued theelin injections on the body growth and organ weights of young female rats. Anat. Rec., 68, 133-44 (1937).

Freudenberger, C. B. and F. W. Clausen: Quantitative effects of theelin on body growth and endocrine glands in young albino rats. Anat. Rec., 69, 17I-77 (1937).

Freudenberger, C. B. and E. I. Hashimoto: A summary of data for the effects of ovariectomy on body growth and organ weights of the young albino rat. Amer. J. Anat., 62, 93-I I 9 (I 937).

Freudenberger, C. B. and P. M. Howard: Effects of ovariectomy on body growth and organ weights of the young albino rat. Proc. Soc. exp. Biol., N.Y., 36, 144-48 (1937).

Frey, E.: Worauf beruht die Harnvermehrung nach Hypophysin? Arch. exp. Path. Pharmak., 187, 22 I-29 (1937).

Freyberg, R. H. and R. L. Grant: Calcium and phosphorus metabolism in a verified case of pituitary basophilism. Arch. intern. Med., $58,213-28(1936)$. 
Freyberg, R. H. and L. H. Newburgh: The obesity and energy exchange in a verified case of pituitary basophilism. Arch. intern. Med., 58, 229-34 (1936).

FriedgoOd, H. B.: Experimental exophthalmos and hyperthyroidism in guinea-pigs. Johns Hopk. Hosp. Bull., 54, 48-73 (1934).

Friedgood, H. B.: The effect of an alkaline extract of the anterior hypophysis upon the weight of the spleen and adrenal glands and upon the blood calcium level. Endocrinology, 20, 159-70 (1936).

Friedgood, H. B.: Similarity of the iodin remission in experimental anterior hypophyseal hyperthyroidism, the hyperthyroidism of acromegaly and that of exophthalmic goiter. Endocrinology, 20, 526-36 (1936).

Friedgood, H. B.: Cortico-adrenal and neural effects on gonadotropic activity of the pituitary. Science, 86, 84-85 (1937).

Friedgood, H. B. and W. B. Cannon: Studies on the sympathetic nervous control of the anterior hypophysis. Amer. J. Physiol., II6, 54 (1936).

Friedgood, H. B. and M. A. Foster: The effect of adrenalectomy upon experimental ovulation and luteinization in anestrous cats. Amer. J. Physiol., I I9, 31 2 (1937).

Friedgood, H. B. and R. MCLEAN: The effect of anterior hypophyseal extract upon the serum calcium and phosphorus. Amer. J. Physiol, I I 8, 588-93 (1937).

Friedgood, H. B. and G. Pincus: Studies on conditions of activity in endocrine organs. XXX. The nervous control of the anterior hypophysis as indicated by maturation of ova and ovulation after stimulation of cervical sympathetics. Endocrinology, 19, 710-18 (1935).

Friedman, M. H. and G. L. Weinstein: The excretion of gonadotropin by normal human males after the ingestion and injection of extracts of pregnancy urine. Endocrinology, 21, 489-94 (1937).

Friedrich, B.: Nachprüfung und Vereinfachung der Schwangerschaftsreaktion nach Visscher und Bowman. Mschr. Geburtsh. Gynäkol., 103, 211-16 (1936).

Frommel, E. and D. Zimmet: Etude sur l'action cardiaque de l'extrait hypophysaire postérieur. II. Ischémie coronarienne et aire cardiaque. Arch. internat. Méd. exp., I2, 323-27 (1937).

FRY, E. G.: The effect of adrenalectomy and thyroidectomy on ketonuria and liver fat content of the albino rat following injections of anterior pituitary extract. Endocrinologv, 21, 283-9I (I937).

Fukushima, K. and H. Kameda: Über den Einfluss von Schwangerenharn auf den überlebenden Kaninchenuterus. Zbl. Gynäkol., 6o, 378-84 (1936).

Gaebler, O. H. and W. H. Price: Effects of an anterior pituitary growth preparation on protein metabolism. J. Biol. Chem., I2I, 497-506 (1937). 


\section{BIBLIOGRAPHY}

Gagel, O. and W. Mahoner: Zur Frage des Zwischenhirn-Hypophysensystems. Z. Neur., I56, 594-613 (1936).

GAGYI, J.: Du contenu de l'hypophyse en vitamine C. Nourrisson, 24, $371-73$ (I 936).

Gaillard, P. J.: An experimental contribution to the origin of the pars intermedia of the hypophysis (by combined culturing of anterior and posterior lobe explants). Acta neerl. Morph. norm. path. I, 3-I I (I937).

Gaillard, P. J.: Die Glandula hypophysis von Kaninchen in der Gewebezüchtung, ihre Strukturveränderungen und ihr Einfluss auf das Wachstum von mit diesen zusammengezüchteten Kulturen osteogenetischer Zellen. Protoplasma, 28, I-I 7 (1937).

Gallien, L.: Action comparée des extraits hypophysaires et des substances gonadotropes de l'urine sur l'ovulation chez Rana temporaria L. C. R. Soc. Biol., Paris, r 24, 874-77 (1 937).

GARDNER, W. U.: The effect of ovarian hormones and ovarian grafts upon the mammary glands of male mice. Endocrinology, r9, 656-67 (1935).

Gardner, W. U., E. T. Gomez, and C. W. Turner: Further studies of the effects of the estrogenic and the galactopoietic hormones upon the mammary gland of the rabbit. Amer. J. Physiol., I I2, 673-83 (I935).

Gaunt, R., J. W. Remington, and M. Schweizer: Some effects of intraperitoneal glucose injections and excess water in normal, adrenalectomized, and hypophysectomized rats. Amer. J. Physiol., 120, 532-43 (I 937 ).

Gaunt, R. and C. E. Tobin: Lactation in adrenalectomized rats. Amer. J. Physiol., i i 5, 588-98 (1936).

GAUPP, R., JR.: Die histologischen Befunde und bisherigen Erfahrungen über die Zwischenhirnsekretion des Menschen. Z. Neur., I54, 3 I 4-30 (I 935 ).

Gaupp, R., Jr. and E. Scharrer: Die Zwischenhirnsekretion bei Mensch und Tier. Z. Neur., I53, 327-55 (I 935).

Gegerson, H. J., A. R. Clark, and R. Kurzrok: Studies on gonadotropic antihormones. Proc. Soc. exp. Biol., N.Y., 35, 193-95 (1936).

Geiling, E. M. K. and M. R. Lewis: Further information regarding the melanophore hormone of the hypophysis cerebri. Amer. J. Physiol. I $3,534-37$ (I935).

Geiling, E. M. K., L. N. Tarr, and A. DE. L. Tarr: The hypophysis cerebri of the finback (Balaenoptera physalus) and sperm (Physeter megalocephalus) whale. Johns Hopk. Hosp. Bull., 57, I23-4 I (1935).

Gentile, F. and G. Amato: L'Ipofisi nella ablazione del pancreas. Ricerche sperimentali. Riv. Pat. sper., 6, 4 I-44 (I936).

Gerbilsky, N. I. and L. A. Kashchenko: The effect of the hypophysis upon the gonads in Teleostei. Bull. Biol. Méd. exp. URSS, 3, I 58-59 (I 937 ).

Gerschman, R. and A. D. Marenzi: Action de l'extrait alcalin antéhypophysaire sur les substances minérales du plasma. C. R. Soc. Biol., Paris, I20, 8 I 7-20 (1935). 


\section{THE PITUITARY BODY}

Gersh, I.: "Glandular" cells in the pars nervosa and stalk of the hypophysis. Proc. Soc. exp. Biol., N.Y., 37, 395-96 (1 937 ).

Gersh, I. and A. D. TARR: The so-called hyaline bodies of Herring in the posterior lobe of the hypophysis. Anat. Rec., 63, 23I-38 (1935).

Grschickter, C. F. and D. Lewis: Lactogenic substance in the human breast. Its use in experimental stimulation of mammary secretion and its assay in cases of cystic disease. Arch. Surg., 32, 598-6I7 (1936).

Gessler, C.: Influence of folliculin on the basal metabolic rate. Arch. int. Pharmacodyn., 54, 263-7 I (I 936).

Gessler, C.: Activité antithyroïdienne de la folliculine. Rôle de l'hypophyse. Arch. int. Pharmacodyn., 55, 267-8I (1937).

Gıęposz, B.: Influence de la thyréostimuline sur l'aspect histologique des glandes endocrines. C. R. Soc. Biol., Paris, r 20, 555-57 (I935).

GiEdosz, B.: Influence des vitamines A et C sur l'aspect histologique des glandes endocrines. C. R. Soc. Biol., Paris, r20, 557-59 (1935).

GiLlARD, J. L.: The effects of hysterectomy on mammary gland development in a rabbit. Amer. J. Physiol., I 20, 300 303 (I 937).

Gilman, A. and L. Goodman: Effect of pituitrin injection in rabbits on serum osmotic pressure and blood picture. Proc. Soc. exp. Biol., N.Y., $33,238-40$ (1935).

Gilman, A. and L. Goodman: The secretion of an antidiuretic hypophyseal hormone in response to the need for renal water conservation. Science, 84, 24-25 (1936).

Gilman, A. and L. Goodman. Pituitrin anemia. Amer. J. Physiol., I I8, $241-50$ (1937).

Gilman, A. and L. Goodman: The secretory response of the posterior pituitary to the need for water conservation. J. Physiol., 90, 113-23 (1937).

Giordano, C. and P. Zeglio: Sulla presenza, nell'urina di soggetti ipertesi, di una sostanza ad azione surrenalotropa. Med. contemp. ('Torino), 2, 35 I-7 I ( 1 936).

Giordano, C. and P. Zeglo: Sulla presenza, nell'urina di soggetti ipertesi, di una sostanza ad azione surrenalotropa. II. Determinazione del contenuto in adrenalina delle surreni di cavie iniettate con urina di soggetti ipertesi. Med. contemp. (Torino), 2, 39I-96 (1936).

Giordano, C. and P. Zeglio: Sulla presenza, nelle urine di soggetti ipertesi, di una sostanza ad azione surrenalotropa. III. Esame istologico. Med. contemp. (Torino), 2, 456-63 (i 936 ).

Giuffrida, F.: Modivicazioni strutturali dell'ingluvie nei piccioni in seguito a trattamento con prolactin. Fol. demogr. gynaec. (Genova), 34,68 I-70I (1937).

Glick, D. and G. R. Biskind: The histochemistry of the hypophysis cerebri. The quantitative distribution of vitamin C. J. biol. Chem., I 10, 583-88 (1935).

Gomez, E. T. and C. W. Turner: Effect of hypophysectomy on development and function of the mammary gland. J. Dairy Sci., I9, 450-52 (1936). 


\section{BIBLIOGRAPHY}

Gomez, E. T. and C. IV. Turner: Non-effect of estrogenic hormones on mammary gland of hypophysectomized guinea pig. Proc. Soc. exp. Biol., N.Y., 34, 320-22(1936).

Gomez, E. T. and C. W. Turner: Effect of hypophysectomy and replacement therapy on lactation in guinea-pigs. Proc. Soc. exp. Biol., N.Y., 34, 404-6 (1936).

Gomez, E. T. and C. W. Turner: Initiation and maintenance of lactation in hypophysectomized guinea pigs. Proc. Soc. exp. Biol., N.Y., 35, $365-67$ (1936).

Gomez, E. T. and C. W. Turner: The adrenotropic principle of the pituitary in relation to lactation. Proc. Soc. exp. Biol., N.Y., 36, 7880 ( 1937 ).

Gomez, E. T. and C. IV. Turner: Effect of thyroxine and galactin on lactation in hypophysectomized guinea pigs. Proc. Soc. exp. Biol., N.Y., 36, 80-81 (1937).

Gomez, E. T. and C.W. Turner: Further evidence for a mammogenic hormone in the anterior pituitary. Proc. Soc. exp. Biol., N.Y., 37, $607-9$ (1 938$)$.

Gomez, E. T., C. W. Turner, W. U. Gardner, and R. T. Hill: Oestrogenic treatment of hypophysectomized male mice. Proc. Soc. exp. Biol., N.Y., 36, 287-90 (1937).

Gomez, E. T., C. IV. Turner, and R. P. Reece: Growth of mammary gland of hypophysectomized guinea pig. Proc. Soc. exp. Biol., N.Y., 36, 286 (1937).

Gordon, A. S., W. Klejnberg, and H. A. Charipper: Relation of reticulo-endothelial system to refractoriness developed in response to gonadotropic hormone. Proc. Soc. exp. Biol., N.Y., 36, 484-86 (1937).

Gordon, A. S., W. Kleinberg, and H. A. Charipper: The reticuloendothelial system and the concept of the "anti-hormone." Science, 86, 62-63 (1937).

Granaat, D. and J. Hillesum: The effect of hypophysis extracts upon the distribution of water in the tissues of the frog. Acta brev. neerl., 7, 1 I1-I3 (I937).

Granati, D., and J. Hillesum: Über den Einfluss von Hypophysenextrakten auf den Wasserstoffwechsel bei Rana esculenta. Arch. néerl. Physiol., 22, 347-58 (1937).

Grant, G. A.: The metabolism of galactose. III. I. Lactose synthesis from (a) a glucose-galactose mixture, $(b)$ phosphoric esters, by slices of the active mammary gland in vitro. 2. The effect of prolactin on lactose synthesis by the mammary gland. Biochem. J., 30, 2027-35 (1936).

Grant, G. A.: The influence of hormones on the secretory activity of the regressing mammary gland. Biochem. J., 31, I 538-43 (1937).

GraY, J. H.: Preliminary note on the mast cells of the human pituitary and of the mammalian pituitary in general. J. Anat., London, 69, I 53-58 (1935).

Greeler, P. O.: Sugar utilization in hypophysectomized rabbits. Proc. Soc. exp. Biol., N.Y., 32, 1070 72 (1935). 


\section{THE PITUITARY BODY}

Greep, R. O.: Functional pituitary grafts in rats. Proc. Soc. exp. Biol., N.Y., 34, 754-55 (1936).

Greep, R. O. and H. L. Fevold: The spermatogenic and secretory function of the gonads of hypophysectomized adult rats treated with pituitory FSH and LH. Endocrinology, 2I, 6I I-I 8 (1937).

Greep, R. O., H. L. Fevold, and F. L. Hisaw: Effects of two hypophyseal gonadotropic hormones on the reproductive system of the male rat. Anat. Rec., 65, 26I-7I (I936).

Grollman, A. and W. M. Firor: The rôle of the hypophysis in experimental chronic adrenal insufficiency. Amer. J. Physiol., II2, 310-I9 (I935).

Gruber, C. M., V. H. Moon, and E. Sufrin: A study of the response of the heart to pitressin following the administration of thyroid extract. Endocrinology, 19, 447-52 (I 935).

Grumbrecht, P.: Die Ausscheidung thyreotrop wirksamer Substanz im Harn klimakterischer Frauen. Zbl. Gynäkol., 59, I33I-36 (I 935).

Guercio, F.: Eliminazione di ormoni sessuali e preipofisari con la saliva. Osservazioni comparative di tecnica tra la A.Z. e la B. e S. Clin. ostetr., 38, 397-408 ( 1936 ).

Gulland, J. M., N. S. Lucas, M. Freeman, and S. S. Randall: The oxytocic hormone of the posterior lobe of the pituitary gland. VII. Sect. A. Ultraviolet absorption spectra. Sect. B. and C. Adsorption and electrodialysis. Biochem. J., 29, 2208-20 (1935).

Gulland, J. M. and S. S. Randall: The oxytocic hormone of the posterior lobe of the pituitary gland. $V$. Recognition as an oxidationreduction system. Biochem. J., 29, 378-90 (I 935).

Gulland, J. M. and S. S. Randall: The oxytocic hormone of the posterior lobe of the pituitary gland. VI. Further studies of the action of oxidising and reducing agents. Biochem. J., 29, 39I-96 (I935).

GuRD, M. R.: The effect of oxytocin and vasopressin on the action of insulin. Quart. J. Pharm. Pharmacol., 7, 66 I-7 I (1934).

Gustus, E. I.., R. K. Meyer, and J.H. Dingle: Relationship of precipitin titers to gonadotropic inhibitory action of monkey sera. Proc. Soc. exp. Biol., N.Y., 33, 257-6 I (1935).

Gustus, E. L., R. K. Meyer, and O. R. Woods: Preparation of the gonadotropic hormone of pregnant mare blood. J. biol. Chem., I I4, 59-63 (I 936$)$.

Guthrie, J. S. and J. A. Bargen: The effect of drugs on different segments of the intestine of man. Surg. Gynecol. Obstetr., 63, 743-49 (I936).

Guyénot, E., E. Held, A. Mozskowska, and H. de Stoutz: L’Urine de femme ovariotomisée ne contient que le facteur auxogène. C. R. Soc. Biol., Paris, 122, I $152-54$ (1936).

Guyénot, E., K. Ponse, and E. Dottrens: Action physiologique et séparation des hormones auxogène, crinogène et thyréostimulante de l'hypophyse. Arch. Anat., 20, I 5-218 (1935). 


\section{BIBLIOGRAPHY}

Guyer, M. F. and P. E. Claus: Vacuolation of the anterior pituitary gland following castration, implantation of cancer tissue and thyroidectomy. Anat. Rec., 67, 145-56 (1937).

Hagen, F. von: Die wichtigsten Endokrinen des Flussaales. Thyreoidea, Thymus und Hypophyse im Lebenscyclus des Flussaals (Anguilla vulgaris); nebst einigen Untersuchungen über das chromophile und chromophobe Kolloid der Thyreoidea. Zool. Jb. Abt. Anat. Ontog., 6r, $467-538$ ( 1936 ).

Hahndel, H.: L'Influenza del lobo anteriore dell'ipofisi sul metabolismo degli jdrati di carbonio dell'uomo. Nota prelim. La ricerca biologica dell'ormone contra-insulare nel liquido cefalo-rachidiano dell'uomo sano. Riv. Pat. nerv., 45, $464-70$ (1935).

HaIN, A. M.: The physiology of pregnancy in the rat: further data on the passage of hormones via the placenta and the mother's milk. Quart. J. exp. Physiol., 26, 29-43 (1936).

Hain, A. M.: The physiology of pregnancy in the rat. The combined action of male and female hormones (testosterone propionate and oestrone). Quart. J. exp. Physiol., 26, 293-98 (1937).

HAIR, G. W.: The nerve supply of the hypophysis of the cat. Anat. Rec., $71,141-60$ ( 1938 ).

HALPERn, A. C.: Cytological responses of rat thyroid to treatment with anterior pituitary and potassium iodide. Proc. Soc. exp. Biol., N.Y., $32,854-57$ (1935).

HaLpern, S. R. and F. E. D'Amour: Studies on the gonad-hypophyseal complex in estrin-injected rats. Amer. J. Physiol., I I5, 229-38 (I 9.36).

Halpern, S. R. and I. E. Hendryson: Comparative eflects of dinitrophenol and thyroid on pituitary-gonadal complex of female rats. Proc. Soc. exp. Biol., N.Y., 33, 263-65 (I 935 ).

Hamblen, E. C. and R. A. Ross: Responses of the human ovary to gonadotropic principles. Endocrinology, 21, 722-26 (I937).

Hamburger, C.: Weitere Untersuchungen über die gonadotropen Hormone bei der trächtigen Stüte. Endokrinologie, I7, 8-2 I (1936).

Hamlett, G. IV. D.: The effects of antuitrin $\mathrm{S}$ and pituitary extract upon the armadillo ovary. Anat. Rec., 62, 201-7 (1935).

Hamlett, G. W. D.: Positive Friedman tests in the pregnant rhesus monkey, Macaca mulatta. Amer. J. Physiol., r 18, 664-66 (1937).

Handovsky, H. and A. SAmaAn: Effet des extraits posthypophysaires sur le débit sanguin rénal et sur la diurèse des chiens normaux et des chiens narcotisés. C. R. Soc. Biol., Paris, 122, I22-25 (1936).

HANn, F. von.: Über die Bedeutung der Hypophysenveränderungen bei Diabetes insipidus. Frankf. Z. Path., 21, 337-65 (1918).

HARE, K.: Degeneration of the supra-optic nucleus following hypophysectomy in the dog. Amer. J. Physiol., I 19, 326 (I937).

Harington, C. R. and I. W. Rowlands: Fractionation of antithyrotropic and antigonadotropic sera. Biochem. J., 31, 2049-54 (I937). 


\section{THE PITUITARY BODY}

HARRIS, G. W.: The induction of pseudo-pregnancy in the rat by electrical stimulation through the head. J. Physiol., 88, 361-67 (1936).

HARRIS, G. IV.: The induction of ovulation in the rabbit, by electrical stimulation of the hypothalamohypophysial mechanism. Proc. Roy. Soc., B, 122, 374-94 (1937).

Harris, G. W. and G. T. Popa: A technique for operations on the hypothalamohypophysial region of the rabbit. J. Anat., London, 72, 226$33(1938)$.

Harrow, B., A. Mazur, I. M. Chamelin, and A. Lesuk: Concentration of a hyperglycemic factor from urine. Proc. Soc. exp. Biol., N.Y., 34, 688-90 (1936).

Hartman, C. G.: Menstruation inhibiting action of testosterone. Proc. Soc. exp. Biol., N.Y., 37, 87-89 (1937).

Hartman, C. G. and M. W. Firor: Possible posterior pituitary involvement in menstruation. Anat. Rec., Suppl., 51, 55-56 (1935).

Haterius, H. O. and A. J. Derbyshire, Jr.: Ovulation in the rabbit following upon stimulation of the hypothalamus. Amer. J. Physiol., I 19, 329-30 (1937).

Haterius, H. O., M. Schweizer, and H. A. Charipper: Experimental studies of the anterior pituitary. III. Observations on the persistence of hypophyseal transplants in the anterior eye chamber. Endocrinology, I9, 673-8i (1935).

Haupstein, P.: Zum Wirkungsmechanismus des Sexual- (Follikel-)Hormons. II. Mitt. Klinische Beobachtungen über die Stellung der Hypophyse. Klin. Wschr., 14, I IO3-7 (1935).

Hauptstein, P. and E. Bühler: Experimentelle Untersuchungen über die Stellung des Uterus im sexualhormonal System. Arch. Gynäkol., $162,1-13(1936)$.

HAWKING, F.: Differential cell counts of the pituitary gland in hypertension and endocrine disturbances. J. Path. Bact., London, 42, 689-702 (1936).

HAYWARD, S. J. and J. LoEB: Effects of sugar, glycerin and urea on hormones of cattle anterior pituitary glands. Proc. Soc. exp. Biol., N.Y., $36,250-53$ ( 1937 ).

Heinbecker, P., M. Somogyi, and T. E. Weichselbaum: Quantitative assay of insulin effect. Proc. Soc. exp. Biol., N.Y., 36, 399-401 (1937).

Heinbecker, P., M. Somogyi, and T. E. Weichselbaum: Effect of diet on insulin response in normal and hypophysectomized dogs. Proc. Soc. exp. Biol., N.Y., 36, 804-5 (1937).

Heinbecker, P. and T. E. Weichselbaum: Blood sugar response to intraperitoneal epinephrine injections in normal and hypophysectomized dogs. Proc. Soc. exp. Biol., N.Y., 37, 527-29 (1937).

Heinemann, K.: Experimentelle Untersuchungen an Meerschweinchen und Ratten zur Frage der Organveränderungen durch thyreotropes Hormon. Endokrinologie, 19, 1-9 (1937). 


\section{BIBLIOGRAPHY}

Hellbaum, A. A.: The gonad-stimulating activity of pituitary glands from horses of different ages and sex types. Anat. Rec., 63, 147-57 (I935).

Hellbaum, A. A.: Augmentation of ovary-stimulating action of gonadotropic preparations. Proc. Soc. exp. Biol., N.Y., 33, 568-70 (1936).

Hellbaum, A. A.: The fractionation, and a study of the interaction, of the gonadotropic factors in pregnant mares' blood. Amer. J. Physiol., I I9, 33 I (I 937).

Hellbaum, A. A., H. L. Fevold, and F. L. Hisaw: Method for concentrating the gonadotropic activity in pregnancy urine. Proc. Soc. exp. Biol., N.Y., 32, I 566-67 (1935).

Hellbaum, H. W.: The cytology of snake thyroids following hypophysectomy, activation and ultra-centrifuging. Anat. Rec., 67, 53-68 (I 936$)$.

Heller, C. G., H. Lauson, and E. L. Sevringhaus: The immature rat uterus as an assay end-point for gonadotropic substances. Amer. J. Physiol., 121, 364-78 (1938).

Heller, H.: The state in the blood and the excretion by the kidney of the antidiuretic principle of posterior pituitary extracts. J. Physiol., 89, $8 \mathrm{I}-95$ (1 937$)$.

Heller, H. and F. F. UrBan: The fate of the antidiuretic principle of postpituitary extracts in vivo and in vitro. J. Physiol., 85, 502-18 (1935).

Hemmingsen, A. M. and N. B. Krarup: Rhythmic diurnal variations in the oestrous phenomena of the rat and their susceptibility to light and dark. Biol. Medd. danske Vidensk. Selsk., I3, 3-6i (I937).

Herold, L.: Nachweis und Auswertung von antithyreoiden Schutzstoffen im Blute von Basedowkranken und Schwangeren. Klin. Wschr., I3, $1242-43$ (I934).

Hertz, R. and R. K. MEYER: The effect of testosterone, testosterone propionate and dehydroandrosterone on the secretion of the gonadotropic complex as evidenced in parabiotic rats. Endocrinology, 2I, 756-6I (1937).

Hertz, S. and E. G. OAstler: Assay of blood and urine for thyreotropic hormone in thyrotoxicosis and myxedema. Endocrinology, 20, 520-25 (1936).

Hess, J. H., R. H. Kunstadter, and W. Saphir: Urinary excretion of gonadotropic hormone in cryptorchidism. J. Amer. med. Ass., I08, 352-54 (I937).

Hesselberg, C. and L. Loeb: The retrogression of the lactating mammary gland in the guinea pig. Amer. J. Physiol., I I8, 528-31 (1937).

Hidaka, J.: Über den Einfluss des Follikelhormons auf die Degeneration des Corpus luteum spurium bei Kaninchen. I. Mitt. Histologische Untersuchung. Mitt. jap. Ges. Gynäkol., 32, 34-35 (1937).

Hill, M. and A. S. PARKEs: Effect of absence of light on the breeding season of the ferret. Proc. Roy. Soc., B, I15, I4-17 (I934). 


\section{THE PITUITARY BODY}

HIL, R. T. and W. U. Gardner: Function of pituitary grafts in mice. Proc. Soc. exp. Biol., N.Y., 34, 78-79 (1936).

Hill, R. T. and A. S. PArkes: Hypophysectomy of birds. IV. Plumage changes in hypophysectomized fowls. Proc. Roy. Soc., B, I17, 202-9 (1935).

Hill, R. T. and A. S. PARkes: Hypophysectomy of birds. V. Effect of replacement therapy on the gonads, accessory organs and secondary sexual characters of hypophysectomized fowls. Proc. Roy. Soc., B, I17, $210-18$ (1935).

Himsworth, H. P. and D. B. McN. ScotT: The relation of the hypophysis to changes in sugar tolerance and insulin sensitivity induced by changes of diet. J. Physiol., 91, 447-58 (I938).

Hinman, F. and T. O. Powell: The management of tumor of the testicle. J. Amer. med. Ass., i Io, i 88-90 (I938).

Hiroshi, O.: Über die Veränderungen der Amphibienhypophyse bei Knochenfraktur. Trans. Jap. path. Soc., 26, 499-502 (I936).

Hisaw, F. L.: The physiology of menstruation in Macacus rhesus monkeys. I. Influence of the follicular and corpus luteum hormones. II. Effects of anterior pituitary extracts. Amer. J. Obstetr., 29, 638-59 (I935).

Hisaw, F. L., R. Hertz, and H. L. Fevold: Experimental production of ovarian refractoriness to anterior hypophyseal stimulation in the monkey. Endocrinology, 20, 40-46 (1936).

Hodler, D.: Surrénales et masculinisation. Thesis. Geneva (I937).

Högler, F. and F. Zell: Hypophysenhinterlappen und Kohlehydratstoffwechsel. WVien. Arch. inn. Med., 27, I4 I-58 (I935).

Hofrmann, F.: Über die Entstehung der Lactation. Zbl. Gynäkol., 6o, 2882-86 (1936).

Hoffmann, F.: Über die Darstellungsmethoden einer gonadotropen Substanz aus der Nebennierenrinde. Endrokrinologie, I9, 145-48 (1937).

Hoffmann, F.: Über die gonadotrope Wirkung von Nebennierenrindenextrakten. Klin. Wschr., 16, 79-81 (1937).

Hogben, L.: The pigmentary effector system. VII. The chromatic function in elasmobranch fishes. Proc. Roy. Soc., B, 120, 142-58 (1936).

Hogben, I. and D. Slome: The pigmentary effector system. VIII. The dual receptive mechanism of the amphibian background response. Proc. Roy. Soc., B, I 20, 158-73 (1936).

HoHLweg, IV.: Corpus luteum-Hormon und Kastrationshypophyse. Klin. W'schr., I4, I027-28 (I935).

Hohlweg, W.: Der Mechanismus der Wirkung von gonadotropen Substanzen auf das Ovar der infantilen Ratte. Klin. Wschr., I5, I 832-35 ( 1936 ).

Hohlweg, W.: Männliche Wirkstoffe und Corpus luteum-Bildung. Klin. Wschr., I6, 586-87 (1937).

Hohlweg, W. and A. Chamorro: Über die luteinisierende Wirkung des Follikelhormons durch Beeinflussung der luteogenen Hypophysenvorderlappensekretion. Klin. W'schr., I6, I96-97 (1937). 


\section{BIBLIOGRAPHY}

Hohlweg, W. and J. Sснмidt: Zur Chemie und Biologie des reinen Corpus luteum-Hormons "Progesteron." Klin. Wschr., I5, 265-67 (1936).

Holden, R. and E. W. Thurston: Effects of cattle anterior pituitary extracts and KI on liver glycogen in guinea pigs. Proc. Soc. exp. Biol., N.Y., 32, $14^{17^{-19}}$ (1935).

Holt, H., R. W. Keeton, and B. Vennesland: The effect of gonadectomy on body structure and body weight in albino rats. Amer. J. Physiol., II4, 51 5-25 (1936).

Holtz, P. and G. Jancke: Die Wirkung der Hormone des HypophysenHinterlappens auf den intraokularen Druck. Arch. exp. Path. Pharmak., I8I, 494-502 (1936).

Holtz, P. and K. Wöllpert: Die Reaktion des Katzen- und Meerschweinchenuterus auf Adrenalin während der verschiedenen Stadien des Sexualcyclus und ihre hormonale Beeinflussung. Arch. exp. Path. Pharmak., 185, 20-4I (1937).

Hoover, E. E.: Experimental modification of the sexual cycle in trout by control of light. Science, 86, 425-26 (1937).

Houssay, B. A.: Modifications hypophysaires produites par les lésions tubériennes. Bull. Acad. Méd. Paris, I 14, 371-73 (1935).

Houssay, B. A.: Action de l'hypophysectomie sur la grossesse et la sécrétion lactée, chez la chienne. C. R. Soc. Biol., Paris, I 20, 496-97 (1935).

Houssay, B. A.: Sécrétion lactée provoquée par l'extrait anté-hypophysaire chez le chien. C. R. Soc. Biol., Paris, I20, 502-3 (1935).

Houssay, B. A.: Diabetes as a disturbance of endocrine regulation. Amer. J. med. Sci., I93, 58 I-606 (1937).

Houssar, B. A. and A. Biasotri: Rôle de l'hypophyse et de la surrénale dans le diabète pancréatique du crapaud. C. R. Soc. Biol., Paris, I23, 497-500 (1936).

Houssay, B. A. and J. M. Lascano Gonzalez: La rate des chiens hypophysoprives. C. R. Soc. Biol., Paris, II8, 487-88 (1935).

Houssay, B. A. and J. M. Lascano Gonzalez: Hypophyse et hypertrophie compensatrice du testicule chez le crapaud. C. R. Soc. Biol., Paris, I 20, 362-63 (1935).

Houssay, B. A. and L. F. Leloir: Action diabétogène antéhypophysaire indépendante des surrénales. C. R. Soc. Biol., Paris, I 20, 670-72 (1935).

Houssay, B. A. and C. T. RieTt1: Action cétonémiante de l'extrait antérohypophysaire dans les insuffisances endocrines du rat. C. R. Soc. Biol., Paris, I26, 620-22 (1937).

Hove, E., C. A. Elvehjem, and E. B. Hart: The physiology of zinc in the nutrition of the rat. Amer. J. Physiol., II9, 768-75 (1937).

Howard, N. J.: Comparative studies of gonadotropic hormones. $\mathrm{V}$. Growth response of rat mammary glands in chronic experiments. Proc. Soc. exp. Biol., N.Y., 34, 732-34 (1936).

Howes, N. H.: A study of the histology of the pituitary gland of the skate. Quart. J. micr. Sci., 78, 637-51 (1936). 


\section{THE PITUI'TARY BODY}

Howet, F.: Effet immédiat de l'hypophysectomie sur la diurèse du chat. C. R. Soc. Biol., Paris, 122, 798-802 (1936).

Huberman, J., H. H. Israeloff, and B. Hymowitz: Effects on spermatogenesis of a follicle stimulating extract obtained from menopausal or castrate urines. Endocrinology, 21, 67-7 I (1937).

Ihering, R. von and P. De Azevedo: Über die Wirkung des SäugetierHypophysenhormons auf den Laichakt der Fische. Zool. Anz., I20, $71-75$ (1937).

Ikuta, H.: Über experimentelle Studien der Zirbeldrüse. I. Mitt.: Veränderungen des Zentralnervensystems bei Exstirpation derselben. Trans. Jap. path., Soc., 27, 498-500 (1937).

Ingelbrecht, P.: Influence du système nerveux central sur la mamelle lactante chez le rat blanc. C. R. Soc. Biol., Paris, I20, I369-7i (I935).

Ingle, D. J. and E. C. KEndall: Atrophy of the adrenal cortex of the rat produced by the administration of large amounts of cortin. Science, 86, 245 (1937).

Ingram, W. R. and R. W. BARRIs: Evidence of altered carbohydrate metabolism in cats with hypothalamic lesions. Amer. J. Physiol., II4, 562-7 I ( 1936$)$.

InGRAM, W. R. and C. Fisher: The relation of the posterior pituitary to water exchange in the cat. Anat. Rec., 66, 271-93 (1936).

Ingram, W. R. and C. Fisher: The effects of thyroidectomy, castration, anterior lobe administration and pregnancy upon experimental diabetes insipidus in the cat. Amer. J. Physiol., II9, 34 I (1937).

INGRAM, IV. R. and C. FISHER: The effects of thyroidectomy, castration, anterior lobe administration and pregnancy upon experimental diabetes insipidus in the cat. Endocrinology, 21, 273-82 (1937).

Ingram, W. R., C. Fisher, and S. W. Ranson: Experimental diabetes insipidus in the monkey. Arch. intern. Med., 57, 1067-80 (1936).

Inohara, S.: Einfluss des Schwangerenharns auf die Nebenniere. IV. Mitt. Die Veränderungen der Nebennierenrinde der verschiedenen Tiere bei Schwangeren und Injektion des sogenannten Hypophysenvorderlappenhormons und eine Frage über Vakuolisierung in der Rinde. Mitt. jap. Ges. Gynäkol., 30, 20 (1935).

Ito, M., S. Hajazu, and F. Ueno: Uber das gonotrope Hormon aus Schwangerenharn. Studien über die Beziehungen der verschiedenen Reinigungs- und Fällungsverfahren zur biologischen Wirkung des Hormons. Zbl. Gynäkol., 6o, 375-78 (1936).

Ivanova, S.: Über den Mechanismus der Wirkung von Licht auf die Hoden der Vögel (Passer domesticus). Arch. exp. Path. Pharmak., I79, 349-59 (1935).

Jacobs, H. R. and A. R. Colwell: Lesions in the pancreas and in the anterior hypophysis with fatal acidosis following prolonged intravenous administration of glucose (in dogs). Amer. J. Physiol., I I6, 194-200 $(1936)$.

$$
\left[33^{6}\right]
$$


Jacobsen, A. P.: Norsk Mag. Laegevidensk., 97, 224-48 (1936) (abstract). Jacobsen, A. W. and A. J. Cramer, JR.: Clinical results of anterior pituitary therapy in children. J. Amer. med. Ass., I09, IOI-8 (I937).

JadAssohn, W., E. Uehlinger, and W. Zürcher: Zur Vergrösserung der Meerschweinchenbrustwarze durch Hormone. (I. Mitt.) Helvet. med. Acta, 4, 199-208 (1937).

JaEgher, M. DE and A. van Bogaert: Hypertension hypothalamique expérimentale par excitation chimique. C. R. Soc. Biol., Paris, II8, I033-35 (1935).

Jafgher, M. De and A. van Bogaert: Hyperglycémie provoquée par excitation électrique de l'hypothalamus. C. R. Soc. Biol., Paris, I 8 , 1035-37 (1935).

Janssen, S.: Reflektorische Einflüsse auf die Ausscheidung von Wasser und Kochsalz. Pflügers Arch., 235, 523-33 (1935).

Jones, A. M. and W. Schlapp: The action and fate of injected posterior pituitary extracts in the decapitated cat. J. Physiol., 87, I44-57 (1936).

Jones, M. E. and F. R. Steggerda: Effects of light and dark environment on weight changes in normal and hypophysectomized frogs. Proc. Soc. exp. Biol., N.Y., 32, I369-71 (I935).

Jones, M. S. and T. N. MACGregor: Inhibitory effect of follicular hormone on the anterior pituitary in humans. Lancet, 23I, 974-76 (1936).

Jores, A.: Änderungen des Hormongehaltes der Hypophyse mit dem Wechsel von Licht und Dunkelheit. Klin. W'schr., I4, I7I3-I6 (1935).

Jores, A.: Untersuchungen über die Funktion des Pigmenthormons im Warmblüterorganismus. I. Mitt. Die Wirkungen des Hormons auf Temperatur und Blutzucker bei intraventrikulärer Injektion beim Kaninchen. Z. ges. exp. Med., 97, 207-13 (1935).

Jores, A.: Die Bedeutung der Hypophyse für die Entstehung des Hochdruckes insbesondere der essentiellen Hypertonie. Klin. W'schr., $\mathbf{1 5}_{5}$ $841-6$ (1936).

Jores, A.: Welche Schlüsse lassen sich aus einer mit menschlichem Harn positiven Melanophorenreaktion ziehen? Klin. Wschr., I5, I+33-3+ (i 936 ).

Jores, A.: Experimentelle Untersuchungen über die Wirkung der Nebennieren auf die Hypophyse. I. Mitt. Änderungen in dem Gehalt der Hypophyse und des Blutes an Melanophorenhormon unter der Wirkung von Adrenalin und Cortidyn. Z. ges. exp. Med., 97, 805-12 (1936).

Jores, A. and H. Beck: Eine biologische Testmethode für das corticotrope Hormon. Z. ges. exp. Med., 97, 622-29 (1936).

Jores, A. and W. Boecker: Welche Rolle spielt die Schilddrüse für die Wirkungen des corticotropen Hypophysenvorderlappenhormons? Z. ges. exp. Med., I00, 332-36 (I937).

Jores, A. and K. G. CAEsAR: Über die Wirkung des Melanophorenhormons auf Pigmentwanderung und Pupillenweite des Froschauges. Pflügers Arch., 235, 724-32 (1935).

Jores, A. and K. Hoeltje: Untersuchungen über die das Melanophorenhormon bindende Substanz im Blut von Tieren (Fröschen und Kanin- 


\section{THE PITUITARY BODY}

chen) und des Menschen nach Dunkelaufenthalt. Z. vergl. Physiol, 23, $571-77$ (1936).

Junkmann, K.: Thyreotropes Hormon und verwandte Hormone. Handb. der biologischen Arbeitsmethoden. Hrsg. von Emil Abderhalden. Abt. V. 'Tl. 3B, H.7, Liefg. 476. Berlin (1936).

Kabat, H., B. J. Anson, H. IV. Magoun, and S. W. Ranson: Stimulation of the hypothalamus with special reference to its effect on gastrointestinal motility. Amer. J. Physiol., II2, $214^{-26}$ (1935).

KAHN, K.: Action de l'hyperinsulinisation chronique sur l'histostructure de la pars antérieur de l'hypophyse du cobaye. Bull. Histol. appl., I2, 300-317 (1935).

Kahn, K. and L. K. Waledinskaja: Die Wirkung der partiellen Pankreatomie auf den Blutzucker und die Histostruktur des Hypophysenvorderlappens. Arch. Anat., 15, $121-23$ (1936).

Kampelmann, F.: Über die Wirkung von arseniger Säure auf Schilddrüse und Hypophysenvorderlappen. Arch. exp. Path. Pharmak., r84, I395 I (1937).

Kampelmann, F. and E. Schulze: Kohlenoxyd und Hypophysenvorderlappen. Arch. exp. Path. Pharmak., 184, 152-55 (1937).

Kapran, S. K.: Med. Ž. vseukraïn. Akad. Nauk., 4, 625-27 (1935).

Karlik, L. N.: Über Wechselbeziehung zwischen Hypophyse und Pankreas. Z. ges. exp. Med., 98, 314-25 (I936).

Karlik, L. N. and J. A. Robinson: Arch. argent. Neur., 12, 6I-79 (1935) (abstract).

Kasamara, S.: On the cultivation in vitro of the hypophysis. Arch. exp. Zellforsch, I8, 42-76 (I 935).

KASAHARA, S.: Further contribution to the tissue culture of the hypophysis. Redifferentiation of epithelial cells in vitro. Arch. exp. Zellforsch., $18,77^{-8} 4$ (1935).

KATER, J.: The insulin sensitiveness of hypophysectomized rats. Acta brev. neerl., 6, 97-99 (1936).

KATz, K.: Hypophysenvorderlappen und Krebs. Z. Krebsforsch., 45, 139$70(1936)$.

KatzMan, P. A.: The quantitative determination of the gonadotropic material of urine of women after castration and the menopause and of normal men. Endocrinology, 21, 89-95 (I937).

Katzman, P. A., N. J. WAde, and E. A. Dolsy: Effects of chronic implantation of rats with pituitaries of the same species. Endocrinology, 2I, 1-7 (1937).

Kaufmann, C. and E. Steinkamm: Über die Wirkung fortgesetzter Zufuhr unphysiologischer Mengen Follikelhormon auf das Genitale weiblicher Ratten. Arch. Gynäkol., 162, 553-94 (1936).

KEHL, R.: Mode d'action des extraits hypophysaires antérieurs de mammifère sur les caractères sexuels secondaires du discoglosse mâle. C. R. Soc. Biol., Paris, 120, 10-12 (1935). 


\section{BIBLIOGRAPHY}

Keller, A. D.: Hypophyseal thyrotropic mechanism essential for occurrence of diabetes insipidus in its maximal form. Proc. Soc. exp. Biol., N.Y., 36, 787-89 (I 937).

Keller, A. D. and M. C. D'Amour: Ulceration in the digestive tract of the dog following hypophysectomy. Arch. Path., 2 I, I85-201 (1936).

Keller, A. D. and J. W. Hamilton, JR.: Degeneration of the infundibular nerve fibers in the cat without eliciting diabetes insipidus. Amer. J. Physiol., rig, 348 (1937).

Keller, A. D. and J. W. Hamilton, Jr.: Normal sex functions following section of the hypophyseal stalk in the dog. Amer. J. Physiol., i r9, 34950 (1 937).

Keller, A. D., W. Noble, and J. W. Hamilton, Jr.: Effects of anatomical separation of the hypophysis from the hypothalamus in the dog. Amer. J. Physiol., II7, 467-73 (1936).

Kelly, G. L. and E. B. Woods: A quantitative study of the Friedman test for pregnancy. J. Amer. med. Ass., 108, 61 5-17 (1937).

KEMP, T. and L. MARx: Beeinflussung von erblichem, hypophysärem Zwergwuchs bei Mäusen durch verschiedene Hypophysenauszüge und durch Thyroxin. I. Wachstum und Geschlechtsfunktion. Acta path. scand., 13, 5 I2-3 I (1936).

Kemp, T. and L. MARx: Beeinflussung von erblichem hypophysären Zwergwuchs bei Mäusen durch verschiedene Hypophysenauszüge und Thyroxin. II. Endokrine Organe. Acta path. scand., I4, I97-227 (1937).

KÉpinov, L.: L’Adrénalino-sécrétion chez les chiens hypophysectomisés. C. R. Soc. Biol., Paris, 122, 351-54 (1 936).

Képinov, L.: Rôle du pancréas, de l'hypophyse et des surrénales dans la genèse de l'hyperglycémie du diabète pancréatique expérimental. Presse méd., 44, I652-54 (1936).

Képinov, L.: Système glycogénolytique hormonal. Sur le mécanisme de l'action glycogénolytique de l'adrénaline et le rôle de l'hormone hypophysaire dans ce mécanisme. C. R. Soc. Biol., Paris, I26, I084-87 (1937).

Kido, I.: Die menschliche Placenta als Produktionsstätte des sogenannten Hypophysenvorderlappenhormons. (Experimentelle Untersuchungen.) Zbl. Gynäkol., 61, I 55 I-55 (I 937 ).

Kindermann, V. and F. Eichiaum: Untersuchungen über die antigenen Funktionen von Hormonpräparaten. Ia. Weitere Mitteilung über das gonadotrope Hypophysenvorderlappenhormon (Prähormon). Z. Immunitätsforsch., 89, 230-38 (1936).

Kinoshita, S.: Experimental studies on the oestrus cycles of parabiosed animals. Jap. J. exp. Med., 15, 49-77 (1937).

Kippen, A. A. and L. Loe B: The relation between the quantity of thryoidstimulating hormone of the anterior pituitary gland administered and the proliferative activity and hypertrophy of the thyroid acini in guinea pigs. J. Pharmacol. exp. Therap., 54, 246-57 (1935). 
Kippen, A. A. and L. Loeb: The effect of gonadectomy on the thyroid gland in the guinea pig. Endocrinology, 20, 20I-9 (I 936).

KIRKMAN, H.: A cytological study of the anterior hypophysis of the guinea pig and a statistical analysis of its cell types. Amer. J. Anat,, $6 \mathbf{r}, 233-87$ ( 1937 ).

Kirschbaum, A. and A. R. Ringoen: Seasonal sexual activity and its experimental modification in the male sparrow, Passer domesticus Linnaeus. Anat. Rec., 64, 453-73 (I 936).

Kiyohara, K. I. and S. Izawa: Über die Wirkung des Harns schwangerer Frauen auf die Sauerstoffatmung des Ovariums. J. Biochem., 24, 337$54(1936)$.

Klaften, E.: Über Bau und Funktion der durch Follikelhormon erzeugten Gelbkörper. Z. Geburtsh. Gynäkol., I I5, 64-99 (1937).

KLEIN, F.: Über den Einfluss von Schwangerenharn und Prolan auf die Meerschweinchenthymus. Klin. W'schr., I5, 37I-75 (I936).

KLein, F.: Über den Einfluss von Schwangerenharn auf die Thymus und Schilddrüse kastrierter Meerschweinchen. Klin. Wschr., I5, I56I-62 (1936).

Kleinholz, L. H.: The melanophore-dispersing principle in the hypophysis of Fundulus heteroclitus. Biol. Bull. Wood's Hole, 69, 379-90 (I935).

Kleinschmidt, A.: Der Einfluss der Hypophysektomie auf die Geschlech tsorgane des männlichen kleinen 'Teichmolches ('Triturus vulgaris L.). Z. mikroskop.-anat. Forsch., 41, 151-76 (1937).

KLodT, W.: Zur Einwirkung des gonadotropen Hypophysenvorderlappenhormons (Prolan) auf das Elektrolytsystem. Arch. exp. Path. Pharmak., I86, 28 I -86 (I 937 ).

Koвауазнi, S.: Studies on the influence of various endocrine organ cell constituents on the development of the chick embryo and chick. Jap. J. $\exp$. Med., I5, 8.3-120 (19.37).

Koch, C., B. Schreiber, and G. Schreiber: Primi risultati di impianti abbinati di organi endocrini in camera anteriore dell'occhio di cavia. Riv. Biol., 22, 40-58 (1937).

Koch, W.: Über den Einfluss von Prolan auf die Legetätigkeit der Vögel. II. Die Beeinflussung der Legetätigkeit normal legender Hennen. Klin. Wschr., 14, 1850-51 (1935).

Koch, W. and L. Scheuring: Die Wirkung von Hypophysenvorderlappenhormon auf den laichakt von Fischen. Zool. Anz., Ir6, 62-64 (1936).

Konsuloff, S.: Die Fischschuppen als Testobjekt für die Melanophorenreaktion. (Ein Beitrag zu der Diagnose-Reaktion der Addisonschen Krankheit durch den Urin.) Z. ges. exp. Med., 99, I09-1 I (1936).

Korenchevsky, I., M. Dennison, and S. L. Simpson: Assay of the gonadotropic hormone of pregnancy urine on male rats. Biochem. J., $29,2522-33(1935)$.

Kotchneff, N. P. and E. S. London: Fiziol. Ž., 22, 372-76 (1937).

$$
[3+0]
$$




\section{BIBLIOGRAPHY}

Kozelka, F. L. and H. J. Tatum: The influence of theelin and the gonad stimulating principle of the anterior pituitary on calcium metabolism in rachitic dogs. Amer. J. Physiol., I I9, 356-57 (1937).

Kraatz, C. P.: A possible rôle of the eosinophil leucocytes in the endocrine complex of the female rat. Amer. J. Physiol., I I7, 250-56 (I 936).

Krane, W.: Untersuchungen über den Einfluss der operativen Entfernung des Uterus bei der geschlechtsreifen Frau auf die hormonale Tätigkeit des Ovariums und des Hypophysenvorderlappens, zugleich eine neue Methode zur Follikulinbestimmung. Arch. Gynäkol., I64, IоI-32 (1937).

KraUl, L.: Untersuchungen über die Funktion des Uterusmuskels. Z. Geburtsh. Gynäkol., I I I, 273-92 (I 935).

Kraus, E. J.: Durch welche endokrinen Vorgänge wird das Einschiessen der Milch post partum ausgelöst? Klin. Wschr., I4, I7 I8-I9 (1935).

KraUs, E. J.: Über die pathogenetische Bedeutung der Basophilie der Hypophyse, insbesondere für die Eklampsie. Med. Klin., 31, I6 $4{ }^{1-42}$ ( I 935).

Kraus, E. J.: Wie lässt sich die Annahme eines corticotropen Hyperpituitarismus beim Menschen morphologisch stützen? Klin. Wschr., 16, I 528-32 (1937).

KrichesKy, B.: Relation of anterior pituitary to the volume of islet tissue in the male rat. Proc. Soc. exp. Biol., N.Y., 34, 126-27 (1936).

Kun, H. and O. Peczenik: Geschlechtsspezifische Wirkung der Sexualhormone auf den Kreatinstoffwechsel. Pflügers Arch., 236, 471-80 (1936).

Kunkel, P. and L. Loeb: Effects of human anterior pituitary gland on sex organs and thyroid of the guinea pig. Proc. Soc. exp. Biol., N.Y., 32, I4I3-17 (1935).

KuP, J. von: Der Zusammenhang zwischen der Zirbel und den anderen endokrinen Drüsen. Frankf. Z. Path., 50, 152-89 (I936).

Kurzrok, R., H. Wiesbader, M. G. Mulinos, and B. P. Watson: The action of pituitrin, estradiol and progesterone on the human uterus in vivo. Endocrinology, 21, 335-42 (1937).

Kusakabe, S. and H. Kusano: Über den Einfluss des Hypophysenvorderlappens auf den Cholesterin- und Calciumgehalt im Blut des normalen, des thyreoidektomierten sowie des thyreoid-splenektomierten Kaninchens. II. Mitt. Fol. endocrin. jap., I2, 15-17 (1936).

Kuschinsкy, G.: Über den Einfluss verschiedener Temperaturen auf die Sekretion des thyreotropen Hormons. Arch. exp. Path. Pharmak., I79, 726-37 (1935).

Kuschinskr, G. and TANg-sü: Über die Wirkung luteinisierender Substanz auf die Funktion der lipoidhaltigen Zellen des Ovariums. Arch. exp. Path. Pharmak., I79, 717-21 (I935).

Kuschinsky, G, and TANG-sü: Über die Wirkung luteinisierender Substanz auf den Hoden. Arch. exp. Path. Pharmak., I79, 722-25 (19.35). 


\section{THE PITUITARY BODY}

KYlıN, E.: Hypophysentransplantationer. Tierexperimentelle Untersuchungen und klinische Ergebnisse. Acta med. scand., 9I, 428-34 (1937).

LAcassagne, A.: Über den Einfluss von Hypophysenbestrahlungen (mit Radonspickung oder Röntgenstrahlen) auf das Ovarium. Strahlentherapie, 54, 477-92 (1935).

Lacassagne, A. and W. NYKa: Sur les processus histologiques de la destruction de l'hypophyse par le radon. C. R. Soc. Biol., Paris, II7, 956-58 (1934).

Lacassagne, A. and W. Nyka: Essais de destruction de l'hypophyse du coq par le radon. C. R. Soc. Biol., Paris, I I9, 354-56 (I 935 ).

L.acassagne, A. and W. NYka: Différence de réaction de l'hypophyse à l'administration prolongée de substances ostrogènes, dans diverses lignées sélectionnées de souris. C. R. Soc. Biol., Paris, 126, 1 I I2-I 5 (1937).

Lacassagne, A. and A. Raynaud: Action de l'œestrine sur la zone $\mathrm{X}$ et sur le cortex de la surrénale chez la souris. C. R. Soc. Biol., Paris, r24, I $186-89$ ( ( 937 ).

LAHR, E. L. and O. RidDle: Temporary suppression of estrous cycles in the rat by prolactin. Proc. Soc. exp. Biol., N.Y., 34, 880-83 (1936).

LAMBADARIDÈs, A.: Einige biologische Ergebnisse aus der Röntgenbestrahlung der Hypophyse. Strahlentherapie, 56, 273-77 (1936).

LAmBie, C. G. and V. M. TRikojus: The preparation of a purified thyrotropic hormone by chemical precipitation. Biochem. J., 31, 843-47 (1937).

LANE, C. E. and R. O. Greep: The follicular apparatus of the ovary of the hypophysectomized immature rat and the effects of hypophyseal gonadotropic hormones on it. Anat. Rec., 63, 139-46 (1935).

LANGE, N.: Morphologisch-physiologische Untersuchungen an der Hypophyse von Fischen. Allat. Közlem., 33, 65-83 (1936) (available as an abstract).

Langeron, L., M. Paget, and A. Danès: Action des extraits posthypophysaires sur la sécrétion gastrique. C. R. Soc. Biol., Paris, I I , 33-35 (I936).

Laporta, M., M. Pepe, and P. Marinelli: Sulle correlazioni umorali fra ipofisi e mucosa gastroenterica. Arch. di Sci. Biol., 22, 379-98 (1936).

Laroche, G. and H. Simonnet: Sur l'existence d'un pouvoir antigonadotrope dans le sérum humain. C. R. Soc. Biol., Paris, I21, 416-19 (1936).

Laroche, G., H. Simonnet, and E. Bompard: Influence de la progestérone sur l'élimination urinaire des principes gonadotropes. C. R. Soc. Biol., Paris, 1 26, I I 59-60 (1937).

LARSON, E.: Depressor substances in the posterior lobe of the pituitary. J. Pharmacol. exp. Therap., 56, 396-416 (1936). 


\section{BIBLIOGRAPHY}

Latyszewski, M.: Tests morphologiques de la réaction du cortex surrénal à l'injection d'extraits préhypophysaires chez le cobaye et le lapin. C. R. Soc. Biol., Paris, 126, 468-70 (1937).

Lauson, H., C. G. Heller, and E. L. Sevringhaus: The effect of graded doses of estrin upon the pituitary, adrenal, and thymus weights of mature ovariectomized rats. Endocrinology, 2I, 735-40 (1937).

Lebedewa, N. S.: Der histophysiologische Effekt der Thyreoidektomie im Hypophysenvorderlappen der Ratte. Arch. exp. Path. Pharmak., I83, I 5-29 ( 1936$)$.

Leblond, C. P. and E. Allen: Méthode rapide de recherche de la prolactine (hormone lactogène de l'hypophyse). C. R. Soc. Biol., Paris, I24, I I90-9I (I 937).

Leblond, C. P. and W. O. Nelson: L'Instinct maternel après hypophysectomie. C. R. Soc. Biol., Paris, I22, 548-49 (1936).

I.eblond, C. P. and W. O. Nelson: Maternal behavior in hypophysectomized male and female mice. Amer. J. Physiol., 120, I67-72 (1937).

Leblond, C. P. and IV. O. Nelson: Modifications histologiques des organes de la souris après hypophysectomie. C. R. Soc. Biol., Paris, I 24, 9-I I (I 937).

Leblond, C. P. and G. K. Noble: Prolactin-like reaction produced by hypophyses of various vertebrates. Proc. Soc. exp. Biol., N.Y., 36, $517-18$ (1937).

Lederer, J. A.: Action de l'hormone thyréotrope sur le glycogène cardiaque. Arch. int. Méd. exp., ro, 137-51 (1935).

Lederer, J. A.: L'Hormone thyréotrope et le métabolisme de base dans les syndromes hypophysaires. Rev. belge Sci. méd., 7, 369-93 (I 935).

LeE, M. and G. B. Ayres: The composition of weight lost and the nitrogen partition of tissues in rats after hypophysectomy. Endocrinology, 20, 489-95 (1936).

LEIN, A.: Augmentation of the gonadotropic hormone from the pregnant mare. Proc. Soc. exp. Biol., N.Y., 36, 609-I I (I 937).

LEONARD, O. L. and S. L. LEONARD: Failure of thyroidectomy to influence the follicular components of the immature rat ovary. Anat. Rec., 68, 249-52 (I 937).

Leonard, S. L.: Hypophysis-thyroid-gonad relationship. Proc. Soc. exp. Biol., N.Y., 34, 599-600 (1 936 ).

LEONARD, S. L.: Changes in the relative amounts of the follicle stimulating and luteinizing hormones in the hypophysis of the female rat under varying experimental conditions. Endocrinology, 21, 330-34 (I937).

Leonard, S. L.: Lutenizing hormone in bird hypophyses. Proc. Soc. exp. Biol., N.Y., 37, 566-68 (1 937).

Leonard, S. L. and J. B. Hamilton: Comparison and rate of testicular degeneration in rats after cryptorchidism and hypophysectomy. Anat. Rec., 68, 497-502 (1937). 


\section{THE PITUITARY BODY}

Leonard, S. L. and I. B. Hansen: The influence of thyroidectomy on the effectiveness of gonad stimulating hormones. Anat. Rec., 64, 203-9 (1936).

Leonard, S. L., F. L. Hisaw, and H. L. Fevold: Pituitary hormone antagonism. Proc. Soc. exp. Biol., N.Y., 33, 319-2I (1935).

Leonard, S. L. and J. IV. Righter: Endocrine weights of the bantam fowl. J. Hered., 27, 363-66 (1936).

Levin, L. and H. H. Tyndale: The concentration and purification of the gonadotropic substance of the urine of human female castrates. J. biol. Chem., rog, liv-lv (1935).

Levin, L. and H. H. Tyndale: A new method for the quantitative assay of "follicle-stimulating" substances. Amer. J. Physiol., I 19, 360 (1937).

Levin, L. and H. H. Tyndale: The quantitative assay of "follicle stimulating" substances. Endocrinology, 21, 619-28 (1937).

Levitr, G.: 'The problem of an antidiuretic substance in the blood of patients with eclampsia and other hypertensive diseases with observations on spinal fluid. J. clin. Invest., I5, 135-4I (1936).

Lewis, D. and C. F. Geschickter: The demonstration of hormones in tumors. Ann. Surg., I04, 787-97 (1936).

Lewis, D., F. C. Lee, and E. B. As'Twood: Some observations on intermedin. Johns Hopk. Hosp. Bull., 6I, 198-209 (1937).

Lewy, F. H. and F. K. Gassmann: Experiments on the hypothalamic nuclei in the regulation of chloride and sugar metabolism. Amer. J. Physiol., I I2, 504-10 (1935).

LI, R. C.: The effect of posterior pituitary extract, epinephrine and acetylcholine on the isolated fallopian tube of the macaque at different stages of the menstrual cycle. Chinese J. Physiol., 9, 315-27 (1935).

Liang, T. Y. and S. W. WU: Über eine hypophysäre, humorale Steurung des Eiweissdepots in der Leber. Chinese J. Physiol., I2, I 25-38 (1937).

LiARD, J.: Sur la présence d'une substance lactogène dans les urines de la femme en lactation. C. R. Soc. Biol., Paris, I26, 512-I4 (1937).

Lippross, O.: Untersuchungen über den Einfluss von Cortin-Degewop und von Suprareninchlorid auf die Struktur der Hypophyse, der Keimdrüsen und Nebennieren von Ratten. Endokrinologie, I8, 18-26 (1936).

Lipschüтz, A.: Différences préhypophysaires spécifiques du sexe, chez le cobaye. C. R. Soc. Biol., Paris, I I8, 33 I-33 (1935).

Lipschürz, A.: On the influence of oestrin injections on the balance between the prehypophyseal gonadotropic hormones of the male rat. Quart. J. exp. Physiol., 25, 109-20 (1935).

Lipschütz, A.: Préhypophyse et ovaire chez le cobaye avec troubles expérimentaux du cycle sexuel. Arch. Biol., Paris, 47, I 8 I-204 (1936).

LıPschüтz, A.: Croissance atypique et destructive des glandes utérines après des interventions ovariennes expérimentales. C. R. Acad. Sci., Paris, 203, 1025-28 (1936).

Lipschüтz, A.: Déséquilibre expérimental entre ovaire et préhypophyse, chez la femelle du cobaye. C. R. Soc. Biol., Paris, I23, 545-47 (1936). 


\section{BIBLIOGRAPHY}

LiPschütz, A. and M. DEL-PINo: Différences préhypophysaires spécifiques du sexe, chez l'homme. C. R. Soc. Biol., Paris, I2 I, 208-I I (I 936).

Lipschütz, A., A. Fuente-Alba, and T. Vivaldi: Disparition du prolan du sang de la lapine néphrectomisée. C. R. Soc. Biol., Paris, x 20, 32326 (1 935 ).

Lıpschütz, A. and C. Ovıedo: Sur le pouvoir gonadotrope de la préhypophyse de Myocastor (Myoptamus) Coypu. C. R. Soc. Biol., Paris, I $18,333^{-} 35$ ( I 935 ).

L.ipschütz, A., L. Palacios, and J. Akel: "Féminisation" de la préhypophyse du rat mâle par injection de folliculine. C. R. Soc. Biol., Paris, I2 I, 205-8 (I 936).

Lipschütz, A. and G. Villagran: Neutralisation de la préhypophyse par castration, chez le rat. C. R. Soc. Biol., Paris, I2I, 203-5 (1936).

L Jachowezki, A. M. and B. P. Chwatow: Der Einfluss von Gonadenstimulatoren auf die Einheilung von transplantierten Geschlechtsdrüsen. Bull. Biol. Méd. exp. URSS., 4, I I-12 (1937).

Loeb, L., J. SAxton, and S. J. HAYwARD: The relationship of the anterior pituitary gland to thyroid and ovary. Endocrinology, 20, 5II-I9 (1936).

Lofser, A.: Die Bedeutung der Hypophyse für die antithyreotrope Schutzkraft des Blutes. Arch. exp. Path. Pharmak., I80, 458-65 (I936).

Loeser, A.: Die Schutzwirkung des Blutes gegen das thyreotrope Hormon des Hypophysenvorderlappens. Zbl. inn. Med., pp. 569-8I, 585-93 $(1936)$.

Loeser, A. and V. M. Trikojus: Der Einfluss des thyreotropen Hormons der Hypophyse auf den C-Vitamingehalt der Nebennieren und der I.cber von Meerschweinchen. Arch. exp. Path. Pharmak., r85, 227-34 (1937).

Long, C. N. H.: Recent advances in carbohydrate metabolisin with particular reference to diabetes mellitus. Ann. int. Med., 9, 166-74 (1935).

L.ONG, C. N. H.: The influence of the pituitary and adrenal glands upon pancreatic diabetes. Harvey Lect., 32, 194-228 (1937).

LONG, C. N. H., and B. Katzin: Effect of adrenal cortical hormone on carbohydrate stores of fasted hypophysectomized rats. Proc. Soc. exp. Biol., N.Y., 38, 5 I6-18 (1938).

Long, C. N. H. and F. D. W. Lukens: 'The effects of adrenalectomy and hypophysectomy upon experimental diabetes in the cat. J. exp. Med., $63,465-90$ ( I 936$)$.

Long, C. N. H., F. D. W. Lukens, and F. C. Dohan: Adrenalectomizeddepancreatized dogs. Proc. Soc. exp. Biol., N.Y., 36, 553-54 (I 937 ).

Lucke, H.: Bestehen Beziehungen des kontrainsulären Vorderlappenhormons zu der auf den Kohlehydratstoffwechselwirkenden Substanz des Hypophysenhinterlappens? Z. ges. exp. Med.. roo, 73-77 (1936).

Lucke, H.: Die Stellung des Hypophysenvorderlappens in der Regulation des normalen Kohlehydratstoffwechsels. Verh. dtsch. Ges. inn. Med., I33-35 (1937). 


\section{THE PITUITARY BODY}

Lucke, H. and E. KRöger: Untersuchungen über den Wirkungsmechanismus des kontrainsulären Hormones des Hypophysenvorderlappens. VII. Mitt.: Der Einfluss des Hormons auf den Glykogenbestand der Leber und den Milchsäurespiegel des Blutes. Z. ges. exp. Med., Ioo, $69-72$ (1936).

LüTH, K. F.: Endokrine Störungen bei eineiigen Zwillingen. Z. menschl. Vererb.-u. Konstitutionslehre, 21, 55-67 (1937).

Lyons, W. R.: Preparation and assay of mammotropic hormone. Proc. Soc. exp. Biol., N.Y., 35, 645-48 (1937).

Lyons, W. R.: 'The hormonal basis for "witches' milk." Proc. Soc. exp. Biol., N.Y., 37, 207-9 (1937).

LYons, W. R. and E. PAGE: Detection of mammotropin in the urine of lactating women. Proc. Soc. exp. Biol., N.Y., 32, 1049-50 (1935).

Macchiarulo, O.: Sui rapporti intercorrenti tra l'ipofisi e la mammella. Effetti della ipofisectomia in cavie allattanti. Ann. Ostetr., 58, 3-16 (1936).

MAcDonald, I. G.: The response of the mammary gland to prolonged stimulation with ovarian hormones. Surg. Gynecol. Obstetr., 63, I3844 (1936).

MACKAY, E. M. and R. H. BARNes: Influence of adrenalectomy on the ketosis of fasting and on the action of the anterior pituitary ketogenic principle. Amer. J. Physiol., I 18, 184-89 (1937).

MAcKAY, E. M. and R. H. BARnes: The effect of adrenalectomy on liver fat in fasting and after the administration of anterior pituitary extracts. Amer. J. Physiol., I18, 525-27 (1937).

Magath, M. A. and E. Lebenson: Vopr. Onkol., 7, 217-24 (1935) (abstract).

Magath, M. A. and E. J. Smollowskaia: Vopr. Onkol., 7, 225-35 (1935) (abstract).

Mahaux, J.: Action dynamique spécifique des protéines et hormone "thyréotrope." C. R. Soc. Biol., Paris, 1 23, 1266-67 (1936).

MaHaux, J.: Action de l'hormone "thyréotrope" et de l'administration successive de thyroxine et d'hormone "thyréotrope" sur le métabolisme du cobaye. C. R. Soc. Biol., Paris, 125, 379-82 (1937).

Mahoney, IV. and D. Sheenan: The effect of total thyroidectomy upon experimental diabetes insipidus in dogs. Amer. J. Physiol., I I2, 250-55 (1935).

Mahoney, W. and D. Sheehan: The pituitary-hypothalamic mechanism: experimental occlusion of the pituitary stalk. Brain, 59, 6i-75 ( I 936).

Makepeace, A. W., G. IV. Corner, and W. M. Allen: The effect of progestin on the in vitro response of the rabbit's uterus to pituitrin. Amer. J. Physiol., I I5, 376-85 (1936).

Makepeace, A. W., G. L. Weinstein, and M. H. Friedman: Effect of progestin and progesterone on ovulation in the rabbit. Proc. Soc. exp. Biol., N.Y., 35, 269-70 (1936). 


\section{BIBLIOGRAPHY}

Makepeace, A. W., G. L. Weinstein, and M. H. Friedman: The effect of progestin and progesterone on ovulation in the rabbit. Amer. I. Physiol., I 19, 5 I 2-16 (1937).

Maloberti, U.: Influenza dell'ormone tireotropo sul glutatione del sangue e di alcuni organi del coniglio e della cavia. Osp. psichiatr., 4, 240-48 (1936).

Mandelstamm, A.: Biologischer Nachweis von Melanophorenhormone im Harn Schwangerer am Fischtest. (Ein Beitrag zur hormonalen Schwangerschaftsdiagnostik.) Zb]. Gynäkol., 59, 2737-4' (1935).

Marburg, O. and K. F. Wenckebach: Über Veränderungen der Hypophyse bei Beriberi. (Ein Beitrag zur Kenntnis der basophilen Hypophysen-Zellen.) Wien. Arch. inn. Med., 29, I-24 (1936).

Marcano, A. G.: Der Hypophysenhinterlappen bei Hypertonie. Klin. Wschr., I4, I 525-29 (1935).

Marchesi, F.: Vitamina E ed ormoni. I. L'azione dell'ormone enteipofisario e dell'urina di donna gravida per ristabilire la fecondità in ratti femmine in stato di avitaminosi. Sperimentale, 89, 166-75 (19.35).

Marchesi, F.: Vitamina E ed ormoni. III. Possibilità di ristabilire la fecondità in ratti femmine in stato di avitaminosi $\mathrm{E}$, mediante somministrazione di estratto totale di placenta. Sperimentale, 89, 622-35 (1935).

Marenzi, A. D.: Chemical changes in the muscle of the hypophysectomized toad. Endocrinology, 20, 184-87 (1936).

Margitay-Becht, E. and E. Wallner: Gewichtsänderung mit Wachstumshormon behandelter Ratten im Zustande der Avitaminose. ' . Vitaminforsch., 6, 119-25 (1937).

Margulis, Z. S.: Interaction between folliculine and the lactogenic hormone of the hypophysis. Bull. Biol. Méd. exp. URSS, 2, 27-28 (1936).

MARkeE, J. E. and J. C. Hinsey: Observations on ovulation in the rabbit. Anat. Rec., 64, 309-19 (I936).

Marrian, G. F. and W. H. Newton: The synergism between oestrin and oxytocin. J. Physiol., 84, I33-47 (1935).

Marshall, F. H. A.: On the change over in the oestrus cycle in animals after transference across the equator, with further observations on the incidence of the breeding seasons and the factors controlling sexual periodicity. Proc. Roy. Soc., B, I22, 4I 3-28 (1 937 ).

Marshall, F. H. A. and F. P. Bowden: The further effects of irradiation on the oestrous cycle of the ferret. J. exp. Biol., I3, 38,3-86 (1936).

Marshall, F.H. A. and E. B. Verney: The occurrence of ovulation and pseudopregnancy in the rabbit as a result of central nervous stimulation. J. Physiol., 86, 327-36 (1936).

Martin, S. J. and J. F. FAzEKAS: Effect of sodium chloride therapy on oestrous cycle and hypophysis of bilaterally suprarenalectomized rats. Proc. Soc. exp. Biol., N.Y., 37, 369-72 (1937).

Martins, T.: Sur la question des "antihormones." C. R. Soc. Biol., Paris, II9, $753-55$ (1935). 


\section{THE PITUITARY BODY}

Martins, T.: Altérations histologiques et fonctionnement des greffes de l'hypophyse. C. R. Soc. Biol., Paris, r23, 699-701 (1936).

Martins, T.: Action des hautes doses d'œstrine sur l'hypophyse in situ, ou greffée dans la chambre antérieure de l'œil du rat. C. R. Soc. Biol., Paris, I23, 702-4 (1936).

Marx, R., H. R. Catchpole, and B. J. Mckennon: Ovarian function and occurrence of menopausal symptoms following hysterectomy. Surg. Gynecol. Obstetr., 63, 170-78 (1936).

MÂrza, V. D. and A. V. Blinov: L'Hypophyse antérieure et la thyroïde au cours de l'ovulation. C. R. Soc. Biol., Paris, I2I, I438-40 (1936).

Matuo, K.: Über die Pigmentwanderung der Pigmentzellen der Froschnetzhaut. (III. Mitt.) Über die Wirkung des Hinterlappenhormons der Hypophyse auf die Pigmentwanderung der Netzhautpigmentzellen des Frosches. Okay. Igak. Zasshi, 47, 2387-91 (1935).

Max, P., M. M. Schmeckebier, and L. Loeb: Acquired resistance to the thyroid-stimulating and pseudoluteinizing hormone of cattle anterior pituitary. Endocrinology, 19, 329-41 (1935).

Maxwell, L. C. and F. Bischoff: Chemical studies on the pituitary gonadotropic hormone. J. biol. Chem., I I2, 21 5-2 I (1935).

MAY, R. M.: Fonctionnement sexuel normal et durable obtenu grâce à la greffe bréphoplastique de l'hypophyse chez des rats hypophysecsectomisées. C. R. Soc. Biol., Paris, 124, 920-23 (I937).

MAYor, J. M.: Die hemmende Wirkung des Follikelhormons auf die Milchsekretion der Wöchnerin. Zbl. Gynäkol., 6o, 2379-83 (1936).

Mazer, C. and S. L. Israel: Studies on the optimal dosage of estrogens. An experimental and clinical evaluation. J. Amer. med. Ass., ro8, 16369 (1937).

Mazer, C., S. L. Israel, and B. J. Alpers: The time element in the pituitary-ovarian response to large doses of the estrogenic hormone. Endocrinology, 20, 753-61 (1936).

Mazer, C., D. R. Meranze, and S. L. Israel: Evaluation of the constitutional effects of large doses of estrogenic principle. J. Amer. med. Ass., r05, 257-63 (1935).

McCullagh, D. R.: Dual endocrine activity of the testes. Science, 76, I 9-20 (1932).

McCullagh, D. R. and E. L. Walsh: Experimental hypertrophy and atrophy of the prostate gland. Endocrinology, I9, 466-70 (1935).

McCullagh, E. P. and W. K. Curler: The Friedman test and pituitary tumor. Endocrinology, 21, 8-18 (1937).

McEuen, C. S., H. Selye, and J. B. Collip: Some effects of prolonged administration of oestrin in rats. Lancet, 230, 775-76 (1936).

McEuen, C. S., H. Selye, and J. B. Collip: Effect of the testis on the mammary gland. Proc. Soc. exp. Biol., N.Y.. 35, 56-58 (1936).

McEuen, C. S., H. Selye, and J. B. Collip: Role of pituitary in effect of testosterone on the mammary gland. Proc. Soc. exp. Biol., N.Y., 36, 2 I $3-15$ ( 1937$)$. 


\section{BIBLIOGRAPHY}

McEuen, C. S., H. Selye, and J. B. Collip: Effect of testosterone on somatic growth. Proc. Soc. exp. Biol., N.Y., 36, 390-94 (1937).

McFarlane, IW. D. and M. K. McPhail: Pituitrin injections and the blood picture in the normal and hypophysectomized guinea-pig. Amer. J. med. Sci., r93, 385-89 (1937).

McGinty, D. A., and N. B. McCullough: Thyrotropic hormone in nonpituitary tissue. Proc. Soc. exp. Biol., N.Y., 35, 24-26 (1936).

McPhaIl, M. K.: 'The assay of progestin. J. Physiol., 83, I45-56 (1934).

McQuarrie, I., W. H. Thompson, and M. R. Ziegler: Effects of posterior pituitary extract on the water and mineral exchanges in edema. J. Ped., 8, 277-91 (1936).

McQueen-IVilliams, M.: Is thyrotropic hormone of beef ant. pituitaries identical with indirect interrenotropic factor? Proc. Soc. exp. Biol., N.Y., 32, 1050-51 (1935).

McQueEn-WILliams, M.: Sex comparison of gonadotropic content of anterior hypophysis from rats before and after puberty. Proc. Soc. exp. Biol., N.Y., 32, I051-52 (1935).

McShan, W. H. and H. E. French: The chemistry of the lactogenic hormone extracts. J. biol. Chem., II7, II I-I7 (1937).

McShan, W. H. and R. K. MEYer: Heme-containing fractions of blood as related to the augmentation of pituitary gonadotropic extracts. Amer. J. Physiol., I19, 574-79 (1937).

MCShan, W. H. and R. K. Meyer: The effect of trypsin and ptyalin preparations on the gonadotropic activity of pituitary extracts. J. bjol. Chem., I26, 36 I-65 (1938).

McShan, W. H. and C. W. Turner: Further purification of galactin, the lactogenic hormone. Proc. Soc. exp. Biol., N.Y., 32, 1655-56 (19.35).

McShan, W. H. and C. W. Turner: Bioassay of galactin, the lactogenic hormone. Proc. Soc. exp. Biol., N.Y., 34, 50-51 (i 936).

Meessen, H.: Zur Pathologie der Hypophyse. Beitr. path. Anat., 95, 39-59 (1935).

Mellish, C. H.: The effects of anterior pituitary extract and certain environmental conditions on the genital system of the horned lizard (Phrynosoma cornutum, Harlan). Anat. Rec., 67, 23-33 (1936).

Mellish, C. H. and R. K. Meyer: The effects of various gonadotropic substances and thyroxine on the ovaries of horned lizards (Phrynosoma cornutum). Anat. Rec., 69, I79-89 (1937).

Mello, R. F. DE: Synergie des hormones des glandes sexuelles au cours d'expériences de greffe et de parabiose. C. R. Soc. Biol., Paris, 123, 213I6 (1936).

Melville, K. I.: Pressor and oxytocic fractions of posterior pituitary extract. Comparative effects on blood pressure and intestinal activity. J. Amer. med. Ass., ro6, 102-5 (1936).

Melville, K. 1.: The influence of salt saturation upon the urinary response to pituitary (posterior lobe) extract. J. Physiol., 87, 129-4.3 (1936). 


\section{THE PITUITARY BODY}

Merklen, P., M. Aron, L. Israël, and A. Jacob: Tests histologiques de l'hyperfonctionnement préhypophysaire chez certains obèses. Bull. Soc. méd. Hôp., Paris, 5I, 1402-6 (1935).

Merkulow, L. G.: Narkotica und sekretorische Darmfunktion. III. Mitt. Wirkung des Pituitrins auf die Darmsekretion. Fiziol. Ž., 20, I 273 I (1936).

MEYER, H. S., L. J. WADE, and C. F. CORI: Influence of extracts of anterior lobe of pituitary on glucose oxidation and glycogen storage. Proc. Soc. exp. Biol., N.Y., 36, 346-48 (1937).

Meyer, R. K. and R. Hertz: The effect of oestrone on the secretion of the gonadotropic complex as evidenced in parabiotic rats. Amer. J. Physiol., I20, 232-37 (1937).

Meyer, R. K., L. C. Miller, and G. F. Cartland: The biological activity of theelol. J. biol. Chem., I I2, 597-604 (I936).

Migliavacca, A.: Ricerche sulla modalità di influenzamento e di regolazione ormonica fra l'ipofisi e le ghiandole sessuali. Arch. Entwmech. Org., 134, 653-93 (1936).

Migliavacca, A.: Inkretogene heterotype Epithelwucherungen im Uterus. Arch. Gynäkol., I62, 595-644 (1936).

Miller, E. G., J. R. Cockrill, and R. Kurzrok: Reactions of human uterine muscle in vitro to pituitrin, adrenalin and acetylcholine and their relations to the menstrual cycle. Amer. J. Obstetr., 33, $154-56$ (1937).

Minclotti, G.: Correlazioni endocrine della preipofisi e morbo di Cushing. Fisiol. Med., 6, 685-726 (1935).

Mirskr, I. A.: The site and mechanism of the antiketogenic action of insulin. Amer. J. Physiol., I 16, 322-26 (1936).

Mirsky, I. A.: Influence of adrenalectomy on anterior pituitary ketogenesis in rats. Science, $88,332-33$ (1938).

Miyagawa, S.: Experimentelle Untersuchung über die biologische Bedeutung der Hypophyse: über meine Methode der Hypophysenexstirpation. Trans. Jap. path. Soc., 26, 494-95 (1936).

Moffat, W. M.: Treatment of menstrual migraine with small doses of gonadotropic extract of pregnancy urine. J. Amer. med. Ass., 108, 612I 5 ( I 937 ).

Molitch, M. and S. Poliakoff: Pituitary disturbances in behavior problems. Amer. J. Orthopsychiatry, 6, 125-33 (1936).

Mǿller-Christensen, E.: Studien über das Zusammenspiel von Hypophysen- und Ovarialhormonen, insbesondere im Lichte von Parabioseversuchen. Copenhagen (1935).

Monnier, M.: Recherche de l'hormone préhypophysaire gonadotrope dans les cas de tumeurs cérébrales et d'affections encéphaliques. C. R. Soc. Biol., Paris, I23, $1116-18$ (1936).

Moon, H. D.: Preparation and biological assay of adrenocorticotropic hormone. Proc. Soc. exp. Biol., N.Y., 35, 649-52 (1937).

Moon, H. I).: Inhibition of somatic growth in castrate rats with pituitary extracts. Proc. Soc. exp. Biol., N.Y., 37, 34-36 (1937). 


\section{BIBLIOGRAPHY}

Moon, H. D.: Effect of adrenocorticotropic hormone on the sexual development of spayed rats. Proc. Soc. exp. Biol., N.Y., 37, 36-37 (1937).

Moore, C. R.: Responses of immature rat testes to gonadotropic agents. Amer. J. Anat., 59, 63-88 (1936).

Moore, C. R. and D. Price: Some effects of synthetically prepared male hormone (androsterone) in the rat. Endocrinology, 2I, 3I $3^{-29}$ (I937).

Morgan, T. N.: Studies on the movements of the uterus. III. The action of gonadotropic extracts on the movements of the uterus of the unanesthetized rabbit. J. Obstetr., 42, 84-87 (I 935 ).

Morgan, T. N.: An analysis of the action of posterior pituitary extracts on the uterus. J. Pharmacol. exp. Therap., 59, 2 I I-2 I (1937).

Moricard, R.: Actions prolongées d'urines de femme enceinte et d'extraits dits antéhypophysaires d'origine urinaire suivi de perte de réceptivité de l'ovaire de lapin à ces actions hormonales. Gynéc. Obstétr., 3I, 102-6 (1935).

Moricard, R.: Développement folliculaire provoqué dans l'ovaire humain en greffe vulvaire par injection de mitosine d'origine urinaire. Bull. Soc. Obstétr., Paris, 25, 422-26 (1936).

Morosowa, A.: Akuš. i Ginek., pp. 93I-35 (I936) (abstract).

Morosowa, T. E.: Die Wirkung des Prolans und des unsterilisierten Harns Schwangerer auf die Reifung der Geschlechtsprodukte des Barsches. Zool. Ž., I5, I69-74 (1936).

Morrin, K. C. and L. Loeb: Effect of various anterior pituitary gland preparations on thyroidectomized guinea pigs. Proc. Soc. exp. Biol., N.Y., 32, 1425-27 (1935).

Mortimer, H.: Pituitary and associated hormone factors in cranial growth and differentiation in the white rat: a roentgenological study. Radiology, 28, 5-39 (I937).

Moruzzi, G. and P. Guareschi: Il Contenuto in bromo dell'ipofisi. Boll. Soc, ital. Biol. sper., I I, 28-29 (1936).

Moszkowska, A.: Extraits alcalins de lobes postérieurs de l'hypophyse de bœuf. C. R. Soc. Biol., Paris, I 19, I 239-40 (1935).

Mouriquand, G.: Hypophyse et prépuberté. Arch. méd. Enf., 39, 407-20 (I936).

MüLLER, C.: Untersuchungen über das integrative Zusammenwirken von Hypophysen-, Thymus- und Ovarialhormon. Endokrinologie, 19, 28992 (I937).

Müller, J. H. and C. Müller: Über morphologische Veränderungen der Adenohypophyse der Ratte bei E-Avitaminose. Endokrinologie, 18, 369-74 (1937).

Müller, M.: Über Adenome im Hypophysenvorderlappen, insbesondere bei Fettsucht. Endokrinologie, r8, I I4-27 (1936).

Müller, R., H. Eitel, and A. Loeser: Der thyreotrope Wirkstoffgehalt der menschlichen Hypophyse. Arch. exp. Path. Pharmak., I79, 427-39 (1935).

MugnaI, U.: Follicolina e secrezione lattea. Monit. ostetr.-ginec., 9, 240$56(1937)$. 


\section{THE PITUITARY BODY}

Murayama, F., C. Gurchot, and O. E. Guttentag: The effect of anterior pituitary growth hormone on the root lengths of seedlings of Lupinus albus. J. Pharmacol. exp. Therap., 6o, I13-I4 (I937).

Mussio-Fournier, J. C., A. Albrieux, and IW. Buño: Action locale de la folliculine sur la mamelle du cobaye mâle. Bull. Acad. Méd., Paris, I I7, 64-66 (1 937).

Nagayama, A.: Studien über die Hormonbildung in der Gewebskultur des Hypophysenvorderlappens. Nagasaki Igak. Zasshi, I5, 2692-270 I (1937).

NAKamURA, H.: Veränderungen der Kaninchenhypophyse durch dauernde Applikation von Jodnatriumlösung. Trans. Jap. path. Soc., 26, 497-98 (1936).

Nathanson, I. T., H. L. Fevold, and D. B. Jennison: Inhibition of estrous cycle in the rodent with post-partum urine and commercial prolactin. Proc. Soc. exp. Biol., N.Y., 36, 48 I-83 (I937).

Necheles, H., M. Maskin, S. Strauss, A. A. Strauss, and E. Taft: Effect of posterior pituitary extracts on motility of the gastro-intestinal tract. Arch. Surg., 33, 780-91 ( 1936).

Nelson, W. O.: Studies on the anterior hypophysis. III. The anterior. hypophysis in vitamine E-deficient rats. Anat. Rec., 56, 24I-53 (I933).

Nelson, W. O.: Changes in the hypophysis of castrate and cryptorchid guinea pigs. Proc. Soc. exp. Biol., N.Y., 32, I605-7 (1935).

NELSON, W. O.: The effect of hypophysectomy upon mammary gland development and function in the guinea pig. Proc. Soc. exp. Biol., N.Y., 33, 222-24 (1935).

Nelson, IV. O.: Studies on the physiology of lactation. VI. The endocrine influences concerned in the development and function of the mammary gland in the guinea pig. Amer. J. Anat., 60, 34 I-65 (I937).

Nelson, W. O. and T. F. Gallagher: Studies on the anterior hypophysis. $I V$. The effect of male hormone preparations upon the anterior hypophysis of gonadectomized male and female rats. Anat. Rec., 64, I2945 (1935).

Nelson, W. O. and T. F. Gallagher: Some effects of androgenic substances in the rat. Science, 84, 230-32 (19.36).

Nelson, W. O. and R. Gaunt: Initiation of lactation in the hypophysectomized guinea pig. Proc. Soc. exp. Biol., N.Y., 34, 671-73 (1936).

Nelson, IV. O. and J. Hickman: Effect of oestrone on hypophyses and reproductive organs of thyroidectomized rats. Proc. Soc. exp. Biol., N.Y., 36, 828-30 (1937).

Nelson, W. O., H. E. Hımwich, and J. F. Fazekas: Studies on the physiology of lactation. $V$. The induction of lactation in depancreatized dogs. Anat. Rec., 66, 20I-I 5 (1936).

Nelson, W. O. and C. G. Merckel: Effects of androgenic substances in the female rat. Proc. Soc. exp. Biol., N.Y., 36, 82.3-25 (1937). 


\section{BIBLIOGRAPHY}

Nelson, W. O. and C. G. Merckel: Maintenance of spermatogenesis in testis of the hypophysectomized rat with sterol derivatives. Proc. Soc. exp. Biol., N.Y., 36, 825-28 (I937).

Nelson, W. O. and M. D. Overholser: The evaluation of gonadotropic hormone preparations on the basis of the rat-mouse ratio assay. J. Pharmacol. exp. Therap., 54, 378-92 (19.35).

Nelson, W. O. and M. D. Overholser: The effect of oestrogenic hormone on experimental pancreatic diabetes in the monkey. Endocrinology, 20, 473-80 (1936).

Nelson, W. O. and C. E. Toвin: Studies on the physiology of lactation. VII. Lactation in thyroidectomized rats and guinea pigs. Endocrinology, 2r, 670-76 (1937).

Nelson, IV. O., C. W. Turner, and M. D. Overholser: The effect of lactogenic hormone preparations on the blood sugar level of rabbits and monkeys. Amer. J. Physiol., II2, 7I 4-17 (1935).

Neubach, L.: Recherche d'une sécrétion vasoconstrictrice hypophysaire dans le sang circulant du crapaud. C. R. Soc. Biol., Paris, r26, 623-24 ( 1937 ).

Newell, R. R. and A. V. Pettit: Effect of irradiation of the pituitary in dysmenorrhea. Radjology, 25, 424-28 (1935).

Newton, IV. H.: Reciprocal activity of the cornua and cervix uteri of the goat. J. Physiol., 8r, 277-82 (19.34).

Newton, W. H.: The insensitivity of the cervix uteri to oxytocin. J. Physiol., 89, 309-15 (1937).

Nicolle, P.: Recherches sur le rôle des hormones dans les variations physiologiques du taux réticulocytaire chez la lapine. C. R. Acad. Sci., Paris, 203, I $84-86$ ( 1936 ).

Nițescu, I. I. and D. Timuş: Die Ausscheidung des thyreotropen Hormons aus dem Hypophysenvorderlappen durch den Harn bei Akromegalie. Spital., 58, 3I (1938) (cited by Ber. ges. Physiol., ro6, 248 [1938]).

Nitschke, A.: Über die Beziehungen zwischen D-Vitamin und innerer Sekretion. Dtsch. med. Wschr., 62, 629-32 (1936).

Nitzescu, I. I. and S. Bratiano: Les Effets des fortes doses de vitamine D sur la structure histologique des glandes à sécrétion interne. C. R. Soc. Biol., Paris, I 21, I 5.33-35 (1936).

Nitzescu, I. I. and I. Gontzea: Hormone gonadotrope préhypophysaire et créatinurie. C. R. Soc. Biol., Paris, 125, 80-81 (1937).

Nitzescu, I. I. and I. Gontzea: Hormone somatotrope et créatinurie. C. R. Soc. Biol., Paris, 125, 291-93 (1937).

Nizza, M. and E. Berutti: Particolare reazione nell'ovaio di coniglia, trapiantato dopo trattamento con ormoni gonadotropi. Ginecologia (Torino), 2, 981-90 (1936).

O'Donovan, D. K.: The influence of pituitary extracts on oxygen consumption. Amer. J. Physiol., r19, 38 I (1937). 


\section{THE PITUITARY BODY}

O'Donovan, D. K. and J. B. Collip: The production of an increase in metabolic rates of thyroidectomized rabbits by certain pituitary extracts. West. J. Surg., 45, 564-66 (1937).

Оенме, C.: Zur antithyreoiden Wirkung der Nebennierenrinde. Klin. Wschr., I5, 512-I4 (1936).

Österreicher, W.: Gonadotrope Hormone des Hypophysenvorderlappens und menstrueller Cyclus. Quantitative Bestimmungen von Sexualhormonen (Hypophysenvorderlappen- und Follikelhormone) bei Gesunden, Geistes- und Nervenkranken. IV. Mitt. Klin. Wschr., I4, I 570-74 (1935).

Oestreicher, T.: Der Stoffwechsel des isolierten Fettgewebes. IV. Mitt.: Fettgewebsstoffwechsel und Hormone. Arch. exp. Path. Pharmak,, I82, 589-6 I6 (1936).

Oettel, $H$. and H. BachmanN: Untersuchungen am puerperalen Säugeruterus über Hypophysin, Ergometrin und Mutterkornextrakte. Arch. exp. Path. Pharmak., 185, 242-58 (1937).

Окамото, Т.: Hat die Hypophysenextirpation bei der Kröte auf die Verschiebung der Netzhautpigmente irgendeinen Einfluss? Z. ges. exp. Med., гог, I 55-65 (1937).

Okkels, H.: 'The culture of whole organs. III. The problem of antihormones studied on isolated living thyroid glands. J. exp. Med., 66, 305-16 (I 937 ).

Oldham, F. K.: The action of the preparations from the posterior lobe of the pituitary gland upon the imbibition of water by frogs. Amer. J. Physiol., I I5, 275-80 (1936).

Orent-Keiles, E., A. Robinson, and E. V. McCollum: The effects of sodium deprivation on the animal organism. Amer. J. Physiol., I I9, 651-6i (1937).

Os, P. M. van: The influence of the gonadotropic hormone from the urine on the testis with degenerated seminal tubules. Acta brev. neerl., 6, 1 $51-55$ (1936).

Osada, S.: Über den Einfluss des sogenannten Hypophysenvorderlappenhormons auf den Leberglykogengehalt. Fol. endocrin. jap., I I, 36-37 (1935).

Osborn, C. M.: The inhibition of molting in Urodeles following thyroidectomy or hypophysectomy. Anat. Rec., 66, 257-69 (1936).

Osima, M.: Experimental studies on the function of the anterior hypophysis. I. Induced sexual activity in the frogs. Sci. Rep. Tôhoku Univ., IV, 12, 195-202 (1937).

Oudet, P.: Rôle éventuel de la sécrétion thyroïdienne dans la mise en jeu des propriétés antithyréostimulantes du sérum d'animaux traités par la thyréostimuline préhypophysaire. C. R. Soc. Biol., Paris, I23, I I808 I ( 936 ).

Oudet, P.: Recherches sur les propriétés antithyréostimulantes du sang d'animaux traités, durant une courte période, par un extrait purifié de préhypophyse. C. R. Soc. Biol., Paris, I26, 710-I I (1937). 


\section{BIBLIOGRAPHY}

Oudet, P.: Recherches sur les propriétés antithyréostimulantes du sang d'animaux traités longuement par des extraits bruts et des extraits purifiés de préhypophyse. C. R. Soc. Biol., Paris, 126, 7 I 2-I 4 (1937).

Owen, S. E.: The reaction of fish to sex hormones. Endocrinology, 20, 214-18 (1936).

Owen, S. E. and M. Cutler: Sex hormones and prostatic pathology. Amer. J. Canc., 27, 308-15 (1936).

Padootcheva, A. L., P. A. Vunder, L.S.Simon, and M. M. Zawadowsky: Trudy Dinam. Razvit., 9, 97-1 I 2 (1935).

PAge, I. H.: Vaso-pressor action of extracts of plasma of normal dogs and dogs with experimentally produced hypertension. Proc. Soc. exp. Biol., N.Y., 35, i $12-16$ ( I 936$)$.

PAge, I. H.: Physiological properties of a central excitatory agent in fluid obtained by occipital puncture of man and animals. Amer. J. Physiol., I 20, 392-400 (1 937).

PAge, I. H. and J. E. Sweet: Extirpation of pituitary gland on arterial blood pressure of dogs with experimental hypertension. Proc. Soc. exp. Biol., N.Y., 34, 260-62 (1936).

PAGE, I. H. and J. E. SwEET: 'The effect of hypophysectomy on arterial blood pressure of dogs with experimental hypertension. Amer. J. Physiol., I20, 238-45 (I937).

Pagliani, F.: Le Modificazioni dell'ipofisi, pancreas e tiroide negli animali adrenalinizzati. Ann. ital. Chir., 16, 407-22 (1937).

Pallot, C.: Réactions de la glande mammaire de la lapine à la folliculine, au corps jaune et à la préhypophyse. Bull. Histol. appl., 13, 90-105 (1936).

PAlmer, A.: A source of error in gonadotropic hormone determinations. Proc. Soc. exp. Biol., N.Y., 37, 295-96 (1937).

PaPADATo, L. and B. SA PKowa: Les Glandes endocrines intracraniennes et les hormones du liquide céphalo-rachidien. Acta med. scand., 88, 204I6 (1936).

Parhon, C. I. and M. CAhane: Recherches sur l'antagonisme intermédine-adrénaline et sur les rapports de ces substances ou des substances qui dilatent les mélanophores, avec les glandes génitales et la glande thyroïde. Bull. Sect. Endocrin. Soc. roum. Neur., 2, 59-61 (19.36).

Parhon, C. I. and B. CoBan: L'Influence de la thymectomie sur la croissance chez les oiseaux (Gallus domesticus). Bull. Sect. Endocrin. Soc. roum. Neur., 2, I 45-46 (I 936).

Parhon, C. I. and C. Parhon-Stefanescu: Essais de phytoendocrinologie. L'indice de croissance ou d'assimilation. Ses rapports possibles avec une hormone de croissance. Orientation des recherches. Bull. Sect. Endocrin. Soc. roum. Neur., I, 22-24 (1935).

Parker, G. H.: An oil-soluble neurohumour in the catfish Ameiurus. J. exp. Biol., I2, 239-45 (I935). 


\section{THE PITUITARY BODY}

PARker, G. H.: The chromatophoral neurohumors of the dogfish. J. gen. Physiol., 18, 837-46 (1935).

PARKER, G. H.: The cellular transmission of substances, especially neurohumors. Quart. Rev. Biol., ro, 25 I-7I (1935).

PARKER, G. H.: Color changes of animals in relation to nervous activity. Philadelphia (19.36).

PARkes, A. S.: Terminology of sex hormones. Nature, r4I, 36 (I938).

PARKES, A. S. and I. W. Rowlands: Inhibition of ovulation in the rabbit by antigonadotropic serum. J. Physiol., 88, 305-I I (1936).

PARKES, A. S. and I. W. Rowlands: Ineffectiveness in birds of antisera for mammalian gonadotropic and thyrotropic substances. J. Physiol., 90, 100-103 (1937).

Paschkis, K. and A. Schwoner: Hypophyse und Eiweissstoffwechsel. Wien. klin. Wschr., 50, I516-I9 (1937).

Pasqualini, R. Q.: La Diurèse des crapauds privés de l'hypophyse ou de son lobe principal. C. R. Soc. Biol., Paris, 120, 498 (1935).

Paulson, D. L.: Experimental exophthalmos in the guinea pig. Proc. Soc. exp. Biol., N.Y., 36, 604-5 (I9.37).

Pencharz, R. I., C. F. Cori, and J. A. Russell: Relation of anterior and posterior lobe of the hypophysis to insulin sensitivity in the rat. Proc. Soc. exp. Biol., N.Y., 35, 32-35 (1936).

Pencharz, R. I., J. Hopper, Jr., and E. H. Rynearson: Water metabolism of the rat following removal of the anterior lobe of the hypophysis. Proc. Soc. exp. Biol., N.Y., 34, 14-17 (1936).

PERLA, D.: Effect of anterior hypophysis emulsion on natural resistance of hypophysectomized and normal rats to histamine poisoning. Proc. Soc. exp. Biol., N.Y., 33, I 21-24 (I935).

PERla, D.: Relation of the hypophysis to the spleen. I. Effect of hypophysectomy on growth and regeneration of spleen tissue. IJ. The presence of a spleen-stimulating factor in extracts of anterior hypophysis. J. exp. Med., 63, 599-615 (1936).

PERLA, D.: Effect of adrenotropic hormone on the natural resistance of hypophysectomized rats. Proc. Soc. exp. Biol., N.Y., 34, 75 I-54 (I936).

PERLA, D. and S. H. Rosen: Effect of hypophysectomy on natural resistance of adult albino rats to histamine poisoning. Arch. Path., 20, 222-32 (1935).

Perla, D. and M. Sandberg: The effect of complete and partial hypophysectomy in adult albino rats on nitrogen, calcium and phosphorus metabolism. Endocrinology, 20, 48 I-88 (1936).

Peters, G.: Über das Vorkommen von "Kolloid"-Einschlüssen in den Zellen der Medulla oblongata beim Menschen. Z. Neur., I53, 779-83 (I935).

Peters, G.: Die Kolloidproduktion in den Zellen der vegetativen Kerne des Zwischenhirns des Menschen und ihre Beziehung zu physiologischen und pathologischen Vorgängen im menschlichen Organismus. Z. Neur., I 54, 33I-44 (I935). 


\section{BIBLIOGRAPHY}

Pfeiffer, C. A.: Sexual differences of the hypophyses and their determination by the gonads. Amer. J. Anat., 58, I95-225 (1936).

Pfeiffer, C. A.: Hypophyseal gonadotropic hormones and the luteinization phenomenon in the rat. Anat. Rec., 67, I 59-75 (I937).

Preiffer, C. A.: Some factors influencing the vitalization of the ovarian graft and the production of sex hormones in the male rat. Endocrinology, 2I, 260-67 (I 937 ).

Pfeiffer, C. A.: Alterations in the percentage of cell types in the hypophysis by gonad transplantation in the rat. Endocrinology, 2I, $812-20$ (1937).

Philipp, E. and H. Huber: Die hormonale Rolle der Decidua. Ein Beitrag zur Frage der Herkunft des Schwangerschaftshormons. Z Zbl. Gynäkol., 6o, 2706-ıo (1 936).

Phillips, IV. A.: The inhibition of estrous cycles in the albino rat by progesterone. Amer. J. Physiol., I19, 623-26 (1937).

Picado, C.: Evolution des précipitines normales "anti-glandes endocrines" en relation avec l'âge et l'espèce animale. C. R. Soc. Biol., Paris, I21, 528-29 (1936).

Picinelli, G.: Azione sul pancreas degli ormoni contenuti nelle urine di gravida. Atti Soc. ital. Ostetr., 32, 398-405 (1936).

PICKFORD, M.: The inhibition of water diuresis by pituitary (posterior lobe) extract and its relation to the water load of the body. J. Physiol., 87,29 I-97 ( I 936 ).

Pierson, H.: Experimentelle Erzeugung von Uterusgeschwülsten bei Kaninchen durch Prolan. Z. Krebsforsch., 45, I-27 (I 936).

Pighini, G.: The anterior pituitary hormone content of the hypothalamus of dogs. Endocrinology, 19, 293-97 (1935).

Pighini, G.: Esperienze di innesto di ipofisi anteriore sullo sviluppo dell'embrione di pollo. Biochim. Ter. sper., 24, 187-90 (1937).

Pincus, G. and R. E. Kirsch: The sterility in rabbits produced by injections of oestrone and related compounds. Amer. J. Physiol., I I5, 2 I $9^{-}$ 28 (1 936 ).

Pitzorno, P. and A. Serra: Sull'azione dell'ormone gonadotropo (prolan) sulle gonadi e sui surreni di animali tiroidectomizzati. Riv. Pat. sper., 5, $101-12$ (1936).

PLAUT, A.: Investigations on the pars intermedia of the hypophysis in anthropoid apes and man. J. Anat., 70, 242-49 (1936).

PodleschKa, K.: Untersuchungen über die Pituitrinempfindlichkeit der menschlichen Uterusmuskulatur am überlebenden Organ. Z. Geburtsh. Gynäkol., I I I, 293-3 Iо (1935).

Podleschka, K. and H. Dworzak: Vergleichende Untersuchungen über die Pituitrin- und Adrenalinerregbarkeit des Uterus, der Harnblase und des Dickdarmes des Kaninchens in verschiedenen Phasen der Ovarialfunktion. Arch. Gynäkol., 162, 340-49 (1936).

Poppi, U.: Le Cellule nervose del tuber cinereum secernono? Riv. Neur., $8,354^{-64}$ ( 1935$)$. 


\section{THE PITUITARY BODY}

Pribram, E. and M. Raschke: Weitere Versuche zur Frage der Wirkung transplantierter Gewebe auf den Oestrus der weissen Maus. Z. Krebsforsch., 44, 93-107 (I936).

Price, D.: Normal development of the prostate and seminal vesicles of the rat with a study of experimental post-natal modifications. Amer. J. Anat., 60, 79-127 (1936).

Pugsley, L. I.: The effect of thyrotropic hormone upon serum cholesterol. Biochem. J., 29, 513-I6 (1935).

R $а$ Aв, W.: Das Hypophysen-Zwischenhirnsystem und seine Störungen. Erg. inn. Med., 51, I25-84 (1936).

Ranson, S. W. and W. R. Ingram: Hypothalamus and regulation of body temperature. Proc. Soc. exp. Biol., N.Y., 32, 1439-4 I (I935).

RANZI, S.: Ghiandole endocrine, maturità sessuale e gestazione nei Selaci. Atti Accad. naz. Lincei, 24, 528-30 (1937).

Rasmussen, A. T.: The relation of the basophilic cells of the human hypophysis to blood pressure. Endocrinology, 20, 673-78 (I936).

Rasmussen, A. T.: Reaction of the supraoptic nucleus to hypophysectomy. Proc. Soc. exp. Biol., N.Y., 36, 729-3 I (1937).

Ratner, J. A.: Sborn. naučno-izsledov. Rab., pp. I7-29 (1936) (abstract).

RAZA, S. H. and W. R. Spurrell: Some characteristics of the action of urine upon amphibian melanophores. J. Physiol., 90, 429-34 (1937).

ReEce, R. P. and C. IV. Turner: Influence of suckling upon galactin content of the rat pituitary. Proc. Soc. exp. Biol., N.Y., 35, 367-68 (1936).

Reece, R. P. and C. IV. Turner: Effect of stimulus of suckling upon galactin content of the rat pituitary. Proc. Soc. exp. Biol., N.Y., 35, 62 I-22 (I 936 ).

Reece, R. P. and C. W. Turner: Experimentally altering galactin content of the rat pituitary. Proc. Soc. exp. Biol., N.Y., 36, 283-85 (1937).

Reece, R. P., C. W. Turner, I. L. Hathaway, and H. P. Davis: Lactogen content of pituitary glands from rats on vitamin deficient rations. Proc. Soc. exp. Biol., N.Y., 37, 293-94 (1937).

Reece, R. P., C. IV. Turner, and R. T. Hill: Mammary gland development in the hypophysectomized albino rat. Proc. Soc. exp. Biol., N.Y., $34,204-7$ (1936).

RÉgnier, M. T.: Etude de la fixation du sulfonal sur les glandes endocrines. C. R. Soc. Biol., Paris, I23, IO4 $\mathrm{I}^{-42}$ (1936).

Reichert, F. L. and W. E. Dandy: Polyuria and polydipsia (diabetes insipidus) and glycosuria resulting from animal experiments on the hypophysis and its environs. Johns Hopk. Hosp. Bull., 58, 418-27 (I 936).

Reiss, M.: Hormone und Wachstum. Endocrin., Gynec., Obstetr., I, $271-89$ (1936).

Reiss, M., J. Bálint, and V. Aronson: Das Zustandekommen der kompensatorischen Hypertrophie der Nebennieren und Beiträge zur 


\section{BIBLIOGRAPHY}

Standardisierung des Nebennierenrindenhormons an Ratten. Endokrinologie, 18, 26-3i (1936).

Riess, M., J. Bálint, F. Oestreicher, and V. Aronson: Zur morphogenetischen Wirkung und biologischen Eichung des corticotropen Wirkstoffes. Endokrinologie, 18, I-IO (1936).

Reiss, M., H. Epstein, and I. Gothe: Hypophysenvorderlappen, Nebennierenrinde und Fettstoffwechsel. Z. ges. exp. Med., I01, 69-86 ( 1937 ).

Reiss, M. and S. Fischer-Popper: Über die Thyroxinempfindlichkeit hypophysektomierter Ratten. Endokrinologie, I8, 92-96 (1936).

Reiss, M., L. Schwarz, and F. Fleischmann: Beiträge zur Beziehung zwischen Hypophysenvorderlappenwachstumshormon und Eiweissstoffwechsel. Endokrinologie, 17, I67-70 (1936).

Remington, J. W.: Low-iodine goiter and the resistance of the rat to thyreotropic stimulation. Proc. Soc. exp. Biol., N.Y., 37, 175-78 (1937).

RepetTi, M.: Ricerche sugli stimoli che influenzano l'attività secretoria della mammella. Fol. Gynaecol. (Genova), 33, 503-8 I (i936).

REy, P.: L'Action des extraits post-hypophysaires sur les échanges d'eau chez Rana temporaria. C. R. Soc. Biol., Paris, I18, 949-5 I (1935).

Reynolds, S. R. M., W. M. Firor, and W. M. Allen: Relative effectiveness of progestin in hypophysectomized and normal rabbits. Endocrinology, 20, 68 I-82 (1936).

Riccitelli, L.: Sulle variazioni funzionali dei centri encefalici vegetativi per apporto locale di ormoni e sostanze farmaco-dinamiche: conseguenti modificazioni elettrocardiografiche. Sperimentale, $89,348-70$ (1935).

Richardson, K. C. and F. G. Young: The "pancreotropic" action of anterior pituitary extracts. J. Physiol., 9I, 352-64 (I937).

Richter, C. P.: The primacy of polyuria in diabetes insipidus. Amer. J. Physiol., II2, 48 I-87 (1935).

Richter, J.: Der Einfluss der Kastration auf die Milch- und Fleischleistung der Kühe. Berl. tierärztl. Wschr., pp. 277-80, 293-98 (เ936).

Riddle, O.: Contemplating the hormones. Endocrinology, I9, I-I3 (I935).

Riddle, O., R. W. Bates, E. L. Lahr, and C. S. Moran: On the identity of the hormone causing ovulation in the rabbit. Amer. J. Physiol., I 6 , I 28-29 (1936).

Riddle, O. and L. B. Dotti: Blood calcium in relation to anterior pituitary and sex hormones. Science, 84, 557-59 (1936).

Riddle, O., L. B. Dott, and G. C. Smith: Blood sugar and basal metabolism in pigeons following administration of prolactin and cortin. Amer. J. Physiol., I 19, 389-90 (1937).

Riddle, O. and J. P. Schooley: Absence of follicle-stimulating hormone in pituitaries of young pigeons. Proc. Soc. exp. Biol., N.Y., 32, I610$I_{4}(19.35)$. 
Riddle, O., G. C. Smith, R. W. Bates, C. S. Moran, and E. L. Lahr: Action of anterior pituitary hormones on basal metabolism of normal and hypophysectomized pigeons and on the paradoxical influence of temperature. Endocrinology, 20, I-I6 (1936).

Riddle, O., G. C. Smith, and C. S. Moran: Effects of complete and incomplete hypophysectomy on basal metabolism of pigeons. Proc. Soc. exp. Biol., N.Y., 32, 1614-16 (1935).

Rihl, J., F. Oestreicher, and M. Reiss: Vergleich der Wirkung von Thyroxin und thyreotropem Wirkstoff auf die Herzschlagfrequenz. Endokrinologie, 18, 88-92 (1936).

Ringoen, A. R. and A. Kirschbaum: Correlation between ocular stimulation and spermatogenesis in the English sparrow (Passer domesticus). Proc. Soc. exp. Biol., N.Y., 36, i I I-13 (1937).

Rivoire, R.: Les Corrélations hypophyso-endocriniennes. V. Hypophyse et parathyroïdes. Presse méd., 43, 528-30 (1935).

Robertson, E. M.: A study of the contractions of the non-pregnant human uterus. Edinb. med. J., 44, 20-27 (1 937).

Robinson, V. E.: Hypophysectomy and its consequences in the pig. Bull. Biol. Méd. exp. URSS, 4, 23-25 (1937).

Robson, J. M.: The effect of oestrin on the uterine reactivity and its relation to experimental abortion and parturition. J. Physiol., 84, I $21-32$ (I 935 ).

Robson, J. M.: The action of oestrin on the uterus of the hypophysectomized and of the pregnant rabbit. J. Physiol., 84, 148-6I (I935).

Robson, J. M.: The action of the ovarian hormones on the uterine muscle measured in vivo and in vitro. J. Physiol., 85, I 45-58 (1935).

Robson, J. M.: Hormones and pregnancy. Brit. med. J., I, 1033-38 (1936).

Robson, J. M.: The rôle of the luteal hormone in the maintenance of gestation. Edinb. med. J., 43, 395-401 (1936).

Robson, J. M.: Uterine changes in experimental abortion and their relation to parturition. J. Physiol., 86, 171-82 (1936).

RoBson, J. M.: Maintenance of pregnancy in the hypophysectomized rabbit with progestin. J. Physiol., 86, 41 5-24 (1936).

Robson, J. M.: The action of progesterone on the uterus of the rabbit and its antagonism by oestrone. J. Physiol., 88, 100-11 I (1936).

Robson, J. M.: The physiology of the endometrium and uterine muscle, and of the ovarian cycle. Brit. med. J., I, 512-I 4, 566-68 (I937).

Robson, J. M.: Maintenance of ovarian and luteal function in the hypophysectomized rabbit by gonadotropic hormones. J. Physiol., 90, I 25-44 (I 937).

Robson, J. M.: Maintenance of pregnancy and of the luteal function in the hypophysectomized rabbit. J. Physiol., 90, 145-66 (1937).

Robson, J. M.: Maintenance by oestrin of the luteal function in hypophysectomized rabbits. J. Physiol., 90, 435-39 (19.37).

Robson, J. M.: Action of testosterone on lactation. Proc. Soc. exp. Biol., N.Y., 36, 153 (19.37). 


\section{BIBLIOGRAPHY}

Robson, J. M. and W. R. Henderson: The action of oestrin on the bitch. Proc. Roy. Soc., B, 120, 1-14 (1936).

Robson, J. M. and H. Schild: Effect of drugs on the muscular activity and blood supply of the uterus. J. Physiol., 90, 17P (1937).

Rochlina, M. L..: Bull. Biol. Méd. exp. URSS, 3, 366-69 (1937) (abstract).

Rodewald, W.: Der Einfluss der Dunkelheit auf den das Melanophorenhormon bindenden Stoff im Froschblut. Z. vergl. Physiol., 22, 43133 (I 935 ).

Rodewald, W.: Über einen das Melanophorenhormon bindenden Stoff im Serum Krebskranker. Dtsch. med. Wschr., 62, 726 (1936).

Romodanowskaja, S. A.: Das Gewicht der innersekretorischen Drüsen des Menschen und ihre wechselseitigen Gewichtskorrelationen. Arch. Anat., 15, 149-5+ (1936).

Rosen, S. and M. C. Shelesnyak: Induction of pseudopregnancy in rat by silver nitrate on nasal mucosa. Proc. Soc, exp. Biol., N.Y., 36, 83234 (1937).

Rostand, J.: Hypophyse et ovulation chez les batraciens. C. R. Soc. Biol., Paris, xi 7, 1079-8 I (1934).

Rostand, J.: Sur l'ovulation provoquée, chez quelques anoures. C. R. Soc. Biol., Paris, I19, 697-98 (1935).

Rostand, J.: Limites saisonnières de l'ovulation chez les batraciens. C. R. Soc. Biol., Paris, r20, 336-38 (1935).

Rothschild, F. and H. Staub: Verhalten von Fett- und Lipoidstoffen in Blut und Organen, Blutzucker, Diurese, Kochsalz- und Harnstoffiusscheidung nach Zufuhr von thyreotropem Hormon des Hypophysenvorderlappens beim Kaninchen. Arch. exp. Path. Pharmak., r78, I8996 (1935).

Rothschild, F., H. Staub, and K. MezeY: Wirkung eines thyreotropen Hormonpräparates des Hypophysenvorderlappens auf die Diurese der weissen Maus und den Blutdruck der Katze. Antagonismus Vorderlappen-Hinterlappenhormone. Arch. exp. Path. Pharmak., r79, 6I-71 (1935).

Roussy, G. and M. Mosinger: Sur la neuronolyse physiologique dans l'hypothalamus des mammifères. C. R. Soc. Biol., Paris, I18, $4^{1-16}$ (I9.35).

Roussy, G. and M. Mosinger: La Neurocrinie pigmentaire hypophysaire et la neurocrinie périphérique. C. R. Soc. Biol., Paris, rr9, 795-97 (1935).

Roussy, G. and M. Mosinger: Sur les réactions neuronales de l'hypothalamus consécutives à l'hyperneurocrinie hypophyso-hypothalamique expérimentale. C. R. Soc. Biol., Paris, I19, 797-99 (1935).

Roussy, G. and M. Mosinger: Le Jeu de neurorégulation de l'hypophyse. C. R. Soc. Biol., Paris, i19, 931-33 (1935).

Roussy, G. and M. Mosinger: Plurinucleose neuronale expérimentale consécutive à l'injection répérée d'extraits antéhypophysaires. C. R. Soc. Biol, Paris, r22, I 290-92 (1936). 


\section{THE PITUITARY BODY}

Roussy, G. and M. Mosinger: La Régulation nerveuse du fonctionnement hypophysaire. Ses conséquences physio-pathologiques et thérapeutiques. Presse méd., 44, I 52 I-23 (1 936).

Roussy, G. and M. Mosinger: La Neurocrinie hypophysaire et les processus neurocrinies en général. Ann. Anat. path., I4, I65-89 (I 937 ).

Roussy, G. and M. Mosinger: Sur la neurocrinie pancréatique et sa stimulation par l'extrait antéhypophysaire. C. R. Soc. Biol., Paris, I26, $1064-66$ ( I 937 ).

Rowan, W.: Relation of light to bird migration and developmental changes. Nature, II5, 494-95 (I925).

Row LANDS, I. W.: Changes in the thyroid gland of certain mammals and birds following hypophysectomy. J. exp. Biol., I2, 337-47 (I935).

Rowland, I. W.: 'The stability of anterior pituitary extract in aqueous solution. Quart. J. Pharm. Pharmacol., 8, 642-45 (1935).

Row LANDS, I. W.: The relative activity of different gonadotropic preparations on oestrous rabbits, pregnant rabbits and immature rats. Quart. J. Pharm. Pharmacol., 8, 646-50 (I935).

Rowlands, I. W.: Species variation in thyrotropic activity of the pituitary gland. J. Physiol., 88, 298-304 (I936).

Rowland, I. IV.: Specificity of antisera to gonadotropic extracts. J. Physiol., 90, I9P-20P (I937).

Rowlands, I. W.: The effect of anti-gonadotropic serum on the reproductive organs of the normal animal. Proc. Roy. Soc., B, I2I, 517-32 (1937).

Rowlands, I. W.: The assay of lactogenic extracts of the anterior pituitary gland. Quart. J. Pharm. Pharmacol., ro, 216-2 I (1937).

Rowlands, I. W.: Pro-gonadotropic sera. Proc. Roy. Soc., B, I24, 492503 (1938).

Rowlands, I. W.: The specificity of antigonadotropic sera. Proc. Roy. Soc., B, I24, 503-21 (1938).

Rowlands, I. W. and M. K. McPhail: The action of progestin on the uterus of the cat. Quart. J. exp. Physiol., 26, 109-I I 8 (1936).

Rowlands, I. W. and A. S. PARKES: A study of anti-thyrotropic activity. Proc. Roy. Soc., B, I20, I I4-25 (I 936).

Rowlands, I. W. and A. S. Parkes: Inhibition of the gonadotropic activity of the human pituitary by antiserum. Lancet, 232, 924 (1937).

Rowlands, I. W. and E. Singer: Gonadotropic activity of the pituitaries of vitamin E deficient rats. J. Physiol., 86, 323-26 (I936).

Rowntree, L. G., J. H. Clark, and A. M. Hanson: Biologic effects of thymus extract (Hanson). Accruing acceleration in growth and development in five successive generations of rats under continuous treatment with thymus extract. Arch. intern. Med., 56, I-29 (I935).

Rubino, A.: Cataratta in soggetto con diabete mellito, nanismo e cachessia ipofisaria. Boll. Ocul., I4, I 297-I306 (I935).

Rugh, R.: A quantitative analysis of the pituitary-ovulation relation in the frog (Rana pipiens). Physiol. Zoöl., 10, 84-100 (1937). 


\section{BIBLIOGRAPHY}

Rugh, R.: Release of spermatozoa by anterior pituitary treatment of the male frog, Rana pipiens. Proc. Soc. exp. Biol., N.Y., 36, 4 I 8-20 (1937).

Rugh, R.: Ovulation induced out of season. Science, 85, 588-89 (I937).

Russell, J. A.: Carbohydrate levels in fasted and fed hypophysectomized rats. Proc. Soc. exp. Biol., N.Y., 34, 279-81 (1936).

Russell, J. A.: Effects of hypophysectomy and of anterior pituitary extracts on disposition of fed carbohydrate in rats. Proc. Soc. exp. Biol., N.Y., 37, 31-33 (1 937).

Russel., J. A.: Production of refractoriness to action of anterior pituitary extracts in depressing oxidation of fed carbohydrate. Proc. Soc. exp. Biol., N.Y., 37, 33-34 (1937).

Russel., J. A.: Effect of thyroxin on carbohydrate metabolism of hypophysectomized rats. Proc. Soc. exp. Biol., N.Y., 37, 569-70 (1937).

Russell, J. A.: The relation of the anterior pituitary to carbohydrate metabolism. Physiol. Rev., 18, I-27 (1938).

Russell, J. A. and L. L. Bennetr: Carbohydrate storage and maintenance in the hypophysectomized rat. Amer. J. Physiol., I18, I96-205 (I937).

Russell, J. A. and G. T. Cori: A comparison of the metabolic effects of subcutaneous and intravenous epinephrine injections in normal and hypophysectomized rats. Amer. J. Physiol., I19, I67-74 (1937).

Russo Marchese, F.: Contributo alle conoscenze circa le correlazionj funzionali dei testicoli e della ipofisi in rapporto alla crescenza. Clinica chir., 12, 261-77 (1936).

SACCHI, U.: Sulla diversa reazione dei centri bulbari all'adrenalina ed alla pituitrina. Arch. Fisiol., 34, 503-14 (1935).

SAGER, V. J. and S. L. LeOnaRD: Relation of oestrin and pregnancy urine hormone in influencing uterine motility. Proc. Soc. exp. Biol., N.Y., 35, $242-44$ (1936).

Salle, A. J. and I. I.. Shechmeister: Effect of lactogenic hormone on embryonic tissues cultivated in vitro. Proc. Soc. exp. Biol., N.Y., 34, $603-6$ (1936).

SALmon, U. J.: Effect of testosterone propionate upon gonadotropic hormone excretion and vaginal smears of human female castrate. Proc. Soc. exp. Biol., N.Y., 37, 488-9 I (1937).

SALMON, U. J. and R. T. FRANK: An improved method for determination of the gonadotropic hormone. Proc. Soc. exp. Biol., N.Y., 32, I 236-37 (1935).

SALMON, U. J. and R. T. FRANK: Hormonal factors affecting vaginal smears in castrates and after the menopause. Proc. Soc. exp. Biol., N.Y., 33, 6I2-I4 (1936).

SamaAn, A.: The effect of pituitary (posterior lobe) extract upon the urinary flow in non-anaesthetized dogs. J. Physiol., 85, 37-46 (1935).

SAmuels, L. T. and H. A. BALL: Hypophysectomy and tumor growth: a supplementary statement. Amer. J. Canc., 23, 80I-3 (1935). 


\section{THE PITUITARY BODY}

Samuels, L. T., H. F. Schott, and H. A. Ball: The relation of the hypophysis and adrenal cortex to the removal of excess glucose from the blood of rats. Amer. J. Physiol., 120, 649-56 (1937).

Sanchez-Calvo, R.: Einfluss der Dunkelheit auf das Zellbild der Hypophyse. Virchows Arch. path. Anat., 300, 560-63 (1937).

Sandberg, M., D. Perla, and O. M. Holly: The effect of complete and partial hypophysectomy in adult albino rats on water, chloride, sodium, potassium and sulfur metabolism. Endocrinology, 21, 346-5 I (1937).

SAnto, E.: Die Beeinflussung der Langerhansschen Inseln durch das sogennante pankreatrope Hormon der Hypophyse. Z. ges. exper. Med., I02, 390-406 (1938).

SAPHiR, W.: Vitamin E and the gonads. Endocrinology, 20, 107-8 (1936).

SARDI, J. 1.: Sécrétion lactée sous l'action de l'extrait antéhypophysaire chez les cobayes mâles. C. R. Soc. Biol., Paris, 120, 503-4 (1935).

Sıtô, K.: Studien über die Morphologie und Histologie der Anurenhypophyse. (III. Mitt.) Okay. Igak. 'Zasshi, 47, I-23 (I935).

Saunders, F. J. and H. H. Cole: Two gonadotropic substances in mare serum. Proc. Soc. exp. Biol., N.Y., 32, I 476-78 (I 935 ).

Saunders, F. J. and H. H. Cole: Age and the qualitative ovarian response of the immature rat to mare gonadotropic hormone. Proc. Soc. exp. Biol., N.Y., 33, 504-5 (1936).

Saunders, F. J. and H. H. Cole: Means of augmenting the ovarian response to gonadotropic substances. Proc. Soc. exp. Biol., N.Y., 33, $505^{-8}(1936)$.

SAvona, B.: Ormoni preipofisari (urina di gravida e prolan) e modificazioni della corteccia surrenale. Riv, ital. Ginec., 18 , 286-99 (1935).

SAxton, J. and L. Loeв: Thyroid stimulating and gonadotropic hormones of the human anterior pituitary gland at different ages and in pregnant and lactating women. Anat. Rec., 69, 26I-79 (1937).

Saxton, J. and L. Loeb: Serial implantation of anterior lobes of bovine and human pituitary glands into guinea-pigs. Arch. Path., 24, I.35-42 (1937).

Scharrer, E.: Über die Zwischenhirndrüse der Säugetiere. Sitzgsber. Ges. Morph. Physiol. Münch., 42, 36-4I (I935).

Scharrer, E. and R. Gaupp: Neuere Befunde am Nucleus supraopticus und Nucleus paraventricularis des Menschen. Z. ges. Neurol. Psychiat., I48, 766-72 (1933).

Scharrer, E. and R. Gaupp: Bemerkungen und Versuche zur Frage der Beziehungen zwischen Schilddrüse und Zwischenhirndrüse. Klin. Wschr., I4, I65I-52 (1935).

Schellong, F.: Hypophyse und Kreislauf. Verh. dtsch. Ges. Kreislaufforsch., pp. 62-78 (1937).

Schildmacher, H.: Histologische Untersuchungen an V'ogelhypophysen.

I. Die Zelltypen der Amsel, Turdus merula L. J. Ornithol., 85, 587-92 (1937).

Schliffer, W.: Die Entwicklung der Hypophyse bei Larven von Bufo vulgaris bis zur Metamorphose nebst Untersuchungen über den Ein- 


\section{BIBLIOGRAPHY}

fluss hormonaler Substanzen auf die äussere Entwicklung der Larven und die Genese der Hypophyse. Zool. Jb. Abt. Anat. Ontog., 59, 383454 (1935).

Schmidt, I. G.: The effects of hypophyseal implants from normal mature guinea pigs on the sex organs of immature guinea pigs. Endocrinology, 2I, 46I-68 (1937).

Schmidt, I. G.: The effects of hypophyseal implants from guinea pigs with irradiated ovaries on the sex organs of immature guinea pigs. Endocrinology, 21, 469-75 (1937).

Schmirz, A.: Über den Einfluss der Schwangerschaftshormone auf die

- Bewegungen des Harnleiters im Hindblick auf die Schwangerschaftsatonie der Ureteren. Z. Urol., 31, 387-400 (1937).

Schneider, B. and A. E. Cohen: Nonspecificity of gonadotropic factor of pregnancy urine intradermally as a test for pregnancy. J. Amer. med. Ass., ro9, I I 5-I7 (I9.37).

Schockaert, J. A. and J. Lambillon: Sur la présence d'une substance antagoniste de la vasopressine dans le sérum de femmes enceintes. C. R. Soc. Biol., Paris, I19, I 194-97 (1935).

Schockaert, J. A. and J. Lambillon: Observations complémentaires sur la substance antagoniste de la vasopressine dans le sérum de la femme enceinte. C. R. Soc. Biol., Paris, I22, 478-80 (1936).

Schockaert, J. A. and J. Lambillon: Spécificité de la substance inhibitant l'action hypertensive de la vasopressine dans le sérum de la femme enceinte. C. R. Soc. Biol., Paris, r22, 48 I-84 (1936).

Schockaert, J. A. and J. Lambillon: Différence de sensibilité à l'injection intraveineuse de vasopressine entre la femme gravide des trois derniers mois et la femme non gravide. C. R. Soc. Biol., Paris, 123, 309I I (1936).

Schockaert, J. A. and A. Lejeune: La Teneur en vasopressine de l'hypophyse de cobaye normal, et après injections de folliculine ou de thyroxine. C. R. Soc. Biol., Paris, I I9, I $197-99$ (1935).

Schoeller, IV., M. Dohrn, and W. Hohlweg: Die Überlegenheit des weiblichen Hormons in seiner Wirkung auf die männliche und weibliche Kastratenhypophyse gegenüber männlichen Hormonen. Klin. Wschr., I5, 1907-8 (1936).

Schönholzer, G.: Zur Frage der Wirkung der Schilddrüsenüberfunktion auf den Eiweissstoffwechsel. Z. ges. exp. Med., I00, 80I-7 (1937).

Schofield, N. D. and R. F. Blount: Changes in the alimentary canal of Urodele larvae associated with an excess or absence of hypophyseal tissue. Anat. Rec., 68, 169-92 (1937).

Schooley, J. P., O. Riddle, and R. W. Bates: Effective stimulation of crop-sacs by prolactin in hypophysectomized and in adrenalectomized pigeons. Proc. Soc. exp. Biol., N.Y., 36, 408-1 I (1937).

Schott, H. F., L. T. Samuels, and H. A. BALL: Effect of hypophysectomy on glycogen distribution in tumor-bearing rats. Proc. Soc. exp. Biol., N.Y., 37, 410-I 2 (1937). 


\section{THE PITUITARY BODY}

Schreiber, B.: Tentative di maturazione sperimentale dell'anguilla con ormoni ipofisari. Ist. Lombardo, Rend., 68, 669-72 (1935).

Schuntermann, C. E.: Experimentelle Untersuchungen über den Synergismus zwischen Calcium und Digitalis am intakten Warmblüter. IV. Mitt. Z. ges. exp. Med., 96, 520-25 (1935).

Schwartz, H. G.: The meningeal relations of the hypophysis cerebri. Anat. Rec., 67, 35-44 (1936).

Schwartz, H. G. and C. L. Buxton: The effect of sympathetic denervation upon ovulation and estrus in the rat. Amer. J. Obstetr., 31, 132$34(1936)$.

Schwartzbach, S. S. and E. Uhlenhuth: Die Physiologie des Thyreoaktivators bei Amphibien. III. Erhöhung des Sauerstoffverbrauchs durch Thyreoaktivatoreinspritzungen. Endokrinologie, I6, 412-22 (1936).

Schweizer, M., H. A. Charipper, and H. O. Haterius: Experimental studies of the anterior pituitary. IV. The replacement capacity and the non-cyclic behavior of homoplastic anterior pituitary grafts. Endocrinology, 21, 30-39 (1937).

Scipiades, E.: Effect of testosterone injections upon the course of pregnancy in unoperated and in castrated rats. Proc. Soc. exp. Biol., N.Y., 37, 242-44 (1937).

Scoтt, A. H.: Effect of hypophysectomy on blood lactic acid of rhesus monkeys. Proc. Soc. exp. Biol., N.Y., 36, 540-42 (1937).

Scowen, E. F.: Effects of the thyrotropic hormone of the anterior pituitary in man. Lancet, 233, 799-802 (1937).

Scowen, E. F. and A. W. Spence: The effect of antithyrotropic serum on the thyroid gland of guinea-pigs treated with thyrotropic hormone. J. Physiol., 86, 109-1 I6 (1936).

ScriBA, K.: Die basophilen Zellen des Hypophysenhinterlappens und ihre Beziehungen zum Hochdruck und zur Eklampsie. (Zugleich ein Beitrag zur Frage: Hat der Mensch eine dem tierischen Zwischenlappen vergleichbare Pars intermedia?) Arch. exp. Path. Pharmak., 297, 22 I-5 I ( I 936$)$.

Sealock, R. R. and V. du Vigneaud: Studies on the reduction of pitressin and pitocin with cysteine. J. Pharmacol. exp. Therap., 54, 433-47 (1935).

Seekles, L.: Über das gleichzeitige Vorkommen eines Prinzips im Blutserum, das den Glykogenbestand der Leber herabsetzt, und Störungen des vegetativ-endokrinen Systems. Z. ges. exp. Med., Ioo, $324^{-3}$ I (1937).

Seligsonn, A.: Über die zentrale Blutdruckwirkung der Hypophysenhormone und nichtisotonischer Lösungen. Wien. Arch. inn. Med., 29, $42 \mathrm{I}-34$ (1936).

Selye, H.: Influence of the uterus on ovary and mammary gland. Proc. Soc. exp. Biol., N.Y., 3I, 488-90 (1934). 


\section{BIBLIOGRAPHY}

Selye, H., J. S. L. Browne, and J. B. Collip: Effect of large doses of progesterone in the female rat. Proc. Soc. exp. Biol., N.Y., 34, 472-74 (1936).

Selye, H. and J. B. Collip: Fundamental factors in the interpretation of stimuli influencing endocrine glands. Endocrinology, 20, 667-72 (1936).

Selye, H., J. B. Collip, and D. L. Thomson: Effect of oestrin on ovaries and adrenals. Proc. Soc. exp. Biol., N.Y., 32, I377-8 I (1935).

Selye, H. and T. Mckeown: Studies on the physiology of the maternal placenta in the rat. Proc. Roy. Soc., B, II9, I-3I (1935).

Severinghaus, A. E.: Cellular changes in the anterior hypophysis with special reference to its secretory activities. Physiol. Rev., I7, 556-88 (I937).

Shapiro, B. G. and H. Zwarenstein: The relation of the pituitary gland to muscle creatine. Proc. Roy. Soc., Edinb., 56, I6 $4-68$ (1936).

Shapiro, B. G. and H. Zwarenstein: The posterior pituitary and muscle creatine. S. Afr. J. med. Sci., 2, I5-17 (1937).

Shapiro, H. A.: Induction of ovulation by testosterone and certain related compounds. Chem. Indus., 55, 1031-32 (1936).

Shapiro, H. A.: The biological basis of sexual behaviour in amphibia. I. The experimental induction of the mating reflex (coupling) in Xenopus laevis (the South African clawed toad) by means of pregnancy urine and of anterior pituitary extracts, with the production of fertilised ova. J. exp. Biol., 13, 48-56 (1936).

Shapiro, H. A.: The biological basis of sexual behaviour in amphibia. II. The independence of ovulation and of coupling (the mating reflex) in Xenopus laevis (the South African clawed toad). J. exp. Biol., 13, 5759 (I 936 ).

Shapiro, H. A.: The influence of the pituitary-like substance in human pregnancy urine on the motor components of sexual behaviour in the South African clawed toad (Xenopus laevis). S. Afr. J. med. Sci., I, $107^{-13}$ (1936).

Shapiro, H. A.: The biological basis of sexual behaviour in amphibia. IV. J. exp. Biol., I4, 38-47 (1937).

Shapiro, H. A.: The effect of prolactin-containing extracts of sheep anterior pituitary on the histological structure of the amphibian testis. S. Afr. J. med. Sci., 2, 121-23 (1937).

Shapiro, H. A. and H. Zwarenstein: Effects of progesterone and testosterone on Xenopus and on its excised ovary. J. Physiol., 89, $3^{8^{\text {P }}}$ (I937).

Shelton, E. K., L. A. Cavanaugh, and M. L. Long: Studies on the effect of human blood serum upon the growth of the rat. Endocrinology, 19, $5+3^{-} 4^{8}$ ( 1935 ).

Shen, T. C. R.: The pharmacology of melanophores in frogs (Rana temporaria) and the role of the hypophysis. Arch. int. Pharmacodyn., $57,289-334$ (1937). 


\section{THE PITUITARY BODY}

SHERwood, T. C. and L. M. Bowers: The effect of ovarian hormone on the basal metabolism of experimental hyperthyroid rats. Amer. J. Physiol., I I5, 645-50 (1936).

Shorr, E., H. B. Richardson, and J. E. Sweet: The respiratory metabolism of lactic acid cycle in the excised skeletal muscle of the depancreatized-hypophysectomized dog. Amer. J. Physiol., I I6, I42-43 (I 936).

Shumacker, H. B. Jr., and A. Lamont: Lack of effect of theelin upon somatogenic, thyreotropic, and adrenotropic activity of hypophysis. Proc. Soc. exp. Biol., N.Y., 32, I 568-70 (1935).

Shute, E.: The relation of deficiency of vitamin $E$ to the anti-proteolytic factor found in the serum of aborting women. J. Obstetr. Gynecol., 43, $74-86$ ( I 936$)$.

Sievert, C.: Über den Einfluss des Fettstoffwechselhormons des Hypophysenvorderlappens auf die Acetonkörperausscheidung von Ratten unter verschiedenen Versuchsbedingungen. 7. ges. exp. Med., 96, 42941 (1935).

Sil.BERBERG, M.: Effects of extract of cattle anterior pituitary gland on endochondral ossification in young guinea pigs. Proc. Soc. exp. Biol., N.Y., 32, I 423-25 (I 935 ).

Silberberg, M.: Effect of cattle anterior pituitary extract on bone and cartilage of the joint (acromegalic arthropathia). Proc. Soc. exp. Biol., N.Y., 34, 333-34 (1936).

SILBERBERG, M.: Influence of cattle ant. pituitary extract on the joints of thyroidectomized guinea pigs. Proc. Soc. exp. Biol., N.Y., 35, 66-67 (I 936$)$.

Silberberg, M. and R. Silberberg: Effect of acid extract of cattle ant. pituitary on bone repair in thyroidectomized guinea pigs. Proc. Soc. exp. Biol., N.Y., 34, 108-10 (1936).

Silberberg, M. and R. Silberberg: Changes in ribs of guinea pigs following administration of cattle anterior pituitary extract (acromegalic rosary). Proc. Soc. exp. Biol., N.Y., 36, 622-25 (1937).

Silberberg, M. and R. Silberberg: Influence of cattle anterior pituitary extract on endochondral ossification in young ovariectomized guinea pigs. Proc. Soc. exp. Biol., N.Y., 37, 446-50 (1 937 ).

SILER, K. A.: The cytological changes in the hypophysis cerebri of the garter snake (Thamnophis radix) following thyroidectomy. J. Morph., $59,603 \sim 23(1936)$.

Silvestroni, E.: Effetti della surrenalectomia mono- o bilaterale sulle grandezze nucleari di alcuni organi endocrini. Atti Accad. naz. Lincei, 22, 540-44 (1935).

Siluestroni, E.: Richerche istologiche e citometriche sulle ghiandole endocrine di ratto albino surrenalectomizzato bilateralmente. II. Ipofisi (lobo anteriore). Atti Soc. med.-chir. Padova ecc., 13, 59-70 (1935).

Simon, A.: Über die Wirkung verschiedener Hormone und des Aufenthaltes im Dunkeln auf den Gehalt des Hypophysenhinterlappens an blutdruck- und uteruswirksamen Stoffen. Arch. exp. Path. Pharmak., I $82,5^{8} 4^{-88}(1936)$. 


\section{BIBLIOGRAPHY}

Simon, A.: Die Wirkung der Hormone und der Dunkelheit auf den Gehalt der blutdruck- und uteruswirksamen Stoffe des Hypophysenhinterlappens. Ber. ges. Physiol., 94, 665 (1936).

Simon, A.: Zur Frage des Vasopressinnachweises im Blute. Arch. exper. Path. Pharmak., 187, 672-77 (1937).

Simon, A.: Über Vasopressin-Nachweis im Blute. Ber. ges. Physiol., ror, 674 ( 1937 ).

Simpson, J. W. and J. C. Burch: Experimental production of glandular cystic hyperplasia of endometrium with estrogenic substances of parturient urine. Proc. Soc. exp. Biol., N.Y., 32, I 570-71 (1935).

Singer, K. and M. Taubenhaus: Zur Frage des Kohlehydratstoffwechselhormons des Hypophysenvorderlappens. Wien. Arch. inn. Med., 31, 59-66 (1937).

SinHA, K. N.: Reactivity of the thyroid of the rabbit to the thyrotropic hormone of the anterior pituitary. Quart. J. exp. Physiol., 26, 33I-37 (I 937 ).

Slome, D.: The diabetogenic hormone of the pituitary gland. J. exp. Biol., I3, I-6 (1936).

Smelser, G. K.: Experimental production of exophthalmos resembling that found in Graves's disease. Proc. Soc. exp., Biol., N.Y., 35, 128$30(1936)$.

Smelser, G. K.: Assay of thyrotropic hormone on day-old chicks, Proc. Soc. exp. Biol., N.Y., 37, 388-90 (1937).

Smith, F. M. and E. M. MAcKAY: Influence of posterior pituitary extracts on sodium balance in normal subject and in patient with diabetes insipidus. Proc. Soc. exp. Biol., N.Y., 34, 116-18 (1936).

SMITH, G. van S. and O. W. SmITH: The inhibition of lactation in rabbits with large amounts of oestrin. Amer. J. Physiol., 103, 356-6i (1933).

Sмith, G. van S. and O. W. Sмгтн: Further quantitative determinations of prolan and estrin in pregnancy, with especial reference to late toxemia and eclampsia. Surg. Gynecol. Obstetr., 6I, 27-35 (1935).

Smith, P. E., L. Dotti, H. H. Tyndale, and E. T. Engle: Effect of hypophysectomy on blood sugar of rhesus monkeys. Proc. Soc. exp. Biol., N.Y., 34, 247-49 (1936).

Smith, P. E., H. H. Tyndale, L. Dotti, and E. T. Engle: Response of normal and hypophysectomized rhesus monkeys to insulin. Proc. Soc. exp. Biol., N.Y., 34, 250-51 (1936).

Smith, P. E., H. H. Tyndale, and E. T. Engle: The reproductive system and its responses to ovarian hormones in hypophysectomized rhesus monkeys. Proc. Soc. exp. Biol., N.Y., 34, 245-47 (I936).

SNOEck, J.: Action inhibitrice de la folliculine sur la montée laiteuse et la sécrétion lactée. Contribution à l'étude de la physiologie de la lactation. Bull. Soc. belge Gynécol., rI, i $38-48$ (1935).

Somogyi, M., T. E. Weichselbaum, and P. Heinbecker: Hyperglycemia in response to hypoglycemia in normal and hypophysectomized dogs. Proc. Soc. exp. Biol., N.Y., 37, 62-65 (1937). 


\section{THE PITUITARY BODY}

Soskin, S., I. A. Mirsky, L. M. Zimmerman, and N. Crohn: Influence of hypophysectomy on gluconeogenesis in the normal and depancreatized dog. Amer. J. Physiol., I I4, I IO-1 8 (1935).

SPARK, C.: Relation between basophilic invasion of the neurohypophysis and hypertensive disorders. Arch. Path., I9, 473-50I (1935).

Spence, A. W., E. F. Scowen, and I. W. Rowlands: The absence of antigonadotropic substances in the blood serum of man injected with gonadotropic extracts. Brit. med. J., I, 66-67 ( 938 ).

Speransky-Stepanova, E. N.: The relationship between the hypophysis and the parathyroid glands. Arch. biol. Nauk., 40, 74-75 (1935).

Speranskaja-Stepanowa, E. N.: Der Einfluss der Nebenschilddrüsen auf den Wasserwechsel. IV. Mitt. Die Diureses hypophysektomierter Hunde bei parathyreoidem Tetanus. Fiziol. Ž, 20, 422-23 (1936).

Sрото, P.: Lo Studio quantitativo della eliminazione delle sostanze gonadotrope urinarie nella valutazione di manifestazioni fisio-patologiche dell'attività sessuale femminile. Ginecologia (Torino), 2, 553$620,645-795$ (1936).

Spreng, A.: Beeinflussung der Schwangerschaftsdauer durch Hypophysenvorderlappenhormon. Endokrinologie, I9, 161-64 (I937).

Stamler, C. M.: The fate of prolane in the organism. Bull. Biol. Méd. exp. URSS, 3, 35-38 (I 937 ).

SteHLE, R. L.: A method for obtaining a preparation of the melanophore hormone of the pituitary gland. J. Pharmacol. exp. Therap., 57, I-5 (1936).

Stehle, R. L. and A. M. Fraser: The purification of the pressor and oxytocic hormones of the pituitary gland and some observations on the chemistry of the products. J. Pharmacol. exp. Therap., 55, I36-5 I (1935).

Stein, K. F.: A sex difference in gonad-stimulating potency of young gonadectomized rats. Proc. Soc. exp. Biol., N.Y., 33, 95-97 (I9.35).

STEIN, S. I.: Experimental studies on the hypophysis cerebri. IV. The effect of vitamin $\mathrm{E}$ deficiency in the female albino rat. J. Nutrit., 9, 6II-I9 (I935).

Steinbach, M. M., and S. J. Klein: The effects of gonadotropic hormones in the treatment of experimental tuberculosis. J. exper. Med., $65,205^{-17}$ (1937).

Stevens, H. M.: A study of the vascularity of the pituitary body in the cat. Anat. Rec., 67, 377-94 (1937).

Stimmel, B. F., D. R. McCullagh, and V. Picha: The thyrotropic hormone of the pituitary gland and iodine metabolism. J. Pharmacol. exp. Therap., 57, 49-55 (1936).

Sturm, A. and W. Schöning: Nachweis des thyreotropen Hormons in nichthypophysären Geweben. Zugleich ein Beitrag zur Frage des Tropismus in der Endokrinologie. Endokrinologie, 16, I-8 (1935).

Sulman, F.: Does the gonadotropic hormone induce antibodies or antihormones? J. exp. Med., 65, I-I4 (I 937 ). 


\section{BIBLIOGRAPHY}

Sulzberger, M. B.: Zur Frage des Pigmenthormons und des antidiuretischen Prinzips der Hypophyse. Klin. Wschr., I5, 489 ( 936 ).

Susman, W.: Adenomata of the pituitary, with special reference to pituitary basophilism of Cushing. Brit. J. Surg., 22, 539-44 (I 935).

Susman, $\mathbf{W}$.: The significance of the different types of cells of the anterior pituitary. Endocrinology, 19, 592-98 (1935).

Swezy, O.: Hormones and evolution. Amer. Naturalist, 70, 498-500 ( 1936$)$.

Swingle, W. W., W. M. Parkins, A. R. Taylor, and J. A. Morrella: Effect of oestrus and certain gonadotropic hormones on life-span of adrenalectomized animals. Proc. Soc. exp. Biol., N.Y., 34, 94-96 (1936).

Swingle, IV. W., IV. M. Parkins, A. R. TAylor, H. W. Hays, and J. A. Morrell: Effect of oestrus (pseudopregnancy) and certain pituitary hormones on the life-span of adrenalectomized animals. Amer. J. Physiol., II9, 675-83 (1937).

Sylla, A.: Störungen des Grundumsatzes und der Nahrungsmittelwirkung bei Erkrankungen der Einsonderungsorgane. Wirkung des thyreotropen Hormons. Z. klin. Med., 129, 296-318 (1935).

SzPidBaum, H.: Influence de l'hormone gonadotrope sur la cholestérolémie et évolution de la fièvre typhoïde. C. R. Soc. Biol., Paris, I I9, 66872 (1935).

TAKÁcs, L.: Der Einfluss der Epiphysis auf das Wachstum. Orv. Hetil., pp. 828-29 (1935) (available as an abstract).

TAKÁcs, L.: Der Einfluss der Zirbeldrüse auf das Wachstum. Z. ges. exp. Med., 97, 204-6 (1935).

Takahisa, K.: Einfluss der Parathyreoidektomie, Parathormon- und Calcium-injektion auf die Vorderlappenzellen der Rattenhypophyse. Trans. Jap. path. Soc., 26, 490-94 (1936).

TAKEWAKI, K.: Effects of injections of pregnancy urine and its gonadotropic extract on mouse adrenal. J. Fac. Sci. Univ. Tokyo IV, 4, 83-98 (1935).

TARKHAN, A. A.: Zur Frage der hormonalen Wirkung der Zirbeldrüse. Endokrinologie, I8, 234-42 (1937).

Taubenhaus, M.: Untersuchungen über das Kohlehydrat- und Fettstoffwechsel-Hormon der Hypophyse bei Diabetikern und bei Hypophysentumoren. Wien. Arch. inn. Med., 29, 25 I-58 (I936).

Teilum, G.: L'Hypercholestérinémie primaire après l'administration d'hormone de lutéinisation (Prolan B). C. R. Soc. Biol., Paris, I22, $981-84$ (1936).

Teilum, G.: Sur l'hypercholestérinémie après castration, chez l'homme. C. R. Soc. Biol., Paris, 125, 577-80 (1937).

Tenney, B., Jr. and F. Parker, Jr.: Some observations of the gonadotropic hormones of pregnancy. Endocrinology, 21, 687-88 (1937).

Teresa, S. J.: Die Bedeutung der Ernärungsfaktoren in der Vermehrungsbiologie. I. Vergleichend-physiologische Untersuchung der gona- 


\section{THE PITUITARY BODY}

dotropen Hypophysenaktivität bei B-Avitaminose. Bull. Biol. Méd. exp. URSS, 3, 175-76 (1937).

Tesauro, G.: Contributo allo studio dell'ormone galattogeno. (Ricerca nel sangue e nelle urine di donna in allattamento.) Pediatria Riv., 44, 665-88 (1936).

TheоваLD, G. W.: A centre, or centres, in the hypothalamus controlling menstruation, ovulation, pregnancy, and parturition. Brit. med. J., I, 1038 -4I (1936).

Theовадd, G. W. and E. B. Verney: The inhibition of water diuresis by afferent nerve stimuli after complete denervation of the kidney. J. Physiol., 83, 341-51 (1935).

Thomas, F.: Technik der Hypophysektomie bei der Maus. Acta brev. neerl., 7, 99-102 (1937).

Thompson, K. W.: Inability of sheep to develop antihormone to the gonadotropic hormone from sheep-pituitary glands. Proc. Soc. exp. Biol., N.Y., 35, 634-37 (1937).

Thоmpson, K. W.: Non-specificity of thyrotropic antihormone. Proc. Soc. exp. Biol., N.Y., 35, 637-40 (1937).

Thompson, K. W.: The augmentary factor in animal sera after injections of pituitary extract. Proc. Soc. exp. Biol., N.Y., 35, 640-44 (I 937 ).

Thompson, K. W. and H. Cushing: Inhibition of action of pituitary hormones by animal sera. Proc. Roy. Soc., B, I2I, 50I-I7 (I937).

Thompson, W. O., S. G. Taylor III, P. K. Thompson, S. B. Nadler, and L. F. N. Dickie: The calorigenic action of extracts of the anterior lobe of the pituitary in man. Endocrinology, 20, 55-63 (1936).

Thompson, W. O., P. K. Thompson, S. G. Taylor III, and L. F. N. DickiE: Interrelations of pituitary and thyroid. West. J. Surg., 44, 507-I 2 (1936).

Thomsen, O. and K. Pedersen-Bjergaard: Une méthode pour révéler de petites quantités d'hormone gonadotrope dans l'urine des sujets normaux. C. R. Soc. Biol., Paris, I20, I I $43-47$ (I 935 ).

Thomsen, O. and K. Pedersen-Bjergaard: Nachweis kleiner Mengen gonadotropen Hormons in Harn besonders von Normalen. Z. Geburtsh. Gynäkol., I I2, 202-33 ( 1 936).

Tiliney, F.: The development and constituents of the human hypophysis. Bull. neur. Inst. N.Y., 5, 387-436 (I 936).

Tislowitz, R. and S. Chodkowska: Influence des extraits de thymus sur le poids du lapin et le poids de ses glandes surrénales. C. R. Soc. Biol., Paris, I22, $841-4+$ (1936).

Tongeren, F. C. van: Nederl. Tijdschr. Verloskde, 39, 17-28 (1936) (abstract).

Toxopéus, M. A. B.: Der Einfluss von Schilddrüse und Hypophysis auf die Chlorverteilung. Arch. exp. Path. Pharmak., 178, 412-15 (I935).

Tramontana, F.: Colesterinemia, lipemia ed ormoni sessuali. Arch. Ostetr., 43, 469-80 (1936).

Trettenero, M.: Azione degli ormoni gravidici sull'ovaio umano e sul ciclo mestruale. Riv. ital. Ginec., I9, 380-404 (1936).

$$
[372]
$$




\section{BIBLIOGRAPHY}

Trossarelli, A.: Eclaircissements sur l'histologie de la neurhypophyse. Bull. Histol. appl., I2, 29-44 (1935).

Tuchmann, H.: Lésions du testicule à la suite d'injection des substances œestrogènes. C. R. Soc. Biol., Paris, I22, I 239-4I (1936).

Tuchmann, H.: Modifications de la structure histologique de l'hypophyse du cobaye normal et castré à la suite d'injections hormonales. C. R. Soc. Biol., Paris, 125, 635-38 (1937).

Tuchmann, H. and M. Demay: Modifications prolongées après injection de benzopyrène concernant spécialement l'appareil génital. C. R. Soc. Biol., Paris, I23, I 1 39-4I (1936).

Turner, C. D.: The effects of antuitrin-S on the male genital organs of the lizard (Eumeces laticeps) during seasonal atrophy. Biol. Bull. Wood's Hole, 69, I43-58 (1935).

Turner, H. H.: Diabetes insipidus: treatment with intermedin and pitmelanin. Endocrinology, 19, 275-83 (1935).

Twомвly, G. H.: Studies of the nature of antigonadotropic substances. Endocrinology, 20, 31 I-17 (1936).

Trndale, H. H. and L. Levin: Ovarian weight responses to menopause urine injections in normal, hypophysectomized and hypophysectomized thyroxin-treated immature rats. Amer. J. Physiol., 120, 486-93 (1937).

Uhl, E., J. Engelbreth-Holm, and A. Rothe-Meyer: Über die Wirkung gonadotropen Hormons (Antex L.eo) auf das Kammwachstum bei Hennen. Endokrinologie, 18, 242-50 (1937).

Uhlenhuth, E.: The thyreoactivator hormone: its isolation from the anterior lobe of the bovine pituitary gland and its effects on the thyroid gland. Ann. int. Med., ro, I459-86 (1 937).

Uhlenhuth, E. and S. S. Schwartzbach: Die Physiologie des Thyreoaktivators bei Amphibien. I. Beschleunigung der Metamorphose bei den Larven von Salamandern. Endokrinologie, I5, 329-42 (I935).

Uhlenhuth, E., S. S. Schwartzbach, and G. P. Thompson: Die Physiologie des Thyreoaktivators bei Amphibien. II. Die Strukturveränderungen der Schilddrüse der mit Vorderlappen eingespritzten Salamander. Endokrinologie, I6, 9-19 (1935).

UnNa, K. and L. WALterskirchen: Über die Wirkung des antidiuretischen Hypophysenhinterlappenhormons am gewässerten und nichtgewässerten Hund. Arch. exp. Path. Pharmak., 178, 639-48 (1935).

UnNA, K. and L. WALterskirchen: Über den Zusammenhang zwischen Chlorid- und Wasserausscheidung nach Pituitrin. Arch. exp. Path. Pharmak., I8I, 68 I-88 (1936).

VALLE, J. R.: Test de la prolactine basé sur le frottis de la muqueuse du jabot. C. R. Soc. Biol., Paris, 126, 134-36 (1937).

van DYke, H. B.: The physiology and pharmacology of the pituitary body. Chicago (I936). 


\section{THE PITUITARY BODY}

VAN DYke, H. B. and G. Chen: The production of ovulation by the anterior lobe of the pituitary of the thyroidectomized rabbit. Chinese J. Physiol., 9, 63-68 (1935).

VAN DYKe, H. B. and R. C. L1: The secretion of progesterone by the cat's ovary following the formation of corpora lutea due to the injection of anterior pituitary extract or prolan. Chinese J. Physiol., I3, 213-28 (I9.38).

VAN Wagenen, G.: The effects of oestrin on the urogenital tract of the male monkey. Anat. Rec., 63, 387-403 (I935).

VEIL, C.: Hypophysectomie et changement de couleur chez le poisson chat. C. R. Soc. Biol., Paris, I 24, 1 I I-I3 (I 937 ).

VEIL, C. and R. M. MAY: Hypophysectomie et changement de couleur chez la torpille (Torpedo marmorata). C. R. Soc. Biol., Paris, I24, 91 $7^{-}$ 20 (19.37).

Vercesi, C. and F. Guercio: Erste Mitteilung über die Förderung des Wachstums von Ovarienexplantaten durch gonadotropes Hormon. Arch. exp. Zellforsch., I8, 2 10-18 (1935).

Victor, J. and D. H. Andersen: The effects of oestrus and spaying on pituitary metabolism. Amer. J. Physiol., I 15, I30-37 (1936).

Victor, J. and D. H. Andersen: Stimulation of anterior hypophysis metabolism by theelin or dihydrotheelin. Amer. J. Physiol., I 20, I $54^{-}$ 66 (1937).

Vilter, V.: Réglage sympathico-hypophysaire de la pigmentation mélanique chez les sélaciens. C. R. Soc. Biol., Paris, I26, 794-95 (I937).

Vinals, E.: Renforcement de l'action gonadotrope de l'urine de la femme gravide, par association avec la glande épiphysaire. C. R. Soc. Biol., Paris, I19, 259-61 (1935).

Visscher, J. P. and D. E. Bowman: Chemical determination of pregnancy. Proc. Soc. exp. Biol., N.Y., 3I, 460-6I (1934).

Voitkevič, A. A.: C. R. Acad. Sci. URSS, I4, 403-7 (i 937 ) (abstract).

Voitkevič, A. A.: C. R. Acad. Sci. URSS, I4, 408-10 (i 937) (abstract).

Voitkevič, A. A.: C. R. Acad. Sci. URSS, I5, 395-98 (I937) (abstract).

Voitkevič, A. A.: C. R. Acad. Sci. URSS, r5, 399-404 (i 937 ) (abstract).

Voltkevič, A. A.: Die morphogenetische Aktivität der verschiedenen Teile der Hypophyse. V. Versuche mit Implantation von Substanzen der eosinophilen Zone des Hypophysenvorderlappens an Kaulquappen unter natürlichen Verhältnissen. C. R. Acad. Sci. URSS, r5, 525-28 (I 937 ).

Voss, H.: Künstliche Eiablage beim Axolotl, hervorgerufen durch das thyreotrope Hypophysenvorderlappenhormon. Arch. Entwmech. Org., I32, 805 ( I 935 ).

Voss, H. E.: Die Latenzzeit des weiblichen hormonalen Fffekts beim krystallisierten Follikelhormon. Klin. Wschr., I5, 633-36 (1936).

WADE, N. J.: Studies on the function of the pineal gland. Endocrinology, 2I, 68 I-83 ( I 937 ). 


\section{BIBLIOGRAPHY}

Walker, A. M., C. F. Schmidt, K. A. Elsom, and C. G. Johnston: Renal flood flow of unanesthetized rabbits and dogs in diuresis and antidiuresis. Amer. J. Physiol., I I8, 95-1 IO (1937).

WANG, Y., H. WU, and C. Y. Chou: Effect of male sex hormone on respiration of sex organs in castrated rats. Chinese J. Physiol., I0, 403-5 (1936).

Waterman, L., J. Freud, and N. Vos-de Jongh: Influence of oestradiolbenzoate and prolactin on the milk production of the cow. Acta brev. neerl., 6, 84-85 (

IVAtrin, J. and R. FrançoIs: Hypertrophie expérimentale du cour de cobaye par injections répétées de posthypophyse. C. R. Soc. Biol., Paris, I 26, 357-58 (1937).

WATTS, R. M.: The effect of administration of preparations of growth hormone of the anterior lobe of the pituitary upon gestation and the weight of the newborn (albino-rats). Amer. J. Obstetr., 30, $174^{-85}$ (1935).

Weichselbaum, T. E., P. Heinbecker, and M. Somogyi: Effect of diet on glucose tolerance of normal and hypophysectomized dogs. Proc. Soc. exp. Biol., N.Y., 36, 802-3 (1937).

Weir, R. and C. Bernheim: Action de l'iodure de potassium, de la diiodotyrosine, de la thyroxine, seuls ou combinés à la préhypophyse, sur les transplants thyroidiens. C. R. Soc. Biol., Paris, I2I, 449-5 I (1936).

Weinstein, G. I. and A. W. Makepeace: The influence of pseudopregnancy on follicular sensitivity to pregnancy urine extracts. Amer. J. Physiol., I 19, 508-I i (1937).

WEIs, M.: Les Modifications de la glande pituitaire du cobaye au moment de l'accouchement et après la mise-bas. Rev. franç. Endocrin., I3, I95-20I (1935).

Weisman, A. I., I. S. Kleiner, and E. Allen: Cow's milk as a possible excretory source of the anterior pituitary-like hormone. Endocrinology, I9, 395-97 (I 9.35).

IVeisman, A. I. and C. C. Yerbury: An investigation of the hormone content of saliva. Endocrinology, 20, $\mathrm{IO}_{3-4}$ (1936).

Weller, D., M. D. Overholser, and W. O. Nelson: The effect of estrin on the prostate gland of the albino rat and mouse. Anat. Rec., $65,149-63$ ( 1936 ).

WELLS, L. J.: Reproductive organs of two mammalian hermaphrodites and their response to injections of pregnant mare serum. Anat. Rec., 67, 233-5 I (I937).

WeLLS, L. J. and E. T. Gomez: Hypophysectomy and its effects on male reproductive organs in a wild mammal with annual rut (Citellus). Anat. Rec., 69, 213-27 (1937).

Wells, L. J. and C. R. Moore: Hormonal stimulation of spermatogenesis in the testis of the ground squirrel. Anat. Rec., 66, I8I-200 (1936). 


\section{THE PITUITARY BODY}

Werner, A. A., D. Kelling, D. Ellersieck, and G. A. Johns: Effecl of gonadotropic extract of the pituitary in cryptorchidism. J. Amer. med. Ass., 106, I 54I-43 (1936).

Werner, S. C.: Prolonged injection of a thyrotropic extract without development of refractoriness. Proc. Soc. exp. Biol., N.Y., 34, 390-92 (1936).

Werner, S. C.: Antibody nature of refractoriness to injections of hypophyseal extracts containing thyrotopic hormone. Proc. Soc. exp. Biol., N.Y., 34, 392-94 (1936).

Wesselow, O. L. V. DE and W. J. Griffiths: On the possible role of the anterior pituitary in human diabetes. Lancet, 230, 991-94 (1936).

Westman, A. and D. JАсовsонn: Über Ovarialveränderungen beim Kaninchen nach Hypophysektomie. Acta obstetr. scand. (Stockh.) I6, 483-508 (1936).

Westman, A. and D. Jасовsонn: Über Oestrinwirkungen auf die Corpus luteum-Funktion. II. Mitt. Acta obstetr. scand. (Stockh.) I7, 13-22 (1937).

Westman, A. and D. Јасовьонn: Experimentelle Untersuchungen über die Bedeutung des Hypophysen-Zwischenhirnsystems für die Produktion gonadotroper Hormone des Hypophysenvorderlappens. Acta obstetr. scand. (Stockh.), I7, 235-65 (1937).

Wetzler-Ligeti, C. and B. P. Wiesner: Restropic effects of anterior pituitary extracts. Nature, I40, 892-93 (1937).

Whitaker, W. L.: Effect of light on reproductive cycle of Peromyscus leucopus noveboracensis. Proc. Soc. exp. Biol., N.Y., 34, 329-30 (1936).

White, A., H. R. Catchpole, and C. N. H. Long: A crystalline protein with high lactogenic activity. Science, 86, 82-83 (1937).

White, H. L.: Pituitary gland influences on water balance in the rat. Amer. J .Physiol., I I9, 5-6 (1937).

White, H. L. and T. Findley, Jr.: Time relations in renal excretion of threshold and no-threshold substances. Amer. J. Physiol., I I9, 419-20 (1937).

White, H. L. and T. Findley, JR.: Time relations in renal excretion of threshold and no-threshold substances. Amer. J. Physiol., I I9, 74048 (1937).

White, H. L. and P. Heinbecker: Pituitary regulation of water exchange in the dog and monkey. Amer. J. Physiol., ir8, 276-84 (1937).

WIEGAND, M.: Über den Einfluss der Ovarialfunktion auf die lactogene Wirkung der Hypophyse. Arch. Gynäkol., 165, 149-54 (1937).

Wiegand, M.: Über die lactogene Wirkung der Hypophyse von normalen, schwangeren und lactierenden Tieren. Zbl. Gynäkol., 6r, 1887-90 (1937).

WIEGAND, M.: Über den Einfluss des Follikelhormons auf die lactogene Wirkung des Hypophysenvorderlappens von lactierenden Ratten. Zbl. Gynäkol., 6r, 2391-93 (1937). 


\section{BIBLIOGRAPHY}

Wilcke, J.: Einfluss der Fixierung auf das histologische Bild der Schilddrüse bei Meerschweinchen. Acta brev. neerl., 5, 99 (I935).

Wilkins, W. E., J. A. Calhoun, C. Pilcher, and E. M. Regen: The influence of pituitary growth hormone on the phosphatase activity of bone and kidney. Amer. J. Physiol., I I 2, 477-80 (I 935).

Williams, G. E. and R. Nomland: Gonadotropic substance in the treatment of acne. J. Amer. med. Ass., I09, 564-65 (1937).

Wilson, D.: The effect of anterior pituitary-like hormone on the blood picture in rabbits. Endocrinology, 21, 96-100 (1937).

Winter, C. A. and F. E. Emery: Compensatory adrenal hypertrophy in the rat as influenced by sex, castration, time and thyroidectomy. Anat. Rec., 66, 40I-9 (1936).

Wislocki, G. B.: The meningeal relations of the hypophysis cerebri. II. An embryological study of the meninges and blood vessels of the human hypophysis. Amer. J. Anat., 6r, 95-I30 (1937).

Wislocki, G. B.: The meningeal relations of the hypophysis cerebri. I. The relations in adult mammals. Anat. Rec., 67, 273-94 (1937).

Wislocki, G. B.: The vascular supply of the hypophysis cerebri of the cat. Anat. Rec., 69, 36I-87 (1937).

Wislocki, G. B. and E. M. K. Geiling: The anatomy of the hypophysis of whales. Anat. Rec., 66, 17-42 (1936).

Wistocki, G. B. and L. S. King: 'The permeability of the hypophysis and hypothalamus to vital dyes, with a study of the hypophyseal vascular supply. Amer. J. Anat., 58, 42 I-72 (1936).

Witschi, E. and WV. N. KEck: Differential effect of some gonadotropic substances on development of cyclical sex characters in the English sparrow. Proc. Soc. exp. Biol., N.Y., 32, 598-603 (I 935 ).

Witschi, E. and C. A. Pfeiffer: The hormonal control of oestrus, ovulation and mating in the female rat. Anat. Rec., 64, 85-105 (1935).

Witschi, E., A. J. Stanley, and G. M. Riley: Gonadotropic hormones of the hypophysis of the turkey. Proc. Soc. exp. Biol., N.Y., 36, 647-5I (1937).

Wolf, O. and R. Greep: Histological study of thyroid gland of hypophysectomized rats exposed to cold. Proc. Soc. exp. Biol., N.Y., 36, $856-$ 60 (1937).

Wolfe, J. M.: The normal level of the various cell types in the anterior pituitaries of mature and immature rats and further observations on cyclic histologic variations. Anat. Rec., 6I, 32 I-30 (1935).

Wolfe, J. M.: Morphologic reaction of the anterior pituitaries of mature female rats to prolonged injections of pregnancy urine extracts. Anat. Rec., 63, 3-1 I (1935).

Wolfe, J. M.: Quantitative studies on the reaction of the anterior pituitaries of immature female rats to extracts of pregnancy urine. Endocrinology, I9, 471-79 (I935).

Wolfe, J. M.: Reaction of anterior pituitaries of mature female rats to injections of large amounts of oestrin. Proc. Soc. exp. Biol., N.Y., 32, 1192-95 (1935). 
Wolfe, J. M.: The action of a synthetic oestrogenic agent or the anterior pituitary of the castrated female rat. Amer. J. Physiol., I I5, 665-69 ( 1936$)$.

Wolfe, J. M.: Ovaries of immature female rats receiving pregnancy urine extract and combinations of pregnancy urine extract and oestrin. Proc. Soc. exp. Biol., N.Y., 34, 26-29 (1936).

Wolfe, J. M.: Comparative action of injections of oestrin and a combination of oestrin and anterior pituitary-like substance on the anterior hypophysis. Anat. Rec., 68, 237-48 (1937).

Wolfe, J. M. and C. S. Chadwick: Quantitative studies on the structural changes induced in the anterior hypophysis by injections of oestrin. Endocrinology, 20, 503-10 (1936).

Wolfe, J. M. and C. S. ChaDwick: Reaction of anterior pituitaries of immature female rats to injections of various amounts of oestrin. Proc. Soc. exp. Biol., N.Y., 34, 56-58 (1936).

Wolfe, J. M. and J. B. Hamilton: Comparative action of testosterone compounds, of esterone and of combinations of testosterone compounds and esterone on the anterior hypophysis. Endocrinology, 2x, 603-10 (1937).

Wolfe, J. M. and J. B. Hamilton: Response of anterior pituitary of immature castrated rat to testosterone and related compounds. Proc. Soc. exp. Biol., N.Y., 36, 307-10 (1937).

Wolfe, J. M. and J. B. Hamilton: Action of male sex hormone with and without estrin in the female rat. Proc. Soc. exp. Biol., N.Y., 37, I8993 (1937).

Wolfe, J. M. and D. Phelps: Reactions of ant. pituitaries of male rats to administration of ant. pituitary-like substance and to oestrin. Proc. Soc. exp. Biol., N.Y., 32, 1 305-9 (1935).

Wolff, E.: I.'Hypophyse et la thryoïde jouent-elles un rôle dans le déterminisme expérimental de l'intersexualité chez l'embryon de poulet? C. R. Soc. Biol., Paris, I26, I 2 17-19 (1937).

Wolff, E. and R. Stoll: Le Rôle de l'hypophyse dans le développement embryonnaire du poulet, d'après l'étude des cyclocéphales expérimentaux. C. R. Soc. Biol., Paris, 126, I $215^{-17}$ (1937).

Wolff, Etienne and Emilienne. Wolff: Sur les différences de sensibilité des embryons femelles de deux races de poules à une hormone sexuelle: l'androstérone. C. R. Soc. Biol., Paris, I23, I I9I-93 (I936).

Wolff, Etienne and Emilienne Wolff: L'Action de différentes substances du groupe de l'androstérone sur les organes génitaux de l'embryon de poulet. C. R. Soc. Biol., Paris, r24, 367-69 (1937).

Wolff, R.: Recherches sur la réaction du jabot chez le pigeon sous l'influence d'extraits de lobe antérieur d'hypophyse. C. R. Soc. Biol., Paris, I 24, 673-76 (1937).

Wrman, I. C. and C. Tum Suden: Factors determining and limiting the growth of transplanted suprarenal cortical tissue. Endocrinology, 2I, $523-28$ (1937). 


\section{BIBLIOGRAPHY}

Wyman, L. C. and C. Tum Suden: Homotransplantation of adrenal cortical tissue. Science, 85, 589-90 (1937).

YANAGI, K.: The effect of posterior pituitary preparations upon the colloid osmotic pressure of serum protein, water and mineral metabolism of dogs. J. Pharmacol. exp. Therap., 56, 23-38 (I936).

YANAGITA, T.: Effects of androsterone, methyl-dihydro-testosterone, testosterone, methyl-testosterone, oestrone and oestriol upon accessory reproductive organs and anterior pituitaries in gonadectomized rats. Mitt. med. Ges. Tokyo, 51, 90I-2 (1937).

Yokoyama, E.: On the influence of the hypophysis upon the purine metabolism. Jap. J. med. Sci., Trans. IV. Pharmacol., 8, $98 \dagger$-IoI $\dagger$ (1935).

Young, F. G.: Glycogen and the metabolism of carbohydrate. Lancet, 231, 237-42, 297-302 (1936).

Young, F. G.: The influence of glycotropic pituitary extracts on liver glycogen. J. Physiol., 90, 20P-22P (I937).

Young, F. G.: Attempts to produce antisera to the lactogenic and glycotropic substances of the anterior pituitary gland. J. Physiol., 90, 22 $\mathrm{P}-$ $23^{\mathrm{P}}$ (1937).

Young, F. G.: Permanent experimental diabetes produced by pituitary (anterior lobe) injections. Lancet, 233, 372-74 (1937).

Young, J. Z. and C. W'. BELlerby: The response of the lamprey to injection of anterior lobe pituitary extract. J. exp. Biol., I2, 246-53 (I935).

Youngken, H. W.: The comparative pharmacognosy of the anterior and posterior lobes of the pituitary of cattle. J. Amer. pharmaceut. Ass, 26, $108-$ I $_{4}$ (1937).

ZAHL, P. A.: Cytological changes in frog pituitary considered in reference to sexual periodicity. Proc. Soc. exp. Biol., N.Y., 33, 56-58 (19.35).

Zajíc, F.: Grundumsatz und das thyreotrope Hormon des Hypophysenvorderlappens. Pflügers Arch., 235, 575-8I (I935).

Zavadovsky, B. M. and E. G. Nesmeyanova-Zavadovskaya: Fiziol. Z., 22, 347-55 (1937) (abstract).

Zavadovsky, B. M., E. G. Nesmeyanova-Zavadovskaya, E. A. Romanovskaya, E. P. Rosen, and L. A. Ivanova: 'The effect of prolonged injections of blood-serum of pregnant mares upon the development of endocrine and reproductive functions of the sexual glands of young cocks. Bull. Biol. Méd. exp. URSS, 4, 19-22 (1937).

Zawadowskr, M. M., P. A. Vunder, A. L. Padootcheva, and S. G. Margvelashvili: Trudy Dinam. Razvit., 9, 62-63 (i 935) (abstract).

Zeckwer, I. T.: Thyrotropic effect of pituitaries from cretin rats. Amer. J. Physiol., i I7, 5 I 8-24 (1936).

ZECKWER, I. T.: Morphological changes in the pituitaries of rats resulting from combined thryoidectomy and gonadectomy. Amer. J. Path., r3, 985-92 (1937). 


\section{THE PITUITARY BODY}

ZECKWER, I. T.: The adrenals of rats following combined thyroidectomy and gonadectomy, considered in relation to pituitary histology. Amer. J. Physiol., II9, 426-27 (1937).

ZECKWER, I. T.: The adrenals and gonads of rats following thyroidectomy considered in relation to pituitary histology. Amer. J. Physiol., I2I, $224-30$ ( 1938 ).

Zeckwer, I. T., L. W. Davison, T. B. Keller, and C. S. Livingood: The pituitary in experimental cretinism. I. Structural changes in the pituitaries of thyroidectomized rats. Amer. J. med. Sci., I90, 145-57 (1935).

ZONDEK, B.: The inhibitory effect of follicular hormone on the anterior lobe of the pituitary gland. Lancet, 230, I0-I 2 (1936).

Zondek, B.: Tumour of the pituitary induced with follicular hormone. Lancet, 230, 776-78 (1936).

ZONDEK, B.: Impairment of anterior pituitary functions by follicular hormone. Lancet, 23I, 842-47 (1936).

Zondek, B.: Hemmung der Menstruation durch Follikelhormon. Wien. klin. Wschr., 49, 455-61 (1936).

ZONDEK, B.: The effect of artificial pseudomenstruation and menstruation on the increased elimination of prolan $A$ in the absence of ovarian function. Amer. J. Obstetr., 33, 96-102 (1937).

ZoNDEK, B.: Impairment of anterior pituitary functions by follicular hormone. Fol. clin. Orient., I, I-36 (1937).

ZoNDEK, B.: Gonadotropic hormone in the diagnosis of chorionepithelioma. J. Amer. med. Ass., 108, 607-I I (1937).

Zondek, B.: Tumour growth in hypophyseal dwarfism. Lancet, 232, 689 (I 937$)$.

Zondek, B. and F. Sulman: The antigonadotropic factor. Origin and preparation. Proc. Soc. exp. Biol., N.Y., 36, 708-12 (1937).

Zondex, B. and F. Sulman: The antigonadotropic factor. Species specificity and organ specificity. Proc. Soc. exp. Biol., N.Y., 36, 7 I 2-1 7 (I 937 ).

Zondek, B. and F. Sulman: Some properties of the antigonadotropic factor. Proc. Soc. exp. Biol., N.Y., 37, 193-98 (1937).

Zondek, B. and F. Sulman: Mechanism of prolan-antiprolan-reaction in simultaneous and unsimultaneous application of both active principles. Proc. Soc. exp. Biol., N.Y., 37, I98-202 (1937).

Zondek, B. and F. Sulman: The antigonadotropic factor. Reversibility of the prolan-antiprolan effect. Proc. Soc. exp. Biol., N.Y., 37, 343-48 (I937).

Zunz, E. and J. LA BARRE: Contributions à l'étude des variations physiologiques de la sécrétion interne du pancréas. XV. Action de la substance thyréotrope d'origine antéhypophysaire sur l'insulinosécrétion. Arch. int. Physiol., 42, I-23 (1935).

Zunz, E. and J. LA BARRE: Contributions à l'étude des variations physiologiques de la sécrétion interne du pancréas. IVI. Action de la 


\section{BIBLIOGRAPHY}

substance pancréatrope d'origine antéhypophysaire sur l'insulinosécrétion. Arch. int. Physiol., 42, 95-I IO (1935).

Zunz, E. and J. LA BARRE: Action de la substance thyréotrope d'origine antéhypophysaire sur la teneur du sang en thyroxine. C. R. Soc. Biol., Paris, I18, 1622-24 (1935).

Zunz, E. and J. LA BARrE: Action de l'hormone pancréatrope d'origine an téh ypophysaire sur la glycémie. C. R. Soc. Biol., Paris, I19, I I 74-77 (I935).

Zwarenstein, H.: Experimental induction of ovulation with progesterone. Nature, I39, I I 2-I3 (I 937 ).

Zwarenstein, H.: Gonadotropic activity of amphibian anterior pituitary. Nature, 140, 588 (1937). 

INDEX 



\section{INDEX}

Abortion

caused by combined action of oestrogen and oxytocic principle, 26I

Acetone bodies; see Metabolism, lipoid

Acne vulgaris

treatment of, by prolan, 138

Acromegaly

changes in bones and, 37

changes in joints and, 37

Addison's disease anatomy of pituitary in, 23

Adenoma; see Neoplasms

Adrenal cortical hormone effect of, on adrenals, 204 and anterior pituitary, $20 \mathrm{I}-2$

and metabolism, carbohydrate, 2192 I

and metabolism, water, 202

and thyrotropic hormone, 187

Adrenal cortical stimulating hormone, $198 \mathrm{ff}$.

and adrenal hypertrophy caused by

formaldehyde, 203

oestrone, 203

assay of, 205

chemistry of, $2 \mathrm{O}_{4}$

distribution of, 199, 204

and growth, $4^{\mathrm{I}}$

and toxicity of histamine, 203

in relation to cortical lipoids, 200 , $204-5$

metabolism of, $203^{-4}$

sexual difference in secretion of, 203

in relation to thyrotropic hormone, 202 , n. 5

Adrenal glands

acetone bodies, and anterior pituitary, 231

effect of adrenal cortical hormone on, 204

and anatomy of pituitary, 22-23

deficiency of secretion of, in relation to anterior pituitary, $20 \mathrm{I}-2$

after gonadectomy, 202, n. 5 gonadotropic effect of extracts of, 97$9^{8}$

and gonadotropic hormone, pituitary, 95-98

relation to hypertension, $287-88$

compensatory hypertrophy of, and anterior pituitary, 202-3

after hypophysectomy, 31, 36, 198201

and lactation, 152,160

lipoids of cortex in relation to adrenal cortical stimulating hormone, $200,204-5$

in relation to lipoid metabolism of liver and anterior pituitary, 229$3 \mathrm{I}$

and metabolism, carbohydrate as related to pituitary, 2I 8-2 I

and metabolism, lipoid, 228

degenerative changes in, caused by oestrone, 19

action of prolan on, I 35

hypertrophy of, after injection of thyrotropic hormone, 190

and action of thyroxine, $180, n .7$ transplantation of,

effect of adrenal cortical hormone on, 203-4

and anterior pituitary, 203-4

$\mathrm{X}$-zone of, and pituitary, $20 \mathrm{I}$

Adrenalin; see Epinephrine

Adrenine; see Epinephrine

Adrenotropic hormone; see Adrenal cortical stimulating hormone

Albumin, egg

augmentation of action of gonadotropic hormone by, II 8

Allopregnanediol, 294, 296

Allopregnanolone, 294, 296

Amniotic fluid

prolan in, 127

Anatomy of pituitary

and adrenal glands, 22-23

comparative, 2 , 10-I I 


\section{THE PITUITARY BODY}

Anatomy of pituitary-Continued

after artificial culture, 28

correlation with disease, $11,24^{-26}$

after gonadectomy, $17^{-18}$

androgens and, 18

androstane diol and, 18

androstene dione and, 18

benzpyrene and, 18

dibenzanthracene and, 18

oestrogens and, 18

progesterone and, 18

gonads, internal secretion of, and, I $5 \mathrm{ff}$.

lactation and, I6-17

microscopic

pars glandularis, I $2-15 \mathrm{ff}$.

pars intermedia, 10-I I

pars neuralis, I I $-\mathbf{I} 2$

effect of oestrogens on, I 8-2I

oestrous cycle and, 15-16; see also sexual cycle and

and pancreas, 23-24

and parathyroid glands, $2+$

parturition and, $16-17$

pathological, 23, 24-26

pregnancy-cells, 26

effect of progesterone on, 21

effect of prolan on, 2 I

sexual cycle and

in frog, 15

in pigeon, 15

effect of testosterone or its propionate on, 21

and thymus, 24

and thyroid gland, $21-22,178-79$

after transplantation, $27-28$

vitamins and, $26-27$

\section{Androgens}

effect on anatomy of pituitary after gonadectomy, 18

and development of breasts, 156

castration changes in pituitary corrected by, $91^{-}-92$

and action of chromatosome-dispersing hormone, $252, \mathrm{n} .10$

in relation to gonadotropic hormones, pituitary, 89-94

effect of, on male gonads, 74

effects on lactation of, $16_{3}-64$

and activity of oxytocic principle, 262 effects in parabiotic animals, 79

maintenance of spermatogenesis by, after hypophysectomy, 93-94

Androstane diol

effect on anatomy of pituitary after gonadectomy, i 8

Androstene dione

effect on anatomy of pituitary after gonadectomy, 18

Androsterone, 294, 296; see also Androgens

Aneurin; see Vitamin $B_{1}$

Antagonism; see also Antihormone of gonadotropic hormone, pituitary, by pituitary extract, 106, I I 5-17 of hormone of pregnant-mare serum by pituitary extract, 106, II 5-16 of prolan by pituitary extract, 106, I $15-16$

Anterior lobe; see Pars glandularis

Anterior pituitary; see Pars glandularis

Antihormone; see also Antagonism of diabetogenic hormone, $227-28$ of gonadotropic hormone, pituitary, 109-I 5, II 7

detection of, I I 2, n. 7 I

immune substances and, II1-13

effect on secretion of hormone, $113^{-}$ 15

source specificity of, II I-I 2

species specificity of, I I I-I 2

of gonadotropic hormone, pregnantmare, I II-I 5, I4I

and growth-promoting extracts, $4+-45$ of ketogenic principle, 232

of lactogenic hormone, 168-69

of prolan, I IO-I $5,139-4 \mathrm{I}$

of thyrotropic hormone, 192-94

Ascorbic acid

anatomy of pituitary and, 26

distribution of, in pituitary, 238

and action of thyrotropic hormone, 190

Assay

of adrenal cortical stimulating hormone, 205

of chromatosome-dispersing hormone, 253, n. 12

of gonadotropic hormone, pituitary, $67-70,107-9$ 


\section{INDEX}

\section{Assay-Continued}

of gonadotropic hormone, pregnantmare, 142

of grow th-promoting extract, $44^{-4}+5$

of lactogenic hormone, 169-71

of pars neuralis principles, 259-60

of prolan, 69-70, $141-42$

of thyrotropic hormone, 194-95

Atropine

effect of, on chromatophores, 25 I

Augmentation

of action of gonadotropic hormone, pituitary, II6-19

by blood, i 18

by casein, I 8

by copper salts, 1 i 8

by egg-albumin, is 8

by heme, 118

by hemoglobin, i 8

as result of injections of hormone, II 7

by luteinizing hormone, 117

by merthiolate, 1 I $8-19$

by tannic acid, II 8

by yeast ash or extract, I 18

by zinc sulphate, 1 i 8

of effects of prolan, I 38

of action of vasopressor principle, $270-71$

Auxogenic hormone, 106

Basedow's disease; see Graves's disease

Basophilism, pituitary; see Cushing's syndrome

Benzpyrene

effect on anatomy of pituitary after

Blood gonadectomy, 18

augmentation of action of gonadotropic hormone, by, i 18

cells of, in relation to pituitary, 237 action of pars neuralis extracts on, $267-68$

sugar of, after hypophysectomy, 205$7,209-10$

Blood vessels of pituitary, $2-5$

Bones

changes in, in acromegaly, 37

effects of anterior pituitary extract on, $37-38$

effects of hypophysectomy on, 38 effect of oestradiol benzoate on, to repair of, facilitated by thyrotropic hormone, 190

\section{Breasts}

cystic disease of, and lactogenic hormone, 165,168

development of

and androgens, 156

growth of nipples and pituitary, 154

and oestrogens, $152-55,157-58$

and pars glandularis, $151 \mathrm{ff}$.

in relation to pregnancy, 155

progesterone and, $155^{-5} 58$

effect of prolan on, $\mathrm{I} 34$

Bromine

in pituitary, 238

Broodiness in fowls and lactogenic hormone, 166-67

Cachexia, pituitary; see Simmonds' disease

Calcium, I02; see also Metabolism, mineral, and Gonadotropic hormones, pituitary

Cancer; see Neoplasms

Carbohydrate-metabolism hormone, 227

Carcinoma; see Neoplasms

Casein

augmentation of action of gonadotropic hormone by, i 8

\section{Castration; see Gonadectomy}

Central nervous system; see also Innervation of pituitary, Nervous system

after hypophysectomy, 3 I

Cervix of uterus action of oxy tocic principle on, 260

Cholesterol; see Metabolism, lipoid

Chloralosane effect of, on chromatophores, 251

Chlorine; see Metabolism, mineral

Chloroform effect of, on chromatophores, 25 I

\section{Chorion}

as source of gonadotropic hormones, $124^{-27}$

Chorionepithelioma; see Gonadotropic hormones of neoplasms 


\section{THE PITUITARY BODY}

\section{Chromatophores}

physiology of, and pituitary, $244 \mathrm{ff}$.

Chromatosome-dispersing hormone; see also Chromatosome distribution effect of androgens on action of, 252, n. 10

assay of, 253, n. 12

chemistry of, $255-56$

crustacean eye-stalk hormone and, 245, n. 1

in relation to culture of isolated pituitary, $254^{-55}$

distribution of, $253-55$

inactivation of, by serum of patients with cancer, 255

metabolic effects associated with, I 83 , n. 10

metabolism of, 253-55

and metabolism, carbohydrate, possible interrelationship, $217, \mathrm{n} .19$ metabolism, water, and, 252-53

effect of oestrogens on action of, 252 , n. 10

pharmacology of, 25I-52

and diagnosis of pregnancy, 127, n. 5 effect of progesterone on action of, 252, n. 10

and position of retinal pigment, 250 thyroid and action of, 252, n. 10

Chromatosome distribution; see also

Chromatosome-dispersing hormone effect of hypophysectomy on, $244 \mathrm{ff}$. in amphibia, $249^{-50}$

in fishes, $245^{-}+9$

in reptiles, $249, \mathrm{n} .5$

Colostrum; see Milk

Comparative anatomy of pituitary, 2

Copper salts

augmentation of action of gonadotropic hormone by, 118

Corpus luteum hormone; see Progesterone

Corticosterone, 294, 296; see also Adrenal cortical hormone, Adrenal glands

Corticotropic hormone; see Adrenal cortical stimulating hormone, Adrenal glands

Crinogenic hormone, Io6
Crop-glands

effect of lactogenic hormone on growth of, $167,169-7 \mathrm{I}$

\section{Crustacea}

eye-stalks of, and chromatosomedispersing hormone, $245, \mathrm{n}$. I

Cushing's syndrome

hypertension of, and adrenal glands, $287-88$

metabolism, mineral, associated with, 235 , n. 35

obesity of, 232, n. 32

pathology of pituitary, $24^{-25}$

Dehydroisoandrosterone, 294, 296; see also Androgens

Diabetes insipidus and pars glandularis, $184,278 \mathrm{ft}$.

and pars neuralis, $276 \mathrm{ff}$.

and pars tuberalis, 28 I

Diabetes mellitus; see also Insulin, Metabolism, carbohydrate, Pancreas, etc.

and anatomy of pituitary, 25

and diabetogenic hormone, 222-24, 226-27

Dibenzanthracene

effect on anatomy of pituitary after gonadectomy, 18

Diencephalon

"glands" of, Io

neurons of nuclei of, $9^{-10}$

Dihydroequilenine, 294, 296

Diiodoty rosine

antagonism of thyrotropic hormone by, 192

\section{Eclampsia}

and anatomy of pituitary, 25

titer of prolan in, 127

relation of vasopressor principle to, 276,287

experimental, and vasopressor principle, $263-64$

Embryology of pituitary, I-2

Ephedrine

effect of, on chromatophores, 251, n. 9

Epi-allopregnanol-3-one-20, 294, 296 


\section{INDEX}

Epinephrine, 294, 296

effect of, on chromatophores, 247, n. 3 , 251

and action of diabetogenic hormone, 2I $19-21$

effects of, on metabolism, carbohydrate, after hypophysectomy, 2II-I 4,2 I 6

and action of thyrotropic hormone 186

Epiphysis

and gonadotropic hormone, pituitary, 98

and growth, 42

and growth of neoplasms, $43^{-4} 4$

in relation to action of prolan, 135

Equilenine, 294, 296

Equilin, 295-96

Ergometrine

effect of, on chromatophores, 251, n. 9

Ergotamine

effect of, on chromatophores, 251

Ether

effect of, on chromatophores, 251

hyperglycemia of, after hypophysectomy, 2I 4, n. I7

Exophthalmic goiter; see Graves's disease

Exophthalmos

and anterior pituitary extract, 183 , I $85-86$

sympathetic nervous system and, I 86

Eye

$$
\begin{aligned}
& \text { action of pars neuralis extracts on } \\
& \text { intraocular pressure and iris, } \\
& 264-65
\end{aligned}
$$

Fallopian tube action of oxytocic principle on, 260

Fat; see Metabolism, lipoid

Fat-metabolism hormone; see Metabolism, lipoid

F 883 effect of, on chromatophores, 25 I

Fetal growth and pituitary, 36-37

F 933

effect of, on chromatophores, 25 I

Follicle-stimulating hormones; see Gonadotropic hormones, pituitary
Formaldehyde

action of, on adrenals and anterior pituitary, 203

Galactin, 158

Gastrointestinal tract, action of vasopressor principle on, 265

Glutathione

of tissues after hypophysectomy, 232, n. 33

in muscle in relation to pituitary, 207

and action of thyrotropic hormone, I 89

Glycogen

metabolism of, and anterior pituitary in liver, 209-14, $216,218,219$, n. 22, 224,230

in neoplasms, $210, \mathrm{n} .14$

in striated muscle, 207, 209-1 4,224

Gonadectomy

effect of, on adrenals, 202, n. 5

effect of, on anatomy of pars glandularis, $17-18,22$

correction of changes in pituitary

by androgens, $9 \mathrm{I}-92$

by oestrogens, $9 \mathrm{I}-92$

by progesterone, 88

Gonadotropic hormone, pregnant-mare, $1+4^{-4}+8$

antagonism of effects of,

by antihormone, I I I-15, I 4 I

by pituitary extract, 106, $115^{-16}$

assay of, 142

biology of, or effects of, on gonads of birds, $1+6$

of mammals, $73^{-74},{ }^{1} 44^{-} 4^{8}$

chemistry of, $1+5^{-}+6$

metabolism of, 146

and growth of neoplasms, 43

pregnancy after injection of, 148

principle (s) of, $145^{-}+6$

effect of, after thyroidectomy, I 48

Gonadotropic hormones

and growth-promoting hormone, 3334

and growth of neoplasms, $43^{-44}$

of neoplasms, $142-44$

amount of, in neoplasms, I 44

prostatic tumors and, 143

testicular neoplasms and, $1+3$

uterine motility and, I 32 


\section{THE PITUITARY BODY}

Gonadotropic hormones-Continued pituitary

and adrenal cortical hormone, $20 \mathrm{I}-2$ and adrenal glands, 95-98, 20I-2 replacement of, by androgens in hypophysectomized males, 9394

in relation to androgens, $89-94$

antagonism of

by specific hormone, 106, I I 5-I 7

by antihormone, 109-15, 117

detection of, II2, n. 7I

immune substances and, I I I-I 3

effect on secretion of hormone, II $3^{-1} 5$

source specificity of, I II-I 2

species specificity of, I I I-I 2

assay of, $69-70$, 107-9

augmentation of effects of, II6-I9

by blood, 118

by casein, I 18

by copper salts, i 8

by egg-albumin, i 8

by heme, 118

by hemoglobin, I I 8

as result of injections of hormone, I 77

by luteinizing hormone, $1 \mathrm{I} 7$

by merthiolate, I18-19

by tannic acid, 118

by yeast ash or extract, 118

by zinc sulphate, I I 8

biology of

in amphibia, 49-51

in birds, 52-62

in fishes, 49

in mammals

female, $62-73$

male, $67-68,73-76$

in reptiles, $51^{-5} 52$

and calcium, 102

chemistry of, $105^{-7}$

in cultures of isolated pituitary, 103

epiphysis and, 98

extraction of, $105^{-7}$

follicle-stimulating hormone, $7 \mathrm{I}-$ $75,78-79,84-87,99,105-9$, I 30

response to, after hypophysectomy, 95

specific stimulation of interstitial cells, 106, i 6 lactogenic hormone and, 98-99

light as a means of controlling secretion of, 58-62

in birds, $58-62$

in fishes, 58

in mammals, 6o-62

in reptile, $5^{8}$

luteinizing hormone, $71-75,78-79$, $84-87,98,105-7$, i $16-18$

male gonads, atrophy of, after hypophysectomy, 73

metabolism of, $\mathrm{IO}_{3}-5$

and growth of neoplasms, $102-3$

nervous control of secretion of, 58-62, 99-101

number of, $7 \mathrm{I}-72$

in relation to oestrogens, $80-88$

and oestrous cycle, 68-69

ovarian deficiency and, 67

ovulation and, 72

parabiosis in relation to, $78-80$, $87^{-88}, 90, \mathrm{II}_{4}^{-15}$

pregnancy and, $65,69^{-70}, 104$

in relation to progesterone, $88-89$

sexual differences in secretion of, $72,75-78$

and spleen, 105

and thyroid, 60, 94-95

and thyrotropic hormone, I 86-87

transplantation of pituitary and, $65-66$

and vitamins, IOI-2

Gonads

anatomy of pituitary and, I $5 \mathrm{ff}$.

and growth, $39^{-} 40$

atrophy of, after hypophysectomy, 36 in relation to thyroid, $185^{-87}$

Graves's disease; see also 'Thyroid gland,

Thyrotropic hormone

and anatomy of pituitary, 25-26

experimental, $183-86$

Growth; see also Growth-promoting ex-

tract, Growth-promoting hormone effect of oestradiol benzoate on, 40 effect of oestrone on, 40

Growth-promoting extract; see also

Growth-promoting hormone

and antihormone production, $44^{-45}$

assay of, $44^{-45}$

effect of, on biochemistry of blood and tissues, 39 


\section{INDEX}

Growth-promoting extract-Continued effect of, and carbohydrate metabolism, 45

effects of, 36-39

and growth of chick embryo, 36

and fetal growth, 36-37

and growth of Lupinus albus seedlings, 36

and duration of pregnancy, 36

preparation of, $45^{-4} 6$

properties of, 46

Growth-promoting hormone, $32 \mathrm{ff}$; see also Bones, Growth, Growth-promoting extract, Hypophysectomy, Joints

and adrenals, 41

and epiphysis, 42

gonadotropic hormones as factor in effects of, 33-34

and gonads, $39^{-40}$

identity of, $32-35$

lactogenic hormone as factor in effects of, $32-35,45$

neoplastic growth and anterior pituitary, $43^{-4} 4$

and sodium deficiency, 42

and thymus, $4 \mathrm{I}$

and thyroid, $40-41$

thyrotropic hormone as factor in effects of, $32-35,45$

transplants of pituitary in sella and, 37

and vitamins, 42

and zinc deficiency, $42-43$

\section{Heart}

effect of oxytocic principle on, 263

effect of vasopressor principle on, 263

behavior of, after administration of thyrotropic hormone or thyroxine, $187-89$

Heme

augmentation of action of gonadotropic hormone by, 118

\section{Hemoglobin}

augmentation of action of gonadotropic hormone by, 118

Hippuline, 295

Histamine

toxicity of, and pituitary, 203

"Houssay dogs," 217
Hydatidiform mole; see Gonadotropic hormones of neoplasms

Hypertension

adrenals and, $287-88$

anatomy of pituitary and, 11, 25

experimental (renal ischemia) and pituitary, 188, 288-90

and pars neuralis, $287-90$

thyroid gland or thyrotropic hormone and, $188,288-89$

Hyperthyroidism

thyrotropic hormone in blood or urine in, 191

Hypophysectomy

effect of, on adrenals, $31,36,198-201$

blood sugar after, 205-7, 209-10

bones after, 38

chemical changes in tissues after, $38-$ 39

effect of, on chromatosome distribution, $244 \mathrm{ff}$.

in amphibia, $249-50$

in fishes, $245^{-49}$

in reptiles, $249, \mathrm{n} .5$

cutaneous changes after, $35,176,178$

effect of, in fowl, 55

gastrointestinal tract after, 237

general effects of, 31

glutathione in tissues after, 232, n. 33

response to gonadotropic hormone, pituitary, after, 95

atrophy of gonads after, 36, 63-65, 73

growth after, $35-36$

lactic acid

of blood, after, 205, n. 9

and action of epinephrine, $211-14$

of striated muscle, after, 205, n. 9

metabolism, carbohydrate, after, 205I 6

absorption of carbohydrate, 207

action of anterior pituitary extract, $223-24$

blood sugar, 205-7, 209-10

effects of epinephrine, $211-14,216$

ether hyperglycemia, 214, n. 17

and action of insulin, 208-9, 21 4-16

and metabolism, fat, 208-10

and metabolism, glycogen

liver, 209-14, 216

muscle, 207, 209-14 


\section{'THE PITUITARY BODY}

Hypophysectomy-Continued

metabolism, ca:bohydrate, after-

Continued

morphine hyperglycemia, 21 4, n. I7

ketogenesis and, 209-10

and metabolism, lactic acid, 207,

n. $9,2 \mathrm{II}-\mathrm{I} 4$

oxidation of carbohydrate, 208

and metabolism, phosphorus, 207,

n. $9,211-14$

and metabolism, protein, 210-II

sugar tolerance, $207 \mathrm{ff}$.

and action of thyroxine, 208

metabolism, creatine-creatinine, after,

233-34

metabolism, lipoid, after, 228

metabolism, mineral, after, 234-36

metabolism, protein, after, 38-39, 233

metabolism, water, after, $276 \mathrm{ff}$.

adrenal cortical hormone and, 202

effect of, on metamorphosis, $174-75$

effect of, on molting

in amphibia, 176,178

in snakes, 178

growth of neoplasms after, $43^{-}-4$

effect of, on central nervous system, 3 I

effect of oestrogen after, 8I

morphology of ovary after, $63^{-65}$

ovogenesis after, 62

anatomy of pancreas after, $216, n$. 18

and pancreatectomy, metabolism, carbohydrate, after, 216-18

carbohydrate oxidation, 217-18

glycogen deposition, 218

ketogenesis and, 218

metabolism, tissue, 217

in parabiotic animals, $79^{-80}$

pregnancy and, 65

progesterone and pharmacology of uterus after, 64

progesterone in pregnant animals after, 65

effect of, on skin, $35,176,178$

effect of, on spleen, 35, 236-37

technic of, $3 \mathrm{I}, 73$

thyroid after, $36,176-80,183, n$. 10

uterine bleeding in monkey after, 64 ,

290-9!

Hypophysial deficiency

caused by oestrone, I 19-2I

Hypophysio-portal system of blood vessels, 2-4
Hypothalamus; see also Diencephalon and pars neuralis secretion, $279 \mathrm{ff}$. general relationship to pituitary, 3 I

Hypothyroidism; see also Thyroidectomy, etc.

thyrotropic hormone in blood or urine in, 191

treatment of, by thyrotropic hormone, I $84-85$

Innervation of pituitary, 6-10; see also Nervous system, control by, of pituitary secretion

Insulin; see also Diabetes mellitus, Metabolism, carbohydrate, Pancreas, etc.

acetone bodies, and anterior pituitary, 230

effect of, on diabetes mellitus caused by anterior pituitary extract, $222-23$

action of, in relation to pars glandularis, 208-9, 2I 4 - -16

pars neuralis extracts and action of, $269-70$

Intermediate lobe; see Pars intermedia

Intermedin; see Chromatosome-dispersing hormone

Interstitial cells of gonads

effect of prolan on, 129, I 31

specific stimulation of, 106, 116

Iodides

prevention of adrenal cortical stimulation by, 202, n. 5

prevention of splenomegaly by, 202, 11. 5

antagonism of thyrotropic hormone by, 190,192

Iodine

effect of, on molting in amphibia, 176,178

Joints

changes in, acromegaly, 37

effects of anterior pituitary extract on, $37-38$

Ketogenesis; see also Metabolism, carbohydrate, Metabolism, lipoid after hypophysectomy and pancreatectomy, 21 8 


\section{INDEX}

Ketogenic hormone; see Metabolism, lipoid

Ketone bodies; see Metabolism, lipoid

Kidneys

action of pars neuralis extracts on, $265-69,276 \mathrm{ff}$.

Lactation: see also Breasts, development of, Lactogenic hormone and adrenals, 152,160

and anatomy of pars glandularis, I6-I7

effects of androgens on, $163^{-6} 4$

effect of lactogenic hormone on, 159$60,164-65$

and metabolism, carbohydrate, 152, 160

nervous control of, I $58-59$

effect of oestrogens on, $160-63$

effects of progesterone on, 163

effect of prolan on, ${ }_{3} 33-34,161, n .14$ and thyroid, 160, n. $12,165-66$

effect of thyroxine on, 166

Lactic acid

of blood

and action of epinephrine, $2 \mathrm{II}^{-} \mathrm{I}_{4}$ effect of hypophysectomy on, 207, n. 9

of striated muscle

effect of hypophysectomy on, 207, n. 9

effect of pituitary on, 207, n. 9

Lactogenic hormone, I $5 \mathrm{I} \mathrm{ff}$; see also

Breasts, development of, Lactation antihormone of, $168-69$

assay of, $169-71$

and broodiness in fowls, I66-67

chemistry of, $171-72$

effect of, on growth of crop-gland, I67, $169-71$

cystic disease of breasts and, $165, \mathbf{I} 68$ distribution of, 164

and gonadotropic hormone, pituitary, 98-99

and growth-promoting hormone, 3235,45

effect on lactation, I 59-60

and maternal behavior, 167

metabolism of, I 67-69

and metabolism, carbohydrate, 165 , n. 17 secretion of milk induced by, $164_{4}-65$

effect on growth of neoplasms, 167

termination of pregnancy by, I67

effect of suckling on secretion of, $158-59$

in relation to thyroid gland, $165-66$

amount of, in pituitary in vitamin deficiencies, $\mathrm{s} 68$

Lipids; see Metabolism, lipoid

Lipoids of adrenal cortex

behavior of, $200,202,204^{-5}$

Liver; see also Glycogen, etc.

acetone bodies, and anterior pituitary, 230

fat metabỏlism, and anterior pituitary, 228-31

adrenals and, 229-31

pancreas and, 229

thyroid and, 228-29

Lobeline

effect of, on chromatophores, 25 I, n. 9

Lungs

action of pars neuralis extracts on bronchi and vessels of, 264

Luteinizing hormone; see Gonadotropic hormones, pituitary

Magnesium; see also Metabolism, mineral

effect of prolan on metabolism of, 137

Mammogenic hormone, I 55

Manmmotropic hormone, 158

Maternal behavior and lactogenic hormone, 167

Medulla of adrenal glands after hypophysectomy, 200, n. 2, 201 effect of pituitary extract on, 200-20I

Medulla oblongata action of vasopressor principle on, 264

Melanophore hormone; see Chromatosome-dispersing hormone

Meningeal relations of pituitary, 5

Menstrual migraine, see Migraine, menstrual

Menstruation; see also Uterine bleeding and administration of oestrogens, 83 , 290-91

pars neuralis and, $290-91$ 


\section{THE PITUITARY BODY}

Merthiolate

augmentation of action of gonadotropic hormone by, 118-19

Metabolism

of adrenal cortical stimulating hormone, 203-4

of chromatosome-dispersing hormone, $253-55$

of gonadotropic hormones, pituitary, $103^{-5}$

of lactogenic hormone, $167-69$

of pars neuralis principles, $270-7 \mathrm{I}$, $274-76,286-87$

of thyrotropic hormone, $190-92$

Metabolism, basal or gaseous

and chromatosome-dispersing hormone, $183, \mathrm{n} .10$

effect of prolan on, 135

and thyrotropic hormone, $183-85$

Metabolism, carbohydrate

adrenals and pituitary and, 2I 8-2 I

effect of anterior pituitary extract on, $222-24$

effects of extracts of cerebrospinal

fluid, serum, and urine on, 226-27

diabetogenic hormone and, 222-24

glycogen and anterior pituitary

liver, 209-14, 216, 218, 219, n. 22, 224,230

neoplasmis, $210, \mathrm{n} .14$

striated muscle, $207,209^{-1} 4,224$

effect of growth-promoting extract and, 45

after hypophysectomy, 205-I 6

absorption of carbohydrate, 207

action of anterior pituitary extract,

$$
223^{-24}
$$

blood sugar, 205-7, 209-10

effects of epinephrine, $211^{-1}+, 216$

ether hyperglycemia, 214 , n. 17

and action of insulin, 208-9, 2I $4^{-16}$

and metabolism, fat, 208-10

and metabolism, glycogen

liver, $209^{-14}, 216$

muscle, 207, 209-14

morphine hyperglycemia, 214 , n. I 7

ketogenesis and, 209-10

and netabolism, lactic acid, 207,

$$
\text { n. } 9,2 \mathrm{I} \mathrm{I}-\mathrm{I} 4
$$

oxidation of carbohydrate, 208 and metabolism, phosphorus, 207, n. $9,2 \mathrm{I} 1-14$

and metabolism, protein, 210-11

sugar tolerance, $207 \mathrm{ff}$.

and action of thyroxine, 208

after hypophysectomy and pancreatectomy, 216-18

carbohydrate oxidation, $217-18$

glycogen deposition, 218

ketogenesis and, 218

metabolism, tissue, 217

hypothalamus and, 218, n. 21

and lactation, 152,160

lactogenic hormone and, 165, n. 17

effect of oestrogens on, 22 I-22

pancreas after glucose infusion, 216 , n. 18

and stimulation of islet tissue of pancreas by pars glandularis, $224^{-26}$ pars glandularis after glucose infusion, $216, n .18$

and pars neuralis extracts, $269-70$

insulin and, 269-70

and pituitary, $205 \mathrm{ff}$.

effect of prolan on, $135-36$

and thyrotropic hormone, I 88-89

Metabolism, creatine-creatinine gonads and, 233, n. 34,234 and pars neuralis extracts, 270 and pituitary, 233-34

after hypophysectomy, 233-34

effect of prolan on, 136

and thyrotropic hormone, 189

Metabolism, fat; see Metabolism, lipoid

Metabolism, lipoid

acetone bodies, and anterior pituitary, $230-32$

adrenal glands and, 23I

effects of anterior pituitary extract, $230 \mathrm{ff}$.

antihormone of, 232

similar effects of extracts of blood and urine, $231-32$

insulin and, 230

relation of liver, 230

thyroid and, $23 \mathrm{I}$

in adrenal cortex, 200, 202, 204-5

cholesterol

and anterior pituitary, 228, 232

and prolan, 232 


\section{INDEX}

Metabolism, lipoid-Continued and chromatosome-dispersing hormone, $183, \mathrm{n} .10$ after hypophysectomy, 228

of liver and anterior pituitary, 228-3I adrenals and, 229-31

pancreas and, 229

thyroid and, 228-29

and metabolism, carbohydrate after hypophysectomy, 208 after hypophysectomy and pancreatectomy, 218

in relation to metabolism, carbohydrate and pituitary, 209-10, 218

and pars neuralis extracts, 270

phosphatide, and anterior pituitary, 228

and pituitary, 228-32

effect of prolan on, $136-37$

and thyrotropic hormone, 189

Metabolism, mineral

and gonadotropic hormones, pituitary, 102

effect of oestrogen on, 162

and pars neuralis extracts, or hormone, $265-69,276$ ff., 285

and pituitary, $234-36$

effect of prolan on, 137

Metabolism, phosphorus

and carbohydrate in relation to pituitary, 207, n. 9, 21 j-1 4

Metabolism, protein

after hypophysectomy, 38-39, 233

metabolism, carbohydrate, and pituitary, 210-11

and pars neuralis extracts, 270

and pituitary, 232-33

and thyrotropic hormone, 189

Metabolism, tissue

after hypophysectomy and pancreatectomy, 217

of pituitary, effect of oestrogen on, 88

of pars glandularis in relation to oestrous cycle, 69

of thyroid, effect of thyrotropic hormone on, 188

Metabolism, water

and adrenal cortical hormone, 202

and chromatosome-dispersing hormone, 252-53 and pars neuralis extracts, or hormone, $265-69,276-85$

effect of prolan on, I 37

Metamorphosis

and thyrotropic hormone, $174^{-76}$, $195-96$

Migraine, menstrual

treatment of by prolan, 138

Milk

composition of, after lactogenic hormone, $164-65$

composition of, after oestrogen, 162

lactogenic hormone in, 168

prolan in, 125

Molting

effect of hypophysectomy on

in amphibia, 176,178

in snakes, 178

effect of iodine on, in amphibia, 176 , I 78

Morphine, hyperglycemia of, after hypophysectomy, 214, n. 17

Myxedema; see Hypothyroidism

Neoplasms; see also Gonadotropic hormones of neoplasms

inactivation of chromatosome-dispersing hormone by serum of patients with, 255

glycogen of, after hypophysectomy, $210, \mathrm{n}_{14}$

growth of, in relation to anterior pituitary, 43-44

and epiphysis, $43^{-4} 4$

and gonadotropic hormones, pituitary, 102-3

and gonadotropic hormone, pregnant-mare, 43

and gonads, 43-44

and hypophysectomy, 43-44

and lactogenic hormone, 167

and oestrogen, 44

and prolan, 43, 103, 137

of pituitary, $24^{-25}$

Nerves of pituitary; see Innervation of pituitary

Nervous system

and control of metabolism, carbohydrate, 218 , n. 21

regulation of pars neuralis by, $277^{-82}$, 286 


\section{THE PITUI'TARY BODY}

Nervous system, control by, of pituitary secretion; see also Innervation of pituitary

of gonadotropic hormone, 58-62, 99IOI

Nicotine

effect of, on chromatophores, 251

Nipples

growth of, and pituitary, 154

Nitrite, amyl

effect of, on chromatophores, 251

Oestradiol; see also Oestrogens

alpha, 295, 297

beta, 295, 297

Oestradiol benzoate; see also Oestrogens effect on bones, 40

effect on growth, 40

and growth of neoplasms, 44

Oestriol, 295, 297; see also Oestrogens

Oestrogens

action of, on adrenals and anterior pituitary, 203

effect on anatomy of pituitary, I 8-2 I after gonadectomy, 18

and development of breasts, I 52-55, I $57-58$

castration changes in pituitary corrected by, $9 I^{-}-92$

and action of chromatosome-dispersing hormone, $252, \mathrm{n}$. IO

in relation to gonadotropic hormones, pituitary, $80-88$

effect of, after hypophysectomy, 8I

effect on lactation, 160-63

and metabolism, carbohydrate, 221-22

effect on composition of milk, 162

effect on minerals of serum, I62

action on uterus and response to oxy tocic principle, 261-62

effect of, on metabolism of isolated pituitary, 88

effects in pregnant animals, 87

alteration of effect of prolan by, I 33

effect of, on thyroid, 187

in relation to thyrotropic hormone, I $85-87$

relation to vasopressor principle and experimental eclampsia, $26_{4}$

effects of, on $\mathrm{X}$-zone of adrenals, 201
Oestrone, 295, 297: see also Oestrogens degenerative changes in adrenals caused by, 19

effect on growth, 40

action on male after hypophysectomy, 93-94

hypophysial deficiency caused by, I 9-2I

Oestrous cycle

and anatomy of pars glandularis, $15^{-}$ I6, 26

and secretion of gonadotropic hormone, 68-69

and metabolism of isolated pars glandularis, 69

Ovaries

deficiency of secretion of, and gonadotropic hormone, 67

morphology of, after hypophysectomy, $63-65$

\section{Ovogenesis}

after hypophysectomy, 62

effect of prolan on, 130

Oxy tocic principle; see also Pars neuralis extracts, Pars neuralis, physiological significance of

assay of, $259-60$

chemistry of, 258-59

effect of, on heart, 263

metabolism of, $270,274^{-76}$

pharmacology of, 260-62

and abortion, 26I

androgen and, 262

response of cervix uteri, 260

response of fallopian tube, 260

oestrogens and, 261-62

parturition and, 26I

progesterone and, 261-62

Pancreas; see also Diabetes mellitus, Insulin, Metabolism, carbohydrate, etc.

in relation to fat of liver and anterior pituitary, 229

effect of hypophysectomy on anatomy of, 216, n. I 8,226

stimulation of islet tissue of, by anterior pituitary, $224^{-26}$

and anatomy of pituitary, $23-24$ 


\section{INDEX}

Pancreatectomy

and hypophysectomy, metabolism, carbohydrate, after, 216-18

carbohydrate oxidation, $217^{-18}$

glycogen deposition, 218

ketogenesis and, 218

metabolism, tissue, 217

Pancreatropic hormone, $22 \psi^{-26}$

Parabiosis, experimental

in relation to gonadotropic hormones, pituitary, $78-80,87-88,90$, $11_{4-}^{-}$ 15

Parathyroid glands

and anatomy of pituitary, 24

and pars glandularis, $235-36$

Parathyrotropic hormone, 236

Pars anterior; see Pars glandularis

Pars buccalis; see appropriate division as Pars glandularis, Pars intermedia, or Pars tuberalis

Pars glandularis; see also all topic headings as Anatomy, etc.

cytogenesis in, $13^{-15}$

and diabetes insipidus, $278 \mathrm{ff}$.

Pars intermedia

anatomy of, IO-1I

and chromatosome dispersion, $244 \mathrm{ff}$.

embryology of, 249

Pars nervosa; see Pars neuralis

Pars neuralis

anatomy of, 11-12

basophils of, $I 1$

innervation of, 6-9

Pars neuralis extracts; see also Oxy tocic principle, Pars neuralis, physiological significance of, Vasopressor principle

assay of, $259^{-60}$

chemistry of, 258-59

metabolism of, $270-71,274^{-76}, 286-$ 87

and metabolism, carbohydrate, 26970

and metabolism, cholesterol, 270

and metabolism, creatine-creatinine, 270

and metabolism, mineral, 265-69

and metabolism, protein, 270

and metabolism, water, $265-69,276-$ 85 effect of, on metamorphosis and growth of tadpoles, 1

Pars neuralis, physiological significance of, $274 \mathrm{ff}$; see also Oxytocic principle, Pars neuralis extracts, Vasopressor principle, etc.

in cardiovascular regulation, 285-90

and central nervous system, $277-82$, 286

diabetes insipidus and, $276 \mathrm{ff}$.

in relation to hypertension, $287-90$

clinical, $287-88$

experimental (renal ischemia), 28890

in menstruation, 290-91

metabolism of active principles and, $270-71,274-76,286-87$

in relation to metabolism of water, $276-85$

in relation to oxytocic principle, 290

Pars tuberalis

anatomy of, 12

and regulation of chromatosome dispersion, 249-50

and diabetes insipidus, 281

Pathology of pituitary, 23-26

Parturition

in relation to oxytocic principle, 261 , 290

and anatomy of pars glandularis, 16I 7

Phosphate, inorganic; see also Phosphorus, etc.

effect of prolan on metabolism of, $\mathrm{J} 37$

Phosphatide; see Metabolism, lipoid

Phosphocreatine

in muscle in relation to pituitary, 207

Phospholipin; see Metabolism, lipoid

Phosphorus; see Metabolism, mineral,

Metabolism, phosphorus

Pilocarpine

and action of thyrotropic hormone, 186

Pineal body; see Epiphysis

Pituitary basophilism; see Cushing's syndrome

Placenta; see Chorion, Prolan, metabolism of

Polydipsia; see Diabetes insipidus 


\section{THE PITUITARY BODY}

Polyuria; see Diabetes insipidus

Posterior lobe; see Pars neuralis or Pars intermedia

Potassium

effect of prolan on metabolism of, I 37

Potentiation; see Augmentation

\section{Pregnancy}

effect of androgens on duration of, 90-9I

in relation to development of breasts, 155

diagnosis of, 127, n. 5

gonadotropic hormones of, $124 \mathrm{fi}$.

and gonadotropic hormones, pituitary, 65, 69-70, 104

after injection of gonadotropic hormone of pregnant mare, $\mathrm{I}+8$

duration of, and growth-promoting extract, 36

hypophysectomy and, 65

termination of, by lactogenic hormone, 167

effects of oestrogens in, 87

progesterone in maintenance of, after hypophysectomy, 65

effect of prolan during, $69-70,132$

and action of vasopressor principle, 286-87

Pregnancy-cells of pituitary, 26

Pregnanediol, 295, 297

Progesterone, 295, 297

effect on anatomy of pituitary, 21

after gonadectomy, 18

and development of breasts, I 55-58

castration changes in pituitary not corrected by, 88

and action of chromatosome-dispersing hormone, $252, \mathrm{n}$. IO

in relation to gonadotropic hormones, pituitary, $88-89$

effects on lactation of, $16_{3}$

and maintenance of pregnancy after hypophysectomy, 65

inhibition of action of prolan by, I 33 effect on pharmacology of uterus after hypophysectomy, 64

action on uterus and response to oxy tocic principle, $26 \mathrm{~s}-62$

Prolactin, I 58
Prolan

"A" and "B," I 29-30

acne vulgaris, treatment of, by, 138

and adrenals, $134^{-35}$

in amniotic fluid, 127

effect on anatomy of pituitary, 21

antagonism of effects of

by antihormone, I10-1 5, I $39^{-41}$

by pituitary extract, I06, I15-16, I 39

assay of, $69^{-70}, \mathrm{I}^{\mathrm{I}-42}$

augmentation of effects of, $\mathrm{J} 38$

biology of, or effects on gonads in

amphibia, 49-50, 128

birds, $52,58, \mathrm{I} 28$

fishes, 128

mammals

female, 67, 129-34

male, $73-74,128-29$

reptiles, $5 \mathrm{I}-52, \mathrm{I} 28$

in blood; see metabolism of

effect of, on blood, $137-38$

effect on breasts, I 34

chemistry of, $\mathrm{I}_{4} 2$

and cholesterol metabolism, 232

chorion and, $124-27$

clinical use of, 132-33

lack of effect in dietary deficiency, I02, n. 60

titer in eclampsia, 127

and epiphysis, 135

effect on ovary after hypophysec-

$$
\text { tomy, } 130-32
$$

effect on interstitial tissue

of ovary, 131

of testis, 129

effect on lactation, $133-34,161, n .14$ luteinizing effects of, $\mathrm{r} 30 \mathrm{ff}$.

menstrual migraine, treatment of, by, 138

metabolism of, $125-28$

effects of, on metabolism

of carbohydrate, $135-36$

of cholesterol, $136-37$

of creatine and creatinine, 136

gaseous, 135

of magnesium, 137

of phosphorus, I 37

of potassium, 137

of sodium, 137

of water, 137 


\section{INDEX}

\section{Prolan-Continued}

in milk, 125

effect of, on growth of neoplasms, 43 , 103,137

effect of, modified by oestrogen, I 33

and culture of isolated ovary, 132

ovulation caused by, $69-70$, I 31

and ovogenesis, 130

in placenta; see metabolism of

and diagnosis of pregnancy, $127, \mathrm{n} .5$

effect of, in pregnancy, $69-70,132$

action inhibited by progesterone, 133

in saliva, 125

action of, and spleen, 105

action of, on thymus, 135

thyroid and, $13+-35$

tuberculosis, experimental, treatment of, by, ${ }_{1} 38$

effect of, on movements of ureter, ${ }_{1} 38$

in urine; see metabolism of

uterine motility and, $70-71,132$

lack of effect in vitamin A deficiency, 129

and deficiency of vitamin E, I37

\section{Prostate}

tumors of, and gonadotropic hormones, I 43

\section{Radon}

effects of, on pituitary, 31, 62

Reticulo-endothelial system

in relation to pars glandularis, 237

and production of prolan antihormone, 139 , n. 16

Retina, pigment of

effect of chromatosome-dispersing hormone on position of, 250

Saliva

prolan in, 125

Sarcoma; see Neoplasms

Simmonds' disease and adrenal cortical stimulating hormone, 204

in identical twins, 36

Skin

changes in, after hypophysectomy, $35,176,178$

effect of pars neuralis extract on chloride of , 267, n. I3
Sodium; see also Metabolism, mineral deficiency of, and gonadotropic hormones, pituitary, I02

deficiency of, and growth, $4^{2}$

effect of prolan on metabolism of, 137

Spaying; see Gonadectomy

Spleen

and action of gonadotropic hormone, pituitary, 105

of prolan, 105

effect of hypophysectomy on, 35, 23637

jodides and prevention of enlargement of, by anterior pituitary extract, 202, n. 5

in relation to pars glandularis, 35 , $236-37$

Strychnine

effect of, on chromatophores, $25 \mathrm{I}$

Suckling

effect of, on secretion of lactogenic hormone, I 58-59

Sugar of blood; see Blood sugar

Sulfonal, in pituitary and tissues, 238

Sulphur; see Metabolism, mineral

Supraoptico-hypophysial tract, 6-8; see also Pars neuralis, physiological significance of

Synergism; see Augmentation

Tannic acid augmentation of action of gonadotropic hormone, by, 118

Teratoma; see Gonadotropic hormones of neoplasms

Testis, neoplasms of; see Gonadotropic hormones of neoplasms

Testosterone, 295, 297; see also Androgens

Testosterone or testosterone propionate, effect on anatomy of pituitary of, 21

Thiamin; see Vitamin $B_{I}$

Thymus

and anatomy of pituitary, 24

and growth, +1

action of prolan on, I 35 


\section{THE PITUITARY BODY}

Thyroid gland; see also Graves's disease, Thyroidectomy, Thyrotropic hormone, Thyroxine

acetone bodies, and anterior pituitary, 23I

and adrenal-pituitary interrelationship, 202

and action of chromatosome-dispersing hormone, $252, n$. 10

cytological changes in response to thyrotropic hormone, 182

in relation to diabetes insipidus, $278 \mathrm{ff}$.

in relation to fat of liver and anterior pituitary, 228-29

and gonadotropic hormone, pregnantmare, 148

and gonadotropic hormones, pituitary, 94-95

in relation to gonads, 60 , $186-87$

and growth, $4 \mathrm{O}^{-} \mathrm{I}$

and experimental hypertension, I 88 , 288-89

after hypophysectomy, 36, I $76-80$, I 83, n. 10

and lactation, I60, n. I2, I65-66

effect of oestrogens on, 187

anatomy of pituitary and, $21-22$

and action of prolan, $134-35$

and thyrotropic antihormone, 182, 193

pharmacology of, and vasopressor principle, 263

Thyroidectomy; see also Thyroid gland,

Thyrotropic hormone

anatomy of pituitary after, $178-79$

Thyrotropic hormone, 174-97; see also

Graves's disease, Thyroidectomy,

Thyroid gland, Thyroxine

action of, $174 \mathrm{ff}$.

and adrenal cortical extract, 187

hypertrophy of adrenals after injection of, $190,202, n .5$

antagonism of, by

antihormone, $192-94$

diiodotyrosine, 192

iodides, I90, 192

oestrogens, 185, 18

thyroxine, 185,192

vitamins, 190

assay of, 194-95 biology of, in

amphibia, $17 t^{-78}$

birds, 178

fishes, 174

mammals, I $78-8 \mathrm{I}$

reptiles, 178

and bone or wound repair, 190

chemistry of, 195-96

cytology of thyroid after injection of, 182

in relation to diabetes insipidus, $278 \mathrm{ff}$.

exophthalmos associated with effect of $, 183,185-86$

action of, and glutathione, 189

and gonads or gonadotropic hormones, $186-87$

and growth-promoting hormone, 3235,45

heart after administration of, I 87-89

and experimental hypertension, 188 , $288-89$

treatment of hypothyroidism by, $184^{-}$ 85

metabolism of, 190-92

comparison with thyroxine, $187-88$

and metabolism, carbohydrate, 18889

and metabolism, creatine-creatinine, 189

and metabolism, gaseous, $183^{-85}$

and metabolism, lipoid, I 89

and metabolism, protein, 189

and metabolism of isolated thyroid, I 88

metamorphosis and, $174^{-76}, 195-96$

nervous system and, 186

in relation to oestrogens, $186-87$

amount of, in pituitary, $179^{-81}$

specificity of, 174

effect of, on thyroid transplants, 182 action of, and

vitamin $A$, I 90

vitamin $C, 190$

vitamin $\mathrm{D}$, 190

Thyroxine, 295, 297

adrenal hypertrophy caused by, 202, n. 4

action of, after hypophysectomy, I 80, n. 7 


\section{INDEX}

Thyroxine-Continued

effect of, on lactation, I66

action of, on metabolism, carbohydrate, after hypophysectomy, 208 antagonism of thyrotropic hormone by, 185,192

effects of, compared with thyrotropic hormone, I 84, I $87-88$

Tissue culture

of ovary

effect of prolan on, I 32

of pituitary, 28

and chromatosome-dispersing hormone, 254-55

and vasopressor hormone, $254-55$

gonadotropic hormone in, 103, n. 6I

Tissue metabolism; see Metabolism, tissue

Transplantation

of pars glandularis, $237-38$

of pituitary

anatomical changes, $27-28$

secretion of gonadotropic hormone and, $65-66$

Tuberculosis

effect of prolan on course of, ${ }_{1} 38$

Tumors; see Gonadotropic hormones of neoplasms, Neoplasms

Ureter

effect of prolan on movements of, 138

Urine

action of pars neuralis hormones or extracts on secretion of, $265-69$, $276 \mathrm{ff}$.

Uterine bleeding

in monkey after hypophysectomy, ${ }_{4}$, 290-9I

\section{Uterus}

motility of

anterior pituitary extract and, $70-$ $7 \mathrm{I}$

progesterone and, 64

prolan and, 70-7I, I32

hormone of testicular neoplasm and, I 32

and action of oxy tocic principle, $260-$ 62,290
Vasopressor principle; see also Pars neuralis extracts, Pars neuralis, physiological significance of, assay of, 259-60 chemistry of, 258-69

in relation to culture of isolated pituitary, 254-55

metabolism of, 270-71, 274-76, 28687

pharmacology of, $262-65$

and eclampsia, $26_{3}-64,287$

and eye, $264-65$

and gastrointestinal tract, 265

and heart, 263

and lungs, 264

and medulla, 264

oestrogens and, $26_{4}$

and pregnancy, 286-87

thyroid extract and, 263

Vitamin A

deficiency of

and lactogenic hormone in pituitary, 168

and prolan, 129

and growth, 42

anatomy of pituitary and, 26

and action of thyrotropic hormone, I 90

Vitamin B (complex)

deficiency of

and lactogenic hormone in pituitary, 168

Vitamin $B_{I}$

anatomy of pituitary and, 26-27

Vitamin C; see Ascorbic acid

Vitamin D

deficiency of

and lactogenic hormone in pituitary, $\mathrm{I} 68$

anatomy of pituitary and, 27

and action of thyrotropic hormone, I90

Vitamin E

deficiency of

and lactogenic hormone in pituitary, 168

and prolan, 137

and gonadotropic hormones, pituitary, IOI-2

anatomy of pituitary and, 27 


\section{THE PITUITARY BODY}

Vitamins

and growth, 42

X-rays

effects of, on pituitary, 3I, 62

$\mathrm{X}$-zone of mouse adrenal

and anterior pituitary, 200-201

effect of oest rogens on, 20 I

Yeast

augmentation of action of gonadotropic hormone by ash or extract of, 1 I 8
Yohimbine

effect of, on chromatophores, 25I

Zinc

deficiency of, and growth-promoting hormone, $42^{-}+3$

Zinc salts

augmentation of action of gonadotropic hormone by, I 18

augmentation of action of vasopressor principle by, 270-71 



